\title{
Die rhetorische Konstruktion von Führung und Steuerung
}

Eine argumentationsanalytische Untersuchung deutschsprachiger Führungsgrundsätze

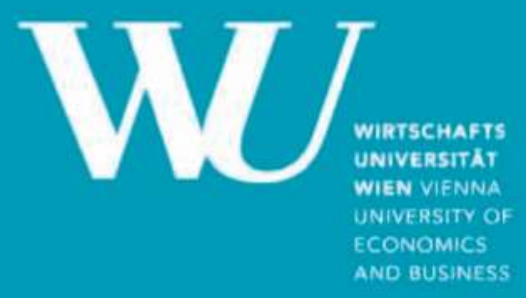


Die rhetorische Konstruktion von Führung und Steuerung 


\section{Forschungsergebnisse der WU Wirtschaftsuniversität Wien

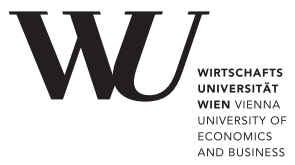

Band 60 


\section{Die rhetorische Konstruktion von Führung und Steuerung}

Eine argumentationsanalytische Untersuchung deutschsprachiger

Führungsgrundsätze 
Bibliografische Information der Deutschen Nationalbibliothek Die Deutsche Nationalbibliothek verzeichnet diese Publikation in der Deutschen Nationalbibliografie; detaillierte bibliografische Daten sind im Internet über http://dnb.d-nb.de abrufbar.

Gefördert durch die WU Wirtschaftsuniversität Wien.

\author{
Umschlaggestaltung: \\ Atelier Platen, nach einem Entwurf \\ von Werner Weißhappl. \\ Universitätslogo der WU Wirtschaftsuniversität Wien: \\ Abdruck mit freundlicher Genehmigung \\ der WU Wirtschaftsuniversität Wien.
}

ISSN 1613-3056

ISBN 978-3-631-63795-1 (Print)

E-ISBN 978-3-653-03328-1 (E-Book)

DOI 10.3726/978-3-653-03328-1

(C) Peter Lang GmbH

Internationaler Verlag der Wissenschaften

Frankfurt am Main 2013

Alle Rechte vorbehalten.

PL Academic Research ist ein Imprint der Peter Lang GmbH. Peter Lang - Frankfurt am Main - Bern - Bruxelles · New York .

Oxford · Warszawa - Wien

Das Werk einschließlich aller seiner Teile ist urheberrechtlich geschützt. Jede Verwertung außerhalb der engen Grenzen des Urheberrechtsgesetzes ist ohne Zustimmung des Verlages unzulässig und strafbar. Das gilt insbesondere für Vervielfältigungen, Übersetzungen, Mikroverfilmungen und die Einspeicherung und Verarbeitung in elektronischen Systemen.

www.peterlang.de 


\section{Vorwort und Danksagung}

Nachdem eine Dissertation - mit all ihren Freuden und Mühen - erfolgreich abgeschlossen worden ist und in Form einer Publikation vorliegt, gerät allzu leicht in Vergessenheit, dass hinter dem Autor eine Gruppe von Menschen und Institutionen steht, welche deren Fertigstellung überhaupt erst ermöglicht hat. Diesen Menschen will ich an dieser Stelle meinen Dank aussprechen.

Zunächst möchte ich meiner Erstbetreuerin Renate Meyer danken, welche mich über die Jahre hinweg professionell begleitet und freundschaftlich unterstützt hat. Sie hat alle Höhen und Tiefen des Projekts miterlebt und mich aus so mancher Talsohle mit inspirierenden Ideen und motivierenden Hilfestellungen herausgehoben. Dank gilt auch meinem Zweitbetreuer Manfred Lueger für seine zahlreichen methodologischen und methodischen Hinweise und die engelsgleiche Geduld, mit welcher er manchmal auf Fortschrittsmeldungen wartete. Darüber hinaus haben mich auch viele Kolleginnen und Kollegen sowohl an der WU Wien als auch im breiteren wissenschaftlichen Umfeld mit kritischem Feedback und konstruktiven Ideen unterstützt. Dies gilt vor allem für Markus Höllerer, Monika Knassmüller und Isabell Egger-Peitler, mit denen ich am Institut für Public Management an der WU Wien viele bereichernde Gespräche führen durfte. $\mathrm{Zu}$ danken habe ich aber auch all jenen, die mir wertvollen Input im Rahmen von diversen Kolloquien, Konferenzen und Präsentationen gespendet und damit wesentlich zum Erfolg der Arbeit beigetragen haben, deren namentliche Erwähnung aber den Rahmen dieses kleinen Dankeschöns sprengen würde.

Auch dem Kreis meiner Familie und Freunde bin ich zu Dank verpflichtet. Vor allem meiner Mutter Heidrun und meinem Bruder Jonathan möchte ich für ihre moralische Unterstützung, die Möglichkeit, Gedanken und Ideen im familiären Kreis „,auszutesten“, aber auch für zahlreiche Formulierungs- und Korrekturvorschläge im fertigen Text danken. Leider gilt auch hier, dass der große Kreis all jener, die mir in den letzten Jahren in vielfältiger und wertvoller Art und Weise Unterstützung haben zukommen lassen, zu umfangreich ist, um namentlich genannt zu werden. Ein herzliches Dankeschön Euch allen!

$\mathrm{Zu}$ guter Letzt sollen noch jene Institutionen erwähnt werden, welche zur Fertigstellung des Projekts wesentlich beigetragen haben. Mein Dank gilt daher der WU Wien, welche stets ein förderndes Umfeld für meine Forschung geboten hat, aber auch dem Jubiläumsfonds der OeNB, welcher durch die Förderung des Projektes Nr. 8457 „Führungsgrundsätze als Steuerungsinstrument“ die Grundlage für die Arbeit legte.

Dennis Jancsary

Wien, im Oktober 2011 



\section{Inhaltsverzeichnis}

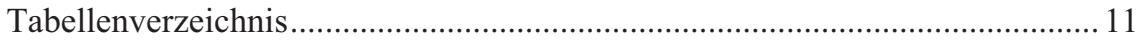

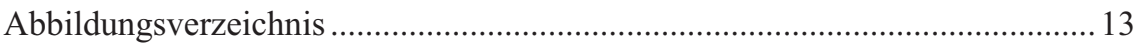

1 Problemstellung und Aufbau der Arbeit ...................................................... 15

1.1 Problemstellung und Anspruch ......................................................... 15

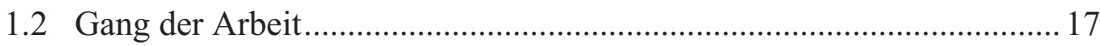

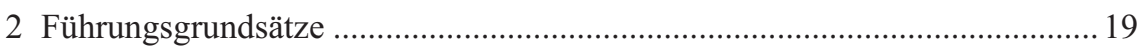

2.1 Begriffsbestimmung und -abgrenzung.................................................. 19

2.1.1 Definitionen in der einschlägigen Literatur ............................... 19

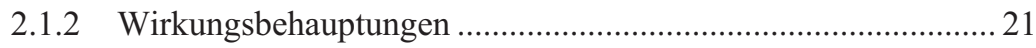

2.1.3 Abgrenzung von verwandten Konzepten.................................. 23

2.2 Historischer Abriss der Forschung ........................................................2 29

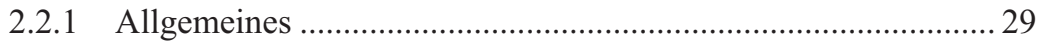

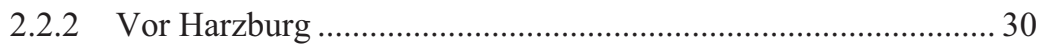

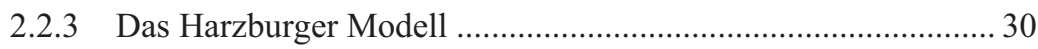

2.2.4 Führungsgrundsätze zwischen 1975 und 2000 .......................... 34

2.2.5 Führungsgrundsätze im 21. Jahrhundert................................... 35

2.3 Themen in Theorie und Empirie ........................................................ 35

2.3.1 Theoretische Einbettung des Konzeptes .................................... 35

2.3.2 Konzeptionelle Schwerpunkte in der Literatur........................... 37

2.3.3 Empirische Schwerpunkte in der Literatur ............................... 44

2.3.4 Kritik am Konzept der Führungsgrundsätze............................... 54

2.3.5 Schlussfolgerung: Lücken in der wissenschaftlichen

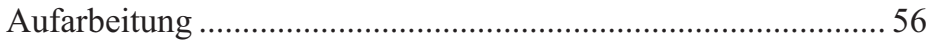

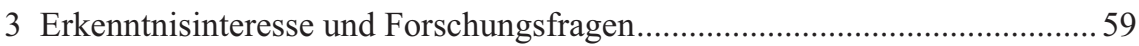

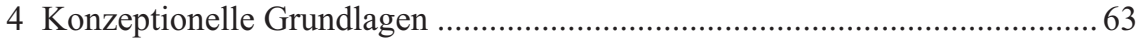

4.1 Grundsätzliche Ausführungen zum konzeptionellen Hintergrund der

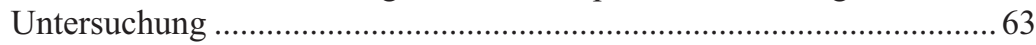

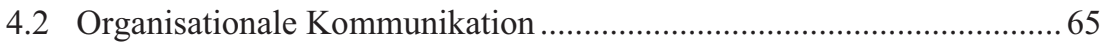

4.2.1 Gegenstand und Begriffsabgrenzung ...................................... 65

4.2.2 Führungsgrundsätze als kommunikatives Genre ....................... 67

4.2.3 Metaphern organisationaler Kommunikation ............................ 69 
4.3 Macht, Herrschaft und Steuerung ….................................................... 71

4.3.1 Begriffsabgrenzungen ........................................................... 72

4.3.2 Die relationale Machtperspektive ............................................ 76

4.3.3 Indirekte, unsichtbare und diskursive Macht.............................. 84

4.3.4 Die diskursive Konstruktion von Identität................................ 91

4.3.5 Machtaspekte in Führungsgrundsätzen....................................... 95

4.4 Rhetorik als Instrument der Überzeugung .......................................... 101

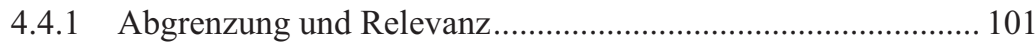

4.4.2 Wirkungsweise rhetorischer Strategien ................................... 104

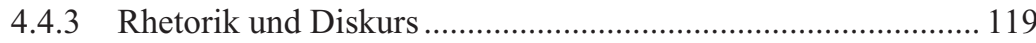

4.4.4 Zur Eignung rhetorischer Analysen für die Untersuchung....... 121

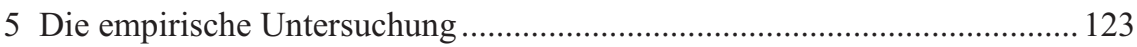

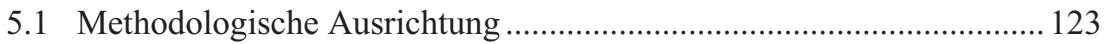

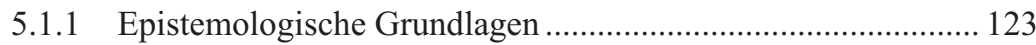

5.1.2 Methodologische und forschungspraktische Aspekte ............. 127

5.2 Diskursanalyse als Forschungsstrategie............................................. 133

5.2.1 Verortung der Arbeit in der Diskursforschung......................... 134

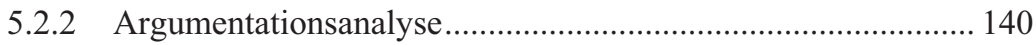

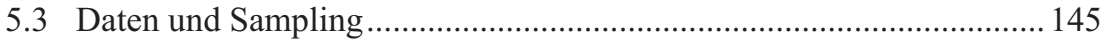

5.4 Vorgehensweise und Untersuchungsablauf ........................................ 147

5.4.1 Grundsätzliches zur Vorgehensweise .................................... 147

5.4.2 Schritt 1: Identifikation und Codierung relevanter TätigkeitsCluster und Rollenaspekte ...................................................... 148

5.4.3 Schritt 2: (Re-)konstruktion von Beziehungstypen als Kombinationen von Tätigkeiten und grammatikalischen

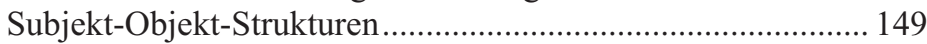

5.4.4 Schritt 3: (Re-)konstruktion der besonderen Topoi ................... 153

5.4.5 Schritt 4: Erstellung einer diskursiven ,Landkarte' .................. 155

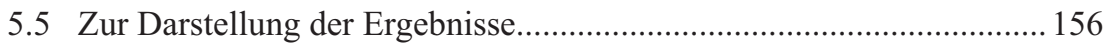

5.6 (Re-)konstruktion der Handlungsmuster.............................................. 157

5.6.1 Grammatische Handlungsmuster im Material .......................... 157

5.6.2 Rollenaspekte als Bündel typischer Handlungen ..................... 161

5.6.3 Identifikation distinktiver Beziehungstypen........................... 177 
5.7 (Re-)konstruktion der Argumentationsmuster ..................................... 179

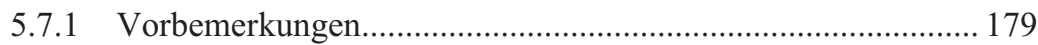

5.7.2 Die konstitutiven Topoi .............................................................. 180

5.7.3 Die ergänzenden Topoi ......................................................... 187

5.7.4 Topoi mit geringem Bezug zu den Akteursbeziehungen.......... 196

5.7.5 Topoi ohne Bezug zu den Akteursbeziehungen ......................... 198

5.7.6 Die rhetorische Steuerungswirkung von Führungsgrundsätzen 199

5.8 Beziehungstypen - eine integrierte Analyse...................................... 203

5.8.1 Die Anspruchs-Beziehung .................................................... 203

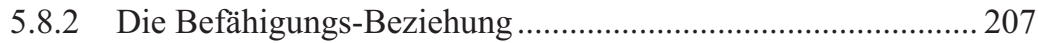

5.8.3 Die Bereitstellungs-Beziehung …………............................... 211

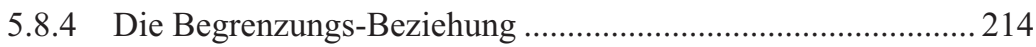

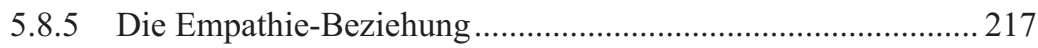

5.8.6 Die Entwicklungs-Beziehung .................................................. 220

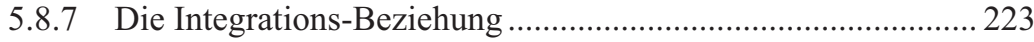

5.8.8 Die Kontroll- und Bewertungsbeziehung ................................ 226

5.8.9 Die Motivations-Beziehung ..................................................... 229

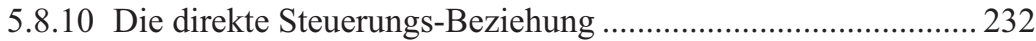

5.8.11 Die Unterstützungs-Beziehung ............................................... 234

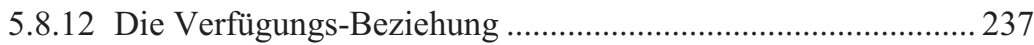

5.8.13 Häufigkeit und Verteilung der Beziehungstypen ..................... 239

5.8.14 Eine konzeptionelle Topos-Karte des Diskurses ...................... 241

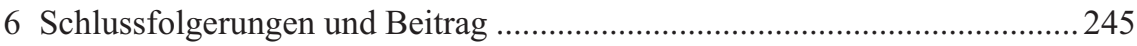

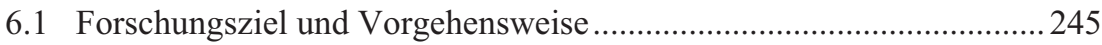

6.2 Beantwortung der forschungsleitenden Fragen .................................247

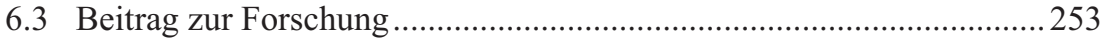

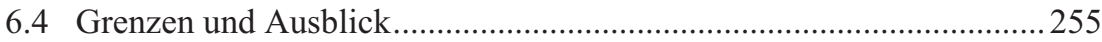

6.4.1 Mögliche Erweiterungen innerhalb der Dokumentanalyse ...... 255

6.4.2 Erweiterungen durch Einbezug von Kontextfaktoren .............. 256

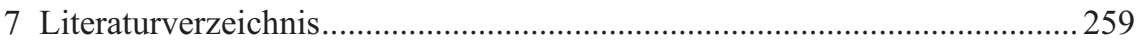

Anhang: Überblick über alle Topoi im Material............................................. 277 



\section{Tabellenverzeichnis}

Tabelle 1: Wirkungskanäle von Führungsgrundsätzen nach Gauger (2000: 104ff.)

Tabelle 2: Verbreitung im deutschsprachigen Raum

(Jancsary et al., 2006: 27) 46

Tabelle 3: Metaphern organisationaler Kommunikation

(in Anlehnung an Putnam \& Boys, 2006)......

Tabelle 4: Zwei Arten strittiger Angelegenheiten

(in Anlehnung an Kopperschmidt, 1989: 33ff.).

Tabelle 5: Kategorienschema der grammatischen Handlungsstrukturen im Korpus

Tabelle 6: Rekonstruktion besonderer Topoi

(in Anlehnung an Froschauer \& Lueger, 2003).............................. 154

Tabelle 7: Die Anspruchs-Beziehung (Überblick).......................................... 207

Tabelle 8: Die Befähigungs-Beziehung (Überblick) ...................................... 211

Tabelle 9: Die Bereitstellungs-Beziehung (Überblick).................................... 214

Tabelle 10: Die Begrenzungs-Beziehung (Überblick).................................... 216

Tabelle 11: Die Empathie-Beziehung (Überblick) ........................................... 219

Tabelle 12: Die Entwicklungs-Beziehung (Überblick).................................. 222

Tabelle 13: Die Integrations-Beziehung (Überblick)...................................... 226

Tabelle 14: Die Kontroll- und Bewertungsbeziehung (Überblick).................. 229

Tabelle 15: Die Motivations-Beziehung (Überblick) ....................................... 231

Tabelle 16: Die Steuerungs-Beziehung (Überblick) .......................................... 234

Tabelle 17: Die Unterstützungs-Beziehung (Überblick) .................................. 237

Tabelle 18: Die Verfügungs-Beziehung (Überblick)....................................... 239

Tabelle 19: Die konstitutiven Topoi ............................................................. 277

Tabelle 20: Die ergänzenden Topoi ............................................................... 280

Tabelle 21: Topoi mit geringem Bezug zu den Akteursbeziehungen................ 284

Tabelle 22: Topoi ohne Bezug zu den Akteursbeziehungen............................. 291 



\section{Abbildungsverzeichnis}

Abbildung 1: Inhalte der Allgemeinen Führungsanweisung in Anlehnung

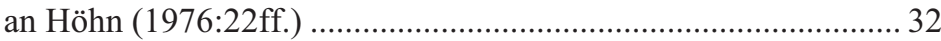

Abbildung 2: Inhalte von Führungsgrundsätzen in Anlehnung an Wunderer und Grunwald (1980:429) .......................................................... 33

Abbildung 3: Verschiedene Perspektiven auf Führungsgrundsätze (eigene Darstellung)

Abbildung 4: Inhalte von Führungsgrundsätzen (in Anlehnung an Wunderer, 2009: 390)

Abbildung 5: Themenfrequenz und Themenbetonung in Führungsgrundsätzen (vgl. Jancsary et al., 2006: 46).

Abbildung 6: Wirkungsbereiche von Führungsgrundsätzen (in Anlehnung an Wunderer und Klimecki, 1990: 110) ............... 53

Abbildung 7: Konzeptioneller Hintergrund der Untersuchung 65

Abbildung 8: Die Ebenen der Macht in Führungsgrundsätzen (eigene Darstellung)

Abbildung 9: Struktur des Enthymemarguments (vgl. Ottmers 2007: 74)...... 114

Abbildung 10: Die zirkuläre Organisierung der Forschung im

Wissenschaftsprozess (M. Lueger, 2001: 363)

Abbildung 11: Die Codierung grammatischer Strukturen 151

Abbildung 12: Rollenaspekte im Material 176

Abbildung 13: Verteilung der Beziehungstypen im untersuchten Material $(\mathrm{n}=53)$

Abbildung 14: Eine konzeptionelle Karte von verwendeten Topoi und Beziehungstypen 



\section{Problemstellung und Aufbau der Arbeit}

\subsection{Problemstellung und Anspruch}

Führung und Steuerung sind zwei Grundkonzepte der organisationstheoretischen Forschung. Fragen danach, wie Menschen in Organisationen dazu bewegt werden können, auf bestimmte Art und Weise zu handeln oder wie arbeitsteilige Prozesse der Leistungserstellung etabliert, aufeinander abgestimmt und stabilisiert werden können, obwohl individuelle Akteure spezifische, nicht unbedingt kongruente Ziele verfolgen, beschäftigen die Betriebswissenschaft seit Jahrzehnten (vgl. Fischer, 2009: 15). Ziel der Führung ist solch eine Einflussnahme auf das Verhalten anderer zum Zwecke der Erreichung organisationaler Ziele (vgl. Steyrer, 2002: 159; Wunderer, 2009: 4). Diese Einflussnahme kann entweder direkt durch unmittelbare Interaktion mit der/dem Geführten bzw. der/dem MitarbeiterIn oder indirekt über die Steuerung und Manipulation technischer, organisationaler und kultureller Rahmenbedingungen (vgl. Wunderer, 2009: 77ff.) geschehen. Im deutschsprachigen Raum zeigt sich eine klare Tendenz zur indirekten oder strukturellen Führung (vgl. Müller, 1995: 575).

Führungsgrundsätze als Instrument der Steuerung sind Teil einer solchen strukturellen Form der Führung (vgl. Wunderer, 2009: 383ff.). Sie stellen ein spezifisches Genre der Unternehmenskommunikation dar, welches im deutschsprachigen Raum seit den späten 1960er Jahren existiert und „die Führungsbeziehungen zwischen Vorgesetzten und Mitarbeitern im Rahmen einer ziel- und werteorientierten Führungskonzeption zur Förderung eines erwünschten organisations- und mitgliedergerechten Sozial- und Leistungsverhaltens" normiert und standardisiert (Wunderer, 2009: 385). Ihnen werden in der präskriptiven Literatur verschiedene Wirkungen unterstellt, welche sich vor allem auf die Orientierung, Motivation und Steuerung von Organisationsmitgliedern, aber auch auf eine positive Darstellung des Unternehmens nach außen beziehen und damit insgesamt eine positive Wirkung für den Unternehmenserfolg postulieren (vgl. z.B. Finzer \& Mungenast, 1989; Grawert, 1997; Lattmann, 1975; Wunderer, 2009). Trotz eines schwindenden wissenschaftlichen Interesses an diesem Dokumentgenre seit dem Ende der 1990er Jahre sind die Dokumente in der betrieblichen Praxis nach wie vor weit verbreitet (vgl. Jancsary, Knassmüller \& Sandner, 2006). Eine wissenschaftliche Einbettung des Instruments in organisationstheoretische Überlegungen sowie eine (konzeptionelle oder empirische) Fundierung ihrer Wirkungen blieben jedoch bisher aus. Wirkungsannahmen existieren folglich Großteils auf dem Niveau ,plausibler' Überlegungen.

Die vorliegende Untersuchung zielt deshalb darauf ab, eine dieser Lücken in der wissenschaftlichen Aufarbeitung des Themas zu schließen. Es soll eine soli- 
de konzeptionelle Basis geschaffen werden, welche es erlaubt, die (hypothetische) Wirkung von Führungsgrundsätzen fundiert und systematisch zu diskutieren. Zu diesem Zweck stützt sich die vorliegende Arbeit in erster Linie auf Konzepte und Theorien aus den Forschungsbereichen der organisationalen Kommunikation (vgl. z.B. Putnam \& Boys, 2006; Yates \& Orlikowski, 1992), der Machtforschung (vgl. z.B. Clegg, Courpasson \& Phillips, 2007; Lukes, 2005; Sandner, 1990; Sandner \& Meyer, 2004; van Dijk, 2008) und der Rhetorik (vgl. z.B. Cheney, 1983; Cheney, Christensen, Conrad \& Lair, 2004; Kopperschmidt, 1989; Ottmers, 2007). Auf der Grundlage dieser konzeptionellen Basis soll geklärt werden, welche Formen von Führung und Steuerung in deutschsprachigen Führungsgrundsätzen propagiert und damit im organisationalen Diskurs stabilisiert werden sollen und mithilfe welcher rhetorischer bzw. argumentativer Strategien diese Steuerungswirkung erreicht werden soll.

Das Steuerungsverständnis innerhalb der gegenständlichen Untersuchung ist folglich ein stark diskursiv-kommunikatives bzw. indirektes (vgl. z.B. Sandner, 1990). Die methodologische Grundlage der Untersuchung bilden die phänomenologische Wissenssoziologie von Berger und Luckmann (2000) sowie ihre von Keller (2008) entwickelte Weiterführung im Bereich der ,Wissenssoziologischen Diskursanalyse'. Wirklichkeit wird als kommunikative bzw. diskursive Leistung sozial handelnder Akteure begriffen, welche in dialektischer Form von Akteuren konstruiert wird, diese aber gleichzeitig auch wiederum konstituiert. Führungsgrundsätze sind somit ein institutionalisierter kommunikativer Akt, welcher durch die Externalisierung bestimmter Weltvorstellungen potentiell konstitutive Macht auf das Weltverständnis und die Rollenidentitäten ihrer Adressaten ausübt. Diese Weltverständnisse äußern sich konkret in Form von Führungsverständnissen (oder - noch präziser - in spezifischen Beziehungen zwischen Führungskräften und MitarbeiterInnen) in den Regelungsinhalten der Dokumente. Diese Regelungsinhalte bzw. die aus ihnen abgeleiteten Handlungsempfehlungen stellen Geltungsansprüche (,claims') dar, welche argumentativ gestützt werden, indem sie sich innerhalb eines institutionalisierten und legitimierten Bezugssystems (,Rahmen“, ,Deutungsmuster') verorten und damit verständlich werden bzw. ,normal' wirken (vgl. z.B. Kopperschmidt, 1989; R. E. Meyer, 2004). Vor diesem Hintergrund wird ein interpretatives Forschungsdesign (vgl. z.B. Froschauer \& Lueger, 2009; Lamnek, 2005; M. Lueger, 2001) vorgestellt, welches sich auf diskursanalytische (vgl. z.B. Keller, 2008; Keller, 2011; van Dijk, 1997a, 1997b; Wodak \& Meyer, 2009) und argumentationsanalytische (vgl. z.B. Kopperschmidt, 1989; Ottmers, 2007; Wengeler, 2003b) Überlegungen stützt. 
Mithilfe dieses Methodenkanons beantwortet die Untersuchung zwei grundlegende Fragen:

- Welche Verständnisse von Führung und Steuerung zeigen sich in deutschsprachigen Führungsgrundsätzen?

- Wie werden diese Verständnisse rhetorisch und argumentativ untermauert?

Die Beantwortung dieser Fragen wird durch eine (Re-)konstruktion und Diskussion von Akteursrollen, Beziehungsmustern und argumentativen Strategien geleistet. Abschließend werden Schlussfolgerungen über Führungsverständnis und Steuerungspotential der Dokumente gezogen.

\subsection{Gang der Arbeit}

Die vorliegende Arbeit gliedert sich in sechs Hauptkapitel. Im folgenden zweiten Kapitel werden die theoretischen Grundlagen zu Führungsgrundsätzen als Steuerungsinstrument erläutert und ein Überblick über die bisherige Forschung in diesem Phänomenbereich hergestellt. Dabei wird die spezifische Entstehungsgeschichte der Dokumente im deutschsprachigen Raum vorgestellt und ihre kulturelle Spezifizität argumentiert. Außerdem werden sowohl die praktischen Empfehlungen der normativ-präskriptiven Literatur als auch die Erkenntnisse empirischer Untersuchungen vorgestellt. Auf Basis dieses State-of-theField der Führungsgrundsatz-Forschung werden wichtige Lücken in der wissenschaftlichen Aufarbeitung identifiziert und diskutiert. Diese Lücken werden im dritten Kapitel der Arbeit aufgegriffen und in Form forschungsleitender Fragen und eines distinktiven Forschungsprogramms dargestellt.

Das vierte Kapitel der Arbeit widmet sich anschließend der Aufarbeitung und Diskussion der theoretisch-konzeptionellen Grundlagen der Arbeit. Als zentrale Konzepte werden Kommunikation, Macht, Herrschaft, Steuerung und Rhetorik vorgestellt und in Bezug auf den Anspruch und die Ziele der Arbeit ausführlich diskutiert. Im Anschluss wird ausgeführt, wie vor dem Hintergrund einer wissenssoziologischen Perspektive verschiedene Formen der Steuerung und Machtausübung über indirekte, unsichtbare und kommunikative Kanäle realisiert werden können.

Das fünfte Kapitel stellt das empirische Design und die konkreten Ergebnisse vor. Zunächst wird ein Überblick über die interpretativ-qualitativen Grundlagen des Forschungsvorhabens geleistet. Des Weiteren werden verschiedene Formen der Diskurs- und Argumentationsanalyse diskutiert, ein Einblick in das Datenmaterial gegeben und die konkrete Vorgehensweise erläutert. Anschließend werden die einzelnen Ergebnisse im Detail vorgestellt. Im sechsten und 
letzten Kapitel werden die Ergebnisse im Hinblick auf die forschungsleitenden Fragen ausführlich diskutiert und einer abschließenden Würdigung und Interpretation unterzogen. Eine Diskussion des Beitrags zur Forschung sowie der Grenzen der Untersuchung, welche in einen Ausblick mündet, beschließen die Arbeit. 


\section{Führungsgrundsätze}

\subsection{Begriffsbestimmung und -abgrenzung}

\subsubsection{Definitionen in der einschlägigen Literatur}

Führungsgrundsätze bzw. deren Vorgängerkonzepte existieren im deutschsprachigen Raum bereits seit den späten 1960er Jahren. Insofern ist es nicht weiter verwunderlich, dass man in der einschlägigen Literatur eine Vielzahl von Definitionsversuchen vorfindet, die in einzelnen Punkten jedoch deutlich voneinander abweichen. Einigkeit herrscht darüber, dass Führungsgrundsätze ein Ausschnitt der Unternehmensgrundsätze sind, bzw. sich aus diesen ableiten (vgl. u.a. Breisig, 1987: 162; Finzer \& Mungenast, 1989: 405; Grawert, 1997: 89) und dass sie als primären Regelungsinhalt „mitarbeiterbezogene Aussagen, in deren engeren Zusammenhang auf die Führungskräfte eingehende Bestandteile stehen" thematisieren (Oechsler, 2000: 379). Gabele (1992: 27) bezeichnet sie als „[m]itarbeiterorientierte Unternehmensgrundsätze“, die „,eine besondere Stellung im Kreis der adressatenspezifischen Unternehmensgrundsätze“ einnehmen. Sie „regeln das Verhältnis zwischen Vorgesetzten und unterstellten Mitarbeitern. Sie zielen auf eine gemeinsame Basis der Führungskräfte im Umgang mit ihren Mitarbeitern ab" (Hoffmann, 1989: 168). In diesem Zusammenhang ist auch erwähnenswert, dass der für diese Untersuchung gewählte Begriff der ,Führungsgrundsätze" nicht das einzige Label für diese Dokumentgattung ist. Er wird oft synonym mit Bezeichnungen wie z.B. ,Leitsätze für Führung und $\mathrm{Zu}$ sammenarbeit in der Unternehmung', ,Allgemeine Führungsleitsätze', ,Leitlinien zur Führung', ,Leitsätze für Führungskräfte', ,Grundsätze des Führungsverhaltens', ,Verhaltensleitsätze', ,Führungshilfen für Vorgesetzte', ,Pflichtenheft für das Vorgesetztenverhalten', ,allgemeine Führungsanweisung' sowie ,Führungsrichtlinien“ (Knebel \& Schneider, 1994: 19) verwendet. Dabei können die einzelnen Termini nicht klar voneinander abgegrenzt werden. Allenfalls lassen harte Formulierungen (,Grundsätze', ,Pflichtenheft', ,Führungsanweisung ') auf eine stärker proklamierte Verbindlichkeit schließen als weichere Labels (,Leitlinien', ,Richtlinien', ,Leitsätze').

Ein früher Versuch der umfassenden Definition von Führungsgrundsätzen stammt von Lattmann (1975):

„Unter Führungsrichtlinien ist der Zusammenhang der vom obersten Willensträger der Unternehmung formalisierten, d.h. schriftlich niedergelegten, Grundsätze zu verstehen, welche von den Führungskräften ihrer Aufgabenerfüllung zugrunde zu legen sind“ (Lattmann, 1975: 47). 
Etwas später erweiterten Grunwald und Lilge (1981) den Wirkungsbereich der Grundsätze insofern, als sie das Verhältnis zwischen MitarbeiterInnen der gleichen Hierarchieebene mit einbeziehen. Interessant ist auch, dass diese Definition außerdem informale Beziehungen thematisiert, sich also nicht nur strikt auf die bestehende Ablauforganisation der Unternehmung bezieht:

„Unter Führungsgrundsätzen verstehen wir normative Aussagen über sozial erwünschte beziehungsweise unerwünschte formale und informale Beziehungen zwischen Vorgesetzten und Mitarbeitern sowie zwischen Gleichgestellten auf vertikaler und horizontaler Ebene im Rahmen betrieblicher Leistungserstellung“" (Grunwald \& Lilge, 1981: 413).

Besonders intensiv hat sich Wunderer (1985, 1995, 2009, 1983; Wunderer \& Klimecki, 1990) mit der Thematik beschäftigt. Seine aktuellste Definition von Führungsgrundsätzen lautet:

„Führungsgrundsätze beschreiben und/oder normieren die Führungsbeziehungen zwischen Vorgesetzten und Mitarbeitern im Rahmen einer ziel- und werteorientierten Führungskonzeption zur Förderung eines erwünschten organisations- und mitgliedergerechten Sozial- und Leistungsverhaltens.

Sie können in schriftlicher Form (explizit) verbindlich fixiert werden oder als ungeschriebene Normen zur Verhaltensorientierung in den Führungsbeziehungen dienen. Häufig werden zusätzlich Prinzipien für die laterale Kooperation einbezogen. In diesem Fall sprechen wir von Führungs- und Kooperationsgrundsätzen“. (Wunderer, 2009: 385)

Dazu sind einige Anmerkungen notwendig. Für das geplante Forschungsvorhaben ist das von Wunderer formulierte ,oder ' ${ }^{\star}$ zwischen formaler und informaler Regelung durch ein ,und/oder ${ }^{`}$ zu ersetzen. Wie an verschiedener Stelle gezeigt wurde, verhindert eine formale Einführung von Normen oder Praktiken keineswegs deren informelle Anpassung oder, Entkopplung' (vgl. z.B. J. W. Meyer \& Rowan, 1977; Westphal \& Zajac, 1998, 2001). Es ist deshalb grundsätzlich davon auszugehen, dass die offizielle, schriftlich festgelegte Version der Führungsgrundsätze der betrieblichen Realität entsprechen kann, dies aber nicht zwangsläufig tun muss (vgl. dazu auch die Ausführungen zu potentieller Macht in Kapitel 4.3.2.2). Es ist durchaus vorstellbar, dass ein Unternehmen sowohl über ein schriftlich formuliertes Grundsatzdokument als auch gleichzeitig über ein Repertoire rein impliziter Regelungen zur Führungsthematik verfügt. Diese beiden ,Regelungsebenen' müssen sich inhaltlich nicht entsprechen. 


\subsubsection{Wirkungsbehauptungen}

Führungsgrundsätzen wird in der Literatur ein Katalog von Wirkungsweisen zugeschrieben, die sich in erster Linie auf eine Optimierung der Abläufe und eine effizientere Kommunikation zwischen (Top)-Management und nachgeordneten Hierarchieebenen beziehen. Die ursprünglichen - und auch aktuell noch oft genannten - Wirkungszuschreibungen lassen sich folgendermaßen zusammenfas$\operatorname{sen}^{1}$ :

- Deklarationsfunktion: Durch die schriftliche Formulierung von Führungsgrundsätzen deklariert die Unternehmensleitung ihren Willen, sich um ihre Einhaltung zu bemühen (vgl. Lattmann, 1975: 54).

- Orientierungs- und Koordinationsfunktion: Führungsgrundsätze sollen alle MitarbeiterInnen des Unternehmens über das gewünschte Führungsverhalten informieren (vgl. Grawert, 1997: 91; Lattmann, 1975: 55). Außerdem sollen sie eine einheitliche Ausrichtung des Führungsverhaltens bewirken und Widersprüchlichkeiten und Reibungsverluste vermeiden (vgl. Finzer \& Mungenast, 1989: 407; Lattmann, 1975: 56). Die Normen sollen ein „gemeinsames und systematisches Vorgehen der Führungskräfte sicherstellen“ (Finzer \& Mungenast, 1989: 407) und eine Stabilisierung der wechselseitigen Verhaltenserwartungen zwischen Vorgesetzten und MitarbeiterInnen bewirken (vgl. Tschirky, 1981: 25).

- Appell- und Aktionsfunktion: Führungsgrundsätze sollen dafür sorgen, dass der Wille der Unternehmensleitung in klare Forderungen an Führungskräfte übersetzt wird. Sie sollen deshalb in Form von Appellen gekleidet sein (vgl. Finzer \& Mungenast, 1989: 407; Lattmann, 1975: 56). Lattmann (1975: 56) fordert zusätzlich, dass Führungsgrundsätze als Muss- und nicht als Sollnormen zu formulieren sind. Eine solche Formulierung findet sich empirisch jedoch nicht durchgängig wieder (vgl. Jancsary et al., 2006).

- Motivationsfunktion: Die enthaltenen Normen sollen von Führungskräften ,innerlich bejaht` werden können, um ihre Einhaltung zu vereinfachen. Deshalb soll durch die Grundsätze eine leitbildliche Vorstellung angesprochen werden (vgl. Lattmann, 1975: 56), welche die Bereitschaft Ziele als die eigenen anzunehmen stimuliert (Finzer \& Mungenast, 1989: 407). Motivation wird außerdem durch die mit dem Erreichen der Ziele in Aussicht gestellte Anerkennung erreicht (vgl. Grawert, 1997: 91).

1 Diese Wirkungsbehauptungen sind zumeist eher wenig differenziert und nur in wenigen Fällen empirisch nachgewiesen. So lassen sich ähnliche Wirkungsbehauptungen z.B. auch für eine starke Organisationskultur finden. Diese tragen teilweise sogar dieselben Labels: Identifikationsfunktion, Integrationsfunktion, Koordinationsfunktion, Motivationsfunktion, Lern- und Entwicklungsfunktion (vgl. z.B. Kasper \& Mühlbacher, 2002). 
- Darstellungsfunktion: Durch die Veröffentlichung von Führungsgrundsätzen kann ein positiver Eindruck des Führungsverhaltens nach außen erreicht werden (vgl. Grawert, 1997: 91). Somit sind Führungsgrundsätze auch ein Instrument der Selbstinszenierung und der Vermittlung eines Idealbildes an relevante Anspruchsgruppen des Unternehmens.

Eine abweichende Systematik findet sich bei Tschirky (1981: 25):

- Pragmatische Funktion: Führungsgrundsätze sollen sich auf ein tatsächliches Führungsgeschehen beziehen und nicht zu abstrakt sein.

- Effizienzfunktion: Führungsgrundsätze sollen die Führungseffizienz steigern.

- Modellfunktion: Die Aussagengesamtheit der Führungsgrundsätze soll den Prinzipien der Vollständigkeit und der Erheblichkeit unterworfen sein.

- Schulungsfunktion: Führungsgrundsätze sollen die Grundlage einer sorgfältigen Schulung der Vorgesetzten sein.

Die aktuellste Darstellung verschiedener Funktionen von Führungsgrundsätzen findet sich bei Wunderer (2009: 391). Er unterscheidet vor allem drei Funktionsbereiche:

- Strukturelle Organisationsentwicklung: Führungsgrundsätze treffen Aussagen zur Gestaltung von Teilsystemen der Führung. Zu diesem Zweck sollen sie erwünschte Einstellungen und Verhaltensweisen generalisieren und Gestaltungshinweise für instrumentelle Maßnahmen zur Internalisierung und Anwendung von Führungs- und Kooperationsnormen liefern. Auch die Verbindung von Organisations- und Personalentwicklung gehört zu den Funktionen von Führungsgrundsätzen.

- Strukturelle Organisationssteuerung: Hier unterscheidet Wunderer (2009) die Funktionen ,Information und Orientierung', ,Motivation und Appell', ,Aktivierung und Legitimation" und ,Beurteilung und Sanktion".

- Organisationsdarstellung und Imageaufbau: Dieser Bereich entspricht weitestgehend der Deklarationsfunktion. Die positive Wirkung sollte jedoch nicht überschätzt werden, da bei zu starker ,Entkopplung‘ von Außendarstellung und Realität intern Glaubwürdigkeitsprobleme entstehen können.

Während der Bezug auf solche Wirkungsbehauptungen in der existierenden Literatur weit verbreitet ist, werden diese theoretisch und konzeptionell nur sehr schwach fundiert. Die Ergebnisse dieser Arbeit tragen zu dieser Diskussion insofern bei, als sie einige Schlussfolgerungen bezüglich der rhetorisch-performativen ,Wirkungen' von Führungsgrundsätzen erlauben. Im Folgenden werden Führungsgrundsätze nun von artähnlichen Dokumentgenres und Konzepten abgegrenzt. 


\subsubsection{Abgrenzung von verwandten Konzepten}

\subsubsection{Im deutschsprachigen Raum}

Im deutschsprachigen Raum existieren vor allem zwei Dokumentgenres, welche eine gewisse Ähnlichkeit zu Führungsgrundsätzen aufweisen. Es sind dies einerseits Unternehmensgrundsätze und andererseits Unternehmensleitbilder. Beide sollen kurz von Führungsgrundsätzen unterschieden werden.

Unternehmensgrundsätze werden laut Breisig (1987: 161) fälschlicherweise oft synonym mit Führungsgrundsätzen verwendet. Trotz der ähnlichen Dokumentform, dem gleichartigen Entwicklungsprozess und den teilweise überlappenden Bezugsgruppen, gibt es jedoch einige Unterschiede. So versteht man unter Unternehmensgrundsätzen „die Zusammenfassung der grundlegenden, normativen und alle internen und externen Funktionsbereiche betreffenden Wertvorstellungen des Unternehmens; sie sollen den Unternehmensangehörigen die für ihre Arbeit notwendige Orientierung an der Gesamtzielsetzung geben“ (Knebel \& Schneider, 1994: 18). Sie stellen im Vergleich zu Führungsgrundsätzen eher allgemeine Verhaltensaussagen für unternehmungsinterne wie -externe Bereiche dar (vgl. Hoffmann, 1989: 168). Außerdem sind sie zumeist wesentlich umfassender (vgl. Breisig, 1987:162) und beschäftigen sich mit mehreren Adressaten zugleich (vgl. Gabele \& Kretschmer, 1986:17). Die Existenz formaler Unternehmensgrundsätze ist für die Einführung von Führungsgrundsätzen keine Voraussetzung. Diese können auch völlig unabhängig von eventuell existierenden Unternehmensgrundsätzen entwickelt werden (vgl. Oechsler, 2000: 379).

Unternehmensleitbilder gehören seit den 1980er Jahren zum Standardrepertoire moderner Unternehmensführung (vgl. Knassmüller, 2005). Wie Führungsgrundsätze können sie zu den Grundsatzdokumenten gezählt werden (vgl. Gabele \& Kretschmer, 1986; Knassmüller, 2005). Knassmüller (2005: 14) unterscheidet Leitbilder von Führungsgrundsätzen in erster Linie aufgrund der Tatsache, dass Unternehmensleitbilder umfassender und an eine größere Anzahl externer und interner Anspruchsgruppen gerichtet sind. Führungsgrundsätze hingegen fokussieren das Verhältnis zwischen Vorgesetzten und MitarbeiterInnen. Unternehmensleitbilder, Unternehmensgrundsätze und Führungsgrundsätze können damit grob in eine Hierarchie von Abstraktion und Breite der Zielgruppen gebracht werden und bauen grundsätzlich aufeinander auf.

\subsubsection{Außerhalb des deutschsprachigen Raums}

Die Suche nach vergleichbaren Dokumenten und Konzepten außerhalb des deutschsprachigen Raums (vor allem im angelsächsischen Raum) gestaltet sich als wenig fruchtbar. Dokumente, welche sich in erster Linie auf die Beziehung zwischen Vorgesetzten und ihren MitarbeiterInnen im Sinne der Steuerung, 
Standardisierung und Effizienzsteigerung beziehen, finden sich in der wissenschaftlichen Literatur nicht. Im Folgenden sollen eine Dokumentgattung und zwei breitere Konzepte diskutiert werden, die aus dem angelsächsischen Raum stammen, sich aber auch im deutschsprachigen Raum immer stärker durchsetzen. Mögliche Anschlusspunkte und Parallelen zu Führungsgrundsätzen werden jeweils aufgezeigt und diskutiert.

Codes of Conduct bzw. Codes of Ethics bezeichnen Grundsatzdokumente, welche vor allem im angelsächsischen Raum hohe Verbreitung finden. Im Gegensatz zu Führungsgrundsätzen betonen diese jedoch nicht in erster Linie Effizienzüberlegungen, sondern werden vielmehr von juristischen und ethischen Motiven geleitet (vgl. Cassell, Johnson \& Smith, 1997; Schwartz, 2001; Weller, 1988). Langlois und Schlegelmilch (1990) subsumieren Unternehmens- und Führungsgrundsätze zwar unter das Label der Codes of Ethics, es wird jedoch aufgrund Ihrer Ausführungen klar, dass sie diesen Begriff eher breit fassen. So führen sie z.B. aus, ihre Untersuchung hätte die Kategorien „Employee Conduct, Community and Environment, Relationship to Customers, Relationship to Shareholders, Suppliers and Contractors, Political Interests, and Innovation in Technology“ ergeben (Langlois \& Schlegelmilch, 1990: 526). Die Autoren stellen u.a. fest, dass die deutschsprachigen Dokumente die Beziehungen zu MitarbeiterInnen sehr viel stärker betonen als die englischsprachigen (vgl. Langlois \& Schlegelmilch, 1990: 530f.). Trotz dieser inhaltlichen Unterschiede halten sie die Annahme aufrecht, dass es sich um dasselbe Dokumentgenre handelt, was vor dem Hintergrund des ethischen Bezugsrahmens der Untersuchung auch sinnvoll begründet wird. Im Rahmen der vorliegenden Untersuchung erscheint es jedoch zielführender, Codes of Ethics aufgrund des unterschiedlichen Wirkungsanspruchs klar von Führungsgrundsätzen abzugrenzen.

Compliance wird in der einschlägigen Literatur in mehreren unterschiedlichen Bedeutungen verwendet. So zeigt z.B. Haase (1997: 99), dass Etzionis (1961) Compliance-Begriff sich auf das Verhältnis von ausgeübter Macht und entgegengebrachtem Involvement bezieht, während der in der Psychologie verwendete Compliance-Begriff die Motivation eines Individuums beschreibt, einer Forderung oder einer Norm nachzukommen. Die Betonung der Macht bei Etzioni zeigt auch Conrad (1988: 185) auf: „Konformität als variierende Übereinstimmung von Verhaltensweisen und Einstellungen mit vorgegebenen Normen, Programmen, Strukturen oder Anforderungen beinhaltet ein Herrschaftsgefälle zwischen der Konformität fordernden Organisation und dem sich diesen Erwartungen fügendem (sic!) Organisationsmitglied“. Roth (2000: 1) erweitert diesen Begriff noch, in dem sie ihm auch eine bedeutsame ethische Dimension zugesteht: „Somit bedeutet Compliance nicht nur eine fallweise Betrachtung und Entscheidung ,post festum', sondern ebenso eine proaktive, grundsätzliche und 
ganzheitliche Auseinandersetzung“. Auch in der angelsächsischen Literatur über sogenannte ,Organizational Citizenship Behaviors" spielt der ComplianceBegriff eine nicht unbedeutende Rolle. Compliance wird dort unter anderem als Komponente eben dieser Verhaltensweisen betrachtet. So verstehen z.B. Borman und Motowidlo (1997: 100) in Anlehnung an Organ (1988), generalized compliance“ als „following the rules and procedures of the organization“. In ihrer Untersuchung über ,prosocial behaviors' identifizieren Brief und Motowidlo (1986: 715) unter anderem den Faktor ,complying with organizational values, policies, and regulations“ als „notions of organizational commitment and citizenship behavior“. Compliance bedeutet in diesem Zusammenhang ,adhering to major policies and procedures despite temptations to shortcut or avoid them when they appear personally inconvenient" (Brief \& Motowidlo, 1986: 715). Williams und Anderson (1991: 602) grenzen ,compliance“ von ,altruism‘ in Bezug auf die erwartete Belohnung ab: ,[...] compliance is viewed as behavior that occurs because of expected rewards or the avoidance of punishment". Führungsgrundsätze könnten im Thema ,Compliance“ insofern einen Platz haben, als sie eine Möglichkeit darstellen, eben diese Konformität zu erreichen, und zwar durch schriftliche, verpflichtende und gelebte Niederlegung der einzuhaltenden Grundsätze (Appell- und Aktionsfunktion). Dennoch werden Führungsgrundsätze im Zusammenhang mit Compliance auch in der deutschsprachigen Literatur nicht angeführt.

Commitment kann als Selbstbindung im Sinne einer psychologischen Ausrichtung verstanden werden, welche zu einer erhöhten Einsatzbereitschaft organisationaler Akteure führt (vgl. Gauger, 2000: 55). In der existierenden Literatur werden häufig drei Komponenten des Commitment unterschieden. Diese Unterscheidung geht auf Allen und Meyer (1991: 64ff.) zurück, die drei gängige Motive für Commitment identifizieren: ,affective attachment", ,perceived costs und ,obligation'. Daraus leiten sie drei Komponenten des Commitment ab:

- Affective Commitment: MitarbeiterInnen bleiben im Unternehmen, weil sie es wollen.

- Continuance Commitment: MitarbeiterInnen bleiben im Unternehmen, weil sie es müssen (weil Kosten mit dem Verlassen des Unternehmens verbunden sind).

- Normative Commitment: MitarbeiterInnen bleiben im Unternehmen, weil sie das Gefühl haben, dass sie das sollten (Verpflichtungsgedanke).

Gemäß der oben angeführten Definition von Gauger (2000) liegt es nahe, Commitment mit Führungsgrundsätzen in Verbindung zu bringen. Auch Führungsgrundsätze bezwecken eine erhöhte Einsatzbereitschaft (Motivationsfunktion) sowie eine stärkere Bindung der MitarbeiterInnen an das Unternehmen (Identi- 
fikationsfunktion). Es finden sich dennoch nur sehr wenige Ansätze, welche Führungsgrundsätze in Zusammenhang mit Commitment diskutieren. Gauger (2000: 206) selbst spricht zwar nur ein einziges Mal wörtlich von Führungsgrundsätzen, es wird jedoch klar, dass ihr Begriff der ,humanressourcenbezogenen Grundsätze und Richtlinien ' den Führungsgrundsätzen, wie sie hier verstanden werden sollen, sehr nahe ist. Laut Gauger (2000: 104) haben diese Grundsätze und Richtlinien durchaus eine Wirkung auf das Commitment der MitarbeiterInnen, wenn sie eine positive Haltung zum Ausdruck bringen, die von Fairness und Wertschätzung geprägt ist. Drei Kategorien solcher Grundsätze hält sie für besonders wichtig, nämlich jene, die

- Zuverlässigkeit und Gerechtigkeit,

- Bereitschaft zu Fürsorge und Unterstützung und

- Einbeziehung in Entscheidungsprozesse

behandeln (vgl. Gauger, 2000: 104). Bezüglich der Förderung von Commitment durch partizipatives Management zeigen sich aber Steyrer, Schiffinger und Lang (2007: 426f.) deutlich skeptischer. In ihrer Untersuchung finden sie keinen signifikanten Zusammenhang zwischen Partizipation und Commitment. Die vorliegende Untersuchung wird zeigen, dass diese drei Themen in modernen Führungsgrundsätzen weit verbreitet sind (vgl. v.a. die Diskussionen zur EmpathieBeziehung, Unterstützungs-Beziehung und Integrations-Beziehung in Kapitel 5.8). Im Wesentlichen beziehen sich alle drei genannten Aspekte auf die Form der psychologischen Steuerung (vgl. Sandner, 1990: 197ff.), welche in Kapitel 4.3.3.2 näher erläutert und sich als Steuerungsmodus innerhalb der Führungsgrundsätze als bedeutsam erweisen wird. In Tabelle 1 werden die Wirkungskanäle, über welche Grundsätze auf die verschiedenen Commitment-Komponenten wirken, kurz dargestellt.

Gaugers Ausführungen können sich jedoch nicht auf empirische Untersuchungen im deutschsprachigen Raum stützen. Auch die angelsächsischen Quellen, welche sie zur Stützung ihrer Ausführungen heranzieht (vgl. Ashford, Lee \& Bobko, 1989; Konovsky \& Cropanzano, 1991; J. P. Meyer, Allen \& Gellatly, 1990; Moorman, Blakely \& Niehoff, 1998; Morris, Lydka \& O'Creevy, 1993), sprechen nicht direkt von ,Grundsätzen“ sondern vielmehr von ,procedures' und ,rules'. Dabei beziehen sie sich viel stärker auf die Wirkung der Inhalte (v.a. Fairness) als auf die Wirkung der Form (Grundsätze). Eine Stützung der These, dass schriftlich festgehaltene Regeln das Commitment der MitarbeiterInnen stärken, kann daraus nicht abgeleitet werden. 
Tabelle 1: Wirkungskanäle von Führungsgrundsätzen nach Gauger (2000: 104ff.)

\begin{tabular}{|c|c|c|c|}
\hline & $\begin{array}{l}\text { Zuverlässigkeit und } \\
\text { Gerechtigkeit }\end{array}$ & $\begin{array}{l}\text { Fürsorge und Unter- } \\
\text { stützung }\end{array}$ & $\begin{array}{l}\text { Einbeziehung in Ent- } \\
\text { scheidungsprozesse }\end{array}$ \\
\hline $\begin{array}{l}\text { Affektives } \\
\text { Commitment }\end{array}$ & $\begin{array}{l}\text { Wahrnehmung einer } \\
\text { Kongruenz mit indi- } \\
\text { viduellen Wertvor- } \\
\text { stellungen fördert die } \\
\text { Identifikation mit der } \\
\text { Organisation. }\end{array}$ & $\begin{array}{l}\text { Wahrnehmung eines } \\
\text { Zusammenhangs zwi- } \\
\text { schen einer positiven } \\
\text { Entwicklung und dem } \\
\text { Verhalten der Orga- } \\
\text { nisation führt zu Iden- } \\
\text { tifikation. }\end{array}$ & $\begin{array}{l}\text { Die eigene Wert- } \\
\text { schätzung und Selbst- } \\
\text { definition wird über } \\
\text { die Organisation ge- } \\
\text { fördert, was zu Identi- } \\
\text { fikation führt. }\end{array}$ \\
\hline $\begin{array}{l}\text { Normatives } \\
\text { Commitment }\end{array}$ & $\begin{array}{l}\text { Gerechtigkeit sowie } \\
\text { die Einhaltung von } \\
\text { Versprechen wird von } \\
\text { Mitarbeitern in gewis- } \\
\text { sem Maße erwidert. }\end{array}$ & $\begin{array}{l}\text { Aus der freiwilligen } \\
\text { Leistung der Organi- } \\
\text { sation entsteht die } \\
\text { Wahrnehmung einer } \\
\text { normativen Verpflich- } \\
\text { tung. }\end{array}$ & $\begin{array}{l}\text { Die Möglichkeit der } \\
\text { Partizipation kann als } \\
\text { Vertrauensvorschuss } \\
\text { interpretiert werden, } \\
\text { aus dem eine normati- } \\
\text { ve Verpflichtung ent- } \\
\text { steht. }\end{array}$ \\
\hline $\begin{array}{l}\text { Kalkuliertes } \\
\text { Commitment }\end{array}$ & $\begin{array}{l}\text { Die Reduktion von } \\
\text { Unsicherheiten erhört } \\
\text { die Handlungsfähig- } \\
\text { keit der Mitarbeiter, } \\
\text { was eine positive } \\
\text { Wahrnehmung der } \\
\text { Organisation fördert. }\end{array}$ & $\begin{array}{l}\text { Die Unterstützung re- } \\
\text { sultiert in einer posi- } \\
\text { tiven Wahrnehmung } \\
\text { der Organisation im } \\
\text { Vergleich zu den ande- } \\
\text { ren verfügbaren Alter- } \\
\text { nativen. }\end{array}$ & $\begin{array}{l}\text { Es ergibt sich ein er- } \\
\text { weiterter Raum zur } \\
\text { individuellen Selbst- } \\
\text { verpflichtung. }\end{array}$ \\
\hline
\end{tabular}

Steinle, Ahlers und Riechmann (1999) entwerfen das Konzept eines ,Management by Commitment', das sich zwar nicht direkt auf Grundsätze bezieht, aber doch Elemente beinhaltet, welche durchaus mit der Idee von Führungsgrundsätzen vereinbar sind. So betonen die Autoren unter anderem die Bedeutung der indirekten Führung - des Management by Objectives (MbO) - als Möglichkeit der Motivation zur Selbst-Führung sowie zur Steuerung durch ,Leitplanken“ und die entsprechende Gestaltung von Rahmenbedingungen (Steinle, et al., 1999: 227ff.). In ihrem Fazit führen sie außerdem aus, dass das Management by Commitment eines Instrumentariums bedarf, welches sowohl Planungs-, Kontroll- und Koordinationsprobleme als auch Motivations- und Identifikationsprobleme aufgreifen kann. Als Lösung schlagen sie wiederum MbO vor (vgl. Steinle et al., 1999: 240). Vor allem im amerikanischen Raum wird das Konzept der Codes of Ethics sehr stark mit Commitment in Verbindung gebracht. Fritz, Arnett und Conkel (1999: 297) zeigen auf, dass ,[o]rganizations that make their 
employees aware of what they are doing to enforce their ethical standards and that reward managers' adherence to standards are acting wisely, reinforcing the organization's identity and strengthening employee commitment to that identity“. Valentine und Barnett (2003: 365) betonen, dass Codes of Ethics auch von der Belegschaft wahrgenommen werden müssen, denn ,[t]he existence alone of an ethics code does not ensure that it will affect employees' perceptions of their organizations" values $[\ldots]$ “. Ist dies jedoch gegeben, dann ist ein Einfluss der Codes auf das Commitment zu erwarten. Somers (2001: 194) bestätigt in seiner Studie nicht nur eine Wirkung von Codes of Ethics auf wahrgenommenes Fehlverhalten im Unternehmen, sondern stellt auch fest, dass die höchsten Ausprägungen von Commitment in jenen Organisationen beobachtet werden können, welche Codes of Ethics implementiert haben.

Da sich Codes of Ethics inhaltlich relativ stark von Führungsgrundsätzen unterscheiden, ist fraglich, ob die Ergebnisse dieser Studien von Relevanz für das Thema sind. Da Gauger (2000) jedoch gezeigt hat, dass zumindest theoretisch durchaus Brücken zwischen Führungsgrundsätzen und Commitment geschlagen werden können, und Studien im englischsprachigen Raum eine Wirkung von Grundsätzen bzw. Codes auf Commitment nahe legen, ist eine solche Relevanz nicht generell auszuschließen. In der deutschsprachigen Literatur scheint dieses Thema bedauerlicherweise noch nicht häufig aufgegriffen worden zu sein, was auf ein viel grundlegenderes Problem verweist, nämlich die dürftige empirische Aufarbeitung der Wirkung von Führungsgrundsätzen.

\subsubsection{Führungsgrundsätze als teutonische Angelegenheit?}

Im Vorwort zu seinem Buch „Führungsgrundsätze in Wirtschaft und öffentlicher Verwaltung" stellt sich Wunderer (1983: vi) die Frage, weshalb Führungsgrundsätze in formulierter und organisationsspezifischer Form nur im deutschsprachigen Raum zu finden sind. Der internationale Managementforscher Bernard Bass soll ihm auf diese Frage geantwortet haben, Führungsgrundsätze wären wohl „eine teutonische Angelegenheit“. Eine mögliche Erklärung für dieses Phänomen findet Müller (1995: 575), der feststellt: „Ein im deutschsprachigen Raum besonders intensiv eingesetztes Medium sozialer Kontrolle ist die Steuerung und institutionelle Legitimation von Verhalten durch strukturelle Regelung“. Er führt diese Bestrebungen auf das Welt- und Menschenbild der Aufklärung zurück, da sie inhaltlich Demokratie- und Mündigkeitsidealen verpflichtet sind, auch wenn sie diese im Rahmen eines reibungslos funktionierenden, leistungsfähigen Gesamtsystems zu verwirklichen trachten (vgl. Müller, 1995: 579). Außerdem stellen für ihn die strukturelle Führung und die Führung durch technologische Prozesssteuerung eher versteckte Akte der Machtausübung dar. Er spricht in diesem 
Zusammenhang von einer Institutionalisierung der Führung, die zugleich Machtausgleich und Ordnung erlaubt (vgl. Müller, 1995: 582) ${ }^{2}$. Provokant formuliert Steyrer (2002: 211) die These, dass die deutschsprachige Führungsforschung eine „Führungsforschung ohne Führende“ sei und führt dies auf eine Art ,Führer-Phobie' zurück, die er mit den leidvollen Erfahrungen mit dem ,größten Führer aller Zeiten' begründet. Steyrer zitiert hier Wunderer (1985:252), der von einer historisch verständlichen ,Führerallergie“ im deutschen Sprachraum spricht und diese als „diktatorisches Syndrom der Führungslehre“ interpretiert.

Alle diese Erklärungen deuten darauf hin, dass Führungsgrundsätze tatsächlich ein Phänomen sind, das in dieser spezifischen Ausprägung nur im deutschsprachigen Kulturraum auftritt. Dementsprechend wird in weiterer Folge bei der Besprechung von Führungsgrundsätzen in erster Linie auf deutschsprachige Literatur Bezug genommen.

\subsection{Historischer Abriss der Forschung}

\subsubsection{Allgemeines}

Die Diskussion zu schriftlichen Grundsätzen der Führung ist bis Mitte der 1970er Jahre auf das so genannte Harzburger Modell (vgl. Höhn, 1976, 1977) beschränkt. Ende der 70er Jahre lösen sich Führungsgrundsätze aus diesem Gesamtkonzept und werden in der Literatur gesondert thematisiert. Dennoch bemerkt Kossbiel (1983: 20) noch zu Beginn der 1980er Jahre: „Fragt man nach der wissenschaftlichen Fundiertheit solcher Regeln für das Führungsverhalten, so kommt man zu einem schnellen, überwiegend negativen Urteil. Die meisten in der Praxis entwickelten Führungsgrundsätze entbehren jeder verhaltenstheoretischen und sozialwissenschaftlichen Begründung“. Breisig (1987: 155) konstatiert nur vier Jahre später: „Daß der Bereich Führungsgrundsätze hochaktuell und vieldiskutiert ist, zeigt sich allein schon an der Vielzahl der Veröffentlichungen, die sich gerade in der letzten Zeit mit diesem Phänomen befassen. Dabei handelt es sich in erster Linie um Praktikerbeiträge mit Empfehlungs- oder Fallbeschreibungscharakter. Inzwischen liegen jedoch auch schon erste Ansätze einer empirischen Durchdringung dieses Problembereichs vor" (155).

Diese exemplarischen Zitate illustrieren, dass die Diskussion zu Führungsgrundsätzen Mitte der 1980er Jahre ihren Höhepunkt erreicht. Dieser ,Boom‘ hält bis ca. Mitte der 1990er Jahre an, danach flacht die wissenschaftliche Diskussion wieder ab. Zwischen 1980 und 1995 werden diverse Versuche unter-

2 Vergleiche dazu auch die Ausführungen von Sandner (1990: 182ff. bzw. 189ff.) zur technologischen und bürokratischen Steuerung. 
nommen, das Phänomen sowohl theoretisch zu verorten als auch praktisch potentiellen Anwendern zugänglicher zu machen. Publikationen nach 1995 sind hauptsächlich als Praktikerbeiträge zu bezeichnen, während das theoretischkonzeptionelle Interesse in dieser Zeit wieder stark abnimmt. Im Folgenden werden diese verschiedenen ,Epochen' des Themas näher betrachtet.

\title{
2.2.2 Vor Harzburg
}

Wunderer (2009: 384) bemerkt, dass sich seit über 4000 Jahren Verhaltensmaximen zur Gestaltung von Führungsbeziehungen finden. So führt er Beispiele aus dem alten Ägypten und der Bibel sowie Weisheiten des Lao-tse und eine Regel des heiligen Benedikt an. Ein weiteres Beispiel, das bereits sehr nahe an die heutigen Führungsgrundsätze heranreicht, stammt aus dem Jahre 1872 und findet sich im so genannten ,Generalregulativ` der Firma Krupp:

\begin{abstract}
„Anregungen und Vorschläge zu Verbesserungen sind aus allen Kreisen der Mitarbeiter dankbar entgegenzunehmen ... Eine Abweisung der gemachten Vorschläge ohne eine vorangegangene Prüfung soll nicht stattfinden. Wohingegen dann auch nicht erwartet werden muss, dass eine erfolgte Ablehnung dem Betreffenden ... genüge und ihm keineswegs Grund zur Empfindlichkeit und Beschwerde gebe“ (Krupp, Generalregulativ, § 13, in Wunderer, 2009: 384).
\end{abstract}

Vom Standpunkt einer modernen Organisationstheorie schlägt die Geburtsstunde der Führungsgrundsätze jedoch erst in den 1950er Jahren mit der Entwicklung des so genannten Harzburger Modells.

\subsubsection{Das Harzburger Modell}

Das Harzburger Modell, entwickelt von Reinhard Höhn und MitarbeiterInnen, findet in Deutschland zwischen 1956 und den späten 1970er Jahren in Wirtschaft und Verwaltung große Verbreitung (vgl. Wunderer \& Grunwald, 1980: 288). Höhn (1976: 1) geht davon aus, dass ,die autoritär-patriarchalische Führung nicht mehr der Führungsstil unserer Unternehmen sein kann“. Dieser Führungsstil nehme seine geistige Kraft aus der absolutistischen Vorstellungswelt und sei in einer industriellen Gesellschaft nicht mehr angebracht. Ihm setzt Höhn sein Konzept der ,Führung im Mitarbeiterverhältnis‘ entgegen. Da dieses Modell an der Akademie für Führungskräfte der Wirtschaft in Bad Harzburg entwickelt wird, spricht man bald nur noch vom ,Harzburger Modell'.

Das Kernstück dieser Konzeption ist die Delegation von Verantwortung (vgl. Höhn, 1976: 9). Dies bedeutet vor allem, dass betriebliche Entscheidungen nicht mehr von der Unternehmensspitze allein getroffen werden, sondern von 
den MitarbeiterInnen derjenigen Ebenen, zu denen sie ,ihrem Wesen nach gehören“ (Höhn, 1976: 9). Innerhalb eines klar abgegrenzten Aufgabenbereiches handelt die/der MitarbeiterIn eigenverantwortlich, es ist der/dem Vorgesetzten nur in Ausnahmefällen gestattet, in den Delegationsbereich einzugreifen. Während die/der MitarbeiterIn die ,Handlungsverantwortung' trägt, bleibt der/dem Vorgesetzten nur noch die ,Führungsverantwortung' im delegierten Bereich. Der Delegationsbereich wird in der so genannten ,Stellenbeschreibung' organisatorisch geregelt. Nach dieser Entscheidung herrscht „ein allgemeines Organisationsverbot für alle im Unternehmen beteiligten Kräfte" (Höhn, 1977: 32). Ein weiteres Instrument des Harzburger Modells neben der Stellenbeschreibung ist die ,Allgemeine Führungsanweisung' (vgl. Wunderer \& Grunwald, 1980: 289). Dieses Instrument ist hier von besonderem Interesse, da es „die Spielregeln für das Zusammenwirken zwischen Mitarbeitern und Vorgesetzten sowie anderen Stellen im Unternehmen im Rahmen einer Führung im Mitarbeiterverhältnis [enthält]" (Höhn, 1976: 21; zu den Inhalten siehe Abbildung 1). In der Allgemeinen Führungsanweisung werden die wichtigsten Führungsprinzipien in einer für alle MitarbeiterInnen verbindlichen Form festgelegt (Steinle, 1975: 188). Damit ähnelt die Führungsanweisung nach Höhn in ihrem Kern stark den modernen Führungsgrundsätzen.

Höhn (1976: 1f.) erklärt weiters, die Notwendigkeit einer Führungsanweisung wäre noch vor wenigen Jahrzehnten als ,abwegig' empfunden worden. In der modernen Zeit jedoch sei eine solche unabdingbar, da ein Neben- und Durcheinander verschiedener Führungsformen Spannungen im Unternehmen erzeuge, welche nur durch klare Grundsatzentscheidungen über Führungsprinzipien ausgeschaltet werden können. „Erst durch sie [die Allgemeine Führungsanweisung; Anm. DJ] werden die Grundsätze der Führung im Mitarbeiterverhältnis zur Norm erhoben und damit für alle Mitarbeiter wie Vorgesetzten im Unternehmen verbindlich“ (Höhn, 1976: 21). Die wiederholte Missachtung solle mit Entlassung bestraft werden (vgl. Höhn, 1977: 322).

Am Harzburger Modell wird jedoch auch zunehmend Kritik geäußert. Breisig (1987: 132) führt an, Höhn schweige sich über theoretische Grundlagen und Hypothesenzusammenhänge aus. Es werde aber deutlich, dass das Harzburger Modell von den Erkenntnissen der Human Relations-Bewegung und der Humanistischen Psychologie geleitet werde. Steinle (1975: 23) schließt sich dieser Kritik an, in dem er anmerkt, dass im Harzburger Modell „eine verhaltenstheoretische Fundierung nicht vorgenommen wird“. Außerdem bemängelt er, das Harzburger Modell befriedige hauptsächlich Sicherheitsmotive. Über physiologische Bedürfnisse werde keine Aussage getroffen; das Bedürfnis nach Achtung und Status sowie das Bedürfnis nach Selbstentfaltung würden im Harzburger Modell nur angedeutet (vgl. Steinle, 1975: 210f.). Vor allem Guserl (1973: 159, 
zit. in Wunderer 1980: 290f.) kritisiert, dass der patriarchalisch autoritäre Führungsstil im Harzburger Modell lediglich durch einen bürokratisch-autoritären Führungsstil ersetzt werde. Das Harzburger Modell mit seinen 315 Organisationsregeln erfülle die meisten Kriterien eines starren Bürokratiemodells. Außerdem sei das Harzburger Modell vorgesetztenzentriert und demnach nicht demokratisch oder kooperativ, sondern versteckt autoritär und dirigistisch. Entsprechend dieser verschiedenen Kritikpunkte wird das Harzburger Modell in der Folge auch stetig weiterentwickelt.

- das Wesen der Führung im Mitarbeiterverhältnis

- die Delegation von Verantwortung

- die Pflichten von Mitarbeitern und Vorgesetzten

- das Wesen der Verantwortung

- die Dienstaufsicht und Erfolgskontrolle

- die Information, Querinformation und Selbstinformation

- das Mitarbeitergespräch und die Mitarbeiterbesprechung

- das Dienstgespräch und die Dienstbesprechung

- die Kritik und die Anerkennung

- das Verhältnis von Linie und Stab

- das Zusammenspiel zwischen Stelleninhaber und Stellvertreter

- das Zusammenspiel zwischen Platzhalter und Stelleninhaber

- der Einzelauftrag

- der Dienstweg

- die Fachvorgesetzten

- die Disziplinarvorgesetzten

- die Beschwerde

Abbildung 1: Inhalte der Allgemeinen Führungsanweisung in Anlehnung an Höhn (1976:22ff.)

Es gibt eine beachtliche Anzahl von Hinweisen darauf, dass die Allgemeine Führungsanweisung des Harzburger Modells einen Vorläufer der heutigen Führungsgrundsätze darstellt. So bemerkt z.B. Breisig (1987):

„Viele Autoren sehen Führungsgrundsätze als Bestandteil von Führungsmodellen an. Diese Sichtweise ist zurückzuführen auf die früheren Phasen der Anwendung von Führungsgrundsätzen, als die meisten den Charakter und auch die Bezeichnung der ,Allgemeinen Führungsanweisung ' trugen, die nur einen Teil des viel umfassenderen HM ausmacht“" (Breisig, 1987: 158).

Er stützt diese Aussage u.a. auf die Bemerkungen von Rühli (1975: 45, zit. nach Breisig 1987: 137), der ausführt: „Insbesondere im Zuge der Verbreitung des 
Harzburger Führungsmodells [...] hat ein weiteres Führungsinstrument in den letzten Jahren stark an Bedeutung gewonnen, nämlich die sogenannten Führungsgrundsätze (Führungsrichtlinien)“. Rühli (1992: 80) bemerkt außerdem, Führungsgrundsätze würden vor allem unter dem Einfluss des Harzburger Modells von zahlreichen Unternehmen eingeführt. Gabele und Kretschmer (1981, zit. in Breisig, 1987: 158) bezeichnen die Allgemeinen Führungsanweisungen als die ,geistigen Väter' der Führungsgrundsätze. Viele Aspekte zur Einführung von Richtlinien, die auch in der späteren Führungsgrundsatzdiskussion immer wieder aufgegriffen werden, werden schon von Höhn (1977) in seinem ,Führungsbrevier der Wirtschaft" behandelt. Insbesondere sind dies:

- die notwendige Übereinstimmung verschiedener Grundsätze und Richtlinien im Unternehmen,

- die Anhörung der MitarbeiterInnen vor dem Erlass von Richtlinien,

- die notwendige periodische Überarbeitung der Richtlinien,

- die intensive Schulung der betroffenen Personen, sowie

- die Notwendigkeit der Identifikation des Top-Managements mit den Regelungen.

Dass auch der Inhalt der Führungsgrundsätze zunächst jenem der Allgemeinen Führungsanweisung sehr ähnlich ist, illustriert ein Inhaltskatalog von Baumgarten (1977: 158, zit. in Wunderer \& Grunwald, 1980: 429), der in Anlehnung an Höhn erstellt wurde (siehe Abbildung 2).

- Kriterien des Führungserfolgs

- Grundsätze der Delegation

- Regelung der Pflichten des Vorgesetzten

- Regelung der Pflichten der Mitarbeiter

- Grundsätze für die Information

- Grundsätze zur Kontrolle

- Grundsätze zur Gruppenarbeit

- Regelungen der Stellvertretung

Abbildung 2: Inhalte von Führungsgrundsätzen in Anlehnung an Wunderer und Grunwald (1980:429)

Auch wenn dieser Inhaltskatalog bereits wesentlich weniger detailliert ausfällt als jener der Allgemeinen Führungsanweisung, so lassen sich doch starke Parallelen erkennen. 


\subsubsection{Führungsgrundsätze zwischen 1975 und 2000}

Während in den 1960ern und frühen 1970ern das Harzburger Modell die Führungsdiskussion dominiert, wird dieser bürokratische Typ der Führungsgrundsätze ab Ende der 1970er Jahre durch einen stärker verhaltenswissenschaftlich orientierten Grundtyp abgelöst. Dieser entwickelt sich sowohl aus der wissenschaftlichen Führungsdiskussion als auch aus Erfahrungen der Praxis (vgl. Wunderer, 1995: 726f.). Breisig (1987) konstatiert:

„[Bei der Literatur über Führungsgrundsätze; Anm. DJ] handelt es sich in erster Linie um Praktikerbeiträge mit Empfehlungs- oder Fallbeschreibungscharakter. Inzwischen liegen jedoch auch schon erste Ansätze einer empirischen Durchdringung dieses Problembereichs vor“" (Breisig, 1987: 155).

Diese Aussage charakterisiert treffend die Literatur zwischen 1975 und 2000. Gerade in der Anfangsphase dominieren erste Blicke in die Praxis (vgl. z.B. Fiedler, 1976) und Fallbeschreibungen (vgl. z.B. Fiedler, 1980). In den 1980er Jahren wird die Diskussion dann etwas lebendiger. Verschiedene Autoren nehmen Anläufe, das Konzept zu systematisieren und einzuordnen (vgl. Finzer \& Mungenast, 1989, 1990; Gabele \& Kretschmer, 1986; Kubicek, 1984a; MüllerSeitz, 1988; Töpfer \& Zander, 1982; Tschirky, 1981; Wunderer, 1983) sowie die Grundsätze auf ihre Bedeutung und Wirksamkeit zu überprüfen (vgl. Kossbiel, 1983; Paschen, 1983). Zum Teil wird auch rigoros Kritik geübt (vgl. Breisig, 1987; Kubicek, 1984a). Aber auch Praktikerbeiträge mit Empfehlungs- und Anleitungscharakter finden weiter Anklang (vgl. Franke \& Kühlmann, 1986; Knebel, 1982). Empirische Daten zu Führungsgrundsätzen bieten die Beiträge von Hoffmann (1989), Gabele, Liebel und Oechsler (1982), Albach (1983) und Grunwald und Lilge (1981).

In den 1990er Jahren flacht das Interesse an Führungsgrundsätzen wieder ab. Es dominieren Beiträge von und/oder für Praktiker (vgl. Attenhofer, 1990; Buchmann, 1994; Fiedler-Winter, 1994, 1999; Mohr, 1999; Pietschmann, Huppertz \& Ruhtz, 1999). Wunderer und Klimecki (1990) leisten eine systematische Einordnung der Führungsgrundsätze in die Unternehmensverfassung, die auch eine größere empirische Untersuchung umfasst. Grawert (1997) betont die Bedeutung von Führungsleitlinien zur Durchsetzung der Unternehmenskultur. Gabele (1992) überarbeitet und ergänzt seinen Beitrag von 1982. Knebel und Schneider (1994) geben ein Buch zur praktischen Umsetzung der Führungsgrundsätze heraus. Auffällig ist, dass sich ab den frühen 1990er Jahren die Abschnitte zu den Führungsgrundsätzen in den gängigen Standardwerken zu Management und Führung nur noch marginal verändern. Das Thema scheint in eine Reifephase eingetreten zu sein. 


\subsubsection{Führungsgrundsätze im 21. Jahrhundert}

Führungsgrundsätze werden nach der Jahrtausendwende fast ausschließlich in Standardwerken zu Personalführung, Unternehmensführung und Management behandelt. Einen eigenen Literaturstrang zu dieser Thematik scheint es nicht mehr zu geben. Darauf deutet auch die Tatsache hin, dass in besagten Standardwerken bei der Behandlung von Führungsgrundsätzen zumeist auf Literatur der 1980er und frühen 1990er Jahre verwiesen wird (vgl. exemplarisch Wunderer, 2009). Wunderer (2009) bemerkt dazu:

„Die weitere Entwicklung der Führungsgrundsätze wird sich auf eine Integration mit anderen Konzepten der Unternehmensführung (z.B. Unternehmensleitbilder) und mit Instrumenten des Personalmanagements, der Organisations- bzw. Personalentwicklung sowie einer Evaluation der Funktionen und Wirkungen des normierten Führungsverhaltens ausrichten. Schließlich findet man eine zunehmende Fokussierung auf zentrale Strategien (z.B. Customer Focus oder internes Unternehmertum)“. (Wunderer, 2009: 385)

Beispiele für Literatur zu Führungsgrundsätzen bieten Malik (2003), der einige sehr allgemeine Gestaltungshinweise für die Erstellung von Führungsgrundsätzen formuliert, Scherer (2000), der eine Kritik der Von Werderschen ,Grundsätze ordnungsgemäßer Unternehmensleitung' vornimmt, sich dabei aber nur in Teilaspekten auf Führungsgrundsätze im Sinne dieser Arbeit bezieht sowie die Abhandlungen über Führungsgrundsätze in den Überblickswerken von Wunderer (2009), Macharzina (2003), Oechsler (2000) und Neuberger (2002).

\subsection{Themen in Theorie und Empirie}

\subsubsection{Theoretische Einbettung des Konzeptes}

Kubicek (1984a: 4) geht davon aus, dass Führungsgrundsätze nicht in der wissenschaftlichen Forschung vor einem klaren theoretischen Hintergrund entwickelt wurden, sondern vielmehr durch Übernahme von Praktikertheorien auch in die betriebswirtschaftliche Forschung Einzug fanden. Auch was die theoretische Fundierung der betrieblichen Praxis in diesem Bereich betrifft, kommt Kossbiel (1983: 20) zu einem ,schnellen, überwiegend negativen Urteil. Die meisten in der Praxis entwickelten Führungsgrundsätze entbehren jeder verhaltenstheoretischen und sozialwissenschaftlichen Begründung“.

Dass diese beiden Zitate aus den 1980er Jahren stammen, vermindert in diesem Fall ihre Gültigkeit nicht. Zwar wurden in den vergangenen 20 Jahren immer wieder Anläufe unternommen, Führungsgrundsätze systematisch in der Führungs- bzw. Organisationstheorie zu verankern, es entwickelte sich jedoch in 
der Disziplin diesbezüglich kein breiterer Konsens. Führungsgrundsätze sind auch aktuell noch ein weitgehend ,theorieloses' Konzept. Die betriebswirtschaftliche Forschung entwickelte aber verschiedene Perspektiven, um bestimmte Bereiche näher zu beleuchten. Diese Perspektiven werden in Abbildung 3 kurz zusammengefasst.

Führungsgrundsätze sind ...

- Bestandteil der strukturellen Unternehmensführung (Oechsler, 1997; vgl. Wunderer, 2009; Wunderer \& Klimecki, 1990)

- Bestandteil der Unternehmensverfassung (vgl. Wunderer \& Klimecki, 1990)

- Bestandteil einer Unternehmens,soll'kultur (vgl. Grawert, 1997)

- ,Sanftere“ Ethik Codes (vgl. Neuberger, 2002)

- ein Bestandteil des Personalmarketing (vgl. Aprath, 1993; Macharzina, 2003; Staehle, 1999; Wunderer, 2009)

- Bestandteil des (unternehmenspolitischen) Normensystems (vgl. Gabele, 1982; Lattmann, 1975; Neuberger, 2002; Staehle, 1999; Wunderer \& Grunwald, 1980)

- Steuerungsinstrument (der Unternehmenspolitik) (vgl. Gabele \& Kretschmer, 1986; Rühli, 1992; Wunderer \& Klimecki, 1990)

- Systematisierung der Führungsphilosophie (vgl. Wunderer, 2009)

- Instrument der Personalführung/Personalpolitik (vgl. Breisig, 1987; H. Meyer, 2002; Oechsler, 1997; Staehle, 1999; Wunderer, 1993)

- Bestandteil von Führungsmodellen (vgl. Höhn, 1976; Töpfer \& Zander, 1982; Wunderer, 2009)

- Ausdruck eines ,Führungsparadigmas‘ (A. Meyer, 2000)

Abbildung 3: Verschiedene Perspektiven auf Führungsgrundsätze (eigene Darstellung)

Wie aus Abbildung 3 ersichtlich wird, schließen sich die verschiedenen Kategorisierungen nicht systematisch aus. Führungsgrundsätze können verschiedene Rollen einnehmen. Eine trennscharfe Abgrenzung lässt sich nicht erkennen. Gemeinsam ist jedoch allen Einordnungen und Definitionen, dass Führungsgrundsätze sich auf das Verhältnis zwischen Vorgesetzten und MitarbeiterInnen beziehen (z.T. auch auf das Verhältnis zwischen MitarbeiterInnen untereinander), und dass sie in diesem Bereich eine Standardisierung des Verhaltens erreichen sollen.

Ebenfalls unklar bleibt das Verhältnis zwischen den Begriffen der Führungsgrundsätze, Führungsmodelle und Führungsinstrumente. Diese drei Konzepte werden teilweise synonym gebraucht, oft aber werden Führungsgrundsätze auch als Bestandteil von Führungsmodellen eingeordnet. Breisig (1987: 158) bezeichnet diese Einordnung als veraltet (stammend aus der Zeit des Harzburger 
Modells) und erklärt, moderne Führungsgrundsätze würden vielmehr Aspekte von Führungsmodellen beinhalten, bzw. wären selbst unternehmensspezifische Führungsmodelle. Als solche jedoch seien sie ebenfalls Führungsinstrumente. Diese Bezeichnung von Führungsgrundsätzen als Führungsinstrument ist ebenfalls weit verbreitet, auch unter jenen, welche sie als Bestandteil von Führungsmodellen sehen.

\subsubsection{Konzeptionelle Schwerpunkte in der Literatur}

Trotz der unterschiedlichen Zugänge zum Phänomenbereich existieren Themen, welche in der einschlägigen Literatur wiederholt auftauchen. In einem ersten Schritt sollen die primären konzeptionellen bzw. präskriptiven Aspekte diskutiert werden. Diese stellen in erster Linie Versuche dar, das Thema in einen gröBeren Zusammenhang einzuordnen, zu fundieren und zu systematisieren.

\subsubsection{Gesellschaftlicher Wandel und Führungsverständnis}

Am Beginn dieses Themenstrangs steht die Überlegung, dass Unternehmen nicht (mehr) unabhängig von der öffentlichen Meinung agieren können. Vielmehr sind sie als Leistungsgemeinschaften innerhalb einer Gesellschaft zu sehen, was die Wünsche der Gesellschaft zur Maßgabe für ihr Handeln und Verhalten der Unternehmen macht. Führungsgrundsätze werden als geeignetes Instrument gesehen, um diese gesellschaftliche Rolle glaubhaft darzustellen (vgl. Fiedler, 1980: 122). Die Diskussion über die soziale Verantwortung von Unternehmen ist zwar keine Errungenschaft der 1970er Jahre, sie gewinnt aber in dieser Zeit an Aktualität und nimmt an Intensität zu (vgl. Albach, 1976: 739). Während Unternehmen im 19. Jahrhundert ihre soziale Verantwortung alleine in der Bereitstellung von Gütern und Dienstleistungen sehen, umfasst die Verantwortung später eine zunehmende Palette von ökonomischen und gesellschaftlichen Aufgaben. Albach (1976: 763f.) kommt zu dem Schluss, dass Führungsgrundsätze diesen gesellschaftlichen Wandel abbilden, gesteht jedoch ein, dass manche Schulen von Führungslehren lediglich eine „modische Überspitzung“ des gesellschaftlichen Wandels beinhalten.

Grunwald und Lilge (1981: 416) bestätigen, dass Führungsgrundsätze sich zwischen 1960 und 1980 entschieden weiterentwickeln. Vor allem im Bereich der Akzeptanz partizipativer Führung konstatieren sie große Schritte. Auch Gabele, Liebel und Oechsler (1982: 17f.) gehen von einem starken gesellschaftlichen Einfluss auf die Formulierung von Führungsgrundsätzen aus. Sie unterscheiden inner- wie außerbetriebliche Einflussfaktoren, wobei sich unter den außerbetrieblichen Faktoren auch „sozialer und gesellschaftlicher Druck“ sowie 
„die zunehmende Verschlechterung des Bildes von Unternehmen in der Öffentlichkeit" finden. Die Autoren sind jedoch skeptisch, was die positive Wirkung von Führungsgrundsätzen betrifft und vermuten eine mögliche Diskrepanz zwischen schriftlicher Formulierung und gelebter Praxis. Hoffmann (1989: 170) sieht außerdem in der in den 1980er Jahren aufgekommenen Diskussion über ,soft facts ' einen neuen Stellenwert der Führungsgrundsätze in der Unternehmung, da diese mitbestimmend für Unternehmenskultur und Management sind sowie Einfluss auf das Klima und das Image der Unternehmung gegenüber der Umwelt haben. Als weitere gesellschaftliche Einflussfaktoren auf die Formulierung von Führungsgrundsätzen werden ein zunehmend organisiertes Gruppeninteresse der Arbeitnehmerschaft, ein Trend zur Mündigkeit in der Gesellschaft, ein gestiegenes Bildungs- und Ausbildungsniveau der MitarbeiterInnen und Forderungen nach der Befriedigung der höheren Bedürfnisse nach Maslow genannt (vgl. Knebel \& Schneider, 1994: 14).

\subsubsection{Verbreitung eines partizipativen Führungsstils}

Eine der größten Entwicklungen in der Zielsetzung der Führungsgrundsätze ist der proklamierte Wandel vom ,delegativen“ Führungsstil des Harzburger Modells (vgl. Höhn, 1976, 1977) zu einem zunehmend ,partizipativen' bzw. ,kooperativen' Führungsstil (vgl. Wunderer, 2009: 384). Auch Grawert (1997: 90) betont die Entwicklung „weg von einem autoritären hin zu einem kooperativpartizipativen Führungsstil“. Dass dieser Trend schon sehr früh beginnt, zeigt die Aussage von Albach (1976: 755): „Das Bekenntnis der Führungsgrundsätze zu einem kooperativen oder partizipativen Führungsstil geht sehr weit“. In einer Untersuchung von 36 Unternehmen findet er den Grundsatz des kooperativen Führungsstils in den Führungsgrundsätzen klar verankert. Er gesteht jedoch ein, dass sieben dieser Führungsgrundsätze sich direkt oder indirekt auf das Harzburger Modell berufen, was er angesichts der Beurteilung des Harzburger Modells als ,bürokratisch-autoritär' als interessant bezeichnet. Grunwald und Lilge (1981: 413f.) bestätigen ebenfalls einen Trend von einem autoritär-bürokratischen zu einem partizipativ-kooperativen Führungsstil. Bei Töpfer und Zander (1982: 14) scheint der kooperative Führungsstil sogar in einer Aufstellung über die wesentlichen Inhaltsbereiche von Führungsgrundsätzen auf, und zwar an erster Stelle. Einen Schritt hin zur kooperativen Führung stellt auch Hoffmann (1989: 182) fest. Im Rahmen einer Frage nach Argumenten für die Anwendung von Grundsätzen wird an zweiter Stelle die Annahme genannt, dass ein kooperativer Führungsstil eine gute und vertrauensvolle Zusammenarbeit bewirkt. Auch Ende der 1990er Jahre wird der partizipative Führungsstil noch 
immer als wesentlicher Bestandteil von Führungsgrundsätzen erachtet (vgl. Staehle, 1999: 816).

Eher skeptisch äußern sich hingegen Kubicek (1984a) und Breisig (1987) zu dieser Thematik. Kubicek (1984b: 81) führt aus, dass trotz des proklamierten Wandels vom autoritären zum kooperativen Führungsstil „weder ein Indiz für einen grundlegenden Wandel in den generellen unternehmerischen Zielen noch ein Indiz für grundsätzlich neue Strategien“ vorliege. Vielmehr ließen sich der politische Hintergrund und die theoretische Basis bis in die 1920er Jahre zurückverfolgen. Vor diesem Hintergrund dienen Führungsgrundsätze den Zwecken der Leistungs- und Herrschaftssicherung, indem sie Produktivitätssteigerungen durch die Sicherung der Leistungsmotivation erreichen sollen. Abgesehen davon hält Kubicek (1984b: 83f.) eine Reduktion der Motivations- und Identifikationsproblematik auf Führungsprobleme für kurzsichtig. Diese Sichtweise erkenne nur eine einzige Ursache, die nicht einmal als die wichtigste angesehen werden sollte. Breisig (1987) entwickelt eine Ideologiekritik am Konzept der kooperativen Führung und speziell der Führungsgrundsätze. Er stellt im Verlauf seiner Arbeit fest, dass

„das Konzept kooperativer Führung in der Form, wie es von der Majorität seiner Promotoren sowie der , herrschenden Meinung' innerhalb der Betriebswirtschaftslehre vertreten wird, ein Unternehmerkonzept ist, das in erster Linie ihren Interessen dient. Zumindest nach der ,reinen' Lehre werden die Interessen der Arbeitnehmer dann und nur dann berücksichtigt, wenn sie mit dem ökonomisch erforderlich Erscheinenden kompatibel sind (z.B. das Aussprechen von Anerkennung steigert die Leistung und entspricht dem Bedürfnis der Arbeitnehmer nach Wertschätzung [Hervorhebungen im Original]" (Breisig, 1987: 433).

Breisig (1987: 432) gesteht jedoch ein, eine überkritische Haltung eingenommen zu haben, um ein „bescheidenes Gegengewicht zu den zahllosen ebenso einseitigen Beschönigungen der Apologeten der Konzeption kooperativer Führung " zu erarbeiten. Obwohl die partizipative Führung gemäß Gauger (2000) ein Regelungsinhalt ist, welcher geradezu dafür prädestiniert ist, Commitment zu erzeugen, wird dieses Konzept in der Literatur nicht erwähnt.

\subsubsection{Verbindlichkeit und Konkretisierung}

Dass Führungsgrundsätze einer gewissen Verbindlichkeit bedürfen, um das Führungsverhalten wirksam zu steuern, darüber sind sich alle Autoren einig. Im Gegensatz zu Höhns $(1976,1977)$ Ansatz jedoch, in dem die mehrfache NichtBefolgung der sehr rigorosen Verhaltensvorschriften zu einer Trennung von der/dem MitarbeiterIn führen sollte, ist man in der späteren Literatur eher dazu geneigt, Führungskräften einen Spielraum einzugestehen, innerhalb welchem sie 
auf unerwartete Situationen flexibel und angemessen reagieren können. Dies schlägt sich auch in der Art nieder, wie Führungsgrundsätze formuliert werden sollen, nämlich tendenziell nicht abschließend, sondern in Form von Leitplanken. Diese sollen ,verhindern, daß der Fahrer von der Straße abkommt, lassen ihm aber die Wahl, ob er ganz rechts fahren will oder vielleicht auf der Überholspur“ (Grawert, 1997: 90). Lattmann (1975: 64) sieht in der Verbindlichkeitserklärung der Führungsrichtlinien einen wichtigen Aspekt im Einführungsprozess. Damit Führungsgrundsätze effektiv eine Verpflichtung bekunden können, müssen für ihn folgende Punkte erfüllt sein (Lattmann, 1975: 54f.):

- Der normative Gehalt muss erkennbar sein.

- Die Normen müssen eine grundsätzliche Bedeutung beinhalten.

- Die Normen müssen den ihnen zugrunde liegenden Absichten entsprechen.

- Die Normen müssen einen sinnvollen Gehalt aufweisen.

- Die Normen müssen anwendbar sein.

Auch Kossbiel (1983: 22) sieht die Verbindlichkeit als wichtige Voraussetzung für die Wirksamkeit von Führungsgrundsätzen. Als Maß der Verbindlichkeit von empfiehlt er die Konsequenzen bei Nichtbeachtung und stellt fest, dass diese oft nicht gegeben sind oder lediglich in einer Verwarnung bestehen. Darin sieht er die Gefahr, dass die Erstellung von Führungsgrundsätzen zur reinen Pflichtübung werden könnte. Ein Argument gegen die allzu streng verbindliche Formulierung von Führungsgrundsätzen liefert hingegen Paschen (1983: 31f.). Er hält eine strenge Verbindlichkeit von Führungsgrundsätzen nur dann für sinnvoll, wenn damit das Führungsverhalten bewusst auf eine bestimmte Klasse von Handlungen eingeschränkt werden soll (wenn also z.B. der kooperative Führungsstil auch dann praktiziert werden soll, wenn ein autoritärer Stil in der speziellen Situation effizienter wäre). Sollen Führungsgrundsätze hingegen Hilfsmittel zur besseren Zielerreichung sein, dann erscheint eine strenge Verbindlichkeit als wenig sinnvoll, da das Aufgabenziel sich u.U. in bestimmten Situationen mit anderen Mitteln besser erreichen lässt. Eine spezielle Art der Verbindlichkeit, nämlich die Zweckmäßigkeit und Erwünschtheit einer ,Rechtsverbindlichkeit' von Führungsgrundsätzen (z.B. im Rahmen des Betriebsverfassungsgesetzes) untersuchen Wunderer und Klimecki (1990). Sie kommen dabei zum Ergebnis, dass eine rechtsverbindliche Regelung von Unternehmen weitgehend abgelehnt wird. Größere Zustimmungen erhalten ,quasirechtliche' Regelungsformen; am wirksamsten wird jedoch die Selbstverpflichtung des Unternehmens gesehen (vgl. Wunderer \& Klimecki, 1990: 162).

Bezüglich des Detaillierungsgrades der Führungsgrundsätze stellt Lattmann (1975) einen Trade-Off fest. Er plädiert deshalb für ein mittleres Verallgemeinerungsmaß. 
„Je allgemeiner die Norm gefasst ist, desto eher kann die kategoriale Umschreibung ihres Anwendungsbereichs so vorgenommen werden, dass er alle den Absichten entsprechenden Verhaltensfragen umfasst. Die Norm wird dadurch um so grundsätzlicher. Desto weniger augenfällig wird dabei aber der Bezug zu diesen einzelnen Verhaltenslagen“ (Lattmann, 1975: 57).

Kossbiel (1983: 21) warnt davor, dass Stabilität zu Starrheit und Inflexibilität führen könne. Er führt aus, dass Führungsverhalten nicht immer unabhängig von der Situation und der Person festgelegt werden könne. Auch die Erledigung dringender Aufgaben dürfe nicht an der Zuständigkeitsfrage scheitern.

\subsubsection{Einführung und Verankerung}

Während im Harzburger Modell die Erstellung der Allgemeinen Führungsanweisung alleiniges Aufgabengebiet der Unternehmensführung war, wird ab Mitte der 1970er Jahre ein eher demokratischer Prozess der Erarbeitung und Einführung empfohlen: Zumindest die betroffenen Führungskräfte, bisweilen aber auch die MitarbeiterInnen sind in diesen Prozess einzubinden. Kossbiel (1983) betont, dass Grundsätze nur dann zu einer erfolgreichen Anwendung gelangen können,

„wenn die Erarbeitung und Verbreitung von einem vielschichtigen Prozeß getragen ist, an dessen Ende eine hohe Akzeptanz steht. Dies wird vor allem dann erreicht, wenn Unternehmungsleitungen mit einer positiven Einstellung die Einführung von Grundsätzen unterstützen und Mitarbeiter möglichst früh einbezogen werden“. (Kossbiel, 1983: 177)

Dass diese Idee auch schon in den 1970er Jahren vertreten wurde, zeigt Lattmann (1975: 62), der argumentiert, dass sich Führungsrichtlinien dann am stärksten im Führungsverhalten niederschlagen, wenn die Führungskräfte an ihrer Erarbeitung teilgenommen haben. Eine partizipative und kooperative Erarbeitung der Führungsgrundsätze empfehlen außerdem auch Buchmann (1994: 195), Grawert (1997: 94f.), Rühli (1992: 88), Töpfer und Zander (1982: 23ff.), Gabele (1992: 47) sowie Wunderer und Klimecki (1990: 13).

Als weiteren wichtigen Punkt zur Verankerung von Führungsgrundsätzen wird das aktive Vorleben der Führungsgrundsätze durch die oberste Führungsebene betrachtet. So zitiert z.B. Buchmann (1994: 196) einen Sinnspruch von Petronius aus dem 1. Jahrhundert: „Qualis est dominus talis est servus“ - Wie der Herr, so's Gscherr. Zu derselben Ansicht gelangen auch Grawert (1997: 95f.), Töpfer und Zander (1982: 28), Knebel (1982: 216f.) sowie Gabele (1992: 47). Grawert (1997: 94) betont die Bedeutung der Ableitung von Führungsgrundsätzen aus der eigenen Organisationskultur durch intensive Abstimmungsund Beratungsprozesse und verneint entschieden die Sinnhaftigkeit des Kopierens von Führungsgrundsätzen anderer Unternehmen. Dieser Meinung sind auch 
Knebel und Schneider (1994: 46), die davon ausgehen, dass Menschen Verhaltensleitsätze nur dann wirklich akzeptieren, wenn diese mit ihren persönlichen Vorstellungen übereinstimmen und sie an der Erarbeitung dieser Grundsätze teilgenommen haben. Als wichtig wird außerdem auch eine Abstimmung der Führungsgrundsätze mit anderen betrieblichen Grundsatzdokumenten und betrieblichen Normierungen gesehen (vgl. Lattmann, 1975: 52f.; Rühli, 1992: 88f.; Wunderer, 2009: 398; Wunderer \& Klimecki, 1990: 13). Kubicek (1984a: 12f.) kritisiert, dass Führungsgrundsätze in vielen Fällen im Widerspruch zu geltenden Arbeitsordnungen stehen. Während Führungsgrundsätze z.B. ein Menschenbild nach der Theory Y von McGregor (vgl. z.B. Schreyögg, 2003: 225ff.) vertreten, stellen Arbeitsordnungen den Menschen zumeist gemäß der Theory X dar. Knebel (1984: 883) stellt diese Tatsache in einer Antwort auf Kubiceks Beitrag als Überbleibsel veralteter Führungsgrundsätze dar und erklärt, moderne Führungsgrundsätze würden bereits in ihrer Entwicklung Arbeitsordnung und Betriebsverfassungsgesetz mit einbeziehen. Er stimmt Kubicek jedoch in dem Punkt zu, dass ,zwischen Führungsgrundsätzen und Betriebs- bzw. Arbeitsordnungen als auch zwischen den Behauptungen und Handlungen kein Widerspruch bestehen darf". Für die endgültige Umsetzung der Führungsgrundsätze empfehlen Knebel und Schneider (1994: 49ff.), Rühli (1992: 88) sowie Töpfer und Zander (1982: 25) eine ausgeprägte Informationspolitik sowie spezielle Seminare und Schulungen möglichst vieler MitarbeiterInnen. Anzumerken ist außerdem, dass Führungsgrundsätze keine ewige Gültigkeit beanspruchen können und deshalb je nach Bedarf überarbeitet und der neuen Situation angepasst werden müssen (vgl. exemplarisch dazu Lattmann, 1975: 64).

\subsubsection{Inhalte}

Beinahe in jeder Veröffentlichung findet sich eine Zusammenstellung der wichtigsten Inhalte von Führungsgrundsätzen. Dabei reicht die Palette von sehr detaillierten Dokumenten, die noch eine deutliche Nähe zum Harzburger Modell zeigen (vgl. Baumgarten, 1977: 158, zit. nach Wunderer 1980: 84), bis hin zu in wenigen Sätzen formulierten Slogans (vgl.Knebel \& Schneider, 1994: 84). Die meisten Vorschläge sind jedoch zwischen diesen beiden Polen angesiedelt. Eine idealtypische Struktur moderner Führungsgrundsätze findet sich in Abbildung 4. Weitaus detailliertere Inhaltsvorschläge bieten Knebel (1982: 202ff.) und Knebel und Schneider (1994: 28ff.). Relativ ausführlich äußern sich zu diesem Thema außerdem noch Lattmann (1975: 60f.), Töpfer und Zander (1982: 15), Kubicek (1984a: 6), Wunderer (1995: 726f.) und Tschirky (1981: 339f.). 


\section{Prinzipien}

- partizipatives und delegatives Verhalten (Teilhabe an Entscheidungsprozessen), z.B. Mitwirkung, Mitdenken, Mitentscheiden, Mitverantworten

- prosoziales Verhalten (Teilnahme an Kooperationsprozessen), z.B. Einfühlung, Vertrauen, Akzeptanz, Wechselseitigkeit

- Teammanagement, laterale Kooperation, Management by Objectives

- interner Wettbewerb (Markt) mit sozialem Netzwerk (Kooperation) verbinden

Ziele

- ökonomische Effizienz (Leistungswirksamkeit oder Wirtschaftlichkeit) zentrale Kriterien sind z.B. hohe Dienstleistungsqualität, wirtschaftlicher Umgang mit Ressourcen

- soziale Effizienz (Mitarbeiterzufriedenheit/Bedürfniserfüllung) zentrale Kriterien sind z.B. Sinn in und Spaß an der Arbeit, Erfolgserlebnisse, Anerkennung, Arbeitsbeziehungen, Beschäftigungssicherheit

Führungspolitische Instrumente

- Kooperation und Delegation als Grundregeln für die laterale und vertikale Zusammenarbeit

- Qualifikation (z.B. nach unternehmerischen Schlüsselkompetenzen)

- Kommunikation (z.B. Aussagen über Inhalte, Formen und Bedeutung der Kommunikation)

- Motivation (Bedürfnisse, Anreize sowie Abbau von Motivationsbarrieren) als individuelle Steuerung

- Organisation (Führungs- und Arbeitsorganisation), z.B. Definition von Führungsaufgaben und -kompetenzen, Delegation von Verantwortung, Partizipation und Zusammenarbeit, fördernde Arbeitsorganisation

- Position (z.B. Leitlinien zur Gewinnung, Umsetzung und Freistellung von MitarbeiterInnen, Grundsätze der internen Nachfolge oder Stellvertretung)

- Sanktion (z.B. Steuerung über monetäre und nicht monetäre Anreize, Bewertungsverfahren, Personal- und Führungs-Controlling)

- Promotion (Grundsätze der Personal- und Organisationsentwicklung)

- Evaluation (z.B. Wirkungsanalysen über Personalbeurteilung und Mitarbeiterbefragungen)

Abbildung 4: Inhalte von Führungsgrundsätzen (in Anlehnung an Wunderer, 2009: 390) 


\subsubsection{Empirische Schwerpunkte in der Literatur}

Während im vorherigen Kapitel die normativen und präskriptiven Elemente der einschlägigen Literatur zusammengefasst wurden, widmet sich dieser Abschnitt den existierenden empirischen Ergebnissen. Dabei ist zu erwähnen, dass einige Themen sowohl präskriptiv als auch empirisch behandelt wurden bzw. dass davon auszugehen ist, dass empirische Analysen die Grundlage für spätere präskriptive Artikel darstellten. Nichtsdestotrotz existieren zum Teil interessante Spannungen zwischen empirischen Fakten und normativem Anspruch, so z.B. bei den Themen ,Verbindlichkeit' und ,Einführung'. Wann immer sich solche Widersprüche ergeben, wird in diesem Kapitel explizit darauf hingewiesen.

Grundsätzlich lässt sich feststellen, dass sich die empirisch motivierte Literatur zur Praxis der Führungsgrundsätze als vergleichsweise dünn erweist. Es überwiegen Untersuchungen mit deskriptivem Charakter. Dabei handelt es sich zumeist um Inhaltsanalysen erhobener Dokumente (vgl. Ebert, 1994a; Ebert, 1994b, 1997; Tschirky, 1981) oder Umfragen zu Verbreitung und Einschätzung des Wirkungsgrades. Ein weiteres Problem stellt sich insofern, als in den Publikationen oft nicht klar zwischen Unternehmensgrundsätzen und Führungsgrundsätzen unterschieden wird, was zu einer Verzerrung der empirischen Ergebnisse führt.

\subsubsection{Verbreitung von Führungsgrundsätzen}

Studien zur Verbreitung von Führungsgrundsätzen werden durchgeführt, seit das Thema in der wissenschaftlichen Literatur auf Interesse stößt. Die Ergebnisse variieren über die Zeit stark, und auch die Transparenz der konkreten Erhebungsmethoden ist nicht immer vollständig gegeben, weshalb eine direkte Vergleichbarkeit nicht möglich ist. Dementsprechend sollen die jeweiligen Studien hier lediglich kurz präsentiert und wo nötig kommentiert werden. Fiedler (1976) befragte 360 Unternehmen und kommt zu dem Ergebnis, dass 75 Unternehmen bereits schriftlich fixierte Führungsgrundsätze besaßen, 65 deren Einführung planten, 32 nicht die Absicht hatten, Führungsgrundsätze einzuführen, 18 die Umfrage ablehnten und 14 an die Muttergesellschaft verwiesen. Aus dem Text ergibt sich, dass die fehlende Zahl der Unternehmen nicht antwortete. Fiedler hält jedoch fest, dass der Großteil dieser Unternehmen sich im Anfangsstadium der Entwicklung von Führungsgrundsätzen befindet. Das ergab ein Telefontest (Fiedler, 1976: 313). Anfang 1980 befragte Fiedler (1980) wiederum 359 Unternehmen. Davon hatten 9 Unternehmen Unternehmensgrundsätze formuliert, wollten aber keine Führungsgrundsätze haben, 110 Unternehmen hatten sowohl Unternehmens- als auch Führungsgrundsätze formuliert, 42 Unternehmen hatten ausschließlich Führungsgrundsätze eingeführt, 71 Unternehmen hatten sowohl 
Unternehmens- als auch Führungsgrundsätze geplant, 14 Unternehmen hatten ausschließlich Führungsgrundsätze geplant und 113 wollten weder Unternehmens- noch Führungsgrundsätze einführen (Fiedler, 1980: 125). Hoffmann (1989: 171f.) stelle in seiner Untersuchung fest, dass von jenen Unternehmen, welche schriftlich fixierte Grundsätze besaßen (56\% der von ihm untersuchten Unternehmen) zwei ausschließlich Unternehmensgrundsätze eingeführt hatten, 10 Unternehmen ausschließlich Führungsgrundsätze formuliert hatten, zehn Unternehmen getrennte Unternehmens- und Führungsgrundsätze besaßen und eine Unternehmung beide Formen in einem Grundsatzdokument vereinte. Wunderer und Klimecki (1990: 84f.) erfassten in einer telefonischen Umfrage, dass 660 der von ihnen befragten Unternehmen Führungsgrundsätze schriftlich fixiert hatten, 1.150 Unternehmen die Einführung planten und 4.200 Unternehmen keine Führungsgrundsätze hatten oder planten. Ihre schriftliche Umfrage hatte ein anderes Ergebnis ergeben, nämlich, dass von den Firmen, welche auf den Fragebogen geantwortet hatten, 63 Führungsgrundsätze besaßen, 34 deren Einführung planten und 72 die Einführung nicht planten. Sie schließen daraus, dass in ihrer Stichprobe die Unternehmen mit Führungsgrundsätzen überrepräsentiert waren, während die Unternehmen, welche keine Führungsgrundsätze planten, stark unterrepräsentiert waren. Auch wenn der Anteil der negativ eingestellten Unternehmen hier gewaltig scheint, konstatieren Wunderer und Klimecki eine zunehmende Attraktivität von Führungsgrundsätzen aufgrund der hohen Anzahl der ,Planer' und führen die Ablehnung von Führungsgrundsätzen weniger auf explizite Entscheidungen denn auf Informationsdefizite zurück.

In jüngerer Vergangenheit führten Jancsary, Knassmüller und Sandner (2006) eine Untersuchung über Strukturen und Inhalte von Führungsgrundsatzdokumenten durch. Dabei konnten die Autoren auch einen tendenziellen (wenn auch nicht statistisch repräsentativen) Verbreitungsgrad im deutschsprachigen Raum ermitteln (siehe Tabelle 2). Diese Ergebnisse geben Hinweise darauf, dass das Instrument trotz des gesunkenen wissenschaftlichen Interesses immer noch praktische Relevanz besitzt. 
Tabelle 2: Verbreitung im deutschsprachigen Raum (Jancsary et al., 2006: 27)

\begin{tabular}{|c|c|c|c|c|c|c|}
\hline & \multicolumn{2}{|c|}{ Deutschland } & \multicolumn{2}{|l|}{ Schweiz } & \multicolumn{2}{|c|}{ Österreich } \\
\hline Kontaktierte Unternehmen & 132 & & 104 & & 134 & \\
\hline - Keine Information über FG & 28 & & 23 & & 15 & \\
\hline - Teilnahme verweigert & 12 & & 5 & & 7 & \\
\hline Teilnehmende Unternehmen & 92 & $100,00 \%$ & 76 & $100,00 \%$ & 112 & $100,00 \%$ \\
\hline Unternehmen mit FG & 26 & $28,26 \%$ & 21 & $28,00 \%$ & 25 & $22,32 \%$ \\
\hline Führungsgrundsätze in Arbeit & 11 & $22,96 \%$ & 4 & $5,33 \%$ & 10 & $8,94 \%$ \\
\hline Keine Führungsgrundsätze & 55 & $58,78 \%$ & 51 & $68,00 \%$ & 77 & $68,75 \%$ \\
\hline
\end{tabular}

\subsubsection{Einführungszeitpunkt}

Bezüglich des Einführungszeitpunktes lässt sich feststellen, dass seit den 70er Jahren die Anzahl der Unternehmen mit Führungsgrundsätzen stark zugenommen hat (vgl. Albach, 1976; Fiedler, 1976; Wunderer \& Klimecki, 1990). Informationen über die Häufigkeit von Erneuerungen und Aktualisierungen - und somit über das ,Alter` der aktuellen Fassungen - existieren jedoch nicht.

\subsubsection{Charakterisierung der Einführung}

Die in der präskriptiven Literatur geforderte ,partizipative‘ Einführung mit Beteiligung der MitarbeiterInnen scheint in der Praxis kein großes Echo gefunden zu haben. Die Untersuchungen belegen mehrheitlich, dass im Wesentlichen Vorstand, Personalabteilung und Führungskräfte an der Erstellung beteiligt werden. In der Untersuchung von Fiedler (1976) kamen die Anregungen zur Erstellung von Führungsgrundsätzen vor allem aus der Personalabteilung und dem Vorstand bzw. der Geschäftsleitung, wobei die Personalabteilung bei den ,Planern' eine noch größere Rolle spielte als bei jenen Unternehmen, welche die Führungsgrundsätze bereits eingeführt hatten. Bei der Erarbeitung waren bei den ,Anwendern' wiederum hauptsächlich Vorstand und Personalabteilung beteiligt, während bei den ,Planern' die beiden meistgenannten Gruppen die Personalabteilung und ausgewählte Führungskräfte darstellten, gefolgt vom Vorstand. Während 41 Unternehmen angaben, keine Probleme bei der Einführung gehabt 
zu haben, stellten nur 15 Unternehmen Probleme und 14 Unternehmen, wenig Probleme fest. Gabele und Kretschmer (1986: 96) kamen zu einem ähnlichen Ergebnis. An den Formulierungsprozessen waren hauptsächlich der Vorstand (94\%) und die Führungskräfte (71\%) beteiligt. Dieselbe Tendenz stellten sie in diversen anderen Untersuchungen fest. Allerdings sind diese Aussagen mit Vorsicht zu genießen, da Gabele und Kretschmer nicht klar zwischen Unternehmens- und Führungsgrundsätzen unterscheiden. Hoffmann (1989) findet in seiner Untersuchung zwar heraus, dass Führungsgrundsätze in fast allen Fällen von Teams erarbeitet wurden, welche jedoch nicht - wie in der präskriptiven Literatur gefordert - mit MitarbeiterInnen aus allen Hierarchieebenen besetzt waren. Vielmehr wurde die Erstellung in 78\% der untersuchten Unternehmungen von dominanten Personen aus dem Top-Management geprägt und stark von externen Beratern oder den Grundsätzen anderer Unternehmen beeinflusst. Der Betriebsrat oder Mitglieder aus unteren Hierarchieebenen wurden nur in drei Unternehmungen einbezogen. Ähnlich gestaltet sich das Ergebnis auch bei Wunderer und Klimecki (1990). Auch hier waren die beiden Gruppen, welche den Inhalt der Führungsgrundsätze am stärksten beeinflussten, die Geschäftsleitung und die Personalabteilung, und zwar sowohl in der schriftlichen als auch in der mündlichen Befragung und bei Anwendern und Planern gleichermaßen. Allerdings waren sich bei direkter Nachfrage die Unternehmen nahezu einig, dass Führungsgrundsätze unter optimalen Voraussetzungen mit Beteiligung aller MitarbeiterInnengruppen entwickelt werden sollten.

\subsubsection{Bezugsgruppen}

Die Führungsgrundsätze in den untersuchten Firmen richten sich in erster Linie an die Führungskräfte und erst in zweiter Linie an die MitarbeiterInnen und andere Organisationsmitglieder. Albach (1976: 748) nennt als die wichtigsten Bezugsgruppen in Führungsgrundsätzen die MitarbeiterInnen, die KundInnen und die Öffentlichkeit, wobei anzunehmen ist, dass hier keine trennscharfe Unterscheidung zwischen Unternehmens- und Führungsgrundsätzen vorgenommen wird. Hoffmann (1989: 178) stellt in seiner Untersuchung fest, dass Führungsgrundsätze zu 60\% nur an Führungskräfte und zu 40\% an alle Unternehmensmitglieder adressiert waren. Wunderer und Klimecki (1990: 137f.) kommen ebenfalls zu dem Ergebnis, dass Führungskräfte die hauptsächliche Bezugsgruppe sind. Bei 10\% der Anwender und 20\% der Planer wurden sogar die unteren Führungskräfte aus dem Geltungsbereich ausgenommen.

Jancsary, Knassmüller und Sandner (2006: 89ff.) finden in den von ihnen untersuchten Dokumenten vor allem Führungskräfte und MitarbeiterInnen als dominante Akteure. Führungskräfte werden in 50 von 53 Dokumenten ange- 
sprochen, MitarbeiterInnen tauchen in 48 Dokumenten auf. Führungskräften kommt dabei stets die aktive Rolle zu, während den MitarbeiterInnen größtenteils grammatikalisch ein Objektstatus zugewiesen wird. MitarbeiterInnen handeln nicht, sie werden behandelt (vgl. Jancsary, et al., 2006: 103f.).

\subsubsection{Regelungsinhalte}

Abgesehen von den in der präskriptiven Literatur erwähnten Vorschlägen bezüglich idealtypischer Inhalte von Führungsgrundsätzen haben einige empirische Studien diese Inhalte in der Praxis überprüft. So finden z.B. Grunwald und Lilge (1981: 414) in ihrer Untersuchung die folgenden hauptsächlichen Regelungsinhalte von Führungsgrundsätzen:

- Informationsaustausch

- Zusammenarbeit

- Delegation

- Initiative/Kreativität

- MitarbeiterInnenförderung, Kontrolle (Prozess/Ergebnis) und Verantwortung (funktionale)

Tschirky (1981) wählt in seiner Inhaltsanalyse der Führungsgrundsätze von 42 Schweizer Unternehmungen eine andere Betrachtungsweise. Er unterteilt die Inhalte in technische und soziale Aspekte und kommt zu folgenden Ergebnissen (vgl. Tschirky, 1981: 35f.):

- Die technische Betrachtungsweise überwiegt verglichen mit der sozialen Betrachtungsweise.

- Unter den Aussagen sozialer Art überwiegt die personale Betrachtungsweise im Vergleich zu einer interaktionalen oder soziolokalen Betrachtungsweise.

Auch Wunderer und Klimecki (1990: 151f.) identifizieren dominante Regelungsinhalte, und zwar die folgenden:

- Grundwerte von Führungs- und Kooperationsbeziehungen

- Entscheidungsbeteiligung der MitarbeiterInnen

- Führungs- und Kooperationsinstrumente

- Organisation

Sie betonen außerdem, dass der Punkt ,Sanktion“ nur in sehr wenigen Fällen explizit anfgeführt wird.

Die bisher aktuellste Untersuchung der Inhalte von Führungsgrundsätzen stammt von Jancsary, Knassmüller und Sandner (2006). Die Ergebnisse können hier nicht in voller Länge diskutiert werden, es soll aber ein kurzer Überblick 
über die von den Autoren identifizierten dominanten Themenstränge gegeben werden. Untersuchungseinheiten waren 53 Führungsgrundsätze aus Österreich, Deutschland und der Schweiz. Auffällig ist dabei, dass die Ergebnisse sehr stark jene von Grunwald und Lilge (1981) bestätigen. Auch Jancsary, Knassmüller und Sandner konstatieren eine Dominanz der Themen Information, Zusammenarbeit, Delegation und Mitarbeiterförderung/Personalentwicklung. Trotz der von Wunderer (2009: 384f.) proklamierten stetigen Weiterentwicklung von Führungsgrundsätzen unterscheiden sich somit die Themen zumindest manifest nicht signifikant von jenen vor 25 Jahren.

\section{Themenfrequenz und Themenbetonung im Detail $(n=53)$}

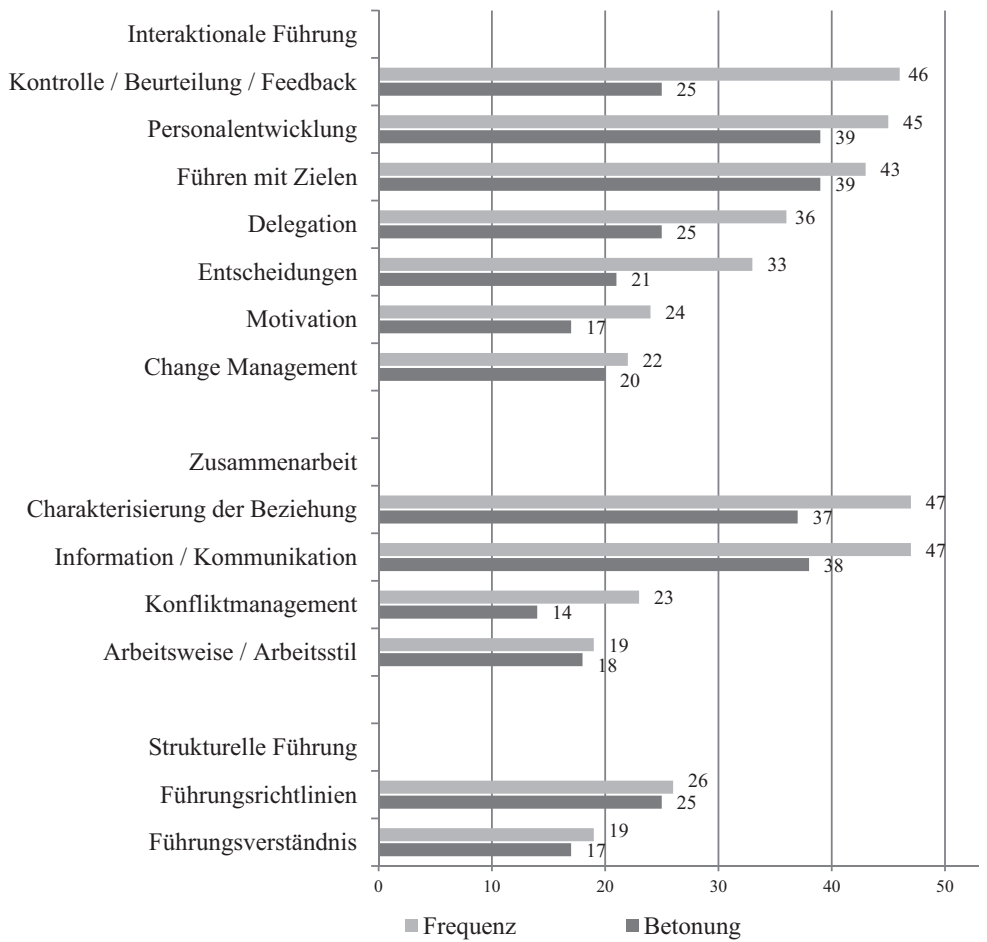

Abbildung 5: Themenfrequenz und Themenbetonung in Führungsgrundsätzen (vgl. Jancsary et al., 2006: 46)

3 Themenfrequenz bezeichnet die absolute Anzahl an Dokumenten, welche ein bestimmtes Thema zumindest einmal erwähnen. Themenbetonung hingegen bezeichnet die An- 


\subsubsection{Verbindlichkeit}

Dass eine angemessene Verbindlichkeit als wichtiges Charakteristikum von Führungsgrundsätzen behandelt wird, wurde bereits besprochen. Empirische Untersuchungen zeigen jedoch, dass die Verbindlichkeit in der Praxis oft nicht so hoch ist, wie dies in der präskriptiven Literatur gefordert wird. Fiedler (1976) kommt zu dem Ergebnis, dass bei den von ihm untersuchten Unternehmen Führungsgrundsätze hauptsächlich als Empfehlungen (24 Anwender/37 Planer), als verbindlich für alle durch Rundschreiben (22 Anwender/0 Planer) und als Bestandteil des Mitarbeitervertrages (15 Anwender/17 Planer) implementiert werden. Hoffmann (1989) hingegen betrachtet die Verbindlichkeit von einer anderen Perspektive aus. Er fragt nicht nach der ,geplanten', sondern nach der ,empfundenen "Verbindlichkeit. Dabei stellt er fest, dass Organisationsmitglieder den Grundsätzen tendenziell keinen ausreichenden Verbindlichkeitsgrad zugestehen. Für 58\% sind Führungsgrundsätze eine Hilfe, 9\% sagen aus, ihre Kollegen würden sich nicht an die Grundsätze halten, bei $80 \%$ der Unternehmensmitglieder hat sich das Verhalten, wenn überhaupt, dann nur geringfügig verändert. Was Sanktionen betrifft, so sehen $60 \%$ keine einschneidenden Karrierehemmnisse bei Nichtbeachtung; und nur von 15\% werden Sanktionen registriert oder vermutet. 52\% der Führungskräfte geben an, dass die Nichtbeachtung überhaupt keine Sanktionen nach sich zieht. Nur 13\% können auf konkrete negative Sanktionen hinweisen. Bei Wunderer und Klimecki (1990: 170ff.) wird das Thema der Verbindlichkeit sehr detailliert dargestellt. Als zweckmäßige Regelungsformen treten zu Tage:

- Gemeinsame Verabschiedung,

- Arbeitsvertragliche Regelungen,

- Anweisung/Direktionsrecht,

- Freiwillige Betriebsvereinbarung.

Bei den Anwendern geben 48 an, eine Einhaltung habe Folgen auf den Personaleinsatz bzw. auf Karriereentscheidungen, 34 führen aus, es gebe überhaupt keine Folgen und bei weiteren 34 gibt es zwar Folgen, diese sind aber nicht konkret fassbar. Bei Nichteinhaltung geben 73 Anwender an, eine Diskussion mit den Betroffenen zu führen, bei 38 gibt es keine konkret fassbaren Folgen, und bei 32 Anwendern hat eine Nichteinhaltung Einfluss auf die Karriereentscheidungen. Eine Einhaltung soll bei 70 Planern Einfluss auf die Karriere haben, bei 44 Planern zu einer Diskussion führen und bei 35 Planern Einfluss auf

zahl an Dokumenten, welche ein bestimmtes Thema auf verschiedene Art und Weise hervorheben, sei dies durch Textstruktur (z.B. Überschriften, Einrückungen, etc.) oder durch Zeichenformatierung (Fettdruck, Kursivdruck, Unterstreichung, Blocksatz, etc.). 
Gehaltsentscheidungen haben. Nichtbeachtung der Führungsgrundsätze soll bei 88 Planern zu einer Diskussion führen, bei 43 Einfluss auf die Karriere haben, und bei jeweils 28 Planern ist eine schriftliche Mitteilung an die Betroffenen bzw. ein Einfluss auf die Gehaltsentscheidungen geplant.

Eine Einschätzung der Verbindlichkeit anhand der Formulierung der Dokumente nehmen Jancsary, Knassmüller und Sandner (2006) vor. Sie unterteilen die verwendeten Formulierungen in akteursunabhängige (Normen, Regeln und Prinzipien) und akteursbezogene (Pflichten, Verbote, Verantwortlichkeiten und Erwartungen) Steuerungsmechanismen, wobei Pflichten die stärkste Verbindlichkeit ausdrücken, Erwartungen die geringste (vgl. Jancsary et al., 2006: 46ff.). Dabei kommen die Autoren zum Schluss, dass verschiedene Themenstränge mit unterschiedlich starker Verbindlichkeit formuliert werden, die grundsätzliche Verbindlichkeit aber als eher niedrig eingestuft werden muss. Eine Einschränkung dieser Aussage stellt das Problem dar, dass viele Führungsgrundsätze als reine Aussagesätze formuliert werden:

Die Führungskräfte informieren ihre Mitarbeiter über getroffene Entscheidungen umgehend sowie mit entsprechender Begründung. (A 04)

Die Verbindlichkeit solcher Aussagen ist ohne Kontextinformationen kaum zu beurteilen, da sie je nach kulturellem Umfeld entweder als äußerst verbindlich (es gibt keine andere Möglichkeit der Handlung) oder als eher unverbindlich (im Sinne einer erwünschten Idealsituation) interpretiert werden können.

\subsubsection{Praktische Erfahrungen mit Führungsgrundsätzen}

Quantitative Wirkungsstudien, wie sie z.T. im angelsächsischen Raum für Codes of Conduct durchgeführt wurden (vgl. z.B. Fritz, Arnett, \& Conkel, 1999; Somers, 2001), finden sich in der empirischen Literatur zu Führungsgrundsätzen nicht. Hingegen gibt es schon sehr früh Studien, die sich mit der grundsätzlichen Zufriedenheit der relevanten Akteure mit Führungsgrundsätzen beschäftigen. Fiedler (1976) interessiert sich dafür, wie sich die Einführung von Führungsgrundsätzen auf das Verhalten von Führungskräften und MitarbeiterInnen auswirkt. Drei Unternehmen beurteilen die Wirkung als ,sehr positiv', 38 Unternehmen als ,positiv', sieben Unternehmen können keine Wirkung feststellen, und bei sechs Unternehmen verlaufen die Führungsgrundsätze nach der Einführung im Sande. Welche konkreten Wirkungen für die Zufriedenheit der Unternehmen verantwortlich sind lässt sich aus der Studie leider nicht ersehen. Weiters fragt Fiedler danach, welche Grundsätze am wenigsten angenommen werden. Hier nennen die Unternehmen vor allem Delegation (31 Stimmen), Information (29 Stimmen), MitarbeiterInnen- bzw. Leistungsbeurteilung (22 Stimmen) sowie Anerkennung und Kritik (20 Stimmen). Auch Hoffmann (1989) 
fragt nach der Zufriedenheit mit Führungsgrundsätzen. Er kommt zu dem Ergebnis, dass der Großteil (63\%) der von ihm befragten Unternehmen die Ziele in hohem Maße erfüllt sehen und auch in hohem Maße mit dem Erreichten zufrieden sind (52\%). Insgesamt 22\% der Führungskräfte sehen die Ziele in eher niedrigem Maße erfüllt, 29\% sind mit dem bisher Erreichten eher unzufrieden. Als Hauptproblemfelder werden genannt (Hoffmann, 1989: 177):

- Führungskräfte sind wenig gewillt, das Risiko der Delegation einzugehen.

- Führungskräfte sind zu sehr auf ihre eigenen Interessen fixiert.

- Kommunikation beschränkt sich auf das absolut Notwendige.

Hier stimmt die Untersuchung von Hoffmann zumindest in den Punkten ,Delegation' und ,Information' mit jener von Fiedler überein, die immerhin 13 Jahre früher durchgeführt wurde.

Diese Studien können oberflächliche Einblicke in den Wirkungsgrad von Führungsgrundsätzen geben. Es stellt sich jedoch die Frage nach der Aussagekraft solcher Stimmenzählungen, da nicht geklärt ist, was genau unter ,positiv` zu verstehen ist, ebenso wenig wie unmittelbar einsichtig wird, die Kategorie ,am wenigsten angenommen' im konkreten Fall bedeutet. Für eine Wirkungseinschätzung, auf der man gegebenenfalls Maßnahmen aufbauen könnte, müsste näher auf den konkreten Kontext und die Problemfelder eingegangen werden. Etwas konkreter sind die Ergebnisse von Wunderer und Klimecki (1990). Die Autoren untersuchen, welche Teile der Führungsgrundsätze eher stärker und welche eher weniger stark angenommen bzw. eingehalten werden (siehe Abbildung 6). In der mündlichen Befragung stellen die Autoren allerdings fest, dass der direkte Interviewkontakt und eine leicht veränderte Frageform zu einer wesentlich kritischeren Grundhaltung führen. Sie bemerken, dass bei einigen Funktionen die Erwartungen der Planer und sogar jene der Ablehner höher sind als die Beurteilung durch die Anwender (vgl. Wunderer \& Klimecki, 1990: 114). 
Hohes Maß an Wirksamkeit:

- Erleichterung der direkten Kommunikation

- Verbesserte Verhaltensbeurteilung

- Verbesserung der organisatorischen Bedingungen des Leistungsprozesses

- Realisierung von Grundwerten der Führung und Kooperation

- Grundlage für Verhaltensschulung

Eingeschränkte Wirksamkeit:

- Klare Kommunikation des gewünschten Verhaltens

- Verbesserte Konflikthandhabung

- Erhöhte Leistungsmotivation

Geringe Wirksamkeit:

- Selbst- statt Fremdkontrolle

- Vorhersehbarkeit des Verhaltens anderer

- Vermeidung / Ersatz für die direkte Diskussion zwischen Vorgesetzten und Mitarbeitern über das erwünschte Führungsverhalten

- Differenzierte Führung in unterschiedlichen Führungssituationen

Abbildung 6: Wirkungsbereiche von Führungsgrundsätzen (in Anlehnung an Wunderer und Klimecki, 1990: 110)

\subsubsection{Die Sprache der Führungsgrundsätze}

In der linguistischen Analyse von Führungs- und Unternehmensgrundsätzen hat sich Ebert (1994a, 1994b, 1997) besonders hervorgetan. In einer sehr ausführlichen Studie (Ebert, 1994a) analysiert er eine Vielzahl von Dokumenten mit dem Hauptziel, die Vielfalt der vorliegenden Textexemplare auf bestimmte Grundtypen von Texten zu reduzieren, um einen Überblick über das Genre zu geben. Dabei stellt Ebert (1994a: 321) eine überraschende Vielfalt an textlichen Darstellungsformen fest, konstatiert aber, dass bis auf wenige Ausnahmen alle mit einem normativen Anspruch versehen sind. Viele der untersuchten Texte erweisen sich als Mischung von Mustern bzw. als ,Textsorten-Komplex', lassen sich also keinem Idealtyp vollständig zuordnen. Dennoch kann Ebert einen Katalog reiner Ideal- oder Prototypen ausfindig machen, nämlich den normativregulierenden, den normativ-orientierenden, den normativ-instruierenden, den informativen und den ausdrucksfunktional-kommisiven Texttyp, sowie kommisive Formen der Verbindlichkeitsregelung, Mikrotexte (Slogans, Formeln) und 
Dokumente an den Grenzen der Textualität (Listen, Graphiken) (Ebert, 1994a: $322 \mathrm{ff}$.). Bei den Strukturmustern überwiegt insgesamt die unverbundene Sammlung von Regeln und Leitsätzen, wobei Grundsätze neueren Datums dazu neigen, Sequenzen des Erklärens, Begründens und Rechtfertigens zu integrieren und somit eher Zusammenhänge aufzeigen. Ebert stellt außerdem fest, dass sich Unternehmens- und Führungsgrundsätze thematisch nicht so stark unterscheiden wie die wissenschaftliche Literatur dies nahe legen würde. Die These, dass sich gesellschaftliche Veränderungen auch in Führungsgrundsätzen wiederfinden, kann Ebert bestätigen.

Einen knappen Einblick in die sprachliche Formulierung von Führungsgrundsätzen geben auch Jancsary, Knassmüller und Sandner (2006). Die Autoren nehmen dabei den Blickwinkel eines (naiven) Lesers ein und stellen fest, dass die untersuchten Führungsgrundsätze durchaus Spielraum für Interpretationen zulassen. So unterbleiben Begriffsklärungen im Großteil der untersuchten Dokumente völlig, und auch verwendete Qualifizierungen durch Adjektive werden kaum näher erläutert (z.B. ,gute‘ Kommunikation). Dadurch spielt bei der Interpretation (sowohl der alltagsweltlichen als auch der wissenschaftlichen) der Grundsätze der Verwendungskontext eine große Rolle, da organisationale Verständnisse den Rahmen für diese Interpretationen liefern (Jancsary et al., 2006: 102). Neben fehlenden Qualifizierungen sind auch missverständliche bzw. ungebräuchliche Definitionen zu finden. Solche mehrdeutigen Anweisungen können in der Praxis zu Irritationen und Double-Binds führen. Auch hier wird eine Interpretationsleistung seitens des Adressaten notwendig.

\subsubsection{Kritik am Konzept der Führungsgrundsätze}

\subsubsection{Kritik an den Wirkungsbehauptungen}

Derschka (1986, zit. in Wunderer/Klimecki, 1990: 15) geht so weit, Führungsgrundsätze als ,in den Wind geschriebene Leerformeln“ zu bezeichnen. Er führt aus, solche Grundsätze wären äußerst interpretationsbedürftig, was einerseits als Notwendigkeit erscheine, da niemals alle Situationen abschließend behandelt werden können. Auf der anderen Seite stellt dies dem Vorgesetzten aber auch einen Freibrief für die Interpretation der Grundsätze aus. Selbst wenn Führungsgrundsätze konkret werden, kann dennoch kein empirischer Beweis für ihre Wirksamkeit erbracht werden, da nicht unmittelbar einsichtig ist, was tatsächlich unter Führungserfolg konkret verstanden wird (vgl. Neuberger, 1984: 23, zit. nach Wunderer/Klimecki, 1990: 15). Zusätzlich äußert Oechsler (2000: 380) die Kritik, dass Führungsgrundsätze aufgrund ihrer Abstrahierung von Individuen 
und Situationen kaum Hinweise auf das tatsächlich angestrebte Führungsverhalten liefern können.

\title{
2.3.4.2 Kritik an der zugrunde liegenden Ideologie
}

Staehle (1999: 817) äußert den Verdacht, dass in Führungsgrundsätzen oft ein breites Spektrum von Führungsverhalten als kooperativ bezeichnet wird, auch wenn einzelne Ausprägungen diese Bezeichnung nicht verdienen. Dadurch wird verschleiert, dass häufig autoritär geführt werden muss. Weiters vermutet er, dass durch die in unverbindlichen Grundsätzen propagierte Partizipation ein Substitut für echte Mitbestimmung als formal-rechtlich gesicherte Teilhabe entwickelt werden soll. Dieser Form der Ideologiekritik widmet sich auch Kubicek (1984a, 1984b). So stellt er fest, dass Führungsgrundsätze bei einer inhaltlichen Erweiterung der Betrachtung als ,ein Element einer komplexeren Gesamtstrategie der Produktivitätssteigerung und Herrschaftssicherung interpretiert werden [können]". Weder die Problemstellung selbst noch die Maßnahmen zur Problembewältigung seien neu, vielmehr entstanden die ersten Ansätze bereits in den 20er Jahren (vgl. Kubicek, 1984a: 16ff.). Er kommt deshalb zu dem Schluss, dass Führungsgrundsätze weder ein Indiz für einen grundlegenden Wandel der unternehmerischen Ziele noch für grundsätzlich neue Strategien seien (vgl. Kubicek, 1984b: 81). Breisig (1987) sieht Bekenntnisse zu Kooperation und MitarbeiterInnenberücksichtigung durch gesellschaftlichen Wandel erzwungen.

\begin{abstract}
„Aus Unternehmersicht ergibt sich [...] die Notwendigkeit, partiell auf über das Materielle hinausgehende Wünsche und Interessen der Mitarbeiter einzugehen. Dieses im Unternehmerinteresse unverzichtbare Strategieerfordernis wird, begünstigt durch seine teilweise Kongruenz mit seit den $60 \mathrm{er}$ Jahren insbesondere bei jüngeren Menschen verstärkt entstehenden ,postmaterialistischen' Wertvorstellungen, offensiv und mit beträchtlichem propagandistischen Aufwand als Beweis für eine demokratische und humanitäre Gesinnung der Unternehmer ins Felde geführt [Hervorhebungen im Original]“ (Breisig, 1987: 420).
\end{abstract}

Es zeige sich dem kritischen Betrachter jedoch schnell, dass das eigentliche Ziel die Effektivität sei, für welche die (partielle) Humanität nur ein Mittel darstelle (vgl. Breisig, 1987: 420). Dies zeige sich u.a. daran, „daß Unternehmer in ,Sonntagsreden“ und Führungsgrundsätzen von Beteiligung und Interessenberücksichtigung der Arbeitnehmer sprechen, bei Fragen der technischorganisatorischen Rationalisierung unter allen Umständen aber trotz Wissens um die möglichen negativen Auswirkungen auf die Arbeitnehmer an ihrer betriebsverfassungsrechtlich kaum eingeschränkten alleinigen Entscheidungskompetenz festhalten" (Breisig, 1987: 422). 


\subsubsection{Kritik an der wissenschaftlichen Aufarbeitung}

Auch bezüglich der wissenschaftlichen Aufarbeitung des Themas zeigt sich Kubicek (1984a) kritisch. So stellt er fest, dass die betriebswirtschaftliche Führungslehre sich bisher darauf beschränkt habe, von Praktikern formulierte Führungsmythen zu reproduzieren und legitimieren (vgl. Kubicek, 1984a: 5ff.). Er bemerkt, dass sich die meisten Aussagen in der Literatur nicht auf Veränderungen in der Führungspraxis an sich, sondern lediglich auf Veränderungen auf der Ebene offizieller Regelungen und dokumentierter Normen beziehen (vgl. Kubicek, 1984a: 8). Dadurch würden die von Praktikern formulierten Ziel- und Mittelaussagen unkritisch übernommen und weitervermittelt. Auf eine eigenständige Rekonstruktion impliziter Prämissen und eine Prüfung der Konsistenz mit anderen Regelungselementen werde weitgehend verzichtet. Durch diese Weitervermittlung der Praktikeraussagen werde ihnen zusätzlich noch eine Art „akademische Weihe“ zuteil (Kubicek, 1984a: 9). Damit spricht Kubicek auch das Problem der generellen ,Theorielosigkeit' von Führungsgrundsätzen an. Führungsgrundsätze werden nicht in der (Betriebs)Wissenschaft erstellt und evaluiert, sondern aus der Praxis unkritisch übernommen (vgl. Kubicek, 1984a: 4). Zu demselben Ergebnis kommt auch Kossbiel (1983: 20), der bemerkt: „Fragt man nach der wissenschaftlichen Fundiertheit solcher Regeln für das Führungsverhalten, so kommt man zu einem schnellen, überwiegend negativen Urteil. Die meisten in der Praxis entwickelten Führungsgrundsätze entbehren jeder verhaltenstheoretischen und sozialwissenschaftlichen Begründung“. Er weist jedoch darauf hin, dass die Praxis auf solche Konzepte angewiesen sei, solange die Wissenschaft keine besseren Konzepte zur Verfügung stelle, und dass auch sich theoriefrei entwickelte Konzepte im Nachhinein durchaus als theoretisch fundierbar erweisen können. Eine solche nachträgliche Fundierung lässt sich aber auch in jüngerer Vergangenheit nicht feststellen.

\subsubsection{Schlussfolgerung: Lücken in der wissenschaftlichen Aufarbeitung}

Ausschlaggebend für die Wahl der Forschungsfrage war sowohl dieser Mangel an einer empirischen Durchdringung des Phänomens als auch das Fehlen von systematischen, qualitativ-interpretativen empirischen Untersuchungen.

Die Untersuchung von Wunderer und Klimecki (1990) ist die letzte ausführliche Studie über Führungsgrundsätze, obwohl Wunderer (2009: 385) selbst von einer ständigen Weiterentwicklung der Dokumente bis in die Gegenwart spricht. Selbst aktuelle Überblickswerke im Bereich Führung und (Personal)management (vgl. z.B. Macharzina, 2003; Oechsler, 2000; Staehle, 1999; Wunderer, 
2009) beziehen sich in der Diskussion von Führungsgrundsätzen auf Studien aus den 1980er und frühen 1990er Jahren. Die nach wie vor große praktische Verbreitung des Instruments legt die Sinnhaftigkeit einer aktuellen Untersuchung nahe. Zudem können die bisher durchgeführten Studien sowohl inhaltlich als auch methodologisch als eher einseitig bezeichnet werden. Rein inhaltsanalytische Untersuchungen ignorieren zumeist den sozialen und diskursiven Kontext, in welchen das Dokument eingebettet ist und vor dessen Hintergrund seine präskriptiven Aussagen als sinnvoll erscheinen. 



\section{Erkenntnisinteresse und Forschungsfragen}

Die vorliegende Untersuchung soll sich diesem Mangel an konzeptionell fundierten qualitativen Studien in der wissenschaftlichen Aufarbeitung von Führungsgrundsätzen widmen. Es ist nicht das Ziel, die Ergebnisse bisheriger Studien zu aktualisieren bzw. zu replizieren. Vielmehr soll mit der vorliegenden Arbeit ein Beitrag geleistet werden, die in der Organisations- und Managementforschung immer stärker geforderte Berücksichtigung der Rolle von Kultur und Bedeutung in organisationalen Kontexten weiterzutreiben. ,Führung' als Phänomen, als eine gesellschaftlich legitimierte Beziehung von Über- und Unterordnung, kann als einer der am stärksten institutionalisierten Aspekte der organisationalen Lebenssphäre bezeichnet werden (vgl. z.B. J. W. Meyer \& Rowan, 1977; Sandner \& Meyer, 2002) und ist deshalb auch nicht begründungsbedürftig, da er als selbstverständlich und normal betrachtet wird. Über die ,optimale“ Art der Führungsbeziehung (bzw. die Möglichkeit einer solchen) hingegen gibt es in Wissenschaft und Praxis bisher keinen breiteren Konsens. Dies zeigt die lange Geschichte der Untersuchung und Diskussion von Führungsstilen, Führungsinstrumenten und Führungsmodellen. Führungsgrundsätze haben das Ziel, die Beziehungen zwischen Führungskräften und MitarbeiterInnen (z.T. auch der MitarbeiterInnen untereinander) im Sinne eines organisationalen Regel- und Wertesystems zu standardisieren und zu normieren (vgl. z.B. Wunderer, 2009: 385). Es ist daher zu erwarten, dass Führungsgrundsätze Elemente verschiedener - wenn auch z.T. impliziter - Führungstheorien beinhalten. Wo immer jedoch die Möglichkeit der bewussten Wahl herrscht, entsteht auch die Notwendigkeit von Begründung und Argumentation. Durch die Analyse der materialen bzw. inhaltlichen Argumentationsmuster im Material - so die Prämisse dieser Arbeit - ist es möglich, die den Führungsgrundsätzen zugrundeliegenden impliziten Theorien bzw. Vorstellungen von Führung und Steuerung zu (re-)konstruieren, vor deren Hintergrund die Texte in ihrem alltagsweltlichen Gebrauch , sinnvoll ${ }^{\circ}$ werden. Eine solche Untersuchung bietet Einblicke in die den Dokumenten zugrundeliegenden Basisannahmen, welche reine Inhaltsanalysen der Regelungsinhalte nicht erreichen können. Die vorliegende Untersuchung geht damit über den bisherigen Korpus an Literatur deutlich hinaus und ergänzt ihn um eine dezidiert diskursanalytische Perspektive. Die forschungsleitende Frage ist zweiteilig und lautet wie folgt: 
Welche (impliziten und expliziten) Vorstellungen von Führung und Steuerung finden sich in kontemporären Führungsgrundsätzen deutschsprachiger Großunternehmen?

Mithilfe welcher rhetorischen Strategien und Ressourcen werden diese Vorstellungen argumentiert und damit im organisationalen Diskurs verankert?

Diese Forschungsfrage wird im Rahmen mehrerer Subfragen konkretisiert.

Subfrage 1: In welchen sozial konstruierten und typisierten Rollen treten Akteure in den Dokumenten auf, und wie werden diese charakterisiert?

Vor dem Hintergrund der Annahme, dass eine Argumentation gewünschter Beziehungsformen immer auf einer entsprechenden Konstruktion der beteiligten Akteure basieren muss, ist zunächst die Frage zu stellen, welche Akteure sich in den Grundsatzdokumenten überhaupt finden und wie deren Rollen jeweils konstruiert werden. Rollen drücken sich über typisierte Tätigkeiten aus (vgl. Berger \& Luckmann, 2000). Ein erster Schritt besteht also darin, genauer zu beleuchten, welche Tätigkeiten mit den jeweiligen Akteurstypen verbunden werden und welche Rollenaspekte sich daraus für diese ergeben.

Subfrage 2: In welche Form(en) der Beziehung treten die dominanten Akteure zueinander?

Führung und Steuerung werden in der gegenständlichen Arbeit als grundsätzlich relationale Phänomene gefasst, welche nur in der Interaktion zwischen verschiedenen Akteuren existieren (vgl. Sandner, 1990; Sandner \& Meyer, 2004). Für eine (Re-)konstruktion der Führungs- und Steuerungsverständnisse ist deshalb zu klären, welche Beziehungsformen zwischen Führungskraft und MitarbeiterIn in den Dokumenten überhaupt zum Ausdruck kommen. Während die grundsätzliche Konstellation von Über- und Unterordnung annahmegemäß nicht hinterfragt wird, ist die Frage nach der Qualität und Art dieser Beziehung von hohem Interesse. Wie im ersten Kapitel ausgeführt, war die Grundidee von Höhns (z.B. 1976) ,Allgemeiner Führungsanweisung ' die Abkehr von einem ,patriarchalisch-autoritären' Führungsstil. In der Folge wurde aber der von ihm vertretene ,bürokratisch-autoritäre' Stil, welcher die persönliche Macht durch die Macht der Regeln ersetzt, stark kritisiert. Moderneren Führungsgrundsätzen wird ein partizipatives bzw. kooperatives Führungsverständnis unterstellt (vgl. Grawert, 1997: 90; Wunderer, 2009: 384) - eine Annahme, die jedoch nicht ohne Widerspruch geblieben ist (vgl. z.B. Breisig, 1987; Kubicek, 1984b). Diese unvollendete Diskussion in der Wissenschaft legt eine differenzierte Untersuchung dieses Aspekts nahe. Ein systematischer Blick soll deshalb auf die Handlungen der Akteure in Bezug zueinander geworfen werden, um die vorgeschlagenen Beziehungsformen zu beleuchten. 
Subfrage 3: Innerhalb welcher inhaltlich-thematischen Argumentationen finden diese Beziehungen statt?

Eine dekontextualisierte Betrachtung von Rollen und Beziehungen greift jedoch zu kurz. Um die (re-)konstruierten Beziehungen im breiteren Kontext des organisationalen (und gesellschaftlichen) Wissensvorrats (vgl. z.B. Keller, 2008: 40), welcher diesen Beziehungen Sinn und Legitimität verschafft, verorten zu können, muss auch die Frage gestellt werden, innerhalb welcher rhetorischen Argumentationsmuster und inhaltlichen Thematiken sich die Führungsbeziehung in den Dokumenten abspielt. An dieser Stelle geht die vorliegende Studie über die bisherige Aufarbeitung inhaltlicher Aspekte von Führungsgrundsätzen (z.B. Jancsary et al., 2006; Tschirky, 1981) hinaus, indem sie nicht nur das Vorkommen eines Themas, sondern vor allem auch dessen - oft nur latent verfügbaren Begründungszusammenhang in Betracht zieht. Warum steht dieses Thema hier? Warum ist es wichtig? Und warum ist die vorgeschlagene Art der Führungsbeziehung innerhalb dieser Argumentation sinnvoll?

Subfrage 4: Wie stehen die in den Subfragen 1-3 untersuchten Aspekte in Beziehung zueinander, und welches argumentative Bild von Führung ergibt sich daraus?

Die ganzheitliche Betrachtung der Rollen(aspekte), Beziehung(en) sowie der argumentative Basis schließlich ermöglicht einen Überblick über die latent repräsentierten Verständnisse von Führung und Steuerung. Organisationale Akteure (vor allem Führungskräfte und MitarbeiterInnen) treten in Dokumenten zueinander in spezifische Beziehungen der Über- und Unterordnung, in welchen sie verschiedene Tätigkeiten in Bezug auf die/den jeweils andere/n durchführen. Diese Beziehungen stehen in einem (mehr oder weniger explizierten) Begründungszusammenhang, welcher auf verschiedene Teilbereiche des gesellschaftlichen Wissensvorrats verweist, in welchen das Individuum ,hineinsozialisiert werden soll (vgl. Berger \& Luckmann, 2000: 148ff.). Eine Betrachtung der vorgeschlagenen Beziehungen unter dem Gesichtspunkt ihrer rhetorischen Argumentation bietet daher Einblicke in die typischerweise evozierten Bezugssysteme, vor deren Hintergrund diese Beziehungen Sinn erlangen und als legitim gelten können.

Das folgende Kapitel gibt durch die Diskussion der Konzepte von Kommunikation, Macht, Herrschaft, Steuerung, Rhetorik und Argumentation einen Überblick über das konzeptionelle Instrumentarium, mit welchem die Forschungsfragen systematisiert und in eine methodologische und methodische Forschungsstrategie übersetzt werden können. 



\section{Konzeptionelle Grundlagen}

\subsection{Grundsätzliche Ausführungen zum konzeptionellen Hintergrund der Untersuchung}

Führungsgrundsätze wurden im ersten Teil der Arbeit als tendenziell eher ,theorielos ' beschrieben. Dies bedeutet einerseits eine größere Freiheit der Perspektive, andererseits geht damit aber auch ein erhöhter Begründungsbedarf einher, da noch kein legitimierter Blick auf das Phänomen existiert. Zunächst soll deshalb festgehalten werden, dass die Ausrichtung der Arbeit eine distinktiv organisationstheoretische ist. Es ist weder das Ziel der Arbeit, Führungsgrundsätze in die Disziplin des Personalmanagements einzuordnen, noch hat die Untersuchung einen führungstheoretischen Fokus (z.B. die Identifikation in der Literatur vorgeschlagener Führungsstile). Vielmehr soll untersucht werden, wie Führungsgrundsätze einen Teil der organisationalen Realität (das Macht- bzw. Herrschaftsverhältnis zwischen Führungskräften und MitarbeiterInnen) konstruieren und welche (diskursiv-rhetorischen) Strategien sie zu diesem Zweck zur Anwendung bringen.

Ausgegangen wird dabei von einer wissenssoziologischen Perspektive (vgl. Berger \& Luckmann, 2000; Keller, 2008; R. E. Meyer, 2006). Dabei werden Prozesse der gesellschaftlichen Objektivierung und der subjektiven Aneignung von Wissensbeständen betont (vgl. Keller, 2008:40). Wissen meint hier alles, was „Bedeutung trägt, Sinn macht oder doch sinnvoll interpretiert werden kann“ (Keller, 2008: 41). Eine Gesellschaft besitzt einen gewissen Vorrat an Wissen, welcher spezifisch (zumeist ungleich) verteilt ist. Es existieren distinktive Strukturen der Wissensverteilung und -differenzierung (vgl. Keller, 2008: 41). Dieser gesellschaftliche Wissensvorrat ermöglicht die sinnvolle Wahrnehmung von Objekten, Handlungen, aber auch der eigenen Person und ihrer Situation innerhalb der Sinnwelt einer (Sub-)Gesellschaft (vgl. Berger \& Luckmann, 2000: 43). Nur wer Teil einer Gesellschaft ist - und somit Zugriff auf deren Wissensvorrat hat kann Dinge, Handlungen, Äußerungen etc. richtig verstehen. Dabei wird Gesellschaft bei Berger und Luckmann (2000) in zweifacher Weise gedacht: sie tritt dem Individuum immer auf subjektive und objektive Art zugleich entgegen. Objektiv ist die Gesellschaft deshalb, weil sie dem Individuum immer schon in Form von Handlungsanweisungen, Regeln, Normen oder Moralvorstellungen als gegeben vorgestellt wird (vgl. Berger \& Luckmann, 2000: 49ff.; Keller, 2008: 42). Objektivierte Wirklichkeit tritt dem Subjekt in Form von Institutionen gegenüber: „Institutionalisierung findet statt, sobald habitualisierte Handlungen durch Typen von Handelnden reziprok typisiert werden“ (Berger \& Luckmann, 2000: 58). Die Gesellschaft ist aber auch subjektive Wirklichkeit, weil das Indi- 
viduum sie im Rahmen der Sozialisierung internalisieren muss. Nur so wird es zum Mitglied einer Gesellschaft (vgl. Berger \& Luckmann, 2000: 140). Die Dialektik der gesellschaftlichen Konstruktion von Wirklichkeit besteht somit in den drei Prozessen der Externalisierung, Objektivation und Internalisierung (vgl. Berger \& Luckmann, 2000: 139). Für Führungsgrundsätze bedeutet dies, dass ihre Aussagen, um sinnvoll zu wirken, sich im sozialen Wissensvorrat einer (Sub-)Gesellschaft verorten müssen. Sie kommunizieren spezifische Regeln, Werte und Normen an die Organisationsmitglieder, um damit eine sekundäre Sozialisierung (vgl. Berger \& Luckmann, 2000: 148) dieser in die spezifische Sinnwelt der Organisation zu erreichen. Die Konzepte der Führung und Hierarchie können in modernen, westlichen Industriegesellschaften als hochgradig institutionalisiert betrachtet werden (vgl. Sandner \& Meyer, 2002). Dennoch benötigen auch diese Institutionen eine gewisse Legitimierung. „Legitimation ,erklärt' die institutionale Ordnung dadurch, daß sie ihrem objektivierten Sinn kognitive Gültigkeit zuschreibt. Sie rechtfertigt die institutionale Ordnung dadurch, daß sie ihren pragmatischen Imperativen die Würde des Normativen verleiht“" (Berger \& Luckmann, 2000: 100). Führungsgrundsätze mögen also spezifische Regeln für das Führungsverhalten beinhalten, gleichzeitig dienen sie aber auch als Instrument der Legitimierung von Führung und Hierarchie an sich. Diese Legitimierung wird innerhalb der Dokumente vor allem durch rhetorische Mittel erreicht.

Das vorgeschlagene konzeptionelle Instrumentarium dieser Arbeit ist somit dreiteilig: (a) Zum einen werden Führungsgrundsätze als spezifisches Genre der organisationalen Kommunikation begriffen. Das folgende Kapitel widmet sich deshalb den Fragen, was in der vorliegenden Arbeit unter den Begriffen der organisationalen Kommunikation und des Genres verstanden wird und was dies für einen kommunikationstheoretischen Blick auf Führungsgrundsätze bedeutet. (b) Zum zweiten werden in der vorliegenden Arbeit die Phänomene der Macht und Herrschaft als wesentliche Elemente jeglicher sozialer Organisation begriffen. Da die (Re-)konstruktion der (impliziten) Steuerungsbeziehungen in Führungsgrundsätzen das erklärte Untersuchungsziel der Arbeit darstellt, wird in einem weiteren Kapitel ausführlich diskutiert, inwiefern und auf welchen Ebenen diese Aspekte Führungsgrundsätze durchdringen und welche Strategien den Verfassern von Führungsgrundsätzen grundsätzlich zur Verfügung stehen, um eine Steuerungswirkung in verschiedenen Bereichen organisationaler Realität (Handlungen/Entscheidungen, Werte/Normen, Rollen/Identitäten) zu erreichen. (c) Schließlich ergibt sich aus der Kombination der ersten beiden Ansätze, dass eine Untersuchung von Machtstrategien in Texten organisationaler Kommunikation notwendigerweise eine Analyse der sprachlichen bzw. rhetorischen Mittel 
der Texte berücksichtigen muss. Eine kurze Diskussion relevanter Theorien und Ansätze im Bereich der Rhetorik bildet somit den Abschluss.

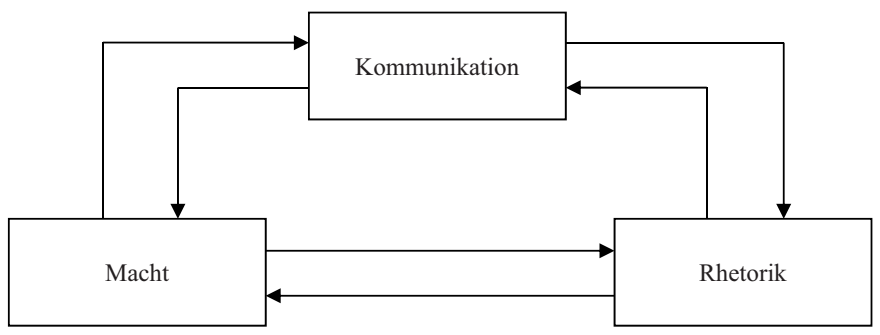

Abbildung 7: Konzeptioneller Hintergrund der Untersuchung

Die lineare Form, in welcher wissenschaftliche Texte verfasst werden, zwingt dazu, die Argumentation ebenfalls in linearer Form vorzubringen. Tatsächlich aber zeigt Abbildung 7, dass die Reihenfolge der Diskussion dieser drei Aspekte im Wesentlichen arbiträr bzw. frei wählbar ist. Kommunikation, Macht und Rhetorik formen eine Triade, welche in der Praxis nicht auflösbar ist. In der vorliegenden Arbeit wurde ein Aufbau gewählt, der grob der Logik der Argumentation vom Allgemeinen zum Spezifischen hin folgt: Kommunikation als performative Konstruktion organisationaler Realität, Machtbeziehungen als Inhalt und Leistung organisationaler Kommunikation und Rhetorik als Arsenal kommunikativer Strategien zur Überzeugung von Organisationsmitgliedern und Herstellung bzw. Stabilisierung von Macht- und Herrschaftsverhältnissen. Da die Linearität aber notwendigerweise eine künstliche ist, lässt es sich nicht vermeiden, in der weiteren Diskussion dieser Elemente an bestimmten Stellen vor und zurück zu springen, um dem ganzheitlichen Verständnis des konzeptionellen Hintergrunds Rechnung zu tragen.

\subsection{Organisationale Kommunikation}

\subsubsection{Gegenstand und Begriffsabgrenzung}

Yates und Orlikowski (1992: 299) bezeichnen menschliche Kommunikation als „central to organizational action“ bzw. noch prägnanter als „the fundamental activity through which social interaction is accomplished" (Orlikowski \& Yates, 1994: 541). Damit ist bereits auf die zentrale Rolle der Kommunikation bzw. Sprache in der Herstellung organisationaler Realität hingewiesen (vgl. auch Berger \& Luckmann, 2000: 36ff.). Als Disziplin blickt Organizational Coтmu- 
nication auf eine lange Geschichte zurück (für einen historischen Überblick siehe z.B. Tompkins \& Wanca-Thibault, 2001). Die Frage nach dem eigentlichen Gegenstand der Disziplin gestaltet sich als entsprechend komplex. So führt z.B. Deetz (2001: 4) aus, die Frage nach einer Definition solle besser durch die Frage „What do we see or what are we able to do if we think of organizational communication in one way versus another?" ersetzt werden. Er trägt damit der Tatsache Rechnung, dass jede Definition notwendigerweise gewisse Möglichkeiten einschließt und andere ausschließt und deshalb fundamental politisch wirkt ${ }^{4}$. Abgrenzungen und Definitionen sind aber für diese Untersuchung dennoch nötig, damit die/der LeserIn die Ergebnisse vor dem Hintergrund eines spezifischen theoretischen Fundaments verstehen kann. Es soll deshalb mit einer sehr grundsätzlichen Definition gestartet und das Argument von dort aus erweitert werden:

„[Discussions about communication processes] have been concerned with the systematic manners by which communication practices can be used to help coordinate and control the activities of organizational members and relations with external constituencies.“ (Deetz 2001: 3)

So breit diese Definition auch ist, sie ermöglicht dennoch die Ableitung einiger tragender Elemente. Zum einen handelt es sich um systematische Kommunikation. Dies legt nahe, dass diese Kommunikationsprozesse intentional geplant sind und auch so ablaufen. Zum anderen ist die Kommunikation zielgerichtet. Sie beabsichtigt eine Koordinierung und Steuerung des Verhaltens von Organisationsmitgliedern sowie des Verhältnisses zu externen Anspruchsgruppen. Führungsgrundsätze als Form organisationaler Kommunikation sind durch diese Definition ganz offensichtlich abgedeckt. Sie beinhalten intentionale, systematische Kommunikationsformen, welche das Verhalten organisationaler Akteure (v.a. Führungskräfte und MitarbeiterInnen) im Unternehmen steuern sollen (vgl. Wunderer, 2009: 385).

Deetz (2001: 4ff.) unterscheidet des Weiteren drei verschiedene Foki und Ausprägungen, wie organisationale Kommunikation konzeptionell abgegrenzt werden kann: (a) organisationale Kommunikation als Tätigkeit spezialisierter Abteilungen und Disziplinen, (b) organisationale Kommunikation als Phänomen in Organisationen, welches isoliert untersucht werden kann und (c) organisationale Kommunikation als konstitutiv für Organisation(en), als spezifischer

4 Auch wissenschaftliche Texte entfalten - in Analogie zu Führungsgrundsätzen und anderen organisationalen Dokumenten - Machtwirkung dahingehend, als die/der AutorIn manche Stimmen zulässt und andere ausblendet und damit bestimmten Konzepten strategisch gewählte Bedeutungen verleiht. Dieser Mechanismus kann nicht ,ausgeschaltet", sehr wohl aber offen gelegt werden. 
Blickwinkel auf Organisation(en) und als Erklärungsansatz für diese (vgl. auch Deetz, 1995). In der vorliegenden Arbeit wird grundsätzlich die dritte Perspektive eingenommen. In der Untersuchung wird die realitätsstiftende Kraft von Sprache und Rhetorik in der Schaffung organisationaler Wirklichkeit betont (vgl. Berger \& Luckmann, 2000: 36ff.). Organisation und Kommunikation sind somit nicht mehr klar voneinander abgrenzbar (vgl. auch Orlikowski \& Yates, 1994: 541). Als dritte Variable - so wird im weiteren Verlauf der Arbeit argumentiert - ist aber auch Macht nicht aus dieser Beziehung wegzudenken. Wer kommuniziert, der organisiert und verdinglicht, stabilisiert oder hinterfragt notwendigerweise bestehende Macht- und Herrschaftsverhältnisse (vgl. z.B. C. Conrad \& Ryan, 1985: 236). Diese Beziehung lässt sich an beliebigen Aussagen im Textkorpus der untersuchten Führungsgrundsätze illustrieren:

Führung stellt sicher, dass jeder Mitarbeiter für den persönlichen Aufgabenbereich Verantwortung übernimmt und im Sinne des Gesamtunternehmens handelt. (D 15)

Die Aussage ist ein kommunikativer Akt. Sie organisiert durch Bezug auf und Stabilisierung eines Diskurses, welcher das Primat der Unternehmensziele (,im Sinne des Gesamtunternehmens"), die Bedeutung individueller Verantwortung (,Verantwortung übernimmt“), das Prinzip der Arbeitsteilung (,den persönlichen Aufgabenbereich“) und die formale Ablaufstruktur („Führung stellt sicher") betont. Die Aussage konstituiert aber auch einen Versuch der Machtausübung, indem sie gegenüber der/dem MitarbeiterIn in zweifacher Weise eine Unterordnungsaufforderung kommuniziert, und zwar unter die Unternehmensziele und unter die Anweisungen einer - in diesem Fall ,entpersönlichten ' Führung. Organisationale Kommunikation - so wie sie in der gegenständlichen Arbeit verstanden wird - ist deshalb immer sowohl performativ (sinn- bzw. wirklichkeitsstiftend) als auch politisch.

\subsubsection{Führungsgrundsätze als kommunikatives Genre}

Führungsgrundsätze werden in der vorliegenden Arbeit als spezifisches Genre der Unternehmenskommunikation verstanden. In der Definition von Yates und Orlikowsky (1992: 301) sind Genres ,a typified communicative action invoked in response to a recurrent situation". Eine solche rhetorische Situation besteht aus drei Elementen: (a) der ,Dringlichkeit“ (exigence), (b) dem Publikum bzw. den AdressatInnen/Bezugsgruppen (audience) und (c) gewissen Einschränkungen (constraints), welche unter Umständen den Ausdruck der Dringlichkeit beeinflussen können (vgl. Yates \& Orlikowski, 1992: 301). Die Dringlichkeit beschreibt dabei etwas, das ,getan werden muss', ein gewisses Bedürfnis bzw. eine Notwendigkeit (vgl. auch Eggins \& Martin, 1997). Ein Genre ist somit immer 
zielgerichtet: sein Zweck (,socially recognized communicative purpose“, vgl. Orlikowski \& Yates, 1994: 543) ist die Lösung eines spezifischen Problems. Dieses Problem ist dabei weder ausschließlich objektiv noch ausschließlich subjektiv, sondern es stellt eine Form sozialen Wissens dar, ,a mutual construing of objects, events, interests and purposes that not only links them but also makes them what they are: an objectified social need“" (Miller, 1983: 157, zit. in Yates \& Orlikowski, 1992: 301). Genres sind also soziale Institutionen der Kommunikation (vgl. auch Knoblauch \& Luckmann, 2004; Luckmann, 2002).

Das in der relevanten Literatur diskutierte spezifische Problem (also die Dringlichkeit), welches Führungsgrundsätze adressieren, stellt die bereits diskutierte Regelung und Standardisierung des Verhältnisses zwischen Führungskräften und MitarbeiterInnen (vgl. Hoffmann, 1989: 168) zur Vermeidung von Spannungen durch verschiedene Führungsformen im Unternehmen (vgl. Höhn, 1977: 322) und somit die Formulierung klarer Grundsätze für das Führungshandeln (vgl. Lattmann, 1975: 47) dar. Letztlich wird als Problem neueren Datums auch die Regelung der Beziehungen zwischen MitarbeiterInnen auf gleicher Ebene (Wunderer, 2009: 385) inkludiert. Das Problem der Steuerung des Verhaltens von Organisationsmitgliedern ist aber weder neu noch auf Führungsgrundsätze beschränkt. Die Frage muss folglich weiter lauten, warum gerade im deutschsprachigen Raum (und nur dort) das Problem der Legitimierung und Steuerung von Führungshandeln über unpersönliche Dokumente ,gelöst' wird. Erste Erklärungsansätze wurden bereits diskutiert. Die empirische Untersuchung soll weitere Aufschlüsse über die konkrete ,exigence' bieten. Als Publikum bzw. AdressatInnen richten sich Führungsgrundsätze an Führungskräfte und MitarbeiterInnen, wobei der Schwerpunkt auf Führungskräften liegt (vgl. Albach, 1976: 748; Hoffmann, 1989: 178; Wunderer \& Klimecki, 1990: 137f.). In Führungsgrundsätzen kann auch die von Yates und Orlikowski (1992: 301) geforderte Ähnlichkeit in „substance and form“ ${ }^{\text {(5 }}$ innerhalb eines Genres festgestellt werden (vgl. auch Orlikowski \& Yates, 1994: 543ff.). Jancsary, Knassmüller und Sandner (2006) stellen eine große Homogenität der Inhalte von Führungsgrundsätzen fest, und Ebert (1997) reduziert Führungsgrundsätze auf eine kleinere Anzahl von Proto- bzw. Idealtypen textlicher Darstellungsform ${ }^{6}$. Damit können Führungsgrundsätze in der vorliegenden Arbeit als Genre der Unter-

5 „When a speaker employs a genre, expectations are created both in the speaker and in the audience [...] An expectation of genre establishes the rhetorical parameters of a text, determining not only its structure but also its vocabulary, syntax, argumentative moves, and narrative appeals. The speaker who oversteps these parameters, betraying audience expectations, often provokes a negative reaction" (Gill \& Whedbee, 1997: 164).

6 Für eine kurze Charakterisierung der materiellen Eigenschaften der Dokumente im Korpus siehe auch Kapitel 5.3. 
nehmenskommunikation klassifiziert werden, und zwar auf der Ebene von Genres ,that are specific to organizations within certain societies or particular cultures“ (Yates \& Orlikowski, 1992: 304). Diese Einschränkung muss getroffen werden, da Führungsgrundsätze nur im deutschen Sprachraum Verbreitung finden.

Genres sind charakterisiert durch spezifische Regeln (,genre rules“, vgl. Yates \& Orlikowski, 1992: 302). Diese Regeln werden von Organisationsmitgliedern explizit oder implizit beachtet, wenn sie sich in der Kommunikation eines bestimmten Genres bedienen. Dabei muss nicht jede Verwendung eines Genres alle Regeln befolgen - gewisse Variationen sind also möglich - aber ein Text muss genügend dieser konstitutiven Regeln beinhalten, um als Teil eines spezifischen Genres erkannt zu werden und Legitimität zu erlangen (vgl. Yates \& Orlikowski, 1992: 302f.). Durch diese institutionalisierten und damit objektivierten Gemeinsamkeiten eines Genres (,institutionalized template for social action“, vgl. Orlikowski \& Yates, 1994: 542) - und damit der Führungsgrundsätze im untersuchten Textkorpus - wird es möglich, durch eine Analyse dieses präskriptiven Genres der Unternehmenskommunikation ein besseres Verständnis der Führungs- und Steuerungsaspekte, die in deutschsprachigen Unternehmen propagiert werden (der ,Problemlösung' durch das Genre), zu erreichen.

\subsubsection{Metaphern organisationaler Kommunikation}

Eine einflussreiche Diskussion verschiedener Möglichkeiten, wie die Konzepte der Kommunikation und Organisation verstanden und zueinander in Beziehung gesetzt werden können, bietet der Überblick von Putnam und Boys (2006). Durch die Rekonstruktion und Analyse verschiedener Metaphern im Feld der Organizational Communication bieten sie einen praktischen Überblick über die verschiedenen Perspektiven auf das Thema. Dabei verstehen sie Metaphern als „ways of seeing as if it were something else“ (Putnam \& Boys, 2006: 542). Der Wert ihrer Systematik für die vorliegende Arbeit besteht darin, dass sie die verschiedenen Möglichkeiten, wie man Kommunikation und Organisation verstehen kann, auf übersichtliche Weise präsentieren. Der Blick auf diese beiden zentralen Konzepte formt und beeinflusst die weitere Analyse, von der Konzeption von Macht angefangen über die notwendigen und relevanten Daten bis hin zu den Methoden der Datenauswertung und -interpretation. Vor allem aber richten die unterschiedlichen Metaphern den Blick der Forscherin/des Forschers auf unterschiedliche Fragen. Es soll daher anhand einer kurzen Diskussion der Metaphern von Putnam und Boys (2006) aufgezeigt werden, welche Konzepte von Organisation und Kommunikation der vorliegenden Arbeit zugrunde gelegt 
werden und in welcher Beziehung diese zu den forschungsleitenden Fragen stehen.

Tabelle 3: Metaphern organisationaler Kommunikation (in Anlehnung an Putnam \& Boys, 2006)

\begin{tabular}{|c|c|c|}
\hline Metapher & Rolle der Kommunikation & Rolle der Organisation \\
\hline Conduit & $\begin{array}{l}\text { Kommunikation als Kanal, der } \\
\text { Nachrichten/Botschaften überträgt }\end{array}$ & $\begin{array}{l}\text { Organisation als Container, in wel- } \\
\text { chem sich die Kanäle befinden }\end{array}$ \\
\hline $\begin{array}{l}\text { Information } \\
\text { Processing }\end{array}$ & $\begin{array}{l}\text { Fokus auf Art und Fluss der Infor- } \\
\text { mation }\end{array}$ & $\begin{array}{l}\text { Organisation als Karte oder Bahn für } \\
\text { die gezielte Übertragung und Verar- } \\
\text { beitung von Kommunikation }\end{array}$ \\
\hline Linkage & $\begin{array}{l}\text { Kommunikation als Verbindung zwi- } \\
\text { schen Individuen, Gruppen und Insti- } \\
\text { tutionen }\end{array}$ & Organisation als Beziehungsnetzwerk \\
\hline Performance & $\begin{array}{l}\text { Kommunikation als soziale Interak- } \\
\text { tion }\end{array}$ & $\begin{array}{l}\text { Organisation als koordiniertes Ver- } \\
\text { halten }\end{array}$ \\
\hline Discourse & $\begin{array}{l}\text { Kommunikation als ,language in } \\
\text { use }\end{array}$ & $\begin{array}{l}\text { Organisation als Beziehung zwischen } \\
\text { Texten }\end{array}$ \\
\hline Symbol & Kommunikation als Sinnkonstruktion & Organisation als literarischer Text \\
\hline Voice & $\begin{array}{l}\text { Kommunikation als Ausdruck und } \\
\text { Unterdrückung }\end{array}$ & Organisation als Arena \\
\hline Contradiction & $\begin{array}{l}\text { Kommunikation als gegensätzliche } \\
\text { Kräfte bzw. Beziehungen zwischen } \\
\text { widersprüchlichen Aussagen }\end{array}$ & $\begin{array}{l}\text { Organisation als ständiger Kampf } \\
\text { zwischen Oppositionen }\end{array}$ \\
\hline
\end{tabular}

Die ersten drei Metaphern, welche sich in erster Linie auf die Übertragungsaspekte von Kommunikation richten (vgl. Putnam \& Boys, 2006: 544) sind für die vorliegende Untersuchung von eher geringem Interesse. Fragen der Informationsübertragung, der Informationsmenge und der Koordination durch Kommunikation werden zwar innerhalb der Dokumente diskutiert und dadurch in der Darstellung der Untersuchungsergebnisse dieser Arbeit wieder aufgegriffen, der Anspruch der vorliegenden Untersuchung besteht jedoch nicht in der Einschätzung der transmissiven Effizienz von Führungsgrundsätzen. Ebenso thematisieren die Discourse Metapher mit ihrem engen Fokus auf Textualität und Intertextualität, die Symbol Metapher mit der Betonung von Geschichten, Ritualen und 
kulturellen Artefakten ${ }^{7}$ und die Contradiction Metapher mit ihrem Fokus auf diametral entgegengesetzte Kommunikationsformen lediglich periphere Aspekte der Arbeit.

Die übrigen beiden Metaphern jedoch, die sich stärker auf die Aspekte von Interaktion, Realitätskonstruktion und Macht konzentrieren, beleuchten zwei der zentralen Aspekte der Untersuchung. Die Performance Metapher zeigt, dass organisationale Realität durch kommunikative Praktiken konstruiert wird (vgl. Putnam \& Boys, 2006: 548). Damit sind Führungsgrundsätze in dieser Perspektive eine Ressource zur Realitätskonstruktion. Die Organisation besteht in einem System koordinierter Handlungen, welches organisationale Wirklichkeit durch Herstellung und Stabilisierung interaktionaler und kognitiver Muster herstellt und stabilisiert. Kommunikation erzeugt organisationale Realität. Die Voice Metapher schließlich betont, dass dieser Prozess der Realitätskonstruktion nicht neutral ist. Sie lenkt den Fokus auf Phänomene der Dominanz und Hegemonie, der Unterdrückung von Konflikt zugunsten von Routine und Herrschaftssicherung, der Abwesenheit unterdrückter Stimmen und des Widerstands gegen dominante Diskurse (Putnam \& Boys, 2006: 556ff.). Damit beschreiben diese beiden Metaphern zwei Grundannahmen der gegenständlichen Untersuchung, welche bereits zu Beginn der Diskussion zu organisationaler Kommunikation offen gelegt wurden: (a) Kommunikation ist performativ und (b) Kommunikation ist politisch. Dieser politische Aspekt von Kommunikation soll nun durch die ausführliche Diskussion von Macht, Herrschaft, Steuerung und in weiterer Folge der Rhetorik weiter erläutert werden.

\subsection{Macht, Herrschaft und Steuerung}

„All forms of organization are forms of organization of social relations. All social relations involve power relations. " (vgl. Clegg, 2009: 327)

Anhand von Führungsgrundsätzen lässt sich sehr gut demonstrieren, wie stark die Themen von organisationaler Kommunikation und Macht miteinander verflochten sind. Nicht nur wird Macht über Kommunikation verbreitet bzw. bedient sich Macht der Kommunikation als Vehikel, sondern jede Form der organisationalen Kommunikation, egal, ob es sich dabei um Communiqués, Firmenzeitungen, Leitbilder, informelle Gespräche in der Kantine oder eben Führungs-

7 Die Betonung symbolischer Kommunikation, welche ebenfalls in der Symbol-Metapher stattfindet, ist hingegen in der vorliegenden Untersuchung hochrelevant. In der Systematik von Putnam und Boys (2006) beinhaltet diese Metapher jedoch in erster Linie Untersuchungen des narrativen Paradigmas, welchem sich die gegenständliche Arbeit nicht primär zugehörig fühlt. 
grundsätze handelt, vermittelt, verhandelt, vergegenständlicht oder hinterfragt bestehende Machtstrukturen, und zwar sowohl durch die Form Ihrer Verwendung (wer wird inkludiert/exkludiert) als auch durch ihre Inhalte und die Werte und Normen, welche sie vermittelt: ,[...] power and communication are essentially identical processes" (C. Conrad \& Ryan, 1985: 236).

Bevor die Diskussion zu Macht, Herrschaft und Steuerung auf den Phänomenbereich der Führungsgrundsätze angewendet werden kann, müssen zunächst die zentralen relevanten Konzepte definiert werden. Dieser Diskussion widmet sich das folgende Kapitel. Im Anschluss daran werden sukzessive die Erkenntnisse der vorgestellten Theorien auf den Forschungsgegenstand übertragen.

\subsubsection{Begriffsabgrenzungen}

Sowohl in der Soziologie als auch in der Organisationsforschung ist das Konzept der Macht nicht unproblematisch. Während ein grundsätzlicher Konsens darüber besteht, dass Macht ein wichtiger, wenn nicht sogar der herausragende Faktor in der Organisationsforschung sein sollte, bleibt die Frage, was sich genau hinter diesem Begriff verbirgt, ein umstrittenes Thema (vgl. z.B. Sandner \& Meyer, 2004: 756). Jedes Verständnis von Macht ist „essentially contested“ (Lukes, 2005: 14). Die meisten Abhandlungen zu Macht und Herrschaft in Organisationen beginnen deshalb zunächst mit der präventiven Klarstellung, dass der Begriff der Macht ebenso komplex wie wichtig ist:

„No concept in sociological and organizational theory is more ubiquitous and perplexing than ,power“" (C. Conrad \& Ryan, 1985: 235).

„Yet, as is the case for many fundamental notions of the social sciences, the notion of power is as complex as it is fuzzy." (van Dijk, 2008: 1).

„There have been countless endeavors to define power more precisely and conclusively, all of their results remain as unsatisfactory as ever" (Göhler, 2009: 27).

Dementsprechend vielseitig stellen sich auch die verschiedenen Versuche der Definition und Operationalisierung von Macht in der Sozialwissenschaft dar. Einen Überblick über verschiedene Ansätze der Macht und deren Ursprünge geben z.B. Sandner und Meyer $(2003,2004,2006)$ in verschiedenen Handwörterbüchern. Sie unterscheiden zwischen (a) personenbezogenen Machtkonzepten, welche Macht an die „Person des Überlegenen, sein Handeln, seine Eigenschaften und Ressourcen“ (Sandner \& Meyer, 2003: 1137) knüpfen; (b) relationalen Machtkonzeptionen, welche die gegenseitige Abhängigkeit der betroffenen Akteure betonen: z.B. die Theorie der strategischen Bedingungen von Hickson et al. (1971), der Resource Dependency Ansatz (vgl. z.B. Pfeffer \& Salancik, 1978) sowie mikropolitische Ansätze (vgl. z.B. Crozier \& Friedberg, 1979); und 
(c) (Post-)Strukturalistische und systemtheoretische Ansätze, welche über interaktionistische Konzepte hinausgehen und das Wirken sozialer Strukturen und Systeme betonen (vgl. z.B. Clegg, 1989; Luhmann, 1977). Haugaard und Clegg (2009) diskutieren weitere Facetten der Macht wie (a) die Unterscheidung zwischen ,power to' (konsensualer Blick auf Macht; Macht als ,Ermächtigung' zur Handlung, vgl. z.B. Arendt, 1970; Parsons, 1964) und ,power over' (Macht als Dominanz, Unterdrückung, Unterordnungsaufforderung) ${ }^{8}$; (b) die Unterscheidung zwischen Macht als direkte Beeinflussung von Handlungen und Macht als systemisches Phänomen, welches soziale Realität schafft und erhält (diese Unterscheidung entspricht in ihrer Grundstruktur jener zwischen relationalen und [post-]strukturalistischen Ansätzen bei Sandner und Meyer); und (c) die Unterscheidung zwischen legitimer und illegitimer Macht (vgl. Haugaard \& Clegg, 2009: 2f.). Diese verschiedenen Forschungsprogramme führen die Autoren zu dem Schluss, dass Macht kein singuläres Konzept ist, sondern vielmehr ein ,Cluster' von Konzepten, welche eine gewisse ,Familienähnlichkeit' aufweisen (vgl. Haugaard \& Clegg, 2009: 3f.). Mumby (2001) unterscheidet für die Disziplin der Organizational Communication verschiedene Herangehensweisen an die Machtthematik. Während sich Arbeiten im Bereich der ,systems-rationality'in erster Linie auf Entscheidungen, Ressourcenkontrolle und Abhängigkeiten konzentrieren, fokussieren sich interpretative Machtkonzepte vor allem auf normative Systeme geteilter Bedeutungen und Werte. Kritische Machttheorien sehen Machtausübung vor allem in Tiefenstrukturen und hegemonischen Praktiken, postmoderne Theorien hingegen betonen die Macht des Diskurses und die positive (sprich: konstruktive) Leistung von Macht. Feministische Theorien schließlich verorten Macht vor allem in ,gendered systems of exploitation“ und im Patriarchat (vgl. Mumby, 2001: 594).

Diese Versuche, das Feld der Machtforschung zu systematisieren, bieten unterschiedliche Blickwinkel auf die Forschung, welche sich zum Teil ergänzen und/oder überschneiden. Aufgrund der eben dargestellten Vielzahl und Diversität der verschiedenen Konzeptionen von Macht ist es im Rahmen dieser Arbeit nicht möglich, sich der Frage nach dem ,richtigen“ Machtbegriff ausführlich zu widmen. Überhaupt muss die Frage gestattet sein, ob es sinnvoll ist, Macht abschließend definieren zu wollen (vgl. Haugaard \& Clegg, 2009). Für die vorliegende Arbeit sollen deshalb in der Folge verschiedene Ansätze der Konzeptionalisierung von Macht diskutiert werden, welche an die wissenssoziologische Aus-

8 „Power over means power over other people, enforcement of one's own intentions over those of others, and is thus only conceivable in a social relation. Power to, on the other hand, is not related to other people. It is an ability to do or achieve something independent of others. It is not a social relation." (Göhler, 2009: 27) Für eine detaillierte Diskussion siehe auch Clegg, Courpasson und Phillips (2007: 190ff.). 
richtung der Arbeit anschlussfähig sind und die für die (Re-)konstruktion von Führungs- und Steuerungsaspekten in Texten der organisationalen Kommunikation fruchtbar erscheinen. Aus der obigen Diskussion der Hauptthemen und Differenzlinien innerhalb der Forschung ergibt sich vor allem die Frage nach dem Ursprung und Ort der Macht: Wird Macht durch den individuellen Akteur oder durch eine soziale Struktur (bzw. den Diskurs) ausgeübt? Für die vorliegende Untersuchung werden nach der Taxonomie von Sandner und Meyer (2003, 2006) sowohl relationale Machtkonzeptionen als auch (post-)strukturalistische als besonders interessant (und nicht unbedingt gegenseitig exklusiv) betrachtet. Erstere lenken den Blick auf die Beziehung zwischen den an einer Machtbeziehung beteiligten Parteien. Konventionelle (und implizite) Theorien der Führung als interaktionale Machtausübung zwischen Führungskraft und MitarbeiterIn können sehr gut mithilfe solcher relationalen Machttheorien diskutiert werden. Im folgenden Kapitel wird deshalb vor allem das Konzept von Sandner (1990) ausführlich diskutiert. Diese handlungsorientierten Machtkonzepte können jedoch nicht alle Aspekte des Machtphänomens erklären (vgl. Sandner \& Meyer, 2003: 1140). Deshalb werden anschließend auch Ansätze diskutiert, welche vor allem die Macht des Diskurses näher erläutern und mit einer wissenssoziologischen Betrachtungsweise kompatibel sind (zu Wissenssoziologie und Diskursforschung vgl. z.B. Keller, 2008, 2011). Diskurse äußern sich in Dispositiven (z.B. Führungsgrundsätzen) und bieten Ressourcen für die Konstruktion von Wirklichkeit und Identität (vgl. Keller, 2008: 258ff.). Während Diskurse nicht direkt gesteuert werden können, haben mächtige Gruppen und Individuen dennoch die Möglichkeit, Diskurse anzupassen oder die (Ir)Relevanz spezifischer Diskurse zu argumentieren, um eine ihnen dienliche Wirklichkeitsauffassung zu propagieren. Solche Diskurse zur Führung beeinflussen letztlich, welche Aussagen in Führungsgrundsätzen überhaupt möglich sind. Auf der anderen Seite sind Führungsgrundsätze Teil des Diskurses und wirken auch wieder auf ihn zurück. Führung und Steuerung werden also nicht nur in der direkten Interaktion zwischen Führungskraft und MitarbeiterIn konstruiert, sondern auch auf einer abstrakteren Ebene durch den Führungsdiskurs.

Die Abgrenzung von Macht und Herrschaft fällt aufgrund der Vielzahl unterschiedlicher Konzeptionen nicht leicht (vgl. Sandner \& Meyer, 2004: 757). Herrschaft lässt sich aber von Macht durch ihre relative Dauerhaftigkeit abgrenzen, während Macht als dynamisches, flüchtiges und fragiles Phänomen betrachtet wird (vgl. Sandner \& Meyer, 2004: 757). Der Begriff der Herrschaft ist untrennbar mit Max Weber verbunden (vgl. Sandner \& Meyer, 2004; Steyrer, 1989; G. Wagner, 2007). Für Weber ist Herrschaft „die Chance, für einen Befehl bestimmten Inhalts bei angebbaren Personen Gehorsam zu finden“ (Weber, 1972: 28, zit. in Sandner \& Meyer, 2004: 762). Dabei unterscheidet er zwischen 
charismatischer (Glaube an die Person des Führers), traditionaler (Gültigkeit überlieferter Gewohnheiten) und legaler (Glaube an die Gültigkeit des gesatzten Rechts) Herrschaft (vgl. Sandner \& Meyer, 2004: 762; Steyrer, 1989). Herrschaft benötigt nach Weber Legitimität (vgl. Sandner \& Meyer, 2004: 762f.) und entsteht nach Lueger (1989: 188) durch eine „Entpersönlichung, Generalisierung und Formalisierung von Macht". Macht löst sich von der konkreten Situation und wird allgemein gültig. Die Führungskraft ist der/dem MitarbeiterIn generell übergeordnet und muss das nicht in jeder Interaktionssituation wieder erreichen. Die Beziehung zwischen Macht und Herrschaft ist eine dialektische: „Erst die Institutionalisierung von Macht bedeutet Herrschaft, welche einerseits aus Machtbeziehungen entspringt, in einmal verfestigtem Zustand aber den Rahmen für diese abgibt“ (M. Lueger, 1989: 188; vgl. auch Sandner, 1990: 172). Herrschaft, welche unmittelbare Machtbeziehungen z.B. durch Faktoren wie Hierarchien, Geschichte oder Verträge substituiert (vgl. M. Lueger, 1989: 188), wirkt quasi als ,Überbau' in unmittelbare Machtbeziehungen hinein. Eine Drohung, ein Versprechen oder ein Überzeugungsversuch erscheinen vor dem Hintergrund einer spezifischen Herrschaftskonfiguration als ,normal' und legitim (vgl. auch G. Wagner, 2007: 17f.). Dies garantiert nicht deren Wirkung, senkt aber annahmegemäß die Wahrscheinlichkeit von Widerstand. Derart institutionalisierte Machtausübung findet sich in Organisationen vor allem durch Regelungen der Hierarchie und Ablauforganisation (vgl. Sandner \& Meyer, 2002). Führungsgrundsätze können folglich in ihren Regelungen darauf aufbauen, dass eine grundsätzliche Unterordnung der MitarbeiterInnen unter ihre Vorgesetzten bereits selbstverständlich und legitimiert ist. Führungsgrundsätze replizieren und verstärken damit Herrschaftsaspekte in Organisationen. Die Hierarchie wiederum verleiht auch den Regelungen in Führungsgrundsätzen Legitimität. Dadurch ist die von Lueger (1989: 188) postulierte Dialektik zwischen Macht und Herrschaft abgebildet.

Steuerung schließlich wird in Anlehnung an Sandner (1990: 159) als jene Handlungen definiert, „die zum Zwecke der Realisierung der Interessen des A auf eine Unterwerfung v.a. kollektiver B unter die Unterordnungsaufforderungen des A gerichtet sind." Diese Definition ist spezifischer als jene von Tompkins und Cheney (1985: 180), welche ,control' als ,exercise or act of achieving a goal" definieren. Fischer (2009: 56) versteht unter Steuerung aus handlungstheoretischer Sicht die ,absichtvolle Beeinflussung sozialer Prozesse“ mit dem Ziel, eine angestrebte Ordnungsbildung unter den Adressaten zu erreichen. Diese Steuerung kann entweder direkt (durch unmittelbare Anweisung bzw. Befehl) oder indirekt erfolgen. Indirekte Steuerung liegt nach dem hier vertretenen Verständnis dann vor, wenn Machtbeziehungen in Strukturen gegossen werden und damit institutionalisiert und über Technologien, (Verfahrens-)Re- 
geln, Sozialtechniken oder Sinnstrukturen auf die potentiell Unterworfenen wirken (vgl. Sandner, 1990: 181ff.; Sandner \& Meyer, 2004: 760).

\subsubsection{Die relationale Machtperspektive}

Zum Einstieg in die Thematik sollen nun die Grundlagen eines relationalen Machtverständnisses im Detail erläutert werden. Die Grundlage dieser Diskussion bildet die Konzeption von Sandner (1990). Dies ist zum einen darin begründet, dass Sandner in sehr ausführlicher Form verschiedene Aspekte der Relationalität von Macht argumentiert, zum anderen aber auch darin, dass er seine Machtkonzeption ausdrücklich für die Disziplin der Betriebswirtschaft entwickelt und dass er in einzelnen Teilen seiner Arbeit explizit einen Anschluss an die wissenssoziologischen Überlegungen von Berger und Luckmann (2000) herstellt. Ausgangspunkt der Diskussion bildet Max Webers vielzitierte ursprüngliche Definition von Macht:

„Macht bedeutet jede Chance, innerhalb einer sozialen Beziehung den eigenen Willen auch gegen Widerstreben durchzusetzen, gleichviel worauf diese Chance beruht.“ (Weber, 1976, zit. in Treiber, 2007: 50)

Macht ist also eine soziale Beziehung (vgl. M. Lueger, 1989; Treiber, 2007) und findet somit zwischen zwei oder mehr Parteien statt. Damit ist aber bereits angelegt, dass Macht weder allein im Ressourcenbesitz noch in der Verfügung über Ressourcen liegen kann (vgl. Sandner, 1990: 9ff.; Sandner \& Meyer, 2004: 759), da jede Theorie, welche in einer sozialen Beziehung die zweite Partei vollkommen ausblendet, notwendigerweise zu kurz greift. Aber auch dann, wenn beide Parteien in einer Konzeption von Macht inkludiert werden, ist es nicht ausreichend, die Macht des A allein aus der Abhängigkeit des B abzuleiten:

„Die Abhängigkeit eines Akteurs von den Ressourcen eines anderen bedeutet nicht von vornherein seine gleichzeitige Machtunterworfenheit. So kann jemand aus allen möglichen Gründen alle möglichen Ressourcen haben wollen und damit vielen Ressourcenkontrollierenden gegenüber dependent sein. Das bedeutet aber noch lange nicht, dass diese deshalb - als Umkehrreaktion - Macht über ihn ausüben könnten.“ (Sandner, 1990: 44)

Jede Theorie - so die Argumentation - welche den Charakter von Macht als soziale Beziehung ernst nimmt, muss notwendigerweise von der Annahme Abstand nehmen, dass Macht etwas ist, das man haben oder besitzen kann, unabhängig von der Akzeptanz dessen, über den Macht ausgeübt werden soll (vgl. Sandner, 1990: 45). Stattdessen hängt Macht in dieser sozialen Beziehung essentiell vom potentiell Machtunterworfenen ab, sie wird dem Mächtigen quasi „als Geschenk überreicht“ (M. Lueger, 1989: 186). Während Sandner (1990) als ei- 
ner der ersten systematisch auf dieses Versäumnis existierender Machtkonzepte hinweist, berücksichtigen und betonen spätere Ansätze die Notwendigkeit der Akzeptanz des Machtunterworfenen viel stärker. So erläutert z.B. Haugaard (2009: 239), Machtausübung benötige ,qualified levels of consent on the part of the relatively powerless“. Auch Clegg et al. (2007) halten fest: „[p]ower is above all a relational effect, not a property that can be held by someone or something." Treiber (2007: 53) argumentiert, dass ,Gehorchen' ein voraussetzungsvolles Verhalten darstellt, bei dem es auf Anerkennung bzw. Akzeptanz ankommt. Und auch van Dijk (2008: 29) betont die Bedeutung der Akzeptanz des Unterworfenen für das Funktionieren einer Machtbeziehung.

\subsubsection{Zur Entstehung von Dependenz und Akzeptanz}

Als erstes Ergebnis für die in der vorliegenden Arbeit herangezogene Definition von Macht lässt sich also festhalten, dass Macht als soziale Beziehung nur dann zustande kommt, wenn die machtunterworfene Partei die Unterordnung auch akzeptiert. Macht kann somit nur dort entstehen, wo auch ein (theoretischer) Freiraum besteht, anders zu handeln (vgl. Lukes, 2005: 43f.; Sandner, 1990: 53). Auf die Frage nach der Entstehung von Akzeptanz, bzw. warum die machtunterworfene Partei einen solchen Versuch der Machtausübung anerkennen sollte, bietet Lukes (2005: 10) verschiedene mögliche Antworten:

1) „The premise is incorrect: subordinates are actually rebelling continuously, but in covert ways.

2) Subordinates actually get something in return for their subordination, something that is sufficient to make them acquiesce most of the time.

3) Through the pursuit of other valued ends such as esteem or identity, subordinates become implicated in systems that exploit or oppress them. (In some versions, no. 3 becomes identical to no. 2)

4) As a result of mystification, repression, or the sheer unavailability of alternative ideological frames, subordinates remain unaware of their true interests.

5) Force and inertia hold subordinates in place.

6) Resistance and rebellion are costly; most subordinates lack the necessary means.

7) All of the above.“ (Tilly, 1991: 594, zit. in Lukes, 2005: 10)

Lukes (2005: 10) konstatiert dabei: „(7) is, clearly, correct: the other answers should not be seen as mutually exclusive (or, indeed, jointly exhaustive)." Sandner hingegen beantwortet die Frage nach der Entstehung von Akzeptanz auf einem höheren Abstraktionsgrad, indem er die Machtbeziehung als Verhandlungsmodell fasst. Für ihn entsteht Macht in einer Beziehung in zwei Schritten (vgl. Sandner, 1990: 94ff.): 
Zunächst kommuniziert das machtausübende Individuum (in der folgenden Argumentation mit A bezeichnet) die Dependenz an das machtunterworfene Individuum (im Folgenden B). Das heißt, A macht B klar, dass sie/er eine Ressource besitzt, welche B benötigt. Unter Umständen kommuniziert A auch direkt, unter welchen Bedingungen B diese Ressource von A erhalten kann. Ressource ist dabei weit zu fassen. Diese kann sowohl materiell (Geld, Güter, usw.) als auch immateriell (Anerkennung, Hilfe, Sicherheit, etc.) sein. A muss B dabei glaubhaft machen, dass (a) die Ressource für B wichtig ist und (b) die Unterwerfung des B diese Ressource für sie/ihn auch tatsächlich generiert. Entscheidend ist, dass an diesem Punkt die Handlung der/des A bereits beendet ist. Über das Zustandekommen der Machtbeziehung entscheidet im Folgenden ausschließlich B. A kann seine Macht über B nicht verursachen (vgl. Sandner, 1990: 94).

In einem zweiten Schritt interpretiert B das Angebot von A und entscheidet über Akzeptanz oder Widerstand. B wägt weiters die entstehenden ,Kosten“, welche die Unterordnung erzeugt, gegen die von A angebotenen Ressourcen ab. Dabei kann es zu drei verschiedenen Ergebnissen kommen: (a) B lehnt die Unterordnung ab, (b) B akzeptiert die Unterordnung und (c) B macht ein Gegenangebot, die Verhandlung geht weiter. Entscheidende Bedeutung kommt dabei dem Bezugsrahmen zu, welchen B für die Entscheidung heranzieht. Hat die Beziehung zwischen A und B bereits eine ,Geschichte', bzw. ist die Beziehung zwischen den Akteuren bereits habitualisiert oder sogar institutionalisiert (vgl. die Ausführungen zur Herrschaft im vorherigen Kapitel), so kann der Verhandlungsprozess entfallen, da die Beziehung bereits in früheren Verhandlungsprozessen geklärt wurde (vgl. Sandner, 1990: 96). Auf einer breiteren (organisationalen oder gesellschaftlichen) Ebene ist es auch möglich, dass die der Entscheidung zugrunde gelegten Rationalitäten als quasi selbstverständlicher Bestandteil unserer (bzw. der organisationalen) Normalität erlebt werden (vgl. Sandner, 1990: 97). Die Unterordnung von B kann sich dann als sozusagen ,zwingend“ darstellen. Dies bedeutet jedoch nichts anderes, als dass sich B auf ein bestimmtes Interpretationsschema eingelassen hat. Theoretisch ist ein Wechsel des Interpretationsschemas - und damit auch eine Ablehnung des Angebots von A dennoch möglich. Auch in einer solchen Konstellation ist die Machtausübung der/des A nicht kausal determiniert (vgl. Sandner, 1990: 97).

Das Zustandekommen solcher (diskursiv vermittelter) Interpretationsschemata, welche Machtausübung als ,normal' und ,unvermeidbar' erscheinen lassen, sowie die Möglichkeit der Schaffung und Beeinflussung dieser werden in der weiteren Argumentation noch diskutiert werden. Zunächst aber soll auf der Mikro-Ebene (in der dyadischen Beziehung zwischen A und B) geklärt werden, welche Möglichkeiten A besitzt, um eine Dependenz herzustellen bzw. den Ressourcenanspruch an B zu kommunizieren. Auch hier bietet Sandner (1990) einen 
umfassenden Katalog an Strategien, welche für die gegenständliche Untersuchung von unterschiedlicher Bedeutung sind:

Im Falle einer Drohung, Bestrafung oder Warnung (vgl. Sandner, 1990: 100ff.) besteht die Gegenleistung von A in einer Unterlassung. Im Falle einer Nicht-Unterordnung von B werden Sanktionen gesetzt. In Führungsgrundsätzen könnte diese Form der Kommunikation von Dependenzen in verschiedener Hinsicht geschehen. Zum einen können Konsequenzen für den Fall angedroht werden, dass die Führungsgrundsätze nicht eingehalten werden. Wie aus der bisherigen Diskussion ersichtlich wurde, ist dies in modernen Führungsgrundsatzdokumenten nur selten der Fall. Zum anderen können aber auch innerhalb der Dokumente Sanktionsmöglichkeiten für Führungskräfte beschrieben werden, falls MitarbeiterInnen sich den Anordnungen widersetzen.

Die Strategie von Versprechen, Belohnung oder Empfehlung (vgl. Sandner, 1990: 105ff.) stellt den Gegenpol zur ersten Strategie dar. Hier wird aber keine negative Sanktion unterlassen, sondern eine positive Handlung in Aussicht gestellt. Führungsgrundsätze versprechen i.d.R. keine Belohnung für den Fall ihrer Einhaltung. Etwas differenzierter stellt sich aber die FührungskräfteMitarbeiterInnen-Beziehung dar. Immer dann, wenn MitarbeiterInnen zu etwas motiviert werden sollen, wird die Unterordnung mit spezifischen Anreizen verknüpft. Innerhalb der Dokumente bzw. der von ihnen proklamierten Führungsbeziehung ist die Dependenzherstellung durch Versprechung und Belohnung sehr wohl relevant.

Die beiden Strategien der Überzeugung und der Manipulation werden innerhalb der vorliegenden Arbeit gemeinsam diskutiert, obwohl sie bei Sandner (1990: 108ff.) analytisch getrennt bleiben. Während bei der Überzeugung B ,in einer Situation freier Wahl den Inhalt einer kommunikativen Handlung des A [anerkennt], ohne positiven oder negativen Sanktionen des A ausgesetzt zu sein" (Sandner, 1990: 109), sind unter Manipulation „(kommunikative) Handlungen zu verstehen, mit denen B solche Situationsinterpretationen nahegelegt werden, die den Erwartungen des A entsprechen, ohne daß B aber die eigentliche, dahinterliegende Absicht des A erkennt" (Sandner, 1990: 108). Aus zwei Gründen wird zwischen diesen Ausprägungen im Folgenden nicht mehr trennscharf unterschieden: (a) im Rahmen einer reinen Textanalyse ist es nicht möglich, die Wahrheit der vermittelten Informationen einwandfrei einzuschätzen. Feststellbar sind allein die rhetorische Argumentation und die sozial geteilte Wissensbasis, auf welche diese sich stützt. Außerdem ist (b) in einer diskursanalytischen Untersuchung der Wahrheitsbegriff ohnehin hochproblematisch. Wahrheit entsteht in der diskursiven Aushandlung zwischen A und B, wobei eine Aussage dann als wahr betrachtet wird, wenn sie von der/dem EmpfängerIn in einen sinnhaften Zusammenhang mit ihrer/seiner Sinnwelt gebracht werden kann. Damit ist 
,Wahrheit" aber nicht zuletzt ein Ergebnis der rhetorischen Fertigkeiten der Sprecherin/des Sprechers (vgl. z.B. Clegg et al., 2007: 296). Jede Äußerung ist damit tendenziell manipulativ, indem sie ein spezifisches Weltbild (bzw. ,Bezugssystem‘, vgl. Kopperschmidt, 1989) evoziert, vor dessen Prämissen sie als sinnvoll erscheint. Die kritische Reflexion der Prämissen und die Aufdeckung des damit verkörperten Weltbildes ist die Aufgabe der in dieser Arbeit vorgenommenen Diskursanalyse. Die Feststellung einer manipulativen Absicht der Verfasserin/des Verfassers hingegen muss notwendigerweise außerhalb der Leistbarkeit eines solchen Ansatzes bleiben. Zu klären bleibt auch die Frage, ob eine Einflussnahme durch Überzeugung überhaupt Macht im eigentlichen Sinne darstellen kann. So führt Sandner selbst an, dass für Etzioni (1975: 378, zit. in Sandner, 1990: 110) aufgrund der freien Wahl von B Überzeugung keine Form der Macht konstituiert. Auch Lukes (2005: 35f.) erkennt dieses Problem, wenn er anmerkt: „The question of whether rational persuasion is a form of power and influence cannot be adequately treated here." Dagegen spricht für ihn die Tatsache, dass es nicht A ist, die/der eine Handlung von B induziert, sondern die ,Begründung' der/des A. Dafür spricht, dass es sich bei der Überzeugung durchaus um eine „form of significant affecting“ (Lukes, 2005: 36) handelt. In der gegenständlichen Arbeit wird der Argumentation von Sandner gefolgt, dass es sich bei Überzeugung durchaus um Macht handelt, da B sich von der Information bzw. der Argumentation von A abhängig macht, wenn er der Überzeugung folgt.

Der Grund, warum der Problematik der Macht durch Überzeugung hier so viel Platz eingeräumt wird, ist jener, dass dieser Strategie in den Führungsgrundsätzen annahmegemäß eine besondere Bedeutung zukommt. Da die Dokumente kaum Gebrauch von Drohung und keinen Gebrauch von Versprechungen machen und physischer Zwang durch schriftlich-textliche Kommunikationsformen nicht möglich ist, bleibt Überzeugung als primäres Mittel der Einflussnahme durch Führungsgrundsätze übrig. Damit die Inhalte der Führungsgrundsätze handlungswirksam werden, müssen sie glaubwürdig eine Dependenz vermitteln. Dies tun sie in erster Linie durch eine nachvollziehbare und überzeugende Darstellung der gewünschten Führungsbeziehung - durch Argumentation. $\mathrm{Ob}$ innerhalb der Führungsbeziehung Überzeugung als sinnvolle Strategie der Handlungsbeeinflussung gesehen wird, bleibt empirisch zu zeigen.

Wie oben angedeutet, identifiziert Sandner noch eine weitere Strategie, nämlich jene des physischen Zwangs. Physischer Zwang, so Sandner (1990: 113) bedarf als einzige Unterordnungsaufforderung keiner Zustimmung von B. Damit ist aber auch schon offensichtlich, dass diese Strategie für Führungsgrundsätze von wenig Relevanz sein dürfte. Weder können Dokumente als solche direkten und physischen Zwang auf den Leser ausüben, noch ist zu erwarten, dass ein Genre, welches sich einen Wandel zur partizipativen Führung auf 
die Fahnen geschrieben hat, in der Führungsbeziehung körperliche Gewalt propagiert.

Unterstützt werden die oben diskutierten Strategien der Dependenzherstellung durch die legale Autorität von A (vgl. Sandner, 1990: 118ff.). Die legale Autorität unterscheidet sich von den bisher diskutierten Strategien darin, dass sie nicht im Moment der Unterordnungsaufforderung hergestellt werden kann. Sie entsteht in der Regel im Vorhinein, z.B. durch das Unterzeichnen eines Arbeitsvertrages:

„B nimmt somit innerhalb einer bestimmten Indifferenzzone (Barnard, 1970: 143 f.) die Anweisungen von Vorgesetzten an, wobei innerhalb dieser Zone gleichgültig ist, was die Anweisung konkret enthält. Während etwa bei einer Überzeugung B die Überlegenheit des A erst dann anerkennt, nachdem er den Inhalt der Kommunikation und die Glaubwürdigkeit des A beurteilt hat, verzichtet B bei der legalen Autorität im voraus auf derartige Beurteilungen." (Sandner, 1990: 118)

Diese Passage könnte nun so verstanden werden, dass legale Autorität im Wesentlichen die vorhin diskutierten Strategien obsolet macht. Mit der Unterschrift unter den Arbeitsvertrag verpflichtet sich der/die MitarbeiterIn, jeglichen Anweisungen der Führungskraft Folge zu leisten, unabhängig von deren Inhalt, solange es sich um Anweisungen handelt, die durch den Arbeitsvertrag legitimiert werden. Dies kann aber nicht die Absicht von Sandner gewesen sein, zeigt doch z.B. eine stetig wachsende Literatur zu Anreizsystemen und Führungsstilen, dass allein mit der Unterschrift unter dem Arbeitsvertrag die Sache mit der Unterordnung noch lange nicht ,gegessen“ ist ${ }^{9}$. Sandner selbst geht auf die Möglichkeit des Zusammenbruchs legaler Autorität ein, wenn B eine Anweisung dennoch ablehnt (vgl. Sandner, 1990: 119). Ein solcher ,Bruch` der legalen Autorität wird - so Sandner - zumeist mit der Strategie der Drohung zu kompensieren versucht. Legale Autorität wird hier folglich als unterstützender Faktor verstanden, welcher die anderen Strategien nicht ersetzt, aber die Wahrscheinlichkeit der Akzeptanz der Unterordnungsaufforderung durch B erhöht. Legale Autorität legitimiert die Unterordnungsbeziehung und damit auch jede konkrete Unterordnungsaufforderung durch den Vorgesetzten. Es erscheint vor diesem Hintergrund also durchaus plausibel, dass selbst bei Existenz legaler Autorität die Verwendung guter Argumente in Weisungen nicht schadet, da sie die Wahrscheinlichkeit der Ablehnung (weiter) verringert. Interessant in diesem Zusammenhang sind auch Sandners (1990: 125ff.) Ausführungen zur Möglichkeit der

9 „Es sei so, dass der durch den Arbeitsvertrag bewirkte Gehorsam nur eine eng begrenzte Zone (der Indifferenz) umreiße; alles darüber hinausgehende Engagement und Leistungsstreben müsse durch andere Maßnahmen (dort: Anreize) sichergestellt werden“ (Schreyögg, 2003: 217). 
Veränderung der Bedingungen: „Sieht sich B in einer Situation, in der A einen Unterordnungsanspruch erhebt, so kann er auf diese Normen, Regeln oder Standards [zur Aufrechterhaltung und Abwicklung des organisatorischen Geschehens; Anm. DJ] rekurrieren, um die soziale Legitimität (in bezug auf die Normen) und die Übereinstimmung des Unterordnungsanspruches mit den Regeln und Standards zu bestreiten." Bestreitbare Ansprüche sind aber die Grundlage argumentativer Kommunikation (vgl. Kopperschmidt, 1989). Dies impliziert, dass Vorgesetzte bei der Durchsetzung von Macht darauf achten müssen, diesem Normensystem nicht zu widersprechen, bzw. den Widerstand der MitarbeiterInnen präventiv zu schwächen, indem bereits die Weisung vor dem Hintergrund dieses Normen- und Regelsystems argumentiert wird.

\subsubsection{Intention, Antizipation und Potentialität}

Mit dem Konzept der Akzeptanz und der verhandlungstheoretischen Basis der Machtbeziehung ist das wesentliche Fundament des Machtverständnisses in dieser Arbeit gelegt. Im Folgenden werden drei weitere relevante Aspekte kurz diskutiert, welche den verwendeten Machtbegriff abrunden.

Intention. Intention betrifft die Frage, ob unbeabsichtigte bzw. im Extremfall unbewusste Beeinflussung der Handlungen anderer dennoch Machtausübung konstituiert. Während Lukes (2005: 42) immer von Macht spricht „whether by individuals or by groups, institutions etc., and whether consciously or not", geht Sandner (1990: 78) davon aus, dass es zweckmäßig ist „(die Ausübung von) Macht als ein beabsichtigtes Tun oder Unterlassen zu definieren." Lukes führt allerdings an, dass es zumindest eine Form der unbeabsichtigten Einflussnahme gibt, welche keine Machtausübung konstituiert, und diese liegt dann vor, wenn A nicht wissen konnte, dass eine Handlung einen gewissen Einfluss auf B ausübt. Wusste A es zwar nicht, hätte sie/er dieses Wissen aber haben können bzw. müssen, so liegt Macht vor (vgl. Lukes, 2005:53f.). In der vorliegenden Untersuchung ist die Frage nach der Intentionalität der Machtausübung eher sekundär. Die Erstellung von Führungsgrundsätzen ist ein intentionaler Akt seitens der Unternehmensleitung mit dem Ziel der „Förderung eines erwünschten organisations- und mitgliedergerechten Sozial- und Leistungsverhaltens" (Wunderer, 2009: 385). Die Machtausübung durch die Führungsgrundsätze ist somit klar intentional (Führungsgrundsätze als Steuerungsinstrument). Nicht-intentional mag u.U. die Legitimierung bestimmter Formen der Machtausübung innerhalb der Führungsgrundsätze sein, sei es wegen unklarer Formulierung oder der Eröffnung eines (allzu) großen Interpretationsspielraums in den Regelungsinhalten. Unbeabsichtigte Machtausübung im eigentlichen Sinne ist daher nicht Teil des Führungsdiskurses. Davon unberührt bleibt aber die Tatsache, dass Füh- 
rungsgrundsätze selbst Ergebnis eines sozialen Prozesses sind, in dem Machtaspekte eine wichtige Rolle spielen. Die Inhalte der Führungsgrundsätze sind weder beliebig, noch sind sie für die Autoren frei wählbar. Für die ,Möglichkeiten und Unmöglichkeiten' der Formulierung von Führungsgrundsätzen zeichnen breitere diskursive Elemente verantwortlich, welche den Textautoren durchaus unbewusst verfügbar sein mögen. Diese diskursiven Kräfte ,always aim to achieve something, but cannot be mastered and controlled by any individual or collective subject or actor" (Torfing, 2009: 113). In der gegenständlichen Arbeit werden diskursive Elemente durch die (Re-)konstruktion rhetorischer Strategien und damit der relevanten Sinnwelt hinter den Führungsgrundsätzen analysiert. Sie zeigen sich in der Typik der Untersuchungsergebnisse, in der (Re-)konstruktion der typischen Führungsbeziehungen und den ,Lücken“ oder ,Silences“, welche diskursive Unmöglichkeiten bzw. Tabus aufzeigen. Die Bedeutung solcher diskursiver Machtaspekte wird in einem späteren Kapitel noch einmal explizit aufgegriffen.

Antizipation. Stark verzahnt mit der Frage nach der Notwendigkeit der Intention der Machtausübung ist der Faktor der Antizipation von Unterordnungsaufforderungen.

„Antizipatorische Reaktionen lassen sich also dadurch charakterisieren, daß der Mitarbeiter B in einer bestimmten Situation zwar die Handlungsalternative b1 bevorzugen würde, aber auf Grund einer in der Folge von A erwarteten Handlung a1 von seiner ursprünglich bevorzugten Alternative Abstand nimmt und die Handlungsalternative b2 wählt, von der er annimmt, daß A sie zulassen wird." (Sandner, 1990: 80)

Eine antizipatorische Reaktion ist folglich eine Machtausübung, welche keiner Unterordnungsaufforderung von A bedarf, da B diese bereits antizipiert. Eine solche antizipatorische Reaktion muss aber nicht mit einer nicht-intentionalen Machtausübung einhergehen. So können Führungsgrundsätze z.B. als Dokumente verstanden werden, welche darauf abzielen, eben solche antizipatorischen Reaktionen hervorzurufen. Führungskräfte (und MitarbeiterInnen) handeln aufgrund ihrer Kenntnis der Regeln in Antizipation der Erwartungen, welche durch diese Regeln kommuniziert werden, in erwünschter Weise, ohne dass die Unterordnungsaufforderung ständig wiederholt werden müsste. Die Machtausübung in diesem Beispiel ist klar intentional, die Unterordnung bedarf aber dennoch keiner Aufforderung.

Potentialität. Ein dritter Punkt betrifft die Frage, ob Macht erst dann entsteht, wenn einer Unterordnungsaufforderung auch tatsächlich Folge geleistet wird (,aktuelle Macht“, vgl. Sandner, 1990: 86) oder schon dann, wenn nur das Potential zu einer solchen Akzeptanz besteht. Dieses Problem der „potentiellen Macht" (vgl. Sandner, 1990: 86) ist für die gegenständliche Untersuchung von 
überragender Bedeutung. Weder durch Führungsgrundsätze noch in Führungsgrundsätzen wird aktuelle/tatsächliche Macht ausgeübt. Sowohl die dargestellten Rollen als auch die propagierten Über- und Unterordnungsverhältnisse entfalten nur dann aktuelle Macht, wenn sich die entsprechenden Akteure daran halten bzw. diese Machtvorstellungen akzeptieren. Damit ist in Führungsgrundsätzen immer nur potentielle Macht angelegt, die laut Sandner (1990: 86) „sich daher nicht auf eine Fähigkeit des A [bezieht], die unabhängig von B besteht, sondern nur auf eine mögliche Handlungskonstellation der Akteure A und B, die neben der Intention des A auf die Zustimmung des B angewiesen ist und zu ihrer Realisierung der aktuellen Ausübung bedarf." Selbst eine direkte Zustimmung des B, in der Zukunft eine Unterordnung vorzunehmen (so z.B. im Arbeitsvertrag) führt nur zu potentieller Macht und muss erst in der konkreten Situation verifiziert werden, bevor von aktueller Macht gesprochen werden kann (vgl. Sandner, 1990: 117). Lukes (2005: 42f.) führt noch eine weitere Unterscheidung ein, welche in dieser Hinsicht von Relevanz ist. Und zwar unterscheidet er einerseits zwischen einer ,operativen' Machtausübung durch A, welche zwar ausreichend (,sufficient ${ }^{`}$ ) ist, um eine bestimmte Handlung zu erreichen, von der aber nicht klar gesagt werden kann, ob sie für die Handlung des B ausschlaggebend war oder ob er diese Handlung auch ohne die Unterordnungsaufforderung von A vorgenommen hätte. Auf der anderen Seite spricht er von einer ,effektiven“ Machtausübung, wenn die Unterordnungsaufforderung von A für die Handlung von $B$ ausschlaggebend war. Eine solche effektive Machtausübung kann nun wiederum erfolgreich sein, wenn die Handlung von B den Erwartungen bzw. den Zielen von A entspricht, oder nicht erfolgreich, wenn B zwar aufgrund der Aufforderung von A eine Handlung vornimmt, diese aber nicht mit den Erwartungen von A übereinstimmt.

\subsubsection{Indirekte, unsichtbare und diskursive Macht}

\subsubsection{Drei Dimensionen der Macht}

,[I]s it not the supreme and most insidious exercise of power to prevent people, to whatever degree, from having grievances by shaping their perceptions, cognitions and preferences in such a way that they accept their role in the existing order of things, either because they can see or imagine no alternative to it, or because they see it as natural and unchangeable, or because they value it as divinely ordained and beneficial?"“(Lukes, 2005: 28)

In der bisherigen, eher allgemeinen Charakterisierung des Machtkonzepts wurden ausschließlich Formen der Machtausübung diskutiert, welche sich sehr explizit und offen zeigen. Diese Formen der Macht, in welcher A gegenüber B ihre/seine Forderungen deutlich macht und B deshalb die Möglichkeit hat, be- 
wusst über Akzeptanz und Ablehnung zu entscheiden, stellen nur einen kleinen Teil des Machtgeschehens in Organisationen dar. Das oben angeführte Zitat von Lukes (2005) dient als Einleitung in die Erweiterung des bisher vorgestellten Machtkonzeptes: Wie kann Macht über B ausgeübt werden, ohne dass ihr/ihm A mit einer offenen Forderung entgegentreten muss bzw. ist es möglich, dass B die Unterordnung gar nicht als solche wahrnimmt? Lukes $(1974,2005)$ geht von der behavioristischen Machtkonzeption Robert Dahls aus und hinterfragt systematisch dessen Annahmen, dass Macht sich a) nur in Entscheidungsprozessen und b) nur in beobachtbaren Konflikten zeige (vgl. Lukes, 2005: 5ff.). Diese Ansicht charakterisiert nach Lukes die erste Dimension der Macht: „Thus I conclude, that this first, one-dimensional, view of power involves a focus on behavior in the making of decisions on issues over which there is an observable conflict of (subjective) interests, seen as express policy preferences, revealed by political participation“ (Lukes, 2005: 19). In seiner weiteren Argumentation zeigt Lukes (2005) auf, dass Macht noch wesentlich mehr - und weniger manifeste - ,Gesichter ${ }^{6}$ hat. Mit Bezug auf Bachrach und Baratz (1962) argumentiert er, dass Macht nicht nur in der Fähigkeit besteht, sich in einer Entscheidungssituation durchzusetzen. Macht kann sich außerdem darin äußern, dass Anzahl und Aus$\mathrm{maß}$ der möglichen und tatsächlich eintretenden Entscheidungssituationen reduziert und gesteuert werden. So kann z.B. häufig festgestellt werden, dass mächtige Parteien dazu in der Lage sind, nur jene Entscheidungen überhaupt zuzulassen, welche relativ unproblematisch und wenig kontrovers sind, während die problematischen Entscheidungen niemals zur Diskussion gelangen (vgl. Lukes, 2005: 6). So genannte Nicht-Entscheidungen sind damit aber ebenso Ausdruck von Machtverhältnissen wie Entscheidungen (vgl. auch Sandner, 1990: 81ff.). Diese zweite Dimension der Macht ,involves a qualified critique of the behavioural focus of the first view [...] and it allows for consideration of the ways in which decisions are prevented from being taken on potential issues over which there is an observable conflict of (subjective) interests, seen as embodied in express policy preferences and sub-political grievances“ (Lukes, 2005: 25). Damit ist die erste Annahme, nämlich dass sich Macht nur in konkreten Entscheidungssituationen äußert, in Frage gestellt. Aber auch bezüglich der zweiten Annahme, dass ein beobachtbarer Konflikt vorliegen müsse, ist Vorsicht angebracht. In seiner Konzeption einer dritten Dimension von Macht stellt Lukes (2005: 7ff.) die Frage: ,[...] why should one exclude the possibility that power may be at work in such a way as to secure consent and thus prevent conflict from arising?" Für diese Argumentation zieht er Gramscis Konzept der Hegemonie heran. In dieser Sichtweise sind es kulturelle und ideologische Elemente, welche die Herrschaft einer Gruppe über eine andere konstituieren und legitimieren (vgl. C. Conrad \& Ryan, 1985; Lukes, 2005: 7). Lukes führt aber weiter an, dass es nicht 
möglich ist, eine große Menge an Individuen langfristig über ideologische ,Täuschungen' zu steuern. Eine Hegemonie kann Über- und Unterordnungsverhältnisse nur dann dauerhaft stabilisieren, wenn sie die Interessen und Ansprüche der dominierten Gruppe berücksichtigt: ,[Consent] is always conditional, and there are limits beyond which it will not be granted" (Lukes, 2005: 9). Damit ist auch in diesem Modell die Akzeptanz der Unterordnungsbedingungen von zentraler Bedeutung (vgl. Sandner, 1990: 94). Dennoch enthält Lukes' Konzeption die Möglichkeit der Manipulation dieser Akzeptanz, und zwar dergestalt, dass sich Individuen ihrer realen Interessen nicht bewusst sein müssen: „A may exercise power over B by getting him to do what he does not want to do, but he also exercises power over him by influencing, shaping or determining his very wants“" (Lukes, 2005: 27). Im Folgenden soll gezeigt werden, welche Möglichkeiten für Organisationen bestehen, durch indirekte Steuerungsstrategien Akzep$\operatorname{tanz}$ zu erreichen, ohne die Steuerungswirkung dabei sichtbar zu machen.

\subsubsection{Indirekte Formen der Steuerung}

Unter der Annahme, dass direkte Anordnungen an Untergebene einen hohen zeitlichen und kommunikativen Aufwand bedeuten, erläutert Sandner (1990: 159), dass „A weniger versuchen wird, Macht direkt im Rahmen seiner legalen Autorität auszuüben, sondern trachten wird, die Tätigkeiten anderer mittels differenzierter und auf mehreren Ebenen angelegter Steuerungsprozesse zu lenken." Dabei spricht er von Versuchen, Situationen so zu gestalten, dass B Interpretationen vornimmt, welche den Interessen von A entgegenkommen und $\mathrm{zu}$ entsprechenden Handlungen führen. Es kann dabei durchaus vorkommen, dass B diese Handlungen nicht als nachteilig, sondern - im Gegenteil - für sich selbst als höchst vorteilhaft empfindet (vgl. Sandner, 1990: 159). Auch in diesen Situationen bleibt es B unbenommen, die Aufforderung von A abzulehnen. A wird aber „tunlichst versuchen, Situationen so zu strukturieren, daß die Handlungskompetenz des B (Verhandlung, Akzeptanz/Ablehnung) diesem als nicht gegeben erscheint und B so den Unterordnungsaufforderungen unproblematisch nachkommt" (Sandner, 1990: 160). Durch diese Möglichkeit der Machtausübung entspricht Sandners indirekte Steuerung der dritten Machtdimension von Lukes (2005). Machtphänomene bedürfen nicht unbedingt eines manifesten Konfliktes. Auch in völlig konfliktfreien Situationen kann Macht ausgeübt werden (vgl. Lukes, 2005: 7ff.; Sandner, 1990: 74). Im Folgenden werden nun die verschiedenen Formen indirekter Steuerung vorgestellt und in ihrer Relevanz für die Betrachtung von Führungsgrundsätzen diskutiert.

Technologische Steuerung. Technologische Steuerung entsteht dort, wo parallel zur Technisierung des Betriebes (Einführung arbeitsteiliger Prozesse, Ver- 
einfachung, Routinisierung, Standardisierung) eine solche auch auf andere Bereiche der Organisation übergreift (vgl. Sandner, 1990: 185f.; vgl. auch Tompkins \& Cheney, 1985). „Mit der Technostruktur wird ein Handlungsrahmen definiert, der vorgibt, welche Handlungen zulässig und welche nicht zulässig sind, welche Handlungen wann, wo, wie und in welcher Intensität erwartet werden und welche nicht" (Sandner, 1990: 186). Damit wirkt die Technostruktur gleichzeitig sinnstiftend: Es wird eine Form der technischen Rationalität kommuniziert (vgl. Sandner, 1990: 186). Dieser Rationalität unterwirft sich nun das Organisationsmitglied. Es ist nicht mehr mit einer/m A konfrontiert, die/der eine Unterordnung fordert: Die Unterordnungs- bzw. Handlungsaufforderung ist bei der technischen Steuerung in den Arbeitsfluss gleichsam ,eingebaut' (vgl. Sandner, 1990: 186). Sie erscheint normal.

Bürokratische Steuerung. Technologische Steuerung erreicht dort ihre Grenzen, wo die ArbeiterInnen realisieren, dass bereits eine kleine Gruppe den Arbeitsprozess stoppen kann, wenn sie die technische Rationalität verweigert (vgl. Tompkins \& Cheney, 1985: 183). Eine noch weniger spürbare Form der Kontrolle stellt die bürokratische Kontrolle dar, welche durch die ,rule of law' (vgl. Tompkins \& Cheney, 1985: 183) steuert. Diese drückt sich durch Regeln, Prozesse und Erwartungen aus, welche eine bestimmte Form der Leistungserstellung nahe legen. Dadurch wird Vorhersehbarkeit und Berechenbarkeit erreicht, welche für das organisationale Miteinander unverzichtbar sind (vgl. Sandner, 1990: 189). Über diese Regeln kann Koordination und Strukturierung von Tätigkeiten erreicht werden, und es entstehen Hierarchien (vgl. Sandner, 1990: 190). Damit dient die bürokratische Steuerung der Herrschaftsausübung und sicherung. Dabei können die Regeln, über welche Steuerungswirkung erzielt wird, sowohl formell als auch informell sein. Die bürokratische Steuerung ist deshalb so wirksam, weil sie einen Beitrag zur Komplexitätsreduktion leistet. Die Einhaltung organisationaler Regeln und Strukturen erscheint normal, weil sie sich scheinbar aus Sachzwängen ableitet (vgl. Sandner, 1990: 192f.). Führungsgrundsätze sind nun als Dokumente, welche gewünschtes Verhalten von Führungskräften und MitarbeiterInnen festlegen, ganz offensichtlich ein Instrument der bürokratischen Steuerung. Sie enthalten präskriptive Regeln (zu Regeln als Element bürokratischer Steuerung vgl. Sandner, 1990: 190), und in der Thematisierung der Beziehung zwischen Führungskraft und MitarbeiterIn verdinglichen sie auch bestehende Hierarchien. Sie stellen folglich „Handlungserwartungen dar, die Teil eines gesamtorganisatorischen Regel- (und Sinn)systems sind und durch den Legalitätsglauben [...] abgesichert sind" (Sandner, 1990: 193). Damit wird Organisationsmitgliedern durch Führungsgrundsätze u.a. vorgeschrieben, wie sie Realität zu konstruieren haben, d.h., es wird ihnen ein Interpretationsrahmen vorgeschlagen, der klärt welche Teile einer Gesamtsi- 
tuation als relevant und welche als irrelevant bzw. illegitim anzusehen sind (vgl. Sandner, 1990: 193). Obwohl Führungsgrundsätze sehr gut in die Kategorie der bürokratischen Steuerung passen - nicht zuletzt deshalb, weil sie in der Vergangenheit als ein Instrument bürokratisch-autoritärer Führung bezeichnet wurden ist hier Vorsicht geboten. $\mathrm{Zu}$ beachten ist, dass Führungsgrundsätze in den Jahrzehnten ihrer praktischen Anwendung einen nicht unwesentlichen Wandel durchgemacht haben. Beinhaltet die ,allgemeine Führungsanweisung“ von Höhn $(1976,1977)$ noch sehr klare und konkrete Anweisungen z.B. zu Dienstaufsicht, Arbeitsauftrag und Dienstweg (vgl. Höhn, 1976: 22ff.), von denen durchaus gesagt werden kann, sie hätten „nicht nur Problemlagen zu Geschäftsfällen geformt“, sondern auch „die möglichen Konfliktlösungen vorgegeben“, sprich „,von der Problemwahrnehmung bis zur Problemlösung [...] die Vorgangsweise in Form von Handlungserwartungen eingegrenzt" (Sandner, 1990: 193), so ist das für neuere Führungsgrundsätze nicht mehr ohne Weiteres postulierbar. Moderne Führungsgrundsätze zeigen eine gewisse Nähe zu Leitbildern (vgl. Wunderer, 2009: 385), indem sie immer stärker erwünschte Werthaltungen parallel zu erwünschten Handlungen thematisieren. Damit können sie zu einem gewissen Grad das Problem umschiffen, dass jedes Regelsystem „im Moment seiner Festschreibung selbst wieder der Veränderungsdynamik ausgesetzt ist. Je rascher sich das faktische Handlungssystem und der Unternehmenskontext ändern, desto obsoleter wird das formale Regelsystem des Unternehmens" (Sandner, 1990: 197). Je konkreter die Handlungsanweisungen innerhalb der Führungsgrundsätze sind, desto stärker erscheinen sie als etwas Organisationsspezifisches, das im nicht-organisationalen Alltag der Organisationsmitglieder keine Relevanz besitzt. Damit werden aber im Kontrast zwischen diesen verschiedenen Sinnwelten (vgl. Berger \& Luckmann, 2000: 148ff.) des Individuums die Konstruiertheit des Regelsystems und somit die inhärenten Machtstrukturen eher sichtbar. Nehmen die Regeln hingegen einen allgemeineren Charakter an, sodass sie sich auf Werte und Normen beziehen, welche - losgelöst von einer konkreten betrieblichen Problemsituation - auch außerhalb der Organisation für das Individuum relevant werden könnten (wie z.B. die Betonung der Bedeutung von Zusammenarbeit, die Forderung nach einer Kultur des Vertrauens usw.), dann wird ein solcher ,Bruch“ vermieden, und die Regeln erscheinen dem Individuum als generell normal und selbstverständlich. Dieser geringe Grad an Konkretion, die Formulierung der Führungsgrundsätze in „oberflächlicher und rudimentärer" Form (Jancsary et al., 2006: 102) ermöglicht außerdem auch in der zeitlichen Dimension eine breitere Gültigkeit: Generelle Werte und Normen müssen seltener angepasst werden als konkrete Problemlösungen. Damit aber gehen moderne Führungsgrundsätze über eine rein bürokratische Steuerungs- 
wirkung deutlich hinaus, indem sie sich nicht nur auf betriebliche, sondern auf gesellschaftlich breitere gültige Werte, Normen und Problemlösungen beziehen.

Psychologische Steuerung. Unter psychologischer Steuerung versteht Sandner grob die Steuerung des Individuums über dessen Wünsche, Neigungen, Ziele, Bedürfnisse, Motive, etc. (vgl. Sandner, 1990: 198). Die Steuerung wird dadurch erreicht, dass MitarbeiterInnen diese Wünsche und Ziele nicht erreichen können, ohne der Unterordnungsaufforderung nachzukommen. Im Gegensatz zur direkten Steuerung durch Belohnung aber findet bei der psychologischen Steuerung die Anreizsetzung systematisch statt. Im optimalen Fall wird eine völlige Gleichschaltung der Ziele von MitarbeiterIn und Organisation erreicht ${ }^{10}$. Sandner (1990: 199) unterscheidet drei dominante Strategien der psychologischen Steuerung: (a) die ,Pflege der sozialen Beziehungen“, (b) die MitarbeiterInnenmotivation und (c) die (partielle) Selbststeuerung der MitarbeiterInnen. Führungsgrundsätze in ihrer Rolle als Steuerungsinstrument machen eher wenig Gebrauch von psychologischen Aspekten. Die von Sandner vorgestellten Strategien sind dort erkennbar, wo Führungsgrundsätzen z.B. eine Motivationsfunktion zugeschrieben wird (vgl. z.B. Grawert, 1997; Lattmann, 1975). In den innerhalb der Führungsgrundsätze vorgeschlagenen Führungs- und Steuerungsbeziehungen hingegen stellt die psychologische Steuerung eine dominante Strategie dar, und zwar in allen drei Ausprägungen. Sowohl die Pflege der sozialen Beziehungen („Charakterisierung der Beziehung") als auch die Mitarbeitermotivation (,Motivation“) und die Gestaltung von Freiräumen („Delegation“) für die MitarbeiterInnen sind wesentliche Elemente der vorgeschlagenen Führungsbeziehungen (vgl. Jancsary et al., 2006: 46). Das Problem der psychologischen Steuerung liegt laut Sandner (1990: 208) darin, dass durch die verstärkte Selbstkontrolle der MitarbeiterInnen das Machtgefälle sich zu deren Gunsten verschiebt. Dies kann von der Organisation dadurch abgefangen werden, dass durch die Kommunikation gemeinsamer Werte und Normen ein noch tiefer gehender Orientierungsrahmen vermittelt wird.

Kulturelle Steuerung. Die kulturelle Steuerung, so Sandner (1990: 209), bezieht sich auf eine Steuerung des Sinnsystems ${ }^{11}$ : „Jede Art von sozialer Ordnung beruht auf einem solchen System gemeinsam geteilter Bedeutungen; ja soziale Interaktionen sind nur unter der Voraussetzung der Annahme sinnvoll, daß ein Mindestbestand gemeinsam geteilten Wissens und gemeinsam geteilter Bedeutungen existiert. Dieser gemeinsam geteilte Bestand an Wissen und Bedeutun-

10 So diskutiert z.B. Schreyögg (2003: 215) die Einführung verschiedener Anreizsysteme explizit unter dem Titel „Integration von Individuum und Organisation“.

11 Die kulturelle Steuerung bei Sandner (1990) ähnelt damit in sehr hohem Ausmaß der ,concertive control` bei Tompkins und Cheney (1985). 
gen ermöglicht dann auch die Bewältigung unproblematischer Alltagssituationen“ (Sandner, 1990: 215, vgl. auch Berger \& Luckmann, 2000). Ansätze der kulturellen Steuerung werden in verschiedenen wissenschaftlichen Disziplinen unter den Labels der ,normative control‘ (vgl. z.B. Barley \& Kunda, 1992; Barley, Meyer \& Gash, 1988; Fleming \& Sturdy, 2009; Hechter, 2008; Kwok, $\mathrm{Au} \& \mathrm{Ho}, 2005)$ bzw. ,cultural control' (vgl. z.B. Grugulis, Dundon \& Wilkinson, 2000; Harris \& Ogbonna, 2011; Robertson \& Swan, 2004) diskutiert. Zusätzlich zur symbolischen Steuerung, der Sandner besonders viel Aufmerksamkeit widmet, werden in dieser Arbeit unter kulturelle Steuerung ganz allgemein alle jene Unterordnungsaufforderungen subsumiert, welche ihre Legitimität weder aus technologischen noch aus bürokratischen Regeln, sondern aus der von den Akteuren geteilten Lebenswelt bzw. ihrem gemeinsamen Wissensvorrat ziehen (vgl. Berger \& Luckmann, 2000; Keller, 2008). Diese Regeln schlagen eine gewisse Form der Wirklichkeitsinterpretation vor: „Within the framework of culture, power is largely exerted by influencing social actors" interpretation. [...] Indirect power may take the form of their conceptions of how the world ought to be, as expressed in norms, values, political loyalties or personal preferences.“ (Engelstad 2009: 211). Diese Unterordnungsaufforderungen speisen ihre Legitimation aus dem „summum totum all dessen, ,was jedermann weiß“, ein Sammelsurium von Maximen, Moral, Sprichwortweisheiten, Werten, Glauben, Mythen und so weiter ..." (Berger \& Luckmann, 2000: 70), und zwar aus dem organisationalen wie aus dem gesamten Wissensvorrat, den die Organisationsmitglieder teilen. Als besonders relevantes Konzept wird hier jenes der ,Rollen' betrachtet, mit welchen bestimmte Handlungserwartungen verbunden sind (vgl. Berger \& Luckmann, 2000: 76ff.). Somit wird über die Zuweisung von solchen Rollen für Vorgesetzte und MitarbeiterInnen Sinn gestiftet. Die/der Einzelne ,erkennt' sich als Teil einer bestimmten Sinngemeinschaft und kann aufgrund der ihr/ihm zugeteilten Rolle auch direkt ihren/seinen ,Platz in dieser Gesellschaft' finden: „Finally, power shapes the interpretational modes of those to be influenced, by affecting actors' worldviews, and epistemological conceptions, their interpretive skills, as well as their self-esteem and ability to make well-founded judgements.“ (Engelstad 2009: 211). Unterordnungsaufforderungen bestehen in einer solchen Sinngemeinschaft quasi zwingend zwischen den Inhabern bestimmter Rollen. Die Aushandlung von Rollenbildern ist somit ebenfalls eine indirekte Form der Steuerung, der sich das Organisationsmitglied nur schwer entziehen kann, solange das vermittelte Rollenbild in seinem sozialen Wissensvorrat ,resoniert'. Ausgehend von obiger Schlussfolgerung, dass Führungsgrundsätze über den Mechanismus einer rein bürokratischen Steuerung hinausgehen, wird argumentiert, dass sie vor allem ein Instrument der kulturellen Steuerung sind, das sich der Konstruktion eben solcher Rollen bedient, um 
Unterordnungsaufforderungen $\mathrm{zu}$ vermitteln und durch den Bezug auf sozial verfügbare Rollenvorstellungen Akzeptanz zu schaffen. Dieser Bezug auf sozial geteilte Werte erhöht die Legitimität der innerhalb der Führungsgrundsätze getätigten Aussagen und verstärkt damit die Unterordnungsaufforderung. Abgesehen von der kulturellen Steuerung durch Führungsgrundsätze sind auch Aspekte der kulturellen Steuerung in Führungsgrundsätzen zu erkennen, und zwar immer dann, wenn von Führungskräften erwartet wird, die Werte und Vision des Unternehmens zu verkörpern oder zu vermitteln bzw. zu kommunizieren.

Alle hier diskutierten indirekten Steuerungsformen haben gemeinsam, dass sie nicht nur die Handlungen der betroffenen Individuen beeinflussen, sondern in hohem Ausmaß auch mit der jeweiligen Rolle bzw. Rollenidentität verbunden sind. So schafft die Arbeitsteilung der technologischen Steuerung in gewisser Weise erst die Kategorie des ,Fließbandarbeiters', ebenso wie die Regeln der bürokratischen Steuerung gewisse Mitarbeiterkategorien (planende und ausführende Rollen, Top-, Middle- und Lower Management, etc.) durch Hierarchien und Strukturen konstruieren. Am klarsten tritt die Identitätskonstruktion durch die Betonung der Sinnstiftung in der kulturellen Steuerung zum Vorschein. All diese konstruktiven Phänomene gehen aber nicht von individuellen Akteuren aus, sondern sind Teil einer breiteren gesellschaftlichen Realitätskonstruktion. Es ist deshalb essentiell, in der Diskussion von Macht und Herrschaft auch die Bedeutung des Diskurses genauer zu beleuchten.

\subsubsection{Die diskursive Konstruktion von Identität}

Die nachfolgende Diskussion entspricht im Wesentlichen Ansätzen der Macht, die bei Sandner und Meyer (2004) unter die post-strukturalistischen Konzepte fallen. Diese lenken einerseits den Blick auf die ermöglichenden und produktiven Qualitäten der Macht und thematisieren außerdem die Konstitution institutioneller Wirklichkeit ${ }^{12}$ sowie des Subjekts und seiner Identitäten in diskursiver Produktion (vgl. Sandner \& Meyer, 2004: 762). Es wird hier argumentiert, dass diese Konzepte den zuvor diskutierten relationalen Ansätzen nicht unbedingt widersprechen müssen. Vielmehr handelt es sich um unterschiedliche Ebenen der Betrachtung von Machtausübung. Die diskursive Ebene wirkt dabei gleichermaßen ermöglichend und einschränkend auf die interaktionale Ebene. Sie ist dieser damit quasi vorgelagert.

12 „Diskursanalyse ist insofern eine alternative Art der Betrachtung von Institutionen, die darauf abzielt, das Funktionieren dieser Institutionen zu beleuchten, darauf, wie Macht sich in institutionellen Arrangements diskursiv manifestiert und wie politischer Wandel sich in solchen Arrangements vollzieht“ (Hajer, 2010: 289). 
Der Begriff des Diskurses ist in der Sozialwissenschaft nicht weniger problematisch oder diffus als jener der Macht. Einer ausführlicheren Diskussion des Begriffs widmet sich Kapitel 5.2, in welchem der diskursanalytische Zugang des Projekts erläutert wird. Für die folgende Diskussion ist es zunächst ausreichend, Diskurs im Sinne der wissenssoziologischen Ausrichtung der Arbeit folgendermaßen zu definieren:

„Der Begriff ,Diskurs' bezeichnet strukturierte und zusammenhängende (Sprach-) Praktiken, die Gegenstände und gesellschaftliche Wissensverhältnisse konstituieren. Einzelne diskursive Ereignisse aktualisieren diesen Zusammenhang. Die Diskursperspektive richtet sich auf die Ebene der gesellschaftlichen Wissensformationen und -politiken, deren Konturen, Genese, Entwicklung, Regulierungen und Folgen (,Machtwirkungen').“(Keller, 2008: 186)

Ein Diskurs ist somit keinesfalls ein machtfreier Raum, und die Entstehung und Erhaltung bestimmter Diskurse ist nicht zwangsläufig ein konsensualer Prozess. Vielmehr unterliegt der Konstruktion sozialer Realität ein ,intense struggle to determine the nature of constructs and to determine which construct applies in which case" (Clegg et al., 2007: 206). Macht ist folglich ein essentieller Bestandteil der diskursiven Realitätskonstruktion und wirkt sich auf mehreren Ebenen auf diskursive Praktiken aus (vgl. van Dijk, 2008: 10ff.): (a) Macht manifestiert sich im Zugang zum Diskurs: Wer kann in welchem Rahmen worüber sprechen und wer ist von der Generierung und Erhaltung des Diskurses ausgeschlossen? (b) Macht manifestiert sich im Inhalt des Diskurses: Worüber kann wem gegenüber was wie gesagt werden? (c) Macht manifestiert sich in der Wirkung des Diskurses: Wie werden Rollen, Identitäten und Verständnisse durch den Diskurs beeinflusst und somit Realitäten geschaffen?

Durch den Fokus auf Diskurs und eine Konzeption von Macht, die auf Bedeutung beruht, kann das Machtkonzept auf eine höhere Ebene gehoben werden als jene der Entscheidung oder der Interessensdurchsetzung dominanter Gruppen (vgl. Mumby \& Stohl, 1991: 314). Außerdem kann durch eine diskursive Machtkonzeption der Tatsache Rechnung getragen werden, dass Macht nicht immer eine isolierbare Kraft ist, die in klar definierten Interaktionen zwischen A und B wirkt, sondern dass jeder Akteur in Organisationen - wie auch die Organisation selbst (vgl. z.B. Fairhurst \& Putnam, 2004) -, wenn auch in unterschiedlichem Maße, diskursiver Macht ausgesetzt ist und durch diese beeinflusst wird. Diskurse wirken sowohl machtermöglichend als auch machtbegrenzend: „, $[\mathrm{A}] \mathrm{n}$ actor is powerful only within a particular discursive context as it is the discourse that creates the categories of power within which actors act" (Clegg et al., 2007: 300). Damit ist aber klargestellt, dass auch A immer und in jeder Interaktion mit B selbst mit einer spezifischen Unterordnungsaufforderung konfrontiert ist, nämlich jener, sich der wirklichkeitsstiftenden Macht des Diskurses 
zu unterwerfen, die sie/ihn als A bzw. als Führungskraft definiert und damit die Möglichkeiten der Machtausübung über B ermöglicht und begrenzt. Auch ManagerInnen können als eine soziale bzw. rhetorische Konstruktion betrachtet werden (vgl. z.B. Barley \& Kunda, 1992; du Gay, 1996). Der Unterschied zu B (bzw. der/dem MitarbeiterIn) liegt annahmegemäß darin, dass in van Dijks (2008) erster Ebene Führungskräfte einen besseren Zugang zur und mehr ,voice‘ (vgl. z.B. Burr, 1995: 120f.) in der diskursiven Konstruktion der organisationalen Realität besitzen. So wurde, wie im ersten Teil der Arbeit bereits dargestellt, in verschiedenen empirischen Untersuchungen zu Führungsgrundsätzen festgestellt, dass vor allem das (Top-)Managementteam an der Erstellung der Dokumente beteiligt ist (vgl. z.B. Gabele \& Kretschmer, 1986; Wunderer \& Klimecki, 1990). Führungsgrundsätze aber sind Teil der diskursiven Konstruktion von Führungs- und MitarbeiterInnenrollen im Unternehmen. Sie stehen in einer dialektischen Beziehung zum breiteren Führungsdiskurs im deutschsprachigen Raum und beeinflussen diesen, während sie gleichzeitig von ihm beeinflusst werden. Führungskräfte haben somit eine viel stärkere Möglichkeit, an der (formal legitimierten) diskursiven Aushandlung organisationaler Realität teilzunehmen als dies für MitarbeiterInnen der Fall ist. „Macht in der Gesellschaft schließt die Macht ein, über Sozialisationsprozesse zu verfügen, und damit die Macht, Wirklichkeit zu setzen“ (Berger \& Luckmann, 2000: 128; vgl. auch Sandner \& Meyer, 2002; Vaara, Tienari, Piekkari \& Säntti, 2005). Insofern wird gemeinsam mit Mumby und Stohl (1991: 313) eine Machtkonzeption vorgeschlagen ,in which discourse is conceived as the principal medium through which power relations are maintained and reproduced. Specifically, power is identified as a pervasive characteristic of organizational life which constitutes the identity of organization members". Hegemonie, wiederum, ist in dieser Perspektive eine Ideologie, welche verschiedene soziale Gruppen in Beziehungen von Dominanz und Unterordnung verbindet. Eine solche hegemonische soziale Struktur wird durch verschiedene diskursive Praktiken artikuliert und stabilisiert (vgl. auch Nonhoff, 2010). Macht geht dadurch weit über einen reinen Zwang hinaus und wird zu einer produktiven Ressource in der Konstruktion sozialer Wirklichkeit (und individueller Identität):

,[...] discourse can be most fruitfully examined as a site of power which produces organizational subjectivity (identity) in a particular way. In other words, discursive practices do not simply provide a context for the exercise of power over organizational members, but rather function as a form of discipline which, within a particular power-knowledge regime, constitutes organization members in a certain manner. Discourse and the rules which constitute it function to establish a particular organizational ,regime of truth " within which organization members are simultaneously objectified (i.e. treated as bodies upon which various disciplinary practices can be 
exercised) and recognize themselves as subjects (i.e. as organization members whose identity is constructed in a particular way." (Mumby \& Stohl 1991: 316).

Mit einem solchen diskursiven Verständnis von Macht wird nun auch das Problem, das Lukes (2005) mit der Unterscheidung von subjektiven und objektiven $b z w$. realen Interessen des Individuums aufwirft, effektiv aufgelöst, denn ,it deconstructs the notion of the subject as something that is a substantial entity, already there and fully formed, except for realization of its real interests which the structure of reality occludes. [...] Subjects are an effect of the play of contingent discursive possibilities, the signification of which is not fixed in advance because all of its possible terms are relational" (Clegg et al., 2007: 220). Nun wurde aber weiter oben bereits ausgeführt, dass auch die Kontrolle des Managements bzw. der Führungskräfte über den Diskurs nicht total ist.

„The power strategies are, intentional and non-subjective" in the sense that they always aim to achieve something, but cannot be mastered and controlled by any individual or collective subject or actor.“ (Foucault, 1976: 95, zit. in Torfing, 2009f.)

Es bleibt also noch zu klären, inwiefern Individuen, deren Rollen (und damit ihre ,agency $\left.{ }^{6}\right)$ selbst durch den Diskurs konstituiert werden ${ }^{13}$, den Diskurs nutzen können, um das Machtverhältnis so zu verschieben, dass sich andere Individuen ihnen in der reziproken Aushandlung von Rollen unterwerfen. Dies kann anhand einer grundsätzlichen Dialektik zwischen sozialer Struktur und Diskurs modelliert werden. Innerhalb dieser dialektischen Beziehung, durch welche Diskurs und soziale Struktur sich laufend gegenseitig beeinflussen und verändern, findet das Individuum eine sogenannte ,subject position'. Diese subject positions sind Orte im sozialen Raum, welche den Individuen spezifische Möglichkeiten zur Textproduktion und somit zur Diskursbeeinflussung einräumen (Clegg et al., 2007: 304). Diese Orte sind aber nicht frei wählbar, sondern werden Individuen durch Diskurs und soziale Struktur quasi ,eingeschrieben“. Die Beziehung zwischen Individuum und Diskurs ist also eine dialektische. Ein wissenssoziologischer Diskursbegriff ,vermeidet gleichermaßen die in Diskurstheorien implizite Ontologisierung bzw. Verdinglichung der Diskurse durch die Einführung eines Akteurskonzepts, mit dem soziale Akteure sowohl als diskursiv konstituierte wie als regelinterpretierend Handelnde, als aktive Produzenten und Rezipienten von Diskursen verstanden werden“ (Keller, 2008: 191). So hat das

13 „Discourse ,disciplines' subjects in that actors are known - and know themselves - only within the confines of a particular discursive context and the possibility that that provides.“ (Clegg, et al., 2007: 299) bzw. „[...] power cannot be viewed as a causal effect of either agency or structure, since these are themselves constructed in and through power strategies that are operating at the level of discourse" (Foucault, 1986a, zit. in Torfing, 2009: 111f.) 
Management in ganz spezifischer Form die Möglichkeit, den Diskurs über die Externalisierung von Regeln und Standards mitzugestalten, während MitarbeiterInnen diese Möglichkeit nicht offen steht. MitarbeiterInnen sind aber dennoch keine rein passiven Empfänger der diskursiven Ressourcen des Managements. Die Konstruktion von Identität ist ein Aushandlungsprozess, in welchem das Individuum die angebotenen diskursiven Ressourcen annehmen kann, aber nicht muss. Auch diskursive Kontrolle ist nicht total: „The different subjectivities always have the possibility of saying no, or acting in a non-prescribed way" (Torfing, 2009: 113).

\subsubsection{Machtaspekte in Führungsgrundsätzen}

Um die ausführliche Diskussion von Macht, Herrschaft und Steuerung zu beschließen, werden im Folgenden die wesentlichen relevanten Schlussfolgerungen für die vorliegende Untersuchung formuliert und zusammengefasst.

\subsubsection{Ebenen der Machtausübung}

Zunächst wird festgehalten, dass Führungsgrundsätze Machtbeziehungen in verschiedener Form betreffen und thematisieren. Diese Formen bedingen einander zwar nicht kausal, stehen aber dennoch in einem engen Zusammenhang. Auf einer der Untersuchung vorgelagerten Ebene stellen Führungsgrundsätze selbst eine Unterordnungsaufforderung dar. Sie bekunden den Willen des Managements und stellen einen Appell an Führungskräfte und MitarbeiterInnen dar (vgl. Finzer \& Mungenast, 1989: 407; Lattmann, 1975:56). Auf dieser ersten Ebene besteht die Unterordnungsaufforderung darin, die Führungsgrundsätze per se, also als (Steuerungs)Instrument der Mitarbeiterführung zu akzeptieren und sich ihnen unterzuordnen. Diese Ebene der Macht entzieht sich einer Untersuchung, welche als Datenmaterial vor allem die Texte selbst heranzieht. Eine Untersuchung der Unterordnungsaufforderungen müsste sich auf begleitende Maßnahmen der Einführung (Partizipation, Schulung, Kommunikation, ...) stützen. Eine solche kann in der vorliegenden Arbeit nicht geleistet werden. Aussagen zur grundsätzlichen Relevanz der Steuerung durch Führungsgrundsätze in deutschsprachigen Unternehmen können deshalb nicht getroffen werden.

Auf einer ersten inhaltlichen Ebene verlangen Führungsgrundsätze eine Unterordnung unter die durch sie kommunizierten Regelungsinhalte. Diese Ebene ist mit der vorgelagerten Ebene insofern verknüpft, als eine Akzeptanz der Inhalte auch die Akzeptanz des Steuerungsinstruments per se bedingt. Umkehrschlüsse können aber nicht argumentiert werden: Weder bedeutet die Akzeptanz einzelner (oder auch aller) Inhalte notwendigerweise die Akzeptanz der Kom- 
munikations- bzw. Steuerungsform (Führungsgrundsätze), noch bedeutet eine hohe Akzeptanz der Form notwendigerweise die Akzeptanz aller Regelungsinhalte. Deshalb empfiehlt es sich, analytisch zwischen den beiden Ebenen eine Grenze einzuziehen. Auch methodologisch sind die Ebenen klar zu unterscheiden. Während die vorgelagerte Ebene eine Untersuchung des Kontextes bedingen würde, kann die zweite Ebene mittels diskursanalytischer Methoden anhand des Textes analysiert werden. (Re-)konstruiert werden damit die spezifischen Steuerungsstrategien - Versprechen, Drohung, Überzeugung, Manipulation (vgl. Sandner, 1990) -, mittels welcher die Inhalte der Dokumente eine Unterordnung zu erreichen hoffen.

Auf einer zweiten inhaltlichen Ebene schließlich stellen Führungsgrundsätze ein Führungsinstrument dar, welches andere Führungsinstrumente thematisiert. Die Beziehung zwischen Führungskraft und MitarbeiterIn ist primärer Regelungsinhalt der Führungsgrundsätze (vgl. Wunderer, 2009: 385). Damit üben Führungsgrundsätze nicht nur selbst potentielle Macht aus, sie schlagen auch eine gewisse Form der Machtverteilung bzw. der Machtausübung innerhalb der Beziehung von Führungskräften und MitarbeiterInnen vor. Führungsgrundsätze bieten Führungskräften ein breites Arsenal verschiedener Strategien und Instrumente, um MitarbeiterInnen zu gewünschtem Handeln zu bewegen (Motivation, Belohnung, Sanktion, Vorbildwirkung, etc.). Die Akzeptanz dieser Strategien und Instrumente ist nun aber nicht zwingend von der Akzeptanz der Führungsgrundsätze per se abhängig. MitarbeiterInnen können sich in konkreten Interaktionen ihrer/m Vorgesetzten unterordnen, unabhängig davon, ob diese Unterordnung in schriftlichen Dokumenten festgeschrieben ist (z.B. aufgrund anderer direkter oder indirekter Steuerungsmechanismen). Die Verständlichkeit und Anschlussfähigkeit der in den Dokumenten vorgeschlagenen Hierarchie- und Machtbeziehungen ist aber Voraussetzung für die Akzeptanz der zweiten Ebene. Nur wenn sich Führungskräfte und MitarbeiterInnen in den dargestellten Führungskontexten ,wiedererkennen', werden die Inhalte des Dokumentes möglicherweise Akzeptanz finden. Die Akzeptanzbedingungen der zweiten und dritten Ebene fallen folglich zusammen, obwohl diese unterschiedliche Akteure und unterschiedliche Abstraktionsgrade der Machtausübung betreffen. Diese zweite inhaltliche Ebene der Steuerung - die Frage also, welche Beziehungen zwischen Führungskräften und MitarbeiterInnen sich in den Dokumenten finden und welche Machtkonstellationen dadurch zum Ausdruck kommen - kann durch inhaltsund diskursanalytische Verfahren ebenfalls aus den Texten (re-)konstruiert werden. Die folgende Graphik zeigt das Zusammenspiel der drei Ebenen schematisch auf: 


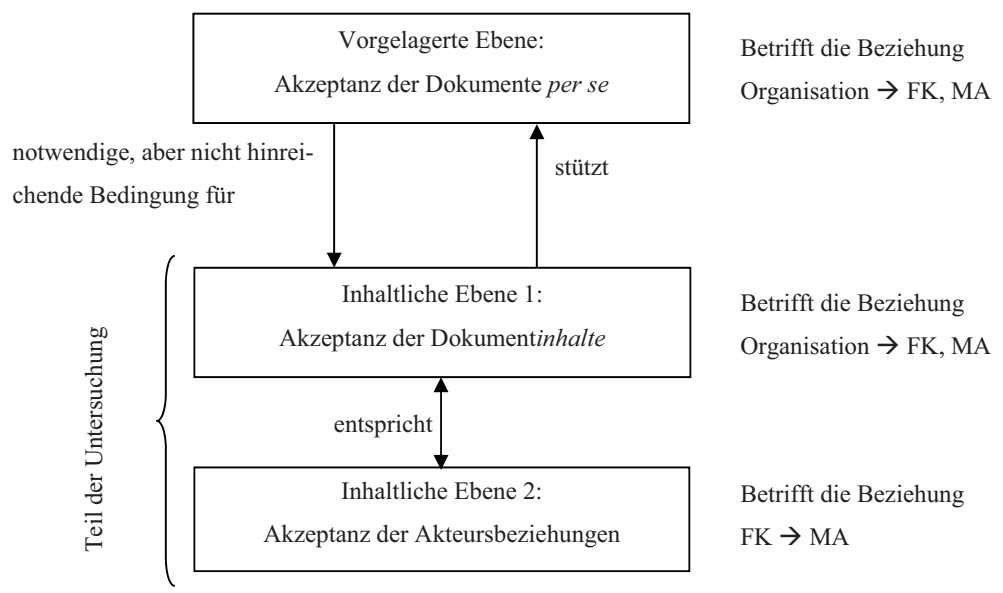

Abbildung 8: Die Ebenen der Macht in Führungsgrundsätzen (eigene Darstellung)

In der graphischen Darstellung wird klar, inwiefern sich die Ebenen überschneiden und in welchen Aspekten sie sich unterscheiden. Die vorgelagerte und die erste inhaltliche Ebene betreffen beide die Unterordnung unter das Dokument und somit die Organisation bzw. das Management. Auf diesen beiden Ebenen sind Führungskräfte (FK) und MitarbeiterInnen (MA) scheinbar gleichgestellt. Sie müssen sich gleichermaßen dem Steuerungsdokument und dessen Regelungsinhalten unterwerfen. Scheinbar ist diese Gleichstellung deshalb, weil annahmegemäß die oberste Ebene der Führungskräfte an der Erstellung der Dokumente beteiligt war und sich deshalb nur den eigenen Vorstellungen zu unterwerfen hat. Die beiden Ebenen unterscheiden sich jedoch bezüglich der Steuerungslogik. Die vorgelagerte Ebene bedingt eine Unterordnungsaufforderung vor und parallel zur Einführung der Dokumente. Es muss sichergestellt werden, dass diese Form der Steuerung (durch schriftliche Regeldokumente) akzeptiert wird. Die erste inhaltliche Ebene betrifft eine Unterordnungsaufforderung innerhalb der Dokumente: Die/der LeserIn bzw. AdressatIn muss die Regeln, welche die Dokumente beinhalten, akzeptieren. Die zweite inhaltliche Ebene schließlich gleicht der ersten in genau diesem Punkt: Werden die Regeln innerhalb der Dokumente akzeptiert, so werden auch zwangläufig die vorgeschlagenen Formen der Unterordnung der MitarbeiterInnen unter die Führungskräfte akzeptiert (diese sind unvermeidbarer Teil der Regeln). Die beiden Ebenen unterscheiden sich aber bezüglich der an der Macht- bzw. Herrschaftsbeziehung beteiligten Akteure. In der zweiten inhaltlichen Ebene verschwindet die Organisation/das Management insoweit, als sie/es nicht deckungsgleich mit den er- 
wähnten Führungskräften ist. Die scheinbare Gleichstellung zwischen Führungskraft und MitarbeiterIn löst sich auf.

\subsubsection{Führungsgrundsätze als Instrument der Machtausübung und Herrschaftssicherung}

Was genau aber beleuchtet nun die Linse von Macht, Herrschaft und Steuerung in der Diskussion von Führungsgrundsätzen? Führungsgrundsätze wurden definiert als spezifisches Genre der Unternehmenskommunikation, welches die Beziehungen zwischen Vorgesetzten und MitarbeiterInnen beschreibt und normiert (vgl. Wunderer, 2009: 385). Führungsgrundsätze sind in diesem Verständnis ein Steuerungsinstrument (vgl. Jancsary et al., 2006). Weiters wurde das Konzept der Macht als zentrales Element jeglichen organisationalen Handelns eingeführt. Macht als ,intentionale Durchsetzung von Zielvorstellungen, die auf das Handeln (die Unterordnung) anderer angewiesen ist" (Sandner, 1990: 94), spielt eine essentielle Rolle in der Diskussion von Führungsgrundsätzen.

„Indeed, it is largely through [these] generalized media of communication that power achieves its reach: hence, the centrality of the bureau to the old models of bureaucracies; the pervasiveness of information systems in newer forms of organization; and the increasing governance of organizations, everywhere, by codified standards [eigene Hervorhebung; DJ].“(Clegg et al., 2007: 237f.)

Durch die Einführung von Führungsgrundsätzen sollen sowohl die Handlungen von Vorgesetzten und MitarbeiterInnen gesteuert (vgl. Lattmann, 1975: 48), aber auch generelle organisationale Ziele und Werte vermittelt werden (vgl. Wunderer, 2009: 383). Darüber hinaus können Führungsgrundsätze auch als organisationale Angebote zur Identitätskonstruktion von Führungskräften und MitarbeiterInnen charakterisiert werden, da sie organisational erwünschte Rollenvorstellungen kommunizieren (vgl. Jancsary, 2007). Unter Rollen werden in dieser Untersuchung Bündel typisierter Verhaltenserwartungen verstanden, welche eine gesellschaftliche Ordnung repräsentieren (vgl. Berger \& Luckmann, 2000: 76ff.). So werden in den Führungsgrundsätzen verschiedene Führungskräfte- und MitarbeiterInnenrollen dadurch konstruiert, dass eben solche typisierten Verhaltenserwartungen kommuniziert werden, und zwar sowohl in der Führungskräfte-MitarbeiterInnen-Beziehung als auch in Bezug auf andere organisationale Tätigkeiten. Akzeptieren die Individuen in der Organisation diese vorgeschlagenen Rollen, so werden die soziale Wirklichkeit in der Organisation - und damit auch die Machtbeziehungen - stabilisiert. Aus diesen verschiedenen Wirkungsvermutungen wird geschlossen, dass Führungsgrundsätze in sehr umfassender Art und Weise Unterordnungsaufforderungen an Führungskräfte und MitarbeiterInnen kommunizieren. Im Extremfall können sie Teil einer hegemo- 
nischen Kontrolle sein, eines Systems diskursiver Konstruktionen, welche eingebunden sind in ,a dense network of institutions and technologies that establish and distribute different roles, identities, rules, norms, calculations, conceptions, values and imaginaries“ (Torfing, 2009: 113). In der Diskussion des Machtbegriffs wurde jedoch festgestellt, dass Machtausübung nur dann tatsächlich vorliegt, wenn die Aufforderung zur Unterordnung akzeptiert wird. Führungsgrundsätze beinhalten jedoch lediglich eine schriftliche Darlegung solcher Aufforderungen. Über die tatsächliche Wirkung der Dokumente kann anhand einer Dokumentenanalyse keine Aussage getroffen werden. Führungsgrundsätze als Dokument und schriftlich formulierter Ausdruck des Willens der Unternehmensleitung (vgl. Lattmann, 1975: 54) können somit ausschließlich potentielle Macht (vgl. Sandner, 1990: 86) konstituieren.

Forschungsleitende Annahme 1: Führungsgrundsätze sind ein Steuerungsinstrument, welches innerhalb eines spezifischen, legitimierten Herrschaftsgefüges (Hierarchie, Aufbauorganisation) existiert und über die Beschreibung und Verordnung erwünschter Handlungen, Werte und Identitäten/Rollen potentielle Steuerungswirkungen auf organisationale Akteure (Führungskräfte und MitarbeiterInnen) auszuüben bezweckt.

Führungsgrundsätze als Instrument der ,strukturellen Führung' (vgl. Wunderer, 2009: 383) zielen auch darauf ab, Führungskräfte in ihrer unmittelbaren, interaktionalen Führungstätigkeit zu entlasten. Sie sind daher den indirekten Steuerungsstrategien zuzuordnen (vgl. Sandner, 1990: 181ff.). Es wurde des Weiteren argumentiert, dass Führungskräfte zur Erreichung der Akzeptanz von Unterordnungsaufforderungen vor allem auf bürokratische (Bezugnahme auf Regeln, Hierarchien, Programme, vgl. Sandner, 1990: 189ff.) und auf kulturelle (Steuerung über Sinn und Symbole, vgl. Sandner, 1990: 209ff.) Ressourcen zurückgreifen. Führungsgrundsätze sind damit sinn- und realitätsstiftend. Dies bedeutet für die vorliegende Untersuchung, dass sie sich in der (Re-)konstruktion von Machtbeziehungen vor allem auf die Analyse jener Sinn- und Bedeutungsstrukturen fokussieren muss, welche die propagierten Machtbeziehungen diskursiv stützen.

Forschungsleitende Annahme 2: Führungsgrundsätze sind ein Instrument der strukturellen Führung. Die Idee der Steuerung durch Grundsatzdokumente verbindet Elemente der bürokratischen und der kulturellen Steuerung.

Führungsgrundsätze sind aber nicht nur selbst ein Führungsinstrument. Der primäre Regelungsinhalt betrifft die Beziehungen zwischen Vorgesetzten und ihren MitarbeiterInnen. Sie beinhalten spezifische Vorstellungen darüber, wie Führung - und damit Macht- und Herrschaftsausübung - in der Organisation zu geschehen hat. Dadurch ergibt sich für die Untersuchung von Machtaspekten in 
Führungsgrundsätzen die weiter oben diskutierte zusätzliche Untersuchungsebene. Führungsgrundsätze sollen nicht nur selbst Steuerungswirkung entfalten, sondern sie enthalten auch Aussagen darüber, wie MitarbeiterInnen von Führungskräften gesteuert werden sollen. Folglich lässt sich in einem diskursanalytischen Forschungsprogramm nicht nur die Steuerungslogik der Führungsgrundsätze selbst (re-)konstruieren, die Dokumente zeichnen auch auf einer viel allgemeineren Ebene inhaltlich ein Bild von Herrschafts- und Führungsverständnissen im Diskurs:

Forschungsleitende Annahme 3: Führungsgrundsätze beinhalten Verständnisse davon, wie die Akteurstypen der Führungskräfte und der MitarbeiterInnen zueinander in Beziehung treten sollen. Über die Analyse dieser vorgeschlagenen Beziehungsmuster lassen sich idealtypische Steuerungsstrategien (re-)konstruieren.

Damit sind die zwei relevanten Ebenen der potentiellen Machtausübung von Führungsgrundsätzen definiert. Die erste inhaltliche Ebene bezieht sich auf die Form der Kommunikation (Rhetorik und Argumentation): Wie erreichen Führungsgrundsätze eine Steuerungswirkung bei den intendierten Adressaten? Die zweite inhaltliche Ebene bezieht sich auf den Regelungsinhalt der Kommunikation: Welche Akteursrollen und -beziehungen bzw. welche erwünschten Handlungen und Werte werden in den Führungsgrundsätzen thematisiert und welche nicht? Gemeinsam mit Clegg et al. (2007) wird hier argumentiert, dass eine Untersuchung beide Ebenen inkludieren muss (das Wie und das Was gleichermaBen): „It is both the reach of the form and the regulatory potential of the content that matter." (Clegg et al., 2007: 237f.)

Forschungsleitende Annahme 4: Eine umfassende (Re-)konstruktion von Aspekten der Macht und der Herrschaft muss sowohl die Form als auch den Inhalt der Führungsgrundsätze, also beide Ebenen der Steuerung berücksichtigen.

Nachdem festgehalten wurde, dass Führungsgrundsätze ausschließlich potentielle Macht entfalten können, stellt sich die Frage nach den Strategien, über welche die Textautoren eine Akzeptanz der Regelungsinhalte möglichst wahrscheinlich machen wollen. Gemeinsam mit Sandner (1990) werden verschiedene Möglichkeiten der Dependenzherstellung unterschieden: körperlicher Zwang, Drohung, Versprechung, Überzeugung und Manipulation. Es wurde argumentiert, dass Führungsgrundsätze nicht in der Lage sind, körperlichen Zwang auszuüben. Die in der präskriptiven Literatur oft geforderte Verbindlichkeit von Führungsgrundsätzen - sprich: die Verbindung von Nicht-Beachtung mit durchsetzbaren Sanktionen - wird in der Praxis kaum vorgefunden (vgl. Fiedler, 1976; Hoffmann, 1989; Wunderer \& Klimecki, 1990). Auch für den in der gegenständlichen Arbeit verwendeten Korpus an Dokumenten konnte die Androhung von konkreten Sanktionen bei Nicht-Beachtung nicht festgestellt werden. Die Versprechung, 
also das in Aussicht Stellen von positiver Sanktionierung bei Beachtung ist in diesem Dokumentgenre ebenfalls unüblich. Es wird folglich festgehalten, dass sich die Regelungsinhalte dieser Dokumente - so sie eine Wirkung erzielen wollen - in erster Linie auf die Strategien von Überzeugung und Manipulation verlassen müssen. Es wurde erläutert, dass die Inhalte der Führungsgrundsätze dann für die organisationalen Akteure ,akzeptabel' sind, wenn sie sich auf breitere diskursive Vorstellungen von Führung und Unterordnung beziehen, wenn sie damit also im sozialen Wissensvorrat (vgl. z.B. Keller, 2008: 40ff.) der spezifischen Diskursgemeinschaft vorhanden sind. Die Überzeugungskraft der Führungsgrundsätze liegt somit darin, dass sie Bilder von Führung zeichnen, mit welchen sich Führungskräfte und MitarbeiterInnen einverstanden erklären können. Das heißt, dass spezifische Vorstellungen von Führung rhetorisch geschickt ,verpackt ${ }^{`}$ bzw. , verkauft' werden müssen, damit die darin enthaltenen Unterordnungsaufforderungen akzeptiert werden. Diese Form der Machtausübung wirkt dann , unauffällig' (unobtrusive): „These are sets of routines, taken for granted schemes, socially inscribed object-distinctions, and self-conceptions that are ready at hand. We feel as if the world is already demarked into this object and act as if socially arbitrary distinctions between things resides (sic!) in nature itself" (Deetz, 1995: 214). Über eine Analyse dieser rhetorischen Mittel lassen sich folglich die Rationalitäten (re-)konstruieren, von welchen die Textproduzenten erwarten, dass sie in der Organisation Zustimmung finden:

Forschungsleitende Annahme 5: Führungsgrundsätze verwenden rhetorische Strategien, um die Adressaten von der Legitimität ihrer Aussagen zu überzeugen. Eine Analyse der zugrundeliegenden Sinnwelt(en) und deren Logiken muss folglich eine Untersuchung dieser rhetorischen Strategien beinhalten.

In diesem Sinne soll im nächsten Kapitel ein kurzer Überblick über die Möglichkeiten rhetorischer Überzeugung gegeben werden, bevor anschließend das methodische Design der Untersuchung vorgestellt wird.

\subsection{Rhetorik als Instrument der Überzeugung}

\subsubsection{Abgrenzung und Relevanz}

„Rhetoric has been the subject of uninterrupted study for at least 2,500 years.“

(Gill \& Whedbee 1997: 158)

Trotz ihrer langen Geschichte ist die Disziplin der Rhetorik in der Gegenwart nicht weniger aktuell als zu ihren Anfängen. Innerhalb dieses Zeitraums ihrer Existenz hat die rhetorische Tradition allerdings einige Entwicklungen und Veränderungen erfahren, welche eine einfache Definition erschweren (vgl. Ottmers, 
2007). Gerade in der Sozialwissenschaft wurde die Rhetorik für verschiedenste Formen von Untersuchungen und Analysen herangezogen und entsprechend auch unterschiedlich konzipiert: „While some of these definitions equate rhetoric with persuasion, others define rhetoric more broadly as any type of instrumental expression. One definition identifies rhetoric with argumentation, another with eloquent language. Finally, while some definitions associate rhetoric exclusively with discourse, at least one leaves open the possibility that rhetoric may include non-discursive activities and objects" (Gill \& Whedbee, 1997: 155). Gemeinsam ist all diesen Theorien aber, dass (a) die grundsätzlichen rhetorischen Aktivitäten in einer politischen Arena stattfinden, und (b) die Rhetorik ein Diskurs ist, der darauf abzielt, ein Publikum in irgendeiner Art und Weise zu beeinflussen (vgl. Gill \& Whedbee, 1997: 155). In der vorliegenden Untersuchung wird die Vielschichtigkeit des Rhetorik-Begriffes zwar anerkannt, wie aber aus dem vorangegangenen Kapitel klar ersichtlich wird, interessiert hier vor allem die Nutzung rhetorischer Strategien, um die Adressaten von der Legitimität einer Unterordnungsaufforderung zu überzeugen. Es wird deshalb in der weiteren Argumentation die Definition von Cheney, Christensen, Conrad und Lair (2004) verwendet:

,[The study of rhetoric] means analysing the art of using symbols to persuade others to change their attitudes, beliefs, values or actions.“ (Cheney et al., 2004: 79)

Die Autoren grenzen in der Folge rhetorische Studien von anderen, verwandten Forschungsprogrammen ab. Der pragmatischen Tradition ähnelt die rhetorische durch den Fokus auf die tatsächlichen und potentiellen Effekte von Aussagen innerhalb eines spezifizierten sozialen Kontextes; von der Konversationsanalyse unterscheidet sie sich durch die Betrachtung von Situationen, welche über die dyadische bzw. die Gruppenebene hinausgehen; mit der Semiotik teilt sie das Interesse an symbolischen Kommunikationsformen, ist aber in ihrem Ansatz weniger strukturalistisch orientiert; narrative Ansätze haben Studien zur Rhetorik durch die Betonung von ,Storytelling' fruchtbar ergänzt; im Gegensatz zu ethnographischen Forschungen beschränkt sich die Untersuchung von Rhetorik auf formalisierte, öffentliche Kommunikation; und mit der kritischen Diskursanalyse schließlich teilt sie sich das Interesse an Phänomenen der Überzeugung und der Macht (vgl. Cheney et al., 2004: 81). Eine detaillierte und allgemeine Diskussion der Rhetorik als Disziplin kann in der vorliegenden Arbeit nicht geleistet werden. In der weiteren Argumentation wird stattdessen im Detail auf die Verbindung der Rhetorik zu den bisher diskutierten Konzepten von Organisation, Kommunikation und Macht bzw. Herrschaft eingegangen. Allgemeine Ausführungen zu den Kernkonzepten der Rhetorik sowie ein Überblick über existierende Forschungsvorhaben finden sich z.B. bei Cheney und Lair (2005), 
eine Diskussion grundlegender Elemente nehmen außerdem Gill und Whedbee (1997) vor. Einen detaillierten und ausführlichen Überblick über Geschichte und Grundlagen der Rhetorik bietet z.B. Ottmers (2007). Rhetorik in ihrer Rolle als Instrument der Überzeugung wurde außerdem in verschiedenen Studien, z.B. zu Strategien der Gewerkschaftsorganisation (Brimeyer, Eaker \& Clair, 2004), Unternehmenskampagnen (Bridges, 2004), zur Steigerung der Identifikation mit einer öffentlichen Organisation (DiSanza \& Bullis, 1999), im Bereich sozialer Bewegungsforschung (Hopkins \& Reicher, 1997) und organisationalen Wandels (Finstad, 1998) beleuchtet.

Wie bereits in den Ausführungen zur organisationalen Kommunikation diskutiert wurde, kann Organisation nicht von Kommunikation und somit von der Verwendung von Sprache getrennt werden. „Much of contemporary communication involves organizations ,speaking', thus invoking the traditional rhetorical situation: speaker $\rightarrow$ message $\rightarrow$ audience" (Cheney \& Lair, 2005: 76). Diese Sprache ist aber nicht neutral, sondern politisch und performativ. Organisationen bezwecken mit ihrer formalen Kommunikation, ihre Mitglieder von gewissen Wirklichkeitskonstruktionen zu überzeugen: „In fact, persuasion is inherent in the process of organizing [...]" (Cheney, 1983: 144). Rhetorik findet sich in allen Bereichen des Designs von Organisationen, sowohl in der Herstellung und Erhaltung von Hierarchie, der Differenzierung organisationaler Subeinheiten als auch der Formalisierung des Organisationsprozesses (vgl. Cheney \& Lair, 2005: 76). Die Untersuchung rhetorischer Elemente erlaubt der/dem ForscherIn eine gewisse Sensibilisierung für die Verwendung und Anpassung von Sprache und anderen symbolischen Elementen, mit denen verschiedene Bezugsgruppen angesprochen werden.

Die zentrale Rolle der Rhetorik für Kommunikation und Organisation wäre damit erläutert. Aber auch mit der Thematik von Macht und Herrschaft ist die Rhetorik stark verknüpft. Cheney und Lair (2005: 67f.) betrachten die Verbindung der beiden Konzepte zunächst etwas zögerlich: „To some scholars strongly allied with critical approaches to communication, power, and institutions, rhetoric lacks the sharp teeth of a power-centered critique [...]. From this perspective, why not talk about power, if it is power you really want to examine?" Diese Trennung von Strategien der Rhetorik und der Machtausübung ergibt sich aber in erster Linie aus ihrer eher engen Machtdefinition als ,[...] instances of brute domination (e.g., in wartime), the significance of material resources (e.g., in the statistical gap between the rich and poor), and the mechanisms that subvert deliberation and persuasion (e.g., literal or figurative contracts between employees and employers that delimit the free speech and ,interactive rights "of members)" (Cheney \& Lair, 2005: 72f.). In Kapitel 4.3 wurde für die vorliegende Arbeit aber eine Machtdefinition erarbeitet, welche explizit Überzeugung als Strategie 
der Machtausübung mit einschließt (vgl. Sandner, 1990: 108ff.). Rhetorik dient dann der klareren und erfolgversprechenderen Kommunikation von Dependenzen und Unterordnungsaufforderungen des A an die/den B. Über die Untersuchung rhetorischer Strategien können somit sowohl gewünschte (potentielle) Machtverhältnisse als auch Strategien der Macht- und Herrschaftssicherung rekonstruiert werden. Hinweise dazu finden sich auch bei Cheney und Lair (2005: $68)$, ,[...] a modified vocabulary and theoretical framework of rhetoric helps us strike to the heart of questions such as the establishment and maintenance of the authority of a ,corporate actor"“. Die Autoren bringen damit die Untersuchung von Rhetorik in einen Zusammenhang mit Foucault'schen Analysen von Institutionen, aber auch mit den hegemonischen Ansätzen von Gramsci - beides Theorien, welche im Kapitel über Macht referenziert wurden. Damit beschäftigen sich rhetorische Studien auch mit Fragen von Institutionen und der Institutionalisierung von Herrschaftsverhältnissen (vgl. Cheney \& Lair, 2005: 68). Die Autoren erkennen auch an, dass über Rhetorik nicht nur Wirklichkeiten sondern auch Identitäten konstruiert werden können (vgl. auch die Diskussion zu Macht, Diskurs und Identität in Kapitel 4.3.4), denn ,[e]specially in the modern industrialized world (and in postmodern society), a great deal of persuasion is tied up with issues of identity" (Cheney \& Lair, 2005: 76) ${ }^{14}$. Die Relevanz der Rhetorik für die vorliegende Untersuchung ist damit geklärt. Im Weiteren soll nun diskutiert werden, wie rhetorische Strategien konkret dazu beitragen können, durch Überzeugung eine Unterordnungsaufforderung zu erwirken.

\subsubsection{Wirkungsweise rhetorischer Strategien}

Rhetorik, so Gill und Whedbee (1997: 157), ,[...] is, in one way or another, a vehicle for responding to, reinforcing, or altering the understandings of an audience or the social fabric of the community." Damit ist die Einflussnahme deutlich mehr als eine bloße oberflächliche ,Überredung“ einzelner Individuen zu spezifischen Handlungen. Durch rhetorische Mittel entfaltet die kommunikative Rede ihre performative und wirklichkeitsstiftende Wirkung. Im Folgenden wird argumentiert, dass Führungsgrundsätze durch rhetorische Mittel über zwei hauptsächliche Kanäle Steuerungswirkung entfalten können: (a) über Prozesse der Identifikation der Organisationsmitglieder mit der Organisation bzw. mit

14 Dies betrifft sowohl die Konstruktion fremder als auch die der eigenen Identität: „,T]o manage one's audience in discourse is also to manage one's identity in discourse, whether that ,one' be an individual or some kind of group. The most profound challenge to advocacy by any organization is to develop a distinct identity while at the same time being recognized as part of the cultural ,crowd“" (Cheney \& Vibbert, 1987: 185). 
ihren Rollen (vgl. z.B. Cheney, 1983; Cheney \& Lair, 2005; Tompkins \& Cheney, 1985) - Organisationen schaffen durch inklusive Strategien eine begrenzte ,Angleichung' der individuellen Interessen ihrer Mitglieder an die Organisationsinteressen, was die weitere Überzeugung durch die ,Einimpfung ' von Entscheidungsprämissen erleichtert; (b) durch die eigentliche rhetorische Formulierung der Aufforderung, die mittels verschiedener Mechanismen an den Adressaten appelliert (vgl. z.B. Gill \& Whedbee, 1997: 158ff.) und diesen durch Bezug auf soziale geteilte Elemente des gemeinsamen Wissensvorrates überzeugt (vgl. z.B. R. E. Meyer, 2004: 200).

\subsubsection{Rhetorik und Identifikation}

Gemäß Cheney und Frenette (1993: 51) liegt die Antwort auf die Frage, durch welche Strategien der Überzeugung Individuen in den organisationalen Alltag integriert werden in den Faktoren, mithilfe welcher spezifische Prämissen für soziale Handlungen und Entscheidungen artikuliert, kontrolliert und den jeweiligen Individuen , eingeimpft' werden. Durch die Identifikation mit der Organisation - und den damit angebotenen Entscheidungsprämissen - wird ein Individuum in einer Entscheidungssituation spezifische Alternativen anderen vorziehen. Dadurch entsteht Steuerungswirkung (vgl. Tompkins \& Cheney, 1985: 191). Durch diesen Prozess findet das Individuum seinen Platz bzw. seine Rolle in der Organisation. Es entwickelt eine ,organisationale Persönlichkeit' (vgl. Tompkins \& Cheney, 1985: 191). Die Vielzahl an möglichen Zielen, Entscheidungsgrundlagen und Werten wird so auf die für die Organisation zentralen reduziert: Identifikation engt den Blick des Entscheidungsträgers ein (vgl. Tompkins \& Cheney, 1985: 192). Die Aufmerksamkeit wird auf zwei Arten gelenkt: (a) es werden nur noch bestimmte Probleme und Alternativen überhaupt gesehen, und (b) es werden jene Alternativen gewählt, welche den organisationalen Zielen am ehesten entsprechen (vgl. Tompkins \& Cheney, 1985: 194).

Für die Entstehung dieser Identifikation gibt es nun verschiedene Erklärungen. Tompkins \& Cheney (1985: 186) führen an, dass Organisationsmitglieder zum einen notwendigerweise einen gewissen Grad an Autonomie aufgeben, wenn sie einer Organisation beitreten (vgl. auch die legale Autorität bei Sandner, 1990: 118ff.). Zusätzlich spielen die Prozesse, welche Sandner (1990: 197ff.) als psychologische Steuerung bezeichnet - nämlich die Befriedigung der Wünsche der MitarbeiterInnen bei entsprechender Leistungserbringung und Regelbefolgung - eine Rolle. Aber auch durch die rhetorische Gestaltung organisationaler Kommunikation kann ein höherer Grad an Identifikation erreicht werden. Cheney (1983: 148 ff.) diskutiert verschiedene Möglichkeiten rhetorischer Strategien: (a) die sogenannte ,common ground technique', in welcher der Spre- 
cher (also die Organisation) eine starke Verbindung zwischen sich selbst und den Adressaten herstellt, um eine weitest gehende Überlappung organisationaler und individueller Interessen zu kommunizieren. In seiner Untersuchung findet Cheney (1983) mehrere Sub-Strategien, wie z.B. die Betonung des Werts des Individuums in der Organisation, die Anerkennung individueller Beiträge zum Organisationserfolg, das Eintreten für (annahmegemäß) geteilte Werte, die Kommunikation von Vorteilen und Leistungen für die Organisationsmitglieder, die Veröffentlichung von Lob durch Externe sowie die Publikation von positiven Bewertungen der Organisation durch MitarbeiterInnen; (b) die Identifikation durch ,Antithese', also die Definition eines ,gemeinsamen Feindes' und (c) die Verwendung eines angenommenen ,Wir' und die damit unterstellte Einheit der Interessen von Organisation und Individuum. Mittels all dieser Techniken kann die Organisation erreichen, dass ihre Mitglieder sich mit ihr selbst bzw. mit den von ihr vorgeschlagenen Rollen und Identitäten identifizieren. Diese Möglichkeit der organisationalen Einflussnahme wird aber dadurch abgeschwächt, dass jedes Individuum zu jedem Zeitpunkt grundsätzlich einer Vielzahl von möglichen wählbaren Rollen und Identifikationsangeboten ausgesetzt ist. Identifikation wird dadurch zu einem Management von „multiple, incongruent selves“ (C. Conrad \& Haynes, 2001: 60).

\subsubsection{Rhetorik und Argumentation}

Laut Gill \& Whedbee (1997: 158f.) gibt es drei klassische Wege, wie rhetorische Sprache überzeugen kann: (a) den Ethos, die Glaubwürdigkeit bzw. der Charakter des Sprechers, (b) den Pathos, die Gefühle, die beim Publikum geweckt werden und (c) den Logos, die Argumentation der Rede selbst. Außerdem ist seit dem griechischen Sophisten Gorgias die sogenannte ,Schicklichkeit (propriety) der Rede von Bedeutung: „At its most basic level, propriety is the adaptation of discourse to extrinsic variables such as the setting, the audience, and the occasion for the speech. Propriety governs the choice of when to speak, when to remain silent, what to say, and how to say it" (Gill \& Whedbee, 1997: 159). Von diesen drei Elementen ist der Logos, die eigentliche Argumentation jene, welche sich der Analyse am deutlichsten erschließt, da ihre Wirkung davon abhängt, dass sie klar und verständlich dargelegt wird: „In order to impinge on the behavior of others, actors must make themselves understood“" (Engelstadt, 2009: 215). Die Argumentationslehre wurde deshalb auch als das Herzstück der rhetorischen Lehre bezeichnet (vgl. Ottmers, 2007: 66).

Es wurde argumentiert, dass Überzeugung das primäre Vehikel darstellen muss, über welches Führungsgrundsätze Unterordnungsaufforderungen an die relevanten Adressaten kommunizieren. Die rhetorische Argumentationslehre 
bietet ein solides Gerüst, wie eine solche Überzeugung durch Argumentation geschehen kann und wie über die Analyse von Argumentationsmustern die dahinterliegenden (Welt-)Verständnisse, Werte und Normen (re-)konstruiert werden können.

„This form of power in communication may be analyzed along the lines of classical theory of rhetoric, which is the earliest reflection over the preconditions of persuasive power.“ (Engelstadt, 2009: 217)

In den folgenden Kapiteln wird deshalb zunächst geklärt, was unter einem Argument zu verstehen ist. Anschließend wird die grundsätzliche Wirkungsweise der Argumentation beleuchtet, bevor mit dem Enthymem und dem Topos zwei zentrale Konzepte der Argumentationslehre diskutiert und für die gegenständliche Untersuchung nutzbar gemacht werden.

\subsection{Zu den Begriffen von Argument und Argumentation}

Damit überhaupt eine Argumentation zustande kommen kann, sind zunächst drei wesentliche Bedingungen zu erfüllen: Zentral ist die grundsätzliche Strittigkeit (bzw. Bestreitbarkeit) eines Sachverhalts oder einer Angelegenheit. Nur dann ist Argumentation nötig (vgl. Kopperschmidt, 1989: 14). Auch Ottmers (2007: 68) bezeichnet die Strittigkeit als „,[d]ie wohl elementarste Vorbedingung für eine Argumentation. “" ${ }^{15}$ Damit ist in erster Linie aber nur die grundsätzliche Bestreitbarkeit eines Sachverhalts gemeint. Ob es zu einer tatsächlichen Bestreitung durch einen Gesprächspartner kommt, ist in vielen Situationen unerheblich. So kann es z.B. in Fällen monologischer Argumentation (vgl. Ottmers, 2007: 70ff.), in welcher ein Autor oder Sprecher die argumentative Rede alleine bestreitet, durchaus möglich und sinnvoll sein, Argumente quasi vorwegzunehmen, bevor ein tatsächlicher Einspruch geschieht. Die Möglichkeit der argumentativen Bewältigung von Problemlagen hingegen beruht in erster Linie auf Faktoren persönlicher und gesellschaftlicher Natur. Überzeugung durch Argumentation funktioniert nur dort, wo „Subjekte fähig und bereit sind, Problemlagen argumentativ zu bewältigen, und wenn die gesellschaftlichen Verkehrsformen diese Form kommunikativer Problembewältigung strukturell fördern" (Kopperschmidt, 1989: 15). Letztlich sind Argumente auch nicht für alle Formen problematisierender Fragen geeignet. Sachfragen, welche nach Ursachen oder Motiven fragen, sind mit Argumenten nicht sinnvoll zu bewältigen. Es sind deshalb in erster Linie Geltungsfragen (vgl. Kopperschmidt, 1989: 68ff.), welche durch Argu-

15 Das bedeutet nicht, dass nur in Kontroversen argumentiert werden muss. Auch dort, wo z.B. der Verlauf bzw. ,Gang‘ einer Rede oder eines Textes plausibel gemacht werden muss, ist Argumentation nötig (vgl. Ottmers 2007: 67). 
mentation beantwortet werden können. Solche Geltungsfragen bieten Geltungsgründe, „mit denen nicht auf empirisch externe Beziehungen zwischen Ursachen und Wirkungen noch auf innerpsychische Beziehungen zwischen Motiven und Handlungen, sondern auf internen (sic!) Beziehungen zwischen GAn [Geltungsansprüchen; Anm. DJ] und Geltungsgründen verwiesen wird, deren überzeugungskräftige Rekonstruktion den GAn ihre Berechtigung garantiert und sie entsprechend akzeptabel macht" (Kopperschmidt, 1989: 70). Diese Geltungsgründe sind eben als Argumente zu bezeichnen (vgl. Kopperschmidt, 1989: 70).

Unter Argumentation versteht Kopperschmidt (1989: 96) somit „,[d]ie spezifische Sprechhandlungssequenz, in der eine Sprechhandlung aufgrund ihrer positionsbedingten Rolle als Argument fungieren und entsprechend als Geltungsgrund für den problematisierten GA einer anderen Sprechhandlung beansprucht werden kann“. Ein Argument hingegen ist dann ein „konstitutives Element jeder Argumentation“ (Kopperschmidt, 1989: 96). Geltungsansprüche (GA) sind definiert als Anspruch, der für Äußerungen über subjektive Erfahrungen erhoben wird. Dieser Anspruch beinhaltet, dass „diese Äußerungen keine bloß subjektiven Gewißheitserlebnisse wiedergeben, sondern subjektiv-übergreifend bzw. intersubjektiv verbindlich sind“ (Kopperschmidt, 1989: 16). Derartige Äußerungen, mit denen ein Wahrheitsanspruch erhoben wird, nennt Kopperschmidt (1989: 17) Behauptungen; die gelungene Einlösung der durch sie eingeforderten Geltungsansprüche bezeichnet er als Gültigkeit (vgl. Kopperschmidt, 1989: 16).

Behauptungen sind aber nicht die einzigen Sprechakte, welche einen Geltungsanspruch vermitteln. Dasselbe gilt auch für Vorschläge und Empfehlungen, die ebenfalls strittig (oder umstritten) sein können (vgl. Kopperschmidt, 1989: 33). Vorschläge und Empfehlungen stellen jedoch keinen Wahrheitsanspruch, sondern einen Richtigkeitsanspruch: Dieser verbürgt im Gegensatz zu Behauptungen keine Verlässlichkeit der Information, sondern die Verbindlichkeit handlungsleitender Orientierungen.

„Während die Verläßlichkeit der Information den individuellen wie kollektiven Handlungsspielraum erweitert, indem sie die Wissens- und Erfahrungspotentiale beliebiger Subjekte instrumentell zu nutzen ermöglicht und die Effektivität eines von theoretischen Annahmen geleiteten intervenierenden Eingriffs in die Wirklichkeit gewährleistet, sichert die Verbindlichkeit handlungsleitender Orientierungen nicht so sehr die Handlungseffizienz, sondern die motivationale Handlungsbereitschaft kooperierender Subjekte, weil sie diese in gemeinsam akzeptierten Normen bzw. Werthaltungen fundiert.“ (Kopperschmidt, 1989: 35)

Empfehlungen und Vorschläge können damit dergestalt argumentiert werden, als ihre handlungsleitende Orientierung in einer ,als gültig akzeptierten Norm verortet werden kann" (Kopperschmidt, 1989: 34). Dies meint, dass nur Normen, „deren Geltung bzw. Existenz legitimiert ist oder als legitimierbar unter- 
stellt wird“ in Handlungsempfehlungen evoziert werden können, um deren Richtigkeitsanspruch einzulösen (vgl. Kopperschmidt, 1989: 38). Die nachfolgende Tabelle zeigt die Unterschiede zwischen den beiden Kategorien schematisch auf:

Tabelle 4: Zwei Arten strittiger Angelegenheiten (in Anlehnung an Kopperschmidt, 1989: 33ff.)
Behauptung
Empfehlung/Vorschlag

\begin{tabular}{l|ll}
\hline Sprechaktkategorie & konstativ & regulativ \\
Geltungsanspruch auf & Wahrheit & Richtigkeit \\
Verbürgt & Verlässlichkeit der Information & $\begin{array}{l}\text { Verbindlichkeit der Handlungs- } \\
\text { empfehlung }\end{array}$ \\
Bildet $a b$ & theoretische Wissensannahmen & praktische Wertentscheidungen
\end{tabular}

Aus der obigen Übersicht wird deutlich, dass innerhalb von Führungsgrundsätzen beide Kategorien relevant werden können. Zum einen werden Behauptungen aufgestellt, wenn eine gewisse Situation oder die Existenz spezifischer Rahmenbedingungen (dynamisches Umfeld, harter Wettbewerb, komplexe Personalstruktur, etc.) glaubhaft gemacht werden soll. Behauptungen treten aber auch immer dann auf, wenn Konzepte definiert werden (Führung ist/bedeutet XY; Eine erfolgreiche Führungskraft ist XY, etc.). Empfehlungen und Vorschläge, also handlungsleitende Orientierungen, werden dort kommuniziert, wo (z.T. aus den Behauptungen abgeleitet) spezifische Verhaltensweisen von den Akteuren verlangt werden.

Für die vorliegende Untersuchung ist ebenfalls die eingangs erwähnte Unterscheidung zwischen einer dialogischen und einer monologischen Argumentationssituation (vgl. Ottmers, 2007: 69ff.) von Interesse. Führungsgrundsätze entfalten ihre Wirkung in einer monologischen Argumentationssituation, welche konstitutiv ist für „Reden und Texte, die von nur einem Rhetor gehalten oder einem Autor verfasst werden“"(Ottmers, 2007: 70). Damit kann auch ihre Geltung nicht direkt während des Prozesses der Argumentation bestritten werden (dies entspricht in hohem Ausmaß auch den Ausführungen zur Potentialität der Machtausübung durch Führungsgrundsätze). Argumente und Gegenargumente müssen daher quasi vorweggenommen werden (vgl. Ottmers, 2007: 72). 


\subsection{Zur Überzeugungskraft von Argumenten}

In einer Situation, in welcher A versucht, den Geltungsanspruch seiner Äußerung durch ein Argument zu stützen, muss nach Kopperschmidt (1989: 111f.) das Argument folgende Eigenschaften besitzen, damit es überzeugungskräftig sein kann: (a) Es muss selbst unstrittig bzw. positiv formuliert sein. Wäre diese Bedingung nicht gegeben, müsste das Argument selbst wieder argumentiert werden (vgl. auch Ottmers, 2007: 79). (b) Es muss geeignet sein, in der Rolle eines Arguments eine These zu stützen, das heißt, es besteht die Notwendigkeit einer materialen Beziehung zwischen These und Argument bzw. der Schluss vom Argument auf die Konklusion muss einen inhaltlich plausiblen Zusammenhang herstellen (vgl. Ottmers, 2007: 79). Dieser wird zumeist durch legitimierte Theorien oder geltende Normen hergestellt, durch welche eine allgemein gültige Annahme auf einen konkreten Fall übertragen wird. (c) Es muss relevant sein, d.h. einem System angehören, welches für die Erklärung des vorliegenden Problemverständnisses innerhalb einer spezifischen sozialen Gemeinschaft als angemessen gilt.

Es ist Punkt (c), der im weiteren Verlauf der Argumentation besonders interessiert, nämlich die Frage, wann ein Argument in einer bestimmten Gemeinschaft als legitim gelten kann. Diese Frage rekurriert nämlich eben auf die spezifischen Wissensvorräte dieser Gemeinschaften. Jede Argumentation stellt grundsätzlich den Versuch dar, die Wahrheit oder Richtigkeit eines Geltungsanspruchs ,durch seine gelingende Rekonstruktion innerhalb eines Systems geltender Überzeugungen nachzuweisen“ (Kopperschmidt, 1989: 105).

„Die Überzeugungskraft von Argumentationen meint im Unterschied zur logischen Gültigkeit keine strikt logischen Deduktionsbeziehungen, sondern graduell differenzierbare Stützleistungen von Äußerungen innerhalb eines kategorial gewählten Systems von Äußerungen (substantielle versus analytische Argumentation). Dieses kategoriale System ist zwar hinsichtlich seiner problemspezifischen Relevanz selbst wieder problematisierbar („Diskursstufen“), doch für die Problematisierung von argumentativ verwendeten Einzeläußerungen ist das gewählte System der Rahmen, den kein Begründungsregreß überschreiben kann“ (Kopperschmidt, 1989: 121).

Die Wahl eines solchen Bezugssystems für die Argumentation impliziert auch eine spezifische Begründungssprache. Die Wahl einer solchen beeinflusst die Argumentation in zweierlei Weise: (a) Jede Begründungssprache bringt ein ganz bestimmtes Problemverständnis mit sich (vgl. Kopperschmidt, 1989: 105). Es macht einen Unterschied, ob Personalführung mittels einer psychologischen, einer ökonomischen oder einer formal-mathematischen Sprache diskutiert wird. (b) Die gewählte Begründungssprache entscheidet damit auch darüber, welche Art von Äußerungen als mögliche Argumente in Frage kommen (vgl. Kopperschmidt, 1989: 105). Wird eine formal-mathematische Begründungs- 
sprache gewählt, so sind Argumente, welche die unsterbliche Seele des Menschen evozieren, nicht mehr anschlussfähig bzw. ,verständlich'. Damit kommt klar zum Ausdruck, dass bereits die Wahl des Bezugssystems bzw. der Begründungssprache politisch sein kann. Ein Argument steht nie für sich allein, es kommuniziert die gesamte Sinnwelt (vgl. Berger \& Luckmann, 2000: 148ff.), aus welcher es stammt und in welcher es Legitimität besitzt, mit. Die Wahl einer angemessenen Begründungssprache bzw. des Bezugssystems ist deshalb grundsätzlich ebenfalls bestreitbar (vgl. Kopperschmidt, 1989: 107).

In ihrer Untersuchung globaler Managementkonzepte leistet Meyer (2004) eine für die vorliegende Arbeit äußerst erhellende Verbindung von Kopperschmidts Argumentationstheorie mit Konzepten der sozialen Bewegungsforschung. Die Anschlussfähigkeit einer Argumentation an eine bestimmte Begründungssprache bzw. ein Sinnsystem macht der von ihr verwendete Begriff des ,Accounts' deutlich. Ein Account ist definiert als ,procedure that utilizes everyday taken for granted knowledge to explain how an apparently problematic action makes sense within a broader framework of legitimate rules and activities“ (Lamertz \& Baum, 1998: 3, zit. in R. E. Meyer, 2004: 176). Somit sind diese Accounts sehr nahe an Kopperschmidts Argumenten, auch wenn sie nicht alle formalen Kriterien erfüllen. Sie sind aber argumentative Kommunikationsformen, welche „die Aufgabe [haben], etwas potenziell Infragegestelltes durch etwas Gültiges/Legitimiertes, auf das sie implizit oder explizit verweisen, abzusichern.“ (R. E. Meyer, 2004: 176). Akteure verweisen in Accounts „auf jene als geteilt unterstellten Wissensvorräte, die sie ihren als problematisch eingeschätzten Praktiken unterlegen möchten“ (R. E. Meyer, 2004: 177). Damit wird die eingangs erwähnte Relevanz des Bezugssystems (vgl. Kopperschmidt, 1989: 111ff.) angesprochen ${ }^{16}$. Diese wird dadurch hergestellt, dass die Argumentation mittels ,framing cues' auf spezifische ,Rahmungen' verweist, welche in einem sozialen Wissensvorrat verfügbar sind (vgl. R. E. Meyer, 2004: 150ff.). Rahmungen stellen über die Deutung und Inszenierung von Themen anschlussfähige Interpretationen zur Verfügung (vgl. z.B. Gamson \& Modigliani, 1989; R. E. Meyer, 2004; Meyer \& Höllerer, 2010). Mithilfe der Aktivität des ,framing wird eine Thematik so dargestellt, dass gewisse Aktivitäten legitimiert werden (vgl. R. E. Meyer, 2004: 151) ${ }^{17}$. Diese beabsichtigte Mobilisierung von Konsens stellt eine Schnittstelle zwischen Führungsgrundsätzen und der Theorie sozialer Bewegungen dar, obwohl die eingenommene Akteursperspektive und die Akti-

16 Vgl. dazu auch die detaillierten Ausführungen in Meyer (2004: 174ff.).

17, ,...] messages acquire a stronger seductive effect when they speak directly to the deep concerns of listeners, mobilizing their well-established frames of interpretation." (Engelstadt, 2009: 218) 
onsrichtung (top-down vs. bottom-up) eine diametral entgegengesetzte ist: Mobilisierung von Konsens bezieht sich auf den „langfristigen, absichtsgeleiteten Versuch von Bewegungsakteuren oder -organisationen, ihre Sichtweise innerhalb einer bestimmten Zielgruppe zu verbreiten" und Unterstützung zu generieren (R. E. Meyer, 2004: 153). Damit entspricht die Grundmotivation sehr stark jener des Managements für den Einsatz von Führungsgrundsätzen. Es ist diese erste Phase - die Herstellung von Konsens - welche hier besonders interessiert, da sie eben die von Kopperschmidt (1989) geforderte Relevanz eines Arguments für ein Problem herzustellen vermag ${ }^{18}$. Die Ableitung einer gewünschten Handlung ergibt sich im Anschluss quasi ,zwingend' aus der vorgebrachten Argumentation, wenn deren Prämissen und deren Eignung anerkannt werden. Rahmungen gehen aber noch weiter. Sie betreffen nicht nur die Identifikation eines Problems, sondern strukturieren als sozial verfügbare Orientierungen von Realität den gesamten Zusammenhang ,von Problemidentifikation bzw. -diagnose, Lösungsvorschlägen, Handlungsanleitungen und Positionierungen für Akteure und Akteursgruppen“ (R. E. Meyer, 2004: 155). Sie bilden damit auch bildlich gesprochen den ,Rahmen“ innerhalb dessen ein Argument sich abspielen muss, um überzeugend zu wirken. Daraus ergibt sich auch logisch, dass jedes Argument mittels der Verwendung spezifischer argumentativer Ressourcen (,framing cues $^{6}$ ) auf einen oder mehrere solcher Rahmungen verweist, welche ihm Legitimation verschaffen. Aufgrund der bisherigen Argumentationslinie der Arbeit ist davon auszugehen, dass Führungsgrundsätze vor allem sogenannte ,consensusframes ${ }^{6}$ evozieren, welche ,eine Wir-Definition entwickeln, aber kein klares Gegenüber definieren“ (R. E. Meyer, 2004: 159). Dies unterscheidet sie von sozialen Bewegungen, welche oft explizit gegen etablierte ,counterframes' antreten. Führungsgrundsätze benötigen für Ihre Argumente eine Legitimationsbasis, welche die gewünschten Macht- und Herrschaftsverhältnisse stützt und selbst möglichst unhinterfragt sind. Die Thematisierung existenter Gegenansätze würde den Hegemonieanspruch schwächen.

Das Problem der Relevanz eines Arguments innerhalb eines Bezugssystems lässt sich somit modellieren. Bevor in der Folge auf die Struktur der Argumentation eingegangen werden wird, soll noch kurz ein von Kopperschmidt (1989: 116) vorgebrachter Punkt berücksichtigt werden, nämlich die Unterscheidung zwischen überzeugen und überreden. Im Gegensatz zur Überzeugung geht es der Überredung nicht „um eine rational motivierte Anerkennung von GAn, sondern um den faktischen Zustimmungserfolg als Mittel der Realisierung von Inte-

18 Vgl. dazu auch den Unterschied zwischen den breiten, cultural themes' und den spezifischeren ,issue cultures‘ als Referenzrahmen bzw. Bezugssystem (vgl. R. E. Meyer, 2004: 154f.). 
ressen, deren Konsensfähigkeit erst gar nicht unterstellt wird. Freilich ist der mögliche Zustimmungserfolg nur zu erreichen, wenn die Überredungsabsicht strategisch verschleiert wird, d.h.: wenn sie sich als Überzeugungsabsicht zu verstellen versteht" (Kopperschmidt, 1989: 117). Diese Unterscheidung wird bei Kopperschmidt vor allem durch die Betonung von Kriterien der Vernunft und der Rationalität in der Argumentation wichtig. Kopperschmidt (1989: 117) selbst gibt aber zu, dass diese theoretisch mögliche Differenzierung in der Praxis schnell an ihre Grenzen stößt. Zudem taucht dieses Problem in der wissenschaftlichen Diskussion nur in spezifischen Sprachräumen auf (so hat sich in der englischsprachigen Diskussion der Begriff der persuasion durchgesetzt, welcher sowohl mit Überzeugung als auch mit Überredung ins Deutsche übersetzt werden kann). Es soll hier deshalb analog zu der in Kapitel 4.3.2 diskutierten Unterscheidung zwischen Überzeugung und Manipulation bei Sandner (1990) argumentiert werden, dass eine Entscheidung, ob in Führungsgrundsätzen überzeugt oder überredet wird, nicht empirisch getroffen werden kann, insbesondere deshalb, weil gemäß der bisherigen Argumentationslinie der Arbeit durch beide Strategien eine Realisierung von Interessen bezweckt wird. Nichtsdestotrotz werden auf einer konzeptionellen Ebene die unterschiedlichen ethischen und moralischen Unterschiede der beiden Strategien anerkannt.

Nachdem also das Bezugssystem einer Argumentation als der zentrale Ansatzpunkt einer (Re-)konstruktion der durch Argumente ausgedrückten und vermittelten Sinnwelt identifiziert wurde, soll im Folgenden kurz angerissen werden, mittels welcher Konzepte aus der Argumentationstheorie ein solches Bezugssystem (re-)konstruiert werden kann. Dazu werden in den nächsten Kapiteln das Enthymem und der Topos vorgestellt.

\subsection{Das Enthymem als Struktur des rhetorischen Arguments}

Das Enthymem ist die einfachste und essentiellste Struktur der Argumentation. Komplexere Formen werden z.B. in Kienpointner (1992: 22ff.) diskutiert. Für den Anspruch dieser Arbeit reicht die einfache Fassung des Enthymems aber vollkommen aus. Bei der Enthymemargumentation wird ein Argument so eingesetzt, dass es eine strittige Aussage glaubhaft macht und eine Konklusion stützt. Die Verbindung zwischen einem Argument und einer Konklusion bildet die sogenannte Schlussregel (vgl. Ottmers, 2007: 74; vgl. auch die Ausführungen zur Struktur von Argumenten bei Toulmin, 2008). 


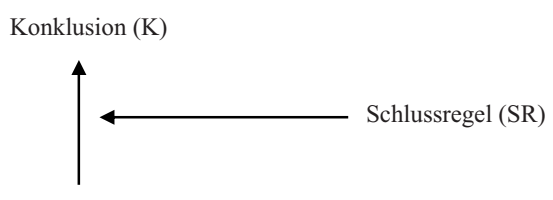

Argument (A)

Abbildung 9: Struktur des Enthymemarguments (vgl. Ottmers 2007: 74)

Das Enthymem weist dabei einige spezifische Kennzeichen und Eigenarten auf:

- Die formale Struktur des Enthymems ist nicht festgeschrieben. Die Reihenfolge und Anordnung der Teilsätze ist frei wählbar (vgl. Ottmers, 2007: 75).

- Ein Enthymem kann auch verkürzt verwendet werden. Nicht immer wird es in allen seinen Schritten explizit gemacht. Besonders verbreitet ist die Vorenthaltung der Schlussregel (vgl. Ottmers, 2007: 75f.). Das Enthymem wird deshalb auch als ,verkürzter Syllogismus“ bezeichnet: „It is ,abbreviated“ because it omits a premise; the audience creates coherence in the incomplete argument by consciously or unconsciously supplying the ,missing link' from the premises in their own belief system" (Gill \& Whedbee, 1997: 171).

- Das Enthymem ist ein ,Plausibilitätsschluss‘. Während die grundsätzliche syllogistische Struktur beibehalten wird, besitzen Enthymeme keine formale Gültigkeit. Vielmehr basiert diese auf der Wahrscheinlichkeit des gezogenen Schlusses (vgl. Ottmers, 2007: 77; Wengeler, 2003b: 178). Wie bereits im vorigen Kapitel ausführlich diskutiert wurde, muss die Argumentation sich in einem verständlichen und legitimierten Begründungssystem verankern. Außerdem muss sich eine enthymemische Argumentation stets auch der Adressaten bewusst sein und deren Voraussetzungen berücksichtigen (vgl. Ottmers, 2007: 77).

- Da das Argument eine strittige Aussage stützen soll, darf es selbst nicht strittig sein. Es kann zwar im Einzelfall hinterfragt werden, darf aber nicht in einen , unendlichen Begründungsregress' fallen (vgl. Kopperschmidt, 1989: 111ff.; Ottmers, 2007: 79).

- Die Schlussregel muss zwischen dem Argument und der Konklusion einen stringenten und inhaltlich möglichst plausiblen Zusammenhang herstellen (vgl. Kopperschmidt, 1989: 111ff.; vgl. Ottmers, 2007: 79).

Enthymeme sind aber nicht nur eine einfache Struktur, um Argumente darzulegen. Durch die obige Charakterisierung des Enthymems wird klar, dass in die Konstruktion eines solchen Arguments eine Menge sinnstiftender Arbeit eingeht: „In creating and responding to enthymemes, speaker and audience reveal their unstated beliefs and values; they reveal their ideology or ,implicit philoso- 
phy' about the nature of reality, the nature of their community, and their conception of appropriate social relations" (Gill \& Whedbee, 1997: 172). Damit wird klar, warum das Enthymem als Untersuchungsgegenstand für die vorliegende Untersuchung angemessen ist. Wenn in die Konstruktion einer solchen Argumentation die impliziten Weltbilder eingehen und wenn diese Weltbilder anschlussfähig an größere diskursive Sinnsysteme (z.B. Rahmungen) sein müssen, um legitim zu wirken - ,[i]n simple terms, the primary characteristic of the enthymeme is that it requires that the rhetor draw upon premises shared by the persuadee(s) in effecting persuasion" (Cheney \& Frenette, 1993: 53) -, dann lassen sich durch die systematische Analyse von Enthymemen und den ihnen zugrundeliegenden Schlussregeln solche Sinnsysteme (re-)konstruieren.

An dieser Stelle ist es notwendig, noch einmal auf die Diskussion des $\mathrm{Zu}$ sammenhangs zwischen Rhetorik und Identifikation zurückzukommen, um die breiteren Zusammenhänge zu klären. Es wurde argumentiert, dass über die ,Einimpfung ' von Entscheidungsprämissen (diese entsprechen in der hier diskutierten Form des Enthymems dem Argument) Organisationsmitglieder insofern gesteuert werden können, als sie in der Folge gewünschte Schlussfolgerungen (Konklusion) ziehen.

„The study works from the basic assumption that a primary persuasive activity of large corporations and other organizations today in the United States is the establishment and maintenance of a set of value premises which serves as a resource for large and small argumentative efforts" (Cheney \& Frenette, 1993: 51).

Die enthymemische Struktur dieses Vorgangs wird von Tompkins und Cheney sogar ausdrücklich betont und diskutiert (vgl. Tompkins \& Cheney, 1985). Die Versuche, über rhetorische Mittel eine Identifikation der Organisationsmitglieder zu erreichen, sind der Überzeugung durch Argumentation folglich vorgelagert. Identifikation unterstützt das Funktionieren einer Argumentation in zweierlei Hinsicht: (a) durch die ,Einimpfung' von Prämissen wird versucht, das Kriterium zu erfüllen, dass die Prämisse/das Argument unstrittig sein muss (vgl. Ottmers, 2007: 79) und (b) durch die verschiedenen Strategien der Erreichung von Identifikation (,common grund technique', Abgrenzung durch Antithese, angenommenes ,Wir', vgl. Cheney, 1983) wird versucht, ein geteiltes Bezugssystem zu schaffen (bzw. die Organisationsmitglieder von dem für die Organisation vorteilhaften Bezugssystem zu überzeugen), innerhalb dessen die eingeimpften Prämissen als gültig erscheinen. Die Überzeugung durch Identifikation erreicht damit selbst keine argumentative Wirkung, sie vereinfacht und unterstützt aber alle folgenden Argumentationen, welche die geschaffenen Bezugssysteme nicht brechen. Es ist also zu erwarten, dass innerhalb der Führungsgrundsätze beide groben Strategien anzutreffen sind, da diese Dokumente eben 
monologisch argumentieren müssen. Zwar besteht die Möglichkeit, die Identifikation der Organisation auch über andere Kanäle zu erreichen, es ist aber dennoch zu vermuten, dass Führungsgrundsätze auch selbst diese Strategien verwenden, um die ebenfalls enthaltenen Argumentationen quasi direkt und vor Ort in ein gewünschtes Bezugssystem (wie sollen die Regeln verstanden werden?) einzubetten.

Das Problem der Verwendung von Enthymemen als Untersuchungseinheiten besteht nun aber in der grundsätzlich fragmentierten Struktur alltäglicher Argumentation. Der größte Teil organisationaler Rhetorik entspricht nicht der Toulmin'schen (vgl. Toulmin, 2008; Van Eemeren, Grootendorst, Jackson \& Jacobs, 1997) Form des Arguments: „If the enthymeme were operating in much of the formal public communication of business, Sproule argued, then it was in an almost unrecognizable form" (Cheney \& Lair, 2005: 73). Ähnlich bemerkt auch Meyer (2004: 197): „Eine funktionale Argumentationsanalyse (Toulmin 1996; Kopperschmid 1989) ist - neben den oben angesprochenen forschungspragmatischen Gründen - auf konzeptioneller und methodologischer Ebene damit konfrontiert, dass sich die Akteure in ihren Alltagskommunikationen nicht an Argumentationsregeln halten und nur , verkürzt ${ }^{\star}$ argumentieren - sie verankern ihre Äußerungen in einen ,Normenhintergrund" oftmals ohne Angabe von Übergangs- bzw. Schlussregeln durch eine Vielzahl unterschiedlicher ,framing-cues", die den Deutungshintergrund als ,bekannte Struktur" , aufrufen" sollen [...]". Argumentationen finden sich also nicht nur in expliziter Form. Gerade aber auch solche Aussagen, in denen eine Argumentation nur implizit enthalten ist, müssen der Analyse zugänglich gemacht werden (vgl. Wengeler, 1997: 124f.). Oder um es wieder mit Meyer (2004) zu sagen:

„Auch wenn Accounts nicht als Begründungen im argumentationstheoretischen Sinne verstanden werden können, liefern sie dennoch die gewünschte Rahmung mit, die sich durch die verwendete Sprache, mit der ein Problem thematisiert wird [...] manifestiert, d.h. in ihren Kommunikationsleistungen transportieren die Akteure - bewusst oder unbewusst - Bedeutungsüberschüsse, indem sie auf diese soziale verfügbaren Wissensbestände rekurrieren, um ,Probleme` zu definieren und Lösungswege aufzuzeigen. Da sich die zugrunde liegenden Wissensstrukturen in verschiedenen Symbolen, diskursiv insbesondere in den verwendeten Argumentationsstrukturen und konkreten Argumentationslinien materialisieren, sind sie über die Äußerungen der Akteure einer Analyse zugänglich“ (R. E. Meyer, 2004: 194).

Im folgenden Kapitel wird nun der Topos als Kategorie eingeführt, welche sich in (unvollständigen) Enthymemstrukturen zeigt und Rückschlüsse über das verwendete Bezugssystem zulassen kann. 


\subsection{Topoi als,Arsenale' rhetorischer Mittel}

Der Topos-Begriff teilt sich schon bei Aristoteles in zwei unterschiedliche, aber eng mit einander verbundene Konzepte (vgl. Ottmers, 2007: 89). Zum einen sind Topoi „nützliche und für jede rhetorische Auseinandersetzung taugliche Arsenale argumentativer Mittel, aus denen der Argumentierende die für seine Zwecke jeweils passenden hervorholt - es sind sozusagen die Quellen, über die jeder Redner oder Autor verfügen muss, um aus ihnen die Argumente ,hervorziehen“ zu können" (Ottmers, 2007: 89). Von daher stammt auch die alternative Bezeichnung der Topoi als ,Orte“ (loci) der Argumentation (Ottmers, 2007: 89). In diesem Verständnis ermöglichen Topoi die Bildung von Enthymemen insofern, als mit ihnen „erforderlichenfalls ohne große Schwierigkeiten geeignete Enthymeme zu finden und anzuwenden" sind (Sprute, 1975: 78, zit. in Wengeler, 2003b:179). Zum anderen bezeichnen Topoi aber auch die eingesetzten Schlussmuster, „deren Plausibilität die ,Kraft‘ (vis) der Argumentation garantiert. Von entscheidender Bedeutung dabei ist, dass der Topos nicht mit der Argumentation selbst, also mit deren Inhalten und Aussagen, identisch ist - dass die Topik also keine schon fertigen, vorstrukturierten Argumentationsmuster bereithält, die sich mehr oder weniger schematisch auf jede nur denkbare Argumentationssituation anwenden lassen. Topisches Denken meint vielmehr die kreative Fähigkeit, die ,gefundenen' Aspekte einer Argumentation miteinander $\mathrm{zu}$ verbinden und daraus überzeugungskräftige Aussagen abzuleiten." (Ottmers, 2007: 90). Als Topos wird in der relevanten Literatur nun z.T. eher unsystematisch entweder „,der in der Schlussregel hergestellte Zusammenhang“ (vgl. Wengeler, 2003b: 181) oder die Schlussregel selbst (vgl. Ottmers, 2007: 88; Wengeler, 2003b: 180) verstanden. In der gegenständlichen Untersuchung werden Topos und Schlussregel nicht gleichgesetzt, sondern der ersten Variante gefolgt, dass der Topos die Idee ist, welche hinter der Schlussregel steht und diese legitimiert und verständlich macht. Dies ist zum einen darin begründet, dass nur dadurch die inhaltliche Unabhängigkeit des Topos von der Argumentation gesichert werden kann, zum anderen bietet auch nur diese Definition die Möglichkeit, dass allgemeine Topoi aus besonderen bzw. inhaltlich spezifizierten Schlussregeln abgeleitet werden können (siehe weiter unten). Wichtig für die gegenständliche Untersuchung ist außerdem die Tatsache, dass die Schlussregel - und damit auch der Topos - „zumeist nicht explizit ausgesprochen wird“ (Wengeler, 2003b: 181, siehe auch die Argumentation im vorherigen Kapitel zur Unvollständigkeit von Enthymemen). Die (Re-)konstruktion von Topoi ist somit ein interpretativer Akt, sie müssen aus den tatsächlich sprachlich realisierten Teilen der Argumentation erschlossen werden (vgl. Wengeler, 2003b: 181). Dazu bieten verschiedene Autoren ein Instrumentarium an, welches im methodi- 
schen Teil der Arbeit genauer erläutert werden wird. Der Topos bietet sich außerdem für die Untersuchung der proklamierten Macht- und Herrschaftsbeziehungen in Führungsgrundsätzen dahingehend an, als das ,Zielen auf Plausibilität und die mangelnde Expliziertheit der Argumentation" (Wengeler, 2003b: 181) ein Kennzeichen der Führungsgrundsätze sind.

Topoi können dabei sowohl in allgemeiner als auch in besonderer Form auftreten. Während allgemeine Topoi ,in ihrer Mehrzahl das kontextabstrakte Strukturprinzip einer Argumentation vorstellen“, sind besondere Topoi ,wesentlich enger an die in einer Argumentation transportierten Inhalte gebunden" (Ottmers, 2007: 93). Da die besonderen Topoi auf konventionalisierten Schlussverfahren beruhen, sind sie stärker als die allgemeinen Topoi den Veränderungen des Meinungs- und Erfahrungswissens unterworfen (vgl. Ottmers, 2007: 93). Ein allgemeiner Topos ist einer, der ,,auf alle Gegenstände in allen Redegattungen anwendbar ist" und mithilfe dessen deshalb über verschiedenste Themenbereiche Enthymeme gebildet werden können (Wengeler, 2003b: 182). Diese allgemeinen Topoi sind den von Kienpointner (1992) identifizierten kontextabstrakten Argumentationsmustern sehr ähnlich. Beide (allgemeine Topoi wie kontextabstrakte Argumentationsmuster) sind für eine Untersuchung der spezifischen Überzeugungskraft von Führungsgrundsätzen zu allgemein (für eine formalere bzw. strukturorientiertere Untersuchung von Unternehmenstexten mithilfe von Topoi vgl. z.B. Diatlova, 2003). Interessanter hingegen sind die sogenannten besonderen Topoi. „Die besonderen Topoi sind also im Gegensatz zu den allgemeinen formalen Schlussmustern inhaltlich spezifizierte ,Schlussregeln', die entsprechend nur in einem bestimmten inhaltlichen Bereich verwendbar sind, um plausible Argumentationen zu realisieren“ (Wengeler, 2003b: 183). In der Regel lassen sich solche besonderen Topoi allerdings auch auf ein formales bzw. abstraktes Schlussmuster zurückführen.

Auch Meyer (2004: 174) bestätigt - wenn auch indirekt - die Eignung von Topoi für die (Re-)konstruktion breiterer diskursiver Sinnstrukturen. Sie versteht Topoi als sehr ähnlich zu ,interpretativen Packages" (vgl. auch Gamson \& Modigliani, 1989; Meyer \& Höller, 2010), auf welchen sie ihre eigene Analyse aufbaut, und zwar insofern, als diese ,inhaltlich bereits angereicherte Muster, die aber dennoch so allgemein sind, daß sie zu verschiedenen Zwecken gefüllt werden können, die also strategisch eingesetzt werden können, was nicht als bewußte Strategie verstanden werden muss", (Wengeler, 1997: 128) darstellen ${ }^{19}$ :

19 Dazu auch Ottmers (2007: 90): „Aufgrund der Tatsache, dass die Topoi zwar inhaltliche Bezüge aufweisen, nicht jedoch mit den Inhalten der Argumentation identisch sind, wird ihr zweites Charakteristikum ersichtlich: jeder Topos ist ,offen', er kann verschiedene, sogar entgegengesetzte Argumentationsmöglichkeiten eröffnen. Das heißt, dass man, ausgehend von ein- und demselben Topos, in Hinblick auf denselben strittigen 
„Die Rekonstruktion solcher Denkfiguren kann zeigen, welche möglichen Wirklichkeitskonstruktionen in einem Diskurs zu einem spezifischen Thema existieren und welchen Entwicklungen diese Muster im Zeitablauf unterliegen (vgl. das Konzept der ,issue cultures' ${ }^{6}$ “. Das Bezugssystem stellt die , organizing idea'; die verwendeten Begründungssprachen, die auf dieses Bezugssystem rekurrieren, machen sich durch die Signifikanz bestimmter Begriffe oder Sprachbilder bemerkbar, mit deren Hilfe ein Problem thematisiert wird, in denen sich ein bestimmtes Problemverständnis artikuliert [... ]“" (R. E. Meyer, 2004: 174).

Bei Wengeler (2003b: 188ff.) findet sich eine sehr ausführliche Diskussion über die Verwendung des Topos-Begriffs in verschiedenen wissenschaftlichen Disziplinen und über die Geschichte hinweg. Für die vorliegende Arbeit reicht es aus, den Topos in der bisher vorgestellten Diskussion zu verwenden, die sich sehr nahe an der ursprünglichen aristotelischen Definition befindet (vgl. Wengeler, 2003b: 188). Eingegangen werden soll aber noch auf die von Wengeler (2003b: 234ff.) diskutierte Verknüpfung von Topos und Lebenswelt, da diese sehr gut zeigt, dass die vorgeschlagene Form der Argumentationsanalyse sich problemlos an die der Arbeit zugrunde liegende Perspektive einer phänomenologischen Wissenssoziologie anschließen lässt. Die Verbindung besteht darin, dass sich „die persuasive Argumentation bei ihren Situationsklärungen auf die topischen Strukturen des von den Beteiligten jeweils geteilten Lebensweltbildes" (Oesterreich 1990: 80, zit. in Wengeler, 2003b: 235) bezieht. Dieses Argument wurde in der konzeptionellen Aufarbeitung der vorliegenden Arbeit bereits mehrfach gemacht: Überzeugend wirkt, was sich in irgendeiner Form auf die Lebenswelt und den geteilten Wissensvorrat der Adressaten bezieht: „Dieser Wissensvorrat bildet ein ,Lebensweltbild', das als Reservoir von Deutungsschemata zur Situationsbestimmung zur Verfügung steht und durch seine sprachliche Verfasstheit ,sozial objektiviert" ist." (Wengeler, 2003b: 235). Die topische Argumentationsanalyse ist also an das Lebenswelt-Konzept anschließbar und mit dessen Grundannahmen vereinbar (vgl. Wengeler, 2003b: 235).

\subsubsection{Rhetorik und Diskurs}

In aller Kürze soll - nach der Betrachtung der Mikro-Ebene der Argumentation - nun noch der Sprung zurück auf die diskursive Ebene geleistet werden. Die Frage, die sich hier stellt, ist jene nach der Reziprozität von Rhetorik und Diskurs. Dass der Diskurs in Form interpretativer Rahmungen Einfluss auf das Ge-

Sachverhalt ganz unterschiedliche, ja sogar gänzlich konträre Argumentationen ableiten kann.“ 
lingen rhetorischer Argumentation nimmt, wurde im vorherigen Kapitel ausführlich diskutiert.

,[...] rhetorical criticism has responded to the contemporary view that linguistic structures or systems of discourse order and give sense to human experience (Foucault, 1972), that is, the way in which we talk about the world affects how we understand or ,see“ it.“(Gill \& Whedbee, 1997: 159)

Kann aber der einzelne Akteur durch seine rhetorischen Handlungen den Diskurs gezielt verändern? Es wird hier argumentiert, dass der individuelle Akteur die soziale Struktur bzw. den Diskurs zwar nutzen, ihn aber nicht beliebig oder gezielt kontrollieren oder verändern kann. Dies ist vergleichbar mit Gramscis (1985, zit. in Haugaard, 2009: 246) Idee der ,privaten Grammatik': „Extending the Greek idea of the ,idiot' as a ,private individual', Gramsci argues that freedom is not freedom to create your private grammars, which is the freedom of an ,idiot"." Stattdessen besteht die Freiheit darin, ,grammars' und Bedeutungssysteme auf neuartige Weise zu benutzen und zu kombinieren, ohne die sozial gültigen Diskurse völlig zu transzendieren (vgl. Haugaard, 2009: 246) ${ }^{20}$.

„[...] meaning is being managed by various institutional actors so as to privilege certain interpretations of the situation and soft-pedal or suppress entirely other, alternative understandings“" (Cheney \& Lair, 2005: 56).

Dadurch kann aber langfristig auch der Diskurs verändert werden: „For example, the work of Czarniawska-Joerges (1995) on the changing relationship between Sweden's public and private sectors shows well how the rhetorical choices of individual agencies and companies both reflect and contribute to broader socio-economic trends regarding privatization of public activities, the withering of the welfare state, and the infusion of business models into decision making and self-representation in just about all types of organizations" (Cheney \& Lair, 2005: 72). Diese Dialektik - welche wiederum die Dialektik zwischen Handlung und Struktur in wissenssoziologischen Ansätzen spiegelt - zeigt sich auch für die einzelne Organisation. Die meisten Organisationen sind sowohl Quelle als auch Ziel rhetorischer und diskursiver Realitätskonstruktion (vgl. Cheney \& Vibbert, 1987).

Auch Foucault thematisiert die Möglichkeit, den Diskurs zu überwinden: ,[...] Foucault's genealogical analysis indicates that while all interpretations are open for relative testing, not all interpretations are equal. In social systems, the acceptance of an interpretation must be built upon a claim of validity, part of which is acquiring legitimacy over alternative interpretations." (Gordon, 2009: 268). Dieser ,claim“ bzw. die dazugehörigen Strategien „are both constrained and enabled by a social system's constitution of knowledge. People reflect upon

Vgl. dazu auch Burrs (1995: 113 ff.) Ausführungen zur „person as a discourse user“. 
their knowledge to make sense of what is happening in their world and to decide how they will position themselves strategically with respect to these happenings. In other words, people position themselves to acquire legitimacy for their viewpoint or preferred course of action" (Gordon, 2009: 268). Dieser Prozess des Legitimitätserwerbs , has a discursive dimension that is distinctly political, strategic, empirical and, thus, context dependent" (Gordon, 2009: 269). Dies bietet eine ausgezeichnete Anschlussmöglichkeit an rhetorische Strategien des Legitimitätserwerbs und damit der Beeinflussung diskursiver Machtverhältnisse.

\subsubsection{Zur Eignung rhetorischer Analysen für die Unter- suchung}

Um den Beitrag der Rhetorik zur gegenständlichen Untersuchung zu diskutieren, soll zunächst noch einmal die zweiteilige Forschungsfrage in Erinnerung gerufen werden:

Welche (impliziten und expliziten) Vorstellungen von Führung und Steuerung finden sich in kontemporären Führungsgrundsätzen deutschsprachiger Großunternehmen?

Mithilfe welcher rhetorischen Strategien und Ressourcen werden diese Vorstellungen argumentiert und damit im organisationalen Diskurs verankert?

Mittels der im konzeptionellen Teil der Arbeit erarbeiteten Grundlage - so die Argumentation - lässt sich die Untersuchung im Wesentlichen als Analyse rhetorischer Strategien verstehen. Die versuchte organisationale Einflussnahme auf organisationale Rollen und die Interaktionen zwischen diesen typisierten Akteuren innerhalb der Führungsgrundsätze (der erste Teil der Forschungsfrage) geschieht über rhetorische Mittel, nämlich die argumentative Stützung bzw. Legitimierung der vorgeschlagenen Beziehungen (der zweite Teil der Forschungsfrage). Die beiden Fragestellungen sind deshalb im gegenständlichen Projekt nicht voneinander zu trennen. Durch die Untersuchung der Begründungs- und Argumentationsbemühungen treten die den Dokumenten zugrundeliegenden basalen Weltverständnisse (bzw. Bezugssysteme) hervor. Dadurch wird es möglich ,[to] employ rhetorical theories and concepts in a heuristic way to examine the functioning of the modern organization itself. Here we return to fundamental aspects of organization such as the individual's relationship to the organization, the organization's status as an actor, and the ongoing construction of authority" (Cheney \& Lair, 2005: 60).

Laut Gill und Whedbee (1997: 161) stehen der/dem ForscherIn in der Untersuchung rhetorischer Elemente verschiedene Leitfragen zur Verfügung: „In answering these or other questions, a critic once again draws on her particular store 
of knowledge; she crawls around and through a text, inspecting it from every angle, from some distance as well as up close, and she makes judgments about how it operates and what it says (and does not say)." Diese Fragen beziehen sich zum einen auf den Kontext der rhetorischen Rede, aber auch auf die Konstruktion des Sprechers, des Publikums sowie der Sprechsituation und letztlich auch auf interessierende Elemente des Textes selbst. Es sind diese textlichen Elemente, oder in der Terminologie von Keller (2008: 234) diskursiven Ereignisse, welchen die hauptsächliche Aufmerksamkeit der gegenständlichen Untersuchung gilt. Die Entscheidung darüber, welche rhetorischen Elemente des Textes in die Untersuchung einbezogen werden, ergibt sich in erster Linie aus der Forschungsfrage. In der vorliegenden Untersuchung liegt das Interesse vor allem in der Überzeugungskraft der Dokumente und damit in der (Re-)konstruktion jener Sinnwelten, vor deren Hintergrund die vorgebrachten Argumente als sinnvoll erscheinen. Der zentrale Untersuchungsgegenstand (neben der Rekonstruktion der Akteursrollen, welche gleichzeitig auch die (Re-)konstruktion des typisierten Publikums bedeutet) ist somit die Analyse der Argumentationsstrukturen innerhalb der Dokumente (vgl. Gill \& Whedbee, 1997: 171), mittels welcher der zweite Teil der Forschungsfrage (die Frage nach dem Wie der Konstruktion von Akteursrollen und -beziehungen) beantwortet werden soll. Dabei greift die Untersuchung auf die in den vorangegangenen Kapiteln vorgestellten Konzepte der Enthymeme und der Topoi zurück. Mittels inhalts- und diskursanalytischer Methoden werden (unvollständige) Enthymeme (re-)konstruiert und die ihnen zugrundeliegenden Topoi identifiziert. Im Anschluss daran werden die vorgefundenen Argumentationsmuster mit den ebenfalls zu (re-)konstruierenden Akteursrollen und -beziehungen zusammengeführt, um ein vollständiges Bild davon zu erreichen, welche Macht- und Herrschaftsbeziehungen im Diskurs deutschsprachiger Führungsgrundsätze als legitim betrachtet werden und mittels welcher diskursiver und argumentativer Ressourcen diese Legitimität gestützt wird.

Das folgende Kapitel der Arbeit widmet sich nun ausführlich der Frage, wie die zentralen Forschungsfragen methodologisch und methodisch behandelt werden können. Dazu wird zunächst ein Überblick über das interpretative Forschungsparadigma geboten. Anschließend werden der diskursanalytische Ansatz des gegenständlichen Projekts expliziert und zentrale Aspekte der Argumentationsanalyse diskutiert. Nach diesem detaillierteren Überblick über die methodologischen Überlegungen folgt eine Erläuterung der konkreten Vorgehensweise und des für die empirische Analyse des Materials eingesetzten Methodenkanons. 


\section{Die empirische Untersuchung}

\subsection{Methodologische Ausrichtung}

Nachdem die konzeptionelle Basis für die Untersuchung gelegt wurde, soll in den folgenden Kapiteln ein Überblick über die methodologischen Grundlagen und die methodische Vorgehensweise der Arbeit gegeben werden. Da sich die gegenständliche Untersuchung mit ihrem Fokus auf Sinnwelten und soziale Wissensvorräte in die Tradition interpretativer Sozialforschung einordnet, sollen in einem ersten Schritt kurz die grundsätzlichen Prämissen und Grundlagen dieser Form der wissenschaftlichen Forschung näher beleuchtet werden. Die Argumentation verläuft grundsätzlich analog zu Lueger (vgl. z.B. Froschauer \& Lueger, 2009; M. Lueger, 2001, 2010) unter Einbezug weiterer zentraler Autoren und Zugänge. Lueger (2001: 19ff.) diskutiert vier zentrale Fragen, mit welchen sich eine methodologische Diskussion grundsätzlich beschäftigen muss:

- Wie gelangen Menschen überhaupt zu ihrer Erkenntnis über die Welt?

- Welche Besonderheiten der sozialen Welt ergeben sich aus dieser erkenntnistheoretischen Perspektive?

- Welche Forschungshaltung liegt einer interpretativen Analysestrategie zugrunde, um verlässliche Erkenntnisse zu produzieren?

- Wie kann die konkrete Organisierung einer interpretativen Forschungsarbeit erfolgen?

Diesen Fragen widmet sich das vorliegende Kapitel. Damit wird ein grundsätzlicher Rahmen geschaffen, innerhalb dessen sich die vorliegende Untersuchung verortet. Die Fragen der Analysestrategien und der Organisierung der Forschungsarbeit werden im Anschluss diskutiert. Danach wird auf die Diskursanalyse als grundsätzliche Ausrichtung der gegenständlichen Untersuchung eingegangen und spezifische Ansatzpunkte der Argumentationsanalyse als Spezialfall der Diskursanalyse vorgestellt. Anschließend werden das Datenmaterial und die Samplingstrategien vorgestellt, bevor die konkrete Vorgehensweise und der eingesetzte Methodenkanon erläutert werden.

\subsubsection{Epistemologische Grundlagen}

Die Notwendigkeit der Frage nach der Möglichkeit der Erkenntnis über die Welt ergibt sich daraus, dass „Methoden epistemologischen und methodologischen Vorstellungen über den prinzipiellen Aufbau der untersuchten Welt und den wissenschaftlichen Zugängen zu dieser untergeordnet sind“ (M. Lueger, 2001: 23). Dies bedeutet, dass sich der vorgeschlagene Methodenkanon einer wissen- 
schaftlichen Untersuchung sinnvoll aus epistemologischen Überlegungen ergibt und aus diesem Zusammenhang heraus begründet werden muss. Die zentralen Voraussetzungen wissenschaftlicher Erkenntnis bestehen dabei in der Fähigkeit zum Erwerb und zur Weitergabe von Wissen, der Möglichkeit der Beobachtung eines Erkenntnisobjekts, einer klaren Vorstellung von sozialer Realität bzw. von Wirklichkeit als Grundlage einer systematischen Analyse, der Möglichkeit der Kommunikation von Wissen (die Bedeutung der Sprache) und letztlich einer Diskussion der Abgrenzung wissenschaftlicher Erkenntnis von anderen Erkenntnisformen (vgl. M. Lueger, 2001: 25). Erkenntnis kann nun aber nicht rein individuell gefasst werden. Sie ist zwar gebunden an eine „letztlich nicht hintergehbare körperliche Situiertheit des Individuums" (M. Lueger, 2001: 33), der Körper des Individuums besitzt jedoch spezifische, sozial mitgeformte Operationsweisen, welche den Erkenntnisgewinn beeinflussen. Erkenntnis kann daher nur als soziale Erkenntnis verstanden werden. Sie greift über die individuelle Ebene hinaus (vgl. M. Lueger, 2001: 37).

„So ist die sensumotorische Ausstattung zentral für das Verständnis organisierter Sozialsysteme, die emotionale Grundstimmung abhängig von kulturellen Regulativen und die Gedächtnisleistung als Wissensstrukturierung eine Frage der Regelung der Zugriffsrechte auf Wissensprozesse.“ (M. Lueger, 2001: 35)

Durch diese Betonung der Ordnung sozialer Prozesse und lebensweltlicher Erfahrungshorizonte ordnet sich das hier vertretene Verständnis in die Tradition konstruktionistischer Perspektiven (vgl. z.B. Dachler \& Hosking, 1995; K. J. Gergen, 1994, 1999; Shotter, 1993) - im Gegensatz zu konstruktivistischen Perspektiven mit einem stärkeren Fokus auf individuelle kognitive Konstruktionsleistungen - ein (zur Unterscheidung vgl. auch M. Gergen, 1995:98f.).

Von Bedeutung ist in diesem Zusammenhang die Unterscheidung zwischen Realität und Wirklichkeit. Erkenntnis kann sich niemals auf die objektive Realität beziehen, diese bleibt immer außerhalb des Bewusstseins (vgl. Froschauer \& Lueger, 2009: 25ff.). Stattdessen erschafft das Individuum in seiner sozialen Interaktion mit anderen eine Wirklichkeit als Modell dieser Realität durch aktive Zuwendung und kognitive Verarbeitung von Erfahrungen (vgl. auch Berger \& Luckmann, 2000). Da diese Wirklichkeit aus der aktiven Erkenntnistätigkeit entsteht, ist ihre konkrete Ausgestaltung ebenfalls an die körperliche Situiertheit des Menschen gebunden (vgl. Froschauer \& Lueger, 2009: 26). Die Konstruktion einer solchen Wirklichkeit entsteht in der Auseinandersetzung mit der Umwelt (vgl. Froschauer \& Lueger, 2009: 27ff.). Diese Auseinandersetzung geschieht in erster Linie durch aktive Beobachtung und das Ziehen von Differenzen: „Beobachten konstituiert eine von der Umwelt unterschiedene Einheit durch Differenzierung und in weiterer Folge Beschreibung“ (M. Lueger, 2001: 
81). Die Bezeichnung eines unterschiedenen Objektes in der Umwelt kann nun aber nur über seine Abgrenzung von anderen Objekten geschehen ${ }^{21}$. Für die Sozialwissenschaft ist wichtig, dass BeobachterInnen sich nicht nur der Differenzkriterien des Beobachteten bewusst sein müssen, sondern auch ihrer eigenen, da diese den Standort der Beobachterin/des Beobachters definieren, und sich das Phänomen von jedem Standort aus anders präsentiert (dialektische Relationierung der Beobachtung zum Beobachteten). Man muss sich also der eigenen Theorien bewusst sein, welche die Beobachtung leiten:

„Genauer gesagt müssen sich sozialwissenschaftliche BeobachterInnen von drei Differenzmomenten anleiten lassen: (a) ihrer eigenen Anwendung von Differenzkriterien anhand der dem Beobachteten impliziten Handlungsoptionen für eine/n BeobachterIn, denn ohne solche sind Beobachtungen unmöglich [also die von den Objekten nahegelegten Differenzkriterien; Anm. DJ]; (b) den von den AkteurInnen im sozialen Feld gezogenen Differenzen, die in allen Handlungen implizit vorhanden (oder beobachtbar) sind ; (c) der Selbstreflexion der von ihnen im wissenschaftlichen Prozeß der Erkenntnisgewinnung zu handhabenden Differenzkriterien, deren Wahl auch von ihrer Anschlußfähigkeit im Wissenschaftssystem abhängt.“ (M. Lueger, 2001: 88)

In der interpretativen Sozialforschung gilt das Primat der Differenzen, welche die beobachteten Akteure selbst ziehen anstelle von theoretischen Vorannahmen, die überprüft werden sollen (M. Lueger, 2001: 92f.). Es interessieren daher vor allem die Beobachtungs- (und damit die Konstruktionsprozesse) der Akteure im Feld. Sozialwissenschaftliche Beobachtung gestaltet sich als ,Beobachtung von Beobachtungen' und somit als Beobachtung zweiter Ordnung (vgl. Giddens, 1984; Hitzler \& Eberle, 2005; Soeffner, 2004). Ein zentraler Aspekt der sozialen Wirklichkeit ist deren Sinn und die damit verbundene Ordnungsleistung (vgl. Berger \& Luckmann, 2000: 104ff.; M. Lueger, 2001: 117). Sinn kann nur im kollektiven Prozess (also intersubjektiv) angeeignet werden und erzeugt soziale Handlungsfähigkeit (vgl. Froschauer \& Lueger, 2009: 34). Soziale Interaktionen sind somit Kernprozesse der Wissenserzeugung. Durch den sozial konstituierten Charakter von Wahrheit und Sinn können diese immer nur unter Berücksichtigung ihrer Entstehungsumstände eingeschätzt werden (vgl. Dachler \& Hosking, 1995:4; K. J. Gergen, 1994: 51f.). Sinn macht spezifische soziale Kategorien

21 Beobachtungen sind offen (sie werden nicht durch die beobachteten Objekte determiniert) und nichtbeliebig (ein Objekt kann nicht beliebig betrachtet und beschrieben werden). Die Forschung hat daher die Aufgabe, ,die Eigenschaften und Grenzen von Analyseobjekten zu umreißen, um die Voraussetzung für wissenschaftliches Beobachten zu schaffen“ (M. Lueger, 2001: 85). Abgrenzungskriterien sind aber immer eine Frage der Relationierung, da jedes Objekt unendlich viele Abgrenzungskriterien nahelegt (z.B. Gewicht, Farbe, Funktion, etc.) (vgl. M. Lueger, 2001: 85). 
erst erlebbar, indem er einen Verweisungshorizont generiert, welcher Möglichkeiten und Unmöglichkeiten definiert sowie Beziehungen zu anderen sinnhaften Objekten herstellt (vgl. Froschauer \& Lueger, 2009: 33ff.). Nur durch Rückgriff auf den sozial konstituierten Sinn lässt sich somit ein Geisterfahrer von anderen Autofahrern und ein Hamburger von einem Cheeseburger , sinnvoll' unterscheiden (vgl. M. Lueger, 2000: 23). Damit ist Sinn ein Ergebnis des Zusammenspiels objektiver Bedingungskonstellationen und der biographisch-lebensgeschichtlichen Situiertheit des Individuums. Sinn reflektiert die Rahmenbedingungen seiner Produktion (vgl. M. Lueger, 2001: 129). Gesellschaftlicher Sinn wird auf dem Wege der Sozialisation vermittelt. Auf der höchsten Ebene bieten symbolische Sinnwelten ,Welterklärungsmodelle‘. Im Zuge der sekundären Sozialisation wird das Individuum aber in spezielle Sinnenklaven integriert, welche als ,Subwelten“ der symbolischen Sinnwelt verstanden werden können (vgl. Berger \& Luckmann, 2000: 104ff. bzw. 148ff.). Verstehende Sozialwissenschaft muss deshalb in ihrer Analyse auf die (Re-)konstruktion sozial konstituierter Sinnstrukturen rekurrieren. Sinn erreicht in der sozialen Welt die Möglichkeit von Komplexitätsreduktion. Diese lässt sich wiederum als Strukturierung fassen, auf welche interpretative Forschung in ihren (Re-)konstruktionsbemühungen abzielt (vgl. Froschauer \& Lueger, 2009: 39). Strukturen treten in der sozialen Welt als Strukturierungsnetzwerk auf, was bedeutet, dass die Sozialwissenschaft Zentrierungen vornehmen muss, welche angeben, ,aus welcher Perspektive bzw. kontextuellen Relationierung heraus ein Phänomen analysiert wird“ (Froschauer \& Lueger, 2009: 42). Von Bedeutung ist auch, dass solche Strukturen eine historische, also zeitliche, Dimension besitzen und nur aus einem historisch-konkreten Zusammenhang heraus verständlich werden können (vgl. K. J. Gergen, 1999: 48; M. Lueger, 2000: 30ff.; Shotter, 1993: 12).

Es stellt sich aber auch die Frage, wie nun Sinn und die ihm zugrundeliegenden Strukturen in einer Gesellschaft erfahrbar werden. Dazu muss die Rolle der Kommunikation als komplexer Prozess der Sinnproduktion (vgl. Froschauer \& Lueger, 2009: 47ff.) diskutiert werden. Erst über Kommunikation wird Sinn vermittelbar:

„Beobachtung erfordert eine zeichenhafte (oder auch sprachliche) Umsetzung als Ausdruck (Signifikant) eines Inhalts (Signifikats), die die Welt in ein komplexes differenzlogisches System faßt, das weit über die Unterscheidung in Ein- und Ausgeschlossenes hinausgeht und durch Oppositionsbegriffe ein Geflecht von Differenzen produziert.“ (M. Lueger, 2001: 82)

Über Kommunikation bzw. Sprache kommt der Prozess der Wirklichkeitskonstruktion und der Formierung gesellschaftlicher Verhältnisse zum Ausdruck (vgl. K. J. Gergen, 1999: 49; Shotter, 1993: 28). Über Zeichensysteme wird Wirklichkeit vermittelt und konstruiert. Sprache ist dabei das wichtigste Zei- 
chensystem der menschlichen Gesellschaft (vgl. Berger \& Luckmann, 2000: 39). Da Worte nicht aufgrund einer gemeinten Korrelation zu präexistenten Bedeutungen, sondern im Sprachspiel (vgl. Wittgenstein, 2006: 241ff.) ihre Bedeutung erhalten, lassen sich kollektive Bedeutungen über die Analyse sprachlicher Äußerungen (re-)konstruieren (vgl. M. Lueger, 2001: 108).

„Für die (Re-)konstruktion sozialer Beziehungen erweist es sich konsequenterweise als sinnvoll, zu überlegen, in welchen Kontexten (d.h. im Fall welcher Sprachspiele) Äußerungen als sinnvoll erscheinen, um die Regeln eines solchen Kontextes schrittweise anhand der Regeln des Sprachspiels zu explizieren. In einer solchen Vorgangsweise unterstellt man, daß:

- Jede Äußerung in ein sinnvolles Sprachspiel eingeordnet werden kann und daher nicht beliebig ist;

- Bedeutungen nicht einfach die Bezeichnung eines Sachverhaltes reflektieren, sondern den sozialen Umgang damit;

- Der lebensweltliche Kontext Wirkungen produziert, die sich in Äußerungen und deren sozial konstituierte Bedeutungen auffinden lassen;

- Handlungsmöglichkeiten nicht nur aufgrund physischer Bedingungen vorgegeben, sondern auch sozial anhand normativer Konstruktionen begrenzt werden;

- Zeichen grundsätzlich nicht mittels einer Dekomposition in die dyadischen Elemente verstanden werden können.“ (M. Lueger, 2001:110)

Die Analyse von Wirklichkeit kann also zur Analyse der Regeln werden, nach denen Sprachspiele gespielt werden (vgl. M. Lueger, 2001: 105). Deshalb ist es zulässig, dass sich die sozialwissenschaftliche Analyse in erster Linie mit Protokollen oder Spuren fixierter lebensweltlicher Äußerungen beschäftigt, welche auf Phänomene, Handlungen, Strukturen oder Prozesse verweisen (vgl. M. Lueger, 2001: 95).

\subsubsection{Methodologische und forschungspraktische Aspekte}

\subsubsection{Maximen und Grundsätze}

Aus den bisherigen Ausführungen leitet Lueger (vgl. Froschauer \& Lueger, 2009; M. Lueger, 2001) nun für die konkrete Ausgestaltung interpretativer Forschungsvorhaben drei zentrale Maximen ab, welche ,auf methodologischer Ebene von der Formulierung von Forschungsfragen, der Gestaltung des Forschungsdesigns, der Organisierung des Forschungsprozesses, der Art der Materialerhebung und der entsprechenden Analyseformen bis hin zur Darstellung der Ergebnisse die gesamte Forschungsarbeit durchdringen“" (Froschauer \& Lueger, 2009: 59): 
Wissenschaft als Spezialfall konstruktiver alltäglicher Interpretation verweist darauf, dass wissenschaftliche Beobachtung im Grunde nach denselben Prinzipien funktioniert wie alltägliche Beobachtung. Sie unterscheidet sich von dieser jedoch zum einen dadurch, dass es sich eben um Beobachtungen zweiter Ordnung handelt (höhere Distanz zum unmittelbar Beobachteten) und zum anderen, dass die Wissenschaft sich bestimmter Verfahrensweisen bedient, welche u.a. eine Systematisierung des Zweifels und damit einen höheren Reflexionsgrad erlauben (vgl. Froschauer \& Lueger, 2009: 59ff.; M. Lueger, 2001: 253ff.).

Orientierung an der Totalität sozialer Phänomene betont, dass ein Erkenntnisgegenstand seine Sinnbezüge als Ordnungsleistung nur über die Einbettung in einen Kontext erschließen lässt. Einzeleinheiten erlangen Bestand erst durch andere Ereignisse, welche die Einzelheit in einen Konstitutionsprozess sozialer Phänomene oder Situationen integrieren. Diese anderen Ereignisse kommen aber selbst durch die Einzelheit erst zum Vorschein. Die Totalität sozialer Phänomene verweist somit auch auf das dialektische Prinzip (vgl. Froschauer \& Lueger, 2009: 63ff.; M. Lueger, 2001: 262ff.).

Die tätige Beobachtung als Ausgangspunkt wissenschaftlicher Forschung schließlich beleuchtet die Tatsache, dass Produktion und Reproduktion rekursiv miteinander verbunden sind, dass also das eine ohne das andere nicht möglich ist. Forschung ist somit ein aktiver Prozess, der niemals ,neutral' wirkt. Tätige Beobachtung beeinflusst den Beobachtungbereich; die spezifische Vorgehensweise der Forscherin/des Forschers konstituiert das analysierte Phänomen. Eine reine Rekonstruktion von Bedeutungen ohne entsprechende ,Co-Konstruktion seitens der Forscherin/des Forschers ist nicht möglich, auch wenn es notwendig ist, sich methodisch auf die Strukturierungsleistungen des Feldes zu fokussieren. Dieser dialektische Prozess von Konstruktion und Rekonstruktion wird deshalb unter dem Begriff der (Re-)konstruktion gefasst (vgl. Froschauer \& Lueger, 2009: 67ff.; M. Lueger, 2001: 272ff. ).

Auf der Grundlage dieser Maximen ergeben sich verschiedene Prinzipien für das Design der vorliegenden Studie:

Offenheit. Um vom Feld getätigte Strukturierungen nicht durch eine Vorstrukturierung der Forscherin/des Forschers von vornherein zu unterdrücken oder zu zerstören, soll die/der ForscherIn möglichst offen an das Feld herangehen, und zwar gegenüber den Untersuchungspersonen, der Situation und auch bezüglich der Methoden. Nur so ist es möglich, die Relevanzstrukturen der Akteure im Feld zu (re-)konstruieren und nicht durch theoretische Vorannahmen zu verstecken (vgl. Lamnek, 2005: 21f.; M. Lueger, 2001: 279ff.).

Forschung als Kommunikation. Die Interaktion zwischen der/dem ForscherIn und der/dem Beforschten wird als konstitutiver Bestandteil des Forschungsprozesses gesehen und nicht als Störvariable. Dialektische Konstruktionsprozes- 
se in der Kommunikationssituation zwischen ForscherIn und Beforschter/m dürfen nicht vernachlässigt werden. Die Beforschten (bzw. die Akteure, deren ,Spuren' betrachtet werden) sind orientierungs-, deutungs- und theoriemächtige Subjekte (vgl. Lamnek, 2005: 22; M. Lueger, 2001: 275f.).

Der Prozesscharakter von Forschung und Gegenstand. Sowohl der Forschungsgegenstand als auch der Akt des Forschens selbst müssen als Prozess begriffen werden. Der Forschungsgegenstand ist keine statische Repräsentation eines unveränderlichen Wirkungszusammenhangs, sondern wird als prozesshafter Ausschnitt der Konstruktion sozialer Realität betrachtet. Die ForscherInnen wirken durch ihre Tätigkeit an dieser Konstruktion mit (vgl. Lamnek, 2005: 23).

Reflexivität von Gegenstand und Analyse. Der Erkenntnisgegenstand lässt seine Sinnbezüge nur über seine Einbettung in seine(n) Kontext(e) erschließen (vgl. M. Lueger, 2001: 262f.). Mit Reflexivität ist dann gemeint, dass jede Bedeutung reflexiv auf das Ganze verweist. Handlungen werden nur unter Bezugnahme auf den relevanten Kontext verständlich (vgl. Lamnek, 2005: 23f.). Reflexivität der Analyse bedeutet, dass diese auf unerwartete Situationsmomente im Prozess des Forschungsaktes abgestimmt werden können muss.

Explikation. Einzelschritte des Untersuchungsprozesses sowie Interpretationsschritte sollten möglichst offen gelegt werden, um eine Nachvollziehbarkeit zu gewährleisten. Interpretative Forschungsvorhaben sind in der Regel weniger stark formalisiert und standardisiert sind als quantitative Designs. Der Herstellung von Nachvollziehbarkeit kommt daher auch im Rahmen der Qualitätssicherung große Bedeutung zu. Eine vollständige Explikation der forschungs- und interpretationsleitenden Strukturen ist jedoch kaum möglich, da diese der/dem ForscherIn (als gesellschaftlich sozialisiertem Individuum) oft selbst nicht in expliziter Form verfügbar sind (vgl. Lamnek, 2005: 25).

Flexibilität. Da bei qualitativen Studien zumeist davon ausgegangen wird, dass der zu untersuchende Aspekt des sozialen Lebens noch nicht hinlänglich bekannt ist, wird von der/dem ForscherIn eine gewisse Flexibilität bei der Vorgehensweise verlangt. Diese wird außerdem bedingt durch die notwendige Offenheit bei der Herangehensweise an das Feld. Eine solche Flexibilität bedeutet vor allem, dass der Blickwinkel auf das Phänomen zuerst notwendigerweise weit sein muss und sich dann im weiteren Forschungsverlauf verengt (vgl. Lamnek, 2005: 25).

\subsubsection{Der interpretative Forschungsprozess}

Qualitativ-interpretative Untersuchungen unterliegen grundsätzlich einem zyklischen Forschungsdesign und sind deshalb nur sehr schwer in linearer Form darstellbar (vgl. M. Lueger, 2001: 363ff.). Diese Zirkularität zeigt sich in drei Di- 
mensionen, und zwar im abstrakten Wissenschaftssystem, in dem Forschungen in den Gesamtzusammenhang der Wissenschaft oder der Disziplin eingebunden werden, in der konkreten Organisierung des Analyseprozesses, in dem die einzelnen Forschungsphasen zyklisch wiederholt werden und als Analysezyklen als Versatzstücke interpretativer Strategien, in denen Interpretationsschritte zyklisch wiederholt werden, um die Zusammenhänge zu einem immer dichteren theoretischen Gefüge zu vernetzen. Diese zyklische Organisierung lässt sich in folgender Abbildung darstellen:

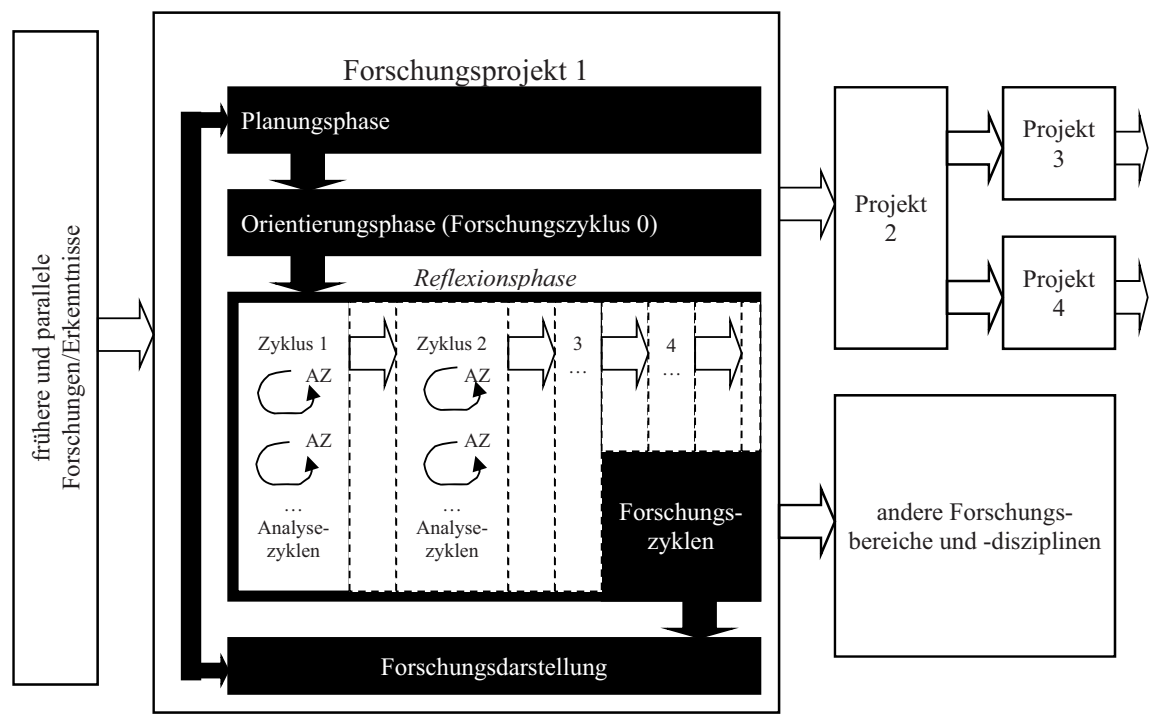

Abbildung 10: Die zirkuläre Organisierung der Forschung im Wissenschaftsprozess (M. Lueger, 2001: 363)

Die vorliegende Untersuchung ist somit eingebettet in einen Zyklus auf der Ebene des Wissenschaftssystems, in dem sie sich früherer Untersuchungen bedient und zur Grundlage späterer Forschungsvorhaben wird. Auf der Ebene der Forschungsorganisierung entsteht ein ,Pseudozyklus' (vgl. M. Lueger, 2001: 364), in dem sich die Phasen der Planung und der Darstellung dem Zyklus zwar in gewissem Grade entziehen, dazwischen aber im Forschungsverlauf permanent Wiederholungen, Modifikationen und Transformationen stattfinden. Innerhalb der Forschungszyklen werden die Prozesse von Datenerhebung, -auswertung 
und -interpretation ständig wiederholt ${ }^{22}$; zwischen den einzelnen Zyklen werden immer wieder Reflexionsphasen eingefügt, in welchen das weitere Vorgehen anhand der bereits vorhandenen Daten geplant wird. In der Planungsphase werden grundsätzliche Entscheidungen bezüglich des gewählten Forschungsparadigmas, der Erkenntnis- und Aussagenreichweite sowie der grundsätzlichen Forschungsorganisierung und der Handlungsbedingungen getroffen (vgl. M. Lueger, 2001: 367f.). In der darauf folgenden Orientierungsphase erfolgt der Feldzugang mit den prinzipiellen Überlegungen zur Feldpositionierung, Methodenanwendung und inhaltlicher und methodischer Forschungsstrukturierung. Diese Phase umfasst im Wesentlichen den hier im Methodenkapitel erarbeiteten Teil der Dissertation. Die eigentliche Zirkularität beginnt dann in der Phase der primären Forschungszyklen. In dieser Phase herrscht ein unentwegtes Ineinandergreifen von Erhebung und Interpretation, das ergänzt wird durch systematische Reflexionsphasen zur weiteren Planung der Forschung. Von Bedeutung ist dabei eine permanente Überprüfung und Modifikation der generierten Interpretationen sowie die ständige und detaillierte Dokumentation aller im Forschungsprozess getroffenen Entscheidungen (vgl. M. Lueger, 2001:367f.). Die abschließende Darstellung der Forschungsergebnisse schließt den Zyklus durch Rückbezug auf die Ausgangssituation und stellt gleichzeitig im Zyklus des Wissenschaftssystems die Anschlussfähigkeit an die Disziplin und somit den Nutzen für spätere Forschungsprojekte sicher (vgl. M. Lueger, 2001: 369).

\subsubsection{Zum Qualitätsbegriff interpretativer Sozialforschung}

Das Thema der Qualitätssicherung hat gerade im qualitativ-interpretativen Paradigma eine breite Aufmerksamkeit erfahren. Zum einen bedarf der Rückgriff auf Interpretationen einer starken Kontrolle, um nicht die Zuverlässigkeit der Forschungsleistungen in Frage zu stellen, zum anderen widerspricht die Art der Analyse in vielerlei Hinsicht dem traditionellen, quantitativ orientierten Wissenschaftsverständnis (vgl. Froschauer \& Lueger, 2003: 166). Die Durchsicht verschiedener Ratgeber zur Durchführung qualitativer Forschung (vgl. z.B. Flick, 2002; Flick, von Kardorff \& Steinke, 2005; Froschauer \& Lueger, 2003; Lamnek, 2005; M. Lueger, 2001) zeigt jedoch, dass sich trotz geteilter Grundannahmen noch keine einheitliche Vorgehensweise für die Qualitätssicherung in der qualitativen Forschung durchsetzen konnte. Dies liegt auch daran, dass die

22 Aufgrund der Tatsache, dass der Datenkorpus im vorliegenden Projekt schon zu Projektbeginn feststand, war eine uneingeschränkte Umsetzung dieser Zirkularität nicht möglich. In die induktive Konstruktion des Codierschemas wurden jedoch innerhalb dieses Korpus nach den Kriterien von Nähe und Differenz sukzessive neue Dokumente einbezogen. 
Verwendung bewährter quantitativer Gütekriterien für qualitative Studien nicht zielführend ist (vgl. Froschauer \& Lueger, 2003: 166). Gegen eine Verwendung allgemeingültiger Qualitätsstandards spricht sich auch Flick (2002: 394ff.) aus. Die wichtigsten Argumente in der Diskussion sollen nun systematisiert dargestellt und auf die vorliegende Arbeit angewandt werden.

Auf der Ebene der Methodologie und der Verfahren empfehlen Froschauer und Lueger (2003: 167f.) die Zerstörung von Vorwissen durch Systematisierung des Zweifels. Dies wird in dieser Arbeit dadurch erleichtert, dass kein systematisches theoretisches Wissen über Führungsgrundsätze vorhanden ist. Diesem Kriterium wird außerdem dadurch Rechnung getragen, dass durch die (Re-)konstruktion der Begründungszusammenhänge innerhalb der Dokumente die ,voice“ den Akteuren überlassen wird. Annahmen der Forscherin/des Forschers (bzw. gängiger Theorien) zur Führung und Steuerung werden somit zunächst systematisch ausgeblendet und erst in der Interpretation der Ergebnisse als Kontrastfolie wieder an das Datenmaterial herangetragen. Die Autoren empfehlen weiters eine extensive Sinnauslegung, um die mögliche Perspektivenvielfalt in der Interpretation zu berücksichtigen. Dieser Aspekt ist vor allem in der Datenanalyse zu beachten und ist inhärenter Bestandteil der (Re-)konstruktion von Topoi durch die Analyse von Enthymemstrukturen. Auch die geforderte Berücksichtigung von Belanglosigkeiten und Anomalien (vgl. dazu auch M. Lueger, 2001: 341) wird geleistet. Die (Re-)konstruktion der Argumentationen im Feld bringt gerade Normalität und Abweichung ins Blickfeld. Die Notwendigkeit der Elaborierung des Kontextes sozialer Phänomene (vgl. M. Lueger, 2001: 339) wird sowohl durch die Rückbindung der Ergebnisse an das Genre als auch durch die (Re-) konstruktion jener Kontexte, in welchen die vorgefundenen Argumente als sinnvoll erscheinen, berücksichtigt. Als problematisch erweisen sich die Forderungen nach der Auslegung im Team und der Trennung von Interviewer und Interpret (vgl. Steinke, 2005: 326). Da eine Dissertation in erster Linie als Einzelleistung konzipiert ist, kann diesen Kriterien nur in eingeschränktem Ausmaß entsprochen werden.

Auf der Ebene des Forschungsprozesses definieren Froschauer und Lueger (2003:168f.) weitere Kriterien: Der zyklischen Organisierung des Forschungsprozesses wurde im Forschungsdesign entsprochen. Reflexionsphasen wurden in regelmäßigen Abständen vorgenommen, um die weitere Vorgehensweise zu planen (vgl. auch Taylor \& Trujillo, 2001: 183). Dies beinhaltet auch die Forderung nach einem theoretischen Sampling der Untersuchungseinheiten (vgl. Glaser \& Strauss, 1998; Locke, 2001) und der Einhaltung des Kriteriums theoretischer Sättigung (vgl. Glaser \& Strauss, 1998; Taylor \& Trujillo, 2001). Reflektiert werden soll jedoch nicht nur über die vorläufigen Ergebnisse, sondern auch über die Bedeutung der Forschungstätigkeit bei der Erzeugung dieser (vgl. M. 
Lueger, 2001: 339). Weiters soll eine systematisch prüfende Intervention zum besseren Verständnis der Strukturierungsprozesse des Untersuchungsbereiches stattfinden. Eine systematische Variation von Erhebungssituationen, Methoden und Perspektiven durch die dreiteilige Analyse von Handlungsmustern, Beziehungstypen und Topoi wird grundsätzlich erfüllt, der Variation von InterpretInnen hingegen kann, wie oben erwähnt, im Rahmen der Dissertation nicht in vollem Ausmaß entsprochen werden. Eine Supervision aus distanzierter Perspektive ist für die Dissertation ohnehin vorgesehen.

Auf der Ebene des Wissenschaftssystems bedarf qualitative Forschung einer strengen Argumentation der Relevanz für die Wissenschaft sowie der Offenlegung der Forschungsstrategie und wichtiger Schlüsselentscheidungen und Verfahren (vgl. Froschauer \& Lueger, 2003: 169f.; M. Lueger, 2001: 341; Steinke, 2005: 324). Weiters wird auch eine exemplarische Erläuterung sensibler Forschungselemente sowie die Darstellung in einer anschlussfähigen narrativen Struktur gefordert (vgl. auch Taylor \& Trujillo, 2001: 183). Diese Elemente gilt es vor allem in der abschließenden Diskussion der Forschungsergebnisse zu beachten.

\subsection{Diskursanalyse als Forschungsstrategie}

Um die rhetorischen Strategien innerhalb deutschsprachiger Führungsgrundsätze adäquat untersuchen zu können, muss sich die Arbeit einer Forschungsstrategie bedienen, welche in der Lage ist, die konstruktive und performative Rolle der Sprache und Kommunikation ausreichend zu erfassen. Grundsätzlich folgt die Arbeit deshalb einer diskursanalytischen Logik. Diskurs ist in dieser Perspektive „the principal means by which organization members create a coherent social reality that frames their sense of who they are" (Mumby \& Clair, 1997: 181). Chilton und Schäffner (1997: 205) verbinden die Studie des Diskurses mit zwei elementaren Konzepten der gegenständlichen Arbeit. Aufgrund der Charakterisierung des Menschen als ,linguistic animal' durch René Descartes und als ,political animal' durch Aristoteles schließen sie, dass diese beiden Aspekte Kommunikation und Politik - nicht getrennt voneinander betrachtet werden können. Ihre Verbindung finden Sie in der Rhetorik als Kunst verbaler Überzeugung. Die Analyse von in sprachlichen Spuren vorliegenden Diskursen ist damit eine sinnvolle Strategie für die (Re-)konstruktion von Führungs- und Steuerungsaspekten im Diskurs deutschsprachiger Führungsgrundsätze. Im folgenden Kapitel soll zunächst ein kurzer Überblick über die Diskursforschung gegeben werden. Das anschließende Kapitel widmet sich dann der Diskussion spezifischer Ansätze zur Untersuchung argumentativer Diskursbestandteile. 


\subsubsection{Verortung der Arbeit in der Diskursforschung}

\subsubsection{Begriff und Reichweite der Diskursforschung}

Die Diskursforschung erlebt in den letzten Jahren - vor allem im deutschsprachigen Raum - eine Konjunktur (vgl. Keller, 2011: 7; Rapley, 2007: xv). Dies zeigt sich deutlich in der wachsenden Literatur zu diskurstheoretischen und diskursanalytischen Ansätzen z.B. bei Keller (1997, 2008, 2011), Jäger (1999), van Dijk (2008, 1997a, 1997b), Fairclough (1997, 2003), Wodak und Meyer (Titscher, Wodak, Meyer \& Vetter, 1998; Wodak, 2008; Wodak \& Meyer, 2009) und in diversen Handbüchern und Überblickswerken (vgl. exemplarisch Grant, Hardy, Oswick \& Putnam, 2004b; Keller, Hirseland, Schneider \& Viehöver, 2010b). Von ,der' Diskursforschung zu sprechen, gestaltet sich deshalb angesichts der Vielfalt und Vielzahl der Disziplinen, AutorInnen und Beiträge mit unterschiedlichen Zielsetzungen, Forschungsinteressen und methodologischen Zuschnitten, welche sich mit dieser Form der Erforschung sprachlicher Artefakte beschäftigen, als schwierig (vgl. Keller, Hirseland, Schneider \& Viehöver, 2010a: 10; Rapley, 2007: 1). Die Anzahl der verschiedenen Beiträge, welche sich mit der Diskursforschung beschäftigen, ist bereits seit einigen Jahren im Steigen begriffen (vgl. Grant, Hardy, Oswick \& Putnam, 2004a). Keller et al. (2010a: 11ff.) diskutieren jedoch einige Gemeinsamkeiten, welche die meisten diskursanalytischen Vorhaben teilen:

- eine grundsätzlich konstruktivistische (bzw. konstruktionistische) Ausgangsposition, welche den performativen und konstitutiven Charakter der Sprache betont (vgl. auch Berger \& Luckmann, 2000) und somit auf den Bedeutungsaspekt der Sprache fokussiert ist (vgl. Fairhurst \& Putnam, 2004: 118).

- eine grundsätzliche Orientierung an textlichem Material mit überwiegend rekonstruktivem, teils aber auch dekonstruktivem Anspruch ${ }^{23}$;

- eine Orientierung weniger am einzelnen Diskursbeitrag, sondern vielmehr an den Beziehungen und Konfigurationen, welche verschiedene diskursive Beiträge und ihre Träger typischerweise ausbilden;

- einen starken Fokus auf qualitative Verfahren, egal ob hermeneutischen oder (post-) strukturalistischen Ursprungs (vgl. auch Keller, 2008: 109);

Die verschiedenen Ansätze zur Diskursanalyse unterscheiden sich hingegen recht stark in Bezug auf den spezifischen Diskursbegriff, die Gegenstandsbereiche und Fragestellungen, methodischen Umsetzungen, einbezogenen Datenma-

23, ,...] texts can be considered to be a manifestation of discourse and the discursive , unit ${ }^{\circ}$ (Chalaby, 1996) on which the organizational discourse researcher focuses. They signify collections of interactions, media of communication (i.e., oral, print, electronic), or assemblages of oral and written forms“ (Grant, et al., 2004a: 3). 
terialien, interessierenden Strukturelemente, gesellschaftlich-historischen Kontexte, sozialen Träger oder Akteure und Diskurs(Macht)effekte (vgl. Fairhurst \& Putnam, 2004; Keller et al., 2010a). Bezüglich des Diskursbegriffs bietet Keller (2008: 99ff.) eine ausführliche Diskussion, welche den Begriff von seinen etymologischen Ursprüngen als ,Hin- und Herlaufen“ in der lateinischen Sprache über seinen Aufstieg zur Fachterminologie im 13. Jahrhundert und seine anschließende Weiterentwicklung und Abwandlung u.a. durch David Hume, Jeremy Bentham, Niccoló Machiavelli, Jean-Jaques Rousseau bis hin zu Charles S. Peirce und George Herbert Mead erläutert. In der Folge wurde der Diskursbegriff stark durch französische Strukturalisten (z.B. Claude Lévi-Strauss, Michel Foucault und Roland Barthes, v.a. unter Rückgriff auf Ferdinand de Saussure) und Poststrukturalisten (z.B. Paul Ricoeur, Julia Kristeva, Jacques Derrida, Pierre Bourdieu oder Gilles Deleuze) geprägt. Diese poststrukturalistischen Ansätze gingen in der sprachphilosophischen Entwicklung einher mit Positionen, welche den Sprachgebrauch als Praktik bzw. Handlung thematisieren, wie z.B. die Sprachphilosophie von Ludwig Wittgenstein, der Pragmatismus von Charles S. Peirce oder die Sprechakttheorien von John Austin und John Searle (vgl. Keller, 2008: 108). In der Gegenwart unterscheidet Keller (2011) die Strömungen (a) der Diskurslinguistik, welche zwischen Geschichtswissenschaften und linguistischer Sprachforschung agiert und sich vor allem mit größeren Textkorpora beschäftigt (vgl. Keller, 2011: 23), (b) der Critical Discourse Analysis (vgl. z.B. Fairclough, 1997; Wodak \& Meyer, 2009) bzw. der deutschsprachigen Variante der kritischen Diskursanalyse (vgl. Jäger, 1999), welche sich vor allem (z.T. mit stark normativem bzw. emanzipatorischem Anspruch) mit ideologie-, gesellschafts- und sprachkritischen Fragestellungen beschäftigt, (c) der kulturalistischen Diskursansätze als Sammelbegriff unterschiedlicher Beschäftigung mit der gesellschaftlichen Bedeutung symbolischer Ordnung (vgl. z.B. Bourdieu, 1974, 1994; Gamson \& Modigliani, 1989), (d) der Diskurstheorien, welche sich im Anschluss an den französischen Poststrukturalismus entwickelten und vor allem um Foucault, Laclau und Mouffe herum bildeten und schließlich (e) der wissenssoziologischen Diskursanalyse, welche Keller (2008) als Ergänzung und Fortführung der phänomenologischen Wissenssoziologie (vgl. Berger \& Luckmann, 2000) und der hermeneutischen Wissenssoziologie (vgl. z.B. Schröer, 1994) entwickelt.

Da der Diskursbegriff somit alles andere als trivial und eindeutig ist, wird es notwendig, für die gegenständliche Untersuchung Abgrenzungen zu treffen. In der Folge wird deshalb in zwei Schritten ein Diskursverständnis entwickelt, welches sich in der phänomenologischen Wissenssoziologie verortet und somit für die vorliegende Arbeit eine brauchbare Grundlage bietet. 


\subsubsection{Sozialwissenschaftliches Diskursverständnis}

Für die vorliegende Untersuchung kommt aufgrund ihrer sozialwissenschaftlichen Ausrichtung auch nur ein sozialwissenschaftlicher Diskursbegriff in Frage. Gemeinsam mit Keller (2011: 8) werden Diskurse deshalb als Versuche verstanden, Bedeutungszuschreibungen und Sinn-Ordnungen auf eine gewisse Zeit zu stabilisieren und damit eine bestimmte soziale Wissensordnung zu institutionalisieren. Damit ergibt sich für die gegenständliche Arbeit folgende grundlegende Definition von Diskursforschung:

„Die sozialwissenschaftliche Diskursforschung beschäftigt sich mit dem Zusammenhang zwischen Sprechen/Schreiben als Tätigkeit bzw. soziale Praktiken und der (Re-) Produktion von Sinnsystemen/Wissensordnungen, den darin eingebundenen sozialen Akteuren, den diesen Prozessen zugrunde liegenden Regeln und Ressourcen sowie ihren Folgen in sozialen Kollektiven“ (Keller, 2011: 8)

Zwischen Diskursen und den ihren Kontext bildenden Sozialstrukturen besteht dabei eine dialektische Beziehung. Diskurse konstituieren die Welt und werden gleichermaßen von ihr konstituiert: „[S]ie (re-)produzieren und transformieren Gesellschaft; sie leisten die Konstruktion sozialer Identitäten, die Herstellung sozialer Beziehungen zwischen Personen und die Konstruktion von Wissensund Glaubenssystemen“ (Keller, 2011: 29). Die Deutungsstrukturen, welche in einem Diskurs aufgebaut und im zeitlichen Verlauf stabilisiert oder modifiziert werden, lassen sich aus einer Analyse der eingesetzten sprachlich-rhetorischen Mittel (re-)konstruieren (vgl. Keller, 2011: 72). Die vorliegende Untersuchung widmet sich insofern dem Zusammenhang zwischen Schreiben als sozialer Praktik und der (Re-)produktion von Sinnsystemen, als Führungsgrundsätze als Ausdruck eines spezifischen Wissensvorrats gesehen werden, welcher beeinflusst, was in diesen Dokumenten gesagt wird, welcher aber durch die spezifische Ausgestaltung der Dokumente auch selbst wieder beeinflusst und laufend verändert wird. Nur vor dem Hintergrund eines solchen Sinnsystems bzw. einer solchen Wissensordnung können Führungsgrundsätze von ihren Adressaten überhaupt verstanden werden. Diese Sinnsysteme sind damit auch Voraussetzung einer möglichen (Steuerungs)Wirkung durch Führungsgrundsätze. Die Untersuchung zielt nun auf die (Re-)konstruktion jener den Regelungsinhalten zugrunde liegenden Regeln und Ressourcen ab, welche Führungsgrundsätze in spezifischen Wissensvorräten verankern und sie damit legitimieren, normalisieren und dadurch für die Adressaten akzeptanzfähig machen. Die Untersuchung teilt damit die von Keller (2011: 9) diskutierten vier Merkmale diskursanalytischer Ansätze: 
- Sie beschäftigt sich mit dem tatsächlichen Gebrauch geschriebener Sprache in gesellschaftlichen Praktiken;

- Sie geht davon aus, dass der Bedeutungsgehalt von Phänomenen (in diesem Fall Führung und Steuerung) im praktischen Zeichengebrauch konstruiert und diese damit in der gesellschaftlichen Wirklichkeit konstituiert werden;

- Sie sieht Führungsgrundsätze als Interpretationsangebote, welche als Teile einer umfassenderen Diskursstruktur (z.B. des deutschsprachigen Führungsdiskurses), die durch spezifische institutionell-organisatorische Kontexte erzeugt und erhalten wird, verstanden werden;

- Sie unterstellt, dass der Gebrauch symbolischer Ordnungen über die Analyse von Regeln des Deutens und Handelns (re-)konstruiert werden kann.

Innerhalb der sozialwissenschaftlichen Diskursforschung erscheint nun die wissenssoziologische Diskursanalyse (vgl. Keller, 2008, 2010, 2011) als besonders interessant, da sie einen Diskursbegriff formuliert, welcher direkt anschlussfähig an die wissenssoziologische Ausrichtung der gegenständlichen Arbeit ist.

\subsubsection{Wissenssoziologische Diskursanalyse}

Die wissenssoziologische Diskursanalyse geht von den bei Berger und Luckmann (2000) formulierten Prämissen aus, dass ,alles, was wir wahrnehmen, erfahren, spüren, über sozial konstruiertes, typisiertes, in unterschiedlichen Graden als legitim anerkanntes und objektiviertes Wissen (Bedeutungen, Deutungs- und Handlungsschemata) vermittelt wird" (Keller, 2011: 59). Sie widmet sich der Untersuchung dieser gesellschaftlichen Praktiken und kommunikativen Konstruktions-, Stabilisierungs- und Transformationsprozesse. Ihr geht es um eine (Re-)konstruktion von Prozessen der sozialen Konstruktion, Objektivation, Kommunikation und Legitimation von Sinnstrukturen auf der Ebene von Institutionen, Organisationen bzw. sozialen Akteuren (vgl. Keller, 2011: 59). In der „Gesellschaftlichen Konstruktion der Wirklichkeit“ kommt der Begriff des Diskurses jedoch nicht explizit vor (vgl. Keller, 2008: 182). Dennoch findet Keller (2008) genügend Anschlusspunkte, um ohne Widerspruch zu Berger und Luckmanns (2000) Konzeption die kommunikative Konstruktion der Wirklichkeit in eine diskursive Konstruktion der Wirklichkeit zu übersetzen, indem von kollektiven Wissensvorräten ausgegangen wird und Diskurse dementsprechend als „handlungspraktische und institutionelle Strukturierungen gesellschaftlicher Wissenspolitiken“ zum Gegenstand der hermeneutischen Wissenssoziologie gemacht werden (vgl. Keller, 2008: 185). Die diskursive Konstruktion der Wirklichkeit bedeutet somit, dass sich die Forschung auf Strukturierungsprozesse konzentriert und damit nicht nur nach Praktiken des Sprachgebrauchs, sondern nach den diskursiven Formationen, in welchen diese erscheinen, fragt (vgl. 
Keller, 2008: 190). Durch die dialektische Auffassung des Verhältnisses zwischen Individuum und Gesellschaft (vgl. Berger \& Luckmann, 2000: 65) vermeidet die wissenssoziologische Diskursanalyse die implizite Ontologisierung des Diskurses, welche den Akteur jeglicher Handlungsfähigkeit beraubt. Das Akteurskonzept der Wissenssoziologischen Diskursanalyse begreift soziale Akteure sowohl als diskursiv konstituierte als auch als regelinterpretierend Handelnde und somit als aktive Produzenten und Rezipienten von Diskursen (vgl. Keller, 2008: 191). Unter dem Begriff des Diskurses werden nun

„[...] strukturierte und zusammenhängende (Sprach-) Praktiken, die Gegenstände und gesellschaftliche Wissensverhältnisse konstituieren [...]“ (Keller, 2008: 186)

verstanden. Diskurse äußern sich über so genannte diskursive Ereignisse als typische Aussagen, welche den Diskurs in einer dialektischen und reziproken Beziehung aktualisieren. Diskurse zeigen sich aber auch in Dispositiven - d.h. der materiellen und ideellen Infrastruktur, die durch Maßnahmenbündel, Regelwerke und Artefakte zum Ausdruck kommt (Führungsgrundsätze konstituieren somit ein solches Dispositiv) - und in Praktiken als Muster für Handeln und Handlungsvollzüge, welche sprachlicher oder nicht-sprachlicher Art sein können (vgl. Keller, 2008: 234f.). Auch der Zusammenhang zwischen Diskurs und Identität lässt sich mit diesem Diskursverständnis diskutieren. So bietet Keller (2008: 235) mit dem Konzept der Subjektposition eine Kategorie, welche ,im Diskurs konstituierte Subjektvorstellungen und Identitätsschablonen für seine möglichen Adressaten" zur Verfügung stellt. Diese Subjektpositionen existieren innerhalb institutioneller bzw. organisatorischer Settings und beinhalten daran geknüpfte Rollenkomplexe. Akteure agieren folglich als Rollenspieler, welche spezifische Positionen einnehmen (vgl. Keller, 2008: 216f.). Diese Positionierungen sozialer Akteure erzeugen Interpretationsschemata und Identitätsangebote, welche den sozialen Akteure angetragen und in Prozessen der Sozialisierung angeeignet werden können (vgl. Keller, 2008: 217). Den Machtaspekt des Diskurses erhält Keller durch den expliziten Bezug auf die Theorien Michel Foucaults: „Diskurse stellen nicht nur die Bedeutungsstrukturen unserer Wirklichkeit her und damit in gewissem Sinne diese selbst, sondern sie haben auch andere reale Folgen, Effekte bzw. ,Machtwirkungen“ im Sinne Foucaults“ (Keller, 2008: 234). Sie bilden die signifikatorischen Grundlage zur Wahrnehmung und Deutung von Phänomenen, befähigen somit zur Handlung, zeigen aber auch , angemessene“ Handlungsformen auf und sind damit an der ,Herstellung' sozialer Akteure beteiligt (vgl. Keller, 2008: 238). Führungsgrundsätze können somit innerhalb dieser theoretischen und methodologischen Position als Dispositive betrachtet werden, welche diskursive Ereignisse in Form typischer Aussagen enthalten und damit angemessene Handlungsformen und gewünschte Subjektpositionen als Identi- 
tätsangebote an ihre Adressaten (Führungskräfte und MitarbeiterInnen) herantragen.

Methodologisch ist mit der (Re-)konstruktion dieser impliziten Diskurse und diskursiven Elemente eine grundsätzlich interpretative Forschungsstrategie verbunden (vgl. Keller, 2011: 76), welche sich an hermeneutischen Prinzipien orientiert, aber im Vergleich zu anderen interpretativen Ansätzen einige Spezifika aufweist:

- Texte und andere Artefakte interessieren nicht als Produkte ,subjektiver oder ,objektiver' Fallstrukturen, sondern ,als materiale Manifestationen gesellschaftlicher Wissensordnungen“" (Keller, 2008: 275; 2011: 78).

- Strukturen der Aussagenproduktion sind textübergreifend und stehen nicht für einzelne ,Typen“. Sie bilden keineswegs nur einen Diskurs ab, sondern können auf verschiedene Diskurse verweisen (vgl. Keller, 2011: 78).

- Es wird nicht davon ausgegangen, dass Texte eine in sich geschlossene Sinnbzw. Fallstruktur aufweisen. Vielmehr stehen Diskurse immer in einem interdiskursiven Kontext. Sie beziehen sich auf andere historisch diachrone und synchrone Diskursformationen. Die Ergebnisse der Analyse einzelner Texte müssen deshalb aufeinander bezogen werden und dürfen nicht isoliert betrachtet werden (Keller, 2008: 275; 2011: 78).

- Durch diese Verweisstruktur zwischen Texten und Diskursen steht die sozialwissenschaftliche Diskursanalyse vor dem Problem großer Textmengen. Dadurch werden verschiedene Strategien der Korpusreduktion relevant (vgl. Keller, 2008: 275; Keller, 2011: 79).

\subsubsection{Zum Textbegriff der Diskursforschung}

Damit wäre das diskursanalytische Forschungsprogramm der vorliegenden Untersuchung ausreichend umrissen. Zu klären bleibt allerdings noch, was in der Arbeit konkret unter dem Begriff eines Textes verstanden wird. Dazu bietet Jäger (1999: 118ff.) einige Kriterien, welche alle gängigen Elemente sonstiger Textdefinitionen enthalten. Dabei hält er fest, dass Texte immer Resultate mehr oder minder ,gelungener' Tätigkeit sind (vgl. Jäger, 1999: 119). Erst durch diesen Tätigkeitsaspekt wird Textanalyse für Jäger (1999: 119) zur Diskursanalyse. Ein Text lässt sich nun gemäß dieser Überlegungen folgendermaßen fassen (vgl. Jäger, 1999: 118; für eine andere Variante vgl. z.B. Phillips \& Brown, 1993): 
- Er ist das sprachlich gefasste Ergebnis einer individuellen Tätigkeit bzw. eines individuellen Denkens.

- Voraussetzung für seine Produktion ist die Existenz von Wissen. Dieses Wissen ist in einem Lernprozess entstanden, in welchen der Mensch lernend verstrickt ist, und zwar in kontinuierlicher Art und Weise.

- Dieses Wissen wird von Menschen in einer konkreten Situation, einem bestimmten Bedürfnis folgend mit einer bestimmten Wirkungsabsicht unter Beachtung der Rezeptionsbedingungen und unter Zuhilfenahme spezifischer überlieferter und konventionalisierter sprachlicher oder gedanklicher Mittel schriftlich oder mündlich in Form von Text(en) als Resultate ihrer Sprechund/oder Denktätigkeit produziert.

Führungsgrundsätze entsprechen diesem Textverständnis. Sie wurden von einem bestimmten Kreis von Organisationsmitgliedern (und u.U. externen Beratern) aufgrund eines spezifischen Wissensvorrates (z.B. über gute/moderne Führung) - und damit unter Berücksichtigung dominanter Diskurse über Führung - in einer konkreten Situation mit einer bestimmten Wirkungsabsicht (Steuerung des Verhaltens der Organisationsmitglieder) mithilfe spezifischer (rhetorischer) sprachlicher Mittel schriftlich in Form des spezifischen Genres der Führungsgrundsätze festgehalten. Die spezifischen diskursiven Ressourcen, welche in die Erstellung dieser Texte Eingang gefunden haben, sind somit einer diskursanalytischen (Re-)konstruktion zugänglich.

Im Verlauf der Arbeit wurde die Bedeutung der Überzeugung und Argumentation in der rhetorischen Ausgestaltung der Dokumente für die Wirkung von Führungsgrundsätzen begründet. Das folgende Kapitel greift nun diese Idee erneut auf und diskutiert, wie Diskursanalyse als Argumentationsanalyse gefasst werden kann und welche spezifischen Herausforderungen und methodische Implikationen sich daraus für die vorliegende Untersuchung ergeben.

\subsubsection{Argumentationsanalyse}

In Kapitel 4.4 wurden die Grundlagen der Rhetorik als Kunst der Überzeugung eines Publikums (vgl. Cheney et al., 2004: 79) und breiter als Prozess der sozialen Konstruktion von Wirklichkeit (vgl. Fairhurst \& Putnam, 2004: 106) ausführlich diskutiert. Führungsgrundsätze wurden dabei als Dokumentgenre beschrieben, welches in hohem Ausmaß die Mobilisierung erwünschter Handlungs- und Denkweisen bei den Organisationsmitgliedern (in erster Linie Führungskräfte und MitarbeiterInnen) beabsichtigt. Solche Mobilisierung kann im Rahmen von Herrschafts- und Machtbeziehungen auf verschiedenste Weise (von körperlicher Gewalt bis hin zu subtiler Manipulation) versucht werden. Es wur- 
de aber argumentiert, dass die Steuerungswirkung durch Führungsgrundsätze auf bürokratische und kulturell-normative Mechanismen beschränkt werden muss. Diese Regeln und Werte müssen aber (auch wenn sie stark durch bestehende Herrschaftssysteme gestützt werden) überzeugen, um Wirkung entfalten zu können. Sie beinhalten Geltungsansprüche bzw. ,claims` (vgl. Kopperschmidt, 1989; R. E. Meyer, 2004), welche in Form von Argumentationen eingelöst werden müssen. Argumentationen sind diskursive Ereignisse (vgl. Fairhurst \& Putnam, 2004: 104). Die Analyse von Argumentationen lässt damit Rückschlüsse auf den sozialen Wissensvorrat bzw. die relevanten Diskurse (das Bezugssystem) zu, vor deren Hintergrund Geltungsansprüche gestellt werden. Es wird folglich ein methodologischer und methodischer Zugang benötigt, welcher in der Lage ist, aus vorhandenen Argumentationsmustern diese diskursiven Sinnsysteme zu (re-)konstruieren. Dazu bietet sich grundsätzlich die Systematik der Argumentationsanalyse nach Kopperschmidt (1989) an, da diese einen vergleichsweise hohen Konkretionsgrad aufweist und ein umfassendes Spektrum verschiedener Möglichkeiten der Betrachtung von Argumenten bietet.

Kopperschmidt (1989) unterscheidet grundsätzlich zwischen mikrostruktureller und makrostruktureller Argumentationsanalyse. Während die mikrostrukturelle Analyse sich auf die detaillierte Untersuchung einzelner Argumente bzw. ihrer jeweiligen prozesshaften Entfaltung beschränkt (vgl. Kopperschmidt, 1989: 123), ist der Gegenstand der makrostrukturellen Argumentationsanalyse die Betrachtung komplexerer Argumentationsstränge mit mehreren argumentierenden Subjekten (Kopperschmidt, 1989: 206ff.). Für die vorliegende Untersuchung ist die makrostrukturelle Analyse aus diesem Grund von wenig Relevanz. Zum einen wurde bereits argumentiert, dass Führungsgrundsätze ein Beispiel monologischer Argumentation (vgl. Ottmers, 2007: 69ff.) darstellen. Monologische Argumentationen betreffen nur einen Sprecher; die Rezeption der Sprechhandlung findet zumeist zeitlich von dieser losgelöst statt. Ein direkter Einspruch, welcher zum Entstehen einer komplexen Kette von Argument und Gegenargument führen könnte, ist somit nicht möglich. Gegenargumente können in der monologischen Argumentation höchstens antizipiert und damit vorweggenommen werden. Zum anderen gestalten sich Führungsgrundsätze als Sammlungen relativ unabhängiger Regelungsinhalte (vgl. z.B. Ebert, 1994b). Komplexe Argumentationsketten, in denen Argumente sich sequentiell aufeinander beziehen, konnten auch in der vorliegenden Analyse nicht identifiziert werden. Diese monologische, in sich abgeschlossene und fragmentarische Form der Argumentation legt einen Fokus auf mikrostrukturelle Analysestrategien nahe. Innerhalb dieser mikrostrukturellen Argumentationsanalyse unterscheidet Kopperschmidt (1989: 122f.) eine funktionale, eine materiale und eine formale Argumentationsanalyse. 
Die funktionale Analyse lehnt sich an die Toulminsche Argumentationstheorie (vgl. Toulmin, 2008, im Original 1958) an und widmet sich der Frage, wie in einer Argumentation Überzeugung und Stützung von Äußerungen konkret geschehen und welche Rollen einzelne Äußerungen in diesem Zusammenhang übernehmen (vgl. Kopperschmidt, 1989: 123). Damit wird auf die zentrale Rolle von Schlussregeln (vgl. Kopperschmidt, 1989: 125; bzw. ,warrants' bei Toulmin, 2008: 91) verwiesen. Das Toulminsche Argumentationsmodell (vgl. dazu auch die Struktur der Enthymeme in Kapitel 4.4.2) stellt somit „ein ,abstraktes Schema' argumentativer Beziehungen bereit, das es erleichtert, einzelne Äußerungen bzw. Äußerungssequenzen - ungeachtet ihres illokutiven Charakters und trotz ihrer kontextuellen Gebundenheit und ihres natürlichsprachlichen Präzisionsdefizits - als funktionsspezifische Einheiten innerhalb dieses Systems argumentativer Beziehungen zu reformulieren und hinsichtlich ihrer Leistung für die Stützung eines problematisierten GAs zu beurteilen“" (Kopperschmidt, 1989: 142). Das Problem der funktionalen Argumentationsanalyse bei der Untersuchung von Alltagssprache ist, dass die Akteure sich „nicht an Argumentationsregeln halten und nur ,verkürzt' argumentieren - sie verankern ihre Äußerungen in einem ,Normenhintergrund' oftmals ohne Angabe von Übergangsbzw. Schlussregeln“ (R. E. Meyer, 2004: 197). Die Herausforderung für die Analyse besteht also darin, methodisch mit dieser argumentativen Verkürzung umzugehen. Meyer (2004: 197f.) schlägt vor, Konzepte der politischen Diskursanalyse heranzuziehen. Sie führt dazu den Begriff des ,claims' ein, welchen sie analog zu Spector und Kitsuse als ,,a demand made by one party to another that something be done about some putative condition" (Spector und Kitsuse 1977: 78, zit. in R. E. Meyer, 2004: 198) definiert. Solche ,claims' versteht sie in weiterer Folge in Anlehnung an Kopperschmidt (1989) als Geltungsansprüche, welche argumentativ durch Rechtfertigungen oder Begründungen (also ,Accounts‘, vgl. z.B. Lamertz \& Baum, 1998) eingelöst werden können. Während solche Geltungsansprüche keine expliziten Stellungnahmen oder Aufforderungen enthalten, aber „Sichtweisen darüber, wie eine Situation ,ist" oder um welche Art von ,Problem' oder ,Konzept' es sich handelt, wie Ursachen und Wirkungen miteinander verbunden sind etc.“ (R. E. Meyer, 2004: 198), stellt jede Sprecheräußerung eine Behauptung dar, die einen Geltungsanspruch und zumindest einen Verweis auf das Bezugssystem, in welchem dieser Geltungsanspruch einlösbar ist (vgl. Kopperschmidt, 1989: 121), beinhaltet. Meyer (vgl. 2004: 199f.) macht außerdem das Konzept der ,idea elements' (vgl. z.B. Gamson \& Modigliani, 1989) für die Argumentationsanalyse nutzbar, indem sie diese als ,freischwebende Begründungen“ charakterisiert, welche (re-)konstruierbar sind, ohne auf kognitive Repräsentationen und Intentionen individueller Akteure zurückgreifen zu müssen (R. E. Meyer, 2004: 199). Über diese kon- 
zeptionellen ,Umwege‘ ermöglicht sie damit eine Analyse von Begründungsstrukturen in fragmentierten Alltagsdiskursen.

Die materiale Argumentationsanalyse fokussiert in einem zweiten Schritt die (Re-)konstruktion des relevanten Bezugssystems, welches die materiale Kohärenz (im Gegensatz zur formalen Konsistenz) des Arguments herstellt. Durch die Wahl eines solchen Bezugssystems wird sowohl ein bestimmtes Problemverständnis ratifiziert als auch bestimmte Problemlösungswege eröffnet (vgl. Kopperschmidt, 1989: 144). Damit bezieht sich die materiale Argumentationsanalyse auf die auch in dieser Arbeit bereits an verschiedenster Stelle betonte Notwendigkeit eines gemeinsamen bzw. geteilten Verständnisses für die Überzeugungskraft eines Arguments (und damit das Wirkungspotentials eines Steuerungsversuches) (vgl. Kopperschmidt, 1989: 154). Solche Bezugssysteme stellen begriffliche Netzwerke bereit, ,innerhalb deren überhaupt etwas als etwas identifiziert werden kann und innerhalb deren Argumentationen und die Kriterien ihrer möglichen Überzeugungskraft immer schon verortet sind“ (Kopperschmidt, 1989: 156). Genau hier liegt auch die Anschlussfähigkeit der Argumentationsanalyse an eine wissenssoziologische Betrachtungsweise. So führt Kopperschmidt aus, dass „,,Gegenstände“ möglichen (Selbst- und Fremd-) Verstehens nicht objektivistisch vorgegeben sind, sondern im Akt des Verstehens durch die in ihm aktualisierten Deutungsschemata erst konstituiert werden, daß mithin soziale Wirklichkeit immer schon eine durch den Verstehenden konstituierte Wirklichkeit ist“ (Kopperschmidt, 1989:160) und somit auch für Argumentationen gilt, dass jeder argumentative Problemlösungsversuch aus der Lebenswelt bzw. einem kulturell überlieferten Vorrat an Deutungsmustern seine mögliche Überzeugungskraft gewinnt. Die konzeptionelle Charakterisierung von Bezugssystemen ist dabei grundsätzlich offen. Kopperschmidt selbst erwähnt Kuhns ,Paradigmen“ (vgl. Kuhn, 1973) ebenso wie ,Deutungsmuster' (vgl. z.B. Lüders, 1991; Lüders \& Meuser, 1997; Meuser \& Sackmann, 1992; Oevermann, 1973), aber auch ,Rahmungen' (bzw. ,frames') können als Bezugssystem konzeptionalisiert werden (vgl. z.B. Goffman, 1977; R. E. Meyer, 2004). Die Verwendung eines spezifischen Bezugssystems selbst kann allerdings ebenfalls begründungsbedürftig, und somit Gegenstand einer Argumentation sein. So wird z.B. bei Meyer (2004: 200) eine spezifische Rahmung dann anerkannt, wenn sie kulturell resoniert bzw. sinnvoll in eine ,story line“ integriert werden kann.

Die formale Argumentationsanalyse, schließlich, ergänzt die funktionale und materiale Analyse durch die Betrachtung der strukturellen Konvergenz ihrer Formprinzipien (vgl. Kopperschmidt, 1989: 178). Damit rückt die formale Argumentationsanalyse in Richtung der allgemeinen Topoi (vgl. Wengeler, 2003b: 182) bzw. kontextabstrakten Argumentationsmuster (vgl. Kienpointner, 1992). Wie bereits im Kapitel zu den Topoi erläutert, ist ein allgemeiner Topos ein sol- 
cher, der ,auf alle Gegenstände in allen Redegattungen anwendbar ist" (Wengeler, 2003b: 182) und mithilfe dessen deshalb über verschiedenste Themenbereiche Enthymeme gebildet werden können. Die allgemeinen Topoi charakterisieren somit eine bestimmte Form der Argumentation, während besondere Topoi bereits stärker inhaltliche und materiale Argumentationsmuster beinhalten. Es sind auch diese allgemeinen bzw. formalen Topoi, welchen Kopperschmidt (1989: 192) die Kraft abspricht, die Rationalität oder Überzeugungskraft einer Argumentation einzulösen. Davon zu unterscheiden sind aber eben jene ,besonderen' bzw. ,kontextspezifischen“ Topoi, welche bereits inhaltliche Elemente besitzen und deshalb auf das spezifische Bezugssystem verweisen, innerhalb dessen sie Gültigkeit besitzen. Diese Topoi haben bei Meyer (2004: 174) eine ähnliche Funktion wie interpretative Packages und sind deshalb zwischen formaler und materialer Argumentationsanalyse angesiedelt.

Sowohl die funktionale als auch die formale Argumentationsanalyse sind im Rahmen der Untersuchung von Führungsgrundsätzen nur schwer vollständig durchzuführen. Die in den Dokumenten verwendeten, claims' sind größtenteils nur fragmentarisch expliziert und folgen keiner spezifischen Form. Auch wenn Handlungsempfehlungen grundsätzlich begründungsbedürftig sind, so bleibt diese Begründung ähnlich diffus wie in dem z.B. von Meyer (2004) und Wengeler (2003b) untersuchten Mediendiskurs. Eine materiale Argumentationsanalyse kann hingegen ein besseres Verständnis der in den impliziten Argumentationsmustern kommunizierten Verständnissen von Führung und Steuerung leisten. Aufgrund seiner ,Hybridstellung' zwischen materialer und formaler Argumentationsanalyse wird im Folgenden der besondere Topos als zentrale bedeutungstragende Kategorie für die gegenständliche Untersuchung als spezifisch geeignet gesehen. Topoi als „Bestandteil kollektiven Wissens und als argumentativ in der Funktion von Schlussregeln gebrauchte[s] Denkmuster" (Wengeler, 2003a:227) stellen für die gegenständliche Untersuchung eine angemessene Analysekategorie dar, da sich über sie latente argumentative Zusammenhänge und geteilte Wirklichkeiten bzw. Wissensvorräte rekonstruieren lassen. Es wird deshalb argumentiert, dass eine (Re-)konstruktion der in den Führungsgrundsätzen verwendeten besonderen Topoi fruchtbare Ansätze für die Beantwortung der forschungsleitenden Fragen bieten kann.

Im folgenden Kapitel wird der Dokumentkorpus kurz beschrieben und argumentiert sowie die grundsätzlichen Sampling-Strategien erläutert. Anschließend wird ein Überblick über die konkrete Vorgehensweise und den eingesetzten Methodenkanon gegeben. 


\subsection{Daten und Sampling}

Der gesamte Korpus an Führungsgrundsätzen, welcher dieser Untersuchung zugrunde liegt, wurde im Rahmen eines vom Jubiläumsfonds der Österreichischen Nationalbank geförderten Projektes am Institut für Public Management der WU Wien (vgl. Jancsary et al., 2006) vom Autor selbst erhoben. Dabei wurden auf Grundlage von Zeitschriftenrankings 370 Unternehmen in Österreich (134), Deutschland (132) und der Schweiz (104) um Zusendung ihrer Führungsgrundsätze gebeten ${ }^{24}$. Auf diese schriftliche Anfrage wurden 38 Dokumente zugesandt, telefonische Erinnerungen zwischen November 2004 und Januar 2005 generierten weitere 63 Dokumente. Außerdem wurden in Eigenrecherche drei zusätzliche Dokumente auf den Websites relevanter Unternehmen gefunden. Insgesamt ergab sich somit eine Sammlung von 104 Dokumenten. Davon blieben nach dem Ausscheiden irrelevanter Texte nach inhaltlichen und formalen Kriterien (für Details, vgl. Jancsary et al., 2006) 16 österreichische, 19 deutsche und 18 Schweizer Dokumente übrig, die als Führungsgrundsätze im eigentlichen Sinne bewertet wurden ${ }^{25}$.

Da mit einem bereits vorhandenen Korpus an Dokumenten gearbeitet wurde, war ein theoretisches Sampling bei der Erhebung der Dokumente in dem Sinne, dass die entstehende Theorie die Auswahl des nächsten Dokuments leiten soll (vgl. Glaser \& Strauss, 1998), nicht möglich. Die Aussagenreichweite des vorliegenden Projekts bleibt damit auf Großunternehmen (in Bezug auf den jährlichen Umsatz) beschränkt. Während eine Kontrastierung mit kleinen und mittleren Unternehmen u.U. interessante Ergebnisse gebracht hätte, ist davon auszugehen, dass Führungsgrundsätze als Instrument der strukturellen Führung vor allem in großen Organisationen mit einem gewissen Grad an Formalisierung Anwendung finden. Ebenfalls ausgeschlossen blieben durch das ökonomische Auswahlkriterium öffentliche und Non-Profit Organisationen.

Der verwendete Dokumentkorpus beinhaltet verschriftlichte Kommunikation verschiedenster Art und Form. Bezüglich der Aufmachung dominieren Hefte und Broschüren (23 Dokumente) und Ausdrucke mit unklarer Herkunft (13

24 Bei den gewählten Zeitschriften handelt es sich um ,Trend“ (Österreich) und ,Welt` (Deutschland). Das Ranking erfolgte aufgrund der Höhe des Jahresumsatzes. In der Schweiz wurde das Ranking der Zeitschrift ,Bilanz' herangezogen, das jedoch eigene Performanceindikatoren anstelle des Umsatzes verwendet. Außerdem wurden noch Unternehmen kontaktiert, die bereits in früheren Untersuchungen Dokumente zur Verfügung gestellt hatten.

25 Die Abgrenzung zwischen relevanten und irrelevanten Dokumenten war in beinahe allen Fällen sehr einfach. Ausgeschieden wurden - abgesehen von fremdsprachigen Texten - zugesandte Unternehmensvisionen, Unternehmensgrundsätze, Leitbilder, ValueStatements, Codes of Conduct und Geschäftsberichte. 
Dokumente). Aber auch Falter, Mappen und Intranet-Ausdrucke tauchen im Dokumentkorpus auf. Was die Materialität der zugesandten Dokumente betrifft, so stellen mit 19 Dokumenten elektronische Formate (Word, PDF, etc.) die häufigste Form dar. Aber auch Ausdrucke auf gewöhnlichem Druck- bzw. Kopierpapier (dreizehn Dokumente), Hochglanzpapier (16 Dokumente) und stärkere Papier- bzw. Kartonsorten (fünf Dokumente) sind möglich. Der Umfang bewegt sich bei den meisten Dokumenten (18) im Bereich zwischen zwei und fünf Seiten. Dreizehn Dokumente sind einseitig, acht umfassen zwischen sechs und zehn Seiten, zehn Dokumente besitzen zwischen zehn und 20 Seiten und vier Dokumente gehen darüber hinaus. Da Dokumente nicht nach Erstellungsdatum, sondern nur nach dem Kriterium der Gültigkeit zum Zeitpunkt der Erhebung eingehoben wurden, weisen die Führungsgrundsätze eine sehr unterschiedliche Existenzdauer aus. Das älteste Dokument wurde 1983 eingeführt, das jüngste 2003. Dazu ist aber anzumerken, dass nur grob die Hälfte der Dokumente einen expliziten Hinweis auf das Erstellungsdatum enthält. Die Dokumente weisen auch unterschiedliche Grade der visuellen Illustration auf, ein Umstand, der jedoch in der vorliegenden Untersuchung nicht in die Analyse mit einbezogen wurde. Bezüglich der durch die Unternehmen abgedeckten Sektoren dominieren der Industrie- (23 Dokumente) und Dienstleistungssektor (22 Dokumente), der Handel ist mit acht Dokumenten vertreten.

Das Sampling bzw. der Einbezug von Dokumenten in die Untersuchung verlief grundsätzlich in zwei Schritten. In einer ersten Phase wurden für die Generierung des Codierschemas verschiedene Dokumente nach den Kriterien maximaler Nähe und maximaler Differenz (vgl. Glaser \& Strauss, 1998) sukzessive in die Untersuchung einbezogen. Nachdem die jeweils entwickelten Kategorien im induktiven Codierprozess stabilisiert und theoretisch gesättigt waren, wurde das dadurch entstandene Codierschema in einer zweiten Phase auf den gesamten Korpus deduktiv (aber mit der nötigen Offenheit für die Generierung notwendiger neuer Kategorien) übertragen. Dies erlaubte die abschließende tentative Betrachtung und Diskussion von Verteilungen von Rollenaspekten, Beziehungstypen und Topoi im untersuchten Material.

Innerhalb der Dokumente wurden grundsätzlich alle Textstellen der Analyse zugeführt, da der Aussage von Meyer (2004: 198) gefolgt wurde, wonach jede Sprecheräußerung in einer öffentlichen Arena grundsätzlich einen ,claim“ sprich: Geltungsanspruch - stellt (zur Bedeutung von Erklärungen und Rechtfertigungen im Alltagsdiskurs vgl. auch W. Wagner, 1994: 110ff.). Ausgeschlossen wurden nach formalen Überlegungen lediglich jene Dokumentteile, in welchen keine expliziten Regeln zum Verhalten von Führungskräften und MitarbeiterInnen erwartet wurden. Dies waren namentlich - wenn vorhanden Vorwort, Inhaltsverzeichnis und Impressum. Codiereinheit war jeweils die ein- 
zelne Äußerung. Äußerungen wurden nach Kopperschmidt (1989: 17, FN 4) als „pragmatische Sinneinheiten kommunikativ funktionalisierter Rede im Unterschied zu "Sätzen" als den linguistischen Sinneinheiten von Sprache" definiert und gemeinsam mit Keller (2008: 234) als „konkret dokumentierte, für sich genommen je einmalige sprachliche Materialisierung eines Diskurses" betrachtet. Eine Äußerung konnte also mehr als einen Satz umfassen, wenn dadurch eine Argumentation bzw. ein Topos vervollständigt wurde. Hinweise darauf fanden sich in linguistischen Konstruktionen und Bindewörtern wie ,und', ,daher', ,deshalb', ,in der Folge‘, etc. Äußerungen in den einzelnen Dokumenten wurden sequentiell codiert, wobei jedoch die Interpretation vorangegangener Äußerungen nicht für die Interpretation der jeweils vorliegenden herangezogen wurde. Jede Äußerung wurde also isoliert analysiert und interpretiert.

\subsection{Vorgehensweise und Untersuchungsablauf}

\subsubsection{Grundsätzliches zur Vorgehensweise}

Das methodische Design lehnt sich an die Grounded Theory (vgl. Glaser \& Strauss, 1998) an und bedient sich vor allem der constant comparative method (vgl. auch Boeije, 2002). Durch den ständigen Vergleich von Daten und Fällen wird es möglich, induktiv Codes und Kategorien zu generieren und zueinander in Beziehung zu setzen (vgl. Boeije, 2002:393; Locke, 2001). Durch ständige Vergleiche werden Erkenntnislücken identifiziert, die durch eine parallel laufende Inklusion zusätzlichen Datenmaterials gefüllt werden, bis ein Kategoriensystem entsteht. Auch hier tritt wieder der grundsätzlich zirkulär gestaltete Untersuchungsablauf qualitativer Studien zu Tage. Diese Vorgehensweise kommt dem Ziel der induktiven Rekonstruktion argumentativer Muster und implizierter Führungsbeziehungen und Steuerungsaspekte stark entgegen. Die Untersuchung verläuft in mehreren analytischen Phasen, die - gemäß der Logik qualitativer Sozialforschung - zirkulär angelegt sind, d.h., der (systematische) Sprung zurück in vorherige Phasen ist stets möglich und erwünscht (vgl. M. Lueger, 2001).

Nicht zu verwechseln mit den Phasen von Dateninklusion, -analyse und -interpretation sind die verschiedenen Schritte, in welchen die gegenständliche Untersuchung durchgeführt wurde. Diese Schritte waren grundsätzlich in sich geschlossen, eine ,Rückkehr' in vorhergehende Schritte war nur in Ausnahmefällen notwendig. In einem ersten Schritt wurden die in den Dokumenten angesprochenen Verben bzw. Tätigkeiten codiert und geclustert. Daraus wurden schließlich unterschiedliche Rollenaspekte (re-)konstruiert, welche den zwei re- 
levanten Akteursgruppen (Führungskräfte und MitarbeiterInnen) zugeordnet wurden. In einem zweiten Schritt wurden die Akteure zueinander in Beziehung gesetzt, indem die jeweilige grammatische Beziehungsstruktur innerhalb der Äußerungen betrachtet wurde. Aus dieser spezifischen Bezugnahme der Akteure aufeinander mittels der zuvor identifizierten Tätigkeiten wurden Beziehungstypen als Kombination spezifischer Akteure und Tätigkeiten (re-)konstruiert, welche unterschiedliche Verständnisse von Führung und Steuerung implizieren und ausdrücken. Diese beiden ersten Schritte wurden in der Praxis parallel zueinander durchgeführt. Sie werden hier aber zur besseren Übersicht sequentiell diskutiert. Sie geben eine Antwort auf den ersten Teil der forschungsleitenden Frage, welcher den expliziten und impliziten Vorstellungen von Führung und Steuerung, welche sich in den konstruierten Beziehungen zwischen Führungskraft und MitarbeiterIn ausdrücken, gewidmet ist.

In einem dritten Schritt schließlich wurden unabhängig von den ersten beiden Schritten mittels hermeneutischer Analysen die relevanten besonderen Topoi im Material identifiziert. Diese wurden in einem letzten Schritt zu den zuvor (re-)konstruierten Beziehungstypen systematisch in Beziehung gebracht, um eine konzeptionelle Landkarte von Beziehungen und den sie argumentativ stützenden Topoi zu erstellen. Damit beantworten der dritte und vierte Schritt den zweiten Teil der forschungsleitenden Frage nach den rhetorischen Strategien und Ressourcen, mittels welcher die vorgeschlagenen Führungs- und Steuerungsbeziehungen argumentiert und damit im organisationalen Diskurs verankert werden. Die vier Schritte sollen nun jeweils im Detail erläutert und diskutiert werden.

\subsubsection{Schritt 1: Identifikation und Codierung relevanter Tätigkeits-Cluster und Rollenaspekte}

Machtausübung - und damit auch Führung und Steuerung - innerhalb interaktionaler Führungsbeziehungen wurde als grundlegend relationales Phänomen vorgestellt. Um diesem relationalen Charakter der Führung Rechnung zu tragen, mussten daher zunächst die im Diskurs hergestellten Rollenverständnisse für die relevanten Akteure (FK und MA) ${ }^{26}$ betrachtet werden. Den Ausführungen von Berger und Luckmann (2000: 77ff.) folgend, dass soziale Rollen durch typisierte Handlungen typisierter Akteure konstituiert werden, lag der Fokus in diesem

26 Aus Gründen der Lesbarkeit werden in der Folge in erster Linie die Abkürzungen FK und MA für die Akteure ,Führungskraft‘ und ,MitarbeiterIn“ verwendet. Es versteht sich von selbst, dass in diesen Abkürzungen jeweils die weibliche und die männliche Form der Bezeichnung gleichermaßen inkludiert sind. 
Schritt der Analyse auf einer Identifikation der im Material dominanten Tätigkeiten und deren Zuordnung zu den jeweiligen Akteuren.

Konkret wurden in den Dokumenten systematisch Verben und Akteure mithilfe der qualitativen Analysesoftware NVivo codiert. Dabei wurde bei den Verben darauf geachtet, in einer ersten Codierrunde möglichst wenig Interpretation einfließen zu lassen. Dies hieß vor allem, dass Verben lediglich in den Infinitiv gebracht, ansonsten aber nicht verändert oder aggregiert wurden. Ergebnis war ein extensiver Katalog an Tätigkeiten, welcher im Anschluss mittels eines Thesaurus und systematischer Diskussionen mit anderen sprachkompetenten Personen durch konstanten Vergleich auf ca. 60 Verbcluster reduziert wurde. Akteure wurden ebenfalls codiert, wobei hier Synonyme zugelassen wurden. So wurden z.B. ,Vorgesetzte/r', ,Führungspersönlichkeit' oder auch nur ,Führung', wenn dieser Begriff eindeutig einen handelnden Akteur bezeichnete, unter dem Label Führungskraft gefasst. Anschließend wurde mittels einer Abfrage in NVivo festgestellt, welche Verbcluster im Material mit welchen Akteursgruppen gemeinsam auftreten. Verben wurden wiederum auf eine höhere Abstraktionsebene aggregiert, um eine handhabbare Anzahl von Rollenaspekten zu generieren. Unter einem Rollenaspekt wird hier ein Bündel an gleichartigen bzw. familienähnlichen (vgl. Wittgenstein, 2006) Tätigkeiten verstanden, welche einen in sich stimmigen Teil des institutionalisierten Aufgabenbereichs eines organisationalen Akteurs darstellen. So ist z.B. AnalystIn ein Rollenaspekt, welcher für FK und MA gleichermaßen relevant ist und die Handlungen (ab)klären, abwägen, infrage stellen, vergleichen, prüfen, kontrollieren, sich mit etwas auseinandersetzen inkludiert. Insgesamt wurden 19 derartige Rollenaspekte identifiziert, die in Kapitel 5.6.2 näher diskutiert werden.

\subsubsection{Schritt 2: (Re-)konstruktion von Beziehungstypen als Kombinationen von Tätigkeiten und grammati- kalischen Subjekt-Objekt-Strukturen}

Bevor die Tätigkeiten bzw. Rollenaspekte dazu verwendet werden konnten, Beziehungstypen zu (re-)konstruieren, war es zunächst notwendig, die codierten Verben in eine Beziehung zum jeweiligen Subjekt und Objekt zu bringen, um die spezifische Verbbedeutung im Satz zu (re-)konstruieren. Dies beruht auf der Tatsache, dass der Verbbezug in der deutschen Sprache einen wesentlichen Unterschied für die Satzbedeutung machen kann. 


\section{Erklärung im Detail:}

Betrachten wir z.B. folgendes (konstruiertes) Beispiel, in welchem das Verb immer dasselbe bleibt (für etwas oder jemanden sorgen):

a) Die Führungskräfte sorgen für die Mitarbeiter.

b) Die Führungskräfte sorgen für das Wohl der Mitarbeiter.

c) Die Führungskräfte sorgen für eine angenehme Arbeitsatmosphäre.

d) Die Führungskräfte sorgen für eine marktgerechte strategische Ausrichtung des Unternehmens.

e) Die Führungskräfte sorgen für eine effiziente Leistungserbringung durch die Mitarbeiter.

f) Die Führungskräfte sorgen gemeinsam mit den Mitarbeitern für herausfordernde Ziele.

g) Die Führungskräfte sorgen für eine rechtzeitige Information der Mitarbeiter.

h) Die Führungskräfte sorgen für sich selbst.

i) Die Führungskräfte sorgen für ihre eigene Weiterbildung.

Bereits an diesem sehr einfachen Beispiel lässt sich zeigen, dass es semantisch einen großen Unterschied macht, WER etwas MIT WEM und in Bezug WORAUF tut. Es lassen sich damit grob zumindest folgende Klassen von Strukturen aus dem obigen Beispiel (re-)konstruieren:

1) Ein Akteur handelt in Bezug auf sich selbst: Beispiel (h)

2) Ein Akteur handelt direkt in Bezug auf einen anderen Akteur: Beispiel (a)

3) Ein Akteur handelt direkt in Bezug auf ein unbelebtes Objekt oder ein abstraktes Konzept: Beispiele (b), (c), (d), (e), (f), (g) und (i)

Eine etwas detailliertere Betrachtung zeigt aber, dass die Beispiele (b) bis (i) auch wiederum systematische Unterschiede aufweisen. Folgende Unterklassen sind erkennbar:

4) Ein Akteur handelt in Bezug auf ein unbelebtes Objekt oder ein abstraktes Konzept, welches diesen Akteur selbst oder einen anderen Akteur betrifft: Beispiele (b), (e), (g), (i); Ein Streitfall könnte hier Beispiel (c) sein. Auch wenn kein zweiter Akteur im Satz explizit genannt wird, ist davon auszugehen, dass die angenehme Arbeitsatmosphäre andere Akteure sehr direkt betrifft. Dasselbe könnte aber auch von Beispiel (d) behauptet werden. Diese Problematik ist noch genauer zu diskutieren.

5) Ein Akteur handelt gemeinsam mit einem anderen Akteur in Bezug auf ein unbelebtes Objekt oder ein abstraktes Konzept: Beispiel (f)

Damit wird das Kategorienschema erneut um einen Schritt detaillierter. Unterschieden wird im Bereich der Handlungen, welche mit unbelebten Objekten oder abstrakten Konzepten vorgenommen werden, ob sie andere Akteure mittelbar betreffen oder nicht. Dies wird wiederum unterteilt in einen Fall, in welchem ein zweiter Akteur an der Handlung mitwirkt und in einen Fall, in welchem ein zweiter Akteur mittelbares Ziel der Handlung ist. Für den unter Punkt (4) angesprochenen Streitfall wird im Rahmen der vorliegenden Arbeit folgende Unterscheidung 
vorgenommen: Da der Untersuchungsgegenstand die Beziehung zwischen FK und MA ist, werden Handlungen, welche diese Beziehung mittelbar betreffen, eher großzügig codiert. Beispiel (c) wäre nach dieser Definition also ein Fall, welcher die MA mittelbar betrifft. Handlungen, welche nicht die Beziehung zwischen FK und MA zum Gegenstand haben, werden hingegen restriktiv codiert. Damit wäre Beispiel (d) keine Handlung, welche die MA (im Rahmen der Führungsbeziehung i.e.S.) mittelbar betrifft. Diese - zugegebenermaßen im Einzelfall oft schwer zu treffende - Differenzierung ist deshalb nötig, da sonst argumentiert werden könnte, dass jede Handlung einer Führungskraft die MA in irgendeiner Art und Weise mittelbar betrifft. Die Betroffenheit wird deshalb bewusst auf die Führungsbeziehung im engeren Sinne reduziert. Beispiel (d) würde in der Kategorisierung verschiedener Beziehungstypen nicht berücksichtigt werden, Beispiel (c) hingegen schon.

Aussagen der Kategorie (4) können nochmals genauer unterschieden werden, und zwar in:

4a) Ein Akteur handelt in Bezug auf ein unbelebtes Objekt oder ein abstraktes Konzept, welches einem anderen Akteur zukommt: Beispiele (b), (c) und (g)

4b)Ein Akteur handelt in Bezug auf ein unbelebtes Objekt oder ein abstraktes Konzept, welches von einem anderen Akteur gefordert wird: Beispiel (e)

4c) Ein Akteur handelt in Bezug auf ein unbelebtes Objekt oder ein abstraktes Konzept, welches von ihm selbst gefordert wird: Beispiel (i)

Abbildung 11: Die Codierung grammatischer Strukturen

Die Erläuterungen in der Box zeigen, dass bereits aus einem einfachen Beispiel mit dem Verb für etwas oder jemanden sorgen ein recht komplexes Kategoriengebilde entstehen kann. Dieses Gebilde wurde nun - anhand zusätzlicher Beispiele - noch weiter verfeinert und geschärft. Aus pragmatischen Gründen kann an dieser Stelle nicht die gesamte (Re-)konstruktion des Kategorienschemas im Detail erläutert werden. Stattdessen soll das aus dem Material generierte finale Schema dargestellt werden.

Tabelle 5 zeigt das abschließende Schema, mit welchem alle relevanten Äußerungen im Material, welche als Akteur entweder FK oder MA betreffen ${ }^{27}$ nach einer Kürzung und Vereinfachung durch Paraphrase dargestellt werden können. Das Symbol,$\rightarrow^{\circ}$ 'ist in diesem Zusammenhang als ,handelt in Bezug auf ${ }^{\star}$ zu le-

27 Ausgenommen sind hier alle Äußerungen, welche keinen der beiden Akteure betreffen, wie z.B. „Neue Ideen und verbesserte Problemlösungen sowie die Schnelligkeit, mit der sie verwirklicht werden, sind Voraussetzungen für den Erfolg im Wettbewerb.“ (A05) bzw. Äußerungen, welche ausschließlich andere Akteure (Kunden, Lieferanten, etc.) betreffen. Diese wurden in der (Re-)konstruktion der besonderen Topoi berücksichtigt, nicht aber in der (Re-)konstruktion der Akteursrollen von Führungskräften und MitarbeiterInnen bzw. derer Beziehungen. 
sen. ,FK` steht für Führungskraft, ,MA` für MitarbeiterIn, ,JEMAND‘ bezeichnet andere möglichen Akteure (Kunden, Lieferanten, etc.) und ,ETWAS‘ steht für alle möglichen unbelebten Objekte und abstrakten Konzepte. Methodisch wurden diese Strukturen rekonstruiert, indem die einzelnen Äußerungen innerhalb der Dokumente auf ein gleiches Abstraktionsniveau paraphrasiert wurden (vgl. z.B. die Technik der ,Zusammenfassenden Inhaltsanalyse' bei Mayring, 2002). Dabei wurde jede Äußerung in die Struktur SUBJEKT VERB OBJEKT gebracht. Alle Äußerungen wurden in die aktive Indikativform gebracht, um eine direkte Vergleichbarkeit zu ermöglichen. Verben wurden jedoch ansonsten nicht verändert. Eine ausführlichere Diskussion der verschiedenen Kategorien findet sich im Rahmen der Diskussion der Ergebnisse in Kapitel 5.6.1.

Tabelle 5: Kategorienschema der grammatischen Handlungsstrukturen im Korpus

Handlungen von FK und MA, welche nicht den jeweils anderen Akteur betreffen

FK $\rightarrow$ ETWAS

FK $\rightarrow$ JEMAND

FK $\rightarrow$ ETWAS für JEMAND

FK $\rightarrow$ ETWAS bei/an JEMAND

$\mathrm{FK} \rightarrow \mathrm{SICH}$ SELBST

$\mathrm{MA} \rightarrow$ ETWAS

$\mathrm{MA} \rightarrow$ JEMAND

MA $\rightarrow$ ETWAS für JEMAND

MA $\rightarrow$ ETWAS bei/an JEMAND

$\mathrm{MA} \rightarrow \mathrm{SICH}$ SELBST

JEMAND $\rightarrow$ FK

JEMAND $\rightarrow$ MA
Handlungen in der Beziehung zwischen FK und $M A$

$\mathrm{FK} \rightarrow \mathrm{MA}$

FK $\rightarrow$ ETWAS für MA

FK $\rightarrow$ ETWAS bei/an MA

$\mathrm{FK} \rightarrow$ ETWAS von MA

$\mathrm{MA} \rightarrow \mathrm{FK}$

MA $\rightarrow$ ETWAS für FK

$\mathrm{MA} \rightarrow$ ETWAS von FK

FK und/mit MA $\rightarrow$ ETWAS

$\mathrm{FK} \leftarrow \rightarrow \mathrm{MA}$ 
Für die (Re-)konstruktion typischer Beziehungen zwischen FK und MA wurden anschließend nur jene Verben verwendet, welche in der direkten Beziehung zwischen den beiden Akteuren (rechte Spalte in Tabelle 5) relevant waren. Wie in Schritt 1 wurde das Zusammenfallen von Verben und grammatischen Beziehungsstrukturen mittels einer Abfrage in NVivo festgestellt. Verben wurden mittels derselben Prinzipien wie in Schritt 1 geclustert, um spezifische Konfigurationen in Form von Beziehungstypen zu generieren. Dabei wurde direkt auf die Verben zugegriffen und nicht auf die zuvor gebildeten Rollenaspekte. So war es möglich, dass ein Rollenaspekt in verschiedenen Beziehungstypen von Relevanz war. Dieser Effekt entstand durch die oben diskutierte Tatsache, dass es z.B. in der Führungsbeziehung einen Unterschied macht, ob die FK für die MA sorgt oder für eine angemessene Leistung der MA sorgt, obwohl dasselbe Verb betroffen ist (und damit derselbe Rollenaspekt der FK).Typen wurden dabei so gebildet, dass sich die Merkmale innerhalb des Typus möglichst ähnlich sind (interne Homogenität) und sich die Typen untereinander möglichst stark unterscheiden (externe Heterogenität) (vgl. Kelle \& Kluge, 2010: 85). Ergebnis war ein Katalog von 12 distinktiven Beziehungstypen, welche in Kapitel 5.6.3 kurz vorgestellt und in Kapitel 5.8 ausführlich diskutiert werden.

\subsubsection{Schritt 3: (Re-)konstruktion der besonderen Topoi}

Während die Analysen der Rollenaspekte und der Beziehungstypen stark ineinandergriffen, wurde die (Re-)konstruktion relevanter besonderer Topoi völlig unabhängig von diesen vorgenommen. Es wurde erläutert, dass Topoi jene Ideen darstellen, welche hinter Schlussregeln in enthymemischen Argumentationen stehen (vgl. Wengeler, 2003b: 181). Die (Re-)konstruktion besonderer Topoi kann also anhand der Analyse verwendeter Enthymemstrukturen (oder ihrer Fragmente) vorgenommen werden.

$\mathrm{Zu}$ diesem Zweck wurde die schematische Vorgehensweise der hermeneutischen Systemanalyse (vgl. Froschauer \& Lueger, 2003: 142ff.; M. Lueger, 2010: 199ff.) adaptiert. Während die hermeneutische Systemanalyse in systematischer Form die (Re-)konstruktion des hypothetischen Äußerungs- und Wirkungskontextes einer Äußerung zum Ziel hat, kann die grundsätzliche Vorgehensweise aber auch genutzt werden, um fragmentarische Enthymeme in Textmaterial zu (re-)konstruieren. 
Tabelle 6: Rekonstruktion besonderer Topoi (in Anlehnung an Froschauer \& Lueger, 2003)

\begin{tabular}{|c|c|c|c|c|c|}
\hline Äußerung & Paraphrase & $\begin{array}{l}\text { Argument } \\
\text { (Daten) }\end{array}$ & Conclusio & Schlussregel & Topos \\
\hline $\begin{array}{l}\text { Wörtliche } \\
\text { Wiedergabe } \\
\text { der im Text } \\
\text { vorgefundenen } \\
\text { Stelle }\end{array}$ & $\begin{array}{l}\text { Reduktion der } \\
\text { Stelle auf die } \\
\text { wesentlichen } \\
\text { Inhalte }\end{array}$ & $\begin{array}{l}\text { Was ist das } \\
\text { Ziel? Was } \\
\text { will die Orga- } \\
\text { nisation errei- } \\
\text { chen? }\end{array}$ & $\begin{array}{l}\text { Welche Hand- } \\
\text { lungen, Ein- } \\
\text { stellungen oder } \\
\text { Fähigkeiten } \\
\text { werden vorge- } \\
\text { schlagen? }\end{array}$ & $\begin{array}{l}\text { Mit welcher } \\
\text { sozial legiti- } \\
\text { mierten ,Re- } \\
\text { gel' lassen } \\
\text { sich Argument } \\
\text { und Conclusio } \\
\text { verbinden? } \\
\text { Was ist die } \\
\text { zugrunde lie- } \\
\text { gende Logik? }\end{array}$ & $\begin{array}{l}\text { Kurze und } \\
\text { prägnante } \\
\text { Bezeichnung } \\
\text { des ausge- } \\
\text { drückten } \\
\text { Topos }\end{array}$ \\
\hline
\end{tabular}

Wie Tabelle 6 zeigt, wurden Äußerungen zunächst wörtlich übernommen und dann in Form einer Paraphrase auf die wesentlichen manifesten Inhalte reduziert (vgl. Froschauer \& Lueger, 2003: 150). Danach wurden in systematischer und extensiver Form die in der Tabelle abgebildeten Fragen an die Paraphrase gestellt. Enthymeme wurden dabei als, weil'-Formulierungen charakterisiert. Da die Äußerungen in erster Linie als Handlungsempfehlungen formuliert sind, wurde das Argument (bzw. das Datum) als Zielzustand formuliert. Die Conclusio stellt somit die vorgeschlagene Handlungsweise dar. Die Schlussregel schließlich stellt die Verbindung zwischen Argument und Conclusio her. Während die Conclusio (als Regel bzw. Handlungsanweisung) in jeder Äußerung bereits vorhanden war, fehlte zumeist die Schlussregel und gelegentlich auch das Argument (bzw. das Ziel). In diesen Fällen wurden in extensiver Auslegung mögliche Argumente und Schlussregeln formuliert. Diese wurden immer stärker eingeengt, je deutlicher sich aus dem Material ein abgrenzbarer Topos herauskristallisierte. Da Topoi als argumentative Ressource in verschiedenen, manchmal sogar gegensätzlichen Argumenten verwendet werden können (vgl. Ottmers, 2007: 90), konnte sich aus verschiedenen Enthymemen derselbe Topos ergeben. Die Formulierung von Argument und Conclusio ist deshalb in dieser Analyse nur als ,Hilfsschritt` zu sehen, um den Topos als eigentliche bedeutungstragende Kategorie zu (re-)konstruieren.

Dieser Prozess wurde solange durchgeführt, bis sich in Bezug auf die relevanten Topoi theoretische Sättigung ergab und keine neuen Topoi mehr auffindbar waren. Dabei wurden zusätzliche Dokumente hauptsächlich nach dem Kriterium der maximalen Differenz in die Analyse aufgenommen, um ein maximales Spektrum unterschiedlicher Führungsgrundsätze zu inkludieren. Anschließend wurde auch der Rest des Materials deduktiv mit den entstandenen Topoi codiert, wobei grundsätzliche Offenheit bewahrt wurde. Es ergaben sich in diesem Pro- 
zess zwar noch einige neue Topoi, diese erwiesen sich aber in der anschließenden Interpretation weder quantitativ noch bezüglich ihres Erklärungswerts als zentral. Eine Diskussion der relevanten Topoi findet sich in Kapitel 5.7, in Kapitel 5.8 wird ihre Bedeutung für die Begründung spezifischer Beziehungstypen erläutert. Im Anhang findet sich außerdem eine abschließende Darstellung aller in der Untersuchung identifizierten besonderen Topoi.

\subsubsection{Schritt 4: Erstellung einer diskursiven ,Landkarte'}

Ein letzter Schritt betraf die Zusammenführung von Beziehungstypen und besonderen Topoi. Die Grundannahme war, dass die (re-)konstruierten Topoi eine argumentative Basis für die im Material vorgefundenen typisierten Beziehungen zwischen FK und MA bereitstellen. Dementsprechend wurde die Relevanz der Topoi für die gegenständliche Arbeit nicht an der Häufigkeit ihres Vorkommens, sondern an dem Grad festgemacht, in welchem sie sich auf die (re-)konstruierten Beziehungstypen beziehen und diese begründen bzw. legitimieren. Die Berechnung statistischer Signifikanzen wäre weder dem Forschungsanspruch noch dem Material gerecht geworden, deshalb wurde auf den Versuch der Berechnung dieser verzichtet. Statt dessen wurde eine zweistufige Überprüfung der Relevanz vorgenommen, indem (a) zunächst konzeptionell überprüft wurde, ob ein spezifischer Topos in plausibler Art und Weise überhaupt dazu geeignet ist, einen Beziehungstypus zu begründen, (b) anschließend anhand einer einfachen Zählung des ,Aufeinanderfallens' von Topos und Beziehungstypus in der Codierung des Materials die tatsächliche Verwendung des Topos im Zusammenhang mit dem Beziehungstypus geklärt wurde. Waren beide Bedingungen in ausreichendem Ausmaß gegeben, wurde eine Relevanz bejaht. Aufgrund des Fehlens einer streng statistischen Überprüfung handelt es sich aber dennoch nur um eine qualitativ festgestellte Relevanz bzw. um typische $\mathrm{Zu}$ sammenhänge. Für die vorliegende Arbeit reicht dies vollkommen aus, da kein Anspruch darauf besteht, universelle Aussagen zu treffen oder kausale Zusammenhänge zu postulieren. Die Ergebnisse bleiben auf dem Niveau einer Typik.

Die in Schritt 2 (re-)konstruierten Beziehungstypen wurden anschließend anhand mehrerer Dimensionen ausführlich interpretiert und diskutiert. Für jede Beziehung wurden die Handlungslogik (bzw. die betroffenen Rollenaspekte), die Argumentationsstruktur im Sinne der jeweils relevanten besonderen Topoi, die Interaktionsstruktur in Form des jeweiligen Handelns der Akteure aufeinander und zueinander innerhalb der Beziehung und die durch die Beziehung und ihre argumentative Basis ermöglichten und propagierten Steuerungsaspekte diskutiert. Diese Ergebnisse werden in Kapitel 5.8 dargestellt. Anschließend wurde 
aufgrund der spezifischen Beziehungen der Topoi zueinander eine konzeptionelle ,Landkarte' erstellt. Diese Landkarte bildet einen Ausschnitt des Diskurses der Führungsgrundsätze über die Verbindung der verwendeten Topoi ab. Konstitutive Topoi wurden zu diesem Zweck den sie ergänzenden Topoi hierarchisch übergeordnet. Diese hierarchischen Beziehungen wurden mittels unidirektionaler Pfeile in einem Diagramm abgebildet. Dadurch lassen sich konzeptionell Zentrum und Peripherie der Topoi im Diskurs (und der durch sie konstituierten Beziehungstypen) als Grad der Vernetzung unterscheiden. Eine Diskussion dieser Landkarte findet sich in Kapitel 5.8.14.

\subsection{Zur Darstellung der Ergebnisse}

Die interpretative Sozialforschung steht grundsätzlich vor dem Problem, dass die lineare Form der Ergebnisdarstellung, welche die meisten Publikationsgenres verlangen, zumeist nicht mit der tatsächlichen, zirkulären Form des Untersuchungsdesigns und damit des Erkenntnisgewinns (vgl. M. Lueger, 2001: 363ff.) übereinstimmt. Die konkrete Vorgehensweise der Untersuchung wurde im vorhergehenden Kapitel im Detail vorgestellt. Die Ergebnisdarstellung analog dieser Vorgehensweise würde entsprechend viele Redundanzen und Wiederholungen aufweisen, welche die Diskussion der Ergebnisse erschweren würde. Die folgende Diskussion steht deshalb vor dem Problem, dass sie Akteursrollen, Beziehungstypen und Topoi grundsätzlich nicht unabhängig voneinander diskutieren kann, da sie nur in ihrer Interaktion die spezifischen Schlussfolgerungen der Arbeit erlauben. Um dem Leser aber die Nachvollziehbarkeit des Vorgehens zu ermöglichen, wird ein Mittelweg gewählt: Grundsätzlich wird versucht, die einzelnen Bestandteile des Gesamtmodells zunächst so gut als möglich voneinander getrennt zu diskutieren, um ihre anschließende Integration klarer argumentieren zu können. Die Diskussion startet deshalb mit der Beschreibung und Analyse der (re-)konstruierten Handlungsmuster und führt von grammatischen Strukturen über Rollenaspekte zu den Beziehungstypen. Wo immer nötig, werden bereits Verweise auf die später diskutierten Ergebnisse (v.a. Topoi) vorgenommen. Anschließend widmet sich die Arbeit der Diskussion der (re-)konstruierten Argumentationsmuster im Sinne der relevanten besonderen Topoi. Kapitel 5.8 schließlich integriert Handlungs- und Argumentationsaspekte in eine integrierte Diskussion von Beziehungstypen, relevanten Topoi und daraus abgeleiteten Führungs- und Steuerungsaspekten. 


\section{6 (Re-)konstruktion der Handlungsmuster}

\subsubsection{Grammatische Handlungsmuster im Material}

In der bisherigen Argumentation wurde gezeigt, dass grammatische Strukturen von Äußerungen innerhalb der Führungsgrundsätze eine wesentliche Rolle vor allem für die (Re-)konstruktion typischer Beziehungen zwischen FK und MA spielen. Im folgenden Kapitel werden die in Tabelle 5 dargestellten Muster näher erläutert und diskutiert sowie konkrete Beispiele aus den analysierten Dokumenten vorgestellt.

5.6.1.1 Handlungen von FK und MA, welche nicht den jeweils anderen Akteur betreffen

Diese Überkategorie von Handlungen zeigt sich in Äußerungen, welche lediglich einen der beiden zentralen Akteure betreffen. Nichtsdestoweniger bezeichnen diese Aussagen Handlungen, welche durch die in den Führungsgrundsätzen konstatierte Typik für die konstruierten Rollenaspekte von hoher Relevanz sind. Während Handlungen dieser Überkategorie nicht dazu verwendet werden konnten, spezifische Beziehungstypen zwischen Führungskraft und MitarbeiterInnen $\mathrm{zu}$ (re-)konstruieren, so sind sie doch wertvolle Ressourcen in der (Re-)konstruktion der propagierten Rollen der beiden Akteure. Handlungen der Kategorie $F K \rightarrow E T W A S$ zeigen somit typische Tätigkeiten von FK in Bezug auf unbelebte Objekte und abstrakte Konzepte. Beispiele dafür sind:

Führungskräfte vertreten die Unternehmensziele und übernehmen Verantwortung. (D06)

[Die Führungskraft] Wägt zwischen kurzfristigen und langfristigen Prioritäten ab. (CH 03)

Als Spiegelbild dessen zeigt die Kategorie MA $\rightarrow$ ETWAS typische Tätigkeiten und Handlungen von MA in Bezug auf unbelebte Objekte und abstrakte Konzepte:

Vertrauen setzt voraus, dass alle Mitarbeiter die für das Unternehmen verbindlichen

Verhaltensregeln beachten und im Sinne des Gesamtunternehmens handeln. (A05)

Ich [der/die MA] nutze die Informationen systematisch. (D16)

Äußerungen der Kategorien $F K \rightarrow$ JEMAND, FK $\rightarrow$ ETWAS für JEMAND, FK $\rightarrow$ ETWAS bei JEMAND bzw. MA $\rightarrow$ JEMAND, MA $\rightarrow$ ETWAS für JEMAND und $M A \rightarrow E T W A S$ bei JEMAND thematisieren Handlungen, welche FK und MA gegenüber dritten Akteuren vornehmen sollen. In erster Linie beziehen sich 
solche Äußerungen auf die Kunden des Unternehmens. Beispiele dafür beinhalten:

Wir [Führungskräfte] richten daher unsere Planungen, Entscheidungen und Handlungen auf den Kundennutzen aus, mit dem Ziel Dienstleistungen bester Qualität zu bieten. (A13)

Wir [Führungskräfte] setzen unsere Fachkompetenz optimal zum Nutzen unserer Kunden und unseres Umfeldes ein. (CH12)

Die Umkehrung, dass dritte Parteien in spezifischer Art und Weise in Bezug auf FK und MA handeln, ist im Diskurs der Führungsgrundsätze peripher und muss deshalb nicht gesondert diskutiert werden. Diese Kategorien (JEMAND $\rightarrow F K$, $J E M A N D \rightarrow M A$ ) wurden der Vollständigkeit halber erfasst, da sie aber keine Rollen von MA und FK beinhalten (diese sind nicht die handelnden Personen in dieser Konstruktion) wurden sie in der weiteren Analyse nicht mehr berücksichtigt.

Von Interesse sind hingegen die beiden Kategorien, in welchen FK und MA in Bezug auf sich selbst Handlungen vornehmen $(F K \rightarrow$ SICH SELBST, MA $\rightarrow$ SICH SELBST). Diese Kategorien erlauben die Identifikation von Handlungen, welche die Akteure mit sich selbst vornehmen, nicht aber mit anderen bzw. auch nicht als Objekt der Handlungen anderer erleben. Beispiele dieser Kategorie von Handlungen sind z.B.:

Ich [Führungskraft] überprüfe kontinuierlich, ehrlich und selbstkritisch meine eigenen Verhaltensweisen. Ich strebe nach persönlicher Verbesserung. (A14)

[Von MitarbeiterInnen wird erwartet] die Verantwortung für Ihre Entscheidungen zu übernehmen. (CH 16)

\subsubsection{Handlungen in der Beziehung zwischen FK und MA}

Äußerungen in dieser Überkategorie betreffen direkt das Verhältnis zwischen FK und MA. Diese Äußerungen sind somit zentral sowohl für die (Re-)konstruktion spezifischer Rollenaspekte als auch der verschiedenen typischen Beziehungen. Wie bereits ausführlich diskutiert, macht es semantisch einen großen Unterschied, wer in Bezug auf wen in welcher Art und Weise einwirkt. Unterschieden werden deshalb die folgenden Handlungsmuster bzw. -kategorien:

$F K \rightarrow M A$ bzw. $M A \rightarrow F K:$ In Äußerungen dieser Kategorie wirkt jeweils ein Akteur auf den anderen direkt und unmittelbar ein. Die Art des Verbs bestimmt sowohl die spezifische Beziehung zwischen den beiden Akteuren als auch die in dieser Beziehung ausgedrückten Rollen der Akteure.

Ich habe Vertrauen in meine Mitarbeiter und rechtfertige das in mich gesetzte Vertrauen. (A14) 
[U]nsere MitarbeiterInnen müssen bereit sein, Verantwortung zu übernehmen und ihre Vorgesetzten und das Team mit ihrem Wissen und Können bei der Arbeit zu unterstützen. (D08)

$F K \rightarrow$ ETWAS für MA bzw. MA $\rightarrow$ ETWAS für FK: In dieser Kategorie wird nicht direkt in Bezug auf den anderen Akteur gehandelt. Vielmehr wird eine andere Variable manipuliert, welche dem Handlungsempfänger letztlich zugutekommt $^{28}$. Etwas wechselt also zwischen den Akteuren, und zwar vom handelnden Akteur weg. Der Einfluss auf den jeweils anderen Akteur ist nicht direkt, sondern eher indirekt bzw. mittelbar.

Kann kein Konsens erreicht werden und ist eine sofortige Entscheidung notwendig, so entscheidet der Vorgesetzte und begründet dies gegenüber den Mitarbeitern. (D10)

Als Grundlage für diese Art der Vorgesetzten-Beurteilung soll alle 2 Jahre das Feedback für Vorgesetzte (Rückmeldung des Mitarbeiters an die Führungskraft über deren Führungsverhalten) durchgeführt werden. (D14)

$F K \rightarrow$ ETWAS bei/an MA: Im Gegensatz zur vorherigen Kategorie geht es hier nicht darum, dass etwas für den anderen Akteur getan, vorgenommen oder hergegeben wird, sondern vielmehr darum, dass etwas (v.a. Leistungen/Handlungen, Werte/Normen und Eigenschaften) bei einem anderen Akteur betrachtet oder manipuliert wird. Als erstes Ergebnis zeigt sich dabei bereits, dass diese Kategorie nur in einer Ausprägung existiert, nämlich in Richtung von FK zu MA. MA handeln im Diskurs folglich nicht in Bezug auf Handlungen, Werte und Eigenschaften der FK.

Sehen Sie bei Ihren Mitarbeitern die Stärken und helfen Sie, diese zu nutzen und weiter auszubauen. (A01)

Wir unterstützen die Eigenverantwortung und Selbständigkeit der Mitarbeiterinnen und Mitarbeiter. (D01)

$F K \rightarrow$ ETWAS von MA bzw. MA $\rightarrow$ ETWAS von FK: Mit der Präposition ,von“ wird in dieser Kategorie ebenfalls ausgedrückt, dass etwas von einem Akteur

28 Die Präposition ,für' in der Bezeichnung der Kategorie ist quasi prototypisch zu verstehen. Selbstverständlich können in den eigentlichen Dokumenten auch andere Präpositionen verwendet werden. Die meisten Satzkonstruktionen können aber in dieses prototypische Schema übersetzt werden. So würde eine mögliche Paraphrase des ersten Beispiels z.B. lauten: „Vorgesetzte begründen Ihre Entscheidungen für die Mitarbeiter (wenn kein Konsens erreicht werden kann und eine sofortige Entscheidung notwendig ist)“. Im zweiten Beispiel könnte sie lauten: „Mitarbeiter geben ein Feedback für Vorgesetzte (alle 2 Jahre als Grundlage für die Vorgesetzten-Beurteilung).“ Damit ist das Schema FK $\rightarrow$ ETWAS für MA bzw. MA $\rightarrow$ ETWAS für FK in allen Beispielen erfüllt. Diese Fußnote gilt für alle weiteren diskutierten Kategorien sinngemäß. 
zum anderen wechselt, allerdings in der entgegengesetzten Richtung als in der Konstruktion mit ,für': Etwas wird vom Subjekt-Akteur erhalten oder genommen. Es wandert also zu diesem Akteur hin.

Ich [Führungskraft] gebe konstruktive Rückmeldungen und nehme sie auch [vom/von der MitarbeiterIn] an. (D 16)

Bei Verwirklichung dieses Prinzips bekommt MitarbeiterIn [von der Führungskraft] den individuellen Gestaltungsspielraum, der Arbeitsfreude ermöglicht, Leistungsreserven freisetzt, Führungskräfte entlastet und objektive Information auf kurzem Wege sicherstellt. (D08)

FK und/mit MA $\rightarrow$ ETWAS: In dieser Kategorie von Äußerungen handeln FK und MA gemeinsam in Bezug auf ein Objekt oder ein Konzept. Diese Kategorie entspricht somit in ihrer grammatischen Struktur am ehesten dem, was in der präskriptiven Literatur als „kooperative Führung“ bezeichnet wird (vgl. z.B. Töpfer \& Zander, 1982: 14).

Die Führungskraft ist gemeinsam mit jedem/r einzelnen MitarbeiterIn sowie mit dem gesamten Team dafür verantwortlich Stärken und Potenziale zu erkennen, zu entwickeln und einzusetzen. (A13)

Bei Fehlentwicklungen müssen Ziele und Maßnahmen möglichst gemeinsam korrigiert oder neu erarbeitet werden. (D10)

$F K \leftrightarrow \rightarrow M A$ : Die letzte Kategorie schließlich umfasst Äußerungen, in welchen FK und MA gegenseitig Handlungen an sich vornehmen. Ähnlich wie die vorhergehende Kategorie impliziert diese ein grundsätzliches Gleichgewicht zwischen FK und MA. Der Unterschied ist der spezifische Bezug der Handlung, der hier in der Person, nicht in einer Sache liegt.

Im Mitarbeitergespräch dokumentieren Führungskraft und MitarbeiterIn die Erwartungshaltungen und geben einander Feedback im Sinne von Leistungsanerkennung. (A11)

Wir geben im delegierten Bereich lösungsorientiert und aufbauend Unterstützung und gegenseitiges Feedback. (CH10)

Diese grammatischen Strukturen und die daraus entwickelten Kategorien geben ein erstes, krudes Bild der Beziehungen, welche in den Dokumenten zwischen FK und MA dargestellt werden. Metaphorisch könnte man davon sprechen, dass diese Kategorien ein ,Skelett' darstellen, auf welches nun noch die konkreten Verben gepackt werden müssen, will man ein vollständiges Bild erhalten. Im folgenden Kapitel wird nun eben jenen Verben nun eine größere Aufmerksamkeit geschenkt, indem der zweite Teil des ,Puzzles' - nämlich die Verbindung einzelner Akteure mit spezifischen Handlungen - diskutiert wird. 


\subsubsection{Rollenaspekte als Bündel typischer Handlungen}

Wie in den Ausführungen zur methodischen Vorgehensweise ausführlich beschrieben, wurden die Rollenaspekte dergestalt gebildet, dass für jeden der beiden relevanten Akteurstypen verschiedene Cluster an Verben (re-)konstriert wurden, welche distinktive Tätigkeitsbereiche beschreiben. In diesem Kapitel sollen nun die dadurch identifizierten Rollen genauer diskutiert werden, um einen Überblick darüber zu geben, welche Tätigkeiten im Diskurs der Führungsgrundsätze den einzelnen Akteuren zugerechnet werden. Um ein Gefühl dafür zu vermitteln, welche quantitative Bedeutung den einzelnen Rollen im Dokumentkorpus zukommt, wird jeweils die Häufigkeit des Vorkommens im Material kurz diskutiert. Dabei besteht die Grundgesamtheit aus dem Sample von 53 Dokumenten $(\mathrm{n}=53)$. Mehrfachnennungen innerhalb eines Dokuments werden nicht berücksichtigt, um diese Form der Verzerrung zu vermeiden. Zunächst werden jene Rollenaspekte vorgestellt, welche von FK und MA gleichermaßen besetzt werden können. Hier wird zuerst jeweils eine kurze allgemeine Beschreibung des Rollenaspekts präsentiert, auf welche eine Diskussion der spezifischen Ausprägung für FK und MA folgt. Im Anschluss daran werden jene Rollenaspekte vorgestellt, welche exklusiv von einem der beiden Akteure eingenommen werden können.

\subsubsection{Geteilte Rollenaspekte von FK und MA}

\subsection{Der Akteur als Analyst und Beurteiler ${ }^{29}$}

Allgemein: Ein zentraler Tätigkeitsbereich innerhalb der Führungsgrundsätze ist das breite Feld der Analyse, Messung, Kontrolle und anschließender Beurteilung sowohl von Sachverhalten als auch von anderen Akteuren. Mit diesem Rollenaspekt assoziierte typische Verben sind einerseits (ab)klären, abwägen, infrage stellen, vergleichen, prüfen, kontrollieren, sich auseinandersetzen, auf der anderen Seite aber auch feststellen, bewerten und beurteilen.

Führungskräfte: Für die FK ist der Aspekt des Analysten und Beurteilers ein sehr typischer innerhalb der Dokumente. In 37 von 53 (69,8\%) Grundsatzdokumenten wird darauf Bezug genommen. Auf der Sachebene bezieht sich die Analyse und Beurteilung z.B. auf Prioritäten (D06) ${ }^{30}$, Veränderungen (D10), Abläu-

29 Zur besseren Lesbarkeit werden die Labels in der männlichen Form gehalten. Die weibliche Form ist immer mitgemeint.

30 Dokumentcodes in Klammern verweisen auf das spezifische Dokument, in welchem eine bestimmte Aussage zu finden ist. Der Buchstabe im Code verweist auf das jeweilige Herkunftsland der Führungsgrundsätze (A für Österreich, D für Deutschland, und $\mathrm{CH}$ für die Schweiz). Diese Referenzen sind grundsätzlich niemals abschließend, son- 
fe (D02), Ziele (D18), Delegation (D10) und Ergebnisse (D18). Auf der Ebene der Personalführung sind die Objekte der Handlung vor allem die Leistungen (D05, D06) und Ergebnisse (A05, CH06) der MA, aber auch die MA selbst (A01) im Sinne der klassischen Vorstellung von Personalbeurteilung (vgl. z.B. G. Lueger, 2002).

MitarbeiterInnen: Die MA werden wesentlich seltener als die FK mit dem Aspekt des Analysten und/oder Beurteilers in Verbindung gebracht, entsprechende Aussagen finden sich in zwölf Dokumenten (22,6\%). In erster Linie geschieht aber auch in diesen Fällen die Analyse und Beurteilung gemeinsam mit den FK (D09, A05). MA alleine prüfen und beurteilen in erster Linie sich selbst (D05, C17). Die FK als Analyst und Beurteiler wird also nicht nur häufiger thematisiert, sie ist auch deutlich stärker ausdifferenziert. Dennoch ist festzustellen, dass vor allem im Sinne gegenseitigen Feedbacks im Diskurs auch die Möglichkeit angelegt ist, dass MA ihre FK beurteilen (D14, CH16).

\subsection{Der Akteur als Entscheider}

Allgemein: Ein zweiter Rollenaspekt, welcher logisch auf jenem als Analyst und Beurteiler folgt ist jener von FK bzw. MA als Entscheider. Der Tätigkeitsbereich in dieser Rolle ist sehr klar abgegrenzt: Die assoziierten Verben sind entscheiden und Konsequenzen ziehen.

Führungskräfte: Interessanterweise wird in den Dokumenten kaum erwähnt, was von FK zu entscheiden ist. Der Fokus liegt stattdessen vielmehr auf der Frage, wie Entscheidungen zu treffen sind. Typische Eigenschaften, welche mit Entscheidungen im Diskurs verbunden werden, sind zeitnah (D12), schnell (D01), nachvollziehbar (D16, A01, A07), sachlich (D16, D01), fair (D16), sorgfältig (D17), mutig (A03) und konsequent (A01). Ein weiteres Kriterium ist das Entscheiden im Sinne der Unternehmensziele (D15). Die Führungskraft als Entscheider taucht in 22 der Dokumente (41,5\%) auf.

MitarbeiterInnen: Die explizite Verwendung des Verbs entscheiden in Verbindung mit MA ist in den Dokumenten eher selten zu finden (fünf Dokumente; 9,4\%). Wo Entscheidungen der MA aber thematisiert werden, da finden diese in einem sehr konkreten und homogenen Kontext statt: Thema ist die selbständige bzw. eigenverantwortliche Entscheidung der/des MA in ihrem/seinem Verantwortungsbereich (D09, D10, D18). Damit bezieht sich dieser Aspekt sehr stark auf das Thema der Delegation, welches das ursprüngliche Hauptthema der all-

dern stellen Beispiele dar, um die Diskussion zu verdichten und den Charakter einzelner Rollen, Beziehungstypen und Topoi besser darzustellen. 
gemeinen Führungsanweisung im Harzburger Modell war (vgl. Höhn 1976, 1977). Die Entscheidungsrolle der/des MA ist also eine von den FK verliehene.

\subsection{Der Akteur als Koordinator und Korrektor}

Allgemein: Auch der Aspekt des Koordinators und Korrektors schließt sehr stark an jenen des Analysten und Beurteilers an. Logischerweise folgt auf die der Phase der Kontrolle und Beurteilung jene der Korrektur und der Koordination. Entsprechend sind die typische Verben, welche dieser Rolle zugeordnet werden, koordinieren, vereinbaren, anpassen und korrigieren.

Führungskräfte: Korrigiert werden z.B. Strukturen und Prozesse (A14), Ziele (D04) und Fehler (CH05), aber im Rahmen der Mitarbeiterführung auch das Verhalten der MA (D19, A06). Koordiniert werden Aufgaben (A15) und Ressourceneinsatz (CH09). Vereinbart werden in erster Linie Ziele (D03, A11, CH08), und zwar sowohl zwischen den Akteuren (die klassische Zielvereinbarung zwischen FK und MA) als auch im Sinne der Koordination gegenläufiger Ziele allgemein. Angepasst hingegen wird die FK in erster Linie selbst, und zwar an Veränderungen (A12), andere Kulturen (A14) und die jeweilige Situation (D02). Für die FK ist der Rollenaspekt in 36 Dokumenten $(67,9 \%)$ relevant, nicht zuletzt wegen der hohen Relevanz von Zielvereinbarungen innerhalb der Dokumente.

MitarbeiterInnen: Wenig überraschend besitzt der Aspekt des Koordinators und Korrektors bei den MA keinen großen Stellenwert. Nur in drei Dokumenten $(5,7 \%)$ wird darauf Bezug genommen. MA übernehmen dann koordinierende und korrigierende Funktionen, wenn sie mit den FK gemeinsam Ziele vereinbaren (D16) oder diese anpassen (D10, D18) bzw. mit der FK zusammen Aufgaben anpassen (D10).

\subsection{Der Akteur als Problemlöser und Verhinderer negativer Zustände}

Allgemein: Ein weiterer Rollenaspekt, welcher in logischer Folge aus jener des Analysten und Beurteilers entsteht, ist jener des Problemlösers bzw. Verhinderers. Relevante Verben bzw. Tätigkeiten, welche dieser Rolle zugehörig sind, beinhalten lösen, beseitigen, bewältigen, überwinden, und beenden, aber auch auf der präventiven Seite dieses Aspekts - verhindern, vermeiden, nicht zulassen und zurückweisen.

Führungskräfte: Angesprochen wird hier die Aufgabe der FK, negative bzw. unerwünschte Zustände, welche sie selbst, das Unternehmen oder die MA betreffen, aufzulösen, zu beenden oder präventiv zu verhindern. Von dem Aspekt als Koordinator und Korrektor unterscheidet sich diese Rolle daher in erster Li- 
nie durch die Erzeugung einer Finalität. Gelöst und überwunden werden Probleme, Widerstände und Konflikte. Verhindert und zurückgewiesen werden hauptsächlich Versuche der MA, delegierte Verantwortung wieder an die FK zurückzugeben. Der Aspekt ist im Diskurs als vergleichsweise peripher zu betrachten (dreizehn Dokumente; 24,5\%).

MitarbeiterInnen: Noch wesentlich unbedeutender aber ist der Aspekt bei den MA. Hier scheint er nur in zwei Dokumenten $(3,8 \%)$ auf. Dabei wird einmal die Möglichkeit der/des MA aufgezeigt, Einmischungsversuche der FK in den eigenen Verantwortungsbereich zurückzuweisen, einmal die Fähigkeit des MA, Aufgaben und Probleme zu bewältigen angesprochen, und in einer dritten Äußerung werden von FK und MA gemeinsam Probleme gelöst.

\subsection{Der Akteur als Bereitsteller bzw. ,Provider ‘}

Allgemein: Der Rollenaspekt des Akteurs als Bereitsteller bzw. Provider bezieht sich auf Handlungen wie geben, ausstatten, zur Verfügung stellen, übertragen und einräumen. Die Logik hinter diesen Handlungen ist, dass etwas den Besitzer wechselt, bzw. allgemeiner von einem Akteur auf den anderen übergeht.

Führungskräfte: Für die FK wird der Aspekt des Bereitstellers bzw. Providers im Dokumentkorpus sehr explizit formuliert (27 Dokumente; 50,9\%). Dabei übertragen sie den MA Kompetenzen (D02, A01) und Freiraum (D07, A06, CH12), geben ihnen Sicherheit (D04) und Orientierung (D06), aber auch Vertrauen (A01), Informationen (D17) und allgemein nötige Ressourcen (A15).

MitarbeiterInnen: Interessanterweise wird der Aspekt des Bereitstellers bzw. Providers bei den MA weitaus seltener explizit angesprochen (2 Dokumente; 3,8\%). Dies liegt daran, dass der hauptsächliche Gegenstand, welche MA ihren FK zur Verfügung stellen, nämlich die individuelle Leistung, als selbstverständlich erachtet wird. Der Aspekt als Bereitsteller wird bei den MA also implizit immer dann angesprochen, wenn FK von ihnen etwas verlangen oder (ein)fordern. Das Entsprechen dieser Forderung wird angenommen, aber nicht expliziert. In den zwei Dokumenten, in denen die Rolle explizit angesprochen wird, geht es hingegen um die Bereitstellung von Informationen (D14, A04).

\subsection{Der Akteur als Beziehungsmanager}

Allgemein: Dieser Rollenaspekt betrifft in erster Linie Handlungen, welche stark auf Eigenschaften rekurrieren. Assoziierte Verben sind vertrauen, hineinversetzen, eingehen, akzeptieren, respektieren, tolerieren, ernstnehmen und wertschätzen. Diese Verben wurden in einer Rolle zusammengefasst, weil sie - obwohl 
sie durchaus unterschiedliche Handlungen und Einstellungen darstellen - insgesamt ein rundes Bild des Akteurs als Beziehungsmanager zeichnen.

Führungskräfte: Dieser Rollenaspekt ist nur schwach mit der Sachebene verbunden, etwa wenn FK kulturelle Elemente, die diverse Belegschaft und die Entscheidungen anderer respektieren. Hauptsächlich aber bezieht er sich auf die Beziehung zu den MA, und dort berücksichtigen Führungskräfte ,[...] Motive, Bedürfnisse, Triebe, (An-)Reize, Wünsche, Emotionen, etc., also psych(olog)ische Faktoren [...]“ (Sandner, 1990: 198). All diese Tätigkeiten sind - auf der Ebene der Personalführung - folglich Elemente in Sandners (1990: 197ff.) psychologischer Steuerung. Die Rolle ist im Diskurs von starker Bedeutung. Sie wird in 33 Dokumenten (62,3\%) angesprochen.

MitarbeiterInnen: Auch bei den MA ist dieser Rollenaspekt im Diskurs vorhanden, wenn auch deutlich schwächer ausgeprägt (vier Dokumente; 7,5\%). In erster Linie besteht die Aufgabe der MA als Beziehungsmanager darin, den FK Vertrauen entgegenzubringen. Aber auch gegenseitige Anerkennung und gegenseitiger Respekt sind Teil des Diskurses. Was im MA-Diskurs jedoch völlig fehlt, ist der Einsatz von Respekt, Wertschätzung und Anerkennung zur Motivation bzw. Steuerung der Führungskräfte. Im Gegenteil - das erwartete Vertrauen des MA in die FK wirkt motivierend auf die/den MA, nicht umgekehrt (bei den FK ist der Vertrauensvorschuss hingegen ein Steuerungsinstrument). Während die assoziierten Verben hier also dieselben sind, stellt der Rollenaspekt für FK und MA quasi zwei verschiedene Seiten einer Medaille dar.

\subsection{Der Akteur als Kommunikator}

Allgemein: In diesen Rollenaspekt fallen alle Tätigkeiten, welche Aspekte der Kommunikation und Information betreffen. Relevante Verben sind kommunizieren, äußern, offenlegen, informieren, argumentieren, thematisieren und ansprechen.

Führungskräfte: FK sind in diesem Aspekt dafür verantwortlich, dass ein konstanter Informationsfluss im Unternehmen gewährleistet ist. Sie sind zentraler Knotenpunkt für Kommunikation und Information. Kommuniziert wird dabei grundsätzlich über alles. Der Katalog an vorgeschlagenen Inhalten ist zu breit, als dass er an dieser Stelle im Detail diskutiert werden könnte. Anfangen von wichtigen Informationen über Leistungsbeurteilungen, Konflikte und Emotionen wird alles von FK angesprochen und thematisiert. Der Aspekt der FK als Kommunikator ist in 32 Dokumenten $(60,4 \%)$ Teil der Regelungsinhalte.

MitarbeiterInnen: Der Rollenaspekt der MA als Kommunikator ist einer der stärkeren im Diskurs. Er ist in dreizehn Dokumenten (24,5\%) enthalten. Ange- 
sprochen werden vor allem gegenseitige Information und ein gutes Gesprächsklima zwischen MA und FK. Spezialfälle stellen Äußerungen dar, welche der/dem MA ausdrücklich ein Recht einräumen, seine Meinung zu äußern. In einem Fall wird auch die Kommunikation von Problemen durch die/den MA an die nächsthöhere FK angesprochen. Der Unterschied zwischen FK und MA ist hier also relativ gering. Information verläuft sowohl top-down als auch bottomup. In der grundsätzlichen Kommunikation wird stets Gemeinsamkeit bzw. Reziprozität betont.

\subsection{Der den Rahmenbedingungen unterworfene Akteur}

Allgemein: Dieser Rollenaspekt thematisiert die grundsätzliche Unterworfenheit der Akteure unter verschiedene Rahmenbedingungen und Kontextfaktoren, seien es nun Standards, Gesetze, Grenzen der Leistungsfähigkeit (die eigenen und jene der anderen), etc. Die mit diesem Rollenaspekt verbundenen Verben sind sich an etwas ausrichten, sich an etwas halten, sich an etwas orientieren, auf etwas achten und etwas berücksichtigen.

Führungskräfte: Der Aspekt zeigt auf, dass auch FK in ihren Gestaltungsoptionen nicht völlig frei sind. Er wird in 16 Dokumenten (30,2\%) explizit thematisiert. Im Wesentlichen lassen sich für FK drei primäre Kontextfaktoren identifizieren, an denen sie sich zu orientieren haben: (a) organisationsexterne Faktoren wie Marktgegebenheiten und Kundenbedürfnisse, (b) allgemeine organisationsinterne Faktoren wie das Arbeitsklima und bestehende Vereinbarungen und (c) Wünsche, Bedürfnisse und Eigenschaften der MA. Die dritte Kategorie schlägt eine Brücke zum Aspekt der FK als Beziehungsmanager, indem sie eine logische Konsequenz der Empathie und der Wertschätzung darstellt, nämlich die Berücksichtigung der festgestellten Bedürfnisse im alltäglichen Handeln.

MitarbeiterInnen: Die Unterworfenheit der MA unter relevante Rahmenbedingungen wird in lediglich drei Dokumenten $(5,7 \%)$ thematisiert und betrifft zum einen die Kundenwünsche und zum anderen generelle Verhaltensregeln im Unternehmen. Dieses Ergebnis mag zunächst überraschen, ist doch anzunehmen, dass MA in der Organisation zumindest ebenso vielen Faktoren unterworfen sind wie FK. Eine mögliche Erklärung dafür ist, dass für MA die Unterworfenheit unter den Willen der FK bereits einen so hohen Grad an Selbstverständlichkeit besitzt, dass dieser in den Dokumenten nicht mehr explizit thematisiert werden muss. Auch kommt diese Form der Unterworfenheit in verschiedensten Ausprägungen ohnehin implizit in einem Großteil der Äußerungen zum Ausdruck. Es ist also nicht notwendig, diese Form der Unterordnung direkt anzusprechen. Auf der anderen Seite ist die Unterordnung von FK unter gewisse 
Faktoren und Bedingungen möglicherweise problematischer, da diese in ihrer Funktion als FK eine Unterordnung eher verlangen als vornehmen. Grenzen werden also explizit da gezogen, wo sie weniger üblich (und somit potentiell problematisch) sind. Die Unterordnung der MA hingegen scheint nicht problematisierungsbedürftig zu sein.

\subsection{Der Akteur als Kenner}

Allgemein: Der Kenner-Aspekt bezieht sich in erster Linie sowohl auf die kognitiven Fähigkeiten als auch auf das Wissen der einzelnen Akteure. Verben, welche mit diesem Aspekt einhergehen, sind (er)kennen und wissen. Im Gegensatz zum Aspekt des Analysten und Beurteilers wird hier aber nicht die aktive Suche nach Wissen und Informationen angesprochen, sondern eher der passive Besitz derselben. Die Bezeichnung der relevanten Verben als Handlungen ist deshalb in dieser Rolle nicht vollständig korrekt. Dennoch bildet das Verbcluster eine klar abgrenzbare Funktion von FK und MA, nämlich jene als ,Besitzer' und ,Hüter' organisationalen Wissens.

Führungskräfte: Der Rollenaspekt der Führungskräfte als Kenner verschiedener Aspekte der Organisation findet sich in 24 Dokumenten (45,3\%). Ähnlich wie beim Aspekt des Kommunikators ist es schwierig, einen Überblick über die vielfältigen Objekte des Wissens der FK zu geben. Diese beziehen sich aber im Wesentlichen auf diejenigen Kategorien, welche Führungskräfte auch zu berücksichtigen haben, nämlich (a) externe Faktoren wie Kunden, Markt, Chancen, Risiken, etc., (b) organisationale Faktoren wie Kultur, Arbeitsweise, Werte etc. und (c) interpersonelle Faktoren wie Wünsche, Bedürfnisse, Eigenschaften und Leistungen der MA und die Eigenheiten einer kulturell diversen Belegschaft. Die Führungskraft als Kenner geht damit logisch sowohl dem Beziehungsmanager als auch der Unterworfenheit unter gewisse Rahmenbedingungen voraus. Nur wer all jene Faktoren kennt, kann sie anerkennen, wertschätzen und letztlich berücksichtigen. Andererseits ist das Wissen um gewisse Sachverhalte auch entscheidend für den Entscheider, Korrektor und Problemlöser. Obwohl der Kenner also vergleichbar selten explizit thematisiert wird, ist er für die Wahrnehmung anderer Rollen im Diskurs zentral.

MitarbeiterInnen: Die MA als Kenner werden in neun Dokumenten (17\%) erwähnt. Das Wissen, welches von MA erwartet wird, ist aber deutlich stärker auf organisationsinterne Faktoren beschränkt, und zwar vor allem auf die eigene Tätigkeit. MA kennen in erster Linie ihre Aufgaben, ihre Verantwortung und die von ihnen erwartete Leistung. Darüber hinaus kennen sie aber auch größere $\mathrm{Zu}-$ sammenhänge im Unternehmen und die Bedürfnisse ihrer Kunden. MA besitzen 
also in erster Linie operatives Wissen, während von FK zusätzlich auch strategisches Wissen sowie Wissen über das eigene Personal verlangt wird. Eine bemerkbare ,Stille" stellt dabei dar, dass MA im Diskurs nichts über die FK wissen. Implizit wird dies allerdings insofern thematisiert, als MA den FK Feedback geben, wofür ein gewisses Wissen vonnöten ist.

\subsection{Der Akteur als Förderer und Unterstützer}

Allgemein: Der Rollenaspekt des Förderers bzw. Unterstützers hat zwei wesentliche Dimensionen. Auf der Sachebene bezieht er sich vor allem auf die Förderung eines bestimmten Umfelds bzw. spezifischer Arbeitsbedingungen und eines erwünschten Arbeitsklimas. Auf der Ebene der Mitarbeiterführung bezieht er sich auf Tätigkeiten im Bereich der Personalentwicklung und Personalbeurteilung. Verben, welche in diesem Zusammenhang vorkommen, sind helfen, unterstützen, fördern, sich für etwas oder jemanden Zeit nehmen und sich um jemanden kümmern.

Führungskräfte: Für die FK ist dies einer der zentralsten Rollenaspekte im Diskurs. Er wird in 38 Dokumenten $(71,7 \%)$ thematisiert und diskutiert. Gefördert und unterstützt werden dabei alle weiter oben genannten organisationalen und personellen Aspekte: FK sind für das Umfeld und die MA verantwortlich und unterstützen gewünschte Zustände. Im Bereich der Mitarbeiterführung sind zwei Ausprägungen der Rolle zu beachten: Zum einen unterstützen die FK ihre MA direkt, indem sie ihnen helfen, sich um sie kümmern und sich für sie Zeit nehmen; in einer anderen, stärker funktional orientierten Ausprägung aber unterstützen die FK vor allem die Weiterbildung (und damit die Qualifikation) sowie die Leistung ihrer MA. Diese beiden Teilaspekte sind in den einzelnen Äußerungen nicht immer klar zu trennen, sind aber analytisch distinkt und betreffen unterschiedliche Beziehungstypen.

MitarbeiterInnen: Der Aspekt des Förderers und Unterstützers bei den MA ist im Diskurs nur sehr schwach ausgeprägt (drei Dokumente; 5,7\%). Sie unterstützen einerseits die FK in deren Tätigkeit, setzen sich aber in einem Fall auch für neue Ideen im Unternehmen ein. Diese schwache Thematisierung des MA lässt sich aus einem eher geringen Pouvoir der MA im Diskurs insgesamt erklären. Um einen anderen Akteur (oder organisationale Ziele) zu fördern und zu unterstützen, benötigt man eine gewisse Basis an Ressourcen und formaler Autorität bzw. Legitimität. Diese wird den MA in den Dokumenten aber nicht zugesprochen. MA sind Empfänger von Unterstützung, sind aber selbst kaum in der Lage, diese zu geben. 


\subsection{Der Akteur als Gewährleister}

Allgemein: Der Rollenaspekt des Gewährleisters zeichnet sich dadurch aus, dass er dafür verantwortlich ist, dass gewisse Dinge ,so sind, wie sie sein sollen'. Damit stellt er die Kehrseite der Rolle des Problemlösers und Verhinderers dar. Während diese Rollenaspekte negative Zustände beenden bzw. verhindern, garantiert der Gewährleister positive Zustände. Typische assoziierte Verben sind sicherstellen, verantworten, gewährleisten, für etwas sorgen und sichern.

Führungskräfte: Für die FK stellt dieser Aspekt einen zentralen Aufgabenbereich dar. 48 Dokumente $(90,6 \%)$ beschäftigen sich explizit damit. Die Palette der zu gewährleistenden Dinge ist sehr breit, es lassen sich aber in Bezug auf die Mitarbeiterführung drei wesentliche Stränge unterscheiden. Zum einen werden zu gewährleistende Faktoren im Umfeld erwähnt, welche die MA zu gewissen Dingen befähigen (v.a. zur Übernahme von mehr Verantwortung und zu höherer Leistung). Dies ist der dominante Strang. Ebenfalls wichtig ist die Förderung der MitarbeiterInnen selbst bzw. die Sicherstellung einer gezielten Entwicklung dieser. Drittens werden auch Faktoren erwähnt, die für die MitarbeiterInnen im Sinne einer Bereitstellung gesichert und gewährleistet werden sollen, wie z.B. Ressourcen und Informationen. Diese Unterschiede werden in der Diskussion der (re-)konstruierten Beziehungstypen wieder aufgegriffen.

MitarbeiterInnen: Für die MA ist der Aspekt des Gewährleisters einer der dominantesten im Diskurs. Er wird in 17 Dokumenten (32,1\%) angesprochen. Dies erklärt sich daraus, dass der Rollenaspekt hier in erster Linie darin besteht, im eigenen Aufgabenbereich Verantwortung für eine angemessen Leistung zu übernehmen. Damit korreliert die Logik der Rolle stark mit jener des Kenners. MA kennen ihren Aufgabenbereich und übernehmen innerhalb der ihnen zugewiesenen Tätigkeiten Verantwortung für die Ergebnisse. Davon abgesehen sind MA aber auch (alleine oder gemeinsam mit den FK) für die eigentliche Entwicklung verantwortlich.

\subsection{Der Akteur als Schöpfer und Gestalter}

Allgemein: Vom Rollenaspekt des Gewährleisters zu trennen ist jener des Schöpfers bzw. Gestalters. In diesem Aspekt tritt das schöpferische Element in den Vordergrund. Es wird etwas (oder jemand) geschaffen, entwickelt, verstärkt, verbessert oder optimiert. Damit ist die Logik eine andere als jene der Sicherung und Gewährleistung.

Führungskräfte: Mit einer Nennung in 38 Dokumenten (71,7\%) gehört der Aspekt als Schöpfer und Gestalter ebenfalls zu den zentralen Rollen der FK im Diskurs. Erschaffen und gestaltet wird auch hier wieder sowohl im Bereich der 
Organisation (Produkte, Strategien, Prozesse, Strukturen, etc.) als auch im Bereich des Personals, und auch dort wieder sowohl im Arbeitsumfeld als auch am MA selbst (Kompetenzaufbau, Personalentwicklung).

MitarbeiterInnen: Auch bei den MA zählt dieser Aspekt mit einem Vorkommen in zehn Dokumenten (18,9\%) zu den breiter diskutierten Rollenaspekten. Zwei Stränge zeigen sich hier als besonders typisch: Zum einen ist die/der MA Mitgestalter organisationaler Aspekte, zum anderen engagiert sie/er sich aktiv für die Entwicklung der eigenen Fähigkeiten und Potentiale. In diesem Zusammenhang sind die beiden Rollenaspekte der MitarbeiterInnen als Gewährleister und als Gestalter sehr eng miteinander verknüpft und unterscheiden sich z.T. nur durch das verwendete Verb.

\subsection{Der Akteur als Verfüger und Nutzer}

Allgemein: Der Rollenaspekt des Akteurs als Verfüger oder Nutzer wird immer dann angesprochen, wenn ein Akteur eine Ressource nutzt oder einsetzt. Dies impliziert, dass der Akteur volle Verfügungsgewalt über die entsprechende Ressource hat.

Führungskräfte: Der Rollenaspekt der FK als Verfüger oder Nutzer wird in 24 Dokumenten $(45,3 \%)$ beschrieben. FK nutzen im Diskurs der Führungsgrundsätze vor allem folgende Dinge: kulturelle Unterschiede, Chancen, ihre eigenen Fähigkeiten und Kompetenzen sowie jene der MA. Weniger häufig werden auch andere Elemente (Fehler, Führungsinstrumente, etc.) genutzt. Eingesetzt werden vor allem MA, wobei diese in einigen Fällen sogar explizit als Ressource bezeichnet werden. Damit kommt eine klare Rollenverteilung zum Ausdruck, die explizit als Verfügungs-Beziehung definiert wird. Die/der MA steht der FK zur Verfügung, diese kann sie/ihn nach seinem Gutdünken einsetzen. Diese Beziehung entspricht in ihrem Wesen sehr stark Sandners (1990: 174ff.) direkter Steuerung.

MitarbeiterInnen: Auch MA können über gewisse Ressourcen verfügen und diese nutzen. Allerdings wird ein solcher Rollenaspekt nur in drei Dokumenten $(5,7 \%)$ explizit thematisiert. MA nutzen dabei vor allem Informationen und die eigenen Kenntnisse und Erfahrungen sowie das im Unternehmen vorhandene Wissen. MA verfügen hingegen nie über andere Akteure oder deren Eigenschaften.

\subsection{Der Akteur als Vertreter und Verfechter}

Allgemein: Der Rollenaspekt als Vertreter oder Verfechter impliziert, dass ein Akteur sich aktiv für eine Sache oder eine Person einsetzt und deren Standpunkt 
bzw. Interessen vertritt. In den Dokumenten wird allerdings nie geklärt, wem gegenüber diese Standpunkte vertreten werden. Die entsprechenden Äußerungen klären damit nur eine Beziehung zwischen Vertreter und Vertretenem, nicht aber der eigentlich notwendigen dritten Partei. Typische Verben, die mit diesem Aspekt assoziiert sind, beinhalten vertreten, repräsentieren, verfechten und sich einsetzen.

Führungskräfte: Die FK als Vertreter und Verfechter ist mit 17 Nennungen $(32,1 \%)$ ein nur vergleichsweise nur schwach verbreiteter Rollenaspekt. Auf der Sachebene vertreten FK vor allem die Unternehmensvision und die Unternehmensziele, aber auch ihre eigene Meinung und ihre Entscheidungen. Sie stehen zu ihrer Verantwortung. Auf der Ebene der Mitarbeiterführung verfechten die FK die Interessen und Standpunkte der MA und setzen sich für diese ein.

MitarbeiterInnen: Mit Nennungen in nur zwei Dokumenten $(3,8 \%)$ ist dieser Rollenaspekt bei den MA sogar noch peripherer als bei den FK. MA stehen vor allem zu eigenen Entscheidungen, setzen sich aber in einem Fall auch für die Interessen der FK ein (dies betrifft aber den gegenseitigen Einsatz für die Interessen des jeweils anderen).

\subsection{Der passiv-abhängige Akteur}

Allgemein: Dieser im manifesten Diskurs äußerst periphere Rollenaspekt diskutiert die grundsätzliche Abhängigkeit des Akteurs von verschiedenen Faktoren. Relevante Verben sind suchen, wünschen, benötigen und brauchen. Wichtig für diesen Aspekt ist, dass er explizit zwar nur sehr peripher auftritt (und zwar für beide Akteure), implizit aber von herausragender Bedeutung ist. Immer dann, wenn ein Akteur eingesetzt, gesteuert, gelenkt oder motiviert wird, aber auch, wenn ihm etwas gegeben oder zur Verfügung gestellt wird, nimmt er diesen Rollenaspekt ein. Der passiv-abhängige Akteur kann also als eine der großen ,Absenzen' bzw. ,Stillen' im expliziten Diskurs identifiziert werden. Seine Bedeutung tritt erst dann zu Tage, wenn man die verschiedenen Rollenaspekte zueinander in Beziehung setzt. Dann nämlich muss festgestellt werden, dass für die meisten Rollenaspekte kein expliziter Gegenpart in den Dokumenten erwähnt wird. Für jeden, der steuert, muss aber nun jemand gesteuert werden. Für jeden, der verfügt, gibt es jemanden, über den verfügt wird. All diese Aspekte werden mit dem Rollenaspekt des passiv-abhängigen Akteurs abgedeckt.

Führungskraft: Explizit wird die Abhängigkeit der FK nur in zwei Dokumenten $(3,8 \%)$ thematisiert. Dann sucht die Führungskraft nach Chancen, und sie braucht Informationen, um ihre Rolle auszufüllen. Implizit ist die Führungskraft aber zumeist der aktive Partner in einer Beziehung. Auch wenn dieses Ergebnis 
nicht mit Zahlen ausgedrückt werden kann, so ist dieser Rollenaspekt für die FK aber doch deutlich peripherer als für die MA. Dies wird in der Diskussion der Beziehungstypen deutlich.

MitarbeiterInnen: Auch bei den MA wird die Abhängigkeit nur in zwei Dokumenten $(3,8 \%)$ direkt diskutiert. Thematisiert wird ausschließlich das Bedürfnis der MA nach Information. Latent besitzen die MA aber viel mehr und viel breitere Bedürfnisse, so z.B. jenes nach Hilfe und Unterstützung, nach Entwicklung, nach Führung und Leitung usw. Sinngemäß gelten deshalb die Ausführungen zu den FK, mit dem Unterschied, dass diese impliziten Abhängigkeiten bei den MA wesentlich breiter und zentraler sind.

\subsubsection{Exklusive Rollenaspekte der FK}

\subsection{Die Führungskraft als Steuermann und Lenker}

Der Rollenaspekt der FK als Steuermann bzw. Lenker des organisationalen Geschehens betont den Charakter der Mitarbeiterführung als nicht delegierbare Führungsaufgabe (explizit festgehalten z.B. in CH17), da dieser Rollenaspekt für MA nicht verfügbar ist. Für die Charakterisierung der FK ist dieser Rollenaspekt im Diskurs allerdings quantitativ von großer Bedeutung (43 Dokumente; 81,1\%). Er kennt zumindest zwei verschiedene Verbgruppen - und damit Handlungsfelder - welche damit quasi Subaspekte konstituieren und in der Diskussion der Beziehungstypen zu verschiedenen Typen gehören. Die erste davon beinhaltet typische Verben wie steuern, lenken, leiten, beeinflussen, führen, zuteilen, fordern, beherrschen und jemanden zu etwas anhalten. Auf der Sachebene beziehen sich diese Verben auf die Unternehmensführung und verschiedene Aspekte derselben. Auf der hier vor allem interessierenden Ebene der Mitarbeiterführung konstituieren diese Tätigkeiten ebenso wie der Rollenaspekt als Verfüger und Nutzer in Sandners (1990: 174ff.) Terminologie vor allem eine direkte Steuerung. Die zweite Gruppe wird assoziiert mit typischen Verben wie ermuntern, ermutigen, motivieren und inspirieren. Diese Tätigkeiten deuten auf eine stärker psychologisch verankerte Form der Steuerung durch den Vorgesetzten (vgl. Sandner, 1990: 197ff.) hin. Die Gemeinsamkeit beider Gruppen liegt darin, dass die FK in dieser Rolle den MA dazu ,bewegt', gewisse Handlungen vorzunehmen. Damit wird mit der Rolle der Führungskraft als Steuermann und Leiter eine klassische Unterordnungsbeziehung impliziert.

\subsection{Die fokussierte Führungskraft}

Der Rollenaspekt der fokussierten Führungskraft stellt einen Spezialfall dar, der sich überhaupt nicht - oder zumindest nur am Rande und sehr mittelbar - mit 
dem Bereich der Mitarbeiterführung auseinandersetzt. Der zentrale Punkt dieses Aspekts besteht darin, dass die Führungskraft in der Lage ist zu fokussieren und zu priorisieren. Kriterium des erfolgreichen Führungshandelns ist hier die Fähigkeit, die richtigen (also organisational gewünschten) Themen und Faktoren in den Vordergrund bzw. Mittelpunkt zu rücken. Dies können z.B. die zu erfüllende Aufgabe (D04), ständige Verbesserung (D05), allgemein die Zukunft (A02), die Unternehmensziele (A13, CH16) oder die eigenen Stärken (A16) sein. Gelegentlich wird auch nur allgemein die Formulierung von Prioritäten gefordert (D02, A14). Dieser Rollenaspekt wird für FK in 12 Dokumenten (22,6\%) diskutiert, ist für MA aber nicht im Diskurs angelegt. Offensichtlich wird die Setzung von Prioritäten vor allem für FK als wichtig erachtet.

\subsection{Die verzichtende Führungskraft}

Schließlich taucht mit der verzichtenden FK noch ein stark peripherer Rollenaspekt (sechs Dokumente, 11,3\%) im Diskurs auf. Relevante Verben sind hier verzichten und verschiedene Formen des Unterlassens von Handlungen. Thematisch bezieht sich dieses Verzichten bzw. Unterlassen z.B. auf Ausreden (D04), auf Kontrolle und Macht im Rahmen der Delegation von Aufgaben (D16) und die Verschwendung verschiedener Ressourcen (D16, CH02, CH04). Der Rollenaspekt der verzichtenden FK thematisiert somit gemeinsam mit den Aspekten der passiv-abhängigen FK und der den Rahmenbedingungen unterworfenen FK die Grenzen der Macht und des Einflusses von FK. Anders als die beiden letztgenannten Rollenaspekte ist der Verzicht auf etwas eine freiwillige Handlung, welche auch anders hätte vorgenommen werden können. Der verzichtenden FK liegt deshalb ein stärker moralisch-ethisches Verständnis zugrunde. Sie unterwirft sich nicht notwendigerweise unabänderlichen Bedingungen, sondern akzeptiert freiwillig einen gewissen Wertekanon.

\subsubsection{Exklusiver Rollenaspekt der MA: der/die MitarbeiterIn als Berechtigter}

Im Diskurs existiert nur ein einziger Rollenaspekt, welchen nur MA einnehmen können, nicht aber FK, und dies ist jener der/des MA als Berechtigter. In gewisser Weise stellt dieser Rollenaspekt das Gegenstück zu jenem der Führungskraft als Steuermann und Leiter dar, und zwar in der Hinsicht, dass er den MA eine Art direkten Steuerungsmechanismus bietet. Die Verbkonstruktion, welche mit diesem Aspekt verbunden ist, ist in erster Linie ein Recht auf etwas haben, aber auch z.B. das Verb verdienen und vereinzelt fordern (können). Dieser Rollenaspekt erlaubt es somit den MA, von der FK etwas zu erhalten, da ihnen ein for- 
males Recht darauf zugesprochen wird. Insofern funktioniert diese Beziehung als eine Art umgekehrter legaler Autorität (vgl. Sandner, 1990: 118ff.). Innerhalb der von diesem Rollenaspekt klar definierten Grenzen hat ein/e MA die Möglichkeit, von der FK Handlungen und Haltungen einzufordern. Dies betrifft z.B. Fairness, Offenheit, Unterstützung, Anerkennung, begründete Kritik, Kooperation und Information (D08, A13), Orientierung (D08, A05), Rückmeldung (D11, D14, D16), Information (D14), konkrete Ziele (A13) und Respekt (CH13). Dieser Rollenaspekt wird im Diskurs in neun Dokumenten (17\%) thematisiert.

\subsubsection{Zusammenfassende Diskussion der Rollenaspekte}

Im bisherigen Verlauf der Arbeit wurden sozial verfügbare Rollen als typisierte Handlungsverrichtungen, welche einen Teil des Selbst im Sinne gesellschaftlich erreichbarer Typisierungen objektivieren (vgl. Berger \& Luckmann, 2000: 77), als besonders relevant für die Konstruktion der Selbstverständnisse organisationaler Akteure diskutiert. Die in den vorhergehenden Kapiteln diskutierten Rollenaspekte stellen also Identitäts- bzw. Identifikationsangebote in Form von Handlungsvorgaben dar, welche die Organisation den Akteuren zur Verfügung stellt. Durch den normativ-präskriptiven Charakter der Führungsgrundsätze bleiben die Rollenaspekte jedoch nicht auf der Ebene reiner Vorschläge, sondern wirken als Aufforderungen an die Akteure. Die Einnahme eines bestimmten Rollenaspekts bestimmt wiederum die Handlungsmöglichkeiten des Individuums und seine Position in Beziehung zu anderen Individuen (vgl. Keller, 2008: 238). Abbildung 12 zeigt noch einmal alle identifizierten Rollenaspekte mit den jeweiligen Häufigkeiten im Überblick.

Auf den ersten Blick fällt auf, dass die (re-)konstruierten Rollenaspekte von FK und MA sich nicht besonders stark unterscheiden. Zwar besitzen die meisten der geteilten Rollenaspekte eine leicht unterschiedliche Ausprägung zwischen den Akteuren, grundsätzlich beinhalten sie aber dieselben Handlungen. Ähnlich unmittelbar kommt aber zum Ausdruck, dass die Rollenaspekte der FK in den Dokumenten wesentlich häufiger und wesentlich differenzierter explizit behandelt werden als jene der MA. Die in Abbildung 12 dargestellten Unterschiede in der quantitativen Nennung der verschiedenen Rollenaspekte sind deshalb mit Vorsicht zu genießen. Ein direkter Vergleich zwischen FK und MA erweist sich als wenig zielführend, da Rollenaspekte der MA insgesamt sehr viel seltener expliziert werden. Die quantitative Verteilung der Rollenaspekte innerhalb eines Akteurstyps ist jedoch sehr wohl aussagekräftig.

Dabei zeigt sich für die FK, dass in erster Linie gewährleistende, gestaltende, lenkende und fördernde Tätigkeiten gefordert und beschrieben werden. Die 
FK ist aktiv, da sie einerseits für das Umfeld bzw. den Arbeitskontext verantwortlich ist (indirekte Führung), andererseits aber auch für die direkte, interaktionale Führung im Dialog mit den MA zu sorgen hat. Diese direkte Auseinandersetzung mit den MA thematisiert vor allem ein zweites Cluster an Tätigkeiten, nämlich die kontrollierenden, koordinierenden, empathischen und kommunikativen. Rollenaspekte, welche die FK jemandem oder etwas anderem unterordnen (Unterworfener, passiv-abhängiger Akteur, Verzichter), sind im Material am unteren Ende der Häufigkeitsskala anzutreffen. Für MA bietet sich ein leicht anderes Bild. Die quantitativ dominanten Rollenaspekte sind sehr einfach zu erkennen, während die Mehrzahl der Aspekte ein vergleichsweise peripheres Dasein führt. Gewährleistende Tätigkeiten sind auch bei MA am stärksten vertreten. Dies erklärt sich daraus, dass hier vor allem Tätigkeiten des Verantwortens und des für etwas Sorgens angesprochen werden. Verantwortlichkeiten aber werden für MA und FK gleichermaßen in den Dokumenten angesprochen. Dann folgen für MA hingegen direkt kommunikative und analytische bzw. bewertende Tätigkeiten. Gestalter, Kenner und Berechtigter schließlich sind die letzten drei Rollenaspekte, welche in einer größeren Häufigkeit (mehr als neun Nennungen) auftreten. Die restlichen Aspekte sind mit maximal fünf Nennungen in 53 Dokumenten eher nebensächlich.

Trotz dieser unterschiedlichen Verteilung der Häufigkeiten kann anhand der (Re-)konstruktion der dominanten Tätigkeiten der beiden zentralen Akteure eine überraschende Homogenität festgestellt werden. Die zu erwartende Zweiteilung des Tätigkeitsspektrums in planende und ausführende Arbeiten ist in den Führungsgrundsätzen so nicht vorzufinden. Dies ist zum einen mit dem hohen Abstraktionsgrad der Dokumente erklärbar. So werden die operativen Tätigkeitsbereiche der MA grundsätzlich aus den Dokumenten ausgeklammert. Wo sie angesprochen werden, werden sie zumindest nicht näher spezifiziert. Grundsätzlich aber bleiben die Tätigkeiten beider Akteure auf einem hohen Abstraktionsniveau und klammern das operative betriebliche Alltagsgeschäft (die eigentliche Leistungserstellung) konsequent aus. Ein zweiter Erklärungsansatz besteht darin, dass das Codieren und Zählen von Tätigkeiten notwendigerweise lediglich die explizierten, also die manifest dargestellten Rollenaspekte zutage fördern kann. Bei der Diskussion verschiedener Aspekte (z.B. Bereitsteller, passiv-abhängiger Akteur) wurde aber bereits erwähnt, dass diese u.U. auch nur implizit - nämlich als notwendiges Gegenstück anderer Rollenaspekte in der Führungsbeziehung auftreten können ${ }^{31}$. Die Identifikations-Aufforderung durch die Führungsgrund-

31 So benötigt z.B. der Rollenaspekt des Unterstützers immer jemanden, der unterstützt wird. Dieser ist aber zumeist untätig und deshalb einer Analyse typischer Tätigkeiten 
sätze entsteht demnach nicht nur in der Diskussion typischer Tätigkeiten, sondern sie ergibt sich auch aus dem konstruierten Verhältnis zum jeweils anderen. Erst in diesen Beziehungen kann ein Machtungleichgewicht - wie es eine Führungsbeziehung bereits impliziert - deutlich gemacht und charakterisiert werden. In einem nächsten Schritt wurden deshalb aus der Kombination der eben diskutierten Akteursrollen und den zuvor diskutierten Subjekt-Verb-ObjektStrukturen distinktive Beziehungstypen (re-)konstruiert, welche das Verhältnis zwischen FK und MA in unterschiedlicher Weise regeln.

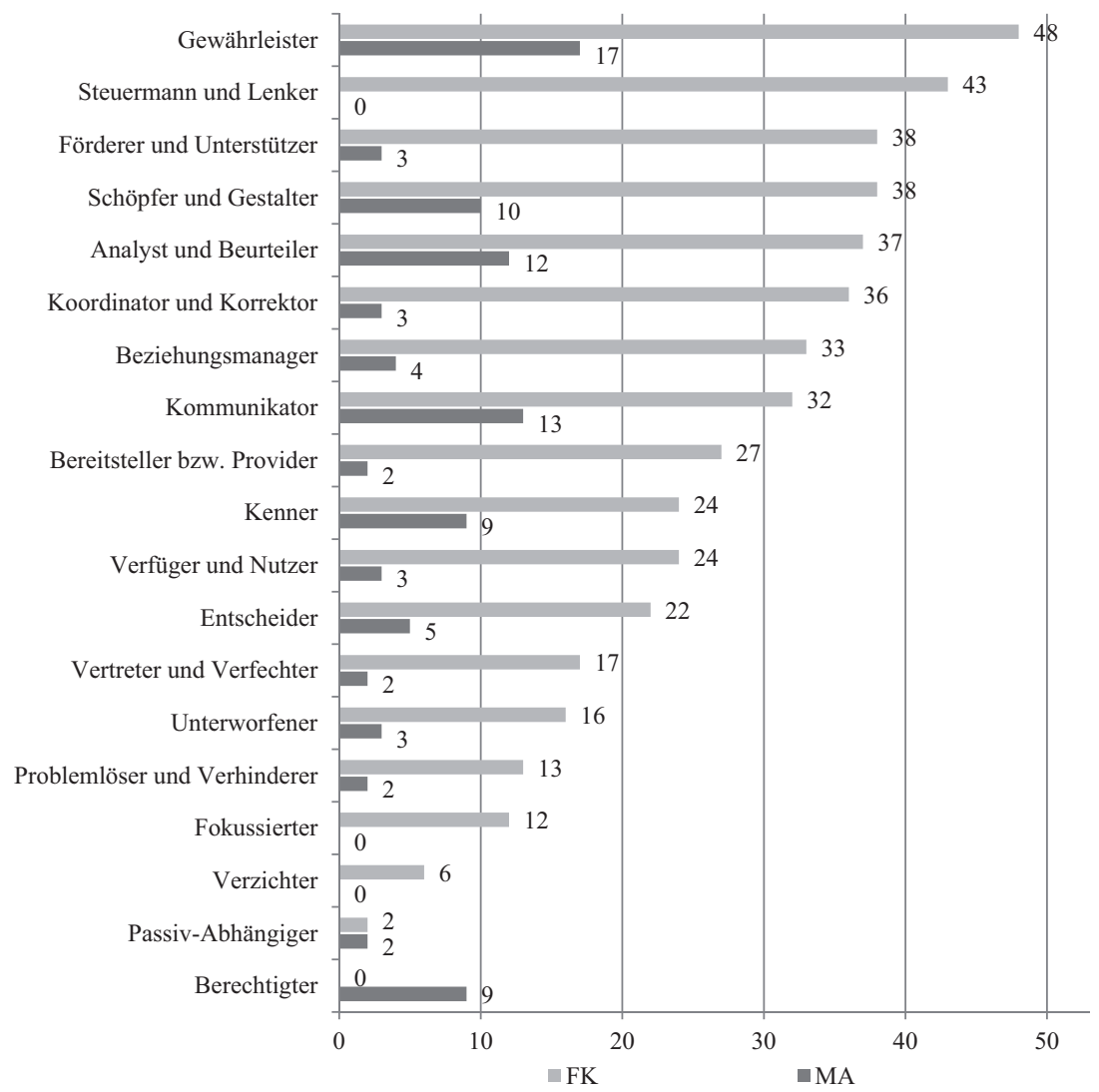

Abbildung 12: Rollenaspekte im Material

nicht zugänglich. Solche Rollenaspekte können nur durch die Juxtaposition der Akteure identifiziert werden. 


\subsubsection{Identifikation distinktiver Beziehungstypen}

In den Ausführungen zur Vorgehensweise wurde gezeigt, wie aus Tätigkeiten und Satzkonstruktionen 12 verschiedene Typen von Beziehungen zwischen FK und MA (re-)konstruiert wurden. Diese sollen hier kurz vorgestellt werden. Eine detaillierte Diskussion jedes einzelnen Typs findet sich in Kapitel 5.8. Da aber eine grundsätzliche Kenntnis der Beziehungstypen in der anschließenden Diskussion der im Material vorgefundenen besonderen Topoi notwendig ist, werden diese hier bereits in aller Kürze vorgestellt:

- Die Anspruchs-Beziehung basiert auf einer reziproken Logik von Fordern und Bereitstellen. Ein Akteur stellt legitime Ansprüche an den anderen, welchen dieser zu entsprechen hat. Explizite Rollenaspekte sind hier Steuermann bzw. Lenker und Berechtigter.

- Die Befähigungs-Beziehung betrifft in erster Linie Führung durch Kontextgestaltung und Verantwortungsübertragung. Die expliziten Rollenaspekte sind dementsprechend Schöpfer und Gestalter, Förderer und Unterstützer sowie Gewährleister.

- Die Bereitstellungs-Beziehung thematisiert ebenfalls Kontextgestaltung, allerdings fokussiert sie sich auf die Bereitstellung von Ressourcen, Werten, Emotionen und anderen Objekten. $\mathrm{Zu}$ einem gewissen Grad ist sie das Gegenstück zur Anspruchs-Beziehung. Zentral sind hier die Rollenaspekte des Bereitstellers bzw. Providers sowie des Schöpfers und Gestalters.

- Die Begrenzungs-Beziehung tritt dann auf, wenn ein Akteur einen Zustand verhindert bzw. einem anderen Akteur Grenzen setzt. Damit ist der zentrale Rollenaspekt jener des Verhinderers.

- Die Empathie-Beziehung thematisiert zusammen mit der UnterstützungsBeziehung dezidiert die zwischenmenschliche Komponente der Führung. Es geht hier vor allem darum, das Gegenüber zu verstehen, anzuerkennen und zu respektieren. Entsprechend sind die explizit genannten Rollenaspekte jene des Kenners, des Beziehungsmanagers und des den Rahmenbedingungen unterworfenen Akteurs, welcher die Wünsche und Bedürfnisse anderer berücksichtigt.

- Die Entwicklung-Beziehung beschreibt, wie ein Akteur den anderen entwickelt, verbessert oder gestaltet. Sie betrifft insofern vor allem den Bereich der Personalentwicklung und Weiterbildung. Diese Tätigkeiten entsprechen den expliziten Rollenaspekten des Schöpfers und Gestalters, des Förderers und des Gewährleisters.

- Die Integrations-Beziehung betrifft Handlungen, durch welche ein Akteur einen anderen inkludiert, einbezieht oder beteiligt. Da dies vor allem durch kommunikative und informative Akte geschieht, sind die zentralen expliziten 
Rollenaspekte in dieser Beziehung jene des Koordinators und des Kommunikators.

- Die Kontroll- und Bewertungsbeziehung hingegen thematisiert die klassischen Bereiche der Personalbeurteilung: Kontrolle, Beurteilung und Rückmeldung bzw. Korrektur. Explizite Rollenaspekte sind Analyst und Beurteiler, Korrektor und Koordinator.

- Die Motivations-Beziehung thematisiert eine Steuerung auf der Ebene von Anreizen. Ein Akteur motiviert, inspiriert oder begeistert den anderen. Diese Tätigkeiten fallen explizit in den Handlungsbereich des Steuermanns oder Leiters.

- Die direkte Steuerungs-Beziehung nutzt im Gegensatz zur MotivationsBeziehung die direkteren Steuerungsformen des Leitens, Lenkens, Führens, Festlegens und -setzens. Auch hier ist der dominante explizite Rollenaspekt jener des Steuermanns oder Leiters. Akteure treten aber auch als Gewährleister auf.

- Die Unterstützungs-Beziehung betrifft ähnlich wie die Empathie-Beziehung vor allem die zwischenmenschliche Ebene. Akteure unterstützen und helfen sich gegenseitig. Die expliziten Rollenaspekte sind Unterstützer und Förderer sowie Vertreter und Verfechter.

- Die Verfügungs-Beziehung schließlich stellt die direkteste Form des Eingriffs bei einem anderen Akteur dar. Der Akteur oder einzelne seiner Fähigkeiten und Eigenschaften werden von einem anderen Akteur genutzt oder eingesetzt. Der entsprechende explizite Rollenaspekt ist der des Verfügers oder Nutzers.

Damit wird deutlich, dass nicht alle der vorgestellten Rollenaspekte auch tatsächlich Relevanz für die Beziehungen zwischen FK und MA besitzen. Der Akteur als Entscheider bleibt ebenso unberücksichtigt wie die fokussierte und die verzichtende Führungskraft. Entscheidungen werden in der Organisation immer und überall getroffen, deshalb kann dieser Rollenaspekt keinem spezifischen Beziehungstypus zugeordnet werden. Ähnlich ist es mit der Setzung von Prioritäten. Die verzichtende Führungskraft könnte insofern der Befähiger-Beziehung zugeordnet werden, als das Verzichten auf Kontrolle im Rahmen der Delegation faktisch einer Erweiterung des Handlungsspielraums der MA gleichkommt. Dieser Teil des Rollenaspekts ist aber eher nebensächlich, da in erster Linie auf die Verschwendung von Ressourcen verzichtet wird, was keinen direkten Bezug zur FK-MA-Beziehung hat.

Nachdem nun aus Rollenaspekten und Akteursbezügen spezifische Beziehungstypen (re-) konstruiert worden sind, wird in einem nächsten Schritt analysiert, mittels welcher rhetorischer (und vor allem argumentativer) Mittel die 
Existenz und Ausgestaltung dieser Beziehungstypen in den Dokumenten legitimiert wird. Dazu werden zunächst die besonderen Topoi diskutiert, welche in den Dokumenten identifiziert wurden. Im Anschluss daran werden die Beziehungstypen noch einmal aufgegriffen und im Detail zusammen mit den sie legitimierenden und konstituierenden Argumentationsformen vorgestellt.

\section{7 (Re-)konstruktion der Argumentationsmuster}

\subsubsection{Vorbemerkungen}

In den folgenden Kapiteln werden die zentralen Ergebnisse der (Re-)konstruktion von relevanten Topoi im Diskurs der Führungsgrundsätze diskutiert. Topoi als ,taugliche Arsenale argumentativer Mittel, aus denen der Argumentierende die für seine Zwecke jeweils passenden hervorholt" (Ottmers, 2007: 89) so wurde argumentiert - erlauben eine Analyse der den propagierten Akteursbeziehungen innerhalb der Führungsgrundsätze zugrundeliegenden Argumentationsmustern und damit von Teilen des Weltverständnisses, innerhalb derer die (re-)konstruierten Beziehungstypen als sinnhaft gelten können. $\mathrm{Zu}$ diesem Zweck wäre die Rekonstruktion allgemeiner Topoi zu abstrakt. Die hier diskutierten Topoi sind also der Variante der besonderen Topoi zuzuordnen, welche bereits inhaltlich stärker spezifiziert und deshalb nur in einem bestimmten inhaltlichen Bereich verwendbar sind (vgl. Wengeler, 2003b: 183). Dadurch lassen sich die konkreten Spezifika der Argumentation in Führungsgrundsätzen viel stärker herausarbeiten.

Die Analyse ergab eine Gesamtheit von 67 distinktiven Topoi im Material. Diese Zahl macht deutlich, dass eine detaillierte Diskussion aller identifizierten Topoi im Detail im Rahmen dieser Arbeit eine Unmöglichkeit darstellt. Die Diskussion beschränkt sich deshalb darauf, die relevantesten Topoi ausführlich zu erläutern, während die anderen summarisch diskutiert werden. Der eingeschränkte Raum erlaubt es auch nicht, die typischen Enthymemstrukturen $\mathrm{zu}$ zeigen, in welchen die diskutierten Topoi jeweils auftreten. Dies wäre aber insofern auch nicht zielführend, da Topoi ja gerade dadurch charakterisiert sind, dass sie in verschiedenen Argumentationsstrukturen verwendet werden können, um z.T. sogar gegensätzliche Auffassungen zu vertreten (vgl. Ottmers, 2007: 90). Die Präsentation von stabilen Enthymemstrukturen könnte hingegen zur Annahme verleiten, die Topoi würden im Material vollkommen homogen verwendet werden, was nicht der Fall ist. Da es aber dennoch stärker und weniger stark verbreitete Enthymeme im Material gibt, findet der interessierte Leser eine volle Darstellung der im Material typischen Enthymeme im Anhang. 
Wie in den methodischen Ausführungen erläutert, wurde eine zweistufige Überprüfung der Relevanz von Topoi vorgenommen, indem (a) zunächst konzeptionell überprüft wurde, ob ein spezifischer Topos in plausibler Art und Weise überhaupt dazu geeignet ist, einen Beziehungstypus zu begründen, (b) anschließend anhand einer einfachen Zählung des ,Zusammenfallens' von Topos und Beziehungstypus in der Codierung des Materials die tatsächliche Verwendung des Topos im Zusammenhang mit dem Beziehungstypus geklärt wurde. Aufgrund dieser groben Relevanzanalyse können vier verschiedene Grade von Relevanz unterschieden werden. Topoi mit der höchsten Relevanz sind folglich jene, welche dazu geeignet sind und im Material auch dazu verwendet werden, spezifische Beziehungen zwischen den Akteuren argumentativ zu untermauern. Diese Topoi werden in der folgenden Diskussion als konstitutive Topoi bezeichnet, da sie die spezifischen Beziehungen im Material rhetorisch konstituieren. Eine zweite Klasse an Topoi ergänzt diese konstitutiven Topoi insofern, als sie zwar nicht die Beziehungen selbst, wohl aber die gewünschte Ausgestaltung dieser Beziehungen argumentiert. Diese beiden Kategorien werden im Folgenden näher diskutiert. Die dritte und vierte Klasse von Topoi, welche einen stets abnehmenden Bezug zu den Beziehungstypen aufweisen, werden nur kurz erwähnt. Allerdings werden auch sie in voller Länge im Anhang dargestellt.

Eine weitere Anmerkung betrifft den Charakter der (re-)konstruierten Topoi in ihrer Typik. Die Zugehörigkeit einer Äußerung zu einem typischen Topos bedingt immer eine interpretative Leistung der Forscherin/des Forschers. Aufgrund des systematischen Charakters der methodischen Vorgehensweise zur (Re-)konstruktion dieser Topoi kann die Konsistenz der Interpretation sichergestellt werden. Dennoch besteht jeder Topos aus einer gewissen Anzahl von Äußerungen, welche den Kern des Topos ausmachen, und aus einer ebenso großen Anzahl von Äußerungen, welche sich in der Peripherie des Topos befinden und deshalb u.U. mehreren Topoi angehören. Da die Dokumente oft Aussagen auf verschiedenen Abstraktionsebenen treffen, sind auch die (re-)konstruierten Topoi in der Abstraktionsebene nicht homogen.

\subsubsection{Die konstitutiven Topoi}

Die in diesem Kapitel vorgestellten Topoi werden insofern als konstitutiv bezeichnet, als sie in den Führungsgrundsätzen dazu verwendet werden, einzelne oder mehrere Beziehungstypen argumentativ zu legitimieren und zu stützen. Während andere Topoi spezifische Ausgestaltungen dieser Beziehungen nahe legen, ist es eben die Leistung der konstitutiven Topoi, die Beziehung selbst als sinnvoll und gerechtfertigt erscheinen zu lassen. Aufgrund der ,Offenheit ${ }^{\star}$ von 
Topoi (vgl. Ottmers, 2007: 90) und ihrer Eigenschaft, für mehrere verschiedene Argumentationen nützlich zu sein, kommt es im untersuchten Material vor, dass ein Topos für zwei oder sogar drei verschiedene Beziehungstypen konstitutiv ist. Dies bedeutet, dass diese Beziehungstypen einer ähnlichen argumentativen Logik folgen. Diese ist jedoch nicht identisch, da eben Topoi für verschiedene, manchmal sogar gegensätzliche Argumentationen eingesetzt werden können. Durch die (Re-)konstruktion der konstitutiven Topoi können aber nicht nur die (oft impliziten) Gründe für das Bestehen der spezifischen Beziehung identifiziert werden, sondern sie geben außerdem auch Hinweise auf das implizit unterstellte Steuerungsverständnis der Textautoren. Dieser Aspekt wird in der integrierten Diskussion der Beziehungstypen näher erläutert werden. Zunächst werden nun abschließend die konstitutiven Topoi im Material diskutiert.

\subsubsection{Der Topos von der Bedeutung des Arbeitsklimas und Ar- beitsumfelds}

Die Leistungsbereitschaft und-fähigkeit von Menschen hängt stark von den Rahmenbedingungen und Kontextfaktoren ab, unter welchen sie diese Leistung zu erbringen haben.

In der Gestaltung der Rahmenbedingungen sind grundsätzlich sowohl FK als auch MA tätig, wenn auch in unterschiedlichem Ausmaß (siehe die Diskussionen zu den Rollen von Schöpfer und Gestalter, Förderer und Unterstützer bzw. Gewährleister in Kapitel 5.6.2). Das Arbeitsumfeld, welches in diesem Topos angesprochen wird, betrifft sowohl Effizienzaspekte (D02), räumliche Arbeitsbedingungen (A10) oder Bürokratieabbau (CH05), aber auch Aspekte der interaktionalen Zusammenarbeit und des ,guten' Umgangs miteinander (D04, D07, A08).

Durch die Betonung von Rahmenbedingungen begründet der Topos von der Bedeutung des Arbeitsklimas und Arbeitsumfelds einen stark indirekten Steuerungsbegriff (vgl. Sandner, 1990: 181ff.). Leistung wird nicht durch direkte Aufforderung gesteigert, sondern durch die mittelbare Steuerung über leistungsfördernde Arbeitsbedingungen. Der Topos von der Bedeutung des Arbeitsklimas und Arbeitsumfelds ist für drei unterschiedliche, wenn auch verwandte Beziehungstypen konstitutiv: Zum einen wird Kontextgestaltung im Sinne von ,Schaffung und Gewährleistung leistungsfördernder Arbeitsbedingungen“ als wichtige Führungsaufgabe betont. Der Topos von der Bedeutung des Arbeitsklimas und Arbeitsumfelds ist somit konstitutiv für die Befähiger-Beziehung ${ }^{32}$. Der zweite Aspekt, nämlich die Ressourcenzuteilung von den FK an die MA macht den To-

32 Alle in der Diskussion genannten Bezeichnungen für Beziehungstypen beziehen sich auf jene Beziehungen, die in Kapitel 5.8 diskutiert werden. 
pos aber auch konstitutiv für einen zweiten Beziehungstypus, nämlich die $B e$ reitstellungs-Beziehung. Der dritte Aspekt dieses Topos, nämlich die Frage des guten Umgangs miteinander, welche vor allem den Umgang der FK mit den MA betrifft, ist konstitutiv für einen verwandten, aber von der Beziehungs- und Handlungslogik her unterschiedlichen Beziehungstypus, nämlich jenen der $U n$ terstützungs-Beziehung.

\subsubsection{Der Topos von der Bedeutung des Einzelnen}

Jeder Einzelne im Unternehmen, sei das Aufgabengebiet auch noch so klein, leistet einen wesentlichen Beitrag (positiv oder negativ) zum Unternehmenserfolg.

Dieser Topos wird in den Dokumenten immer dann evoziert, wenn von der Leistung jedes Einzelnen (D02) oder vom persönlichen Beitrag (D04, D05) die Rede ist. Die zugrundeliegende Argumentationslogik besteht darin, dass dieser individuelle Beitrag erkannt und gefördert werden muss (D05). Deshalb muss auch auf den Einzelnen eingegangen werden (D10, CH04) und er muss individuell behandelt werden (A05). Im Gegenzug wird aber auch von jedem Einzelnen erwartet, dass er sich entsprechend für das Unternehmen einsetzt (A05, CH 16).

Die Bedeutung dieses Topos für die Beziehung zwischen FK und MA ist unschwer erkennbar. Die Gültigkeit dieses Argumentationsmusters erfordert, dass die MA in der Führungsbeziehung als Individuen mit all ihren spezifischen Wünschen, Ängsten und Bedürfnissen erkannt, respektiert und wertgeschätzt werden. Damit ist der Topos von der Bedeutung des Einzelnen konstitutiv für die Empathie-Beziehung. Die stark individualistische Ausrichtung des Topos führt außerdem dazu, dass auch Führung und Förderung individuell und situativ gestaltet werden müssen. Sie führt aber auch dazu, dass der verlangte Beitrag der MA variieren kann, je nach Leistungsfähigkeit des Individuums. Damit verunmöglicht der Topos in seiner harten Auslegung die Möglichkeit einer erfolgreichen kollektiven Steuerung. Jede/r MA bzw. jedes Organisationsmitglied muss individuell behandelt, geführt und gesteuert werden.

\subsubsection{Der Topos von der Einheit von Kompetenz und Verantwor- tung}

Die Leistung einer Person ist dann am höchsten, wenn sie an ihrem Arbeitsplatz weder unternoch überfordert ist. Der höchste Grad an Leistung wird dort erreicht, wo ein hoher ,Fit * zwischen Person und Arbeitsplatz bzw. Verantwortung besteht.

Mit seiner Betonung der Kontextabhängigkeit von Leistung stellt der Topos von der Einheit von Kompetenz und Verantwortung argumentationslogisch eigentlich eine spezifischere Variante des Topos von der Bedeutung des Arbeitsklimas 
und Arbeitsumfelds dar. Er wird aber im Material sehr oft in dieser spezifischen Form verwendet, und es wird noch zu zeigen sein, dass er in dieser Form andere Beziehungstypen zu konstituieren in der Lage ist als in seiner allgemeineren Form. Inhaltlich ist der Topos im Diskurs relativ wenig spezifiziert. Bis auf die Betonung, dass jeder im Unternehmen dort eingesetzt werden soll, wo er die höchste Kompetenz besitzt, wird wenig über Details gesagt. Gesichert werden sollen dadurch z.B. Qualität und Professionalität (D02), Selbstverantwortung und Eigenständigkeit (D03, A10), die Konzentration auf Stärken (A15) und optimale Leistung (CH13).

Auch dieser Topos ist durch seine Offenheit konstitutiv für zwei verschiedene Beziehungstypen: Zum einen bietet er im Rahmen der Verfügungs-Beziehung FK eine Rechtfertigung dafür, MA an entsprechender Stelle im Unternehmen einzusetzen. Der Topos kann also für die Legitimation einer direkten Verfügungsgewalt der FK über die MA (und damit einer direkten Steuerung) verwendet werden. Im Typ der Begrenzungs-Beziehung hingegen begründet er das Recht der MA, Einmischungsversuche der FK in ihre Verantwortungsbereiche zurückzuweisen, da sie dort selbst die höchste Kompetenz besitzen. Eine Machtausübung des FK über die MA wird argumentativ unterbunden. Die beiden Argumentationen müssen sich dabei nicht unbedingt widersprechen. Die entscheidende Frage lautet, wo das Wissen über den besten Einsatz der MA verortet wird, bei den MA selbst oder bei den FK.

\subsubsection{Der Topos vom Fordern und Fördern}

Jeder im Unternehmen besitzt Rechte und Pflichten gleichermaßen. Diese Rechte und Pflichten müssen deshalb im Unternehmen in einem angemessenen Verhältnis zueinander stehen.

Der Topos vom Fordern und Fördern trägt in seinem Kern die Idee der Reziprozität mit sich. Die grundlegende Argumentation dahinter ist jene, dass jemand, der viel fordert, auch viel geben muss, um ein Anreizgleichgewicht zu schaffen. Umgekehrt lässt sich natürlich ebenfalls argumentieren: Wer dem anderen eine starke Förderung zukommen lässt, hat ein Recht darauf, auch entsprechend mehr zu fordern. Die Verbindung von Fordern und Fördern stellt im Diskurs der Führungsgrundsätze quasi eine rhetorische Figur dar. Sie wird in den meisten Fällen nicht weiter expliziert. Ausgeführt wird hingegen sporadisch, was genau gefordert und gefördert werden soll, nämlich z.B. höchste Leistung (D04), Bereitschaft zur Veränderung (D15), Selbständigkeit und Eigenverantwortung (A07), die eigene Entwicklung (A11) sowie Engagement und Initiative (CH07).

Der Topos legitimiert durch seine Schlussregel damit in erster Linie Ansprüche, nämlich sowohl jene, die durch das Fordern entstehen als auch jene, welcher im Fördern begründet sind. Dies ist auch der inhärente Steuerungsmecha- 
nismus, welche durch diesen Topos argumentiert wird: eine Steuerung über das reziproke Verhältnis von Rechten und Pflichten. Der Topos vom Fordern und Fördern ist somit auch konstitutiv für die Anspruchs-Beziehung, welche genau diese Rechte und Pflichten der Akteure untereinander zum Thema macht.

\subsubsection{Der Topos von der Notwendigkeit der Kontrolle}

Vertrauen hat seine Grenzen. Nur eine effiziente Kontrolle sichert die Vermeidung von Fehlern und Abweichungen.

Dieser Topos legitimiert in erster Linie verschiedene Prozesse der Kontrolle und Bewertung. Dies bezieht sich sowohl auf Ergebnisse wie Zielerreichung (D06, A04), Maßnahmenerfolg (D10) und Dienstleistungsqualität (A15) als auch auf das Verhalten der Organisationsmitglieder im Sinne von Leistungsprozessen und Leistungserbringung (CH13, D05).

Steuerungslogisch entspricht dieser Topos der „Theory X“ von McGregor (vgl. z.B. Schreyögg, 2003: 225ff.). Die Kontrolle als „notwendige Voraussetzung für eine effektive Arbeit" (A04) entspringt einem Menschenbild, welches das Individuum als grundsätzlich eher arbeitsscheu charakterisiert. Durch rigorose Kontrolle kann dieser Tendenz entgegengewirkt werden. Die Kontrolle kann aber auf verschiedene Art und Weise geschehen. Von der klassischen topdown Kontrolle durch die FK (D10) müssen die Selbstkontrolle der MA (CH10) bzw. der FK (D18) und die gemeinsame Kontrolle durch FK und MA (CH14) unterschieden werden. Diese Unterschiede werden in der Kontroll- und Bewertungsbeziehung diskutiert, für welche dieser Topos konstitutiv ist.

\subsubsection{Der Topos von der Notwendigkeit von Orientierung und Si- cherheit}

Effiziente Leistungserstellung ist nur dann möglich, wenn ein gemeinsamer Weg und geteilte Verständnisse existieren, damit grundlegende (Routine)Fragen nicht mehr in jedem Einzelfall geklärt werden müssen.

Diese Herstellung von Orientierung und Sicherheit - und damit eines gemeinsamen Handlungsrahmens - geschieht in den Dokumenten grundsätzlich in drei verschiedenen Varianten: (a) durch direkte Kommunikation von Vorgaben an die MA durch die FK (D01, A08) bzw. durch die Vermittlung von expliziten Werten und Regeln (D02, A05), (b) durch die Gestaltung von Rahmenbedingungen (A03) und (c) durch das Vorleben und die Vorbildwirkung gewünschter Verhaltensweisen durch die FK (D04, A02), wobei sich diese drei Varianten zum Teil überschneiden können. 
Orientierung und Sicherheit kann somit über verschiedene Steuerungsmechanismen hergestellt werden. Variante (a) entspricht einer Mischform aus direkter und bürokratischer Steuerung. Variante (b) beinhaltet Elemente technologischer und bürokratischer Steuerung, und Variante (c) schließlich verwendet stärker psychologische Steuerungsmechanismen (vgl. Sandner, 1990). Dementsprechend taucht dieser Topos auch in verschiedenen Beziehungstypen auf. Am stärksten ausgeprägt ist im Diskurs Variante (a). Damit ist der Topos konstitutiv für die Steuerungs-Beziehung, in welcher vor allem direkte Steuerungsformen zur Anwendung kommen. Von eher untergeordneter Bedeutung ist der Topos in der Motivations-Beziehung (durch den Vorbild-Aspekt) und in der BefähigungsBeziehung (durch die Gestaltung von Rahmenbedingungen).

\subsubsection{Der Topos vom Stillstand als Tod}

Da sich das Umfeld (und damit die Anforderungen an das Unternehmen und den Einzelnen) ständig ändert, muss jeder konstant vorwärts gehen und sich verbessern bzw. anpassen. Wer sich nicht mit bewegt, der bleibt auf der Strecke.

In diesem Topos wird der Imperativ der ständigen Verbesserung betont. Stillstand ist im Wesentlichen nicht möglich, denn er führt dazu, dass man in Wahrheit zurückbleibt. Niemand darf sich auf seinen Lorbeeren ausruhen. Ständige Weiterentwicklung und -bildung sind nötig und gefordert. Jeder Aspekt des Tuns und Handelns (D05) ist dieser Logik unterworfen. Verbessert und weiterentwickelt werden auf der einen Seite Produkte und Dienstleistungen (D02), die Position des Unternehmens im Markt (CH03), Problemlösungen und Abläufe (A05) sowie allgemein Innovationen (D02, D03, CH04), aber auch die Unternehmenskultur (A03). Zentral ist selbstverständlich auch die Weiterbildung des Personals, und zwar sowohl der FK (A03, CH14) als auch der MA (D01, A06).

Das Steuerungspotential dieses Topos besteht in erster Linie darin, dass die betroffenen Akteure selbst aktiv werden müssen, um ,gut genug“ zu bleiben. Der Topos aktiviert damit quasi Überlebensinstinkte, welchen das Individuum folgen muss, um nicht hinter andere zurückzufallen. Die Logik des Topos ist jene des Marktes, in welchem die Konkurrenz nicht schläft. Diese Logik wird auch ins Innenverhältnis übertragen. Wer nicht ständig an der Verbesserung der eigenen Person und der eigenen Fähigkeiten arbeitet, ist irgendwann nicht mehr gut genug. Auf der einen Seite wird die Kontrolle und Steuerung also quasi internalisiert bzw. in das Individuum selbst verlagert. Sie liegt im Interesse jedes Einzelnen. Auf der anderen Seite wird damit aber auch ein Unterordnungsverhältnis argumentiert. Die FK als Kenner weiß von den Stärken und Schwächen der MA. Folglich ist sie in ihrer Funktion als Gestalter dazu legitimiert, die 
Entwicklung dieser voranzutreiben. Der Topos vom Stillstand als Tod konstituiert und legitimiert somit die Entwicklungs-Beziehung.

\subsubsection{Der Topos von der Transparenz als Allheilmittel}

Sowohl effizientes Arbeiten als auch ein gutes Arbeitsklima sind nur dann möglich, wenn alle relevanten Faktoren bekannt und allen zugänglich sind. Verschleierung verunmöglicht optimale Lösungen.

Der Topos von der Transparenz als Allheilmittel verlangt von allen Akteuren die grundsätzliche Bereitschaft zur Offenlegung und Kommunikation von Feedback (D02, D14), insbesondere auch Kritik (D14, A04, CH07), allgemeine Meinungen (D02, D09), Erwartungen und Anforderungen (D06, A12, CH02) sowie Beurteilungskriterien (D09, D10), Entscheidungen (D16, A12), Verantwortungen (A02), Veränderungen (D10), Zielen (D18, A10, A14) und Gesamtzusammenhängen (D19, CH06). Offengelegt werden sollen aber auch eher heikle und persönliche Aspekte wie Empfindungen (D16), Konflikte (A05, A13), Probleme und Missstände (CH01, CH02) und (eigene) Fehler (D16, A05). Diese Transparenz im Handeln und Denken führt zu zwei positiven Effekten: Zum einen wird ein besseres Arbeitsklima erreicht, das durch Ehrlichkeit und Offenheit geprägt ist (D01), zum anderen aber wird auch die Effizienz der Arbeit erhöht (D14).

Auch dieser Topos entfaltet Steuerungswirkung in erster Linie über die Argumentation, dass die Einhaltung des geforderten Verhaltens im eigenen Interesse des Akteurs liege. Zwar liegt die erhöhte Arbeitseffizienz in erster Linie im Interesse des Unternehmens, aber das bessere Arbeitsklima nutzt allen. Transparenz wird daher auch als ,Service“ vor allem an die MA kommuniziert, da diese dadurch einen besseren Überblick über das Unternehmen, über die an sie gestellten Anforderungen, die eigene Leistung und die Zufriedenheit der FK mit dieser Leistung bekommen. In diesem Sinne wird sehr subtil eine Unterordnungsaufforderung kommuniziert. Die FK ist ein Kenner, sie besitzt die nötigen Informationen. Diese stellt sie den MA zur Verfügung, damit sie sich wohler fühlen und eine bessere Leistung erbringen können (welche ihnen im Rahmen der Leistungsbeurteilung wieder zugutekommt). In einigen Fällen funktioniert die Argumentation aber auch umgekehrt: Die MA besitzen Informationen, welche die FK benötigt. Diese sind ihr von den MA zur Verfügung zu stellen. Da durch die Transparenz bis dahin ,unwissende ${ }^{6}$ Akteure quasi ins Geschehen ,integriert" werden, ist der Topos von der Transparenz als Allheilmittel konstitutiv für die Integrations-Beziehung. 


\subsubsection{Der Topos vom Zusammenhang zwischen Motivation und Leistung}

Motivierte MitarbeiterInnen sind leistungsbereite MitarbeiterInnen.

Diese seit der Human-Relations-Bewegung etablierte Grundfrage der verhaltenswissenschaftlich orientierten Organisationsforschung (vgl. Schreyögg, 2003: 217ff.) - ob nämlich ein direkter und kausaler Zusammenhang zwischen Motivation und Leistungsgrad besteht - wird im Diskurs der Führungsgrundsätze als sehr unproblematisch gesehen. Motivation und Leistung werden entweder in einem Atemzug erwähnt (D18) oder der Zusammenhang wird explizit behauptet (A04). Zumeist aber wird die Motivation der MitarbeiterInnen ohne konkrete Angabe von Gründen in einer Logik der Effizienz verordnet (A14, CH05).

Die Steuerungslogik hinter diesem Topos liegt folglich in einer Steuerung durch verschiedene Anreizsysteme und folgt der Argumentation von Sandners (1990:197ff.) psychologischer Steuerung. Durch diese Anreize werden die Interessen des Individuums an jene der Organisation angeglichen. Das diesem Topos im Diskurs zugrundeliegende Menschenbild wird dann deutlich, wenn man die Akteursbeziehungen, welche durch diesen Topos begründet werden, näher betrachtet (der Topos ist konstitutiv für die Motivations-Beziehung). Motivationsbedürftig sind nämlich ausschließlich die MA. Diese werden in der Mehrzahl der Fälle durch die FK motiviert; in einigen Äußerungen motivieren sie sich aber auch selbst. Eine relevante ,Stille“ im Diskurs ist jedoch die Frage der Motivation der FK. Diese wird grundsätzlich einfach unterstellt und niemals thematisiert.

Damit ist die Diskussion jener Topoi, welche über die von ihnen gestützten Argumente spezifische Formen der Interaktion zwischen FK und MA konstituieren und legitimieren abgeschlossen. Im nächsten Kapitel werden nun jene Topoi kurz diskutiert, welche ebenfalls einen wichtigen Beitrag zum besseren Verständnis der vorgeschlagenen Beziehungsformen zwischen FK und MA leisten, diese aber stärker in einzelnen Details und nicht wie die konstitutiven Topoi in ihrem Kern betreffen.

\subsubsection{Die ergänzenden Topoi}

Die nun folgenden Topoi sind nicht konstitutiv für einzelne Beziehungstypen in dem Sinne, als sie die Existenz und Sinnhaftigkeit der spezifischen Beziehungsform per se begründen. Sie stützen allerdings verschiedene Aspekte der spezifischen Ausgestaltung einzelner Beziehungstypen. Aus diesem Grunde werden sie einigermaßen detailliert - wenn auch nicht so ausführlich wie die konstitutiven 
Topoi - hier diskutiert. Auch für diese Kategorie von Topoi findet sich eine Darstellung der jeweils typischen Enthymemstrukturen im Anhang.

\subsubsection{Der Topos von der Bedeutung der Kommunikation}

Gute und umfassende Kommunikation steigert sowohl das Arbeitsklima als auch die Effizienz der Arbeit.

Der Topos von der Bedeutung der Kommunikation ist im Diskurs sehr häufig anzutreffen und spiegelt somit die Dominanz des Themas der Kommunikation zwischen FK und MA in der wissenschaftlichen Literatur wider (vgl. z.B. Modaff, DeWine \& Butler, 2008: 196ff.). Betont wird sowohl die Kommunikation als interaktionaler Prozess (D05, A05) als auch die Fähigkeit zur Kommunikation, sowohl bei FK (D03, A12) als auch bei MA (D05). Die Nähe zum Topos der Transparenz als Allheilmittel ist unverkennbar. Auch der Topos von der Bedeutung der Kommunikation betont den doppelten Nutzen seines Gegenstandes, nämlich sowohl in einer Verbesserung der Effizienz als auch der Beziehung. Kommunikation ist im Diskurs eine Form der Herstellung von Transparenz, weshalb die beiden Topoi auch argumentationslogisch aufeinander aufbauen. Dementsprechend ist der Topos von der Bedeutung der Kommunikation auch vor allem mit der Integrations-Beziehung verknüpft, welche durch den Topos von der Transparenz als Allheilmittel konstituiert wird. Diese Transparenz wird durch Kommunikation erreicht.

\subsubsection{Der Topos von der Bedeutung der MitarbeiterInnen}

Die MitarbeiterInnen sind der wichtigste Erfolgsfaktor des Unternehmens.

Dieser Topos erhebt einen stark normativen Anspruch auf den Wert des Menschen (ähnlich dem Topos von der Bedeutung des Einzelnen). Er ist im Gegensatz zu diesem allerdings in einer Hinsicht deutlich spezifischer ausgerichtet (indem er nur eine bestimmte Akteursgruppe, nämlich die MA, inkludiert), in anderer Hinsicht aber auch deutlich allgemeiner formuliert (indem er sich auf die MA als Kollektiv und nicht als Einzelpersonen bezieht). Die MA sind die wichtigste und wertvollste Ressource (D05) bzw. das stärkste Aktivum (D05), ein Vorteil im Wettbewerb (D07), eine Investition in die Zukunft (D12), Voraussetzung für den Erfolg (A04) bzw. „Schlüssel zum Unternehmenserfolg“ (A09). Dadurch wird die instrumentelle Logik dieses Topos klar. Gemäß Cheney (1983: 148ff.) ist davon auszugehen, dass dieser Topos in erster Linie die Identifikation der MA mit der Organisation steigern will, indem diese ihre Wertschätzung gegenüber ihrer Belegschaft explizit zum Ausdruck bringt. Entsprechend stützt der Topos von der Bedeutung der MitarbeiterInnen im Diskurs auch zwei 
Beziehungstypen, welche den Wert bzw. die Rechte der MA betonen, nämlich einerseits die Anspruchs-Beziehung, wo er die spezifischen Ansprüche der MA gegenüber den FK begründet (nicht aber die Ansprüche der FK gegenüber den MA) und andererseits die Empathie-Beziehung, wo er den konstitutiven Topos von der Bedeutung des Einzelnen näher spezifiziert.

\subsubsection{Der Topos ,Gemeinsam sind wir stärker ${ }^{6}$}

Ziele werden leichter erreicht, wenn alle, an einem Strang ziehen ' und Ressourcen gebündelt werden können.

Dieser Topos argumentiert spezifische Beziehungsformen sowohl auf der horizontalen als auch auf der vertikalen Hierarchieebene. Horizontal kommt dies vor allem in einer starken Befürwortung von Teamarbeit zum Ausdruck (D02, D05, A05, CH03). Vertikal betrifft der Topos vor allem die Zusammenarbeit zwischen FK und MA, und dort spezifisch eine Beteiligung der MA an der Entscheidungsfindung (D10, D17, A01, CH02).

Während die Teamwork-Thematik quantitativ den wesentlich größeren Teil der Äußerungen innerhalb des Topos stellt, ist für die gegenständliche Untersuchung die Beteiligung der MA von höherer Relevanz. Der Topos hängt durch die Thematisierung von Teamentwicklung und Förderung von Teamstrukturen als Möglichkeit der Vermeidung von Stillstand stark mit der EntwicklungsBeziehung und über die Forderung nach Beteiligung der MitarbeiterInnen mit der Integrations-Beziehung zusammen.

\subsubsection{Der Topos von der fairen Beurteilung und Belohnung}

Eine Beurteilung ist nur dann sinnvoll, wenn sie fair und objektiv geschieht, da nur dann sinnvolle Maßnahmen abgeleitet werden können und die MitarbeiterInnen die Beurteilung nur dann als gerecht empfinden und akzeptieren werden.

Auch in diesem Topos findet sich eine doppelte Argumentationsstruktur, die sich aber letzten Endes wieder verbindet. Durch eine objektive - und damit faire - Beurteilung werden sowohl Effizienz wie auch die empfundene Gerechtigkeit gesteigert, wobei eine höher empfundene Gerechtigkeit letzten Endes wieder zu höherer Effizienz (durch gesteigerte Akzeptanz von Korrekturmaßnahmen) führt. Die Dokumente bleiben in der konkreten Konstruktion von Argumentationen in diesem Bereich sehr vage, wodurch unentscheidbar wird, welcher der beiden Faktoren hauptsächlich betont wird. Sehr häufig wird nur die Conclusio ausgedrückt, nämlich, dass Beurteilung fair (D02, D08, A12), objektiv (A13, CH14), nachvollziehbar (D07, D11), transparent (D09, A04), konstruktiv (D17, CH07), ausgewogen (D18), sorgfältig (A05) und leistungsgerecht (A15, CH12) 
zu erfolgen hat. Argumente und Schlussregeln werden ausgelassen, weshalb der Topos nur in dieser vagen Form (re-)konstruiert werden konnte. Eindeutig ist hingegen sein Bezug zum Typus der Kontroll- und Bewertungsbeziehung, wo er die Art und Weise der gewünschten Kontrolle und Bewertung erläutert. Aber auch für die Motivations-Beziehung stellt der Topos Argumente zur Verfügung, da eine faire Beurteilung annahmegemäß auch motivierend auf die Belegschaft wirkt.

\subsubsection{Der Topos von der Gleichwertigkeit und Gleichbehandlung}

Grundsätzlich sind alle Menschen gleich. Nur tatsächliche Unterschiede können eine ,,gerechte" Differenzierung begründen.

Ähnlich wie der Topos von der fairen Beurteilung und Belohnung ist Gerechtigkeit eines der zentralen Konzepte in diesem Topos. Die Idee, Gleiches gleich und Unterschiedliches unterschiedlich zu behandeln ist ein zentraler normativer Topos vor allem dort, wo mit Unterschieden und Diversität umgegangen werden muss, so z.B. mit Minderheitsmeinungen (D14), unterschiedlichen Unternehmensbereichen (A04) und natürlich mit MA mit unterschiedlichem Geschlecht und unterschiedlicher Nationalität, Kultur und Religion (A14, CH02, CH 14). Eine weitere, wichtige Ausprägung des Topos stellt aber die Diskussion der hierarchischen Unterschiede zwischen FK und MA dar. Zentral ist hier vor allem die Idee der ,Partnerschaft' (D01, D02, CH01, CH11). Aber auch flache Hierarchien (A13) werden explizit angesprochen. Die Gleichwertigkeit und Gleichbehandlung stellt im Diskurs der Führungsgrundsätze vor allem einen Aspekt der Bewertung und Beurteilung dar. Daher taucht der Topos im Material speziell in der Bewertungs- und Beurteilungsbeziehung auf. Dort spezifiziert er noch näher, was mit dem Topos der fairen Beurteilung und Belohnung gemeint ist, indem er einen Aspekt daraus - die gleiche Beurteilung für Gleiches - explizit macht.

\subsubsection{Der Topos von der internen Mobilität}

Eine Entwicklung in den Fähigkeiten und den Kompetenzen muss auch mit einer Entwicklung im Verantwortungsbereich und der Kompensation einhergehen.

Der Topos von der internen Mobilität erweitert den Topos von der Einheit von Kompetenz und Verantwortung um die dynamische Dimension. Während dieser postuliert, dass jede Person im Unternehmen jene Tätigkeiten vornehmen und jene Verantwortung tragen soll, für welche sie kompetent ist, berücksichtigt der Topos von der internen Mobilität, dass sich diese Kompetenzen und Fähigkeiten im Laufe der Karriere ändern können. Interessant ist hierbei aber, dass der Topos von der internen Mobilität, trotz seiner Nähe zum Topos von der Einheit von 
Kompetenz und Verantwortung, weder mit der Begrenzungs-Beziehung noch mit der Verfügungs-Beziehung stark verbunden ist. Dies liegt daran, dass die Möglichkeit der beruflichen Veränderung im Diskurs der Führungsgrundsätze als Service an die MA bzw. als Unterstützung gerahmt wird. Gemäß diesem Diskurs liegt es eben nicht im Interesse der FK, über die MA dergestalt zu verfügen, dass er sie befördert. Stattdessen wird die ,zu starke' Entwicklung der MA als Gefahr (A10) bezeichnet, da die FK ihre MA gleichsam ,verliert', wenn diese den Bereich wechseln. Interne Mobilität liegt also im Interesse der MA. Ihre Gewährung durch die FK ist folglich eine Unterstützung dieser Interessen und ist Teil des Diskurses der Unterstützungs-Beziehung.

\subsubsection{Der Topos vom Mitunternehmertum}

Der Erfolg des Unternehmens steigt, wenn jeder so handelt, als würde ihm das Unternehmen selbst gehören.

Die häufig strapazierte Idee vom Mitunternehmertum (vgl. z.B.Wunderer, 2009: 387f.) findet sich ebenfalls im Diskurs der Führungsgrundsätze. Sie ist stark mit der Entwicklungs-Beziehung verbunden, da MA in ihrer Weiterentwicklung letzten Endes zu Mitunternehmern werden sollen. Dadurch entsteht eine Angleichung der Interessen von MA und Unternehmensführung. Die MA erkennen ihren persönlichen Beitrag zum Unternehmenserfolg (D04), sie arbeiten aktiv an der Erreichung der Unternehmensziele mit (D01), sie zeigen unternehmerischen Mut (D07), sind kostenbewusst (A07) und handeln allgemein unternehmerisch (CH06, CH07).

\subsubsection{Der Topos ,No Risk, no Gain“}

Nur wer Risiken eingeht, hat die Möglichkeit, einen Vorsprung zu erreichen und andere zu übertreffen.

Dieser Topos ergänzt im Wesentlichen den Topos vom Mitunternehmertum bzw. begründet seine Notwendigkeit, indem er Risikofreudigkeit zur zentralen unternehmerischen Eigenschaft und gleichzeitig zu einem wichtigen Erfolgsfaktor macht. Im Diskurs der Führungsgrundsätze ist dieser Topos Bestandteil der Entwicklungs-Beziehung, da die Erziehung zur Risikofreudigkeit ein Ziel der MA-Entwicklung darstellt. Gefördert wird unternehmerische Risikobereitschaft (D07, CH06), Experimentierfreudigkeit (CH09) und das bewusste Zulassen von Fehlern (CH04). 


\subsubsection{Der Topos von der positiven Diversität}

Unterschiedliche Sichtweisen und Hintergründe sind wertvoll, weil die Einnahme unterschiedlicher Perspektiven die Problemwahrnehmung erweitert und zu kreativeren und besseren Lösungen führt.

Dieser Topos stellt das klassische Argument der präskriptiven Literatur zur Diversität im Unternehmen dar (vgl. exemplarisch Kreitner \& Kinicki, 2004). Interessant ist vor allem, dass eine in erster Linie utilitaristische Perspektive auf das Phänomen eingenommen wird. Unterschiede sind etwas, das von den FK zu nutzen ist (D02, A01, A05, CH03), aus dem Wert geschöpft werden kann (D02) und das für neue Lösungswege eingesetzt werden soll (D09). Erst in zweiter Linie sind Unterschiede wertzuschätzen (D02) und zu respektieren (A12, CH14). Der Topos von der positiven Diversität ist daher stark mit der VerfügungsBeziehung verbunden, indem er Hinweise darauf gibt, welche Aspekte der MitarbeiterInnen eingesetzt und genutzt werden sollen. Durch die Thematisierung von Respekt und Wertschätzung besitzt er aber auch argumentative Kraft in der Empathie-Beziehung.

\subsubsection{Der Topos von der positiven Veränderung}

Jede Veränderung beinhaltet den Kern einer neuen Möglichkeit, wenn man bereit ist, diesen zu sehen.

Der Topos von der positiven Veränderung hängt stark mit dem Topos vom Stillstand als Tod zusammen. Während dieser betont, dass nur Weiterentwicklung und Verbesserung den Wert des Unternehmens bzw. des Individuums aufrecht erhalten, argumentiert der Topos von der positiven Veränderung, dass Veränderung ganz allgemein eine positive Note besitzt. Die gängige Schlussfolgerung daraus ist, dass die Akteure Veränderungen gegenüber offen sind (D01, D02, A09), diese als Chance sehen (D04, D05, A07, CH09) und Veränderungen deshalb von allen getragen werden (D07). Dieser Topos findet sich vor allem in der Entwicklungs-Beziehung.

\subsubsection{Der Topos vom Primat von Information und Wissen}

Information und Wissen machen handlungsfähig und verbessern die Entscheidungsqualität. Information ist deshalb auf allen Ebenen und in allen Bereichen zu fördern.

Ähnlich wie der Topos von der Bedeutung der Kommunikation ist auch der Topos vom Primat von Information und Wissen eng mit dem Topos von der Transparenz als Allheilmittel verknüpft. Die Forderung nach Information (und zwar dem Einholen von Information ebenso wie der Weitergabe dieser; D16, CH02, 
CH07, A04) ist ein zentraler Aspekt im Diskurs der Führungsgrundsätze. Information ist eine Querschnittsmaterie und zieht sich durch einen Großteil der in dieser Arbeit (re-)konstruierten Beziehungstypen. Am wichtigsten ist der Topos aber für die Integrations-Beziehung insofern, als die Information des jeweils anderen Akteurs (ebenso wie die Kommunikation) als wesentliche Bedingung für seine Integration bzw. seinen Einbezug in die eigene Tätigkeit verstanden wird. Auch durch Information (welche faktisch oft mit Kommunikation gleichzusetzen ist) wird Transparenz erreicht. Aber auch für die Empathie-Beziehung ist Information bzw. Wissen zentral, nämlich das Wissen über die Eigenheiten und Bedürfnisse des anderen. Nur dann kann diesen Bedürfnissen auch entsprochen werden. Eine dritte wichtige Beziehung ist die Bereitstellungs-Beziehung. Neben Freiraum ist Information eines der wesentlichen Objekte, welches den anderen Akteuren bereitgestellt wird.

\subsubsection{Der Topos von der situativen, direkten Führung}

Jede/r MitarbeiterIn und jede Situation sind anders und verlangen deshalb ein maßgeschneidertes, kompetentes Führungshandeln.

Aus der Logik der Empathie-Beziehung heraus, dass die Akteure sich gegenseitig kennen, verstehen, respektieren und anerkennen und deshalb ihre jeweiligen Bedürfnisse und Wünsche berücksichtigen, entsteht im Diskurs der Führungsgrundsätze auch die Forderung nach direkter und situativer Führung. Da jedes Individuum im Unternehmen anders ist (siehe auch den Topos von der Bedeutung des Einzelnen), muss auch jedes anders geführt werden. Der Führungsstil muss der Situation angepasst sein (D02, D08, A08, CH18), die FK kennt viele verschiedene Instrumente und Stile (D06, CH08), sie wird den Fähigkeiten und Potentialen der MA gerecht (D12, CH10) und berücksichtigt deren Persönlichkeit (D17).

\subsubsection{Der Topos vom Vertrauen als Basis organisationaler Bezie- hungen}

Ohne ein grundsätzliches Vertrauen ist Zusammenarbeit nicht möglich. Vertrauen ersetzt in einem gewissen Maß Kontrolle und macht dadurch handlungsfähig.

Das Thema vom Vertrauen ist im Diskurs relativ zentral. Dabei bleibt die Argumentation - ähnlich wie im Topos von der fairen Beurteilung und Belohnung - aber stets vage. Vertrauen dient dem Aufbau von Beziehungen (D02), ist allgemein Voraussetzung für erfolgreiche Führung (D08), unterstützt die Zusammenarbeit (D15, A10) und Entfaltung am Arbeitsplatz (CH07), führt zu gegenseitiger Unterstützung (D05) und erzeugt ein ,Wir-Gefühl` (A04). Außerdem 
ermöglicht Vertrauen erhöhte Transparenz (D14) und Sicherheit (D16). Vertrauen wird im Diskurs in beide Richtungen geschenkt, sowohl von der FK zu den MA als auch umgekehrt. Der Topos vom Vertrauen als Basis organisationaler Beziehungen ist in zwei Beziehungstypen relevant. Zum einen stellt er in der Empathie-Beziehung sowohl Bedingung als auch Konsequenz guter Zusammenarbeit dar, zum anderen ist er in der Bereitstellungs-Beziehung einer der Aspekte, welcher gegeben, geschenkt und zur Verfügung gestellt wird.

\subsubsection{Der Topos von der Zielklarheit}

Ziele kann man nur erreichen, wenn man genau weiß, wohin man will und wie man dorthin gelangt.

Der Formulierung von Zielen kommt im Diskurs der Führungsgrundsätze vor allem im Rahmen der Konzeption des MbO eine große Bedeutung zu. Der Topos von der Zielklarheit ist einer von mehreren Topoi, welche sich mit der optimalen Zielsetzung bzw. -vereinbarung auseinandersetzen. Tatsächlich ist er jener Topos, welcher für das Thema des MbO konstitutiv wirkt. Ohne Ziele kann die Organisation nirgends ankommen. Nur über die Formulierung möglichst klarer Ziele kann überhaupt ein sinnvoller Weg beschritten werden. Ziele werden im Diskurs entweder vereinbart (D03, A01, CH04) oder gesetzt (D02, A02, CH16). Obwohl erst zu untersuchen wäre, ob der unterschiedlichen Rhetorik auch eine unterschiedliche Praxis entspricht, findet der Topos in der gegenständlichen Untersuchung durch diese Zweiteilung in zwei verschiedenen Beziehungstypen Eingang. Wenn Ziele vereinbart werden, entspricht dies der Logik der Integrations-Beziehung. Werden Ziele hingegen gesetzt oder vorgegeben, dann fällt dies in die Logik der Steuerungs-Beziehung.

\subsubsection{Der Topos von der Zielkoordination}

Individuen haben unterschiedliche individuelle Ziele. Diese können zu Zerreißproben führen, wenn sie nicht koordiniert werden.

Der Topos von der Zielkoordination thematisiert einen weiteren spezifischen Aspekt der Zielthematik, nämlich das Problem des Widerspruchs zwischen verschiedenen Zielen von Abteilungen (D05, A15), Individuen (D02) bzw. von Zielkonflikten zwischen Individuum und Organisation (D14). Ziel ist es stets, diese Zielkonflikte aufzulösen bzw. abzugleichen (D02, D08, D14), und zwar am besten in Form einer Win-win-Situation (D02, A15, CH02). Der Topos von der Zielkoordination ist mit keinem Beziehungstypus besonders stark verbunden, ist aber grundsätzlich für drei Beziehungen von Bedeutung. In der Empathie-Beziehung begründet er die Notwendigkeit, sich in andere Personen hinein- 
zuversetzen. Dies ist eine notwendige Voraussetzung für den Abgleich von Zielen. In der Integrations-Beziehung ergänzt er den Topos von der Zielklarheit um den Aspekt des Zielkonflikts, und in der Befähiger-Beziehung stellt er einen Rand-Topos dar, welcher die ,befähigende“ Wirkung der Auflösung von Konflikten und Zielwidersprüchen thematisiert.

\subsubsection{Der Topos vom Zusammenhang zwischen Freiraum und Mo- tivation}

Motivation entsteht nur dort, wo man ihr Freiraum zur Entfaltung lässt. Übermäßige Kontrolle erzeugt ihre eigenen Prämissen.

Die Argumentationslinie dieses Topos folgt im Wesentlichen den Ausführungen der „Theory Y“ von McGregor (vgl. z.B. Schreyögg, 2003: 225ff.) und stellt somit den Gegen-Topos zum Topos von der Notwendigkeit der Kontrolle dar. Organisationsmitglieder erbringen eine höhere Leistung, wenn man ihnen den notwendigen Freiraum zuteilt, innerhalb dessen sie selbständig handeln und entscheiden können. Eine grundsätzliche Leistungsbereitschaft des Individuums wird vorausgesetzt. Konsequenzen aus diesem Topos sind vor allem die verstärkte Vornahme von Delegation (D01, A01, CH10), aber auch allgemein die Schaffung von Freiräumen bei der Leistungserstellung (D03, D09, A04). Zum Teil wird gefordert, dieser Freiraum solle möglichst groß sein (CH13), zumindest aber soll er angemessen bzw. sinnvoll sein (CH14, D16).

Zunächst scheint hier ein Steuerungsverzicht vorzuliegen, da gerade das Fehlen von (direkter) Steuerung als leistungssteigernd argumentiert wird. Dennoch unterbricht der Topos nicht die grundsätzliche Logik von Führung und Hierarchie. Freiräume sind etwas, das den MA von den FK gegeben wird. Die MA können sich diesen Freiraum nicht selbst schaffen. Damit funktioniert der Topos innerhalb der Führungsgrundsätze ausschließlich in institutionalisierten Herrschafts- bzw. Hierarchieverhältnissen. Durch diese Betonung des Gebens bzw. des Vertrauens in die MA kann dieser Topos aber gemäß Cheney (1983: 148ff.) als Versuch der Herstellung von Identifikation im Rahmen der ,Common Ground Technique' gesehen werden. Das Vertrauen der Organisation in die MA erzeugt ein gewisses Nähegefühl (,Wir vertrauen darauf, dass $\mathrm{Du}$ in unserem Sinne handeln wirst.') Dieses Nähegefühl kann die gefühlte Verantwortung der MA der Organisation gegenüber steigern und zu höherer freiwilliger Anpassung an die Organisationsziele führen.

Der Vollständigkeit halber - und um den argumentativen Diskurs der Führungsgrundsätze möglichst umfassend zu diskutieren - werden in der Folge noch jene 
Topoi kurz summarisch dargestellt, welche kaum einen oder gar keinen empirischen Bezug zu den (re-)konstruierten Beziehungstypen aufweisen.

\subsubsection{Topoi mit geringem Bezug zu den Akteursbezie- hungen}

Wenn Topoi wenig Bezug zu den hier diskutierten Beziehungstypen aufweisen, dann bedeutet dies, dass sie im Diskurs nur sehr schwach mit typischen Handlungen der Akteure in Verbindung gebracht werden. In der Mehrzahl der Fälle stellen diese Topoi Argumentationsmuster zur Begründung allgemeiner Werte und Normen dar, welche in den Führungsgrundsätzen nicht auf die Interaktionsebene zwischen Führungskraft und MitarbeiterInnen herunter gebrochen wurden. Manche von Ihnen sind für die Führungsbeziehung dennoch von einiger Relevanz, während wiederum andere sich völlig anderen Themen (vor allem finanziellen und Wettbewerbsaspekten) widmen. Da die Führungsbeziehung den primären Regelungsinhalt der Führungsgrundsätze darstellt, sind die anschließend diskutierten Topoi auch in ihrer Quantität und Verbreitung über die Dokumente eher unbedeutend. Wo immer eine Ausnahme von dieser Regel besteht, wird dies explizit angeführt.

\subsubsection{Topoi mit konzeptioneller Relevanz für die Führungsbezie- hung}

Ein zentraler Topos, welcher die Überordnung von Führungskräften und deren Weisungsbefugnis begründet, ist jener von der Komplexität und Bedeutung der Führung. Führung ist ein schwieriges Unterfangen, welches besonderen Einsatz und besondere Kompetenz erfordert. Führung entscheidet außerdem wesentlich über den Erfolg des Unternehmens. Die Ansprüche an FK sind dementsprechend hoch, und aus der hohen Qualifikation und Motivation der FK lassen sich auch deren Überlegenheit und ihre legale Autorität begründen.

Ein weiteres grobes Cluster der für die Führungsbeziehung relevanten Topoi betrifft den Topos von der Goldenen Regel und den Topos von der Win-win Lösung. Beide Topoi betreffen ähnlich dem Topos von der Gleichheit und Gleichbehandlung die Idee, dass Gleiches auch gleich behandelt werden soll, allerdings auf einem höheren Spezifikationsgrad. Der Topos von der Goldenen Regel folgt der Logik, dass man von anderen nur verlangen soll, was man selbst auch bereit ist zu leisten oder zu geben. Der Topos von der Win-win Lösung argumentiert, dass das Gesamtwohl dort maximiert ist, wo von einer Entscheidung beide Parteien gleichermaßen profitieren. 
Ein anderes Cluster, das ebenfalls aus zwei spezifischen Topoi besteht, thematisiert die Fehlertoleranz in der Organisation. Der Topos von der Unvermeidbarkeit von Fehlern argumentiert, dass Fehler grundsätzlich nicht vermieden werden können und dass diese in der Organisation deshalb auch zugelassen werden müssen. Dadurch soll erreicht werden, dass Fehler eingestanden und nicht vertuscht werden. Der Topos vom Lernen aus Fehlern stellt eine logische Erweiterung dar. Wenn Fehler auftauchen, müssen sie besprochen und analysiert werden, damit sich diese in Zukunft vermeiden lassen. Beide Topoi stehen durch die Betonung von Offenheit und Transparenz auch dem Topos von der Transparenz als Allheilmittel nahe, da sie ähnliche Konklusionen nahelegen.

Zwei weitere Topoi bieten Argumentationsgrundlagen im Bereich der Verantwortungsabgrenzung. Der Topos von der Klarheit von Kompetenz und Verantwortung argumentiert, dass Verantwortung klar zugewiesen und abgegrenzt sein soll, um Sicherheit und Orientierung zu ermöglichen. Der Topos von der Einheit von Macht und Verantwortung ergänzt jenen von der Einheit von Kompetenz und Verantwortung dahingehend, als nicht nur die Verantwortung durch eine entsprechende Kompetenz ausgeglichen werden soll, sondern auch die verfügbare Macht durch klar definierte Verantwortlichkeiten abgesichert sein muss. Damit entspricht dieser Topos der Forderung nach einer legitimen Grundlage organisationaler Macht.

Motivationale Aspekte werden schließlich noch vom Topos der Motivation durch Herausforderung und vom Laissez-Faire Management angesprochen. Ersterer bietet neben dem Topos vom Zusammenhang von Freiraum und Motivation eine zweite Einflussgröße der Motivation ins Spiel, nämlich die Herausforderung. Realistische Herausforderungen - so die Argumentation - erhöhen die Leistungsmotivation. Der Rand-Topos vom Laissez-faire Management schließlich stellt eine Steigerung des Topos vom Zusammenhang von Freiraum und Motivation dar, indem er argumentiert, dass die FK sich nur dann in die Angelegenheiten der MA einmischen soll, wenn dies unbedingt erforderlich ist.

Der Topos von der Klarheit der Zielhierarchien thematisiert die Notwendigkeit der Hierarchisierung organisationaler Ziele; der Topos von den Grenzen der Information und Kommunikation wiederum betont die Bedeutung von Vertraulichkeit in bestimmten Aspekten des organisationalen Alltags. Der Topos von der guten Kontrolle schließlich argumentiert, dass Kontrolle nur dann sinnvoll eingesetzt wird, wenn sie zur Informationsbeschaffung und nicht zur Unterdrückung verwendet wird. 


\subsubsection{Topoi ohne besondere konzeptionelle Relevanz für die Füh- rungsbeziehung}

Ein Cluster von Topoi ohne besondere Relevanz für die Führungsbeziehung besteht aus den drei Topoi von der Notwendigkeit einer ganzheitlichen Sichtweise, dem Primat des Gesamtinteresses und dem Topos vom Global Business und der Cultural Awareness. Diese Topoi anerkennen die Notwendigkeit, ,über den Tellerrand' hinauszusehen und breitere Zielsetzungen anzuerkennen. Drei weitere Topoi betrachten die Ziel-Mittel-Relation, wobei der Topos Der Weg ist das Ziel und der Topos von der Notwendigkeit der Prozessoptimierung den Fokus stärker auf die Mittel legen, während der Topos Der Zweck heiligt die Mittel eine alleinige Ergebnisorientierung nahe legt. Die Topoi von der Notwendigkeit von Regeln und Standards sowie von der Notwendigkeit von Stabilität argumentieren für die Erhaltung des Status quo, während die Topoi von der Macher-Mentalität und der Notwendigkeit von Flexibilität stärker für Innovation eintreten. Die Topoi von der Qualitätsorientierung, der Kundenorientierung und der Topos ,Wenig ist wirklich wichtig ' schließlich verlangen die Fokussierung auf verschiedene Aspekte betrieblicher Zielsetzungen. Die Topoi der Vermeidung von Befangenheit, der individuell angepassten Entlohnung, der Notwendigkeit von Emotionen und vom Selbstbewusstsein als Erfolgsfaktor beschließen diese Aufzählung.

\subsubsection{Topoi ohne Bezug zu den Akteursbeziehungen}

Die restlichen Topoi, welche keinen (empirischen oder konzeptionellen) Bezug zu den unmittelbaren Akteursbeziehungen besitzen, betreffen zum einen den Markt bzw. den Wettbewerb (Topos von der Geschwindigkeit als Wettbewerbsvorteil, Topos vom Streben an die Spitze, Topos vom Benchmarking, Topos vom Networking), finanzielle Aspekte (Topos vom Primat des finanziellen Ergebnisses, Topos vom Kostenbewusstsein) bzw. andere Aspekte der Unternehmensführung (Topos von der Risikoaversion). Aber auch Aspekte der Unternehmensethik (Topos von den ethischen Grundlagen des Handelns, Topos von der gesellschaftlichen Verantwortung des Unternehmens, Topos von der Außenwirkung interner Prozesse) und andere allgemeine Normen (Ohne Fleiß kein Preis, Topos vom positiven Denken, Der Mensch ist gut) werden thematisiert. 


\subsubsection{Die rhetorische Steuerungswirkung von Führungs- grundsätzen}

Die Analyse topischer Argumente in den Führungsgrundsätzen wurde als Möglichkeit diskutiert, die grundlegenden Verständnisse hinter den vorgeschlagenen Führungsbeziehungen in deutschsprachigen Führungsgrundsätzen zu analysieren. Während die diskutierten Beziehungstypen eine Antwort auf den ersten Teil der vorgestellten Forschungsfrage bieten,

Welche (impliziten und expliziten) Vorstellungen von Führung und Steuerung finden sich in kontemporären Führungsgrundsätzen deutschsprachiger Großunternehmen?

ermöglicht die Analyse der verwendeten Topoi die Beantwortung des zweiten Teils dieser Forschungsfrage:

Mithilfe welcher rhetorischen Strategien und Ressourcen werden diese Vorstellungen argumentiert und damit im organisationalen Diskurs verankert?

Dabei sind vor allem die konstitutiven Topoi interessant, welche distinktive Formen des In-Bezug-aufeinander-Handelns der FK und MA - und damit spezifische Formen der Über- und Unterordnung - legitimieren. Die neun konstitutiven Topoi im Material weisen deutliche Tendenzen zu einer Rationalisierung und damit Versachlichung bzw. Entemotionalisierung des Themas auf. Damit einher geht auch eine starke Entpersönlichung der Führungsbeziehung. Mit Ausnahme eines Topos (Bedeutung des Einzelnen) exkludieren alle Topoi die beteiligten Personen und rekurrieren stattdessen auf abstrakte Zusammenhänge und Kausalitäten. Einige davon folgen einer funktionalistischen bzw. utilitaristischen Logik (Bedeutung des Arbeitsklimas und Arbeitsumfelds, Notwendigkeit von Kontrolle, Notwendigkeit von Orientierung und Sicherheit, Einheit von Kompetenz und Verantwortung, Zusammenhang zwischen Motivation und Leistung, Transparenz als Allheilmittel, Stillstand als Tod). Andere wiederum beziehen sich stärker auf normative oder ethische Bezugssysteme wie Gerechtigkeit und Humanismus (Bedeutung des Einzelnen, Fordern und Fördern). Führung und damit Über- und Unterordnung bzw. Hierarchie - ist also sowohl (vor dem Hintergrund einer Logik ökonomischer Effizienz) funktional notwendig als auch moralisch vertretbar. Die Ausübung von Herrschaft und Macht im Unternehmen hat damit nichts mit Vorstellungen von der Durchsetzung persönlicher Interessen, der Tyrannei und der Unterdrückung der Arbeiterschaft zu tun. Es gibt faktisch keine Alternative zu Führung. Mit dieser Logik der , guten Unternehmensführung' als zentralem Argumentationspunkt für die Existenz von Herrschaftsund Machtbeziehungen im Unternehmen sichert sich die Organisation Legitimität sowohl nach innen als auch nach außen (vgl. z.B. J. W. Meyer \& Rowan, 
1977). Oder mit anderen Worten: Die Accounts, welche die Organisationen durch die Verwendung dieser Topoi kommunizieren, sind geeignet, ,etwas potenziell Infragegestelltes durch etwas Gültiges/Legitimiertes, auf das sie implizit oder explizit verweisen, abzusichern“ (R. E. Meyer, 2004: 176). Das grundsätzlich erklärungsbedürftige Phänomen der Gestaltung von Über- und Unterordnung wird also vor allem mit Argumenten der ökonomischen Rationalität, gemischt mit einer ,Prise“ Humanismus (die Führung liegt auch im Interesse der MA), gestützt.

Führung als Phänomen kann aber in modernen westlichen Gesellschaften und vor allem in der Sub-Sinnwelt der Wirtschaft, in welcher sich Interaktionen zwischen FK und MA abspielen - bereits als institutionalisiert und damit grundsätzlich legitimiert betrachtet werden (vgl. Sandner \& Meyer, 2002). Die Begründung der Existenz von Über- und Unterordnung per se kann deshalb nicht das einzige bzw. hauptsächliche Ziel von Führungsgrundsätzen sein. Durch die konsequente Verwendung legitimitätssteigernder Argumente und Symbole tragen die Dokumente jedoch zur Herrschaftssicherung bei. Abgesehen von dieser stabilisierenden Funktion haben Führungsgrundsätze aber auch den Anspruch, wünschenswerte Formen von Führung und Unterordnung zu etablieren. Diese vorgeschlagenen Führungsvarianten finden sich in der gegenständlichen Untersuchung in den (re-)konstruierten Akteursrollen und Beziehungstypen wieder. Mittels ergänzender Topoi wird in den Dokumenten die konkrete Ausgestaltung dieser Beziehungen argumentiert. Dabei fällt auf, dass die verwendeten Topoi insgesamt auf einem eher allgemeinen Niveau angesiedelt sind. Hier findet die Logik der ,guten Unternehmensführung' als Erklärung für die verwendeten Argumentationsmuster ihre Grenzen. Die Dokumente vermitteln weder den Eindruck, dass Führung nach besonders modernen Grundsätzen geschieht, noch wird jemals explizit Bezug auf wissenschaftliche Erkenntnisse der Führungsoder Personalforschung genommen. Vielmehr rekurrieren die verwendeten Topoi auf generische Kausalitätsunterstellungen, welche dem ,Hausverstand“ in Form gängiger ,Klischees` zugeordnet werden können (z.B. Gemeinsam sind wir stärker, No Risk, no Gain, Stillstand als Tod) oder aber sie evozieren vermeintlich geteilte Wertvorstellungen, denen man in einer westlichen Gesellschaft kaum (unbegründet) widersprechen kann (z.B. Fordern und Fördern, positive Diversität, positive Veränderung, faire Beurteilung und Belohnung, Gleichwertigkeit und Gleichbehandlung). Während manche dieser Topoi (z.B. Mitunternehmertum, direkte, situative Führung, interne Mobilität) explizit aus einem betriebswirtschaftlichen Kontext stammen und nur in diesem Bezugssystem argumentativ wirken können (vgl. Kopperschmidt, 1989: 105), transzendieren andere Topoi den betriebswirtschaftlichen Kontext und beziehen sich auf 
breitere gesellschaftliche Verständnisse (z.B. Gemeinsam sind wir stärker, Gleichwertigkeit und Gleichbehandlung).

Den Topoi ist gemeinsam, dass sie kaum dazu geeignet sind, spezifische und innovative Formen der Führungsbeziehung zu begründen. Durch ihre Verankerung im Allgemein- bzw. Klischeewissen der Adressaten resonieren sie aber in bekannten Sinnstrukturen und integrieren die thematisierten Führungsaspekte in dieses Wissen.

Weil eine gemeinsame Linie in der Organisation wichtig ist, damit nicht jeder in eine andere Richtung arbeitet, ist Führung notwendig. Weil Führungskräfte eine bessere Ausbildung und aufgrund ihrer Position einen besseren Überblick besitzen, sind die MitarbeiterInnen von deren Unterstützung abhängig. Weil man sich nur verbessern kann, wenn man seine Schwächen kennt, muss man sich von seiner/m Vorgesetzten beurteilen lassen.

Damit wirken Führungsgrundsätze aber in erster Linie stabilisierend auf den Status quo und nicht innovierend im Sinne moderner und entwicklungsorientierter Führungsverständnisse. Sie sind aber auch nicht geeignet, unternehmensspezifische Regeln zu begründen. Die identifizierten Topoi rekurrieren ja gerade auf den kleinsten gemeinsamen Nenner, auf jene Klischees und ,Binsenweisheiten', deren Wahrheits- oder zumindest Plausibilitätsgehalt kaum jemand (weder innerhalb noch außerhalb des Unternehmens) in Abrede stellen kann.

„[...] to reach a multiplex audience, it is necessary to frame the message in a relatively generalized and abstract way, something that on the other hand may weaken its intensity and appeal.“ (Engelstadt, 2009:218)

Wunderer (2009: 397ff.) bestätigt indirekt die Allgemeinheit der in Führungsgrundsätzen vertretenen Positionen, wenn er die Verhaltensleitsätze in den Dokumenten mit jenen in populären Märchen vergleicht und hohe Übereinstimmungen feststellt. Damit stehen die Textautoren vor dem Problem, die Inhalte als etwas darzustellen, was , wir im Unternehmen“ - und damit implizit alle anderen nicht - so machen. Sie tun dies zum einen vor allem durch die explizite Formulierung dieses Anspruchs im Vorwort:

Die Führungsprinzipien geben Orientierung für die Führung unseres Geschäfts und für unsere Zusammenarbeit. Sie beschreiben meine Erwartungen an das Verhalten der Führungskräfte bei [Unternehmen]. (D02)

Führungsgrundsätze geben die angestrebte Unternehmens- und Führungskultur, die in unserem Unternehmen durch die [Unternehmen] Mitarbeiter Charta und die [Unternehmen] Unternehmensverfassung determiniert wird, wieder und dienen auch als Messlatte, in welchem Ausmaß sie in der Praxis schon gelebt werden. (A08) 
Sie tun dies aber auch durch eine intensive Verwendung des Pronomens, wir' im Gegensatz zu einem möglichen ,man', um die Spezifizität der Regelungen für das Unternehmen zu unterstreichen. Dieses Spannungsfeld zwischen der Evokation gängiger sozialer Normen und Klischees und deren Präsentation als unternehmensspezifische Grundsätze wird in den Dokumenten nicht aufgelöst. Sie verstärkt aber die legitimitätssteigernde Wirkung der kommunikativen Handlungen durch das Bekenntnis zur Normalität. Es liegt daher die Schlussfolgerung nahe, dass Führungsgrundsätze nicht unbedingt konkrete und anwendbare Regeln für das tägliche Führungshandeln geben wollen, sondern vielmehr das Konzept von Führung und Hierarchie an sich - und damit alle Handlungen, welche in den Dokumenten als Führung definiert werden - normalisieren und legitimieren, indem sie dieses in einen Argumentationszusammenhang mit hoher Anschlussfähigkeit stellen. Führungsgrundsätze wirken damit vor allem stabilisierend auf bestehende Herrschaftsverhältnisse. Damit werden aber auch die Diffusität der Regeln und die fehlende Verbindlichkeit der Dokumente zu einem funktionalen Charakteristikum des Diskurses. Eine hohe Verbindlichkeit mit Sanktionsandrohung für ohnehin als normal bzw. selbstverständlich unterstellte Normen würde die Normalität sogar eher in Frage stellen (Warum wird etwas Selbstverständliches hier so betont?). Ohne Betonung der Verbindlichkeit hingegen verstärken die Handlungsaufforderungen lediglich institutionalisierte Verständnisse - „Da wären wir wieder einmal“ (Berger \& Luckmann, 2000: 61) und sichern bestehende Herrschaftsverhältnisse ${ }^{33}$.

Eine weitere funktionale Wirkung diffuser Regeln und allgemeiner Begründungsformen besteht in der Möglichkeit einer breiten Identifikationswirkung. Im Rahmen der ,Common Ground Technique' (vgl. Cheney, 1983: 148ff.) finden sich in den (re-)konstruierten Topoi einige der diskutierten Strategien. Die Betonung des Werts des Individuums z.B. wird explizit im Topos von der Bedeutung des Einzelnen festgehalten. Die Anerkennung individueller Beiträge ist wesentlicher Bestandteil der Empathie-Beziehung und des Topos von der fairen Belohnung und Beurteilung. Das Eintreten für geteilte Werte ist ein wichtiger Aspekt der Rolle des Akteurs als Vertreter bzw. Verfechter und damit auch Teil der Unterstützungs-Beziehung, welche ebenfalls die Kommunikation von Vorteilen und Leistungen für die Organisationsmitglieder betont. Insgesamt bieten die Führungsgrundsätze eine Vielzahl von möglichen Normen und Grundsätzen, mit

33 Vgl. dazu auch Luegers (2001) Ausführungen zur Temporalität des kulturellen Gedächtnisses. Durch den Bezug zu einer Kollektivgeschichte - sprich: zu allgemein gültigen und historisch gewachsenen Argumentations- und Begründungsformen - können Führungsgrundsätze ,das Eindringen von Veränderung durch Absichern des Erlangten verhindern, womit sich der Sinn auf das Regelmäßige, die Wiederholung legt (Herrschaftssicherung und Stabilisierung)“(M. Lueger, 2001: 32). 
welchen sich das Individuum problemlos identifizieren kann, da sie im Wesentlichen unproblematisch - wenn nicht sogar (scheinbar) direkt vorteilhaft für das Individuum - sind. Diese argumentativen Identifikationsangebote werden durch die rhetorische Strategie der Verwendung des angenommenen, wir ${ }^{6}$ (vgl. Cheney, 1983: 148ff.) noch verstärkt. Das Publikum wird in die Aussage direkt inkludiert; eine Zustimmung wird rhetorisch quasi vorweggenommen. Führungsgrundsätze wirken also sowohl durch Identifikation als auch durch Argumentation, wobei die beiden Wirkungsformen nicht klar voneinander abgrenzbar sind. Die Argumentation wirkt aber nicht in erster Linie dadurch, dass sie Individuen dazu bringt, etwas zu tun, was diese ursprünglich nicht getan hätten. Vielmehr hilft sie den Individuen, ihre bestehenden Handlungen zu rationalisieren, sie in ein vertrautes Sinnsystem zu integrieren und damit bestehende (Herrschafts)Verhältnisse zu stabilisieren und zu verstärken.

Interessant für die Analyse ist aber nicht nur die Interpretation einzelner Topoi oder ihrer Gesamtpopulation im untersuchten Material. Topoi verweisen außerdem auch auf bestimmte Zusammenhänge zwischen einzelnen Beziehungstypen. So verweist ein ähnliches Muster von Topoi auch auf ähnliche Begründungslogiken innerhalb verschiedener Beziehungen. Teilen sich Beziehungstypen einzelne Topoi, so ist zu klären, welche Funktionen diese innerhalb der Beziehungen erfüllen. Manche Topoi treten außerdem auch als argumentative Stütze für andere auf oder explizieren diese. Im nächsten Kapitel werden deshalb die Beziehungstypen in direkte Beziehung zu den jeweils relevanten Topoi gesetzt und ausführlich diskutiert.

\subsection{Beziehungstypen - eine integrierte Analyse}

Im Folgenden wird für jeden Beziehungstyp erläutert, wie seine spezifische Handlungslogik aussieht, welche Argumentationsmuster er aufweist, wie die Akteure innerhalb dieser Beziehung miteinander interagieren und welches Steuerungspotential bzw. -verständnis durch die spezifische Beziehungsform entsteht und erhalten wird.

\subsubsection{Die Anspruchs-Beziehung}

\section{Handlungslogik und betroffene Rollenaspekte}

Die grundlegende Handlungslogik hinter der Anspruchs-Beziehung ist die Gegenüberstellung von Fordern und Bereitstellen/Versorgen. Eine Partei besitzt ein legitimes Recht, von einer anderen Partei etwas einzufordern. Diese hat das 
Geforderte zur Verfügung zu stellen. Auf der Seite der FK ist damit vor allem der Rollenaspekt des Steuermannes und Leiters betroffen, welcher die Handlung des Forderns beinhaltet. Auf der Seite der MA hingegen ist es der Aspekt des Berechtigten (ein Recht haben, etwas verdienen). Der Unterschied zwischen diesen beiden Rollenaspekten liegt in erster Linie in der Initiative. Während die FK aktiv fordert, wird die Forderung der MA als Zustand ausgedrückt. Die Forderung der MA besteht somit passiv und automatisch; sie muss nicht mehr ausgedrückt werden. Die Anspruchs-Beziehung ist von den relevanten Handlungen her der Bereitstellungs-Beziehung nicht unähnlich. Der Ausgangspunkt ist jedoch ein anderer. Während bei der Bereitstellungs-Beziehung die Interaktion mit der Bereitstellung beginnt (und zumeist auch endet), beginnt sie in der AnspruchsBeziehung mit der Forderung - die Erfüllung der Forderung bleibt meist implizit. Damit wird in der Anspruchs-Beziehung die Steuerungswirkung deutlich expliziter ausgedrückt (siehe unten).

\section{Argumentationsstruktur - relevante Topoi}

Die Identifikation des konstitutiven Topos für die Anspruchs-Beziehung gestaltet sich als eher schwierig. Ein expliziter Topos, welcher spezifisch legitime Ansprüche eines Akteurs gegenüber einem anderen begründet, ist in den Dokumenten nicht zu finden. Der Topos vom Fordern und Fördern ist jedoch in der Lage, solche Ansprüche zu begründen, und er korrespondiert auch vergleichsweise stark mit den Äußerungen innerhalb der Anspruchs-Beziehung:

Jeder im Unternehmen besitzt Rechte und Pflichten gleichermaßen. Diese Rechte und Pflichten müssen deshalb im Unternehmen in einem angemessenen Verhältnis zueinander stehen.

Dieser Topos argumentiert für eine Ausgewogenheit von ,Geben“ und ,Nehmen“ in der Führungsbeziehung. Damit ist die Reziprozität der Ansprüche zentral. Die Führungskräfte sind zwar aufgefordert, die MitarbeiterInnen zu fördern und zu entwickeln, sie dürfen dafür aber auch eine entsprechende Leistung von ihren MitarbeiterInnen einfordern. Die Ansprüche der MA an die FK stellen somit die andere Perspektive auf die Verpflichtung zur Förderung der MA durch die FK dar: Für die erbrachte Leistung haben die MA Anspruch auf Anerkennung (D08), Informationen (D10), Feedback (D11), Orientierung und Unterstützung (A05) und konkrete Ziele (A13). Führungskräfte hingegen fordern von ihren MitarbeiterInnen in erster Linie Leistung (D08, A09), Mitverantwortung (CH07, CH14) und Mitunternehmertum (D07). Jeweils gegenseitig werden Information und Feedback erwartet. Deshalb ist auch der Topos von der Transparenz als Allheilmittel relativ stark mit diesem Beziehungstyp verbunden: 
Sowohl effizientes Arbeiten als auch ein gutes Arbeitsklima sind nur dann möglich, wenn alle relevanten Faktoren bekannt und allen zugänglich sind. Verschleierung verunmöglicht optimale Lösungen.

Transparenz ist ein zentrales Element, welches von beiden Akteuren im Rahmen dieser Beziehung gefordert wird. Damit zeigt sich klar die Beziehung zwischen dem konstitutiven Topos und dem ergänzenden. Der Topos vom Fordern und Fördern erklärt, warum die Anspruchs-Beziehung legitim ist. Der Topos von der Transparenz als Allheilmittel argumentiert innerhalb dieser Beziehung die Bedeutung eines spezifischen Anspruchsinhalts.

\section{Interaktionsstruktur}

Die Anspruchs-Beziehung ist in hohem Grade in gegenseitiger Form vorzufinden. Die Ansprüche der MA und FK sind in einem ähnlichen Ausmaß differenziert und betont. Der Hauptunterschied besteht darin (siehe oben), dass sich die Satzstruktur und somit die Formulierung der Ansprüche stark unterscheiden, je nachdem, ob ein Anspruch der FK oder einer der MA besteht. So werden Ansprüche der FK zumeist in einer aktiven, handelnden Form ausgedrückt:

Ich fordere Zwischenberichte an und erteile nicht ständig gute Ratschläge. Ich beantworte keine Fragen, die Mitarbeiter selbst beantworten können und löse keine Aufgaben, die Mitarbeiter selbst lösen können. (D 16)

Ich erwarte, dass meine Mitarbeiterinnen und Mitarbeiter mitdenken, nehme ihre konstruktive Kritik an und ermutige sie, Lösungsvorschläge zu bringen. (CH 14)

FK gehen somit aktiv auf ihre MA zu und fordern ihr Recht ein. Zumindest aber besteht eine explizite Erwartung, dass die MA die Ansprüche erfüllen. Im Gegensatz dazu sind die berechtigten Ansprüche der MA in erster Linie passiv und als ,Zustand' formuliert, wobei die MA dabei nicht aktiv tätig werden:

Jeder Mitarbeiter hat ein Recht auf alle Informationen, die er benötigt, um zielorientiert handeln und entscheiden zu können. (D 10)

Unsere Mitarbeiter verdienen es, ernst genommen, angehört und respektiert zu werden. Der Vorgesetzte kümmert sich persönlich um sie und ihre Interessen. (CH 13)

Damit zeigt sich in der Anspruchs-Beziehung deutlich, dass MA in den Führungsgrundsätzen eine eher passive Rolle einnehmen. Während bei den FK bereits in der Formulierung angelegt ist, dass sie ihre Ansprüche auch einfordern, werden jene Ansprüche bei den MA in erster Linie nur definiert und festgelegt. MA werden lediglich in einer einzigen Instanz aktiv tätig, und zwar dort, wo es um die gegenseitige Durchsetzung von Ansprüchen geht. 


\section{Steuerungsaspekte}

Die Handlungen innerhalb der Anspruchs-Beziehung (das Fordern bzw. Ansprucherheben) entsprechen einer sehr direkten Steuerungshandlung: ein Akteur wendet sich „ohne Heranziehung technischer, bürokratischer, psychologischer oder kultureller Steuerungsformen" an einen anderen, um dessen/deren Unterordnung zu erreichen (Sandner, 1990: 174; vgl. auch die ,simple control' bei Tompkins \& Cheney, 1985: 181f.). Die Formulierung von Regeln in Führungsgrundsätzen, welche Ansprüche explizit festlegen, legitimiert jedoch erst die Ausübung direkter Steuerung durch die FK (bzw. die MA). Diese Steuerung durch die Festsetzung legitimer Ansprüche und Forderungen entspricht stark einem bürokratisch-regelhaften Steuerungsverständnis. Grundsätzlich ist die Logik dahinter dieselbe wie bei der Steuerung durch die Festlegung von Pflichten. Damit entspricht die Steuerungslogik weitestgehend Sandners (1990) Vorstellung der bürokratischen Steuerung (vgl. auch Tompkins \& Cheney, 1985:183). Es wird ein Rahmen für die legale Ausübung von Macht festgelegt (vgl. Sandner, 1990: 190), innerhalb dessen sich die Akteure bewegen können. Durch die explizite Aufzählung der Ansprüche in schriftlichen Führungsgrundsätzen sind diese Regeln dem formalen Regelsystem der Organisation zuzuordnen. Durch die Legitimierung solcher Ansprüche im Rahmen eines formalen Regelsystems löst sich die Unterordnungsaufforderung vom konkreten Akteur. Es entsteht der Anschein von Objektivität und Sachlichkeit (vgl. Sandner, 1990: 193). Damit wird verschleiert, dass - und dies gilt in besonderem Ausmaß für Führungsgrundsätze - die Machtausübenden (das Management, die Führungskräfte) sich nur an Regeln zu halten haben, welche sie selbst aufgestellt haben (vgl. Sandner, 1990:193). Regelsysteme - zu denen auch die Definition von Ansprüchen gehört - sind somit nicht nur ein Instrument der Herrschaftsausübung sondern auch der Herrschaftssicherung. 
Tabelle 7: Die Anspruchs-Beziehung (Überblick)

Rollenaspekte

FK: Steuermann und Leiter (explizit), Bereitsteller (explizit und implizit)

MA: Berechtigter (explizit), Bereitsteller (implizit)

Handlungslogik

fordern, verdienen, ein Recht haben vs. bereitstellen, versorgen

Konstitutiver Topos

Topos vom Fordern und Fördern

Ergänzende Topoi

Topos von der Transparenz als Allheilmittel

$F K \rightarrow M A$

$\mathrm{Ja}$, vor allem aktiv (fordern etwas ein)

$M A \rightarrow F K$

Ja, vor allem passiv (haben ein Recht, einen Anspruch)

$F K \leftarrow \rightarrow M A$

Ja, gegenseitiges Einfordern

$F K, M A \rightarrow$

FK $\rightarrow$ sich selbst

$M A \rightarrow$ sich selbst

Nein
Nein
Nein

\subsubsection{Die Befähigungs-Beziehung}

\section{Handlungslogik und Rollenaspekte}

Die grundsätzliche Logik der Befähigungs-Beziehung zielt darauf ab, den anderen zu einer Handlung zu befähigen. Die Beziehung tritt im Diskurs in zwei leicht unterschiedlichen Varianten auf. Davon gehorcht die dominante Variante in erster Linie einer Logik der Ermöglichung (das ,Können“). Die zentrale Handlung besteht dabei darin, dass eine Partei für eine andere Partei gewisse Dinge schafft/gestaltet, gewährleistet oder fördert. Die entsprechenden Rollenaspekte sind daher jene des Schöpfers und Gestalters, des Gewährleisters und des Unterstützers und Förderers. Die empfangende Partei wird zwar genannt, ist aber selbst nicht aktiv bzw. tritt so als rein passiver Empfänger auf (der passivabhängige Akteur). Die zweite, periphere Variante thematisiert die Ermächtigung zur Handlung (das ,Dürfen'). In einigen der Äußerungen verschwimmt aber die Grenze zwischen diesen konzeptionell unterschiedlichen Formen der Befähigung. 


\section{Argumentationsstruktur - relevante Topoi}

Die Befähigungs-Beziehung ist eine von drei distinktiven Beziehungen, welche durch den Topos von der Bedeutung des Arbeitsumfelds und Arbeitsklimas konstituiert und legitimiert werden:

Die Leistungsbereitschaft und-fähigkeit von Menschen hängt stark von den Rahmenbedingungen und Kontextfaktoren ab, unter welchen sie diese Leistung zu erbringen haben.

Die ,Befähigung' in dieser Beziehung ist somit keine persönliche Befähigung (wie z.B. in der Entwicklungs-Beziehung), sondern eine Befähigung durch Kontextgestaltung. Die aktive Partei befähigt die empfangende Partei zu höherer Leistung, indem sie ein leistungsförderndes Umfeld schafft/gestaltet, gewährleistet oder fördert. Ein ergänzender Topos ist in dieser Beziehung jener vom Zusammenhang von Freiraum und Motivation:

Motivation entsteht nur dort, wo man ihr Freiraum zur Entfaltung lässt. Übermäßige Kontrolle erzeugt ihre eigenen Prämissen.

Freiraum ist in diesem Zusammenhang also ein entscheidender Kontextfaktor, welcher die Motivation beeinflusst. Die Argumentationskette geht aber noch weiter, denn ebenfalls von Relevanz ist der Topos vom Zusammenhang zwischen Motivation und Leistung, welcher von einer Leistungsperspektive den Topos vom Zusammenhang von Freiraum und Motivation stützt:

Motivierte MitarbeiterInnen sind leistungsbereite MitarbeiterInnen.

Dieser Topos schließt die Lücke zwischen Freiraum und Leistung. Freiraum bzw. Erhöhung des Verantwortungsbereiches motiviert, diese erhöhte Motivation führt zu erhöhter Leistung. Die Frage schließlich, wie hoch der gewährte Freiraum sein soll, wird vom Topos von der Einheit von Kompetenz und Verantwortung argumentiert:

Die Leistung einer Person ist dann am höchsten, wenn sie an ihrem Arbeitsplatz weder unternoch überfordert ist. Der höchste Grad an Leistung wird dort erreicht, wo ein hoher ,Fit “ zwischen Person und Arbeitsplatz bzw. Verantwortung besteht.

Jeder soll also den maximalen Freiraum und Verantwortungsbereich erhalten, für welchen seine Kompetenz ausreicht. Die vier Topoi erzeugen gemeinsam ein klares, stringent argumentiertes Bild der Befähigungs-Beziehung: Um einen möglichst hohen Grad an Leistung zu erreichen, muss ein Akteur (neben anderen Kontextbedingungen) möglichst viel Freiraum für seine Tätigkeit erhalten. Dieser Freiraum steigert seine Motivation, welche wiederum seine Leistung erhöht. Begrenzt wird der mögliche Freiraum durch die Kompetenz des Akteurs, da eine Überforderung den Leistungsoutput wieder senken würde. 


\section{Interaktionsstruktur}

Im Gegensatz zur Anspruchs-Beziehung ist die Befähiger-Beziehung rein unidirektional ausgestaltet. Aktive Partner sind ausschließlich die FK, deren MA sind passive ,Empfänger' bzw. ,Nutzer' des von den Führungskräften geschaffenen Umfelds. Die drei zu unterscheidenden Handlungsformen lassen sich in eine Hierarchie bringen:

Die stärkste Form der Kontextgestaltung ist die schaffend/gestalterische. Hier wird von FK erwartet, dass sie Kontextbedingungen gezielt herstellen bzw. verändern. Der Fokus liegt auf dem schöpferischen Aspekt:

Wir schaffen Rahmenbedingungen und Freiräume, die es den Mitarbeiterinnen und Mitarbeitern ermöglichen, an der Verwirklichung der Unternehmensziele mit ihrer bestmöglichen Leistung mitzuwirken und ihre Talente und Potenziale zu entfalten. (D 01)

In einer etwas abgeschwächten Form treten die FK als Gewährleister in Erscheinung. Sie stellen Kontextbedingungen sicher, sorgen für sie bzw. sind für deren Ausgestaltung verantwortlich:

Führen ist ein sozialer Prozess. Es beeinflusst maßgeblich das Verhalten von Personen und Gruppen und deren Stimmung. Die Qualität der Beziehung ist wichtig für die Unternehmenskultur. Die Führungspersönlichkeit macht den ersten Schritt - Sie ist für das Beziehungsumfeld verantwortlich. (A 15)

In der dritten Ausprägung sind die FK Förderer eines gewünschten Zustandes. Sie wirken auf diesen Zustand hin, schaffen ihn aber nicht direkt und garantieren auch nicht dessen Eintreten:

Sie wirken auf die reibungslose Zusammenarbeit zwischen den Mitarbeitern hin und fördern die Leistungsfähigkeit der Organisationseinheit insgesamt. (D 03)

Schließlich ist auch noch der Ermächtigungsaspekt Teil des Diskurses. Führungskräfte befähigen MitarbeiterInnen zu eigenen Entscheidungen, z.B. durch Delegation:

Ermächtigt Mitarbeitende, Entscheidungen auf der niedrigstmöglichen Stufe zu fällen. (CH 09)

Auch wenn die MA als distinktiver Akteurstypus nicht befähigend aktiv werden, so werden sie doch in Einzelfällen an der Gewährleistung von Rahmenbedingungen beteiligt. Inhaltlich zielt die Befähigungs-Beziehung immer entweder auf ein gutes Leistungsumfeld (CH14) oder ein gutes Beziehungsumfeld (A15) ab. Maßnahmen dafür liegen hauptsächlich im Bereich der Delegation (D01, A07, CH18), Information (D01) oder der Prozessgestaltung (A13). 
Die Steuerung in der Befähiger-Beziehung kann nicht klar einer der diskutierten Steuerungsformen zugeordnet werden. Dazu sind die Einflussmöglichkeiten der FK auf die MA zu vielseitig. Die Befähigung von MA durch die klassische Ausgestaltung des Arbeitsplatzes, also der physischen Faktoren der Leistungserstellung, entspricht im Wesentlichen der technologischen Steuerung (vgl. Sandner, 1990; Tompkins \& Cheney, 1985). Befähigung zu einer spezifischen Art von Tätigkeit kann auch gleichzeitig Beschränkung der möglichen anderen Arten von Tätigkeiten bedeuten. Durch technologische Steuerung wird der Handlungsspielraum der Akteure verengt: „Es wird ein Handlungsrahmen definiert, der vorgibt, welche Handlungen wann, wo, wie und in welcher Intensität erwartet werden, und welche nicht" (Sandner, 1990:186). Die Befähiger-Beziehung argumentiert nun aber gerade in die entgegengesetzte Richtung. Die Handlungsspielräume der MA sollen erweitert, nicht verengt werden. Deshalb liegt hier eine Kombination mit der psychologischen Steuerung vor, vor allem mit der Strategie der ,Pflege des Arbeitsfeldes', bei der es darum geht, „Rahmenbedingungen zu schaffen, um die eigentliche berufliche Tätigkeit von äußeren Beeinträchtigungen möglichst frei zu halten und das Arbeitsumfeld attraktiv zu gestalten“" (Sandner, 1990: 200). Auch durch die Betonung der Erweiterung des Verantwortungsbereichs und damit auch des Handlungsspielraums wird der Wert der MA betont und berücksichtigt. Diese Erweiterung wird als Befriedigung der Wünsche und Bedürfnisse der MA kommuniziert, stellt aber im Wesentlichen eine „strategische, begrenzte Öffnung von Handlungsspielräumen“ (Sandner, 1990: 205) dar, um die Ziele des Unternehmens zu realisieren. Damit ist ein Hauptcharakteristikum psychologischer Steuerung angesprochen: die instrumentelle Befriedigung von MA-Wünschen (vgl. Sandner, 1990: 198). Außerdem kann - wie für den Topos vom Zusammenhang zwischen Freiraum und Motivation diskutiert - diese Betonung des Gebens bzw. des Vertrauens in die MA gemäß Cheney (1983: 148ff.) als Versuch der Herstellung von Identifikation im Rahmen der ,Common Ground Technique، gesehen werden. Die Steuerungswirkung entsteht durch die Kombination von Kontextgestaltung im Sinne organisationaler Zielsetzungen bei gleichzeitiger Kommunikation dieser Kontextgestaltung als Handlung im Sinne der MA. Die Instrumentalität dieser Vorgehensweise wird bei genauerer Betrachtung der relevanten Topoi offensichtlich. 
Tabelle 8: Die Befähigungs-Beziehung (Überblick)

Rollenaspekte

FK: Gestalter und Schöpfer, Gewährleister, Unterstützer und Förderer (explizit)

MA: Der passiv-abhängige Akteur (implizit)

Handlungslogik

Schaffen, gestalten bzw. gewährleisten, verantworten bzw. fördern, unterstützen vs. passive Abhängigkeit

Konstitutiver Topos

Topos von der Bedeutung des Abeitsumfelds und Arbeitsklimas

Ergänzende Topoi

Topos vom Zusammenhang von Freiraum und Motivation

Topos vom Zusammenhang von Motivation und Leistung

Topos von der Einheit von Kompetenz und Verantwortung

$F K \rightarrow M A$

$M A \rightarrow F K$

$F K \leftrightarrow \rightarrow M A$

$\mathrm{Ja}$

Nein

Nein

$F K, M A \rightarrow$

Ja, gemeinsames Gewährleisten

FK $\rightarrow$ sich selbst

Nein

$M A \rightarrow$ sich selbst

Nein

\subsubsection{Die Bereitstellungs-Beziehung}

\section{Handlungslogik und Rollenaspekte}

Sehr ähnlich der Befähigungs-Beziehung tritt in der Bereitstellungs-Beziehung ein aktiver ,Geber' einem passiven ,Empfänger' gegenüber. Die Handlungslogik ist dabei in erster Linie jene des Bereitstellens/Versorgens. Wird für den Empfänger eigens etwas geschaffen, so kann auch die Handlung des Schaffens/Gestaltens relevant sein. Relevante Rollenaspekte sind für den aktiven Akteur deshalb jener des Bereitstellers bzw. Providers und jener des Schöpfers und Gestalters. Der Empfänger tritt implizit als passiv-abhängiger Akteur auf. Im Unterschied zur Befähigungs-Beziehung geht es hier um die direkte Übergabe eines (materiellen oder immateriellen) ,Objekts“ und nicht so sehr um die Gestaltung von Rahmenbedingungen. Im Unterschied zur Anspruchs-Beziehung ist 
der Impetus hier im Bereich des Gebens und nicht im Bereich des Forderns. Die Bereitstellung erfolgt pro-aktiv.

\section{Argumentationsstruktur - relevante Topoi}

Die Bereitstellungs-Beziehung ist die zweite von drei Beziehungen, welche von einer Spielart des Topos von der Bedeutung des Arbeitsklimas und Arbeitsumfelds konstituiert und legitimiert werden:

Die Leistungsbereitschaft und-fähigkeit von Menschen hängt stark von den Rahmenbedingungen und Kontextfaktoren ab, unter welchen sie diese Leistung zu erbringen haben.

Der Unterschied zwischen der Bereitstellungs-Beziehung und der BefähigungsBeziehung liegt deshalb in erster Linie in der oben diskutierten unterschiedlichen Handlungslogik. Die vier zentralen ,Objekte', die bereitgestellt werden, sind Freiraum bzw. Gestaltungsspielraum, Orientierung, Information und Vertrauen. Jedes dieser vier Elemente wird in einem eigenen ergänzenden Topos näher beschrieben und argumentiert, nämlich (a) im Topos vom Zusammenhang von Freiraum und Motivation

Motivation entsteht nur dort, wo man ihr Freiraum zur Entfaltung lässt. Übermäßige Kontrolle erzeugt ihre eigenen Prämissen.

(b) im Topos von der Notwendigkeit von Orientierung und Sicherheit

Effiziente Leistungserstellung ist nur dann möglich, wenn ein gemeinsamer Weg und geteilte Verständnisse existieren, damit grundlegende (Routine)Fragen nicht mehr in jedem Einzelfall geklärt werden müssen.

(c) im Topos vom Primat von Information und Wissen

Information und Wissen machen handlungsfähig und verbessern die Entscheidungsqualität. Information ist deshalb auf allen Ebenen und in allen Bereichen zu fördern.

und (d) im Topos vom Vertrauen als Basis organisationaler Beziehungen:

Ohne ein grundsätzliches Vertrauen ist Zusammenarbeit nicht möglich. Vertrauen ersetzt in einem gewissen Maß Kontrolle und macht dadurch handlungsfähig.

Mit dem Topos vom Zusammenhang von Freiraum und Motivation zeigt sich erneut die Schnittstelle zur Befähigungs-Beziehung. Der Unterschied liegt in der Unmittelbarkeit der Gewährung von Freiraum. Während in der BefähigungsBeziehung Rahmenbedingungen geschaffen werden, welche einen erhöhten Freiraum bewirken, wird in der Bereitstellungsbeziehung Freiraum direkt gewährt oder übertragen. Der Unterschied mag in der Praxis rein semantisch sein, in der hier gewählten Logik der Beziehungs(re-)konstruktion sind die unter- 
schiedlichen Logiken und Grade der Einwirkung eines Akteurs auf den anderen zu unterscheiden.

\section{Interaktionsstruktur}

Die Bereitstellungsbeziehung existiert im Diskurs in erster Linie top-down. Die Führungskraft gibt, schenkt, bietet oder überträgt den MA etwas:

Mitarbeiter mit Kompetenzen ausstatten, um Entscheidungen so kundennah wie möglich zu treffen (D 02)

Wir übertragen unseren Mitarbeitern Verantwortung und geben ihnen den Freiraum und die Unterstützung, diese aktiv wahrzunehmen. (D 07)

Der Diskurs ermöglicht aber auch die bottom-up Bereitstellung:

Neben der Informationspflicht seitens der Führungskräfte besteht für jeden Mitarbeiter die Verpflichtung, wichtige Informationen, soweit sie die Unternehmung bzw. ihr Umfeld betreffen, unverzüglich an die unmittelbaren Vorgesetzten bzw. die betroffenen Stellen weiterzugeben. (A 04)

In einer Äußerung existiert auch das gegenseitige Versorgen mit Informationen. Hier wird die grundsätzliche Verwandtschaft zwischen Anspruchs-Beziehung und Bereitstellungs-Beziehung deutlich, da die Aussage die Handlungen des Forderns und des Gebens miteinander vereint:

Die Führungskraft und der Mitarbeiter bei [Unternehmen] sind gleichermaßen verpflichtet, Informationen rechtzeitig zu geben („,Bring-Schuld“) als auch notwendige Informationen einzufordern (,Hol-Schuld“). (D 14)

Damit sind Bottom-up-Bereitstellung und Gegenseitigkeit im Diskurs zwar angelegt bzw. möglich, die dominante Ausprägung ist jedoch klar die Top-downVariante des Beziehungstyps.

\section{Steuerungsaspekte}

Die Steuerung in der Bereitstellungs-Beziehung entspricht im Wesentlichen jener der Befähigungs-Beziehung. Die Ausstattung der MA mit bzw. die Übertragung von Kompetenzen an die MA entspricht der Logik einer Erweiterung des Handlungsspielraums. Die Bereitstellung anderer Faktoren und Ressourcen wirkt letztlich ebenfalls auf den Handlungsspielraum des Individuums ein. Die Logik der Bereitstellung ist hier aber keine des Belohnens, da nicht die MA, sondern die Organisation davon profitiert. Vielmehr besteht hier eine Gestaltung des Arbeitsprozesses durch Ressourcenzuteilung, die wiederum einem technologischen Steuerungsverständnis (vgl. Sandner, 1990: 182ff.) entspringt. Die Unterordnungsaufforderung kann jedoch im individuellen Verhältnis zwischen FK und MA zu einer Dependenzherstellung durch Versprechen oder Drohung werden (vgl. Sandner, 1990: 105 bzw. 101), wenn im Rahmen einer Krisensituation 
die FK (deren Ressourcenverfügung in den Dokumenten legitimiert wird) dem MA zusätzliche Ressourcen in Aussicht stellen bzw. den Entzug von Ressourcen androhen muss, um dessen Unterordnung weiter zu erhalten.

Tabelle 9: Die Bereitstellungs-Beziehung (Überblick)

Rollenaspekte

FK: Bereitsteller und ,Provider', Schöpfer und Gestalter (explizit) bzw. passiv-abhängiger Akteur (implizit)

MA: passiv-abhängiger Akteur (implizit), aber auch z.T. Bereitsteller und ,Provider` (explizit)

Handlungslogik

Konstitutiver Topos

schaffen, gestalten bzw. bereitstellen, versorgen vs. passive Abhängigkeit

Ergänzende Topoi

Topos von der Bedeutung des Arbeitsklimas und Arbeitsumfelds

Topos vom Zusammenhang von Freiraum und Motivation

Topos von der Notwendigkeit von Orientierung und Sicherheit

Topos vom Primat von Information und Wissen

Topos vom Vertrauen als Basis organisationaler Beziehungen

$F K \rightarrow M A$

Ja

$M A \rightarrow F K$

$F K \leftrightarrow \rightarrow M A$

Ja, gegenseitiges Bereitstellen / Versorgen

$F K, M A \rightarrow$

FK $\rightarrow$ sich selbst

Nein

Ja

$M A \rightarrow$ sich selbst

Nein

\subsubsection{Die Begrenzungs-Beziehung}

\section{Handlungslogik und Rollenaspekte}

Die Begrenzungs-Beziehung ist eine nur sehr schwach verbreitete Beziehung, die aber gerade dadurch, dass sie den ansonsten durchwegs positiven Führungsdiskurs durchbricht, den Diskurs wesentlich erweitert. Die grundlegende Handlungslogik hinter der Begrenzungs-Beziehung ist die Umkehrung der Anspruchs- 
Beziehung - eine Forderung wird zurückgewiesen. Damit ist hier vor allem der Rollenaspekt des Akteurs (sowohl FK als auch MA) als Verhinderer relevant. Die implizite Gegenrolle, die in den Äußerungen aber nie direkt angesprochen wird, ist für FK jene des Steuermannes bzw. Leiters und für MA die des (scheinbar) Berechtigten. Scheinbar ist diese Berechtigung deshalb, weil der sonst klar formulierte Anspruch hier fehlt. Deshalb muss die FK die Forderung zurückweisen.

\section{Argumentationsstrukturen - relevante Topoi}

Die Begrenzungs-Beziehung bezieht sich inhaltlich ausschließlich auf die Abgrenzung von Verantwortlichkeiten und nimmt ihre Begründung und Legitimation deshalb aus dem Topos der Einheit von Kompetenz und Verantwortung.

Die Leistung einer Person ist dann am höchsten, wenn sie an ihrem Arbeitsplatz weder unternoch überfordert ist. Der höchste Grad an Leistung wird dort erreicht, wo ein hoher ,Fit * zwischen Person und Arbeitsplatz bzw. Verantwortung besteht.

Die Argumentation besteht in einer spezifischen Auslegung dieser Schlussregel: Dort, wo Kompetenz und Verantwortung bereits übereinstimmen, ist zu verhindern, dass der kompetente Akteur in seiner Entscheidungsfindung gestört wird. Durch das geringe quantitative Vorkommen des Topos im Diskurs sind keine relevanten ergänzenden Topoi zu identifizieren. Die Begrenzungs-Beziehung regelt einen sehr abgegrenzten, kleinen Teil des Verhältnisses zwischen FK und MA.

\section{Interaktionsstruktur}

Grundsätzlich können FK und MA gleichermaßen die Beziehung initiieren, je nachdem, von wem die (ungerechtfertigte) Forderung stammt:

Durch die angemessene und vertrauensvolle Delegation von Aufgaben und Kompetenzen fördern sie [Anm. die FK] deren [Anm. MA] Selbstverantwortung und Eigenständigkeit. Die Rückdelegation von Aufgaben lassen sie nicht zu. (D 03)

Deshalb hat auch MitarbeiterIn Anspruch darauf, einen klar abgegrenzten Zuständigkeitsbereich zur selbständigen und eigenverantwortlichen Durchführung übertragen zu bekommen; in diesem Rahmen sollte MitarbeiterIn jede versuchte Einmischung einer(s) Vorgesetzten zurückweisen, solange die Erreichung der vereinbarten Ziele hinlänglich gewährleistet ist. (D 08)

Eine etwas abweichende Spielart der Begrenzungs-Beziehung findet sich dort, wo FK und MA gemeinsam etwas beenden bzw. lösen. In diesem Fall tritt der Beziehungs-Aspekt allerdings in den Hintergrund. Beendet wird nicht etwas zwischen den beiden Akteuren, sondern ein Problem, mit dem beide konfrontiert sind. 


\section{Steuerungsaspekte}

Der Charakter der Begrenzungs-Beziehung als ,Spiegelbild“ der AnspruchsBeziehung zeigt sich auch in ihrer Steuerungslogik. Die Begrenzungshandlungen selbst sind Teil eines direkten Steuerungsverständnisses (vgl. Sandner, 1990: 174). Wie bei der Anspruchsbeziehung wird aber diese direkte Steuerung durch eine bürokratische Steuerung in Form expliziter Regeln (vgl. Sandner, 1990: 190) legitimiert und unterstützt. Nicht die individuelle Macht eines Akteurs definiert die Beziehung, sondern die Macht und Legitimation einer abstrakten Regel (Wer darf was in welchem Rahmen zurückweisen bzw. verhindern?). Das erlaubt auch, dass die Akteure bei der Regelbefolgung grundsätzlich gleichberechtigt sind - die Regel gilt für alle gleichermaßen.

Tabelle 10: Die Begrenzungs-Beziehung (Überblick)

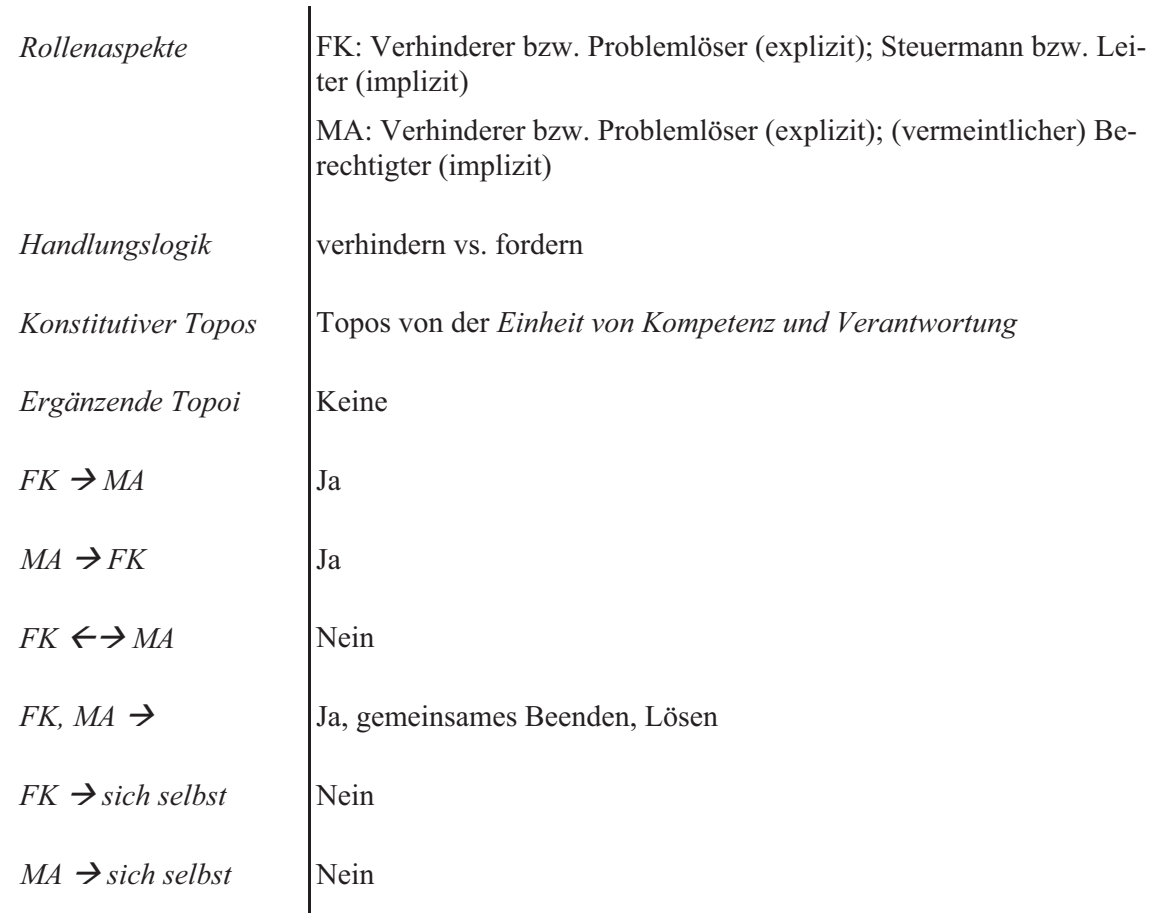




\subsubsection{Die Empathie-Beziehung}

Handlungslogik und Rollenaspekte

Die Empathie-Beziehung folgt in erster Linie der Handlungslogik des Erkennens bzw. Verstehens des anderen. Daraus abgeleitet werden auch noch das Respektieren bzw. Anerkennen des anderen, das Berücksichtigen seiner Eigenheiten und Wünsche sowie das Vertrauen in den anderen in diesen Typus inkludiert. Die dominanten Rollenaspekte sind somit jene des Kenners und des Beziehungsmanagers. Aber auch der den Rahmenbedingungen unterworfene Akteur ist insofern relevant, als er die Eigenheiten und Wünsche anderer Akteure zu berücksichtigen hat. Das Gegenstück stellt für gewöhnlich der passiv-abhängige Akteur dar, der vom Beziehungsmanager angemessen behandelt wird.

Argumentationsstruktur - relevante Topoi

Mit der Betonung individueller persönlicher Eigenheiten, Wünsche und Bedürfnisse folgt die Empathie-Beziehung argumentativ stark dem Topos von der $\mathrm{Be}$ deutung des Einzelnen und wird von diesem konstituiert:

Jeder Einzelne im Unternehmen, sei das Aufgabengebiet auch noch so klein, leistet einen wesentlichen Beitrag (positiv oder negativ) zum Unternehmenserfolg.

Wenn der Einzelne bzw. das Individuum im Unternehmen wichtig für den Erfolg der Organisation ist, dann ist es notwendig, sich entsprechend um das Individuum zu kümmern. Die Grundlogik der Empathie-Beziehung ist also eine stark utilitaristische. Innerhalb dieser Grundannahme besitzt die EmpathieBeziehung zwei hauptsächliche Ausprägungen. Zum einen existiert im Diskurs eine in erster Linie rationale Ausprägung, welche das Erkennen bzw. Verstehen der MA durch die FK zur Voraussetzung für den optimalen Arbeitseinsatz derselben macht. Diese Ausprägung folgt dem Topos der Einheit von Kompetenz und Verantwortung. Die FK kennt und berücksichtigt die Stärken, Schwächen, Potentiale und Neigungen seiner MA und setzt diese entsprechend ein:

Die Leistung einer Person ist dann am höchsten, wenn sie an ihrem Arbeitsplatz weder unternoch überfordert ist. Der höchste Grad an Leistung wird dort erreicht, wo ein hoher ,Fit * zwischen Person und Arbeitsplatz bzw. Verantwortung besteht.

In Zusammenhang damit steht auch ein weiterer, deutlich weniger dominanter Topos, nämlich jener von der direkten/situativen Führung. Um die MA zu kennen und entsprechend einsetzen zu können, ist es nötig, dass sich die Führungskraft direkt und persönlich mit jedem einzelnen auseinandersetzt. In der zweiten, stärker emotional ausgeprägten Variante führt das Erkennen/Verstehen des anderen zu einer Beziehung, die von Respekt, Anerkennung und Vertrauen geprägt 
ist. Dieser Ausprägung entspricht der argumentative Topos vom Vertrauen als Basis organisationaler Beziehungen:

Ohne ein grundsätzliches Vertrauen ist Zusammenarbeit nicht möglich. Vertrauen ersetzt in einem gewissen Maß Kontrolle und macht dadurch handlungsfähig.

Auch der Topos von der positiven Diversität ist von Relevanz. Er verlangt innerhalb der Empathie-Beziehung, dass Unterschiede innerhalb des sozialen Gefüges des Unternehmens wertgeschätzt und respektiert werden (für eine alternative Verwendung dieses Topos siehe die Verfügungs-Beziehung).

\section{Interaktionsstruktur}

Die rationale Ausprägung der Empathie-Beziehung existiert im Diskurs ausschließlich in einer Top-Down Variante. Die FK kennt die MA und kann ihnen deshalb entsprechende Verantwortlichkeiten und Aufgaben übertragen:

Die anfallenden Aufgaben und Aufträge delegieren sie unter Einbeziehung der Stärken und Fähigkeiten ihrer Mitarbeiter nach dem Grundsatz: „Der richtige Mitarbeiter zur richtigen Zeit am richtigen Ort“. (D 03)

Die emotionale Ausprägung hingegen läuft in beide Richtungen. FK vertrauen den MA und respektieren sie und vice versa. Gegenseitiger Respekt wird auch explizit thematisiert:

Ihre Mitarbeiter sind leistungsfähig und leistungsbereit. Wagen Sie es, mit wenig Kontrolle auszukommen. Ihr Vertrauen wird den unternehmerischen Schwung auslösen, den wir alle wollen. (A 01)

Wir respektieren uns gegenseitig als Partner. $(\mathrm{CH} 01)$

Als Spezialfall erscheint schließlich die Empathie gegenüber sich selbst. Ausschließlich FK wird im Diskurs eine selbstkritische und selbstreflektierte Kompetenz zugeschrieben. FK kennen auch ihre eigenen Stärken, Schwächen und Entwicklungspotentiale und stellen ihre Handlungen und Entscheidungen in Frage:

[D]ie eigenen Stärken und Entwicklungsbedürfnisse erkennen (D02)

\section{Steuerungsaspekte}

Ein wesentliches Merkmal der psychologischen Steuerung bei Sandner (1990) ist die Tatsache, dass sie auf symbolischer Ebene durch den ,Mensch-imMittelpunkt-Mythos' ${ }^{6}$ ausgedrückt wird (vgl. Sandner, 1990: 199). Dies ist aber gerade die Hauptaussage des Topos von der Bedeutung des Einzelnen. Während die Befähigungs- und die Bereitstellungs-Beziehung innerhalb der psychologischen Steuerung auf die Selbststeuerung der MA als Strategie setzen, stützt sich die Empathie-Beziehung stark auf die ,Pflege der sozialen Beziehungen“ 
(vgl.Sandner, 1990: 199f.), und dort vor allem auf die Pflege der Aufrechterhaltung der Zusammenarbeit: „Das patriarchalisch-joviale (Schein-)Interesse für außerbetriebliche Angelegenheiten der Mitarbeiter (z.B. ,wie war das Wochenende?', ,was machen die Kinder?') als einleitende oder abschließende Umrahmung (Verpackung) betrieblicher Leistungsaufforderungen ist ebenfalls Ausdruck eines solchen ,pflegenden" Umgangs mit den Mitarbeitern“" (Sandner, 1990: 201).

Tabelle 11: Die Empathie-Beziehung (Überblick)

Rollenaspekte

FK: Kenner bzw. Beziehungsmanager (explizit), den Rahmenbedingungen unterworfener Akteur (explizit), passiv-abhängiger Akteur (implizit)

MA: passiv-abhängiger Akteur (implizit), Beziehungsmanager (explizit)

Handlungslogik

Berücksichtigen, erkennen und wissen, anerkennen und respektieren, vertrauen vs. passive Abhängigkeit

Konstitutiver Topos

Topos von der Bedeutung des Einzelnen

Ergänzende Topoi

Topos von der Einheit von Kompetenz und Verantwortung

Topos von der direkten/situativen Führung

Topos vom Vertrauen als Basis organisationaler Beziehungen

Topos von der positiven Diversität

$F K \rightarrow M A$

$\mathrm{Ja}$

$M A \rightarrow F K$

Ja, MA vertrauen den FK

$F K \leftrightarrow \rightarrow M A$

Ja, gegenseitiger Respekt

$F K, M A \rightarrow$

Nein

$F K \rightarrow$ sich selbst

Ja, selbstreflektiertes Erkennen

$M A \rightarrow$ sich selbst

Nein 


\subsubsection{Die Entwicklungs-Beziehung}

Handlungslogik und Rollenaspekte

In der Entwicklungs-Beziehung besteht das vorrangige Ziel in der Entwicklung, Steigerung oder Verbesserung des anderen. Die zentrale Handlungslogik ist demzufolge das Schaffen/Gestalten im Sinne einer Steigerung des Existierenden. Insofern, als es diesem Ziel dient, sind dabei auch fördernde/unterstützende bzw. gewährleistende Handlungen möglich. Diesen Handlungsformen entsprechen die (re-)konstruierten Rollenaspekte des Schöpfers und Gestalters, des Förderers und des Gewährleisters. Auf der empfangenden Seite steht der passivabhängige Akteur, welcher Potential besitzt, dieses aber selbst (in der Mehrzahl der Äußerungen) nicht zu realisieren imstande ist.

Argumentationsstruktur - relevante Topoi

Das Argumentarium der Entwicklungs-Beziehung kennt einen zentralen konstitutiven Topos, nämlich jenen vom Stillstand als Tod:

Da sich das Umfeld (und damit die Anforderungen an das Unternehmen und den Einzelnen) ständig ändert, muss jeder konstant vorwärts gehen und sich verbessern bzw. anpassen. Wer sich nicht mitbewegt, der bleibt auf der Strecke.

Dieser Topos bezieht sich somit nicht nur auf den Aspekt des Marktes (,die Konkurrenz schläft nicht'), sondern auch auf die interne Personalentwicklung. Wer nicht bereit bzw. in der Lage ist sich weiterzuentwickeln, der stellt einen Problemfaktor für das Unternehmen dar. In der Folge dieser Grundargumentation wird auch hin und wieder der Topos von der positiven Veränderung evoziert:

Jede Veränderung beinhaltet den Kern einer neuen Möglichkeit, wenn man bereit ist, diesen zu sehen.

In Kombination mit diesem Topos wird die Aufforderung zur Veränderung nicht nur zur lästigen Pflicht, sondern zum genuinen Interesse jedes einzelnen Akteurs. Wie schon in der Anspruchs-Beziehung ist auch in der EntwicklungsBeziehung der Topos vom Fordern und Fördern von Relevanz. Allerdings wird hier bewusst die Seite des ,Förderns' betont, während die Anspruchs-Beziehung sich vor allem auf die Seite des ,Forderns' konzentriert. Insofern als Entwicklungsbemühungen oft Team-Building und Zusammenarbeit betreffen, ist auch der Topos Gemeinsam sind wir stärker inkludiert. Inhaltlich steht - neben der grundsätzlichen fachlichen und persönlichen Weiterentwicklung - vor allem die Entwicklung von Eigenverantwortung und Mitunternehmertum im Zentrum. Diese wird von den beiden Topoi des Mitunternehmertums und vom ,No risk, no gain ' abgedeckt und schlägt die Brücke zu Themen im Bereich des Marktes und 
des unternehmerischen Handelns, welche nicht mehr unmittelbar der Führungsbeziehung zugehörig sind.

\section{Interaktionsstruktur}

Die Entwicklungsbeziehung ist im Diskurs der Führungsgrundsätze eindeutig unidirektional. Die FK entwickeln die MA, aber umgekehrt findet keine Entwicklungstätigkeit statt. Die Verantwortungen sind in dieser Hinsicht eindeutig geklärt:

Sehen Sie bei Ihren Mitarbeitern die Stärken und helfen Sie, diese zu nutzen und weiter auszubauen. (A 01)

Es liegt in der Verantwortung der Führungskräfte, die Fähigkeiten der Mitarbeiter zur Wirkung zu bringen. (D 02)

Ein vergleichbar starkes Element in der Entwicklungs-Beziehung ist - im Vergleich zu anderen Beziehungstypen - die Beschäftigung des Akteurs mit sich selbst. Sowohl FK als auch MA wird die Verantwortung auferlegt, für ihre eigene Weiterentwicklung und Weiterbildung aktiv zu werden. Dies ist die einzige Form, in welcher die MA in dieser Beziehung tatsächlich tätig aktiv werden:

[Die Führungskraft] beobachtet sich selbst und holt aktiv Rückmeldungen ein, um Stärken und Schwächen zu erkennen, geht konstruktiv mit eigenen Schwächen um; lernt aus Fehlern und Misserfolgen. Zeigt die Fähigkeit und den Willen, zu lernen und neue Ideen umzusetzen, verbessert laufend die eigenen organisatorischen, führungsbezogenen und technischen Fähigkeiten. (CH 09)

Der Mitarbeiter nimmt an internen und externen Bildungsveranstaltungen teil. (D 14)

Während gegenseitige Entwicklung zwischen FK und MA aus dem Diskurs ausgeschlossen ist, kommt es vor, dass die MA sich selbst gemeinsam mit den FK weiterentwickeln. Diese Ausgestaltung befindet sich jedoch stark an der Peripherie des Beziehungstyps.

\section{Steuerungslogik}

Die Entwicklungs-Beziehung spielt im Wesentlichen damit, dass die Organisation den MA für ihre Unterordnung eine ,Verbesserung' der eigenen Person und der eigenen Leistungsfähigkeit in Aussicht stellt. Wenn die Prämissen der Argumentation - nämlich (a) die MA besitzen Potential, um besser zu werden, (b) die MA müssen besser werden, um ihren Wert zu erhalten und (c) die FK besitzen die Fähigkeit, Entwicklungspotentiale zu erkennen und in Folge auch zu realisieren - von den MA akzeptiert werden, dann ergibt sich daraus quasi ,zwingend" der Schluss, dass sie sich den FK unterordnen und sich von ihnen entwickeln lassen. Die Herrschaftssicherung der FK entsteht hier also in einer klar 
hierarchischen Charakterisierung der Fähigkeiten der beiden Akteure. Die FK sind wissend und handlungsermächtigt, die MA von den FK abhängig. Die metaphorische ,Karotte', welche den MA dieses Bild schmackhaft machen soll, besteht in der Evokation eines gesellschaftlichen Wertes, nämlich der ständigen Verbesserung. Die Steuerungsmechanik ist deshalb hier sowohl psychologisch (Anreizsetzung) als auch kulturell (die Art des Anreizes ist nichts Greifbares, sondern die Erfüllung gesellschaftlicher Erwartungen). Dieser ,kulturell resonierende' Anreiz regelt auch die Selbststeuerung dort, wo von MA und FK erwartet wird, dass sie für ihre eigene Entwicklung sorgen. Die Erfüllung organisationaler Erwartungen wird durch ihre Gleichschaltung mit sozialen Erwartungen verstärkt.

Tabelle 12: Die Entwicklungs-Beziehung (Überblick)

Rollenaspekte

FK: Schöpfer und Gestalter, Förderer, Gewährleister (explizit)

MA: passiv-abhängiger Akteur (implizit)

Handlungslogik

Schaffen, gestalten / fördern, unterstützen / gewährleisten, verantworten vs. passive Abhängigkeit

Konstitutiver Topos

Topos vom Stillstand als Tod

Ergänzende Topoi

Topos von der positiven Veränderung

Topos vom Mitunternehmertum

Topos No Risk, no Gain

Topos Gemeinsam sind wir stärker

Topos vom Fordern und Fördern

$F K \rightarrow M A$

Ja

$M A \rightarrow F K$

$F K \leftrightarrow \rightarrow M A$

Nein

Nein

$F K, M A \rightarrow$

Ja, gemeinsam schaffend, gestaltend

FK $\rightarrow$ sich selbst

Ja, Eigenverantwortung für Entwicklung

$M A \rightarrow$ sich selbst

Ja, Eigenverantwortung für Entwicklung 


\subsubsection{Die Integrations-Beziehung}

\section{Handlungslogik und Rollenaspekte}

Die zentrale Handlungslogik hinter der Integrations-Beziehung ist das Einbeziehen des anderen in einen Prozess oder eine Tätigkeit. Dies geschieht in den Dokumenten in erster Linie auf kommunikative und argumentative Art und Weise - es wird aufgeklärt, erklärt, kommuniziert, informiert, einbezogen, etc. Das zentrale Beispiel hier ist das Vereinbaren von Zielen (im Gegensatz zum Setzen/Vorgeben von Zielen in der Steuerungs-Beziehung). Indirekt kann die Integration auch über Handlungen des Bereitstellens/Versorgens geschehen, indem Informationen und Erklärungen weitergeleitet oder zur Verfügung gestellt werden. Die zentralen Rollenaspekte für den aktiven Akteur sind hier jene des Kommunikators und des Koordinators. Der empfangende Akteur bleibt wiederum passiv-abhängig. Er kann die Integration nicht von sich aus herstellen, sondern muss auf die Bemühungen des aktiven Partners vertrauen.

Argumentationsstruktur - relevante Topoi

Die konstitutive Begründung für die Integration des anderen liefert der Topos von der Transparenz als Allheilmittel:

Sowohl effizientes Arbeiten als auch ein gutes Arbeitsklima sind nur dann möglich, wenn alle relevanten Faktoren bekannt und allen zugänglich sind. Verschleierung verunmöglicht optimale Lösungen.

Damit bietet dieser Topos die Grundmotivation für die Integration anderer Akteure. Nur was gemeinsam gewusst wird, kann auch gemeinsam für das Unternehmen eingesetzt werden. Uninformierte Akteure sind zwar u.U. eher bereit, Unterordnungsaufforderungen zu akzeptieren, sie werden aber in ihrer täglichen Arbeit auch suboptimale Entscheidungen treffen. Die Stärkung des gemeinsamen Wissens wird auch vom Topos Gemeinsam sind wir stärker thematisiert:

Ziele werden leichter erreicht, wenn alle, an einem Strang ziehen ' und Ressourcen gebündelt werden können.

Die Transparenz von Informationen, Entscheidungsprozessen und anderen organisationalen Faktoren ermöglicht aber erst das ,Ziehen an einem Strang'. Stark korrespondiert die Integrations-Beziehung auch mit allen jenen Topoi, welche die Bedeutung des Informationsflusses bzw. der Informationssymmetrie hervorheben. Dies ist vor allem der Topos des Primats von Information und Wissen:

Information und Wissen machen handlungsfähig und verbessern die Entscheidungsqualität. Information ist deshalb auf allen Ebenen und in allen Bereichen zu fördern. 
Im Zusammenhang mit dem Transparenz-Topos sind außerdem noch zwei weitere, am Rande relevante Topoi zu nennen, nämlich jene von der generellen Zielklarheit (Man muss wissen, wohin man will, um den Weg gehen zu können) und der Zielkoordination (Ziele können sich widersprechen und müssen angepasst werden). Der Topos von der Bedeutung der Kommunikation hingegen zielt stärker in die zwischenmenschliche Richtung. Er betont die Relevanz von Kommunikation in Bezug auf ein angenehmes und leistungsförderndes Arbeitsumfeld:

Gute und umfassende Kommunikation steigert sowohl das Arbeitsklima als auch die Effizienz der Arbeit.

Die Integrations-Beziehung argumentiert somit sowohl auf der Ebene einer rationalen Prozess- und Entscheidungsverbesserung als auch auf einer zwischenmenschlich-kommunikativen Ebene.

\section{Interaktionsstruktur}

Die Beziehung ist in erster Linie top-down ausgestaltet. FK besitzen Informationen und die Macht, diese weiterzugeben. Sie sind es auch, welche darüber entscheiden können, ob die MA in eine Entscheidung/einen Prozess eingebunden werden oder nicht. Die MA sind passiv und vom Willen der FK abhängig:

Ich spreche mit jedem Mitarbeiter ab, welche Entwicklungs- und Weiterbildungsmöglichkeiten geplant sind, z.B. zusätzliche Aufgaben, Projektarbeiten und Teilnahme an Seminaren. (D 16)

Die Führungskräfte beziehen ihre Mitarbeiter aktiv in diesen Prozess ein und vereinbaren mit ihnen im Rahmen von Zielvereinbarungsgesprächen (Führen durch Zielvereinbarung) herausfordernde und realistische Ziele. (D 03)

Die Bottom-up-Variante ist leicht unterschiedlich ausgestaltet. Im Bereich der Information wird analog zur Top-down-Beziehung davon ausgegangen, dass die MA Informationen besitzen, welche der FK nicht geläufig sind. MA besitzen also einen Informationsvorsprung, der aus Effizienzgründen aufgelöst werden soll. Davon abgesehen wird aber die Einbeziehung der FK durch die MA nicht nur aus einer Position der Macht, sondern auch aus einer Position der Ohnmacht heraus argumentiert - die MA sind mit der Arbeit überfordert und müssen die FK um Rat bitten:

In schwierigen Situationen soll sich der Mitarbeiter rechtzeitig mit seinem Vorgesetzten beraten, ohne dass es dabei zu einer Rückübertragung der Verantwortung auf den Vorgesetzten kommt. (D 18) 


\section{Steuerungsaspekte}

Ähnlich wie die Befähiger-Beziehung operiert die Integrations-Beziehung vor allem mit Strategien psychologischer Steuerung. Der instrumentelle Einsatz von kooperativen und partizipativen Unterfangen (vgl. Sandner, 1990: 198; Seibold \& Shea, 2001) ist das zentrale Element der Integrations-Beziehung. Durch die Integration der MA in Entscheidungen soll vor allem eine „leistungsfördernde Mobilisierung von ansonsten zurückgehaltenen Ressourcen der Mitarbeiter" (Sandner, 1990: 199) stattfinden. Gleichzeitig wird eine Unterordnungsaufforderung aber auch damit legitimiert, dass die Einbeziehung der MA in deren eigenem Interesse ist. Es entsteht somit auch eine partielle Selbststeuerung der MA (Sandner, 1990: 204), da sie in die Entscheidungsprozesse eingebunden werden und vor allem im Bereich des $\mathrm{MbO}$ die ihnen auferlegten Ziele (zumindest theoretisch) mitbestimmen können. Mitarbeiterpartizipation kann aber auch (vor allem in einer marxistisch inspirierten Interpretation) die Entfremdung der MA von ihrer Tätigkeit bewirken. Management-Tätigkeiten liegen nicht in der eigentlichen Kompetenz der MA. Sie werden also zum ,Mit-Manager' und erhalten eine Rolle, die sie von ihrer Identität als MA trennt. Außerdem wird den MA das Recht zur Mitentscheidung von den FK bzw. dem Management eingeräumt. In dieser neuen Identität zwischen MA und FK bleiben die MA also von den FK völlig abhängig. (vgl. C. Conrad \& Ryan, 1985: 149). Die offen kommunizierte MitarbeiterInnenpartizipation kann auch von der tatsächlichen Praxis entkoppelt sein. Die scheinbar eigenständig getroffenen Entscheidungen der MA werden dann schlicht nicht mehr offen, sondern durch versteckte diskursive Steuerungsmöglichkeiten beeinflusst (vgl. Bisel, Ford \& Keyton, 2007: 136f.; vgl. auch die Ausführungen zur ,unsichtbaren' bzw. ,unauffälligen' Machtausübung im entsprechenden Teil der Arbeit). Das primäre Motiv für Partizipation ist hingegen ökonomischer Natur (vgl. Seibold \& Shea, 2001: 689). Eine andere Steuerungslogik besitzt die Informationspflicht des MA gegenüber seiner FK. Hier herrscht wieder eine klassisch bürokratische Regelsteuerung (vgl. Sandner, 1990: 190). 
Tabelle 13: Die Integrations-Beziehung (Überblick)

Rollenaspekte

Handlungslogik

Konstitutiver Topos

Ergänzende Topoi

$F K \rightarrow M A$

$M A \rightarrow F K$

$F K \leftrightarrow \rightarrow M A$

$F K, M A \rightarrow$

FK $\rightarrow$ sich selbst

$M A \rightarrow$ sich selbst
FK: Kommunikator bzw. Koordinator (explizit), passiv-abhängiger Akteur (implizit)

MA: passiv-abhängiger Akteur (implizit), Kommunikator bzw. Koordinator (explizit)

einbeziehen, argumentieren, kommunizieren, bereitstellen, versorgen vs. passive Abhängigkeit

Topos von der Transparenz als Allheilmittel

Topos Gemeinsam sind wir stärker

Topos vom Primat von Information und Wissen

Topos von der Zielklarheit

Topos von der Zielkoordination

Topos von der Bedeutung der Kommunikation

$\mathrm{Ja}$

$\mathrm{Ja}$

Ja, gegenseitiges Einbeziehen, Kommunizieren

Nein

Nein

Nein

\subsubsection{Die Kontroll- und Bewertungsbeziehung}

\section{Handlungslogik und Rollenaspekte}

In der Kontroll- und Bewertungsbeziehung steht bezüglich der Handlungslogik das Analysieren, Bewerten und anschließende Korrigieren/Anpassen im Zentrum. Dies kann die Person betreffen (z.B. im Rahmen von Feedback-Prozessen und Mitarbeitergesprächen), aber auch deren Tätigkeit bzw. das Ergebnis/die Leistung (im Rahmen von Leistungskennzahlen und -kontrollen). Im ersten Fall ist die Kontroll- und Bewertungsbeziehung in gewisser Weise der EntwicklungsBeziehung vorgelagert (Kontrolle als Grundlage für Entwicklungsgespräche), im 
zweiten Fall bietet sie die Grundlagen für die Steuerungs-Beziehung (Anpassung von Zielvorgaben, Kurskorrekturen, etc.). Die mit der Kontroll- und Bewertungsbeziehung assoziierten Rollenaspekte sind auf der aktiven Seite der Akteur als Analyst und Beurteiler, in der Folge aber auch als Korrektor und Koordinator. Der kontrollierte bzw. beurteilte und korrigierte Akteur verhält sich stets passiv-abhängig.

\section{Argumentationsstruktur - relevante Topoi}

Bezüglich der Argumentationsstruktur wird in der Kontroll- und Bewertungsbeziehung die Notwendigkeit der Kontrolle als konstitutiver und legitimierender Topos verwendet:

Vertrauen hat seine Grenzen. Nur eine effiziente Kontrolle sichert die Vermeidung von Fehlern und Abweichungen.

Der Großteil der argumentativen Äußerungen innerhalb dieser Beziehung bezieht sich auf die konkrete Ausgestaltung der Kontrolle. Dies lässt vermuten, dass Kontrolle und Bewertung grundsätzlich umstrittene Themen sind, deren Durchführung im Einklang mit gesellschaftlichen Werten argumentiert werden muss. So ist z.B. der Topos der fairen Beurteilung und Belohnung in dieser Beziehung zentral:

Eine Beurteilung ist nur dann sinnvoll, wenn sie fair und objektiv geschieht, da nur dann sinnvolle Maßnahmen abgeleitet werden können und die MitarbeiterInnen die Beurteilung nur dann als gerecht empfinden und akzeptieren werden.

Als Spezialfälle bzw. differenziertere Betrachtungen des ,Fairness'-Topos treten außerdem noch der Topos von der Transparenz als Allheilmittel

Sowohl effizientes Arbeiten als auch ein gutes Arbeitsklima sind nur dann möglich, wenn alle relevanten Faktoren bekannt und allen zugänglich sind. Verschleierung verunmöglicht optimale Lösungen.

sowie der Topos von der Gleichwertigkeit und Gleichbehandlung im Diskurs in Erscheinung:

Grundsätzlich sind alle Menschen gleich. Nur tatsächliche Unterschiede können eine, gerechte 'Differenzierung begründen.

Kontrolle und Bewertung sind also Themen, welche argumentativ sehr, vorsichtig' (im Sinne von ,sozial anschlussfähig') behandelt werden.

Interaktionsstruktur

Mit der Vorsicht im Umgang mit diesem Thema lässt sich auch erklären, warum die Beziehung im Vergleich zu anderen Typen der Führungsbeziehung auffällig 
bidirektional diskutiert wird. Sowohl FK als auch MA geben der jeweils anderen Partei Rückmeldung, und auch gegenseitiges Feedback wird explizit erwähnt. Dabei fällt allerdings auf, dass die Initiative, auch wenn es um die Vorgesetztenbeurteilung geht, oft bei der FK liegt.

Zudem geben wir dem/r MitarbeiterIn laufend Feedback, indem wir fördernd und korrigierend die Arbeitssituation besprechen und Orientierung für die Bewertung gewinnen. (A 06)

Wir äussern gegenseitig offenes Feedback im positiven wie im negativen Sinne. (CH 01)

Die anschließende Korrektur bzw. Anpassung von Verhalten im Sinne einer Steuerung ist hingegen wieder alleine den FK überlassen:

Die Steuerung besteht darin, daß der Vorgesetzte Arbeitsergebnisse, Arbeits- und Führungsverhalten bestätigt oder notwendige Korrekturen vornimmt. (D 19)

Einen wichtigen Raum im Diskurs nehmen in diesem Beziehungstypus auch die Prozesse der Selbstkontrolle ein. Diese betriffen sowohl FK als auch MA:

Die Kontrolle ist eine mit der Vorgesetztenfunktion untrennbar verbundene Aufgabe. Sie macht jedoch die Selbstkontrolle der Mitarbeiter nicht überflüssig, sondern will sie sicherstellen und ergänzen. Verantwortung kann nicht delegiert werden. (CH 17)

\section{Steuerungsaspekte}

Wie bereits in der obigen Diskussion erwähnt, kämpft die Argumentation in der Kontroll- und Bewertungsbeziehung damit, dass sie eine sehr direkte Steuerungslogik, nämlich Kontrolle, Beurteilung und Korrektur der MA durch die FK sozial legitimieren muss. Dies geschieht vor allem in argumentativer Weise, aber auch durch die Evokation kulturell akzeptierter Werte. Rationalisiert wird die Kontrolle durch die Betonung ihrer Notwendigkeit. Es besteht keine Alternative, da ohne Kontrolle eine effiziente und effektive Leistungserbringung nicht möglich ist. Das Unternehmen verpflichtet sich und die Führungskräfte aber da$\mathrm{zu}$, diese Kontrolle und Bewertung in Übereinstimmung mit kulturell geteilten Werten vorzunehmen. Diese sind Fairness, Objektivität, Transparenz und Gerechtigkeit im Sinne von Gleichbehandlung. Die Kontroll- und Bewertungsbeziehung wird dadurch sowohl normalisiert als auch legitimiert. Die Beschwörung dieser kulturellen Werte stellt eine symbolische Kommunikation des Unternehmens dar (vgl. Sandner, 1990: 213ff.) und wird hier deshalb als Teil der kulturellen Steuerung betrachtet. In einzelnen Äußerungen finden sich aber auch hier wieder psychologische Elemente - nämlich immer dann, wenn die Beurteilung bzw. die Rückmeldung der FK an die MA als Service für die MA ,verkauft` wird, da sie ihnen dazu dient, sich stetig zu verbessern (zur Steuerungs- 
wirkung der Idee von der kontinuierlichen Verbesserung vgl. auch die Diskussion der Entwicklungs-Beziehung).

Tabelle 14: Die Kontroll- und Bewertungsbeziehung (Überblick)

Rollenaspekte

Handlungslogik

Konstitutiver Topos

Ergänzende Topoi

$F K \rightarrow M A$

$M A \rightarrow F K$

$F K \leftrightarrow \rightarrow M A$

$F K, M A \rightarrow$

FK $\rightarrow$ sich selbst

$M A \rightarrow$ sich selbst
FK: Analyst und Beurteiler, Korrektor und Koordinator (explizit), passiv abhängiger Akteur (implizit)

MA: passiv-abhängiger Akteur (implizit), Analyst und Beurteiler (explizit)

Analysieren, bewerten, anpassen, korrigieren vs. passive Abhängigkeit

Topos von der Notwendigkeit von Kontrolle

Topos von der fairen Beurteilung und Belohnung

Topos von der Transparenz als Allheilmittel

Topos von der Gleichwertigkeit und Gleichbehandlung

$\mathrm{Ja}$

Ja, aber nur Bewertung

Ja, gegenseitiges Bewerten

Ja, gemeinsame Bewertung und gemeinsame Korrektur

\subsubsection{Die Motivations-Beziehung}

\section{Handlungslogik und Rollenaspekte}

Die Motivations-Beziehung benennt jene Form der Interaktion, in welcher eine Partei direkt motivierend auf die andere Partei einwirkt, sei es durch Ermuntern/Ermutigen oder durch Anerkennen/Belohnen gewünschter Handlungen. Diese Handlungslogik unterscheidet sich somit in der Tätigkeit von motivierenden Faktoren der Kontextgestaltung in der Befähiger-Beziehung. Von der Steuerungs-Beziehung unterscheidet sie sich dadurch, dass die Motivations-Beziehung nicht auf direkten Befehl und Zielvorgabe, sondern auf ,weichere‘ Faktoren der 
Steuerung (Gestaltung von Anreizen) zurückgreift. Die mit dieser Beziehung assoziierten Rollenaspekte sind somit vor allem der Steuermann und Leiter, aber auch zu einem gewissen Grad der Beziehungsmanager. Der Gegenpart verhält sich wie in den meisten diskutierten Beziehungstypen abhängig-passiv. Er ist nicht in der Lage oder willens, sich selbst zu motivieren, sondern ist auf extern gesetzte Anreize angewiesen.

\section{Argumentationsstruktur - relevante Topoi}

Der konstitutive Topos für die Motivations-Beziehung ist jener, welcher den Grund für die gewünschte Motivation der Akteure erläutert, nämlich der Topos vom Zusammenhang von Motivation und Leistung. Auch hier zeigt sich wieder das klassisch utilitaristische Prinzip:

Motivierte MitarbeiterInnen sind leistungsbereite MitarbeiterInnen.

Wie diese Motivation geschehen soll, erläutert der Topos von der fairen Beurteilung und Belohnung:

Eine Beurteilung ist nur dann sinnvoll, wenn sie fair und objektiv geschieht, da nur dann sinnvolle Maßnahmen abgeleitet werden können und die MitarbeiterInnen die Beurteilung nur dann als gerecht empfinden und akzeptieren werden.

Anders als in der Kontroll- und Bewertungs-Beziehung rekurriert dieser Topos hier weniger auf den Aspekt der Gerechtigkeit der Beurteilung, sondern stärker auf die motivierende Wirkung angemessener Beurteilung und Belohnung.

\section{Interaktionsstruktur}

Die Motivations-Beziehung ist im Diskurs ausschließlich unidirektional möglich. FK motivieren und ermuntern ihre MA und setzen Anreize durch Belohnung. Motivation ist außerdem ausschließlich fremdinduziert - die aktive Motivation der eigenen Person ist nicht Teil des Diskurses (sehr wohl ist aber der Zustand des ,Motiviert-Seins` von allen Parteien gefordert).

Wir motivieren die Mitarbeitenden mit messbaren Zielvereinbarungen und überprüfen periodisch den Erreichungsgrad. (CH 18)

[Anm: Führungskräfte] begeistern durch ihr Vorbild (A 02)

Steuerungsaspekte

Die Motivations-Beziehung im klassischen Sinn wird in ihrem Steuerungspotential durch die motivationale Steuerung als Substrategie der psychologischen Steuerung umfassend erklärt (vgl. Sandner, 1990: 202ff.). Die Steuerungswirkung erfolgt vor allem durch Anreizgestaltung, wobei die Anreize teilweise institutionalisiert sind, teilweise aber auch von der FK spontan eingesetzt werden können. Jedenfalls sind diese Anreize so gestaltet, dass sie von den MA erst 
dann konsumiert werden können, wenn diese gewisse Bedingungen erfüllen, sprich, die erwartete Leistung zeigen (vgl. Sandner, 1990: 203). Einen Sonderfall dieser Logik stellt aber die in den Dokumenten weit verbreitete Steuerung durch Vorbildwirkung des Vorgesetzten dar, welche in den Bereich der, charismatischen' bzw. ,transformationalen' Führung (vgl. Wunderer, 2009: 242ff.) fällt und mit herkömmlichen Anreizmodellen nicht mehr vollständig erklärbar ist. Die Unterordnung der MA entsteht dann aus dem Bedürfnis heraus, der FK ,gleich zu sein` bzw. es der FK, gleich zu tun'. Die FK tritt gleichsam als ,Verkörperung' gewünschter (organisationaler) Werte auf und wird damit selbst zu Medium und Botschaft symbolischer Kommunikation:

[Die Führungskraft] verkörpert eine Leidenschaft für die Erreichung von Zielen, die über Geld oder Status hinausgeht. (CH09)

Die Steuerung durch Vorbildwirkung der Führungskraft stellt somit eine Mischform zwischen psychologischer und kultureller Steuerung dar, indem sie wiederum das Bedürfnis der MA nach Anerkennung und Selbstbestätigung mit organisational relevanten kulturellen Werten gleichschaltet.

Tabelle 15: Die Motivations-Beziehung (Überblick)

Rollenaspekte

Handlungslogik

Konstitutiver Topos

Ergänzende Topoi

$F K \rightarrow M A$

$M A \rightarrow F K$

$F K \leftarrow \rightarrow M A$

$F K, M A \rightarrow$

FK $\rightarrow$ sich selbst

$M A \rightarrow$ sich selbst
FK: Steuermann und Leiter, Beziehungsmanager (explizit)

MA: passiv-abhängiger Akteur (implizit)

Ermuntern, motivieren, anerkennen, belohnen vs. passive Beeinflussbarkeit

Topos vom Zusammenhang von Motivation und Leistung

Topos von der fairen Kontrolle und Beurteilung

$\mathrm{Ja}$

Nein

Nein

Nein

Nein

Nein 


\subsubsection{Die direkte Steuerungs-Beziehung}

Handlungslogik

Die Handlungslogik der direkten Steuerungs-Beziehung liegt im expliziten und direkten Steuern bzw. Führen. Im Gegensatz zur Motivationsbeziehung, wo die Steuerungswirkung über Anreizgestaltung geschieht, und zur BefähigungsBeziehung, wo über die Gestaltung von Rahmenbedingungen Steuerungswirkung erzielt wird, geht es in der direkten Steuerungs-Beziehung um unmittelbare Einflussnahme durch lenken, leiten, zuteilen, festsetzen, festlegen, usw. Insofern, als etwas BEI den MA sichergestellt wird (im Gegensatz zur Sicherstellung FÜR die MA in der Befähigungs-Beziehung) sind auch gewährleistende Tätigkeiten im Diskurs enthalten. Die relevanten Rollenaspekte für die Akteure sind somit Steuermann bzw. Leiter und Gewährleister für den steuernden Part und passiv-abhängiger Akteur für den gesteuerten Part.

Argumentationsstruktur - relevante Topoi

Zur Legitimation der direkten Steuerungs-Beziehung wird vor allem der Topos von der Notwendigkeit von Orientierung und Sicherheit als konstitutiv verwendet:

Effiziente Leistungserstellung ist nur dann möglich, wenn ein gemeinsamer Weg und geteilte Verständnisse existieren, damit grundlegende (Routine)Fragen nicht mehr in jedem Einzelfall geklärt werden müssen.

Durch (konsistente) direkte Anweisung und Steuerung wird Unsicherheit vermieden und Leistungsfähigkeit erhalten. Führung ist notwendig für das reibungslose Funktionieren des Systems. Die Logik dieses Topos zeigt auch die argumentative Ähnlichkeit zwischen der direkten Steuerungs-Beziehung und der Bereitstellungs-Beziehung. Ob die FK den MA Sicherheit und Klarheit gibt/schenkt oder ob sie diese Klarheit durch Ziel- und Regelsetzung erreicht, mag in erster Linie eine rhetorische Unterscheidung sein, die aber nichts desto trotz ein unterschiedliches Verhältnis zwischen FK und MA impliziert. Das gewünschte Steuerungspotential bleibt aber in beiden Varianten erhalten. Da sich in den Dokumenten die Steuerung stark auf die Zuteilung und Definition von Kompetenzen bezieht, ist als inhaltlich verwendeter Topos auch die Einheit von Kompetenz und Verantwortung von Relevanz:

Die Leistung einer Person ist dann am höchsten, wenn sie an ihrem Arbeitsplatz weder unternoch überfordert ist. Der höchste Grad an Leistung wird dort erreicht, wo ein hoher ,Fit * zwischen Person und Arbeitsplatz bzw. Verantwortung besteht.

Anders als in der Begrenzungs-Beziehung geht es hier nun aber um eine positive Definition von Verantwortung, d.h. um die Frage, was alles in den Verantwor- 
tungsbereich der Person gehört. Wie in der Integrations-Beziehung, so ist auch in der direkten Steuerungs-Beziehung die Transparenz und Klarheit (in diesem Fall bezüglich der Grundlagen von Anweisungen) zentral. Insofern sind auch die beiden Topoi von der Zielklarheit und von der Transparenz als Allheilmittel im Diskurs vertreten.

\section{Interaktionsstruktur}

Die direkte Steuerungs-Beziehung ist ähnlich der Motivations-Beziehung ausschließlich unidirektional und top-down organisiert. Die FK steuert und lenkt ihre MA:

Damit der Mitarbeiter eine optimale Leistung erbringen kann, teilen wir ihm nach Möglichkeit ein Arbeitsgebiet zu, das seiner Ausbildung sowie seinen Fähigkeiten, Kenntnissen und Neigungen entspricht. (CH 13)

Die Steuerung besteht darin, daß der Vorgesetzte Arbeitsergebnisse, Arbeits- und Führungsverhalten bestätigt oder notwendige Korrekturen vornimmt. (D 19)

Ein Sonderfall ist die gemeinsame Steuerung, in welcher FK und MA zusammen etwas festlegen oder definieren:

Die Führungskräfte legen aufgrund der in den Mitarbeiterförderungsgesprächen gewonnenen Informationen und Einsichten gemeinsam mit dem betroffenen Mitarbeiter fest, welche Förderungsmaßnahmen dieser erfahren soll. (A 04)

Außerdem sind im Sinne einer Sicherstellung bzw. Gewährleistung grundsätzlich auch Selbststeuerungsmechanismen im Diskurs angelegt.

\section{Steuerungsaspekte}

Wie bereits in der Bezeichnung angelegt, handelt es sich bei der Steuerungslogik in dieser Beziehung in erster Linie um eine direkte Steuerung (vgl. Sandner, 1990: 174ff.), und zwar innerhalb der legalen Autorität der FK (vgl. Sandner, 1990: 178). Der Topos von der Notwendigkeit von Orientierung und Sicherheit thematisiert zumindest indirekt die Legitimität dieser legalen Autorität. Ohne Führung und Hierarchie wäre keine Sicherheit in der Leistungserbringung möglich. Führung ist somit funktional. Damit begründet der Topos aber in weiterer Folge nicht nur die direkte Steuerungs-Beziehung, sondern die Notwendigkeit von Führung an sich. Es ist wenig verwunderlich, dass dies in einem Beziehungstyp geschieht, in welchem die Führung bzw. Steuerung in ihrem direkten Charakter besonders offen zutage tritt. Damit erfüllt aber die explizite Thematisierung und Legitimierung direkter Steuerung auch im Rahmen der Führungsgrundsätze die Funktion der Herrschaftssicherung (vgl. auch Sandner, 1990: 174). Sie bestätigt den Führungs- und Herrschaftsanspruch der FK und schafft gleichzeitig Handlungsspielräume für direkte Intervention und Machtausübung. 
Tabelle 16: Die Steuerungs-Beziehung (Überblick)

\begin{tabular}{|c|c|}
\hline \multirow[t]{2}{*}{ Rollenaspekte } & FK: Steuermann und Leiter, Gewährleister (implizit) \\
\hline & MA: passiv-abhängiger Akteur (implizit) \\
\hline Handlungslogik & $\begin{array}{l}\text { Steuern, führen, gewährleisten, verantworten vs. passive Abhängig- } \\
\text { keit }\end{array}$ \\
\hline Konstitutiver Topos & Topos von der Notwendigkeit von Orientierung und Sicherheit \\
\hline \multirow[t]{3}{*}{ Ergänzende Topoi } & Topos von der Einheit von Kompetenz und Verantwortung \\
\hline & Topos von der Zielklarheit \\
\hline & Topos von der Transparenz als Allheilmittel \\
\hline$F K \rightarrow M A$ & $\mathrm{Ja}$ \\
\hline$M A \rightarrow F K$ & Nein \\
\hline$F K \leftarrow \rightarrow M A$ & Nein \\
\hline$F K, M A \rightarrow$ & Ja, gemeinsames Steuern, Führen \\
\hline FK $\rightarrow$ sich selbst & Ja, etwas bei sich selbst gewährleisten \\
\hline$M A \rightarrow$ sich selbst & Ja, etwas bei sich selbst gewährleisten \\
\hline
\end{tabular}

\subsubsection{Die Unterstützungs-Beziehung}

Handlungslogik und Rollenaspekte

Die Handlungslogik der Unterstützungs-Beziehung beinhaltet mehrere ähnliche, aber im Detail unterschiedliche Elemente. Zum einen geht es um die direkte Unterstützung des anderen, und zwar im Bereich der Arbeit wie im Bereich der Beziehungsgestaltung. Ein zweiter Punkt betrifft die Unterstützung/Förderung von gewünschten Eigenschaften und Tätigkeiten des anderen. Hier gibt es Berührungspunkte zwischen der Unterstützungs-Beziehung und der EntwicklungsBeziehung. Ein drittes Element schließlich beinhaltet das Vertreten/Verfechten der Interessen des anderen bzw. auch das Einstehen/Eintreten für einen anderen. Relevante Rollenaspekte sind daher Unterstützer bzw. Förderer und Verfechter bzw. Vertreter. Den Gegenpol stellt wieder der passiv-abhängige Akteur dar, 
welcher Unterstützung und die Vertretung seiner Interessen durch Dritte benötigt.

\section{Argumentationsstruktur - relevante Topoi}

Die Unterstützungs-Beziehung ist der dritte Beziehungstyp, welcher seine Legitimation aus dem Topos von der Bedeutung des Arbeitsklimas und Arbeitsumfelds zieht:

Die Leistungsbereitschaft und-fähigkeit von Menschen hängt stark von den Rahmenbedingungen und Kontextfaktoren ab, unter welchen sie diese Leistung zu erbringen haben.

Hier betrifft dieser Topos aber nicht die generellen Rahmenbedingungen der Leistungserstellung wie in der Befähigungs-Beziehung und auch nicht die Versorgung mit arbeitstechnisch wichtigen Faktoren und Objekten wie in der $\mathrm{Be}$ reitstellungs-Beziehung, sondern vielmehr den gegenseitigen Umgang der Individuen in der Organisation miteinander. Gegenseitige Unterstützung schafft ein Arbeitsumfeld, in welchem die Menschen gerne arbeiten und Leistungen erbringen. Weitere relevante - inhaltliche - Topoi sind jene von der internen Mobilität, mit welchem vor allem eine Unterstützung im Bereich der persönlichen Karriere argumentiert wird

Eine Entwicklung in den Fähigkeiten und den Kompetenzen muss auch mit einer Entwicklung im Verantwortungsbereich und der Kompensation einhergehen.

und vom Stillstand als Tod, welcher die Unterstützung bei der persönlichen und fachlichen Entwicklung betrifft:

Da sich das Umfeld (und damit die Anforderungen an das Unternehmen und den Einzelnen) ständig ändert, muss jeder konstant vorwärts gehen und sich verbessern bzw. anpassen. Wer sich nicht mitbewegt, der bleibt auf der Strecke.

\section{Interaktionsstruktur}

Die Unterstützungs-Beziehung ist bidirektional ausgelegt. Sowohl die FK unterstützen ihre MA als auch umgekehrt (wenn auch weit seltener):

Unsere Mitarbeiter verdienen es, ernst genommen, angehört und respektiert zu werden. Der Vorgesetzte kümmert sich persönlich um sie und ihre Interessen. (CH 13)

Unsere MitarbeiterInnen müssen bereit sein, Verantwortung zu übernehmen und ihre Vorgesetzten und das Team mit ihrem Wissen und Können bei der Arbeit zu unterstützen. (D 08)

Auch gegenseitige Unterstützung ist im Diskurs explizit möglich:

Vorgesetzte und Mitarbeiter setzen sich gegenseitig für die Interessen des andern ein (win-win Situation). (CH 02) 
Die Unterstützungs-Beziehung ist somit der Typus mit dem höchsten Grad an Gleichwertigkeit zwischen den Akteuren. Machtgefälle treten hier in den Hintergrund. Betont wird der gegenseitige Umgang miteinander, welcher zumeist von beiden Seiten aktives Handeln benötigt. Unterstützung als Führungsfunktion weist natürlich dennoch klassische Hierarchieaspekte auf.

\section{Steuerungsaspekte}

In der Unterstützungs-Beziehung tritt die Steuerung am wenigsten deutlich erkennbar auf. Auf der Ebene der Steuerung beider Akteure (FK wie MA) durch die Dokumente bzw. die Organisationsführung handelt es sich um einen Aspekt der kulturellen Steuerung. Gegenseitige Unterstützung ist ein kultureller Wert, welcher außerdem auch psychologisch auf die Identifikation der Akteure mit der Organisation wirkt: Es entsteht der Eindruck einer familiären Atmosphäre, in welcher sich jeder für den anderen und dessen Wohlergehen interessiert. Die Unterstützungs-Beziehung ist deshalb auch geprägt von einem vergleichsweise hohen Grad an Emotionalität in der Sprache. Im Gegensatz zur EntwicklungsBeziehung, welche ebenfalls unterstützende Handlungen beinhaltet, tritt in der Unterstützungs-Beziehung das utilitaristische Motiv (zumindest scheinbar) in den Hintergrund. Psychologisch wird damit bei den Akteuren derselbe Effekt erzielt wie in der Empathie-Beziehung: es entsteht der Mythos vom Menschen im Mittelpunkt (vgl. Sandner, 1990: 199). Die Hierarchie und mit ihr die die Herrschaft der FK über die MA treten in der Unterstützungs-Beziehung völlig in den Hintergrund. Da beide Akteure sowohl Unterstützer als auch passiv abhängiger Akteur sein können, entsteht der Eindruck von Gleichwertigkeit. Eine mögliche Erklärung für die unklare Steuerungswirkung dieses Typus könnte sein, dass er nicht direkt ein Steuerungspotential begründen, sondern vielmehr die Identifikation der Akteure mit der Organisation stärken will, um sie für die anderen Prämissen in den Dokumenten (und anderen kommunikativen Handlungen) empfänglicher zu machen. 
Tabelle 17: Die Unterstützungs-Beziehung (Überblick)

Rollenaspekte

FK: Förderer und Unterstützer, Verfechter und Vertreter (explizit), passiv-abhängiger Akteur (implizit)

MA: Förderer und Unterstützer, Verfechter und Vertreter (explizit), passiv-abhängiger Akteur (implizit)

Handlungslogik

Unterstützen, fördern, vertreten, verfechten vs. passive Abhängigkeit

Konstitutiver Topos

Topos von der Bedeutung des Arbeitsklimas und Arbeitsumfelds

Ergänzende Topoi

Topos von der internen Mobilität

Topos vom Stillstand als Tod

$F K \rightarrow M A$

$\mathrm{Ja}$

$M A \rightarrow F K$

$F K \leftarrow \rightarrow M A$

Ja, gegenseitige Unterstützung

$F K, M A \rightarrow$

FK $\rightarrow$ sich selbst

$M A \rightarrow$ sich selbst

Nein

Ja

Nein

Nein

\subsubsection{Die Verfügungs-Beziehung}

\section{Handlungslogik und Rollenaspekte}

Die Verfügungs-Beziehung ist der Steuerungs-Beziehung in der Direktheit des Steuerungsaspekts sehr ähnlich. Ihre Handlungslogik geht jedoch noch über jene der Steuerungs-Beziehung hinaus, indem sie eine Partei direkt über die andere verfügen bzw. die andere nutzen lässt. Der entsprechende Rollenaspekt für den aktiven Beziehungspartner ist somit jener des Verfügers oder Nutzers. Der zweite Partner verhält sich passiv-abhängig. Dadurch, dass der passiv-abhängige Akteur in dieser Beziehung aber nicht nur Empfänger einer Handlung ist, sondern dem anderen Partner vollumfänglich zur Verfügung steht, kann diese spezifische Ausprägung als ,Ressourcen'-Rolle bezeichnet werden.

Argumentationsstruktur - relevante Topoi 
Aufgrund der Ähnlichkeit zur Begrenzungs-Beziehung, was die Direktheit des steuernden Eingriffs betrifft, verwundert es auch nicht, dass der dominante Topos derselbe ist, nämlich jener von der Einheit von Kompetenz und Verantwortung:

Die Leistung einer Person ist dann am höchsten, wenn sie an ihrem Arbeitsplatz weder unternoch überfordert ist. Der höchste Grad an Leistung wird dort erreicht, wo ein hoher ,Fit * zwischen Person und Arbeitsplatz bzw. Verantwortung besteht.

Im Unterschied zur Steuerungs-Beziehung, in welcher die FK die Verantwortung der MA entsprechend ihrer Kompetenz festlegt, ist der Ansatz hier direkter: Die FK setzt die MA ihrer Kompetenz entsprechend ein. Damit ist eine direkte Verfügung der FK über die MA legitimiert, die MA werden zur Ressource:

Führen heißt: Die knappen Ressourcen im Unternehmen - Mitarbeiter - Zeit - Geld - zielgerichtet einzusetzen. (A 15)

Der Vorgesetzte plant aufgrund der ihm gestellten Ziele, den optimalen Einsatz seiner Mitarbeiter und setzt diese nach ihren spezifischen Fähigkeiten ein. (CH 17)

Ein weiterer Topos, der stark mit der Verfügungs-Beziehung korrespondiert, ist jener von der positiven Diversität:

Unterschiedliche Sichtweisen und Hintergründe sind wertvoll, weil die Einnahme unterschiedlicher Perspektiven die Problemwahrnehmung erweitert und zu kreativeren und besseren Lösungen führt.

Dabei wird in dieser Beziehung in erster Linie der utilitaristische Wert von Differenzen erkannt und angesprochen. Differenzen und unterschiedliche Perspektiven werden nicht aus einem Sinn der Fairness oder Gerechtigkeit heraus akzeptiert, sondern weil sie einen unmittelbaren Nutzen für das Unternehmen bedeuten:

[...] Nutzen aus der kulturellen Vielfalt der Mitarbeiter ziehen (D 02)

Interaktionsstruktur

Die Verfügungs-Beziehung ist wie die Steuerungsbeziehung ausschließlich unidirektional bzw. top-down organisiert. MA sind nur dann aktiv, wenn sie mit den FK gemeinsam etwas nutzen bzw. einsetzen:

Die Führungskraft ist gemeinsam mit jedem/r einzelnen MitarbeiterIn sowie mit dem gesamten Team dafür verantwortlich, Stärken und Potenziale zu erkennen, zu entwickeln und einzusetzen. (A 13)

Im Gegensatz zu MA sind FK außerdem angehalten, Eigenschaften und Fähigkeiten, die sie selbst besitzen, einzusetzen und zu nutzen: 
Wir [Anm.: die Führungskräfte] setzen unsere Fachkompetenz optimal zum Nutzen unserer Kunden und unseres Umfeldes ein. (CH 12)

Steuerungsaspekte

Die Steuerungslogik der Verfügungs-Beziehung ist analog zu jener der direkten Steuerungs-Beziehung zu betrachten. Die Mechanismen der Steuerung entsprechen zu einem hohen Grade der direkten Steuerung. Der Beziehungstyp begründet im Wesentlichen völlige Entscheidungsfreiheit der FK beim Einsatz der MA und deren Eigenschaften. Der Typus legitimiert dabei diese Verfügungsgewalt sehr allgemein und stellt die direkte, situative Steuerung den FK frei.

Tabelle 18: Die Verfügungs-Beziehung (Überblick)

Rollenaspekte

FK: Verfüger bzw. Nutzer (explizit)

MA: passiv-abhängiger Akteur (implizit) bzw. Ressource (explizit und implizit)

Handlungslogik

verfügen, nutzen vs. passiv, verfügbar

Konstitutiver Topos

Topos von der Einheit von Kompetenz und Verantwortung

Ergänzende Topoi

Topos von der positiven Diversität

$F K \rightarrow M A$

$\mathrm{Ja}$

$M A \rightarrow F K$

$F K \leftrightarrow \rightarrow M A$

$F K, M A \rightarrow$

Ja, gemeinsam etwas nutzen/einsetzen

FK $\rightarrow$ sich selbst

Ja, eigene Eigenschaften, Fähigkeiten nutzen

$M A \rightarrow$ sich selbst

Nein

\subsubsection{Häufigkeit und Verteilung der Beziehungstypen}

Nicht alle der hier diskutierten Beziehungstypen sind in allen untersuchten Dokumenten vorhanden. Die folgende Graphik gibt einen Überblick über die quantitative Verteilung der Beziehungstypen im Material. 


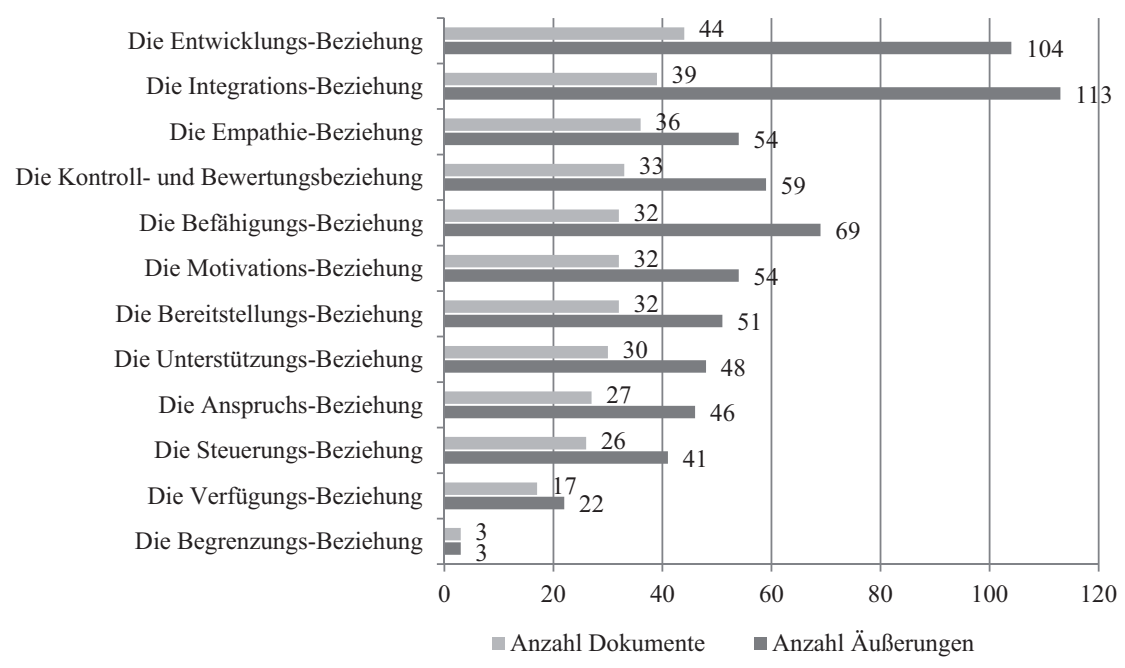

Abbildung 13: Verteilung der Beziehungstypen im untersuchten Material ( $n=53)$

Die Anzahl der Dokumente, in welchen ein Beziehungstyp vorkommt, gibt einen Überblick darüber, in welchem Ausmaß dieser Typ im Diskurs der Führungsgrundsätze aufscheint. Hier zeigt sich ein sehr gleichmäßiges Bild. Der Großteil der (re-)konstruierten Beziehungen kann in 30 bis 40 Dokumenten identifiziert werden. Als peripher im Diskurs können nur die VerfügungsBeziehung und speziell die Begrenzungs-Beziehung bezeichnet werden. Ein zweiter Balken zeigt im Kontrast dazu die Anzahl der Äußerungen, in welchen der spezifische Beziehungstyp angesprochen wird. Diese Zahl ist insofern verzerrt, als Dokumente, welche einen einzelnen Typ sehr stark erwähnen, hier auch stärker zu Buche schlagen. Es zeigt sich aber, dass in dieser Betrachtungsweise deutlichere Unterschiede in der quantitativen Relevanz einzelner Typen zu Tage treten. Die Integrations-Beziehung und die Entwicklungsbeziehung sind die im Material am häufigsten vertretenen Beziehungstypen. Dies stimmt mit den Ergebnissen von Jancsary et al. (2006: 46) überein, nach denen Personalentwicklung und Führen mit Zielen zwei der häufigsten Regelungsinhalte von Führungsgrundsätzen sind. Führen mit Zielen (bzw. management by objectives) korreliert insofern stark mit der Integrations-Beziehung, als die Vereinbarung von Zielen (nicht das Vorgeben derselben) eine wesentliche Tätigkeit innerhalb dieses Beziehungstyps darstellt. Auch die Befähiger-Beziehung und die Kontroll- und Bewertungsbeziehung heben sich deutlich von den anderen ab. Diese korrespondieren lose mit den Kategorien ,Delegation“ respektive ,Kontrolle / Beurteilung / Feedback' bei Jancsary et al. (2006: 46), welche ebenfalls domi- 
nante Diskursstränge darstellen. Peripher hingegen bleiben die BegrenzungsBeziehung und die Verfügungs-Beziehung.

\subsubsection{Eine konzeptionelle Topos-Karte des Diskurses}

Neben der Verteilung der Beziehungstypen über die Dokumente kann auch eine Analyse der Beziehungen zwischen den einzelnen Beziehungstypen durch die Betrachtung geteilter Topoi Aufschluss über strukturelle Elemente des Diskurses geben. Verbindet man alle Beziehungstypen miteinander, indem man die Verteilung der Topoi auf diese Typen abbildet, dann erhält man eine solche netzwerkähnliche Karte, wie Abbildung 14 sie zeigt. Mit den dunkleren Ellipsen wurden dabei die konstitutiven Topoi abgebildet. Diesen konstitutiven Topoi wurden in Form von Rechtecken direkt die entsprechenden Beziehungstypen, welche durch sie legitimiert werden, beigefügt. Bis auf den Topos von der Einheit von Kompetenz und Verantwortung (zwei Beziehungstypen) und den Topos von der Bedeutung des Arbeitsklimas und Arbeitsumfelds (drei Beziehungstypen) konstituiert jeder Topos nur einen einzigen Beziehungstyp. Die Pfeile, welche von diesen konstitutiven Topoi ausgehen, stellen dar, welche ergänzenden Topoi (die helleren Ellipsen) den jeweiligen konstitutiven Topos ergänzen und/oder stützen. Dabei kann der konstitutive Topos einer Beziehung gleichzeitig ein ergänzender Topos einer anderen Beziehung sein (so ist der Topos von der Transparenz als Allheilmittel konstitutiv für die Integrations-Beziehung, er ist aber ein ergänzender Topos innerhalb der Anspruchs-Beziehung). Die Richtung der Pfeile gibt an, welcher Topos sich welcher anderen Topoi bedient. Die Beschriftung der Pfeile hingegen erläutert, innerhalb welches Beziehungstyps diese Bezugnahme stattfindet. 


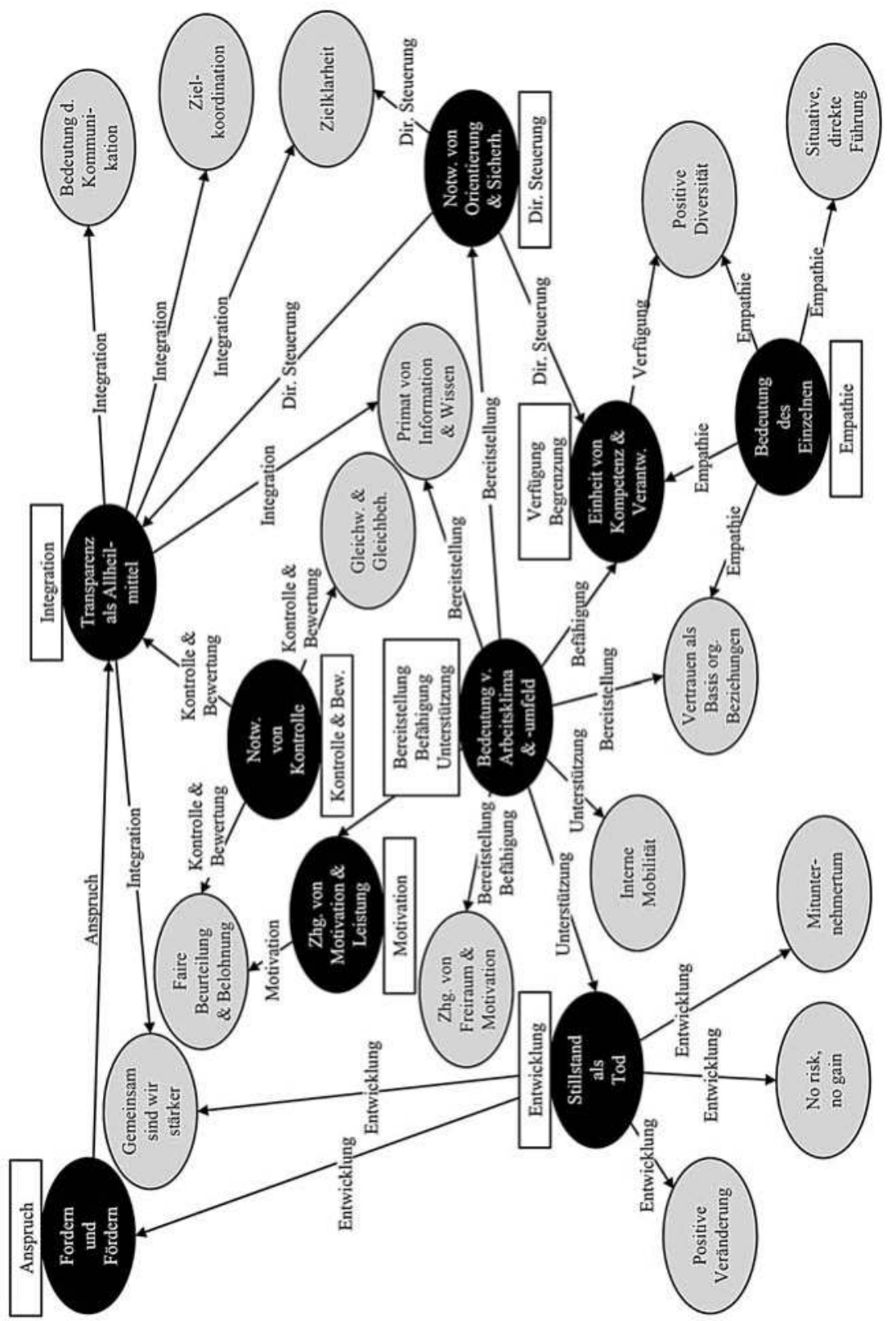

Abbildung 14: Eine konzeptionelle Karte von verwendeten Topoi und Beziehungstypen 
Aus der Abbildung lässt sich relativ deutlich ablesen, welche Topoi im Diskurs in konzeptioneller Hinsicht eine zentrale bzw. periphere Position einnehmen. Der Topos von der Bedeutung des Arbeitsklimas und Arbeitsumfelds stellt sozusagen den inneren Knotenpunkt des Diskurses dar. Er konstituiert drei verschiedene Beziehungstypen und bedient sich entsprechend einer Vielzahl anderer ergänzender Topoi, um diese Beziehungstypen näher argumentativ zu stützen. Er selbst wird aber von keinem anderen Topos ergänzend gebraucht. Diese Eigenschaft ergibt sich nicht zuletzt aus dem eher hohen Abstraktionsgrad des Topos. Alle Topoi, derer er sich bedient, können als Teil des Arbeitsklimas oder Arbeitsumfelds betrachtet werden. Auch der Topos von der Transparenz als Allheilmittel besitzt viele Verbindungen zu anderen Topoi. Im Gegensatz zum Topos von der Bedeutung des Arbeitsklimas und Arbeitsumfelds bedient er sich allerdings in erster Linie peripherer Topoi. Er verwendet auch keinen anderen konstitutiven Topos zur Stützung seiner Ansprüche. Hingegen wird er von drei verschiedenen anderen konstitutiven Topoi ergänzend evoziert. Damit zeigen sich in der Karte zwei verschiedene Formen von Zentralität. Der Topos von der Bedeutung des Arbeitsklimas und Arbeitsumfelds ist insofern zentral, als er einen Großteil des Diskurses aufbaut. Er schlägt Brücken zwischen verschiedenen anderen konstitutiven Topoi (und damit verschiedenen Beziehungstypen), indem er deren Argumente mitverwendet. Der Topos steht damit in der Argumentationshierarchie ganz oben. Der Topos selbst ist aber nur in drei verschiedenen Beziehungstypen von Relevanz. Seine Bedeutung besteht nicht in seiner vielseitigen Verwendbarkeit, sondern in der Möglichkeit der Integration einer Mehrzahl untergeordneter Argumente. Viele andere Topoi im Diskurs ordnen sich der Schlussregel dieses Topos unter. Der Topos von der Transparenz als Allheilmittel hingegen vereint zwei Formen der Zentralität. Wie der Topos von der Bedeutung des Arbeitsklimas und Arbeitsumfelds bedient er sich verschiedener ergänzender Topoi und trägt damit zur ,Breite“ des Diskurses bei. Er selbst ist aber auch anschlussfähig an drei verschiedene andere konstitutive Topoi und unterstützt deren Argumentation. Damit steht er hierarchisch in der Mitte und verbindet verschiedene Abstraktionsebenen. Ein Beispiel für das untere Ende der Topos-Hierarchie ist der Topos von der Einheit von Kompetenz und Verantwortung. Er selbst bedient sich nur eines einzigen anderen Topos, wird aber von drei verschiedenen konstitutiven Topoi herangezogen.

Anhand der ,Karte' lässt sich auch grob die konzeptionelle Nähe verschiedener Beziehungstypen zueinander diskutieren. So fällt vor allem auf, dass die Empathie-Beziehung und die Unterstützungs-Beziehung, wenn man die ihnen zugrundeliegenden Topoi betrachtet, keine Verbindung miteinander besitzen. Dies mag auf den ersten Blick überraschen, da die Konzepte von Empathie und Unterstützung in einem alltagstheoretischen Verständnis sehr nahe beieinander 
liegen. Betrachtet man aber die entsprechenden Topoi, so stellt man fest, dass die Unterstützungs-Beziehung sehr stark mit eher sachlichen Themen und Argumenten in Verbindung gebracht wird. Die Unterstützung bezieht sich in erster Linie auf die Personalentwicklung und die Karriereplanung. Die EmpathieBeziehung hingegen linkt stark zu menschlichen Themen und Topoi, wie der Vertrauens-Thematik, der Bedeutung von Diversität und der Notwendigkeit der individuellen Anpassung von Führungsstilen. Interessant ist auch die Betrachtung der Integrations-Beziehung. Die Argumentation der Notwendigkeit einer Partizipation der MA im Sinne eines höheren Grads an Selbststeuerung und größerem Einfluss auf die eigene Tätigkeit ist in den Dokumenten nur ein Aspekt der Integration von MA unter mehreren. Die Partizipation wird in erster Linie mit Transparenzüberlegungen argumentiert und durch rationale Aspekte wie Zielkoordination, Zielklarheit und Informationssymmetrie ergänzt. Direkt verbunden ist die Integrations-Beziehung mit der direkten Steuerungsbeziehung, der Kontroll- und Bewertungsbeziehung und der Anspruchs-Beziehung, nicht aber mit der Unterstützungs-Beziehung oder der Empathie-Beziehung. Mitarbeiterpartizipation wird im Diskurs also in erster Linie rational bzw. funktional betrachtet. Die ,menschlichen“ Aspekte sind eher sekundär. Die Partizipationsbestrebungen zielen in erster Linie auf höheren Unternehmenserfolg und sind somit Teil einer breiten Tendenz, die Interessen organisationaler Akteure zu rationalisieren und mit den Interessen privilegierter Gruppen (des Managements) zu verbinden (vgl. Seibold \& Shea, 2001: 689ff.)

Insgesamt lässt sich feststellen, dass das konzeptionelle Netzwerk sehr dicht ist. Es gibt keine vollständig isolierten Beziehungen. Jeder Beziehungstyp ist zumindest über einen Topos mit einem anderen Typus verbunden. Dies zeugt zusammen mit der Tatsache, dass der zentrale Diskurs über die FK-MABeziehung von einigen wenigen Topoi konstituiert wird - von einer hohen Dichte des Diskurses. Die in der Abbildung dargestellten Topoi zeigen damit die typischen Argumentationsstrukturen innerhalb der Führungsgrundsätze. Natürlich sagt diese Karte nichts über die Verwendung und Vernetzung von Topoi in einzelnen Dokumenten aus. Die Abbildung zeigt aber auf der Ebene des Gesamtdiskurses, dass die Charakterisierung der FK-MA-Beziehung innerhalb der Dokumente nicht besonders vielseitig oder divers ist. 


\section{Schlussfolgerungen und Beitrag}

\subsection{Forschungsziel und Vorgehensweise}

Führungsgrundsätze wurden als Genre der Unternehmenskommunikation vorgestellt, welches im deutschsprachigen Raum bereits seit den späten 1960er Jahren existiert. In dieser 50-jährigen Geschichte haben die Dokumente verschiedene Labels wie „Leitsätze für Führung und Zusammenarbeit in der Unternehmung“, „Allgemeine Führungsleitsätze“, „Leitlinien zur Führung“, „Leitsätze für Führungskräfte“, „Grundsätze des Führungsverhaltens“, „Verhaltensleitsätze“, „Führungshilfen für Vorgesetzte“, „Pflichtenheft für das Vorgesetztenverhalten“, ,allgemeine Führungsanweisung“ sowie „Führungsrichtlinien“ (Knebel \& Schneider, 1994: 19) erhalten. Sie entwickelten sich von eher starren, regelhaften Pflichtenheften im Rahmen des Harzburger Modells (vgl. Höhn, 1976, 1977) zu werteorientierten Dokumenten mit dem Charakter von Leitlinien bzw. Leitbildern (vgl. Wunderer, 2009: 385). Moderne Führungsgrundsätze wurden folgendermaßen definiert:

„Führungsgrundsätze beschreiben und/oder normieren die Führungsbeziehungen zwischen Vorgesetzten und Mitarbeitern im Rahmen einer ziel- und werteorientierten Führungskonzeption zur Förderung eines erwünschten organisations- und mitgliedergerechten Sozial- und Leistungsverhaltens.

Sie können in schriftlicher Form (explizit) verbindlich fixiert werden oder als ungeschriebene Normen zur Verhaltensorientierung in den Führungsbeziehungen dienen. Häufig werden zusätzlich Prinzipien für die laterale Kooperation einbezogen. In diesem Fall sprechen wir von Führungs- und Kooperationsgrundsätzen“ (Wunderer, 2009: 385).

Ihnen werden verschiedene Wirkungen zugeschrieben, die im Rahmen oft erwähnter ,Funktionen“ - Deklarationsfunktion, Orientierungs- und Koordinationsfunktion, Apell- und Aktionsfunktion, Motivationsfunktion, Darstellungsfunktion - in der präskriptiven Literatur breit diskutiert werden (vgl. z.B. Finzer \& Mungenast, 1989; Finzer \& Mungenast, 1990; Grawert, 1997; Lattmann, 1975; Tschirky, 1981; Wunderer, 2009). Führungsgrundsätze gelten dennoch weithin als ,theorielos'. Die unterstellten Wirkungen sind wissenschaftlich nie in einen systematischen, konzeptionellen Rahmen eingebettet worden (vgl. Kossbiel, 1983: 20; Kubicek, 1984a: 4). Auch empirische Untersuchungen in diesem Feld beschränken sich zumeist auf inhaltsanalytische Designs, Verbreitungsstudien und die Erhebung subjektiver Wirkungseinschätzungen unter Führungskräften.

Die vorliegende Untersuchung hat es sich deshalb zum Ziel gesetzt, sich dieser Lücke in der Literatur anzunehmen und sich der latent hinter den Rege- 
lungsinhalten befindenden Sinn- und Bedeutungsdimension der Dokumente zu widmen. Dazu wurde zunächst durch die Diskussion von Kommunikation, Macht, Herrschaft, Steuerung, Rhetorik und Argumentation ein konzeptioneller Rahmen geschaffen, der es ermöglicht, die potentielle Wirkungsweise bzw. Steuerungswirkung von Führungsgrundsätzen sowie die in den Dokumenten propagierten Führungs- und Machtbeziehungen konzeptionell zu erfassen. Es wurde festgehalten, dass

- Führungsgrundsätze ein Steuerungsinstrument sind, welches in einem spezifischen, legitimierten Herrschaftsgefüge (Hierarchie, Aufbauorganisation) existiert und über die Beschreibung und Verordnung erwünschter Handlungen, Werte und Identitäten/Rollen potentielle Steuerungswirkungen auf organisationale Akteure (Führungskräfte und MitarbeiterInnen gleichermaßen) ausübt;

- Führungsgrundsätze ein Instrument der strukturellen Führung (vgl. Wunderer, 2009: 384) darstellen, welches in erster Linie Elemente der bürokratischen und kulturellen Steuerung (vgl. Sandner, 1990) verbindet;

- Führungsgrundsätze dadurch Verständnisse davon vermitteln, wie die Akteurstypen der Führungskräfte und MitarbeiterInnen zueinander in Beziehung treten sollen und sich daher über die Analyse dieser Beziehungsmuster typische Steuerungsstrategien (re-) konstruieren lassen;

- Führungsgrundsätze in erster Linie rhetorische bzw. argumentative Strategien verwenden, um die Adressaten von der Legitimität ihrer Aussagen zu überzeugen;

- eine umfassende (Re-)konstruktion von Aspekten der Macht, Herrschaft und Steuerung sowohl die Form (Argumentation) als auch den Inhalt der Führungsgrundsätze berücksichtigen muss.

In weiterer Folge wurde ein Untersuchungsdesign vorgestellt, welches in der Lage ist, über die (Re-)konstruktion fragmentarischer und latenter Begründungsmuster in Form von Enthymemen und Topoi (vgl. z.B. Ottmers, 2007; Wengeler, 2003b) dahinterliegende Welt- und Führungsverständnisse zu identifizieren. Dies wurde in einem grundsätzlich interpretativen Forschungsdesign (vgl. z.B. M. Lueger, 2001, 2010), das sich diskursanalytischer (vgl. z.B. Jäger, 1999; Keller et al., 2010a) und argumentationsanalytischer (vgl. z.B. Kopperschmidt, 1989; R. E. Meyer, 2004) Elemente bedient, geleistet. Die zentralen Ergebnisse der Untersuchung sollen nun anhand der eingangs formulierten Forschungs- und Subfragen im Detail diskutiert werden. 


\subsection{Beantwortung der forschungsleitenden Fragen}

Welche (impliziten und expliziten) Vorstellungen von Führung und Steuerung finden sich in kontemporären Führungsgrundsätzen deutschsprachiger Großunternehmen?

Mithilfe welcher rhetorischen Strategien und Ressourcen werden diese Vorstellungen argumentiert und damit im organisationalen Diskurs verankert?

Subfrage 1: Welche Akteure treten in den Dokumenten auf und wie werden diese charakterisiert?

In der Arbeit wurden sozial verfügbare Rollen als typisierte Handlungsverrichtungen, welche einen Teil des Selbst im Sinne gesellschaftlich erreichbarer Typisierungen objektivieren (vgl. Berger \& Luckmann, 2000: 77), diskutiert. Im Rahmen der Analyse wurden in den Dokumenten über die Codierung und Interpretation typischer Tätigkeiten für Führungskräfte und MitarbeiterInnen jeweils typische Rollenaspekte generiert und diskutiert. Dabei zeigten sich folgende Auffälligkeiten:

- Die (re-)konstruierten Rollenaspekte zwischen FK und MA unterscheiden sich nicht besonders stark. Die meisten der vorgefundenen Tätigkeiten werden sowohl FK als auch MA zugeschrieben. Dennoch besitzen die meisten der geteilten Rollenaspekte eine leicht unterschiedliche Ausprägung zwischen den Akteuren. Die Tätigkeiten der FK werden ebenfalls weitaus häufiger und auch detaillierter behandelt als jene der MA.

- Die Betrachtung der Häufigkeiten zeigt, dass für FK in erster Linie gewährleistende, gestaltende, lenkende und fordernde Tätigkeiten relevant sind. Die FK nimmt eine durchwegs aktive Rolle ein; sie ist sowohl für das Umfeld als auch für die direkte, interaktionale Mitarbeiterführung verantwortlich. In diesem Bereich führt sie vor allem kontrollierende, koordinierende, empathische und kommunikative Handlungen aus.

- Ein anderes Bild bietet sich bei der Betrachtung der MA. Die wenigen stark vertretenen Tätigkeiten bestehen in der Übernahme von Verantwortung und Gewährleistung. Es folgen kommunikative und analytische Handlungen.

Damit kann eine Trennung der Tätigkeiten in planende und dispositive Handlungen in den Dokumenten nicht erkannt werden. Dies liegt aber in erster Linie darin begründet, dass sich die Dokumente hauptsächlich auf die Führungsbeziehung konzentrieren. Die operative Geschäftstätigkeit des Unternehmens wird nur in seltenen Fällen angesprochen. Für FK gibt es jedoch einige Tätigkeiten (entscheiden, priorisieren), welche nicht (nur) direkt mit der MitarbeiterInnenführung in Zusammenhang stehen. Die MA hingegen tauchen hauptsächlich dann auf, wenn sie mit den Führungskräften in eine Beziehung treten oder ge- 
wisse Wertvorstellungen verkörpern sollen. Die (Re-)konstruktion von typischen Tätigkeiten und Rollenaspekten durch die Betrachtung von Verben im Material hat den Nachteil, dass damit nur explizit formulierte Rollenaspekte erfasst werden können. In der nachfolgenden Betrachtung der Beziehungen zwischen den Akteuren taucht ein weiterer Rollenaspekt auf, der sich vor allem implizit aus der Gegenüberstellung der Akteure ergibt, nämlich jener des passiv-abhängigen Akteurs, welcher den Handlungen anderer Akteure unterworfen ist, selbst aber nicht handelt. Dieser Rollenaspekt ist im Dokumentkorpus vor allem für die MA relevant, was die These von Jancsary et al. (2006: 103) von der Passivität und dem Objektstatus der MA in den Dokumenten stützt.

Subfrage 2: In welche Form(en) der Beziehung treten die dominanten Akteure zueinander?

Durch den systematischen Vergleich von Rollenaspekten und Tätigkeiten im Material wurden in der Folge 12 distinktive Beziehungstypen (re-)konstruiert. Diese bilden im Wesentlichen die klassischen Führungstätigkeiten und -bereiche wie z.B. Befähigung, Bereitstellung, Entwicklung, Integration, Kontrolle und Bewertung, Motivation, direkte Steuerung und Unterstützung ab. Spezifisch erscheinen lediglich die Empathie-Beziehung, welche die Anerkennung, Wertschätzung und Berücksichtigung der MA durch die FK fordert, die AnspruchsBeziehung, welche den Akteuren gegenseitig bestimmte Rechte einräumt und die Verfügungs-Beziehung, welche der FK die Möglichkeit gibt, direkt und unmittelbar über die MA zu verfügen bzw. diese zu nutzen und einzusetzen. In der Betrachtung der Beziehungstypen wurde festgestellt, dass Führungsgrundsätze im Wesentlichen alle Formen der von Sandner (1990) diskutierten Formen der Steuerung ansprechen, nämlich die direkte Steuerung (direkte SteuerungsBeziehung, Verfügungs-Beziehung, Anspruchs-Beziehung, BegrenzungsBeziehung), die technologische Steuerung (eingeschränkt die BereitstellungsBeziehung), die bürokratische Steuerung (Anspruchs-Beziehung, BegrenzungsBeziehung), die psychologische Steuerung (Befähigungs-Beziehung, Bereitstellungs-Beziehung, Empathie-Beziehung, Entwicklungs-Beziehung, IntegrationsBeziehung, Motivations-Beziehung, Unterstützungs-Beziehung) und die kulturelle Steuerung (Anspruchs-Beziehung, Entwicklungs-Beziehung, MotivationsBeziehung). Dabei zeigt sich sowohl quantitativ als auch qualitativ eine starke Dominanz der psychologischen Steuerung, und zwar in allen von Sandner diskutierten Ausprägungen: Pflege der sozialen Beziehungen, Motivationale Steuerung und Selbststeuerung (vgl. Sandner, 1990: 199ff.). Damit kann auch der proklamierte Wandel von den bürokratisch-autoritären Regelungsinhalten zu einem (manifest) stärker partizipativ und kooperativ ausgerichteten Führungsverständnis (vgl. Wunderer, 2009: 219ff.) bestätigt werden (vgl. z.B. Grawert, 
1997; Grunwald \& Lilge, 1981). Zu beachten ist allerdings, dass diese verstärkte Thematisierung motivationaler, integrativer und kooperativer Elemente in erster Linie instrumentell und funktional begründet wird. Die im Anschluss diskutierte Betrachtung der Begründungsmuster in den Dokumenten zeigt sehr deutlich, dass die meisten Regelungsinhalte aufgrund von Überlegungen ökonomischer Rationalität argumentiert werden. Ob also mit der veränderten Rhetorik auch ein entsprechend verändertes Menschenbild in den Organisationen einhergeht (auch vor dem Hintergrund der MA als ,Ressource“ und ,passiv-abhängigem“ Akteur), darf bezweifelt werden.

Subfrage 3: Innerhalb welcher inhaltlich-thematischer Argumentationen finden diese Beziehungen statt?

Zur Beantwortung dieser Frage wurde im Material durch die (Re-)konstruktion fragmentarischer und latenter Enthymem-Strukturen ein Katalog an besonderen Topoi (vgl. z.B. Ottmers, 2007; Wengeler, 2003a) generiert. Diese Topoi stellen argumentative Ressourcen dar, welche eingesetzt werden können, um Argumentationen in Form von Schlussregeln zu stützen. Topoi wurden in konstitutive Topoi (welche Beziehungstypen konstituieren und legitimieren) und ergänzende Topoi (welche innerhalb der Beziehungstypen verschiedene Aspekte argumentieren) unterteilt. Dabei wurden folgende Schlussfolgerungen gezogen:

- Die neun konstitutiven Topoi im Material zeigen deutliche Tendenzen zu einer Rationalisierung bzw. Versachlichung und Entemotionalisierung des Führungsthemas auf. Die meisten dieser Topoi rekurrieren auf abstrakte $\mathrm{Zu}-$ sammenhänge und Kausalitäten. Alle identifizierten Topoi lassen sich dabei letzten Endes auf zwei dominante Rationalitäten (im Sinne von Letztbegründungen) reduzieren: In erster Linie beziehen sich die Argumente auf eine funktionalistische bzw. utilitaristische Logik (,weil es [ökonomisch] gut für das Unternehmen ist'). In zweiter Linie werden aber auch normative und ethisch moralische Bezugssysteme herangezogen (,weil es [moralisch/ethisch] gut/richtig ist'; z.B. Gerechtigkeit, Gleichheit, Reziprozität). Führung als persönliche Einwirkung zur Durchsetzung organisationaler Interessen wird dadurch größtenteils ausgeblendet. Dieser direkte Eingriff in die Subjektsphäre des Individuums verschwindet vor dem Hintergrund von Sachzwängen und organisationalen Zielsetzungen. Es ist daher in den Führungsgrundsätzen eine starke Tendenz zur Herrschaftssicherung im Sinne der Stabilisierung und Normalisierung von Führung und Hierarchie zu finden.

- Neben der Argumentation von Führung und Steuerung an sich wird aber vor allem durch die ergänzenden Topoi auch die konkrete Ausgestaltung der Beziehung argumentiert. Diese Topoi bleiben ebenso wie die konstitutiven Topoi größtenteils diffus und auf einer sehr allgemeinen Ebene. Sie konstituie- 
ren Kausalitätsunterstellungen, welche auf der Ebene des ,Hausverstandes“ oder gängiger ,Klischees` zu finden sind. Damit evozieren sie vermeintlich geteilte Wertvorstellungen, denen man in der westlichen Gesellschaft kaum widersprechen kann; sie thematisieren den ,kleinsten gemeinsamen Nenner ${ }^{6}$ des sozialen Wissensvorrats (z.B. Gemeinsam sind wir stärker, No Risk, no Gain, Stillstand als Tod).

- Daraus ergibt sich, dass die Regelungsinhalte von Führungsgrundsätzen nicht in der Lage sind, spezifische und innovative Formen der Führungsbeziehung zu begründen. Sie resonieren aber in bekannten Sinnstrukturen und integrieren die thematisierten Führungsaspekte in den geteilten sozialen Wissensvorrat: „Sprecheräußerungen resonieren kulturell, wenn sie als Bausteine von ,bekannten Strukturen“ erkannt werden [...]“ (R. E. Meyer, 2004: 200). Führungsgrundsätze sind damit aber auch nicht in der Lage, ein spezifisches Führungsverständnis der eigenen Organisation zu entwickeln, das sich klar von denen anderer Organisationen abhebt. Dieses Manko wird dadurch zu schmälern versucht, dass die eigene Organisation in den Dokumenten z.T. stark betont und in ein diffuses, wir" integriert wird.

- Durch die Verwendung diffuser Regeln und allgemeiner Begründungsformen steigern die Dokumente auch die Möglichkeit ihrer identifikationsstiftenden Wirkung. Viele der diskutierten Topoi fallen in den Bereich der von Cheney (1983: 148ff.) diskutierten Strategien zur Identifikationserzeugung, vor allem der ,Common Ground Technique‘, welche im Bereich der Strategien und Instrumente stark mit Sandners (1990) psychologischer Steuerung korrespondiert. Durch die (wenig konkrete) Externalisierung von Bemühungen zur Partizipation und ,Fürsorge' für die MA wird annahmegemäß das Identifikationspotential gesteigert, was anschließend eine breitere Akzeptanz für jene Topoi schafft, welche mit dem Argument der Vorteilhaftigkeit für die Organisation arbeiten (vgl. Tompkins \& Cheney, 1985). Damit ist ein möglicher Erklärungsansatz für den ungewöhnlichen Mix an ökonomischen und moralisch/ethischen Begründungslogiken in den Dokumenten gefunden. Durch die Evokation moralisch/ethischer Argumente und Topoi wird den Organisationsmitglieder kommuniziert, dass die Organisation/das Management bereit ist, sich an diese Grundsätze zu halten. Gemeinsam mit der starken Betonung psychologischer Steuerungsformen (Anreize, Selbststeuerung, Mensch im Mittelpunkt) wird dadurch eine Identifikation der Individuen mit der Organisation zu erreichen versucht. Je stärker diese Identifikation ausfällt, desto eher sind diese Individuen im Anschluss dazu geneigt, die Argumente ökonomischer Rationalität (,was gut für das Unternehmen ist, ist auch gut für mich') als Entscheidungsprämissen für ihr Handeln zu akzeptieren (vgl. Tompkins \& Cheney, 1985). 
Insgesamt lässt sich daraus schließen, dass die in der präskriptiven Literatur formulierten Forderungen nach einer stärkeren wissenschaftlichen bzw. handlungstheoretischen Fundierung der Inhalte von FG (vgl. z.B. Kossbiel, 1983) sowie nach einer stärkeren Verbindlichkeit dieser (vgl. z.B. Kossbiel, 1983; Lattmann, 1975) sich vor dem Hintergrund dieser Ergebnisse als nicht zielführend erweisen. Moderne Führungsgrundsätze dienen in erster Linie dem Ziel der Herrschaftssicherung und der Normalisierung von Führung und Hierarchie. Jegliche wissenschaftliche Fundierung bedeutet eine Problematisierung der Alltagslogik der handelnden Akteure und würde im Kontext der organisationalen Sinnwelt die Hierarchiebeziehungen in den Blickpunkt rücken und somit deren Normalität gefährden. Dasselbe gilt für die harte Formulierung von Verbindlichkeiten: Was sanktioniert werden muss, kann nicht normal sein. Die Normalität wird aber eben gerade dadurch hergestellt, dass Führungs- und Hierarchiebeziehungen mit Argumenten untermauert werden, welche dem ,Hausverstand' bzw. gängigen ,Klischees‘ entsprechen. Diese sind unmittelbar anschlussfähig an die Lebenswelt einer Vielzahl verschiedener Akteure und führen daher nicht zur Hinterfragung oder offenen Diskussion der Prämissen. Die Diffusität, Banalität und Unverbindlichkeit der Grundsatzdokumente ist vor diesem Hintergrund durchaus funktional. Dies rückt sie weg von der bürokratischen Steuerung und stärker hin zu einer kulturellen bzw. normativen Steuerung durch symbolische Kommunikation (vgl. Sandner, 1990).

Subfrage 4: Wie stehen die in den Subfragen 1-3 untersuchten Aspekte in Beziehung zueinander und welches argumentative Bild von Führung ergibt sich daraus?

Viele Punkte, welche mit dieser vierten Subfrage angesprochen werden, wurden bereits in der Diskussion der ersten drei Fragen diskutiert. Die vorgestellte ,konzeptionelle Karte' des argumentativen Diskurses der Führungsgrundsätze fasst alle Aspekte zusammen. Dabei wird festgestellt, dass der Kern des Diskurses aus einigen wenigen, dafür aber stark vernetzten Topoi und Beziehungstypen gebildet wird. Die Kontrolle bzw. die Beeinflussung der kontextuellen Arbeitsbedingungen stellt damit das unmittelbare Zentrum eines Netzwerks an Argumentationsmustern dar. Die Betonung struktureller Führung im Gegensatz zur direkt interaktionalen, deren Ausprägung die Existenz der Führungsgrundsätze selbst ist, zeigt sich somit auch im inhaltlichen Diskurs der Dokumente. Gesteuert wird nicht in erster Linie direkt, sondern über die Manipulation von Kontextfaktoren. Diese wird gegenüber den MA hauptsächlich positiv begründet: Sie ermöglicht Empowerment, Ressourcenzuteilung und Unterstützung. Ein zweiter Knotenpunkt findet sich in der Betonung der Transparenz, welche den zentralen Topos für die Argumentation partizipativer und integrativer Führungsbestrebungen darstellt. MA werden integriert, da die erhöhte Transparenz be- 
trieblicher Abläufe ihr Leistungspotential und ihre Motivation steigert. Auch hier zeigt sich wieder der deutlich instrumentelle Charakter psychologischer Steuerungsformen im Material.

Führungsgrundsätze wurden eingangs als spezifisches Genre der Unternehmenskommunikation bezeichnet. Genres als soziale Institutionen (vgl. Knoblauch \& Luckmann, 2004; Orlikowski \& Yates, 1994; Yates \& Orlikowski, 1992) widmen sich der Lösung sozialer Probleme (vgl. das Konzept der 'exigence' bei Yates \& Orlikowski, 1992: 301). Was ist also vor dem Hintergrund der vorliegenden Untersuchung das Problem, und welche Lösungen bieten Führungsgrundsätze dafür? Dazu ist zunächst noch einmal darauf hinzuweisen, dass Führungsgrundsätze als Genre nur im deutschsprachigen Raum Verbreitung finden. Sie sind ,specific to organizations within certain societies or particular cultures" (Yates \& Orlikowski, 1992: 304). Sie bieten also eine kulturell spezifische Lösung für ein Problem. Ansätze zur Erklärung dieser kulturellen Spezifizität wurden mit Steyrers (2002) Begründung einer ,Führer-Phobie' geleistet, welche sich darin äußert, dass im deutschsprachigen Raum vor allem strukturelle Führungspraktiken dominieren (vgl. Müller, 1995: 575; Wunderer, 2009: $77 \mathrm{ff}$.). Dieses ambivalente Verhältnis zu (direkter) Führung zeigt sich auch in den Ergebnissen der vorliegenden Arbeit, und zwar in der absoluten Dominanz indirekter Führung und Steuerung über Kontextfaktoren. Während Beziehungstypen existieren, die einen direkteren Eingriff bzw. eine Verfügung eines Akteurs über den anderen begründen (Begrenzungs-Beziehung, direkte SteuerungsBeziehung, Verfügungs-Beziehung), sind diese im Diskurs eher peripher. Gesteuert wird nicht in erster Linie durch direkten Eingriff, sondern durch Manipulation von Faktoren des Arbeitsklimas und Arbeitsumfelds. Dies zeigt sich auch in der extremen Zentralität des entsprechenden Topos auf der ,Landkarte‘. Der Topos von der Bedeutung des Arbeitsklimas und Arbeitsumfelds konstituiert drei distinktive Beziehungstypen und ist somit einer Vielzahl unterschiedlicher anderer Topoi übergeordnet. Diese ,Vorsicht` mit direkten Führungsformen zeigt sich aber auch in der Rationalisierung und damit Entpersönlichung bzw. Entmenschlichung des Führungsthemas in der Argumentation, die weiter oben diskutiert wurde. Die Entscheidung über bestimmte Führungsformen ist im Diskurs der Führungsgrundsätze keine persönliche oder auch nur wählbare. Bestimmte Beziehungsausprägungen zwischen FK und MA werden stattdessen als notwendig argumentiert, und zwar entweder ökonomisch oder ethisch/moralisch. Damit wird die Entscheidungsverantwortung für die Inhalte von den Verfassern abgegeben, was einen Bruch mit der häufigen Verwendung des Pronomens ,wir' und der Argumentation der Spezifizität der vorgeschlagenen Regelungen erzeugt. Führungsgrundsätze - welche selbst ein Instrument der strukturellen Führung darstellen - sind somit ein in einem Kontext latenter ,Führerallergie' (vgl. 
Wunderer, 1985: 252) legitimiertes Medium zur Kommunikation über Führung. Sie abstrahieren von der direkten Führungsbeziehung in doppelter Art und Weise, nämlich indem sie einerseits demAdressaten selbst als Artefakt (und nicht als Person) entgegentreten und indem sie auch in ihren Regelungsinhalten die direkte, unmittelbare Steuerung herunterspielen und persönliche sowie Machtmotive seitens der FK völlig verschleiern. Nichtsdestotrotz - wie in der bisherigen Argumentation deutlich gemacht - lösen sie das Thema von Macht und Herrschaft damit nicht auf bzw. überführen es nicht in ein System mutueller Kooperation und Gleichwertigkeit von FK und MA. Sie ersetzen deutlich sichtbare, offene Formen von Macht und/oder Steuerung lediglich durch unsichtbare und unauffällige Strategien (vgl. z.B. Lukes, 2005; Tompkins \& Cheney, 1985) der Herrschaftssicherung.

\subsection{Beitrag zur Forschung}

Die Untersuchung leistet somit einen dreiteiligen Beitrag zur aktuellen Forschung. Im Bereich des Phänomens - also als empirischer Beitrag - bereichert sie die Forschung zu Führungsgrundsätzen durch eine aktuelle Studie. Während eine konsequente Weiterentwicklung der Grundsatzdokumente bis heute angenommen wird (vgl. Wunderer, 2009: 385), existieren keine wissenschaftlichen Untersuchungen, welche sich dieser Entwicklung annehmen. Die vorliegende Untersuchung leistet zwar keinen systematischen Vergleich mit älteren Studien, sie zeigt aber den aktuellen Stand gültiger Führungsgrundsätze auf und erweitert damit den Forschungsbereich. Außerdem leistet die gegenständliche Untersuchung eine bisher neuartige Verankerung der Thematik im konzeptionellen Bereich von Herrschaft, Steuerung und Rhetorik und folgt damit dem Ruf einer stärkeren konzeptionellen ,Untermauerung' der Forschung. Obwohl die vorliegende Untersuchung keine systematische Einschätzung der tatsächlichen Wirkung von Führungsgrundsätzen bietet, so liefert sie doch eine systematische und konzeptionell begründete Analyse der potentiellen oder hypothetischen Wirkungskanäle und -formen und geht auch in diesem Aspekt über die bisherige Formulierung von Wirkungsbehauptungen in der präskriptiven Literatur hinaus. Schließlich leistet die Arbeit auch einen kritischen Blick auf den in der Literatur behaupteten Wandel von der bürokratischen zur partizipativen bzw. kooperativen Führung, indem sie den strategischen bzw. instrumentellen Charakter dieser Form der neuen Rhetorik hervorhebt und durch die Analyse der Argumentationsstrukturen zeigt, dass die in der Vergangenheit geäußerte Kritik an der inkonsistenten und z.T. manipulativen Ideologie hinter Führungsgrundsätzen auch 
heute noch von Bedeutung ist (vgl. Breisig, 1987; Kubicek, 1984a, 1984b; Staehle, 1999).

Auf konzeptioneller Ebene leistet die Untersuchung eine stärkere Integration rhetorischer Argumente in die Diskussion von Macht und Steuerung durch die Einnahme einer wissenssoziologischen Perspektive. Während relationale Theorien der Macht (vgl. z.B. Sandner, 1990; Sandner \& Meyer, 2004) dem Akteur (auf beiden Seiten der Machtbeziehung) starke Handlungsmacht zukommen lassen und Intentionalität der Machtausübung erlauben bzw. unterstellen, dafür aber die Machtwirkung sozialer Strukturen und Diskurse größtenteils ausblenden, tendieren (post-)strukturalistische und diskursive Konzeptionen von Macht (vgl. z.B. Haugaard, 2009; Torfing, 2009) dazu, Akteure als Entscheidungssubjekte vollständig auszublenden bzw. lediglich als ,Spielball‘ größerer sozialer Strukturen und Diskurse zu sehen. Ein auf der phänomenologischen Wissenssoziologie von Berger und Luckmann (2000) aufbauendes Verständnis der Beziehung von Individuum und Gesellschaft kann diesen Bruch überwinden, indem durch die Integration diskurstheoretischer Argumente (vgl. Keller, 2008, 2010) eine dialektische Beziehung der gegenseitigen Konstitution zwischen Akteur und Diskurs angenommen wird. Durch diese Konzeption einer diskursiven Konstruktion der Gesellschaft rückt der Fokus auf kommunikative Praktiken der Machtausübung. Rhetorische Strategien werden dabei als vermittelnde Instanz zwischen Akteur und Diskurs gesehen. Akteure nutzen diskursive ,Bausteine ' in Form von Topoi (oder anderer, interpretativer Packages') um argumentativ auf breitere Deutungsschemata im sozialen Wissensvorrat zu verweisen (vgl. z.B. R. E. Meyer, 2004). Diese diskursiven Ereignisse (vgl. Keller, 2008) konstituieren Wirklichkeiten und typische Identitäten bzw. Rollen und üben somit innerhalb gesellschaftlicher Subwelten Macht aus. Mithilfe dieser theoretischen Konzeption wurde die Basis gelegt, um in der vorliegenden Untersuchung die potenzielle rhetorische Wirkung von Führungsgrundsätzen und die dahinter liegenden Weltbzw. Führungsverständnisse zu (re)konstruieren. Sie ist aber grundsätzlich für jede Untersuchung kommunikativer Praktiken in Organisationen anwendbar und lenkt den Blick der Forscherin/des Forschers auf kollektive Prozesse der Sinnkonstruktion und der grundsätzlich politischen Natur organisationalen Handelns und damit organisationaler Kommunikation.

In methodologischer und methodischer Hinsicht leistet die Untersuchung einen Beitrag zur stärkeren Inklusion argumentativer Strukturen in der Organisationsforschung. Während die Diskursforschung eine Blütezeit erlebt, wird die Untersuchung rhetorischer Elemente zwar in den meisten Standardwerken erwähnt (vgl. z.B. Grant et al., 2004b; van Dijk, 1997a, 1997b), führt aber in der Organisationsforschung dennoch ein eher bescheidenes Dasein (Ausnahmen sind z.B. Cheney, 1983; Cheney et al., 2004; Cheney \& Lair, 2005; Green, 2004; R. E. 
Meyer, 2004; Sillince, 1999; Vaara et al., 2005). Besonders die Verwendung des argumentativen Topos als Untersuchungseinheit hat sich in der Organisationstheorie noch nicht durchgesetzt. Dabei liegt die Stärke dieses Konstrukts darin, dass es in der Lage ist, argumentative Elemente abzubilden, ohne dass dafür vollständige formale Argumentationen (vgl. Toulmin, 2008) im Material vorhanden sein müssen. In dieser Hinsicht wirken Topoi wie interpretative Packages, indem sie auf die breiteren Bezugssysteme verweisen, vor deren Hintergrund sie argumentative Wirkung entfalten (vgl. R. E. Meyer, 2004: 174). Die vorliegende Untersuchung zeigt, dass die (Re-)konstruktion von Topoi zur Diskussion organisationaler Diskurse interessante Einblicke in die Begründungsstrukturen dieser Diskurse bieten kann.

\subsection{Grenzen und Ausblick}

Grundsätzlich hat jede empirische Untersuchung Grenzen, ob diese nun aus dem Format der Arbeit, der wissenschaftlichen Fragestellung oder den Ressourcen der Forscherin/des Forschers stammen. Im Folgenden sollen deshalb verschiedene Aspekte des Themas diskutiert werden, welche in der vorliegenden Arbeit nicht abgedeckt werden konnten, für zukünftige Untersuchungen aber möglicherweise von Interesse wären.

\subsubsection{Mögliche Erweiterungen innerhalb der Dokument- analyse}

Aufgrund des Charakters der Untersuchung als Querschnittsstudie kann sie Aussagen ausschließlich auf der Ebene des Gesamtkorpus (bzw. des Diskurses) treffen. Während dieses Untersuchungsdesign vielfältige Erkenntnisse über zentrale und periphere Diskurselemente zulässt, wäre es ergänzend auch interessant, die sequentielle Abfolge von Argumenten und Beziehungstypen innerhalb einzelner Dokumente zu betrachten. Eine solche Vorgehensweise würde es ermöglichen, die Zusammenhänge zwischen Beziehungstypen und Topoi noch stärker zu beleuchten und auch Argumentationsketten innerhalb der Dokumente stärker zu berücksichtigen (Welches Argument folgt typischerweise auf welches, welche kommen nie in direktem Zusammenhang vor, usw.?). Dadurch würde es auch in systematischer Weise möglich, unterschiedliche Ausprägungen bzw. Varianten des Diskurses schärfer herauszuarbeiten.

Von Interesse wäre außerdem der Vergleich der Argumentationsmuster und Beziehungstypen über verschiedene Dokumentgenres hinweg. In der abschließenden Diskussion der Ergebnisse wurde betont, dass Führungsgrundsätze ein 
für den deutschsprachigen Kulturraum spezifisches Instrument des Sprechens über und des Steuerns von Führungshandeln darstellen. Das Problem der Steuerung organisationalen Handelns kann aber als universell bezeichnet werden: „Wie sonst sollen die arbeitsteiligen Prozesse der Leistungserstellung trotz nie ganz konvergierender Ziele und Interessen der Beteiligten etabliert, aufeinander abgestimmt und auf Dauer gestellt werden können?“ (Fischer, 2009: 15). Abgesehen von der Schärfung der Genregrenzen von Führungsgrundsätzen durch Inklusion von Leitbildern, Unternehmensgrundsätzen, Codes of Conduct und anderen im deutschsprachigen Raum ebenfalls verbreiteten Dokumentgenres wäre es deshalb für das Verständnis der Spezifizität der Steuerung durch Führungsgrundsätze von Interesse, kommunikative Lösungen dieses Problems aus anderen Kulturräumen zu untersuchen. Eine zukünftige Untersuchung könnte zeigen, welche Unterschiede und Gemeinsamkeiten diese Genres in Bezug auf die ihnen zugrundeliegenden Begründungsstrukturen aufweisen und damit noch tiefere Einblicke in die soziale Einbettung des Phänomens ,Führungsgrundsätze“ bieten.

Auch der Textbegriff könnte in einem zukünftigen Forschungsprojekt erweitert werden. Während Sprache laut Berger und Luckmann (2000) das wichtigste soziale Symbolsystem zur Konstruktion sozialer Realität ist, so ist es doch bei weitem nicht das einzige. Führungsgrundsätze weisen unterschiedliche Grade der Visualisierung ihres Inhalts mittels Bildern und Graphiken aus. Die (Re-) konstruktion von Bedeutungsmustern über Bilder und visuelle Elemente ist in der Organisationsforschung ein emergentes Feld (vgl. z.B. Bell \& Davison, forthcoming; Bell, Schroeder, \& Warren, 2013; Höllerer, Jancsary, Meyer, \& Vettori, forthcoming; Meyer, Höllerer, Jancsary, \& van Leeuwen, forthcoming). Eine Analyse visueller Elemente könnte die Diskursanalyse des verbalen Textes ergänzen und erweitern.

\subsubsection{Erweiterungen durch Einbezug von Kontextfakto- ren}

Abgesehen von der Erweiterung des Scopes der Untersuchung innerhalb der Dokumentanalyse wäre es aber auch interessant, die Handhabung der Dokumente in situ, also im direkten Organisationskontext zu betrachten. Diskursanalysen verschriftlichter Artefakte können immer nur Aussagen zu typischen oder hypothetischen Bedeutungen dieser Artefakte im organisationalen Alltag treffen. Insofern wäre es auch von Interesse, die konkrete Erstellung eines solchen Dokuments begleitend $\mathrm{zu}$ untersuchen, um die in dialogischen Argumentationsprozessen entstehende Bedeutung von einzelnen diskursiven Ereignissen zu analysieren. Ebenso nützlich wäre aber auch eine Untersuchung der tatsächlichen 
Verwendung der Dokumente im organisationalen Alltag, um neben der Logik der Produktion auch jene der Rezeption zu erfassen: Wie werden die Dokumente gelesen? Wie werden ihre Regelungen empfunden? Wann , nimmt man sie in die Hand"? All diese Fragen mussten notwendigerweise aus der gegenständlichen Untersuchung ausgeklammert werden, könnten aber in zukünftigen Forschungsvorhaben zu einem noch besseren Verständnis des Phänomens und damit der argumentativen Strategien der Konstruktion von Führung und Steuerung beitragen. 



\section{Literaturverzeichnis}

Albach, H. (1976). Welche Aussagen lassen Führungsgrundsätze von Unternehmen über die Auswirkungen gesellschaftlicher Veränderungen auf die Willensbildung im Unternehmen zu? In H. Albach \& D. Sadowski (Hrsg.), Die Bedeutung gesellschaftlicher Veränderungen für die Willensbildung im Unternehmen (S. 739-764). Berlin: Duncker \& Humblot.

Albach, H. (1983). Zum Einfluß von Führungsgrundsätzen auf die Personalführung. In R. Wunderer (Hrsg.), Führungsgrundsätze in Wirtschaft und öffentlicher Verwaltung (S. 2-15). Stuttgart: Poeschel.

Allen, N. J. \& Meyer, J. P. (1991). A three component conceptualization of organizational commitment. Human Resource Management Review, 1(1), 61-89.

Aprath, G. (1993). Führungsgrundsätze. In H. Strutz (Hrsg.), Handbuch Personalmarketing (2. Aufl., S. 482-489). Wiesbaden: Gabler.

Arendt, H. (1970). On violence. New York: Harcourt Brace and Company.

Ashford, S. J., Lee, C. \& Bobko, P. (1989). Content, causes, and consequences of job insecurity: A theory-based measure and substantive test. Academy of Management Journal, 32(4), 803-829.

Attenhofer, M. W. (1990). Führen mit Leitbild, Grundsätzen und Strategien. IO Management Zeitschrift, 59(5), 27-31.

Bachrach, P. \& Baratz, M. S. (1962). Two Faces of power. American Political Science Review, 56(4), 947-952.

Barley, S. R. \& Kunda, G. (1992). Design and devotion: Surges of rational and normative ideologies of control in managerial discourse. Administrative Science Quarterly, 37(3), 363-399.

Barley, S. R., Meyer, G. W. \& Gash, D. C. (1988). Cultures of culture: Academics, practitioners and the pragmatics of normative control. Administrative Science Quarterly, 33(1), 24-60.

Baumgarten, R. (1977). Führungsstile und Führungstechniken. Berlin.

Bell, E., \& Davison, J. (forthcoming). Visual management studies: Empirical and theoretical approaches. International Journal of Management Reviews.

Bell, E., Schroeder, J.E., \& Warren, S. (Eds.). (2013). The Routledge companion to visual organization. London et al.: Routledge.

Berger, P. L. \& Luckmann, T. (2000). Die gesellschaftliche Konstruktion der Wirklichkeit. Frankfurt am Main: Fischer. 
Bisel, R. S., Ford, D. J. \& Keyton, J. (2007). Unobtrusive control in a leadership organization: Integrating control and resistance. Western Journal of Communication, 71(1), 136-158.

Boeije, H. (2002). A purposeful approach to the constant comparative method in the analysis of qualitative interviews. Quality \& Quantity, 36(4), 391-409.

Borman, W. C. \& Motowidlo, S. J. (1997). Task performance and contextual performance: The meaning for personnel selection research. Human Performance, 10(2), 99-109.

Bourdieu, P. (1974). Zur Soziologie der symbolischen Formen. Frankfurt a.M.: Suhrkamp.

Bourdieu, P. (1994). Sozialer Sinn. Frankfurt a.M.: Suhrkamp.

Breisig, T. (1987). Führungsmodelle und Führungsgrundsätze - verändertes unternehmerisches Selbstverständnis oder Instrument der Rationalisierung? Spardorf: Wilfer.

Bridges, J. A. (2004). Corporate issues campaigns: Six theoretical aproaches. Communication Theory, 14(1), 51-77.

Brief, A. P. \& Motowidlo, S. J. (1986). Prosocial organizational behaviors. Academy of Management Review, 11(4), 710-725.

Brimeyer, T. M., Eaker, A. V. \& Clair, R. P. (2004). Rhetorical strategies in union organizing. Management Communication Quarterly, 18(1), 45-75.

Buchmann (1994). Führungsgrundsätze in der Kreditwirtschaft. Zeitschrift Führung und Organisation, 3, 194-196.

Burr, V. (1995). An Introduction to social constructionism. London et al.: Routledge.

Cassell, C., Johnson, P. \& Smith, K. (1997). Opening the black box: Corporate codes of ethics in their organizational context. Journal of Business Ethics, 16(10), 1077-1093.

Cheney, G. (1983). The rhetoric of identification and the study of organizational communication. Quarterly Journal of Speech, 69 (2), 143-158.

Cheney, G., Christensen, L. T., Conrad, C. \& Lair, D. J. (2004). Corporate rhetoric as organizational discourse. In D. Grant, C. Hardy, C. Oswick \& L. L. Putnam (Hrsg.), The SAGE Handbook of Organizational Discourse (S. 79-103). London et al.: SAGE Publications.

Cheney, G. \& Frenette, G. (1993). Persuasion and organization: Values, logics, and accounts in contemporary corporate public discourse. In C. Conrad (Hrsg.), The Ethical Nexus (S. 49-73). Norwood, NJ: Ablex Publishing.

Cheney, G. \& Lair, D. J. (2005). Theorizing about rhetoric and organizations. Classical, interpretive, and critical aspects. In S. May \& D. K. Mumby (Hrsg.), Engaging organizational communication theory \& research: 
Multiple perspectives (S. 55-84). Thousand Oaks et al.: SAGE Publications.

Cheney, G. \& Vibbert, S. L. (1987). Corporate discourse: Public relations and issues management. In F. M. Jablin, K. H. Roberts \& L. W. Porter (Hrsg.), Handbook of Organizational Communication (S. 165-194). Beverly Hills, CA: SAGE Publications.

Chilton, P. \& Schäffner, C. (1997). Dicourse and politics. In T. A. van Dijk (Hrsg.), Discourse studies: A multidisciplinary introduction. Volume 2: Discourse as social interaction (S. 206-230). London et al.: SAGE Publications.

Clegg, S. R. (1989). Frameworks of power. London et al.: SAGE Publications.

Clegg, S. R. (2009). Managing power in organizations: The hidden history of its constitution. In S. R. Clegg \& M. Haugaard (Hrsg.), The SAGE handbook of power (S. 310-331). Los Angeles et al.: SAGE Publications.

Clegg, S. R., Courpasson, D. \& Phillips, N. (2007). Power and organizations. Los Angeles et al.: SAGE Publications.

Conrad, C. \& Haynes, J. (2001). Development of key constructs. In F. M. Jablin \& L. L. Putnam (Hrsg.), The new handbook of organizational communication (S. 47-77). Thousand Oaks, CA: SAGE Publications.

Conrad, C. \& Ryan, M. (1985). Power, praxis, and self in organizational communication theory. In R. D. McPhee \& P. K. Tompkins (Hrsg.), Organizational communication: Traditional themes and new directions (S. 235-257). Beverly Hills et al:: SAGE Publications.

Conrad, P. (1988). Involvement-Forschung. Berlin et al.: de Gruyter.

Crozier, M. \& Friedberg, E. (1979). Macht und Organisation. Königstein: Ts.

Dachler, H. P. \& Hosking, D.-M. (1995). The primacy of relations in socially constructing organizational realities. In D.-M. Hosking, H. P. Dachler \& K. J. Gergen (Hrsg.), Management and organization: relational alternatives to individualism (S. 1-28). Aldershot: Avebury.

Deetz, S. (1995). Representational practices and the political analysis of corporations: Building a communication perspective in organizational Studies. In B. Kovačić (Hrsg.), New approaches to organizational communication (S. 211-243). Albany: State University of New York Press.

Deetz, S. (2001). Conceptual foundations. In F. M. Jablin \& L. L. Putnam (Hrsg.), The new handbook of organizational communication (S. 3-46). Thousand Oaks, CA: SAGE Publications.

Diatlova, I. (2003). Unternehmenstexte: Textsorten, Textcluster, topische Muster. Frankfurt a.M. et al.: Peter Lang. 
DiSanza, J. R. \& Bullis, C. (1999). "Everybody identifies with Smokey the Bear": Employee responses to newsletter identification inducements at the U.S. Forest Service. Management Communication Quarterly, 12(3), 347398.

Du Gay, P. (1996). Making up managers: Enterprise and the ethos of bureaucracy. In S. Clegg \& G. Palmer (Hrsg.), The politics of managment knowledge (S. 19-35). London et al.: SAGE Publications.

Ebert, H. (1994a). Textfunktionen und Textstrukturen von Führungs- und Unternehmensgrundsätzen der Gegenwart. Frankfurt a.M. et al.: Peter Lang.

Ebert, H. (1994b). Zur Sprache von „Führungsgrundsätzen“ in Wirtschaft und öffentlicher Verwaltung. Eine handlungsorientiert-textlinguistische Studie. In T. Bungarten (Hrsg.), Unternehmenskommunikation. Linguistische Analysen und Beschreibungen (S. 43-60). Tostedt: AttikonVerlag.

Ebert, H. (1997). Textfunktionen und Textstrukturen von Führungs- und Unternehmensgrundsätzen der Gegenwart. Frankfurt a.M. et al.: Peter Lang.

Eggins, S. \& Martin, J. R. (1997). Genres and registers of discourse. In T. A. Van Dijk (Hrsg.), Discourse as structure and process (S. 230-256). London et al.: SAGE Publications.

Engelstadt, F. (2009). Culture and power. In S. R. Clegg \& M. Haugaard (Hrsg.), The SAGE handbook of power (S. 210-237). Los Angeles et al.: SAGE Publications.

Etzioni, A. (1961). A comparative analysis of complex organizations. New York: The Free Press.

Fairclough, N. (1997). Critical discourse analysis (2. Aufl.). London: Longman.

Fairclough, N. (2003). Analysing discourse: Textual analysis for social research. London et al.: Routledge.

Fairhurst, G. T. \& Putnam, L. (2004). Organizations as discursive constructions. Communication Theory, 14(1), 5-26.

Fiedler-Winter, R. (1994). Braucht ein Unternehmen überhaupt Führungsgrundsätze? IO Management Zeitschrift, 63(5), 69 - 72.

Fiedler-Winter, R. (1999). Vom „roten Faden der Unternehmenskultur“. Leitlinien - Führungsgrundsätze - Visionen. IO Management Zeitschrift, 68(10), 18-22.

Fiedler, H. (1976). Führungsgrundsätze. Fortschrittliche Betriebsführung und Industrial Engineering, 25(5), 313-317. 
Fiedler, H. (1980). Unternehmensgrundsätze und Führungsleitlinien. Fortschrittliche Betriebsführung und Industrial Engineering, 29(2), 122129.

Finstad, N. (1998). The rhetoric of organizational change. Human Relations, 51(6), 717-740.

Finzer, P. \& Mungenast, M. (1989). Führungsgrundsätze: Führungsinstrument oder Unternehmenszeremonie? 1. Teil: Klassifikation und Funktionen. Zeitschrift Führung und Organisation, 1989, Heft 6, 405-409.

Finzer, P. \& Mungenast, M. (1990). Führungsgrundsätze: Führungsinstrument oder Unternehmenszeremonie? 2. Teil: Inhalte und Gestaltungsansätze. Zeitschrift Führung und Organisation, 1990, Heft 1, 50-54.

Fischer, J. H. (2009). Steuerung in Organisationen. Wiesbaden: VS Verlag für Sozialwissenschaften.

Fleming, P. \& Sturdy, A. (2009). "Just be yourself!" Towards neo-normative control in organizations? Employee Relations, 31(6), 569-583.

Flick, U. (2002). Qualitative Sozialforschung. Eine Einführung (6. Aufl.). Reinbek bei Hamburg: Rowohlt.

Flick, U., von Kardorff, E. \& Steinke, I. (Hrsg.). (2005). Qualitative Forschung. Ein Handbuch. Hamburg: Rowohlt.

Franke, J. \& Kühlmann, T. (1986). Führungsgrundsätze. Sind sie Pflichtübung oder echte Hilfe? IO Management Zeitschrift, 55(1), 80-81.

Fritz, J. M. H., Arnett, R. C. \& Conkel, M. (1999). Organizational ethical standards and organizational commitment. Journal of Business Ethics, 20(4), 289-299.

Froschauer, U. \& Lueger, M. (2003). Das qualitative Interview. Zur Praxis interpretativer Analyse sozialer Systeme. Wien: WUV Universitätsverlag.

Froschauer, U. \& Lueger, M. (2009). Interpretative Sozialforschung: Der Prozess. Wien: Facultas WUV.

Gabele, E. (1982). Führungs- und Unternehmensgrundsätze - Ein Spiegelbild innerbetrieblicher und gesellschaftlicher Entwicklungen. In E. Gabele \& W. A. Oechsler (Hrsg.), Führungsgrundsätze und Führungsmodelle (S. 13-34). Bamberg: Bayrische Verlagsanstalt.

Gabele, E. (1992). Führungs- und Unternehmensgrundsätze - Ein Spiegelbild innerbetrieblicher und gesellschaftlicher Entwicklungen. In E. Gabele, H. J. Liebel \& W. A. Oechsler (Hrsg.), Führungsgrundsätze und Mitarbeiterführung (S. 11-58). Wiesbaden: Gabler.

Gabele, E. \& Kretschmer, H. (1981). Unternehmensgrundsätze in Theorie und Praxis. Bamberg: Univ.

Gabele, E. \& Kretschmer, H. (1986). Unternehmensgrundsätze. Empirische Erhebungen und praktische Erfahrungsberichte zur Konzeption, 
Einrichtung und Wirkungsweise eines modernen Führungsinstrumentes. Zürich: Verlag Industrielle Organisation.

Gabele, E., Liebel, H. J. \& Oechsler, W. A. (1982). Führungsgrundsätze und Führungsmodelle. Bamberg: Bayrische Verlagsanstalt.

Gamson, W. A. \& Modigliani, A. (1989). Media discourse and public opinion

on nuclear power: A constructionist approach. American Journal of Sociology, 95(1), 1-37.

Gauger, J. (2000). Commitment-Management im Unternehmen. Wiesbaden: Deutscher Universitäts-Verlag.

Gergen, K. J. (1994). Realities and relationships. Soundings in social construction. Cambridge, MA et al.: Harvard University Press.

Gergen, K. J. (1999). An invitation to social construction. London et al.: SAGE Publications.

Gergen, M. (1995). The social construction of grievances: constructive and constructionist approaches to a relational theory. In D.-M. Hosking, H. P. Dachler \& K. J. Gergen (Hrsg.), Management and organization: relational alternatives to individualism (S. 98-103). Aldershot et al.: Avebury.

Giddens, A. (1984). The constitution of society. Cambridge et al.: Polity Press.

Gill, A. M. \& Whedbee, K. (1997). Rhetoric. In T. A. van Dijk (Hrsg.), Discourse studies: A multidisciplinary introduction. Volume 1: Discourse as structure and process (S. 157-185). London et al.: SAGE Publications.

Glaser, B. G. \& Strauss, A. L. (1998). Grounded Theory: Strategien qualitativer Forschung. Bern et al.: Huber.

Goffman, E. (1977). Rahmen-Analyse. Ein Versuch über die Organisation von Alltagserfahrungen. Frankfurt a.M.: Suhrkamp.

Göhler, G. (2009). 'Power to' and 'Power over'. In S. R. Clegg \& M. Haugaard (Hrsg.), The SAGE handbook of power (S. 27-39). Los Angeles et al.: SAGE Publications.

Gordon, R. (2009). Power and legitimacy: From Weber to contemporary theory. In S. R. Clegg \& M. Haugaard (Hrsg.), The SAGE handbook of power (S. 256-273). Los Angeles et al.: SAGE Publications.

Grant, D., Hardy, C., Oswick, C. \& Putnam, L. (2004a). Introduction: Organizational discourse: Exploring the field. In D. Grant, C. Hardy, C. Oswick \& L. L. Putnam (Hrsg.), The SAGE handbook of organizational discourse (S. 1-36). London et al.: SAGE Publications.

Grant, D., Hardy, C., Oswick, C. \& Putnam, L. (Hrsg.). (2004b). The SAGE handbook of organizational discourse. London et al.: SAGE Publications.

Grawert, A. (1997). Führungsleitlinien - Ein Instrument zeitgemäßer Personalführung. In U. Kadritzke (Hrsg.), ,, Unternehmenskulturen “ unter 
Druck: neue Managementkonzepte zwischen Anspruch und Wirklichkeit (S. 87-99). Berlin: Edition Sigma.

Green, S. E. Jr. (2004). A rhetorical theory of diffusion. Academy of Management Review, 29(4), 653-669.

Grugulis, I., Dundon, T. \& Wilkinson, A. (2000). Cultural control and the 'Culture Manager': Employment practies in a consultancy. Work, Employment \& Society, 14(1), 97-116.

Grunwald, W. \& Lilge, H.-G. (1981). Auf dem Weg zur partizipativen Führung. Fortschrittliche Betriebsführung und Industrial Engineering, 30(6), 411417.

Guserl, R. (1973). Das Harzburger Modell: Ideen und Wirklichkeit. Wiesbaden. Haase, D. (1997). Organisationsstruktur und Mitarbeiterbindung. Köln: Deutscher Instituts-Verlag.

Hajer, M. A. (2010). Argumentative Diskursanalyse. Auf der Suche nach Koalitionen, Praktiken und Bedeutung. In R. Keller, A. Hirseland, W. Schneider \& W. Viehöver (Hrsg.), Handbuch Sozialwissenschaftliche Diskursanalyse. Band 2: Forschungspraxis (4. Aufl., S. 271-298). Wiesbaden: VS Verlag für Sozialwissenschaften.

Harris, L. C. \& Ogbonna, E. (2011). Antecedents and consequences of management-espoused organizational cultural control. Journal of Business Research, 64 (5), 437-445.

Haugaard, M. (2009). Power and hegemony. In S. R. Clegg \& M. Haugaard (Hrsg.), The SAGE handbook of power (S. 239-255). Los Angeles et al.: SAGE Publications.

Haugaard, M. \& Clegg, S. R. (2009). Introduction: Why power is the central concept of the social sciences. In S. R. Clegg \& M. Haugaard (Hrsg.), The SAGE handbook of power (S. 1-24). Los Angeles et al.: SAGE Publications.

Hechter, M. (2008). The rise and fall of normative control. Accounting, Organizations and Society, 33, 663-676.

Hickson, D. J., Hinings, C. R., Lee, C. A., Schneck, R. E. \& Pennings, J. M. (1971). A strategic contingencies' theory of intra-organizational power. Administrative Science Quarterly, 16(2), 216-229.

Hitzler, R. \& Eberle, T. (2005). Phänomenologische Lebensweltanalyse. In U. Flick, E. von Kardorff \& I. Steinke (Hrsg.), Qualitative Forschung. Ein Handbuch (S. 109-118). Hamburg: Rowohlt.

Hoffmann, F. (1989). Unternehmungs- und Führungsgrundsätze. Zeitschrift für betriebswirtschaftliche Forschung (ZfBf), 41(3), 167-185.

Höhn, R. (1976). Stellenbeschreibung und Führungsanweisung. Bad Harzburg: Verlag für Wissenschaft. 
Höhn, R. (1977). Führungsbrevier der Wirtschaft. Bad Harzburg: Verlag für Wissenschaft, Wirtschaft und Technik.

Höllerer, M. A., Jancsary, D., Meyer, R. E., \& Vettori, O. (forthcoming). Imageries of corporate social responsibility: Visual re-contextualization and field-level meaning. Research in the Sociology of Organizations.

Hopkins, N. \& Reicher, S. (1997). Social movement rhetoric and the social psychology of collective action. A case study of anti-abortion mobilization. Human Relations, 50(3), 261-286.

Jäger, S. (1999). Kritische Diskursanalyse. Duisburg: DISS.

Jancsary, D. (2007). Standard dance or improvisation? The role of Austrian written principles of leadership in the construction of social identities. Paper presented at the 23rd EGOS Colloquium.

Jancsary, D., Knassmüller, M. \& Sandner, K. (2006). Führungsgrundsätze als Steuerungsinstrument (Unveröffentlichter Projektbericht des OeNBJubiläumsfondsprojektes Nr. 8457). Wien: Institut für Public Management an der WU Wien.

Kasper, H. \& Mühlbacher, J. (2002). Von Organisationskulturen zu lernenden Organisationen. In H. Kasper \& W. Mayrhofer (Hrsg.), Personalmanagement, Führung, Organisation (3. Aufl., S. 95-156). Wien: Linde Verlag.

Kelle, U. \& Kluge, S. (2010). Vom Einzelfall zum Typus. Fallvergleich und Kontrastierung in der qualitativen Sozialforschung. Wiesbaden: VS Verlag für Sozialwissenschaften.

Keller, R. (1997). Diskursanalyse. In R. Hitzler \& A. Honer (Hrsg.), Sozialwissenschaftliche Hermeneutik (S. 309-333). Opladen: UTB.

Keller, R. (2008). Wissenssoziologische Diskursanalyse. Grundlegung eines Forschungsprogramms. Wiesbaden: VS Verlag für Sozialwissenschaften.

Keller, R. (2010). Der Müll der Gesellschaft. Eine wissenssoziologische Diskursanalyse. In R. Keller, A. Hirseland, W. Schneider \& W. Viehöver (Hrsg.), Handbuch Sozialwissenschaftliche Diskursanalyse. Band 2: Forschungspraxis (4. Aufl., S. 195-232). Wiesbaden: VS Verlag für Sozialwissenschaften.

Keller, R. (2011). Diskursforschung. Eine Einführung für SozialwissenschaftlerInnen (4. Aufl.). Wiesbaden: VS Verlag für Sozialwissenschaften.

Keller, R., Hirseland, A., Schneider, W. \& Viehöver, W. (2010a). Die vielgestaltige Praxis der Diskursforschung - Eine Einführung. In R. Keller, A. Hirseland, W. Schneider \& W. Viehöver (Hrsg.), Handbuch Sozialwissenschaftliche Diskursanalyse. Band 2: Forschungspraxis (4. Aufl., S. 7-20). Wiesbaden: VS Verlag für Sozialwissenschaften. 
Keller, R., Hirseland, A., Schneider, W. \& Viehöver, W. (Hrsg.). (2010b). Handbuch Sozialwissenschaftliche Diskursanalyse. Band 2: Forschungspraxis (4. Aufl.). Wiesbaden: VS Verlag für Sozialwissenschaften.

Kienpointner (1992). Alltagslogik. Struktur und Funktion von Argumentationsmustern. Stuttgart et al.: Frommann-Holzboog.

Knassmüller, M. (2005). Unternehmensleitbilder im Vergleich: Sinn- und Bedeutungsrahmen deutschsprachiger Unternehmensleitbilder - Versuch einer empirischen (Re-)Konstruktion. Frankfurt a.M.: Peter Lang.

Knebel, H. (1982). Einführung von Führungsgrundsätzen. In A. Töpfer \& E. Zander (Hrsg.), Führungsgrundsätze und Führungsinstrumente (S. 194263). Frankfurt a.M.: Metzner.

Knebel, H. (1984). Führungsgrundsätze in der Diskussion - eine Stellungnahme zu den Thesen von Herbert Kubicek. Zeitschrift für Betriebswirtschaft, 54(9), 881-887.

Knebel, H. \& Schneider, H. (1994). Führungsgrundsätze: Leitlinien für die Einführung und praktische Umsetzung. Heidelberg: Sauer.

Knoblauch, H. \& Luckmann, T. (2004). Genre analysis. In U. Flick, E. von Kardorff \& I. Steinke (Hrsg.), A companion to qualitative research (S. 303-307). London et al.: SAGE Publications.

Konovsky, M. A. \& Cropanzano, R. (1991). Perceived fairness of employee drug testing as a predictor of employee attitudes and job performance. Journal of Applied Psychology, 76(5), 698-707.

Kopperschmidt, J. (1989). Methodik der Argumentationsanalyse. Stuttgart et al.: Frommann-Holzboog.

Kossbiel, H. (1983). Die Bedeutung formalisierter Führungsgrundsätze für die Verhaltenssteuerung von Organisationen. In R. Wunderer (Hrsg.), Führungsgrundsätze in Wirtschaft und öffentlicher Verwaltung (S. 17-27). Stuttgart: Poeschel.

Kreitner, R. \& Kinicki, A. (2004). Organizational Behavior (6. Aufl.): Boston, MA: McGraw-Hill.

Kubicek, H. (1984a). Führungsgrundsätze. Lösungen von gestern für die Probleme von morgen? Teil 1. Zeitschrift Führung und Organisation, 1984, Heft 2, 81-88.

Kubicek, H. (1984b). Führungsgrundsätze. Lösungen von gestern für die Probleme von morgen? Teil 2. Zeitschrift Führung und Organisation, 1984, Heft 3, 182-188.

Kuhn, T. S. (1973). Die Struktur wissenschaftlicher Revolutionen. Frankfurt a.M: Suhrkamp. 
Kwok, C.-K., Au, W. T. \& Ho, J. M. C. (2005). Normative controls and selfreported counterproductive behaviors in the workplace in China. Applied Psychology: An International Review, 54(4), 456-475.

Lamertz, K. \& Baum, J. A. C. (1998). The legitimacy of organizational downsizing in Canada: An analysis of explanatory media accounts. Canadian Journal of Administrative Sciences, 15(1), 93-107.

Lamnek, S. (2005). Qualitative Sozialforschung (4. Aufl.). Weinheim et al.: Beltz.

Langlois, C. C. \& Schlegelmilch, B. B. (1990). Do corporate codes of ethics reflect national character? Evidence from Europe and the United States. Journal of International Business Studies, 21(4), 519-539.

Lattmann, C. (1975). Führungsstil und Führungsrichtlinien. Bern et al.: Paul Haupt.

Locke, K. (2001). Grounded Theory in Management Research. London et al: SAGE Publications.

Luckmann, T. (2002). Wissen und Gesellschaft. Konstanz: Universitätsverlag.

Lüders, C. (1991). Deutungsmusteranalyse. Annäherungen an ein risikoreiches Konzept. In D. Garz \& K. Kraimer (Hrsg.), Qualitativ-empirische Sozialforschung (S. 377-408). Opladen: Westdeutscher Verlag.

Lüders, C. \& Meuser, M. (1997). Deutungsmusteranalyse. In R. Hitzler \& A. Honer (Hrsg.), Sozialwissenschaftliche Hermeneutik (S. 57-80). Opladen: Leske und Budrich.

Lueger, G. (2002). Personalbeurteilung. In H. Kasper \& W. Mayrhofer (Hrsg.), Personalmanagement, Führung, Organisation (S. 447-480). Wien: Linde Verlag.

Lueger, M. (1989). Das Verhältnis von Macht und Herrschaft als politische Wechselbeziehung in Organisationen. In K. Sandner (Hrsg.), Politische Prozesse in Unternehmen (S. 185-203). Berlin et al.: Springer.

Lueger, M. (2000). Grundlagen qualitativer Feldforschung. Wien: WUV Universitätsverlag.

Lueger, M. (2001). Auf den Spuren der sozialen Welt. Methodologie und Organisierung interpretativer Sozialforschung. Frankfurt a.M.: Peter Lang.

Lueger, M. (2010). Interpretative Sozialforschung: Die Methoden. Wien: Facultas WUV.

Luhmann, N. (1977). Macht. Stuttgart: Lucius \& Lucius.

Lukes, S. (1974). Power. A radical view. London: Macmillan.

Lukes, S. (2005). Power. A radical view (2. Aufl.). Hampshire et al.: Palgrave Mcmillan. 
Macharzina, K. (2003). Unternehmensführung. Das internationale Managementwissen. Konzepte - Methoden - Praxis. Wiesbaden: Gabler.

Malik, F. (2003). Führungsgrundsätze. In R. Boutellier, S. M. Wagner \& H. P. Wehrli (Hrsg.), Handbuch Beschaffung. Strategien. Methoden. Umsetzung (S. 133-141). München: Hanser.

Mayring, P. (2002). Qualitative Inhaltsanalyse. Weinheim: Belz - UTB.

Meuser, M. \& Sackmann, R. (Hrsg.). (1992). Analyse sozialer Deutungsmuster. Beiträge zur empirischen Wissenssoziologie. Pfaffenweiler: CentaurusVerlagsgesellschaft.

Meyer, A. (2000). Führende und Geführte im Wandel der Führungsparadigmen des 20. Jahrhunderts: ein Wandel vom Objekt zum selbstverantwortlichen Subjekt? Frankfurt a.M.: Peter Lang.

Meyer, H. (2002). Unternehmensführung. Aufgaben und Techniken betrieblichen Managements (2. Aufl.). Berlin: Herne.

Meyer, J. P., Allen, N. J. \& Gellatly, I. R. (1990). Affective and continuance commitment to the organization: Evaluation of measures and analysis of concurrent and time-lagged relations. Journal of Applied Psychology, 75(6), 710-720.

Meyer, J. W. \& Rowan, B. (1977). Institutionalized organizations: Formal structure as myth and ceremony. American Journal of Sociology, 83(2), 340-363.

Meyer, R. E. (2004). Globale Managementkonzepte und lokaler Kontext. Organisationale Wertorientierung im österreichischen öffentlichen Diskurs. Wien: WUV Universitätsverlag.

Meyer, R. E. (2006). Visiting relatives: Current developments in the new sociology of knowledge. Organization, 13(5), 725-738.

Meyer, R. E., \& Höllerer, M. A. (2010). Meaning structures in a contested issue field: A topographic map of shareholder value in Austria. Academy of Management Journal, 53(6), 1241-1262.

Meyer, R. E., Höllerer, M. A., Jancsary, D., \& van Leeuwen, T. (forthcoming). The visual dimension in organizing, organization, and organization research: Core ideas, current developments, and promising avenues. Academy of Management Annals.

Modaff, D. P., DeWine, S. \& Butler, J. A. (2008). Organizational communication: Foundations, challenges, and misunderstandings. Boston et al.: Pearson.

Mohr, G. (1999). Führungsleitlinien: Ein Leitimpuls für alle Führungssysteme in einem Unternehmen. Sparkasse, 116(3), 134-137.

Moorman, R. H., Blakely, G. L. \& Niehoff, B. P. (1998). Does percived organizational support mediate the relationship between procedural justice 
and organizational citizenship behavior? Academy of Management Journal, 41(3), 351-357.

Morris, T., Lydka, H. \& O'Creevy, M. F. (1993). Can commitment be managed? A longiudinal analysis of employee commitment and human resource policies. Human Resource Management Journal, 3(3), 21-42.

Müller-Seitz, P. (1988). Führungsgrundsätze als Bestandteil von Unternehmensverfassungen? Pro und Contra. Zeitschrift Führung und Organisation(3), 152 - 159.

Müller, W. R. (1995). Führungsforschung/Führung in der Bundesrepublik Deutschland, in Österreich und in der Schweiz. In A. Kieser, G. Reber \& R. Wunderer (Hrsg.), Handwörterbuch der Führung (2. Aufl., S. 573586). Stuttgart: Schäffer-Poeschel.

Mumby, D. K. (2001). Power and politics. In F. M. Jablin \& L. L. Putnam (Hrsg.), The new handbook of organizational communication (S. 585623). Thousand Oaks, CA: SAGE Publications.

Mumby, D. K. \& Clair, R. P. (1997). Organizational discourse. In T. A. van Dijk (Hrsg.), Discourse studies: A multidisciplinary introduction. Volume 2: Discourse as social interaction (S. 181-205). London et al.: SAGE Publications.

Mumby, D. K. \& Stohl, C. (1991). Power and discourse in organization studies: absence and the dialectic of control. Discourse \& Society, 2(3), 313-332.

Neuberger, O. (2002). Führen und geführt werden (6. Aufl.). Stuttgart: Lucius \& Lucius.

Nonhoff, M. (2010). Hegemonieanalyse: Theorie, Methode und Forschungspraxis. In R. Keller, A. Hirseland, W. Schneider \& W. Viehöver (Hrsg.), Handbuch Sozialwissenschaftliche Diskursanalyse. Band 2: Forschungspraxis (Vol. 4., S. 299- 331). Wiesbaden: VS Verlag für Sozialwissenschaften.

Oechsler, W. A. (1997). Personal und Arbeit. München, Wien: Oldenburg.

Oechsler, W. A. (2000). Personal und Arbeit (7. Aufl.). München et al.: Oldenburg.

Oevermann, U. (1973). Zur Analyse der Struktur von sozialen Deutungsmustern.Unpublished manuscript, Franfurt am Main.

Organ, D. W. (1988). Organizational citizenship behavior: The good soldier syndrome. Lexington, MA: Lexington.

Orlikowski, W. J. \& Yates, J. (1994). Genre repertoire: The structuring of communicative practices in organizations. Administrative Science Quarterly, 39, 541-574.

Ottmers, C. (2007). Rhetorik (2. Aufl.). Stuttgart et al.: J.B. Metzler. 
Parsons, T. (1964). Essays in sociological theory. New York: Free Press of Glencoe.

Paschen, K. (1983). Führungsleitsätze - eine modische Erscheinung oder eine Chance für eine effiziente Unternehmenssteuerung? In R. Wunderer (Hrsg.), Führungsgrundsätze in Wirtschaft und öffentlicher Verwaltung (S. 29-34). Stuttgart: Poeschel.

Pfeffer, J. \& Salancik, G. R. (1978). The external control of organizations. New York: Harper \& Row.

Phillips, N. \& Brown, J. L. (1993). Analyzing communication in and around organizations: A critical hermeneutic approach. Academy of Management Journal, 36(6), 1547-1576.

Pietschmann, B. P., Huppertz, S. \& Ruhtz, V. (1999). Was macht Führungsgrundsätze erfolgreich? Personal, 1999, Heft 10, 506-510.

Putnam, L. \& Boys, S. (2006). Revisiting metaphors of organizational communication. In S. R. Clegg, W. Nord \& C. Hardy (Hrsg.), The SAGE handbook of organization studies (S. 541-576). London et al.: SAGE Publications.

Rapley, T. (2007). Doing conversation, discourse and document analysis. London et al.: SAGE Publications.

Robertson, M. \& Swan, J. (2004). 'Control - What Control?' Culture and ambiguity within a knowledge intensive firm. Journal of Management Studies, 40(4), 831-858.

Roth, M. (2000). Compliance. Begriff, Bedeutung, Beispiele. Basel et al.: Helbing \& Lichtenhahn.

Rühli, E. (1975). Die wichtigsten Führungsinstrumente im Betrieb. In E. Rühli (Hrsg.), Beiträge zur Unternehmensführung und Unternehmenspolitik (S. 15-26). Bern, Stuttgart: Haupt.

Rühli, E. (1992). Gestaltungsmöglichkeiten der Unternehmungsführung. Führungsstil, Führungsmodelle, Führungsrichtlinien, Mitwirkung, Mitbestimmung. Bern et al.: Haupt.

Sandner, K. (1990). Prozesse der Macht. Zur Entstehung, Stabilisierung und Veränderung der Macht von Akteuren in Unternehmen. Berlin et al.: Springer.

Sandner, K. \& Meyer, R. E. (2002). Strategic actors and constructed identities: Power and politics in organization theory. Soka Review of Business Administration, 26(2), 47-60.

Sandner, K. \& Meyer, R. E. (2003). Macht. In E. Gaugler, W. A. Oechsler \& W. Weber (Hrsg.), Handwörterbuch des Personalwesens (3. Aufl., S. 11331141). Stuttgart: Schäffer-Poeschel. 
Sandner, K. \& Meyer, R. E. (2004). Macht in Organisationen. In G. Schreyögg \& A. von Werder (Hrsg.), Handwörterbuch Unternehmensführung und Organisation (4. Aufl., S. 756-765). Stuttgart: Schäffer-Poeschel.

Sandner, K. \& Meyer, R. E. (2006). Macht. In Handelsblatt (Hrsg.), Wirtschaftslexikon. Das Wissen der Betriebswirtschaftslehre. Band 7 (S. 3566-3572). Stuttgart: Schäffer-Poeschel.

Scherer, A. (2000). Können die „Grundsätze ordnungsmäßiger Unternehmensleitung (GoU)“ ${ }^{،}$ zu einer Verbesserung der Managementpraxis beitragen? - Kritische Bemerkungen $\mathrm{zu}$ den Vorschlägen von Axel von Werder. Betriebswirtschaftliche Forschung und Praxis (BFuP), 2000, Heft 1, 84-98.

Schreyögg, G. (2003). Organisation. Grundlagen moderner Organisationsgestaltung (4. Aufl.). Wiesbaden: Gabler.

Schröer, N. (Hrsg.). (1994). Interpretative Sozialforschung. Auf dem Wege zu einer hermeneutischen Wissenssoziologie. Opladen: Westdeutscher Verlag.

Schwartz, M. (2001). The nature of the relationship between corporate codes of ethics and behavior. Journal of Business Ethics, 32(3), 247-262.

Seibold, D. R. \& Shea, C. B. (2001). Participation and decision making. In F. M. Jablin \& L. L. Putnam (Hrsg.), The new handbook of organizational communication (S. 664-703). Thousand Oaks, CA: SAGE Publications.

Shotter, J. (1993). Conversational realities: Constructing life through language. London et al.: SAGE Publications.

Sillince, J. A. A. (1999). The organizational setting, use and institutionalization of argumentation repertoires. Journal of Management Studies, 36(6), 795830.

Soeffner, H.-G. (2004). Social scientific hermeneutics. In U. Flick, E. Von Kardorff \& I. Steinke (Hrsg.), A companion to qualitative research (S. 95100). London et al.: SAGE.

Somers, M. J. (2001). Ethical codes of conduct and organizational context: A study of the relationship between codes of conduct, employee behavior and organizational values. Journal of Business Ethics, 30(2), 185-195.

Staehle, W. H. (1999). Management. Eine verhaltenswissenschaftliche Perspektive (8. Aufl.). München: Vahlen.

Steinke, I. (2005). Gütekriterien qualitativer Forschung. In U. Flick, E. von Kardorff \& I. Steinke (Hrsg.), Qualitative Forschung. Ein Handbuch (S. 319-331). Hamburg: Rowohlt.

Steinle, C. (1975). Leistungsverhalten und Führung in der Unternehmung. Berlin: Duncker \& Humblot. 
Steinle, C., Ahlers, F. \& Riechmann, C. (1999). Management by Commitment Möglichkeiten und Grenzen einer ,selbstverpflichtenden' Führung von Mitarbeitern. Zeitschrift für Personalforschung, 1999, Heft 3, 221-245.

Steyrer, J. (1989). Sozioökonomische Rahmenbedingungen politischer Prozesse in Unternehmen. In K. Sandner (Hrsg.), Politische Prozesse in Unternehmen (S. 7-45). Berlin et al.: Springer.

Steyrer, J. (2002). Theorien der Führung. In H. Kasper \& W. Mayrhofer (Hrsg.), Personalmanagement, Führung, Organisation (S. 157-212). Wien: Linde Verlag.

Steyrer, J., Schiffinger, M. \& Lang, R. (2007). Ideal- und Realbild von Führung. Zum Zusammenhang zwischen Führungswahrnehmung, organisationalem Commitment und Unternehmenserfolg. Zeitschrift für Management, 2(4), 402-434.

Taylor, B. C. \& Trujillo, N. (2001). Qualitative research methods. In F. M. Jablin \& L. Putnam (Hrsg.), The new handbook of organizational communication (S. 161-196). London et al.: SAGE Publications.

Titscher, S., Wodak, R., Meyer, M. \& Vetter, E. (1998). Methoden der Textanalyse. Opladen: Westdeutscher Verlag.

Tompkins, P. K. \& Cheney, G. (1985). Communication and unobtrusive control in contemporary organizations. In R. D. McPhee \& P. K. Tompkins (Hrsg.), Organizational communication: Traditional themes and new directions (S. 179-211). Beverly Hills et al.: SAGE Publications.

Tompkins, P. K. \& Wanca-Thibault, M. (2001). Organizational communication: prelude and prospects. In F. M. Jablin \& L. L. Putnam (Hrsg.), The new handbook of organizational communication (S. xvii-xxxi). Thousand Oaks, CA: SAGE Publications.

Töpfer, A. \& Zander, E. (1982). Einordnung der Beiträge. In A. Töpfer \& E. Zander (Hrsg.), Führungsgrundsätze und Führungsinstrumente (S. 1-28). Frankfurt am Main: Metzner.

Torfing, J. (2009). Power and discourse: Towards an anti-foundationalist concept of power. In S. R. Clegg \& M. Haugaard (Hrsg.), The SAGE Handbook of Power (S. 108-124). Los Angeles et al.: SAGE Publications.

Toulmin, S. E. (2008). The uses of argument. New York: Cambridge University Press.

Treiber, H. (2007). Macht - ein soziologischer Grundbegriff. In P. Gostmann \& P.-U. Merz-Benz (Hrsg.), Macht und Herrschaft. Zur Revision zweier soziologischer Grundbegriffe (S. 49-62). Wiesbaden: VS Verlag für Sozialwissenschaften.

Tschirky, H. (1981). Führungsrichtlinien. Zürich: Industrielle Organisation. 
Vaara, E., Tienari, J., Piekkari, R. \& Säntti, R. (2005). Language and the circuits of power in a merging multinational corporation. Journal of Management Studies, 42(3), 595-623.

Valentine, S. \& Barnett, T. (2003). Ethics code awareness, perceived ethical values, and organizational commitment. Journal of Personal Selling \& Sales Management, 23(4), 359-367.

Van Dijk, T. A. (2008). Discourse and power. Hampshire et al.: Palgrave Mcmillan.

Van Dijk, T. A. (Hrsg.). (1997a). Discourse studies: A multidisciplinary introduction. Volume 1: Discourse as structure and process. London et al.: SAGE Publications.

Van Dijk, T. A. (Hrsg.). (1997b). Discourse studies: A multidisciplinary introduction. Volume 2: Discourse as social interaction. London et al.: SAGE Publications.

Van Eemeren, F. H., Grootendorst, R., Jackson, S. \& Jacobs, S. (1997). Argumentation. In T. A. Van Dijk (Hrsg.), Discourse studies: A multidisciplinary introduction. Volume 1: Discourse as structure and process (S. 209-229). London et al:: SAGE Publications.

Wagner, G. (2007). Herrschaft und soziales Handeln - eine Notiz zur Systematisierung zweier soziologischer Grundbegriffe. In P. Gostmann \& P.-U. Merz-Benz (Hrsg.), Macht und Herrschaft. Zur Revision zweier soziologischer Grundbegriffe (S. 17-26). Wiesbaden: VS Verlag für Sozialwissenschaften.

Wagner, W. (1994). Alltagsdiskurs. Die Theorie sozialer Repräsentationen. Göttingen et al.: Hogrefe.

Weller, S. (1988). The Effectiveness of corporate codes of ethics. Journal of Business Ethics, 7(5), 389-395.

Wengeler, M. (1997). Argumentation im Einwanderungsdiskurs. Ein Vergleich der Zeiträume 1970-1973 und 1980-1983. In M. Jung, M. Wengeler \& K. Böke (Hrsg.), Die Sprache des Migrationsdiskurses (S. 121-149). Opladen: Westdeutscher Verlag.

Wengeler, M. (2003a). Argumentationstopos als sprachwissenschaftlicher Gegenstand. Für eine Erweiterung linguistischer Methoden bei der Analyse öffentlicher Diskurse. In S. Geideck \& W.-A. Liebert (Hrsg.), Sinnformeln. Linguistische und soziologische Analysen von Leitbildern, Metaphern und anderen kollektiven Orientierungsmustern. Berlin et al.: Walter de Gruyter.

Wengeler, M. (2003b). Topos und Diskurs. Begründung einer argumentationsanalytischen Methode und ihre Anwendung auf den Migrationsdiskurs (1960-1985). Tübingen: Niemeyer. 
Westphal, J. D. \& Zajac, E. J. (1998). The symbolic management of stockholders: Corporate governance reforms and shareholder reactions. Administrative Science Quarterly, 43 (1), 127-153.

Westphal, J. D. \& Zajac, E. J. (2001). Decoupling policy from practice: The case of stock repurchase programs. Administrative Science Quarterly, 46 (2), 202-228.

Williams, L. J. \& Anderson, S. E. (1991). Job satisfaction and organizational commitment as predictors of organizational citizenship and in-role behaviors. Journal of Management, 17(3), 601-617.

Wittgenstein, L. (2006). Werkausgabe Bd. 1. Tractatus logico-philosophicus, Tagebücher 1914-1916, Philosophische Untersuchungen. Frankfurt a.M.: Suhrkamp.

Wodak, R. (2008). Qualitative Discourse Analysis in the Social Sciences. Hampshire: Palgrave Macmillan.

Wodak, R. \& Meyer, M. (2009). Methods for Critical Discourse Analysis. London et al.: SAGE Publications.

Wunderer, R. (1985). Betriebswirtschaftslehre und Führung Entwicklungslinien, Besonderheiten, Funktionen. In R. Wunderer (Hrsg.), Betriebswirtschaftslehre als Management- und Führungslehre (S. 237267). Stuttgart: Schäffer-Poeschel.

Wunderer, R. (1993). Führungsgrundsätze. In R. Wunderer (Hrsg.), Führung und Zusammenarbeit. Beiträge zu einer Führungslehre (S. 172-183). Stuttgart: Schäffer-Poeschel.

Wunderer, R. (1995). Führungsgrundsätze. In A. Kieser, G. Reber \& R. Wunderer (Hrsg.), Handwörterbuch der Führung (2. Aufl., S. 720-734). Stuttgart: Schäffer-Poeschel.

Wunderer, R. (2009). Führung und Zusammenarbeit (8. Aufl.). München: Luchterhand.

Wunderer, R. (Hrsg.). (1983). Führungsgrundsätze in Wirtschaft und öffentlicher Verwaltung. Stuttgart: Schäffer-Poeschel.

Wunderer, R. \& Grunwald, W. (1980). Führungslehre. Berlin: de Gruyter.

Wunderer, R. \& Klimecki, R. (1990). Führungsleitbilder. Grundsätze für Führung und Zusammenarbeit in deutschen Unternehmen. Stuttgart: Schäffer-Poeschel.

Yates, J. \& Orlikowski, W. J. (1992). Genres of organizational communication: A structurational approach to studying communication and media. Academy of Management Review, 17(2), 299-326. 



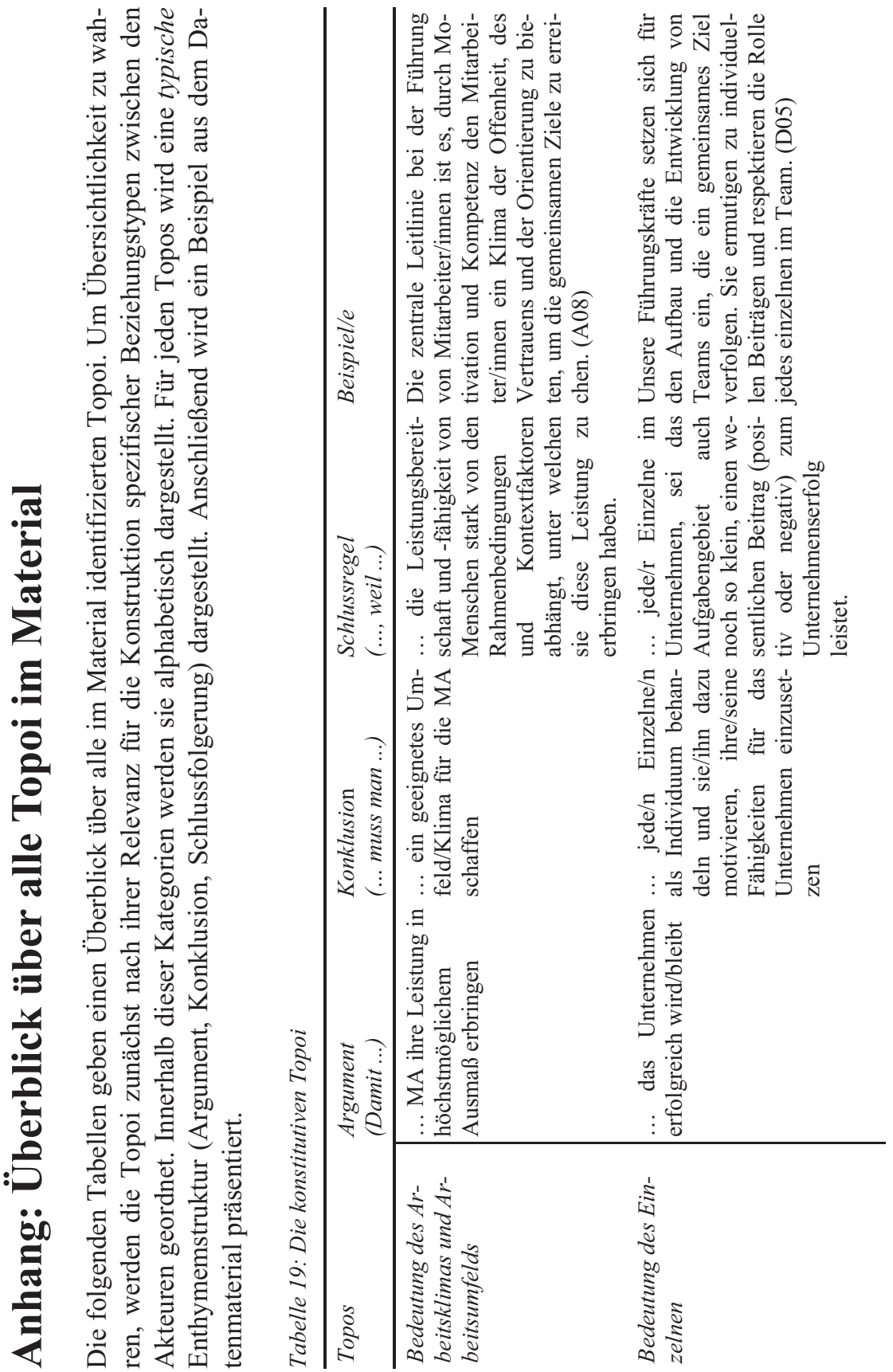


눌

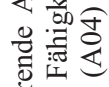

可

产苛

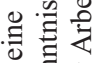

进总

品 卷

表

उ.

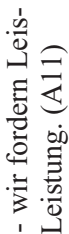

이 동

蛋

플

क्ष.

战
咅

㤩总起

的岛

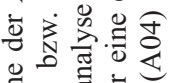

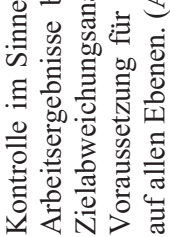

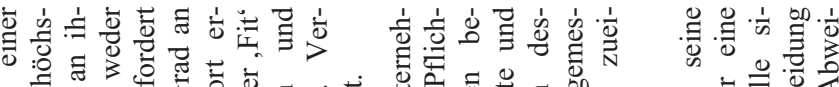

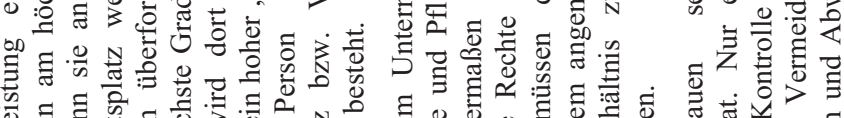

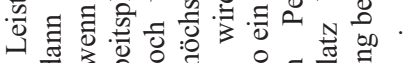

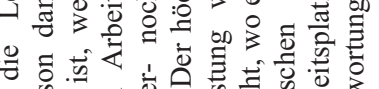

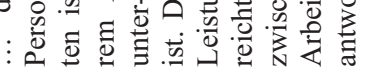

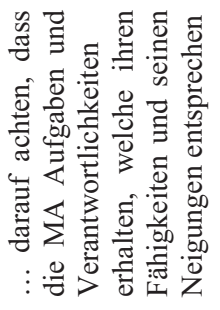

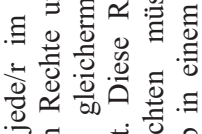

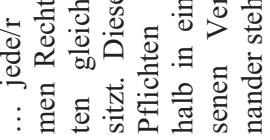

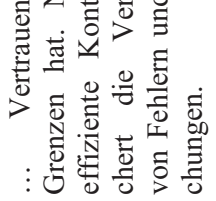

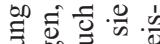

需离

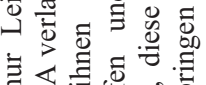

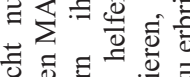

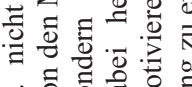

:

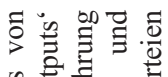

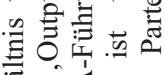

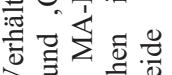

里语

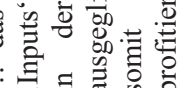

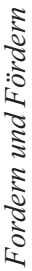

家

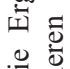

$\because \frac{0}{=}$

를휴

$:$ 包

宛穿㐫

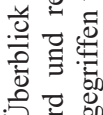

5 . 홉

矛先

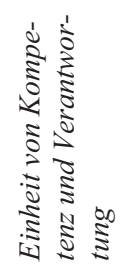




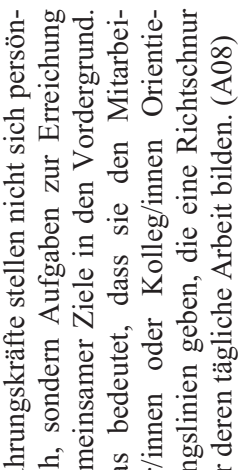

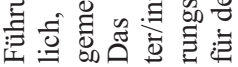

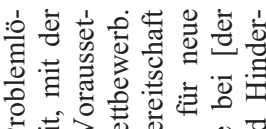

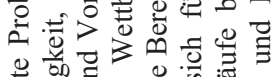

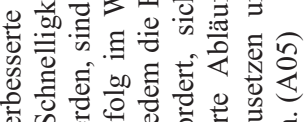

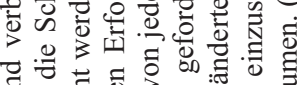

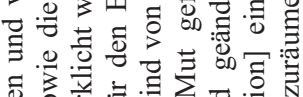

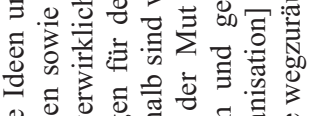

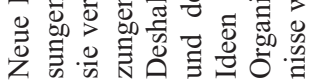

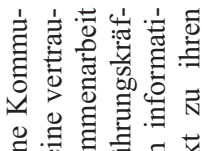

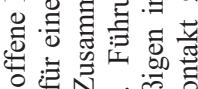

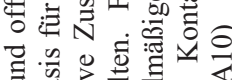

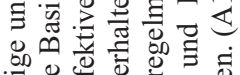

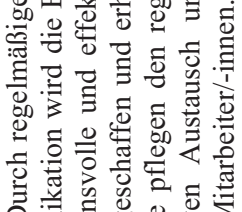

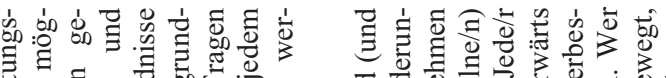

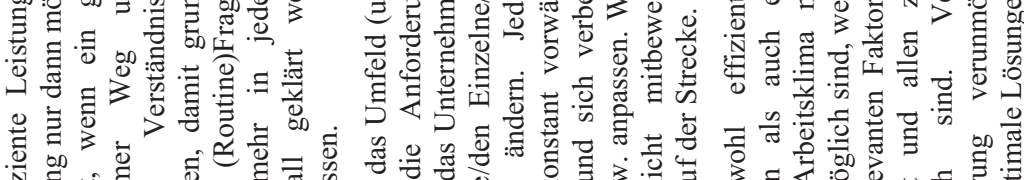

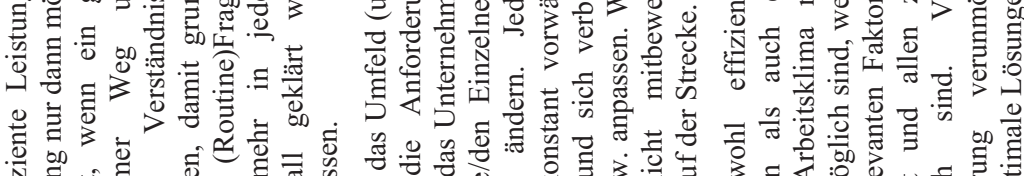

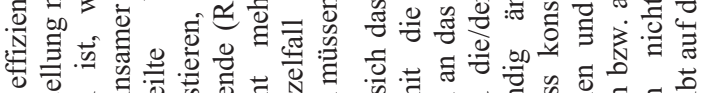

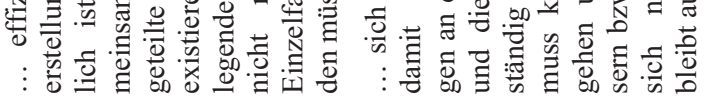

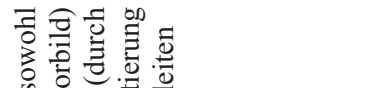

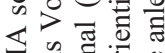

$\sum_{0}^{\frac{n}{\pi}} \sum_{0}^{0} \cdot \frac{0}{0}$

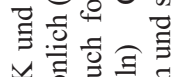

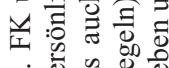

$\vdots$ : $\frac{0}{2} \stackrel{0}{0}$

:

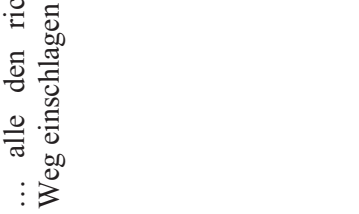

\section{$\geq 8$ i 00}

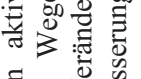

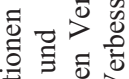

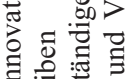

三? :

: 壱寻㤩导

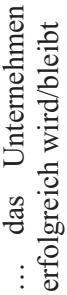

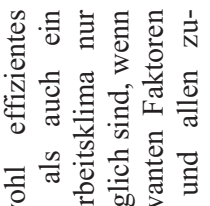

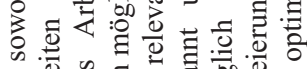

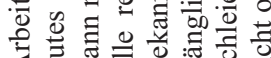

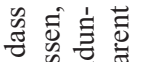

( ) क्ष

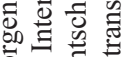

क्ञ

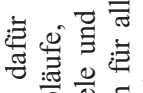

: 过 总常

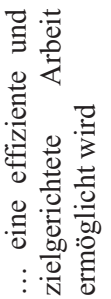

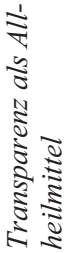

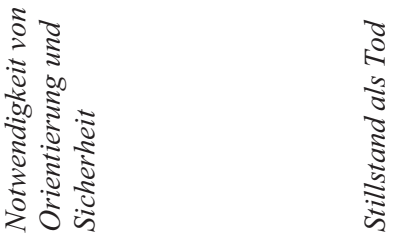

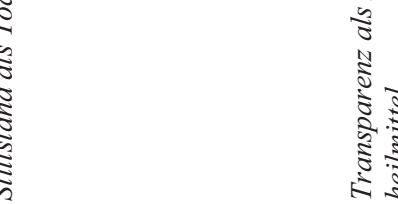




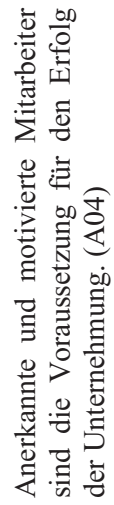

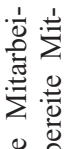

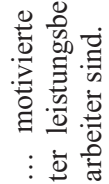

$\stackrel{1}{\Xi}$

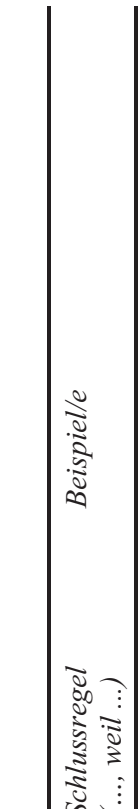

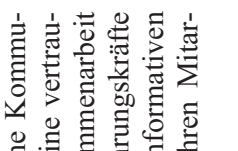

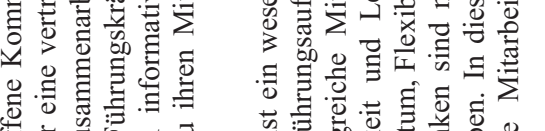

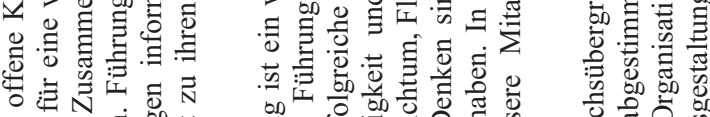

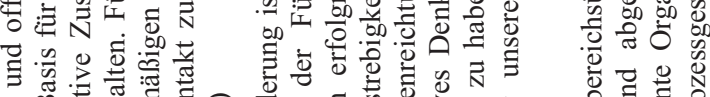

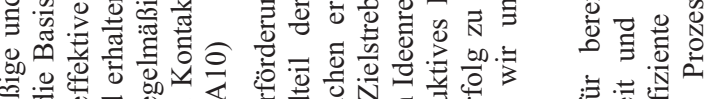

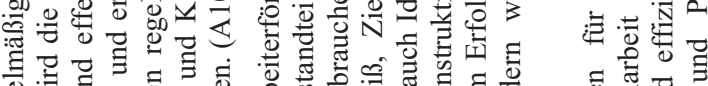

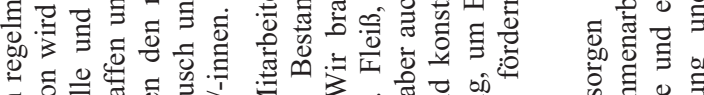

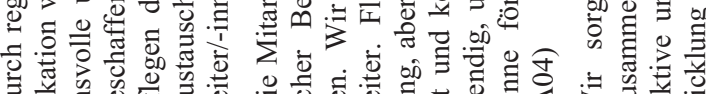

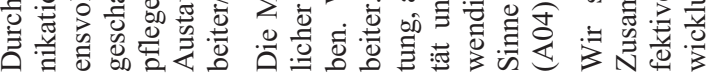

등 步

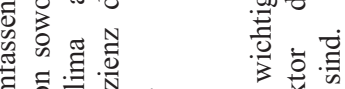

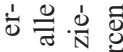

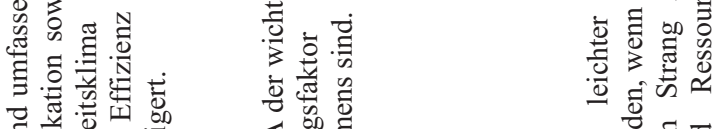

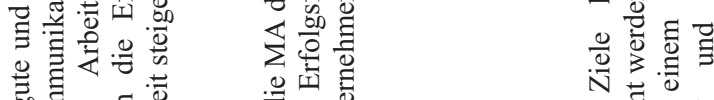

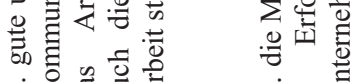

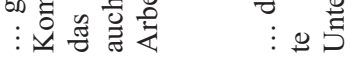

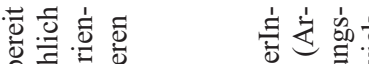

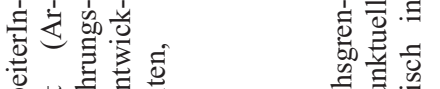

:

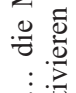

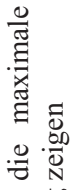

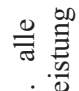

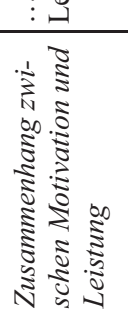

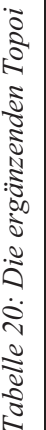

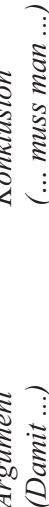

원

䗆

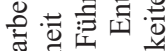

.

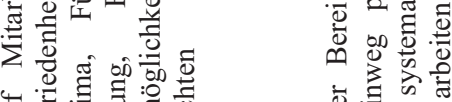

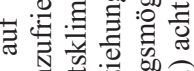

$\vdots$ :

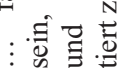

毒:

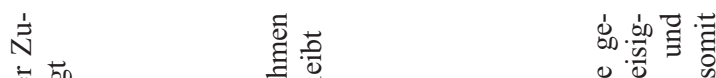

可.

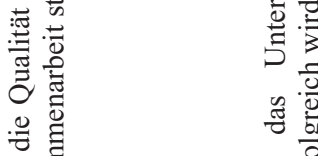

要. 


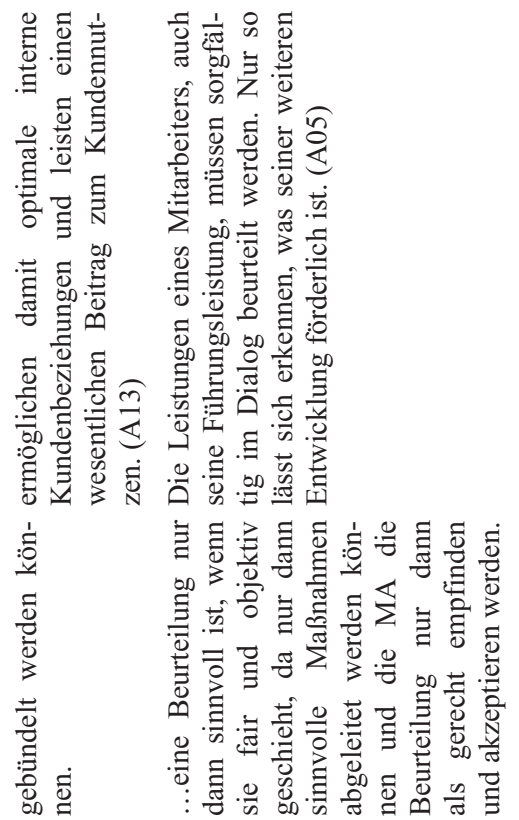

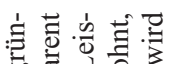

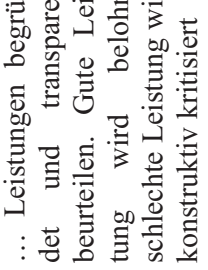

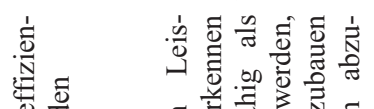

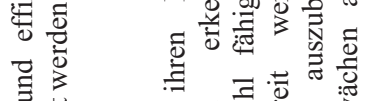

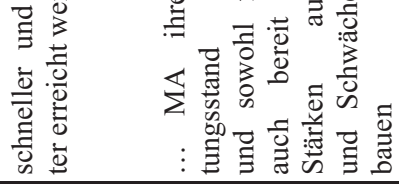

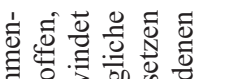

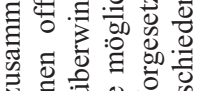

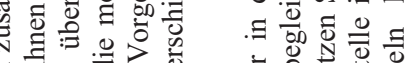

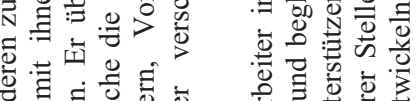

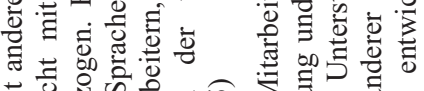

t5

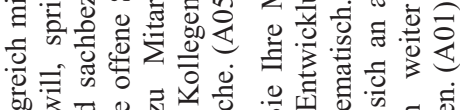

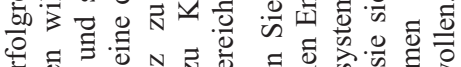

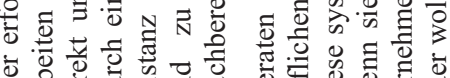

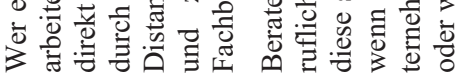

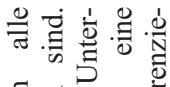

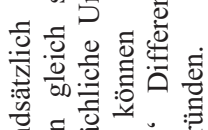

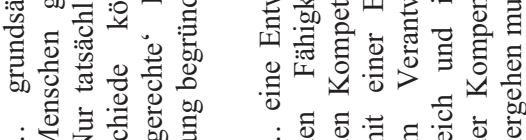

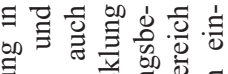

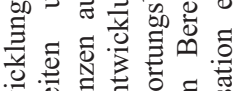

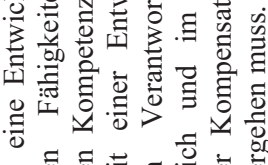

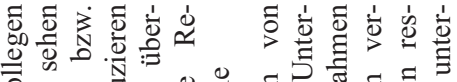

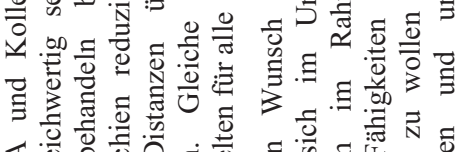

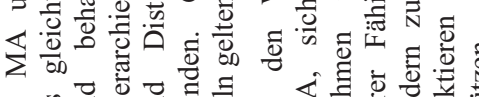

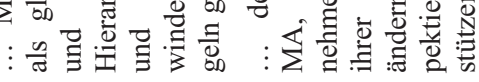

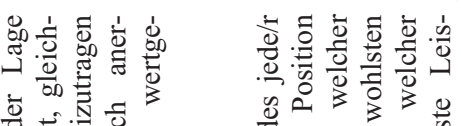

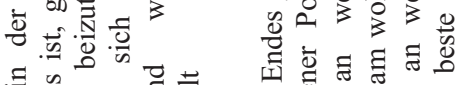

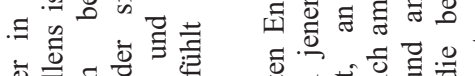

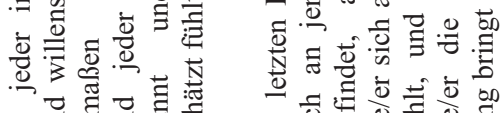

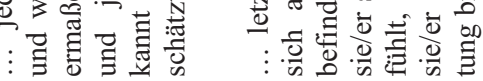




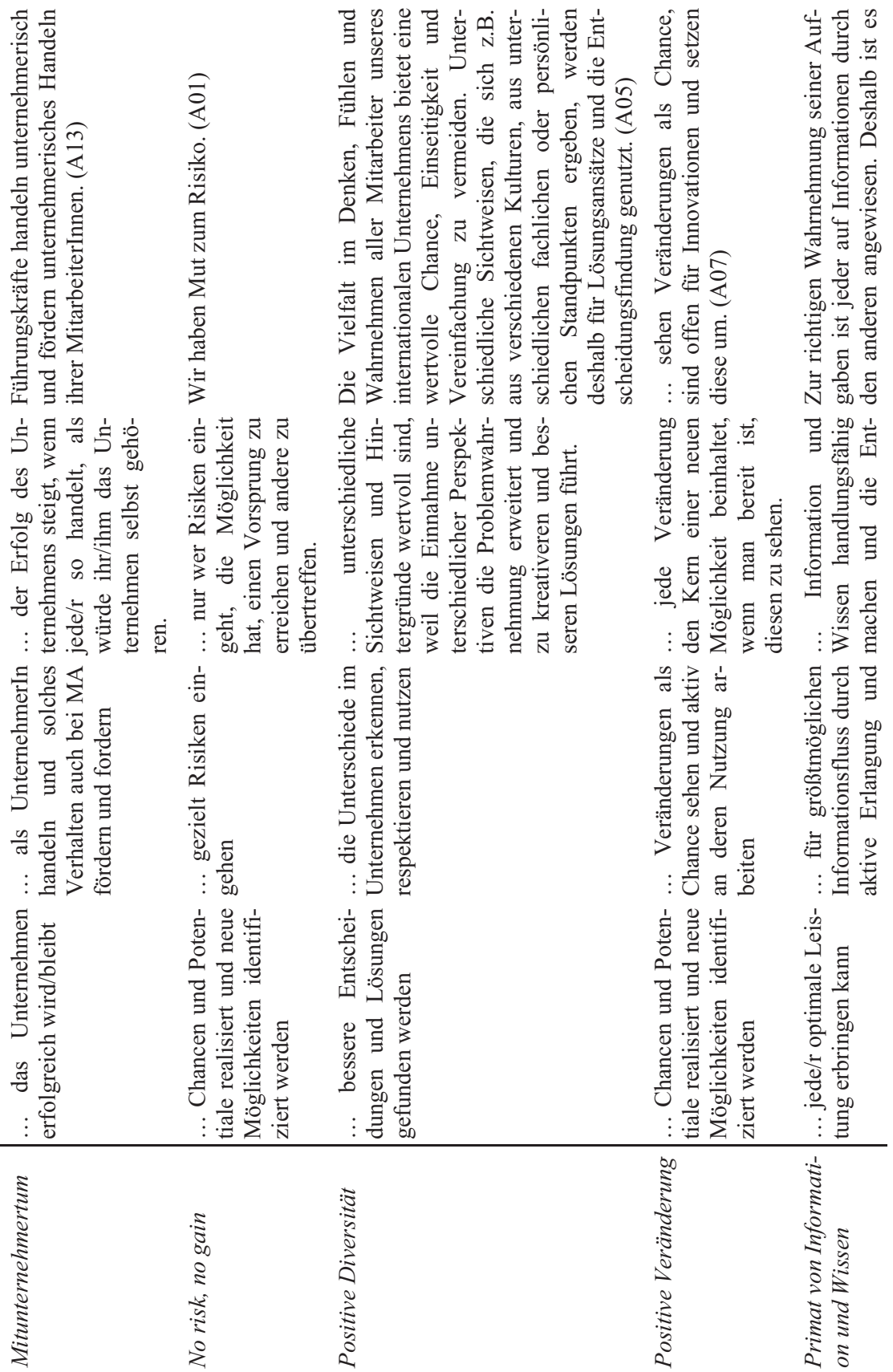



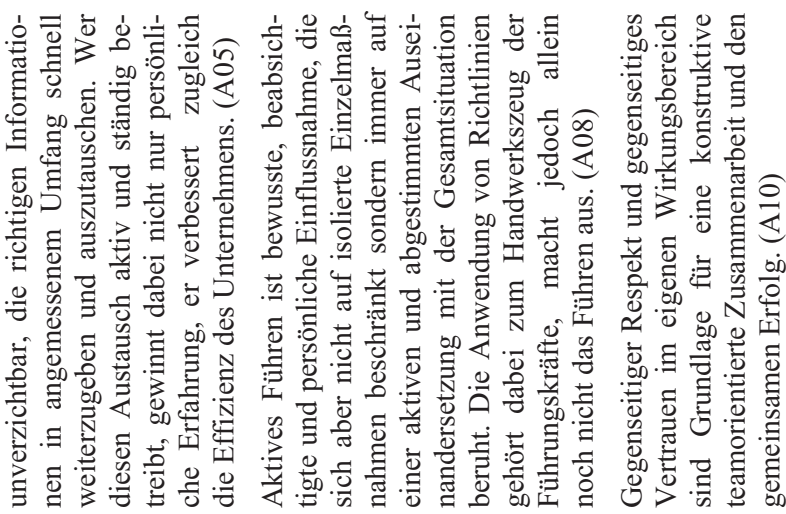

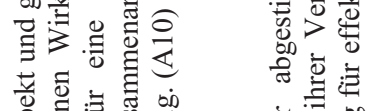
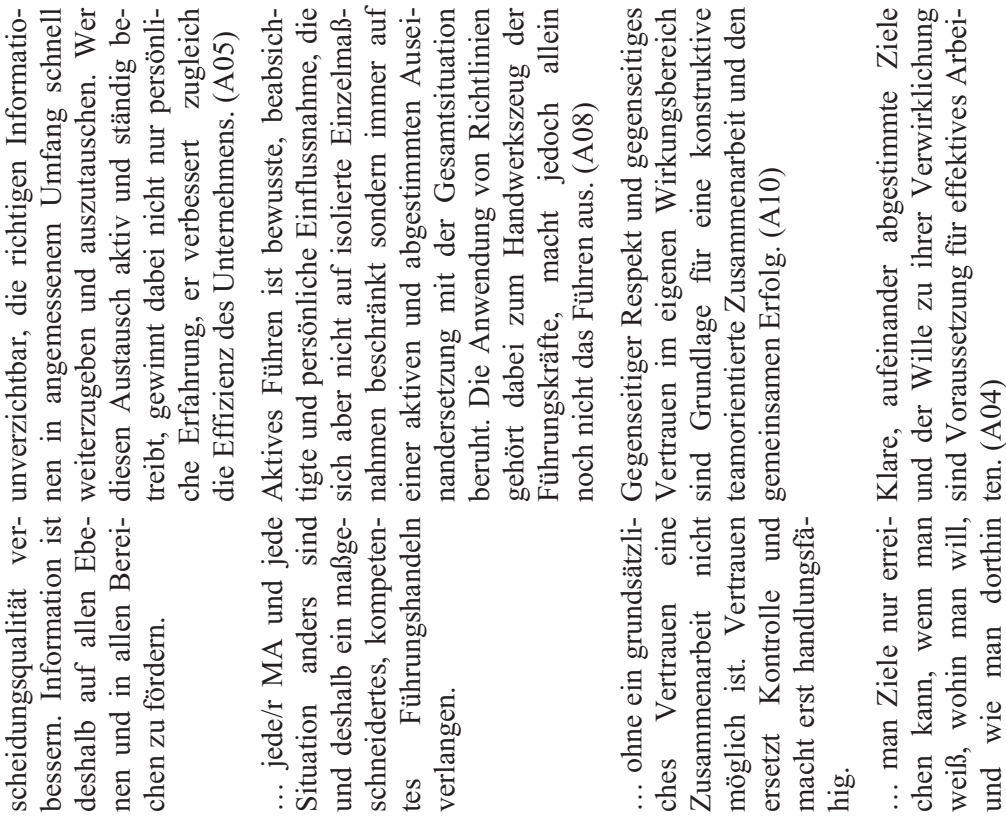

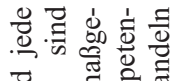

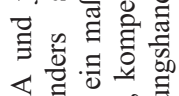

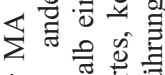

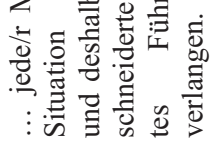

$\stackrel{1}{\Xi}$

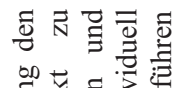

ธี ฮี

\&

密

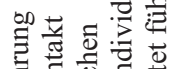

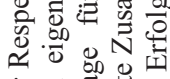

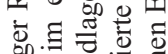

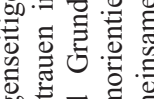

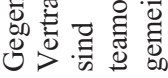

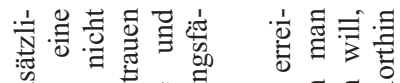

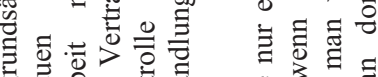

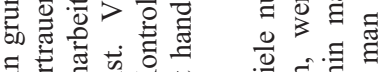

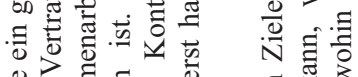

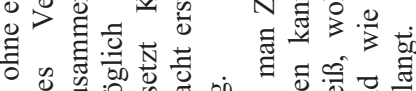

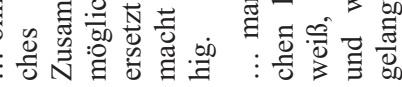

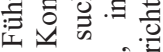

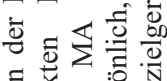

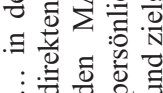

离:

๘ี

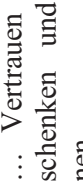

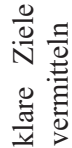

离

它竎.

至导:

害

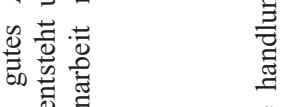

$\vdots \underline{n}$

쿼

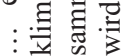

를

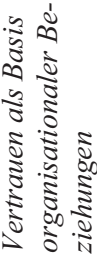

$\frac{\sqrt{\frac{\pi}{2}}}{\frac{\pi}{2}}$ 
Anhang

ฮี.

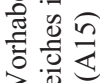

ㄷํㅇ

三荡

은

渮密

돈

音

$=\frac{0}{0}$

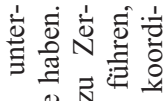

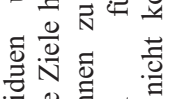

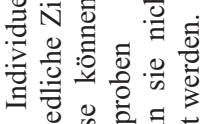

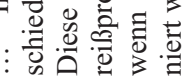

害宕总密.

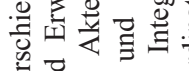

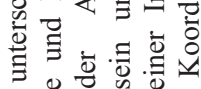

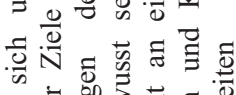

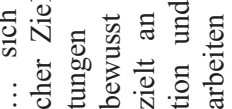

着实

$\frac{\pi}{2}=$

항

क्ष

竞.

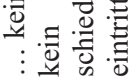

5
$\vdots$
$\vdots$
$\vdots$
$\vdots$
0
0
$\frac{1}{2}$

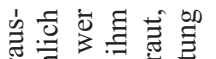

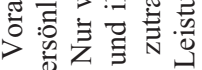

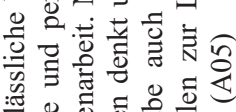

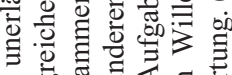

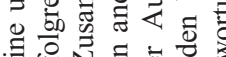

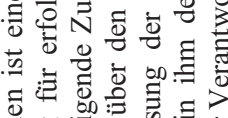

重

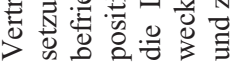

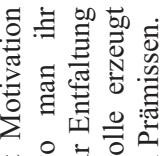

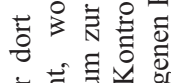

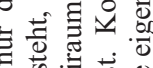

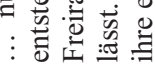

言站

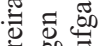

证:

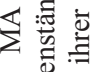

동. $\frac{0.000}{0}$

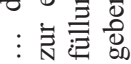

言崖兽

政:

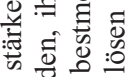

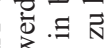

$\sum 3$

亭

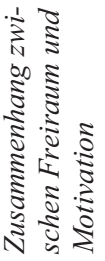

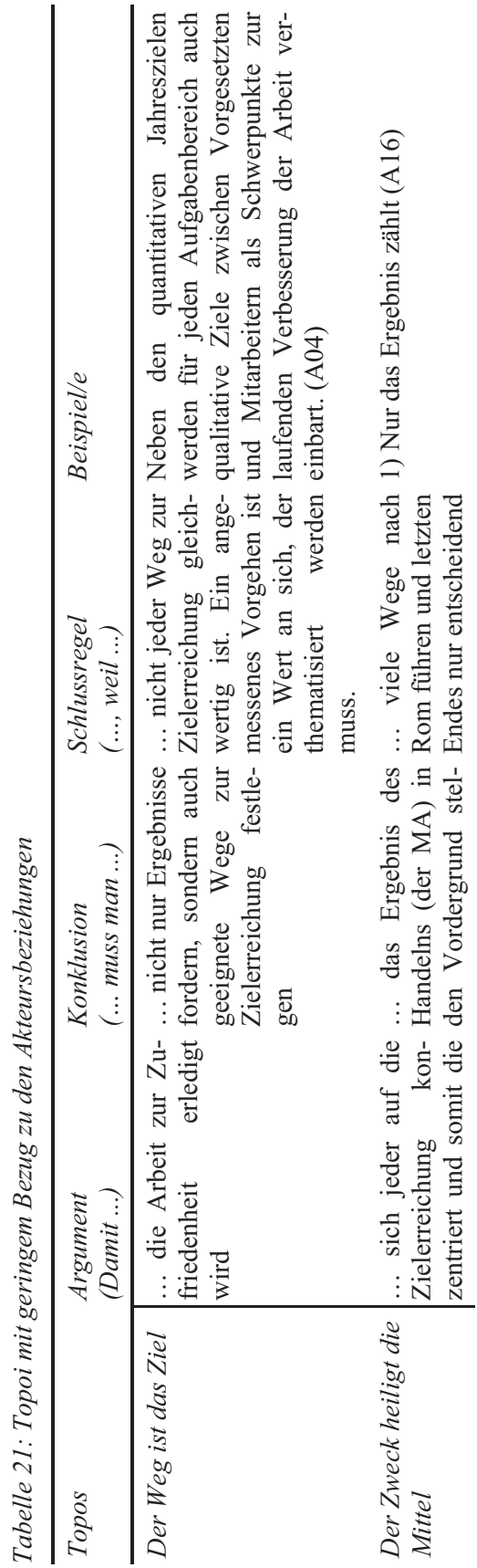



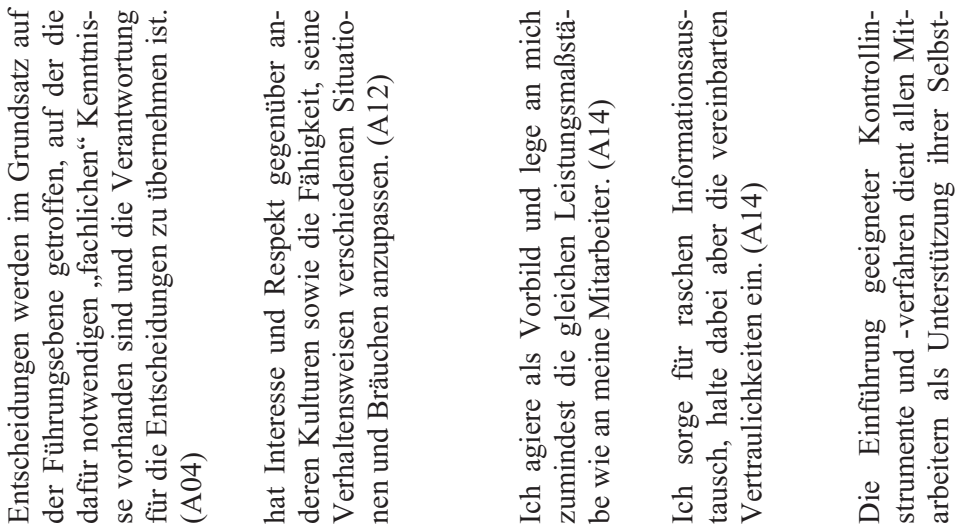

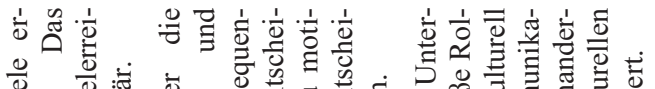

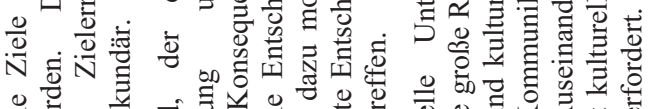

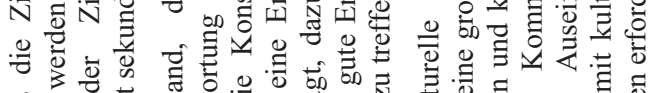

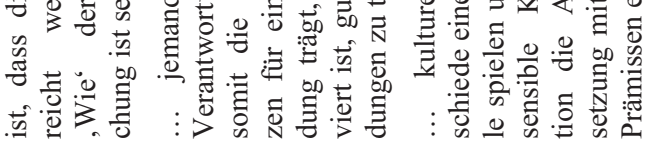

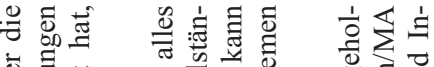

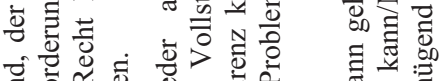

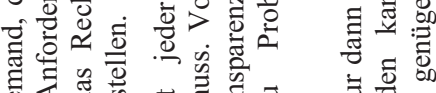

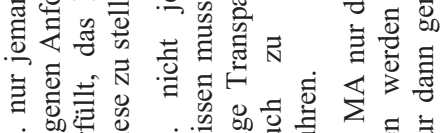

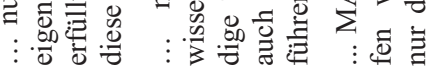

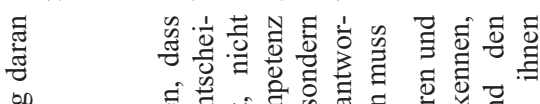

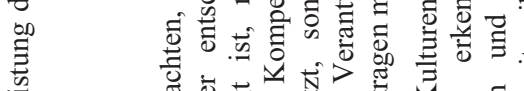
$\frac{\pi}{0}$

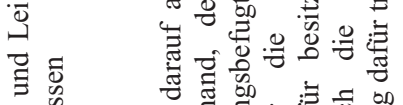

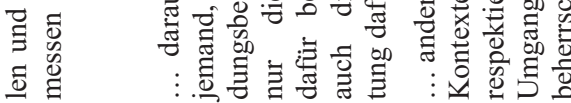

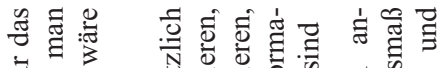

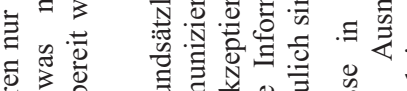

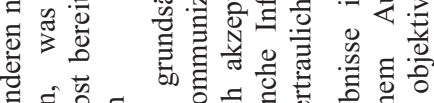

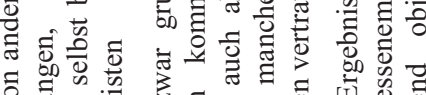

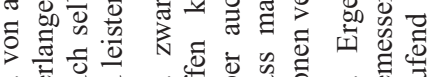

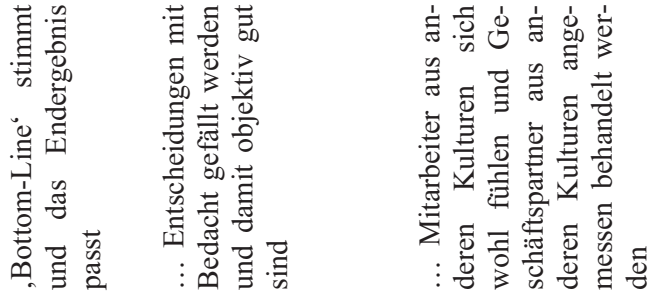

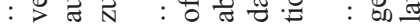

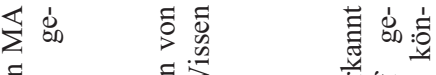

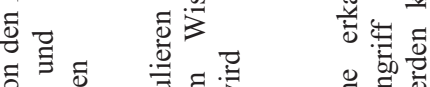

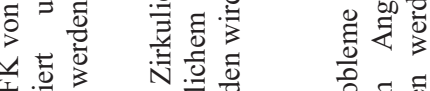

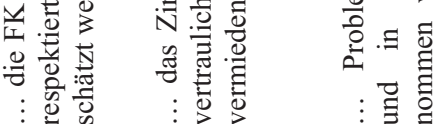

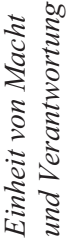

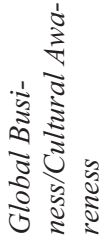

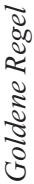

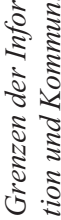

1




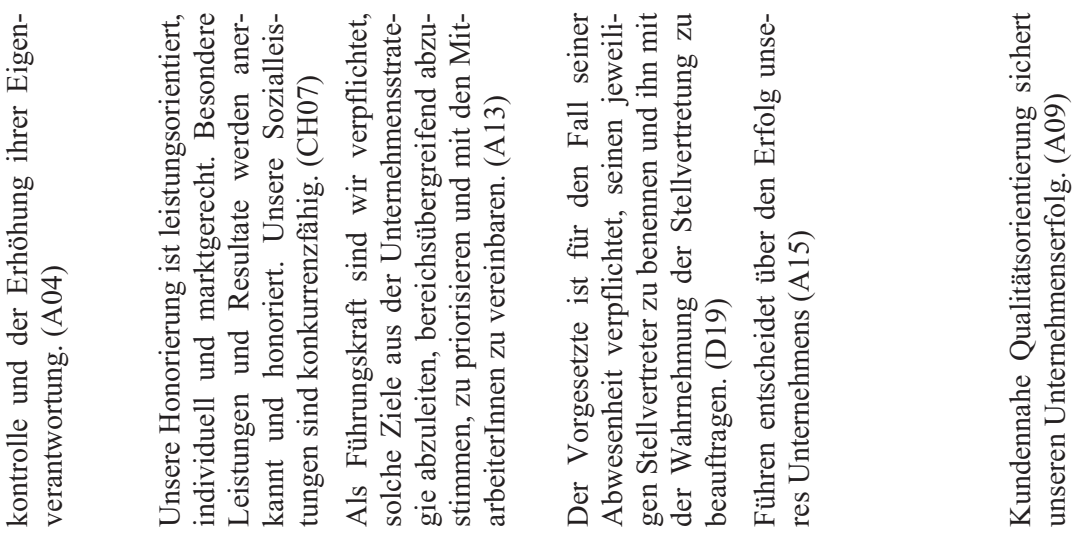

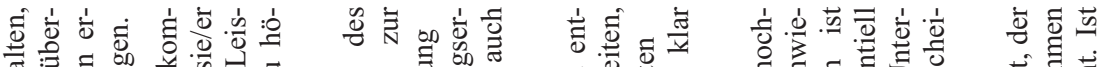

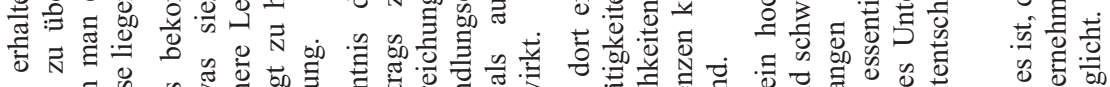

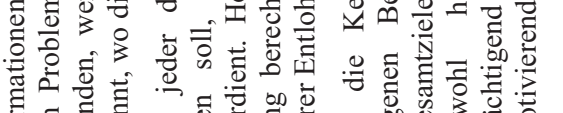

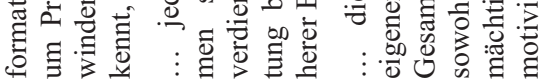

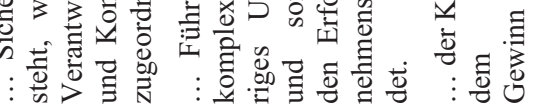

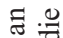

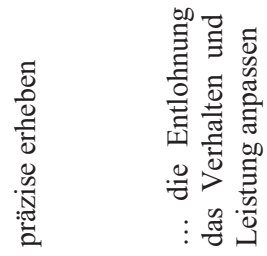

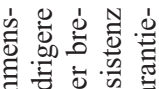

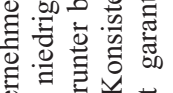

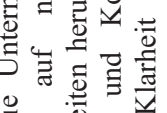

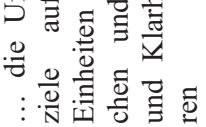

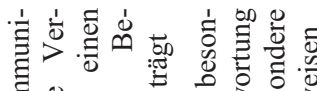

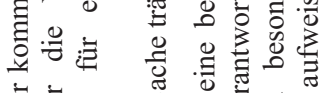

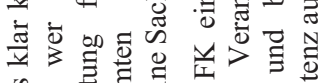

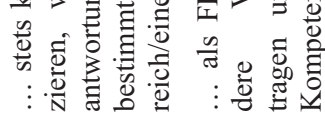

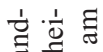
垔㻤 害总 要品 등 洍 咅要

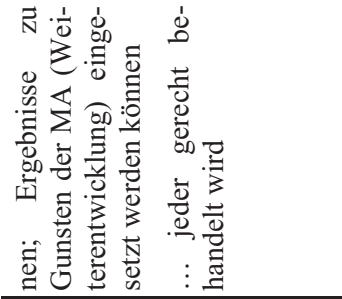

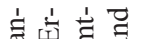

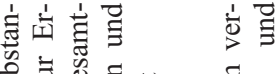

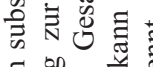

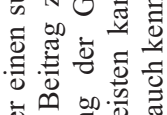

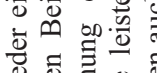

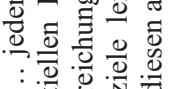

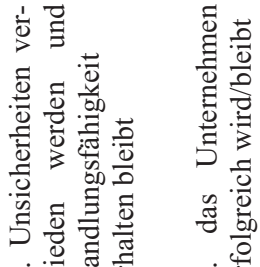
政 응 郭客

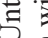

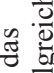

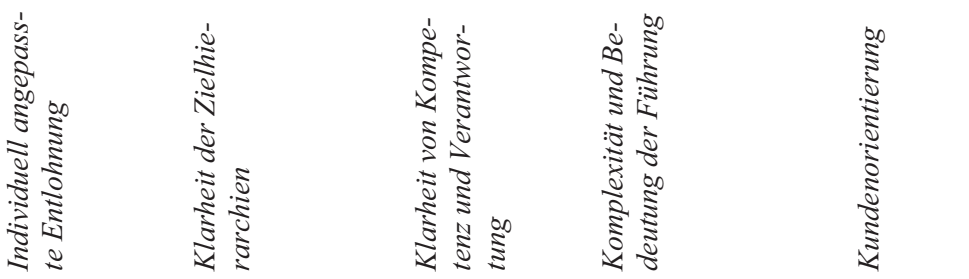




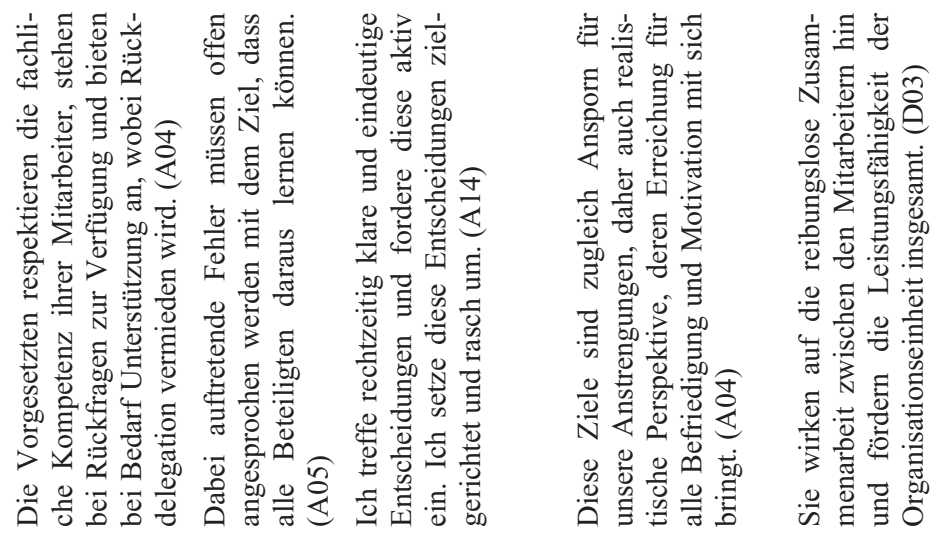

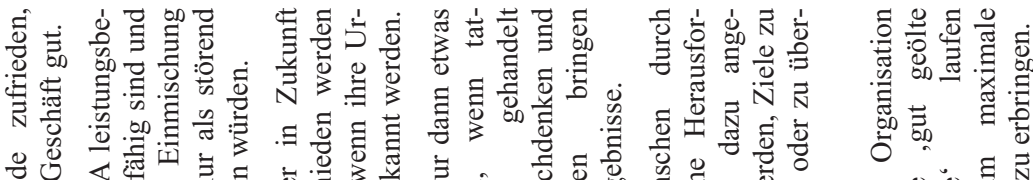

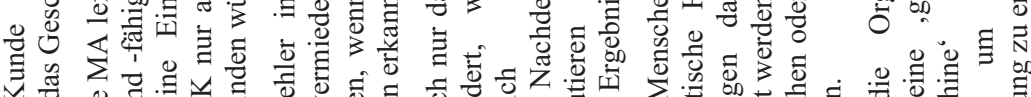

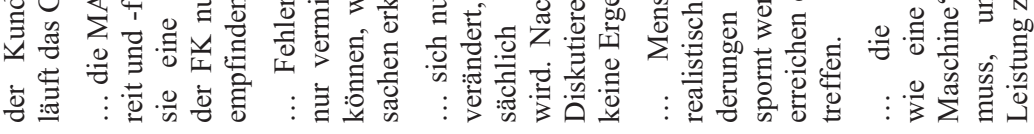

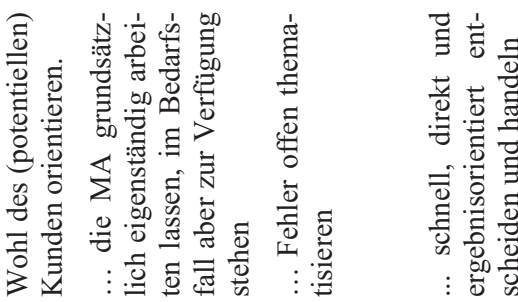

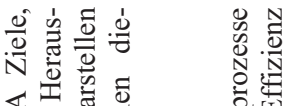

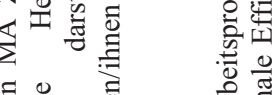

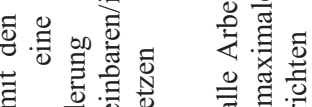

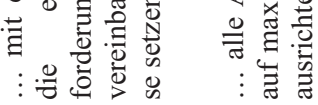
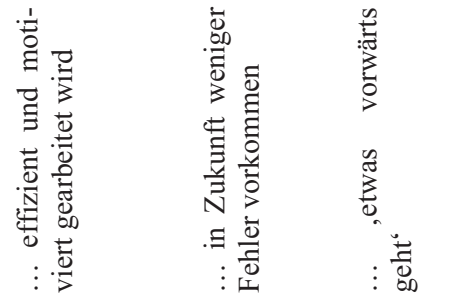

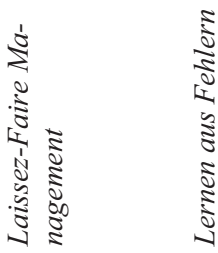

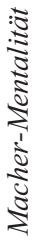

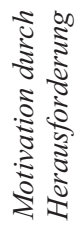

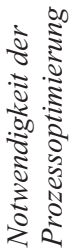


뫀 홈

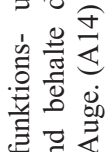

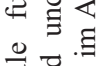

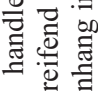

范紫

0 要

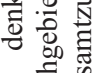

동 현웡

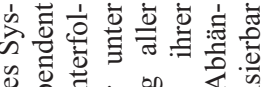

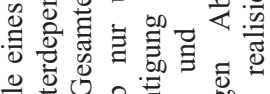

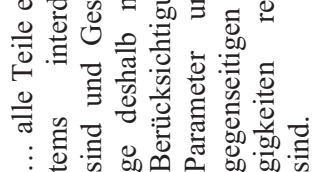

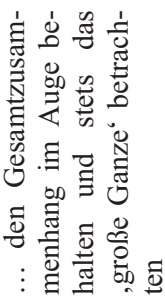

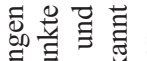

言言紊

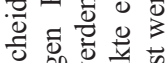

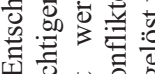

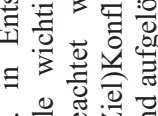

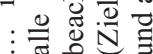

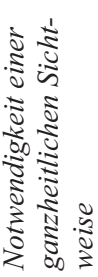

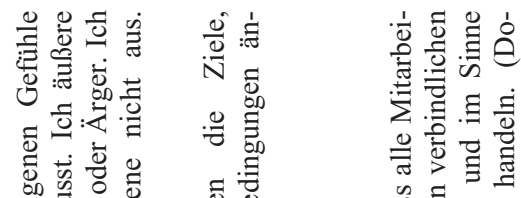

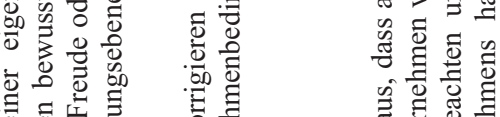

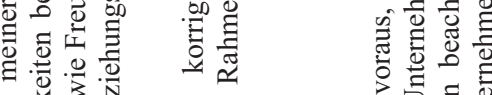

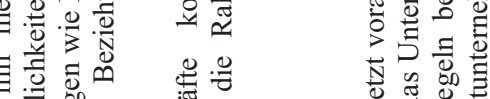

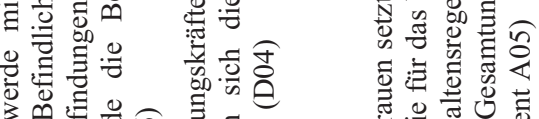

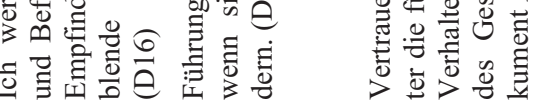

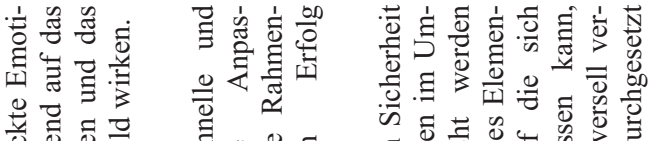

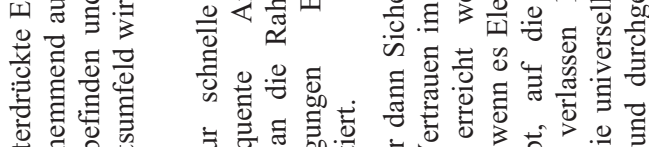

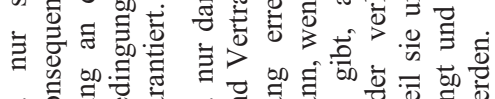

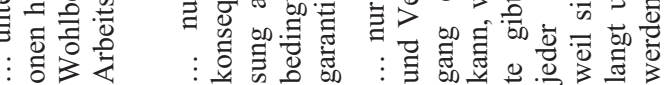

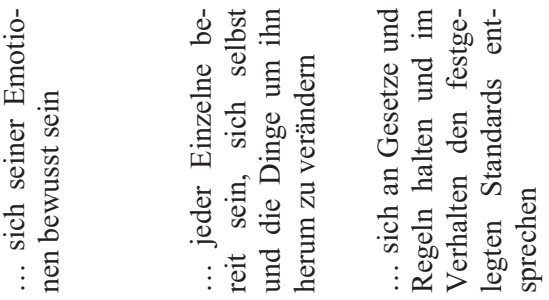

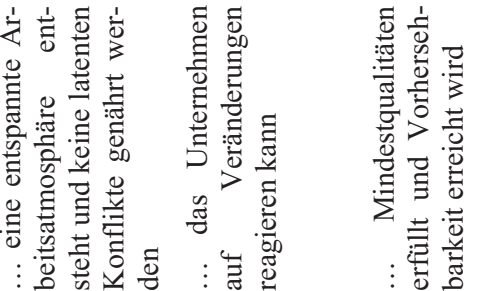

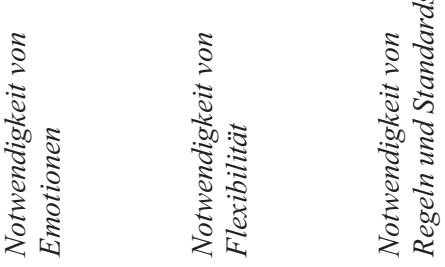




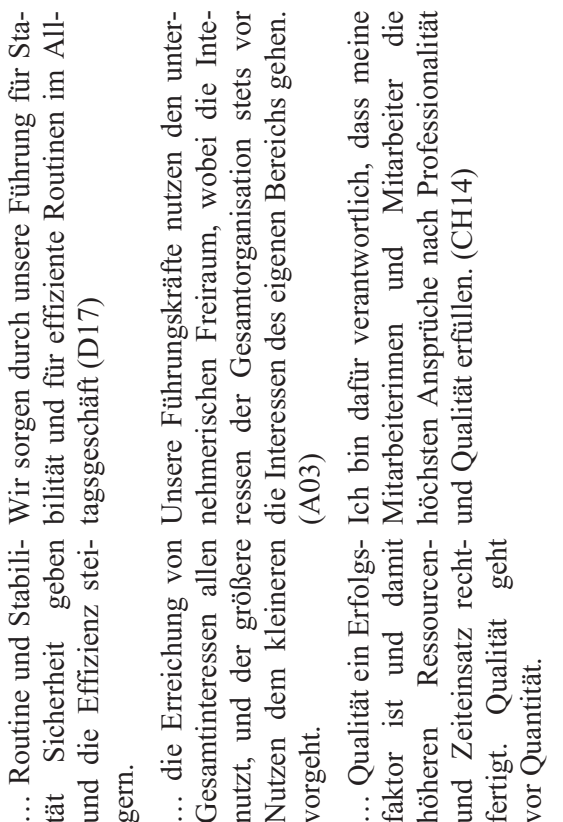

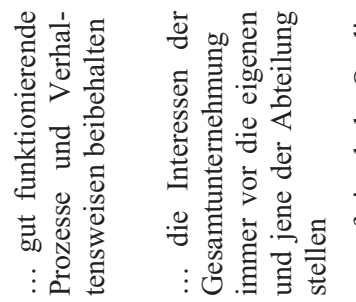

咅离节

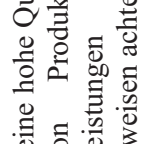

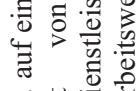

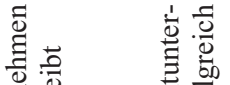

焉.
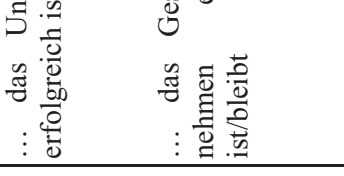

$\frac{2}{2}$

$\frac{2}{\pi}$

: :

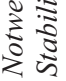

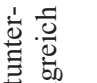

潬哭是

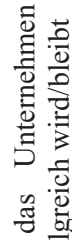

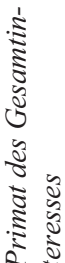

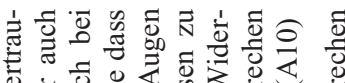

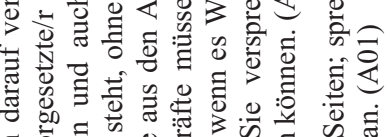

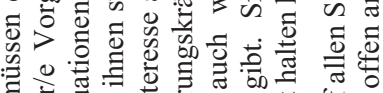

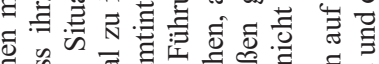

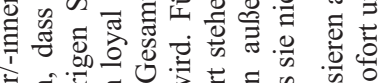

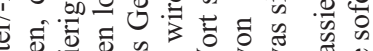

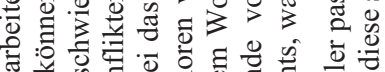

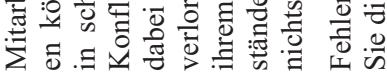

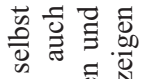

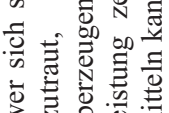

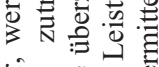

青

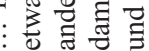

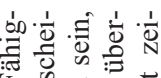

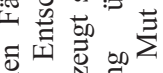

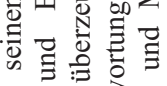

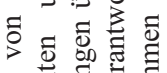

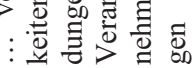

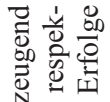

岂壱吉

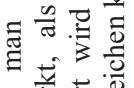

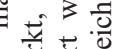

弯

言

$\frac{\bar{t}}{5}$

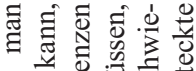
을

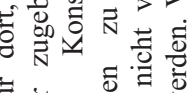

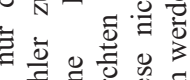

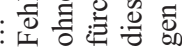
跑主言 호을 흐응 tक

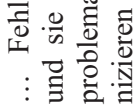
: 듬

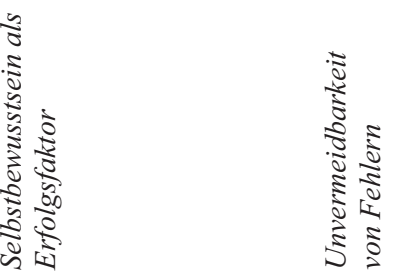



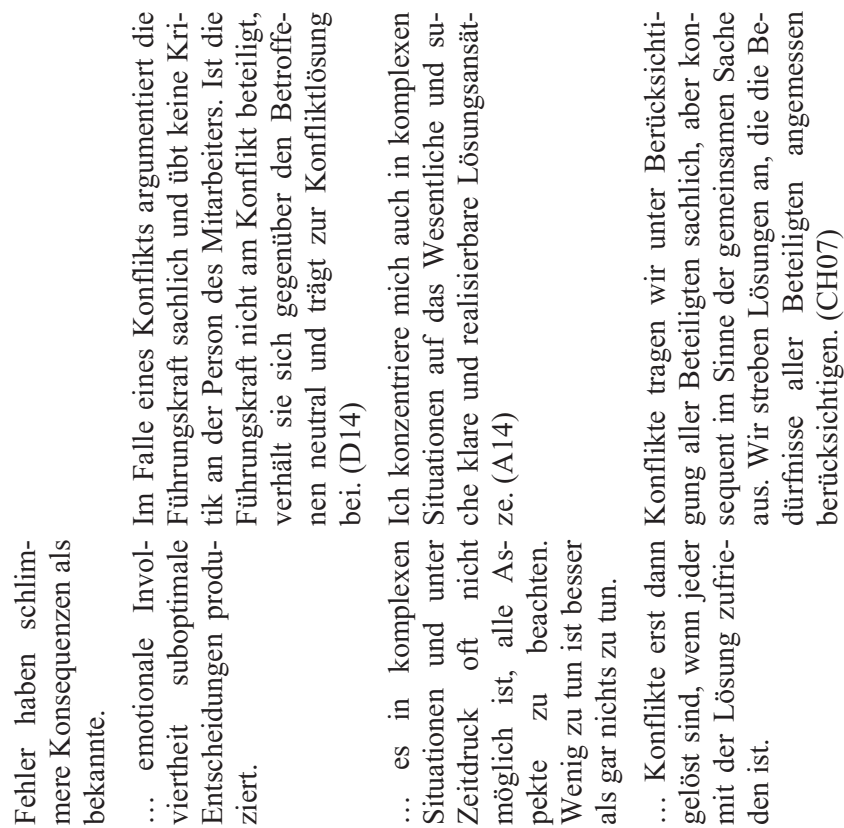

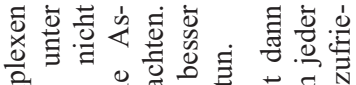

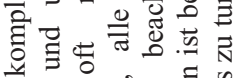

落 不

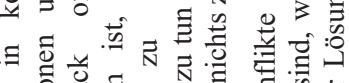

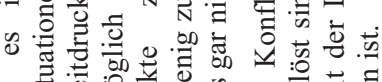

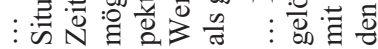

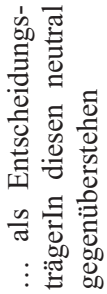

妾衰

害宫

品苛

政

: 형

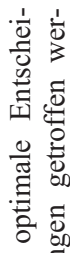

产

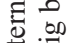

을

吅

들

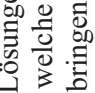

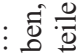

画亭

7

可这

贾

要

五

: ฮ్త

: 坖
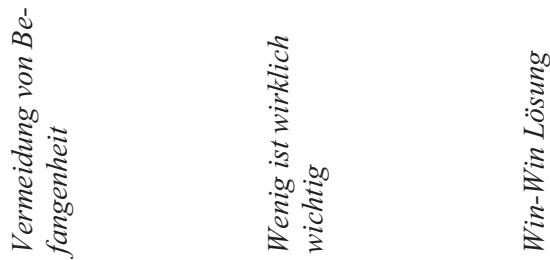


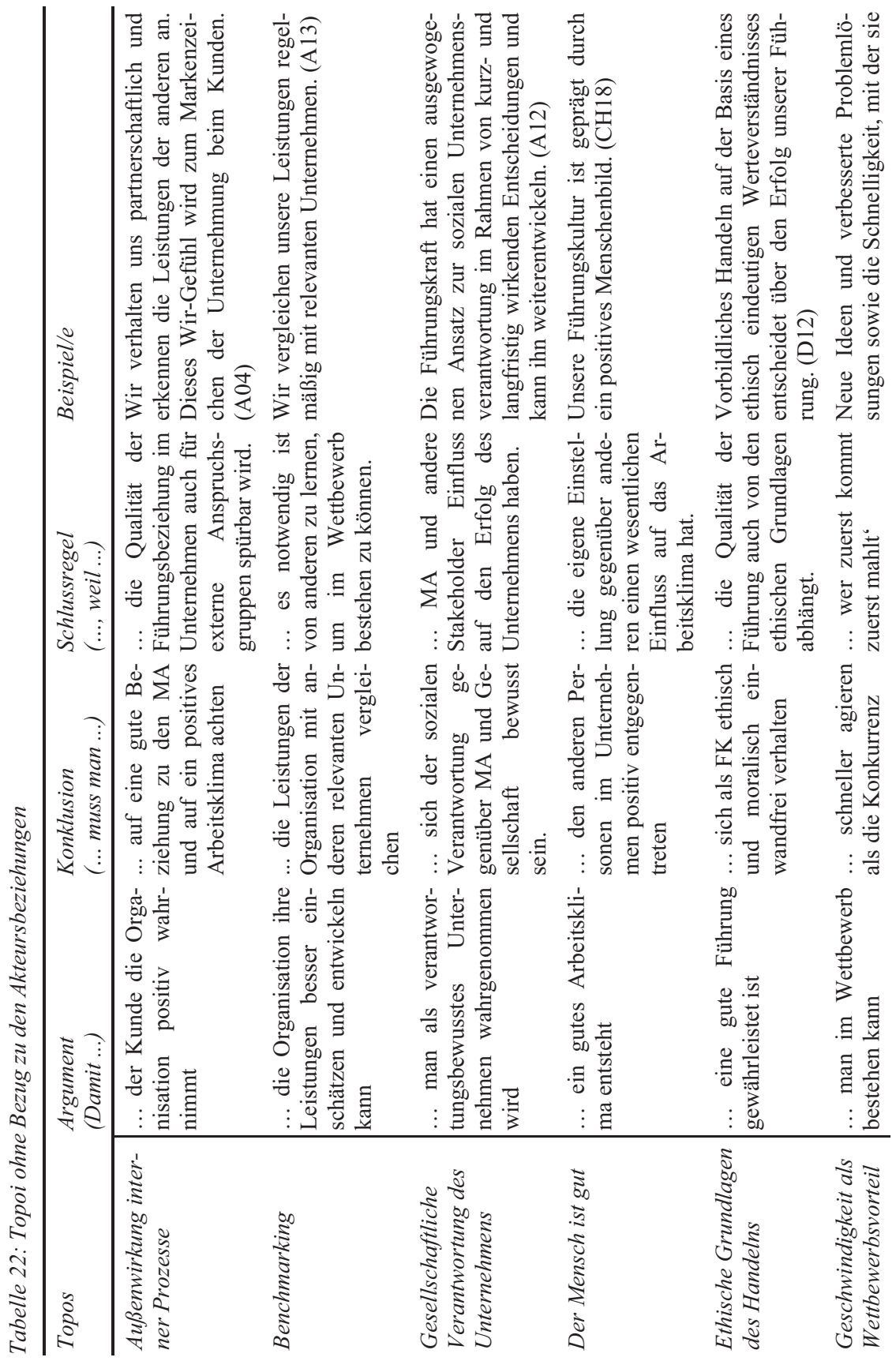




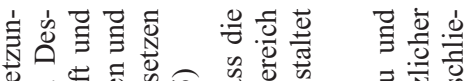

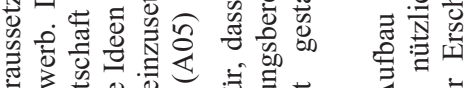

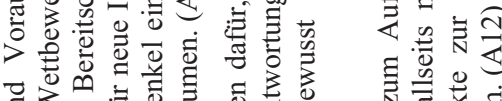

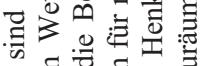
ㅁ.

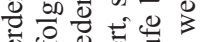

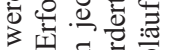

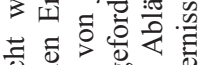
政

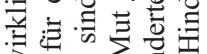

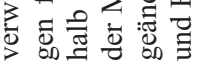

क

万) की on

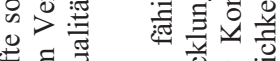

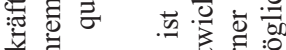

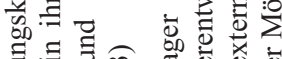

ㅋ.引

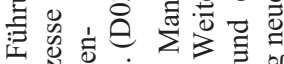

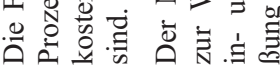

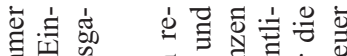

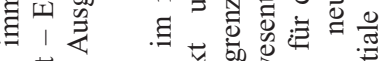

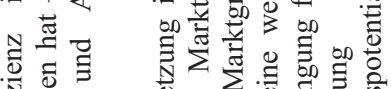

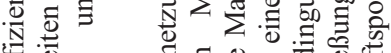

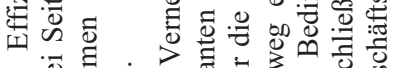

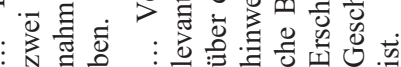

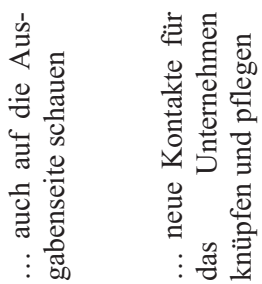

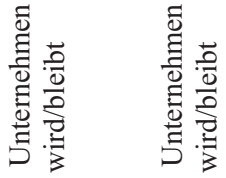

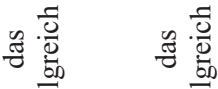

$\frac{20}{\frac{0}{2}}$

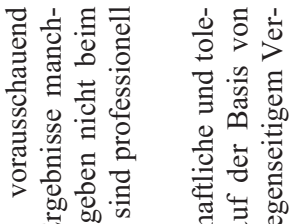

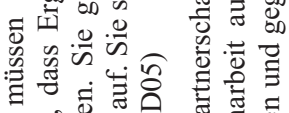

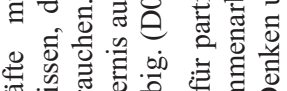

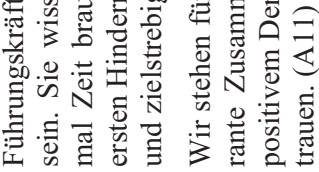

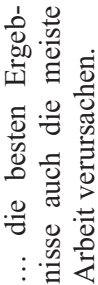

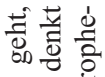

를

喜哭照

要灵

部蜜宗

音

을 항

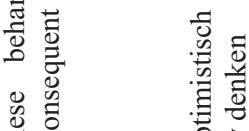

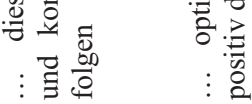

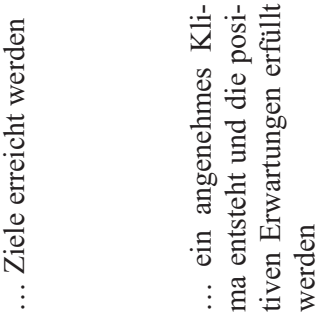

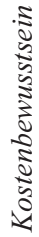

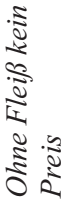

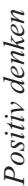




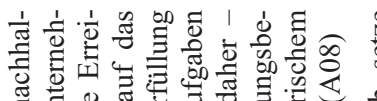

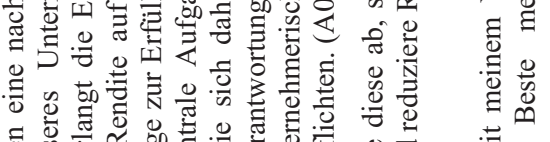

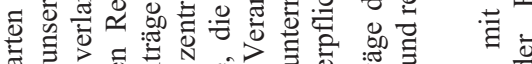

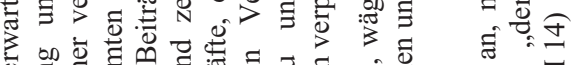

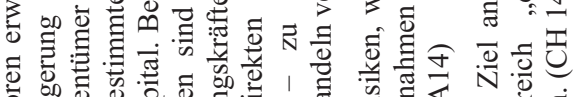

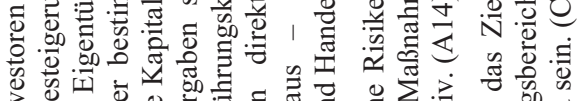

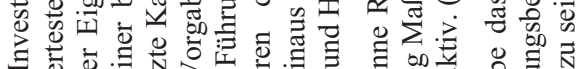

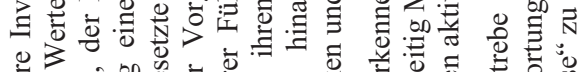

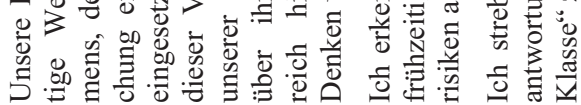

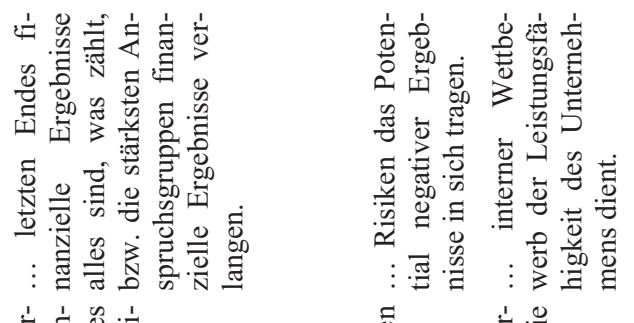

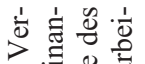

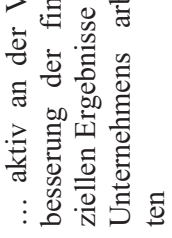

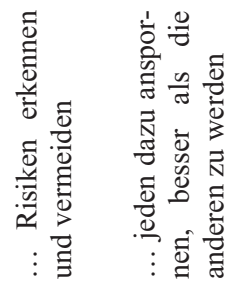

造

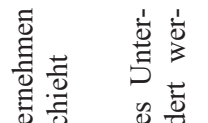

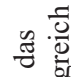

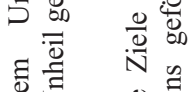
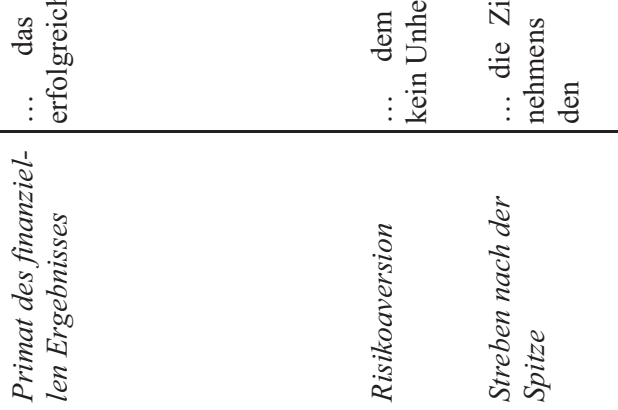


\title{
Forschungsergebnisse der WU Wirtschaftsuniversität Wien
}

\author{
Herausgeber: WU Wirtschaftsuniversität Wien - \\ vertreten durch Univ. Prof. Dr. Barbara Sporn \\ INFORMATION UND KONTAKT: \\ WU Wirtschaftsuniversität Wien \\ Department of Finance, Accounting and Statistics \\ Institute for Finance, Banking and Insurance \\ Heiligenstädter Straße 46-48, 1190 Wien \\ Tel.: 0043-1-313 36/4556 \\ Fax: 0043-1-313 36/904556 \\ valentine.wendling@wu.ac.at \\ www.wu.ac.at/finance
}

Band 1 Stefan Felder: Frequenzallokation in der Telekommunikation. Ökonomische Analyse der Vergabe von Frequenzen unter besonderer Berücksichtigung der UMTS-Auktionen. 2004.

Band 2 Thomas Haller: Marketing im liberalisierten Strommarkt. Kommunikation und Produktplanung im Privatkundenmarkt. 2005.

Band 3 Alexander Stremitzer: Agency Theory: Methodology, Analysis. A Structured Approach to Writing Contracts. 2005.

Band 4 Günther Sedlacek: Analyse der Studiendauer und des Studienabbruch-Risikos. Unter Verwendung der statistischen Methoden der Ereignisanalyse. 2004.

Band 5 Monika Knassmüller: Unternehmensleitbilder im Vergleich. Sinn- und Bedeutungsrahmen deutschsprachiger Unternehmensleitbilder - Versuch einer empirischen (Re-)Konstruktion. 2005.

Band 6 Matthias Fink: Erfolgsfaktor Selbstverpflichtung bei vertrauensbasierten Kooperationen. Mit einem empirischen Befund. 2005.

Band 7 Michael Gerhard Kraft: Ökonomie zwischen Wissenschaft und Ethik. Eine dogmenhistorische Untersuchung von Léon M.E. Walras bis Milton Friedman. 2005.

Band 8 Ingrid Zechmeister: Mental Health Care Financing in the Process of Change. Challenges and Approaches for Austria. 2005.

Band 9 Sarah Meisenberger: Strukturierte Organisationen und Wissen. 2005.

Band 10 Anne-Katrin Neyer: Multinational teams in the European Commission and the European Parliament. 2005.

Band 11 Birgit Trukeschitz: Im Dienst Sozialer Dienste. Ökonomische Analyse der Beschäftigung in sozialen Dienstleistungseinrichtungen des Nonprofit Sektors. 2006

Band 12 Marcus Kölling: Interkulturelles Wissensmanagement. Deutschland Ost und West. 2006.

Band 13 Ulrich Berger: The Economics of Two-way Interconnection. 2006.

Band 14 Susanne Guth: Interoperability of DRM Systems. Exchanging and Processing XML-based Rights Expressions. 2006.

Band 15 Bernhard Klement: Ökonomische Kriterien und Anreizmechanismen für eine effiziente Förderung von industrieller Forschung und Innovation. Mit einer empirischen Quantifizierung der Hebeleffekte von F\&E-Förderinstrumenten in Österreich. 2006. 
Band 16 Markus Imgrund: Wege aus der Insolvenz. Eine Analyse der Fortführung und Sanierung insolventer Klein- und Mittelbetriebe unter besonderer Berücksichtigung des Konfigurationsansatzes. 2007.

Band 17 Nicolas Knotzer: Product Recommendations in E-Commerce Retailing Applications. 2008.

Band 18 Astrid Dickinger: Perceived Quality of Mobile Services. A Segment-Specific Analysis. 2007.

Band 19 Nadine Wiedermann-Ondrej: Hybride Finanzierungsinstrumente in der nationalen und internationalen Besteuerung der USA. 2008.

Band 20 Helmut Sorger: Entscheidungsorientiertes Risikomanagement in der Industrieunternehmung. 2008.

Band 21 Martin Rietsch: Messung und Analyse des ökonomischen Wechselkursrisikos aus Unternehmenssicht: Ein stochastischer Simulationsansatz. 2008.

Band 22 Hans Christian Mantler: Makroökonomische Effizienz des Finanzsektors. Herleitung eines theoretischen Modells und Schätzung der Wachstumsimplikationen für die Marktwirtschaften und Transformationsökonomien Europas. 2008.

Band 23 Youri Tacoun: La théorie de la valeur de Christian von Ehrenfels. 2008.

Band 24 Monika Koller: Longitudinale Betrachtung der Kognitiven Dissonanz. Eine Tagebuchstudie zur Reiseentscheidung. 2008.

Band 25 Marcus Scheiblecker: The Austrian Business Cycle in the European Context. 2008.

Band 26 Aida Numic: Multinational Teams in European and American Companies. 2008.

Band 27 Ulrike Bauernfeind: User Satisfaction with Personalised Internet Applications. 2008.

Band 28 Reinhold Schodl: Systematische Analyse und Bewertung komplexer Supply Chain Prozesse bei dynamischer Festlegung des Auftragsentkopplungspunkts. 2008.

Band 29 Bianca Gusenbauer: Öffentlich-private Finanzierung von Infrastruktur in Entwicklungsländern und deren Beitrag zur Armutsreduktion. Fallstudien in Vietnam und auf den Philippinen. 2009.

Band 30 Elisabeth Salomon: Hybrides Management in sino-österreichischen Joint Ventures in China aus österreichischer Perspektive. 2009.

Band 31 Katharina Mader: Gender Budgeting: Ein emanzipatorisches, finanzpolitisches und demokratiepolitisches Instrument. 2009.

Band 32 Michael Weber: Die Generierung von Empfehlungen für zwischenbetriebliche Transaktionen als gesamtwirtschaftliche Infrastrukturleistung. 2010.

Band 33 Lisa Gimpl-Heersink: Joint Pricing and Inventory Control under Reference Price Effects. 2009.

Band 34 Erscheint nicht.

Band 35 Dagmar Kiefer: Multicultural Work in Five United Nations Organisations. An Austrian Perspective. 2009.

Band 36 Gottfried Gruber: Multichannel Management. A Normative Model Towards Optimality. 2009.

Band 37 Rainer Quante: Management of Stochastic Demand in Make-to-Stock Manufacturing. 2009.

Band 38 Franz F. Eiffe: Auf den Spuren von Amartya Sen. Zur theoriegeschichtlichen Genese des Capability-Ansatzes und seinem Beitrag zur Armutsanalyse in der EU. 2010. 
Band 39 Astrid Haider: Die Lohnhöhe und Lohnstreuung im Nonprofit-Sektor. Eine quantitative Analyse anhand österreichischer Arbeitnehmer-Arbeitgeber-Daten. 2010.

Band 40 Maureen Lenhart: Pflegekräftemigration nach Österreich. Eine empirische Analyse. 2010.

Band 41 Oliver Schwank: Linkages in South African Economic Development. Industrialisation without Diversification? 2010.

Band 42 Judith Kast-Aigner: A Corpus-Based Analysis of the Terminology of the European Union's Development Cooperation Policy, with the African, Caribbean and Pacific Group of States. 2010.

Band 43 Emel Arikan: Single Period Inventory Control and Pricing. An Empirical and Analytical Study of a Generalized Model. 2011.

Band 44 Gerhard Wohlgenannt: Learning Ontology Relations by Combining Corpus-Based Techniques and Reasoning on Data from Semantic Web Sources. 2011.

Band 45 Thomas Peschta: Der Einfluss von Kundenzufriedenheit auf die Kundenloyalität und die Wirkung der Wettbewerbsintensität am Beispiel der Gemeinschaftsverpflegungsgastronomie. 2011.

Band 46 Friederike Hehle: Die Anwendung des Convenience-Konzepts auf den Betriebstyp Vending. 2011.

Band 47 Thomas Herzog: Strategisches Management von Koopetition. Eine empirisch begründete Theorie im industriellen Kontext der zivilen Luftfahrt. 2011.

Band 48 Christian Weismayer: Statische und longitudinale Zufriedenheitsmessung. 2011.

Band 49 Johannes Fichtinger: The Single-Period Inventory Model with Spectral Risk Measures. 2011.

Band 50 Isabella R. Hatak: Kompetenz, Vertrauen und Kooperation. Eine experimentelle Studie. 2011.

Band 51 Birgit Gusenbauer: Der Beitrag der Prospect Theory zur Beschreibung und Erklärung von Servicequalitätsurteilen und Kundenzufriedenheit im Kontext von Versicherungsentscheidungen. 2012.

Band 52 Markus A. Höllerer: Between Creed, Rhetoric Façade, and Disregard. Dissemination and Theorization of Corporate Social Responsibility in Austria. 2012.

Band 53 Jakob Müllner: Die Wirkung von Private Equity auf das Wachstum und die Internationalisierung. Eine empirische Impact-Studie des österreichischen Private Equity Marktes. 2012.

Band 54 Heidrun Rosič: The Economic and Environmental Sustainability of Dual Sourcing. 2012.

Band 55 Christian Geier: Wechselkurssicherungsstrategien exportorientierter Unternehmen. Effizienzmessung von regelgebundenen Selektionsentscheidungen. 2012.

Band 56 Ernst Gittenberger: Betriebsformenwahl älterer KonsumentInnen. 2012.

Band 57 Michael Pichlmair: Miete, Lage, Preisdiktat. Strukturelle Effekte der Lageregulierung im mietrechtlich geschützten Wiener Wohnmarkt. 2012.

Band 58 Anna Katherina Guserl: Internationalisierungsprozesse und Finanzstrategien. Ansätze und empirische Analysen. 2013.

Band 59 Christian Idinger: Konsumentenpreiswissen. Eine empirische Studie im österreichischen Lebensmitteleinzelhandel. 2013.

Band 60 Dennis Jancsary: Die rhetorische Konstruktion von Führung und Steuerung. Eine argumentationsanalytische Untersuchung deutschsprachiger Führungsgrundsätze. 2013.

www.peterlang.de 